
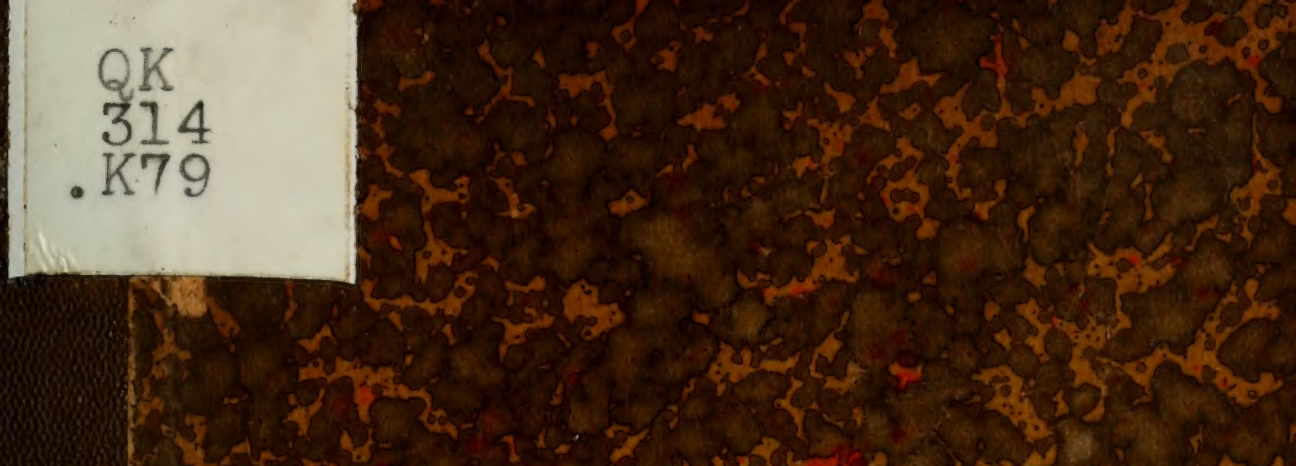

tom 19

- ais 1

전슨

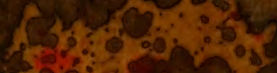

1 (1)

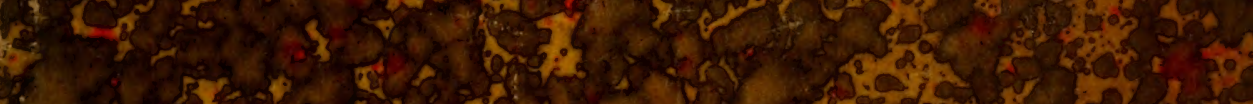

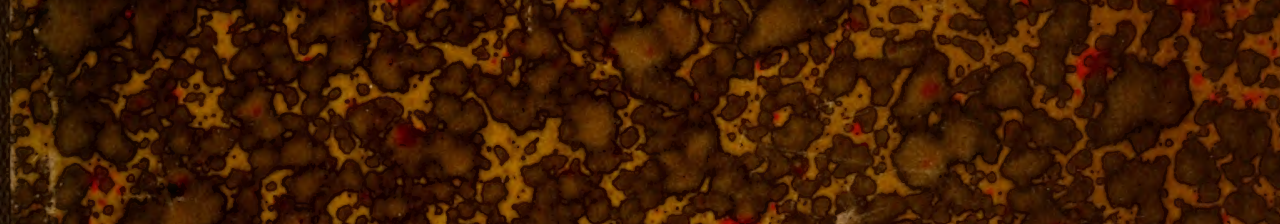

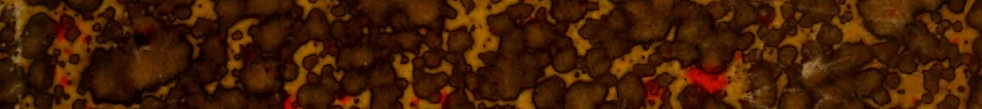

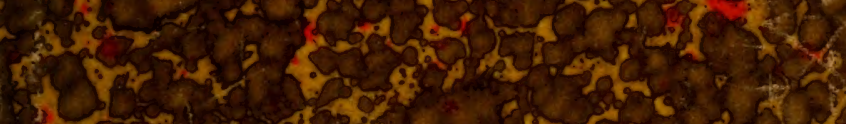

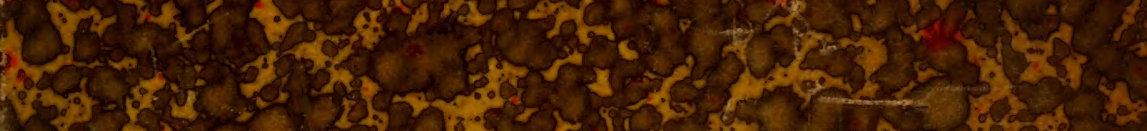

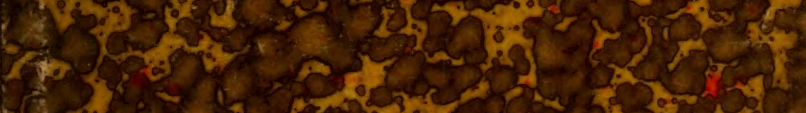
3 T.

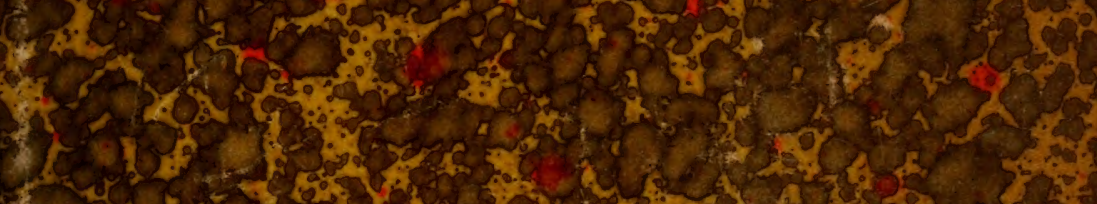

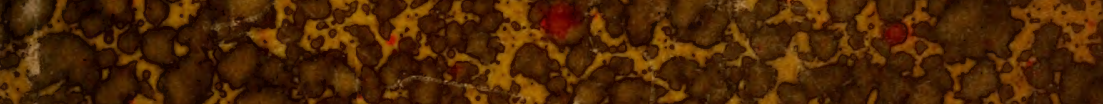
3 in 3 .

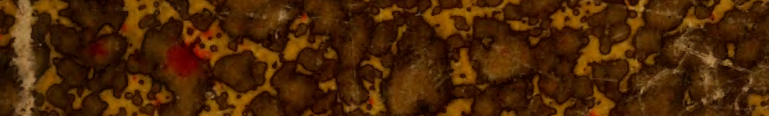

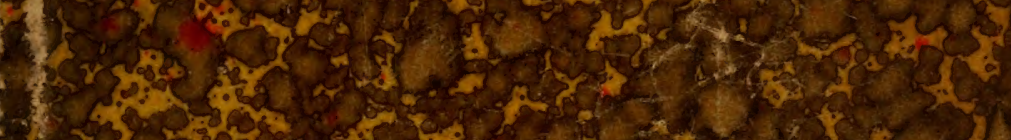

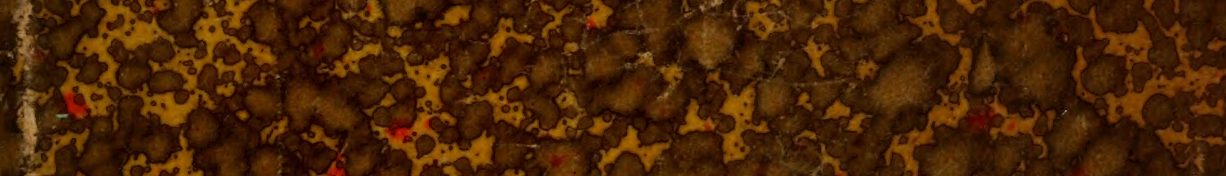
4 is 3 s.

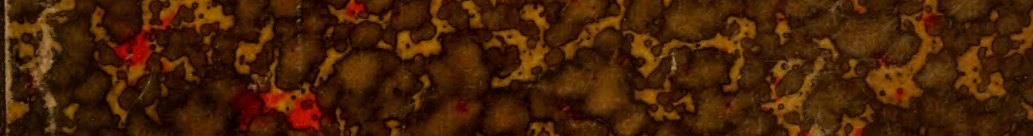

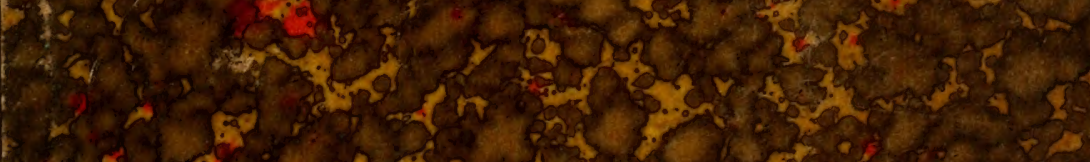

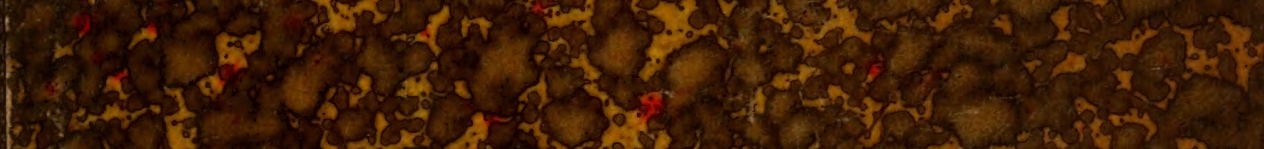
in: ${ }^{2}$ :

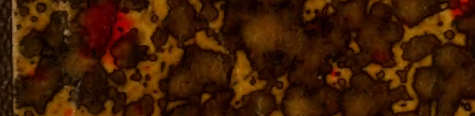
3. 


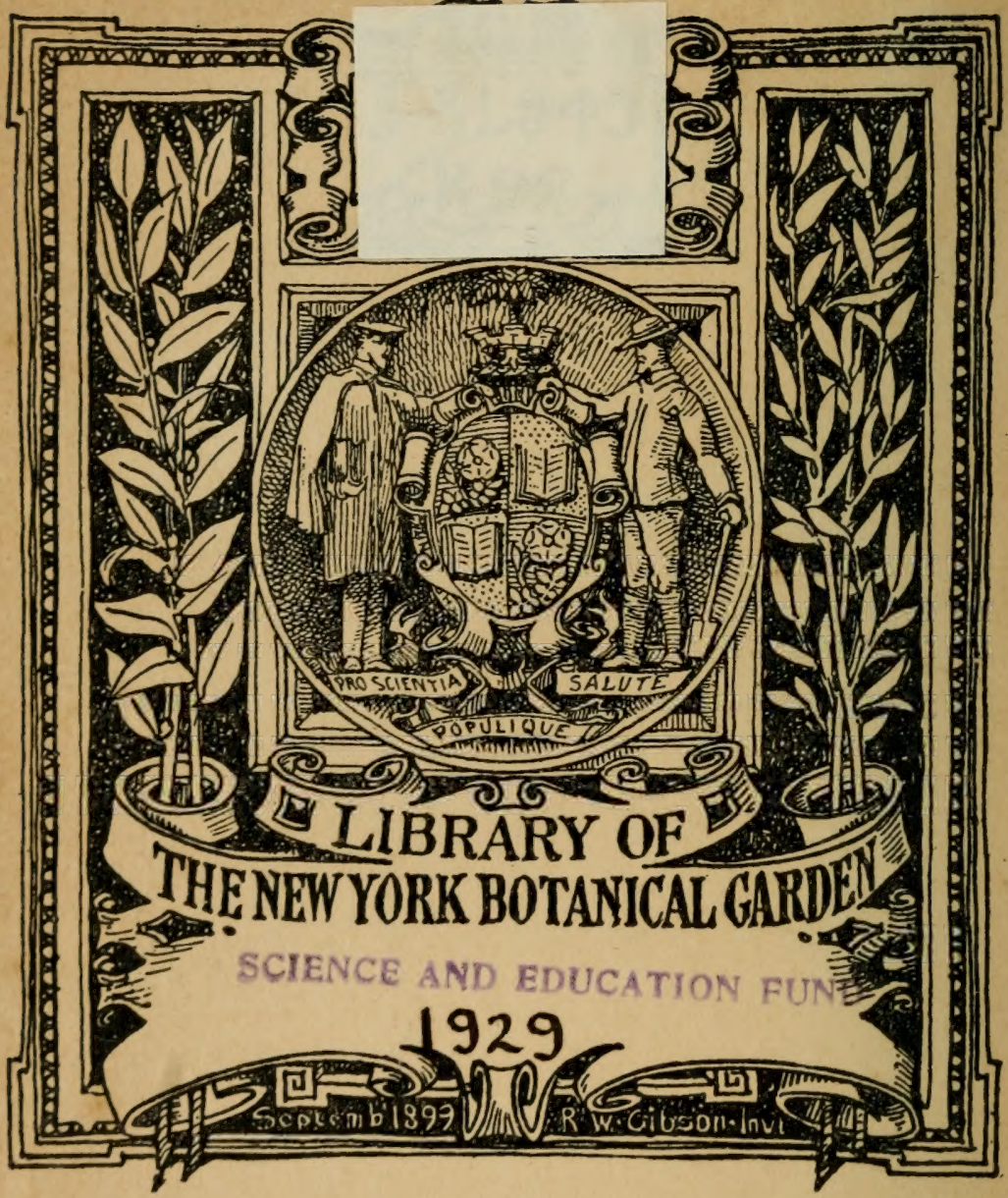






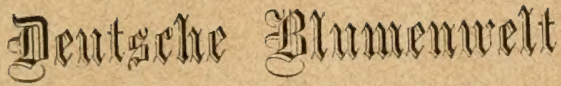

ix 



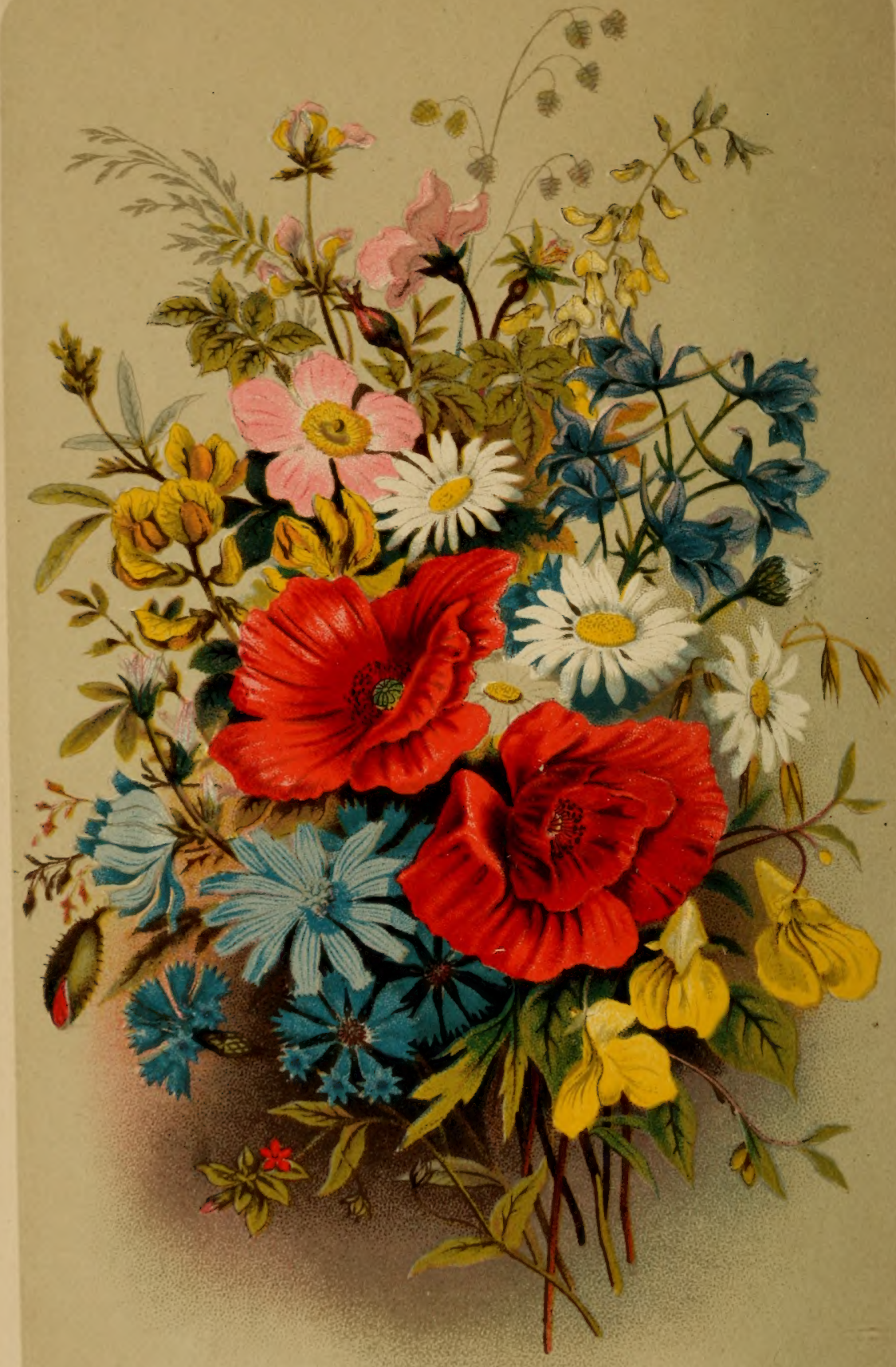




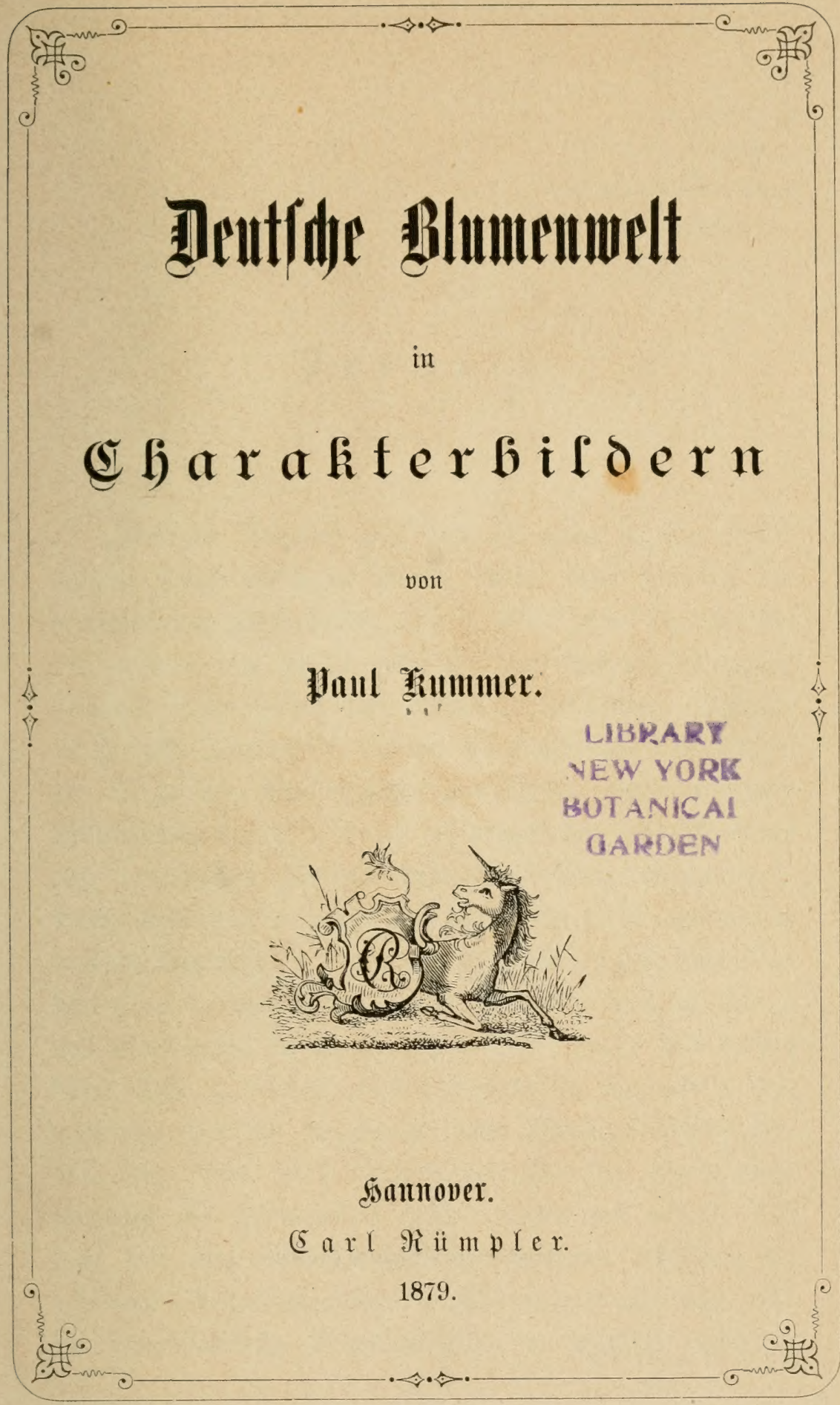




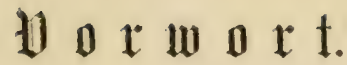

Dentides B(unten nut finto bildocn dicjes Bürdelein bietct.

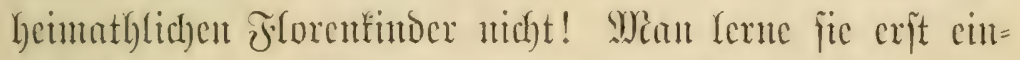
mal femten, un fie z̆t wilroigen mo baburd) bic cigene

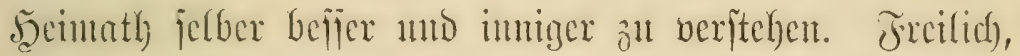

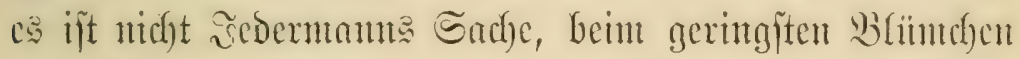
ant Wiscge die Stanbge

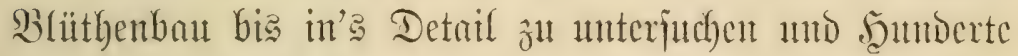

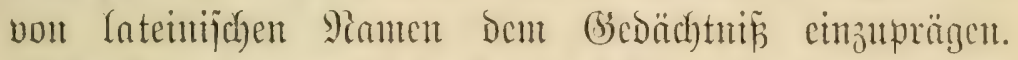

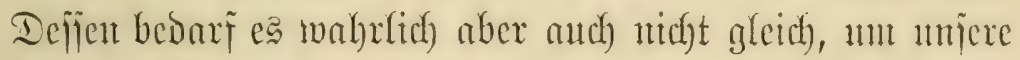

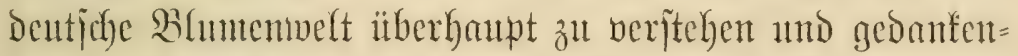

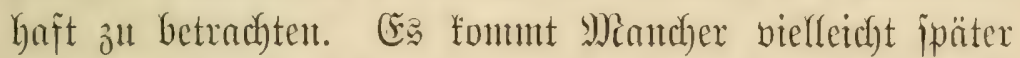

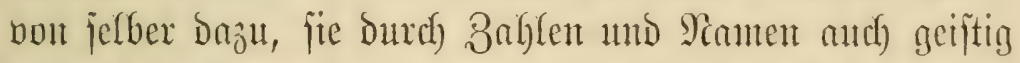

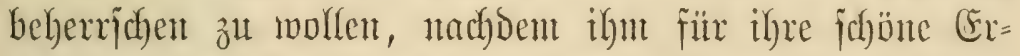

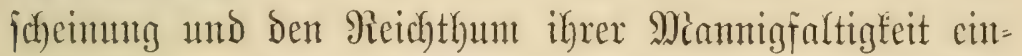

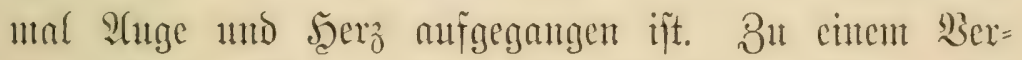

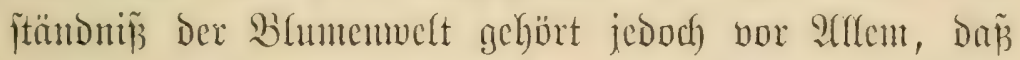

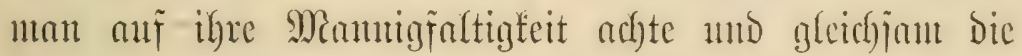

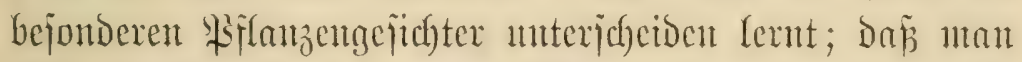

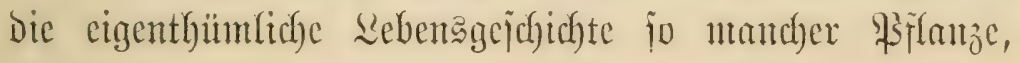




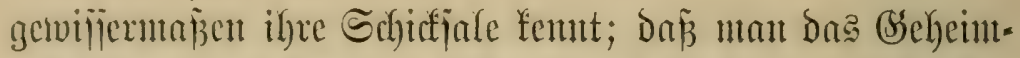

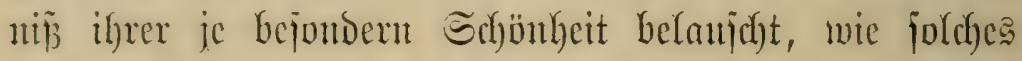

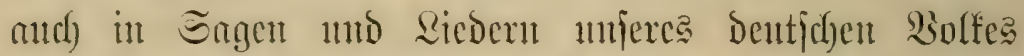

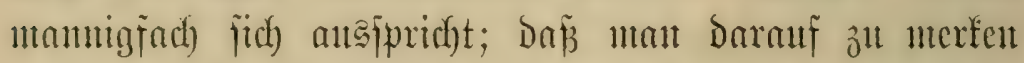

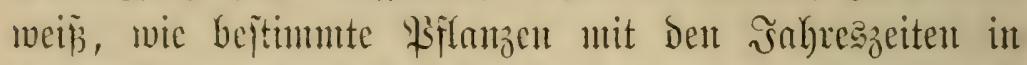

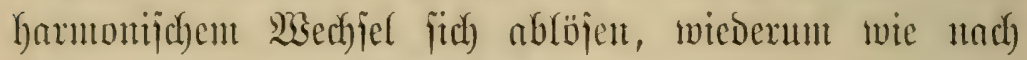

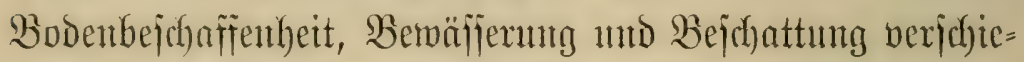
Dene Naturpläbe ifre cigene Flora baben.

Danon gebt and bies Bürdylem aus. Sielleidyt, Dañ Defien bunte Bhantenbitoer aber auth Durch ifre jwangloje Beichnung auf bent lanojichaftlichen Brumbe ictber, unt io

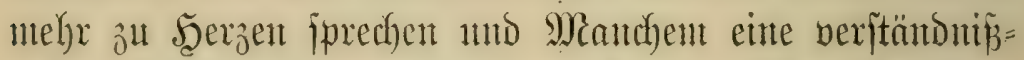

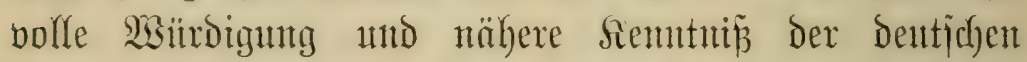

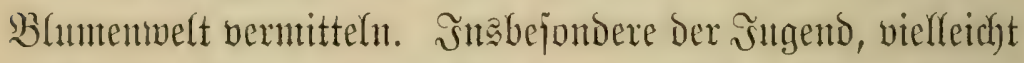

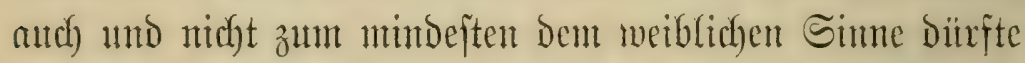

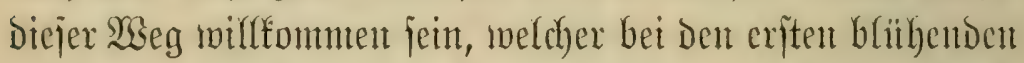

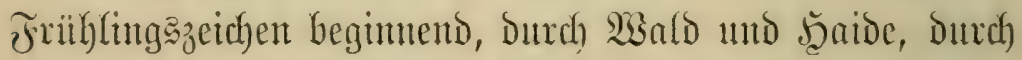

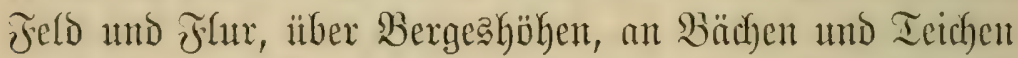
voriiber, Durd) Dorfĩtraß̧en und (şärten, Den Lejer überalt in bie Dentid)e Bhlumenwelt führt.

Mänden, int Wonnentonat 1879.

\section{Der 引exfaller.}




\section{In halt.}

I. Dentiche frühlingszeichen .......... ${ }^{\text {Eeite }} 1$

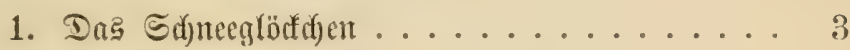

2. Dnక Bsildten................. 13

3. Die \$rintet. . . . . . . . . . . . 20

II. Int Wald und anf der Gaide ......... 27

1. Der Friblingguald . . . . . . . . . . 29

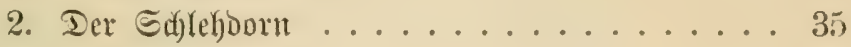

3. Die Mtaiblume . . . . . . . . . . . 40

4. Unjere Maldbeeren.............. 44

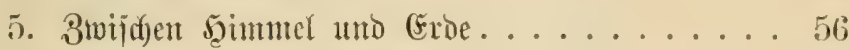

6. Streifereien int 9tadelwaldo ......... 64

III. In feld und flux .............. 73

1. Miniaturbfuntert .............. 75

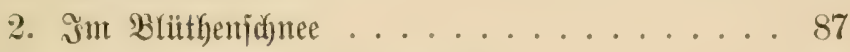

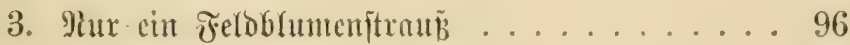

4. Am Feldorain . . . . . . . . . . . 106

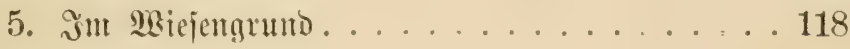

IV. Zuf Bergeshöhen . . . . . . . . . . 127

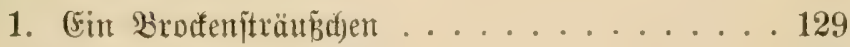

2. Âfferlet Bergftäuter ............ 135

3. $\mathfrak{A}$ rnifa . . . . . . . . . . . . 140 
V. Im Reich der Gewälifer . . . . . . E. 147

1. Brumenfrefīe . . . . . . . . . . . 149

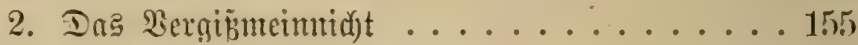

3. Die Röntgiumen Der (Setwäfïer. . . . . . . . . 165

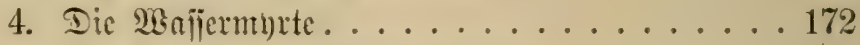

5. Die fleinten B(üthenpflänzd)en . . . . . . 184

II. In Dorf und Stadt . . . . . . . . . . 189

1. Dic Bietreutr De马 Dorie马 . . . . . . . . . 191

2. Begetabilijude Sdulmgen . . . . . . . . . . . 204

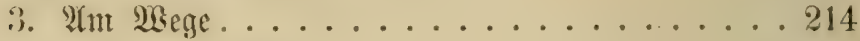

4. Die Etrajentiora ................222

5. Deutiche Gintenthumen . . . . . . . . . 226

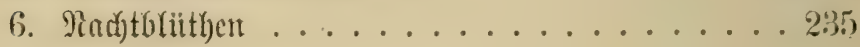

VII. Blicte aus der Göhe . . . . . . . . . . . . 243

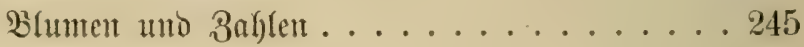




\section{I.}

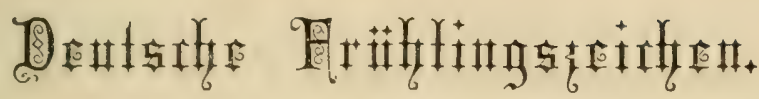

Winterlid Stürnten die Welt nun bezwang: falb ift Der 1 ald und dic Gaide fdon lang, Wo boch jo licblid mand Stmmlein erflantg. Spielten die Mägolein erît Strafen entlang sall, o fo fehrte der Dögel Briang.

Könnt' ich verjd,lafen im winter die Jeit! Wach' idh derweilen, fo thut es mir Ieid, Daf er regieret fo wcit und fo breit.

Endith Doch ficget oer Mtai in Dem Streit: Blumen dann lep' id, wo Shnce nun geiłhncit.

Whalther von Der Vogelmeide. 


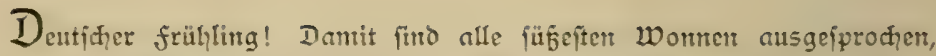

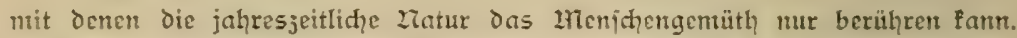
Der deutidye frühling ift aber fo ergreifent, weil auf die Langen, troftlofert Wintermonate das Eeben alsbald in jeiner rührendiften Sicblid,eit hervorbrid\}t. Durch die Pflanjert insbefondere ipridyt der Sharafter der Eebendigfeit in Der STatur fith aus, in ilhnen vor allem crwaht audy der frühling! Die DahI feinter

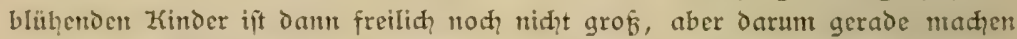
bie eingelnen fith dant um fo inniger gelteno, und die 2luswahl von Eieblingen if teidłter, ja ergiebt fid cigentlidy von jelber, oa die edeliften Blumen gerabe Des frühlings fo uiberaus zart dharaftervoll fint. Darum wohl hat aud die

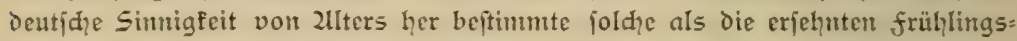
seifhen begrufft und geehrt, wie eintigen vont ilpnen aud fdyon in der nebelfernen

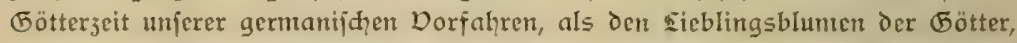
bejondere Euldigung erwiejen wurde. Deren 2luswahl fömnte wohl leiđht ver: meł̧rt und ein vollerer frühlingsf́alender aufgeftellt werden. 2lber die vor:

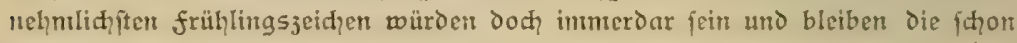
aแรerwählten:

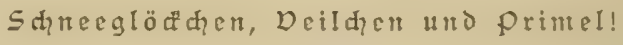

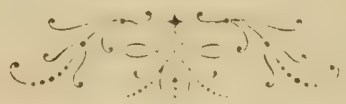




\section{1.

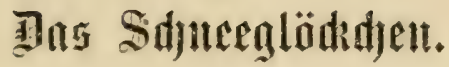

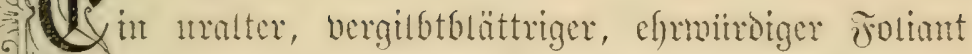

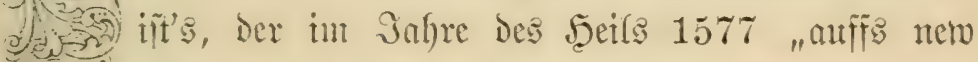

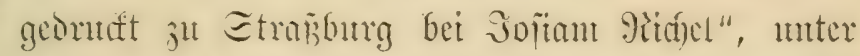

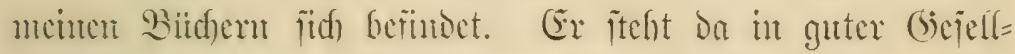

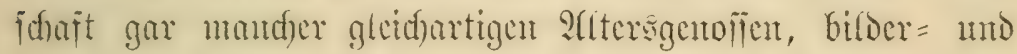
mährecidben Sirünterbïd)er ats jenen exĩten Beiten, als ïber=

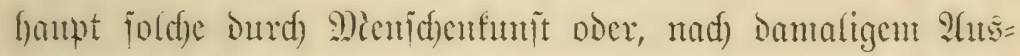
Drutfe, Durdf idfuarje Simit georufft worben find. Ias bejagte uralte Bud) ijt aber gauj bejonders jauber ausgejtattet; alferdings nidft in modernem Sime, ant menigiten in rothen Ealico gebumoen, mit (5olojonitt berjicrt, fondern in geib=

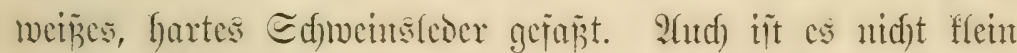

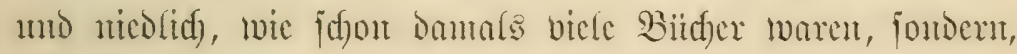
wie fodon erwälnt, cin jolwerer, grofier Foltant.

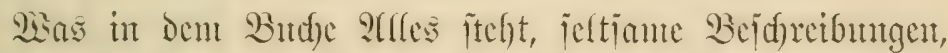
jomic farbige 2tbbilonngen mit Sumberten bentidfer Fiflanzen,

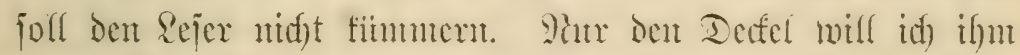
anfïd)lagen uno wenige Eciten weiter blätternt. Da fonm cine Ecite, anf weider der alte, biescre Berfajier in colorirtent

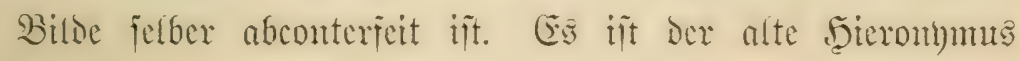




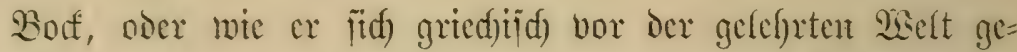
nant hat mo nod) ben hentigen (jeleforten wohlbcfaunt ijt,

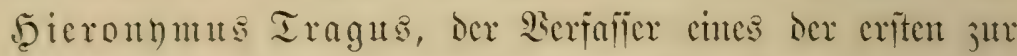

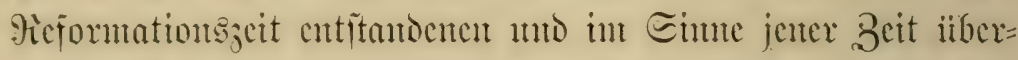

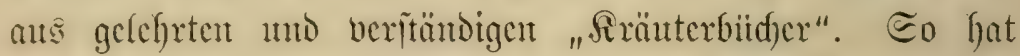
Der Mantn antsejchen! In idfwargem Ialax iteft $c r$, in vollem Bruftbiloc, da mit jeinent fremtolidsen, bon weijent

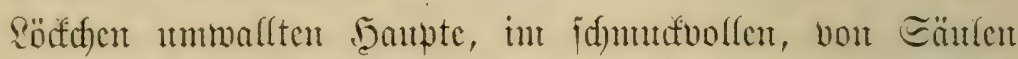
getragenent Fortalrafmen, itber Den fein feingemaltes Wappen mit einent Bodfe thront.

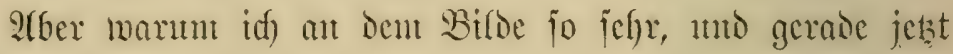
twieder meine Jrende habe?

Meil es wieder Frithling wird anf ber lange werwinterten

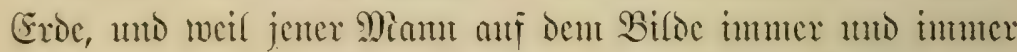

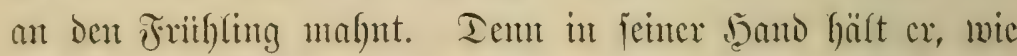
bas Euntbol jeutes Sebens, cin grofics Educeglöctden!

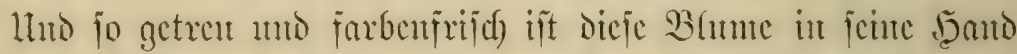

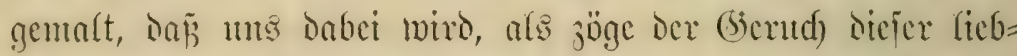

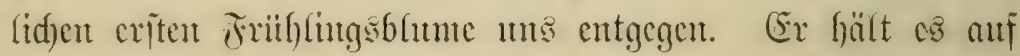
dem Bilo in feiner beato fo tren unt fejt, wic er es wohl

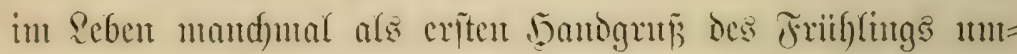

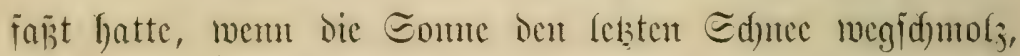
und ex mut in den wald unto anf bie fendete, nod) griutloje

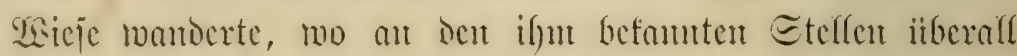

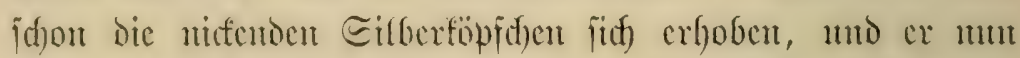

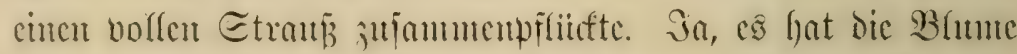

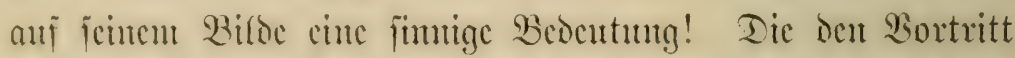

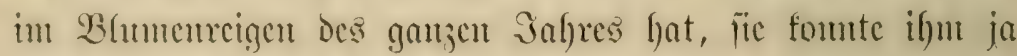

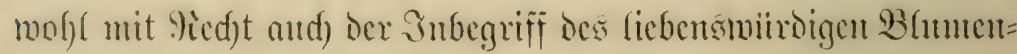




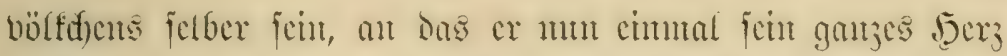

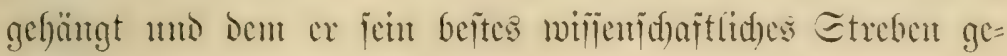
wiomet batte.

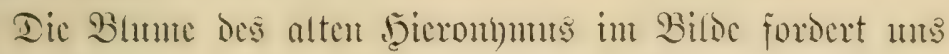
im Frithlutg wox affem anj, if)r lebendiges Driginal felbitt jut

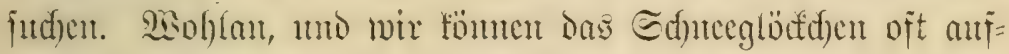

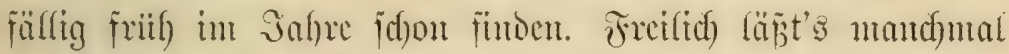

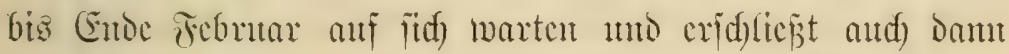

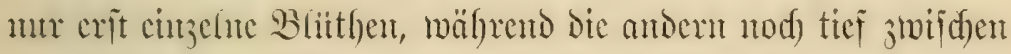

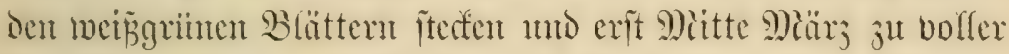

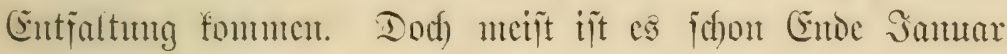

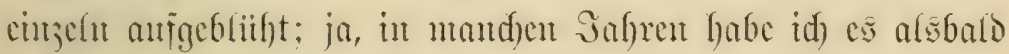
mad) Vienjah)r geöfinct angetroffent. Der 23 inter iît bam frei=

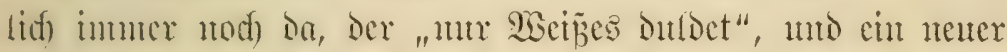

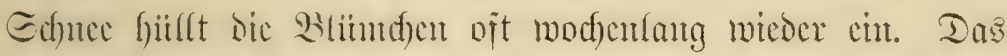

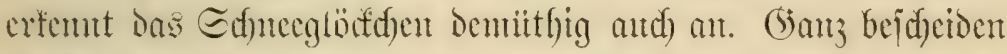

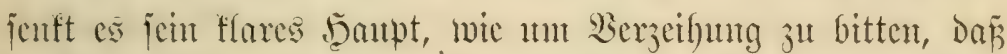

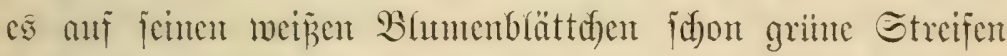
und Fifectach zat tragen wagte.

23ir haben bon vorigen Sabre die Etelfe nod) getwust

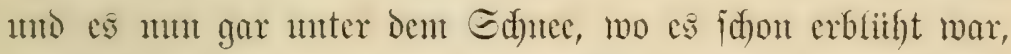

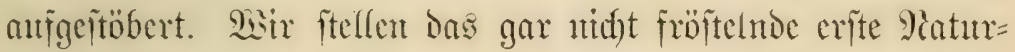
find gar andäd)tig im (sitaje an's Fenfter umo wenoen ifm jeden emfallemon Sommentrahl ju. Es ladt dant die Somme uns fajöner in bic Stube fincin, dic gegen das Fenfer ftöbern=

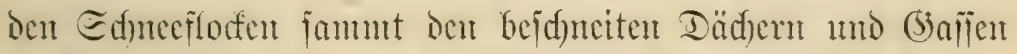

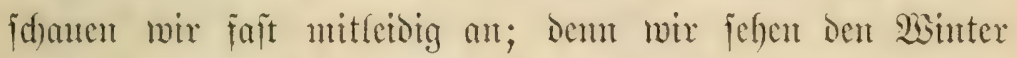

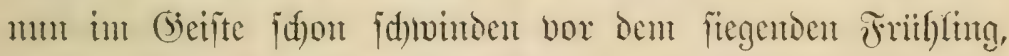
den uns fein exfter Bote berfindigt hat. 
Mite aber cine Forbe, ein Ion oft and) alte Erinnerungen wecft! Ias Edhrecglöifden crjählt mix bon einer alten, cngen,

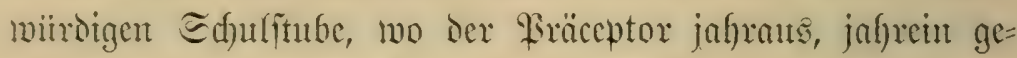
maltig regicrte, abex bei aller Bornesftrenge ab und jut and

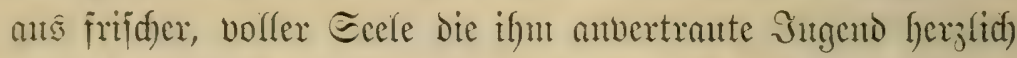
crquicte. In Den Februartagcn war cs; Der Edunce ftöberte

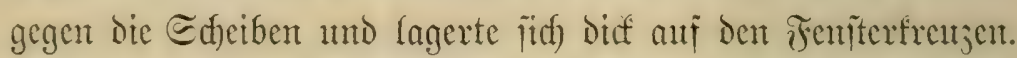

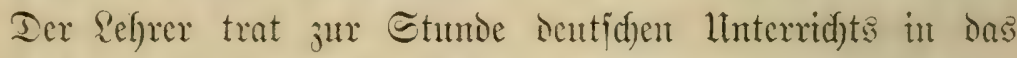
Sdutjimmex uno fono auf Dent fiatheder cinen mëchtigen

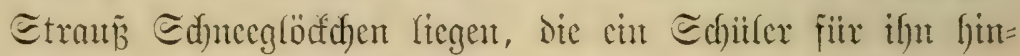
gelegt batte. Er frente jidf jidhtlid) Dariiber, fei cs ïber den

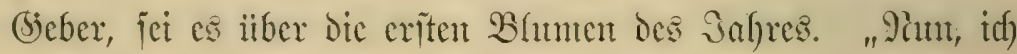
milf fie aber nidgt alfent befalten", äujerte ex nad) furger Weile; "id) theile fie ans unter cud s), Sedcr befonmit cine

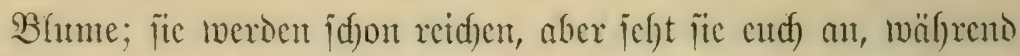

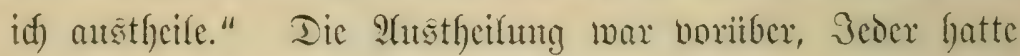
jeine Bfunte, uno die Sungen fahen cinander ganj vermutudert

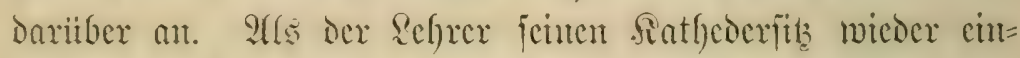
genomment batte, iagte cr: "IAljo bentiche @tumbe ijt jelst;

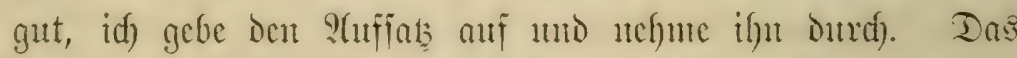

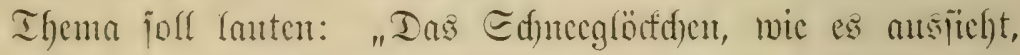

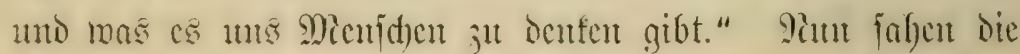
Suntgen fidd) nid)t mel)x an, aber wie cin Eommentrahl jurfte

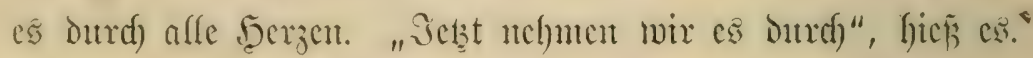
Paun murde crflärt; Seder mun̈te bie Morte an jeiner empjangenen Bame priifen. Sede Wiflange, lautete ctwa bie bon Fragen mo (ntworten unterbrod)ene Gede, bejteft noth)=

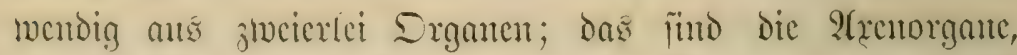

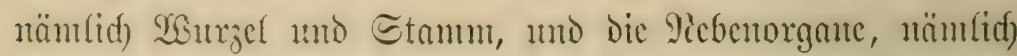




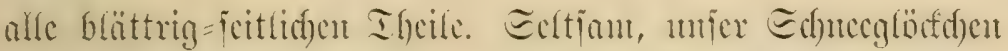

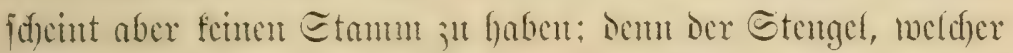

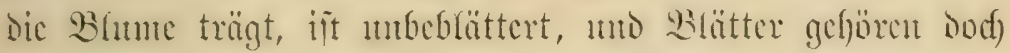

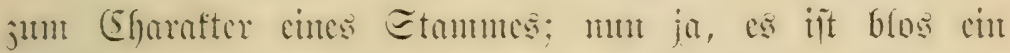
fogentmuter Edhat. Ltho Dod) ift and cin Etamm vorfgantom!

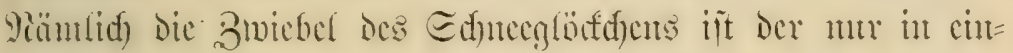

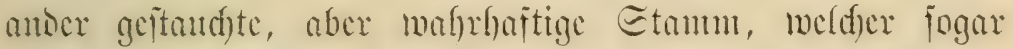

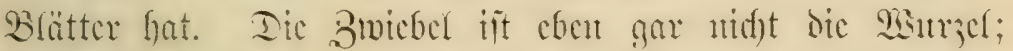

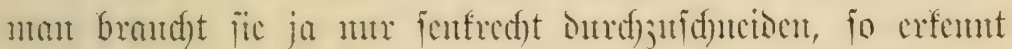

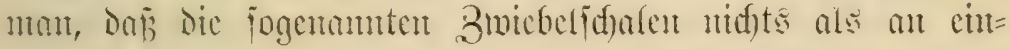

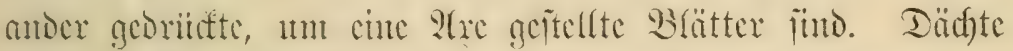
man fich bicje ?lae ansigejogen, fo entitünde cint langer be=

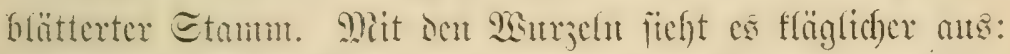

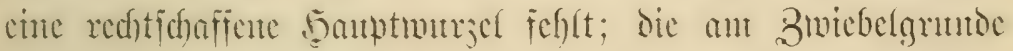

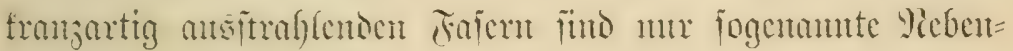

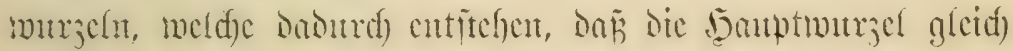

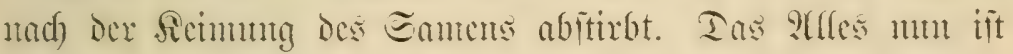
Efarafter cinter ganjen Fiflanjenabthcilung, dex fogentantent

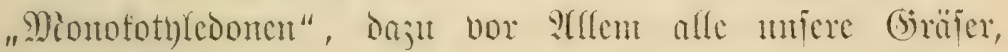

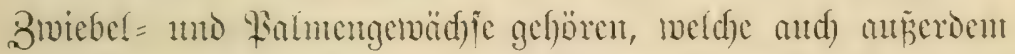

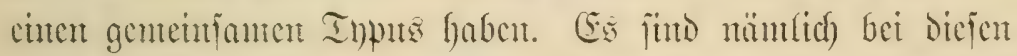

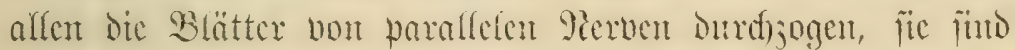

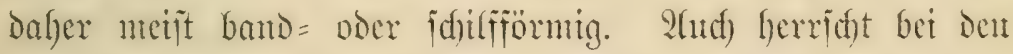
Monofotyledonen in affen ifyen Theilen Die Dreizall oder

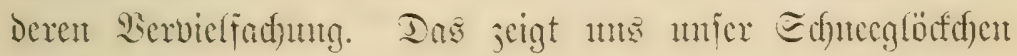

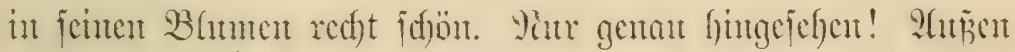

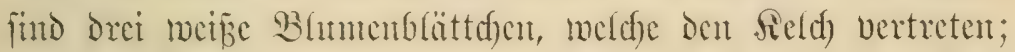

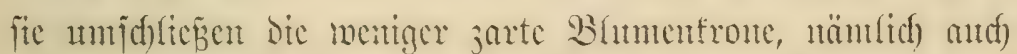
Drei mit grimen Fiscefen betupfte, Elcumere jolde Bfätter. 


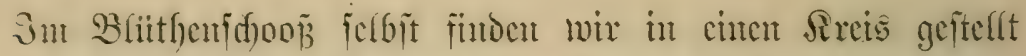
wicoerum fed) (aljo jweimal orei) gologcloc Staubbentel, anf

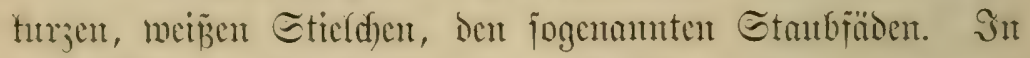

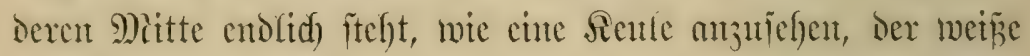
Frutd)tgriffel mit grimer Eptice, Die jogentannte Rarbe, weld)e Den in den Staubgefäp̃en enthaltenen golsigen Blitthentanto

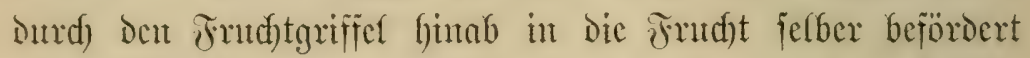

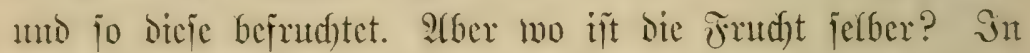

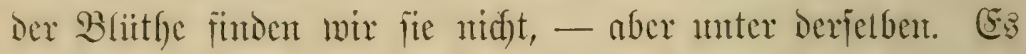

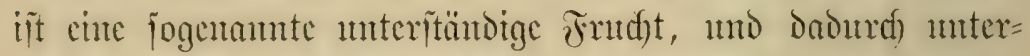

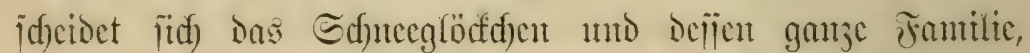

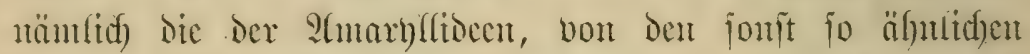

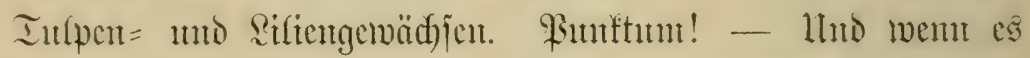

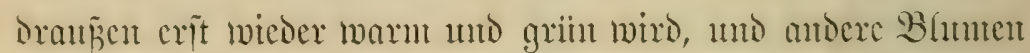

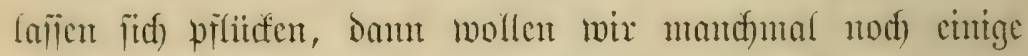

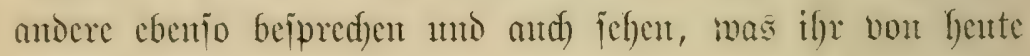
bebaltent babt.

Der Sräceptor fprady's, nuto dic Edfiller larjaten. Der

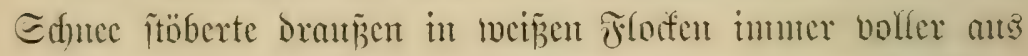

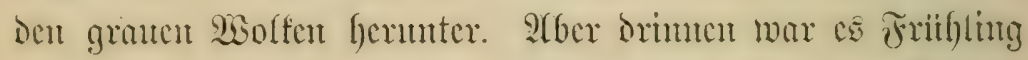

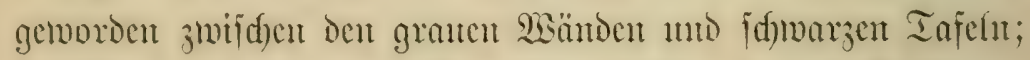

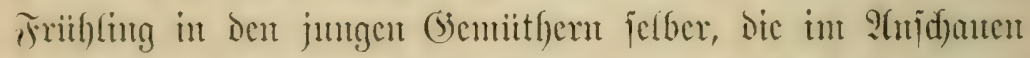

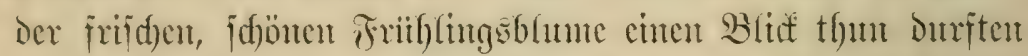
in cine gehemunip̈volle Sromung Der Matur.

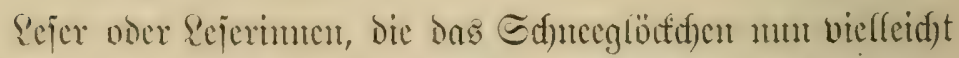

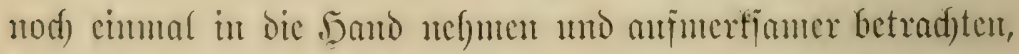

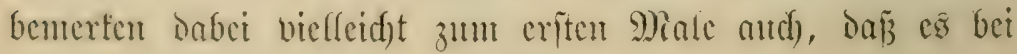




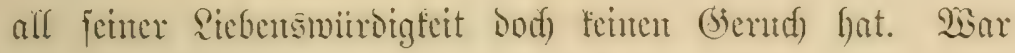
nidyt aber gejagt, Daj es gar lieblid) Dufte?

Plferdings, das cigentlid)e (biartenjd)neeglö dden (Galanthus nivalis), meld)es aนf (Brnspläłsen mo Becten des Gartens ant fritfeften herborfpresent, nit Den weingrinten

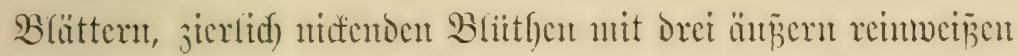
Seld)blättd)en mo oret längeren intueren grintfleffigen Bhunten= blättd)en, - cos ijt böllig gerudflos. Sicịes ijt's aber aud gar nidjt, weldyes der alte bieronumus Iragus fo tuntg

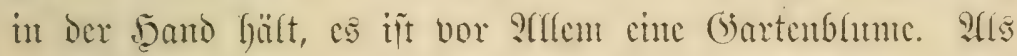
wibmadjend finton wir es fic mo da in ber Edyeciz, aber

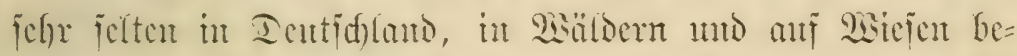

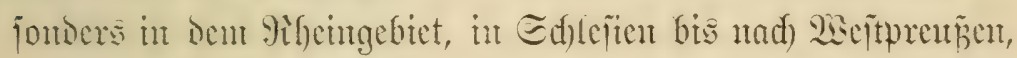

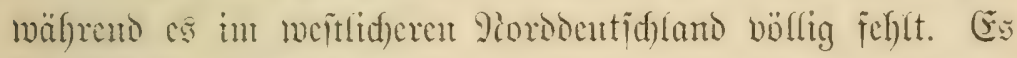
hat jidf allerdings itberall frentislid) an unjer sentides Reben angejodlofien uno gejällt jidod in umiern (särten.

Das deutid) Eduecglö)d den (Leucojum vernum) aber Duftet! Dics ift nod) cine gant; andere Blunte! C5

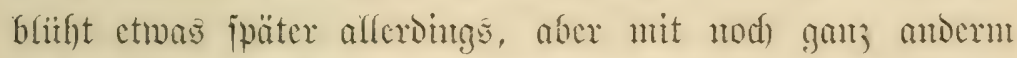
Rebreci; uno wirtfid) weildenartigem Iuft. Ecinc Blätter find ftürfer und faftgrim: ocr Bhmmenjadaft reeft fid) bis weit ïber fingerfodh, uno bie Blithen, aus jedfs gleid)artigen

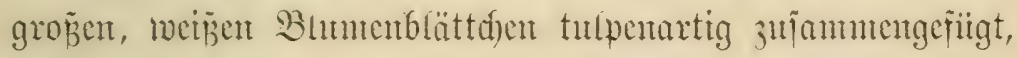
fino weit gröper uto tragen anf jedent ifrex Bfättden cin

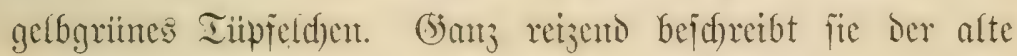
5. Iragus jelbit: "ecute jode Edjell oder Blum bergleidht

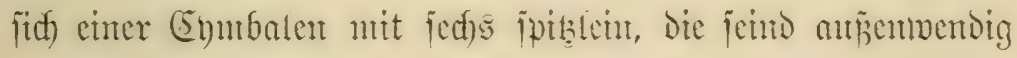
mit gälgrinen tröpfịlein anfi ben fpişen gemalet." 


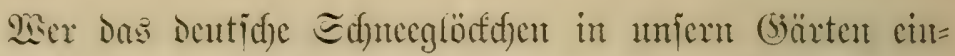
mal ïeft, wo es and biclfad) angepflanst ijt, bält cs wohl

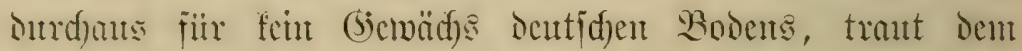

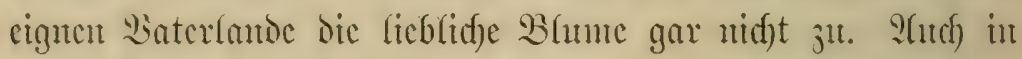

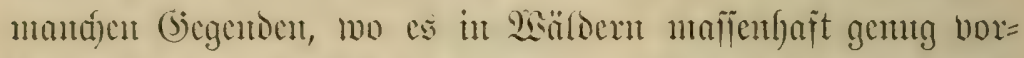

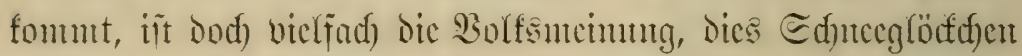
fei Durd) friblere Botanter ans Franfreidy in Den bentichen Sald gepflantht uno num verwiloert; aber freilich lange jei bus her, dent es wadje da, fo lange man fid crintern fömte.

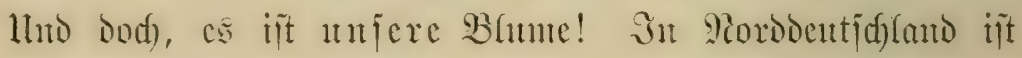
fie allerorten in brudfigen, oder dod) ctwas fatthten Saub= wälocrn, cbenjo unter (sciträud) añ mafjen sisejen ju findent.

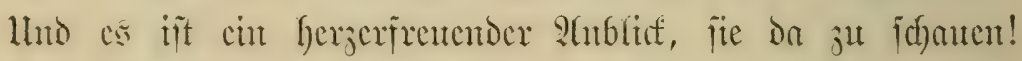
In diduten Irupps oder jerftrent ftelen fic Dort, gern an

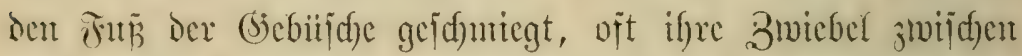

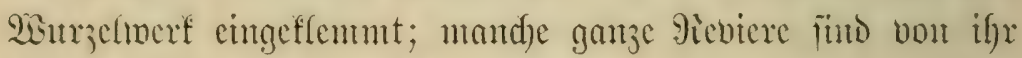

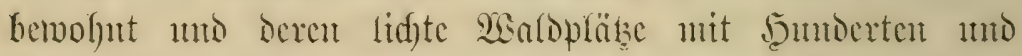

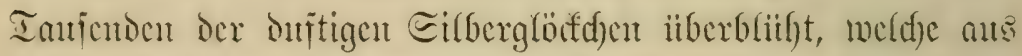
Den dutfelgriuten હdyertblättern gar ftolj bervorragen. Bci

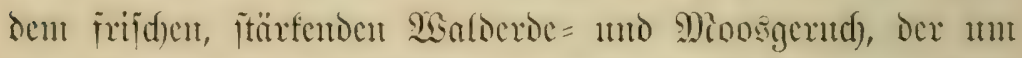

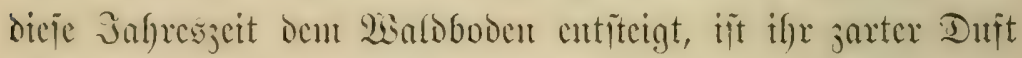
bou Boben ber allerdings wenig mertlid), aber twent wix

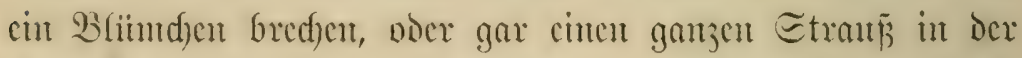

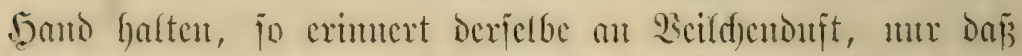
ex ctwas weniger itart ijt. - 2 atd in Mittel = atmo Eito = bentid)(anto, ebenjo in ocr Edfuciz, findot fids nufer Edynce= glördfen mannigjad) berbreitet. Scovd) andere "ä̈tocr, andere

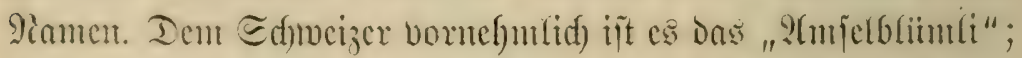

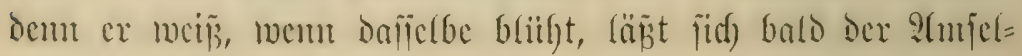




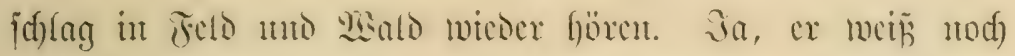

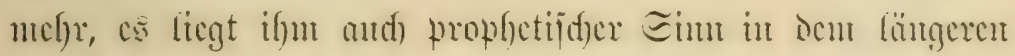

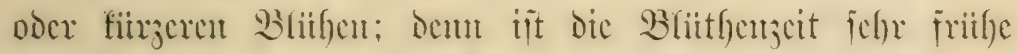

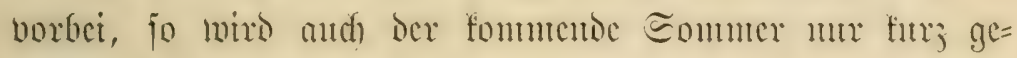
mefien jein.

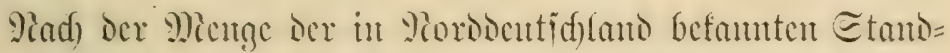

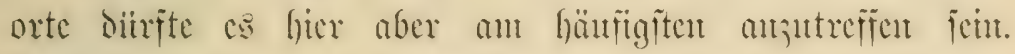

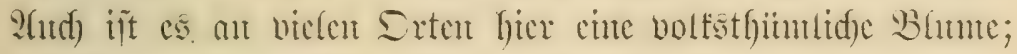

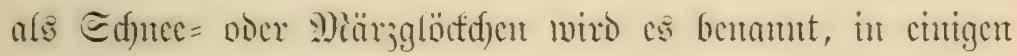

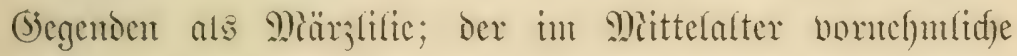

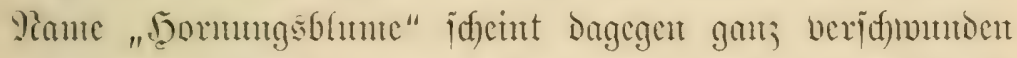

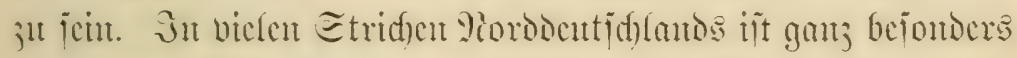

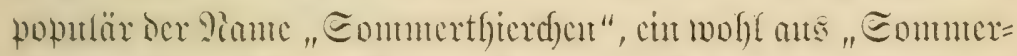

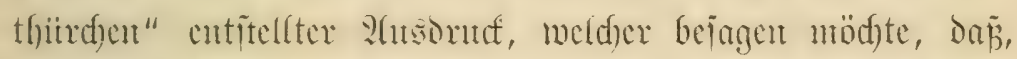
mem dieje Bhtme blitht, ber Eontmer bor ber Thitr ijt atmo

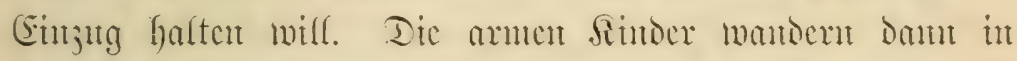

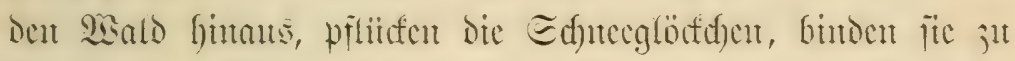

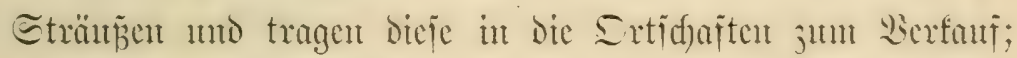
unto dic bejdectonte Bitte: "Sinuten Eic Eommertfierdfut?"

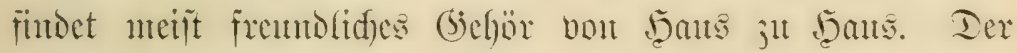

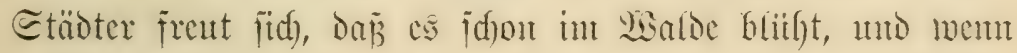
cr die Gabe der armen Sintoer mut an's jomning Fenter ge=

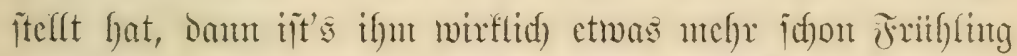

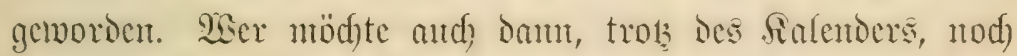
ant ben sinter glauben!

Pium, gechrter \&cjer, wirît Dut geru begreifen, warum dex

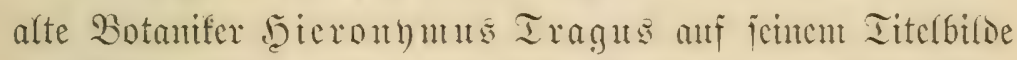

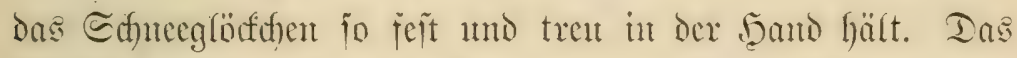
Meife, was Das 3afy in jeinem weitern Werfanj bietet, mag 


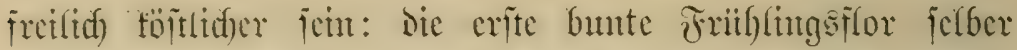

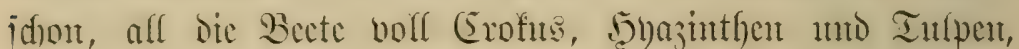

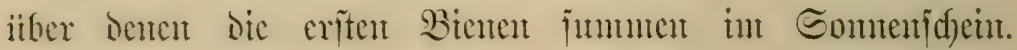

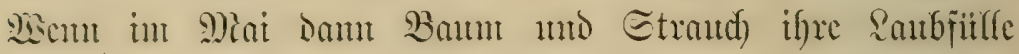

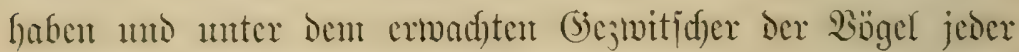

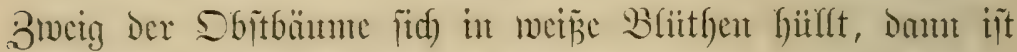

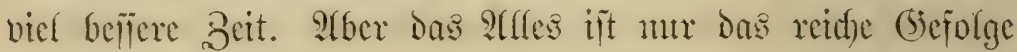

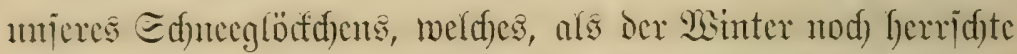

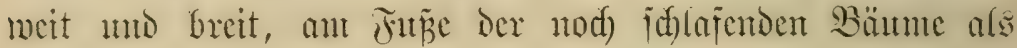

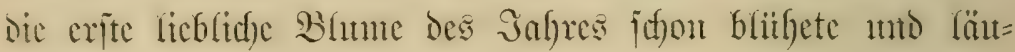
tete, dañ es Beit werde, aufzumadien.

Bon meitem fört' id zarten Ion Mie Eilberglö̈fden Yäuten;

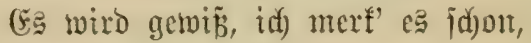
Dos orrüblingajeft bedeuten.

Da fäbrt empor uno ipizt und rectit Das junge Gras Die Dhrent Itro ftrebt, bon biurrem \&aub bebecft, Eid) an Das Ridjt

Da fommt, fid gegen Frülflinggmadid Bei Beiten j̧u verwalyren, Der Minter branjend ilber Madyt Bon Rorben Kergejalyren.

D Minter, fiebjt Dem nidjt bas $\mathbb{R} a u b$ ? Merfit niryt, was Das bedeutet? Du alter Binter, blind und taub,

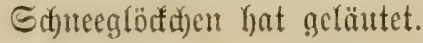




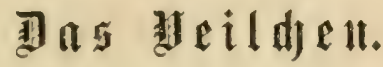

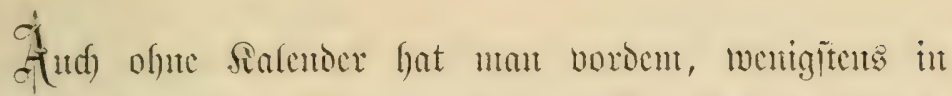

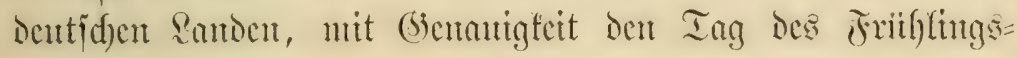

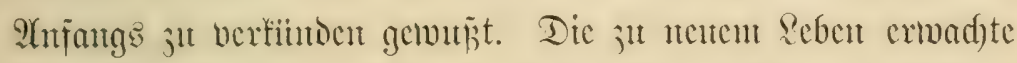

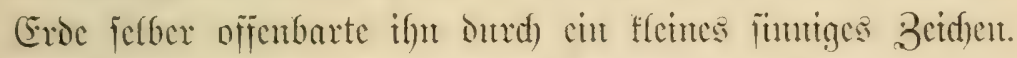

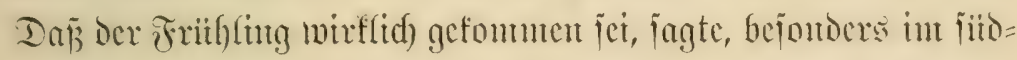
(idf)en Deutjdf(anto, gefeimui

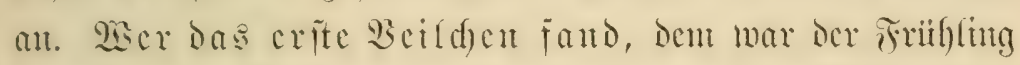

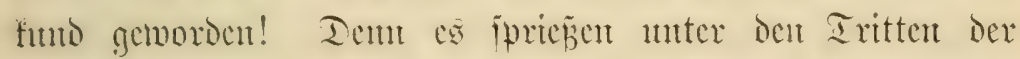

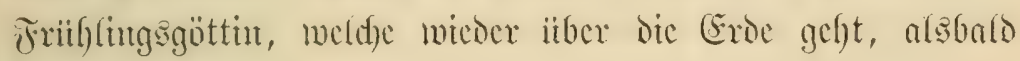
dic erfiten B̧eilden auf.

Es war ein Jutheltag, foweit bie frohe Botidjaft orang.

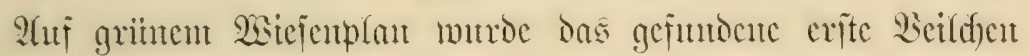

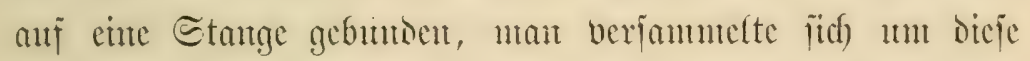
for uno jeierte Den Iag unter Earg uno Epiel. Dic 3utgent

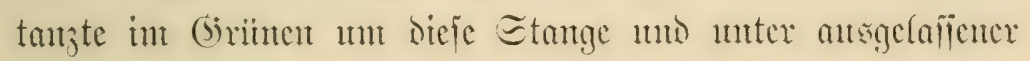

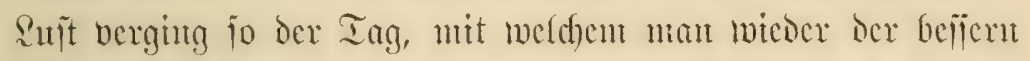
Beit ics 3afres entgegentebte. - Dicje Sitte gab in incu Iagen Stto des Fröffiden in ber llmgegent bon 13 in felt=

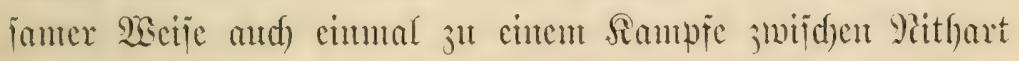

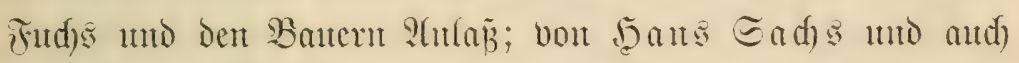

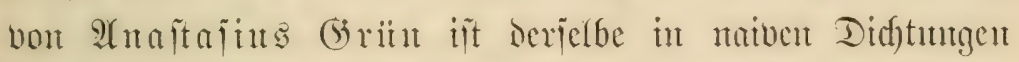

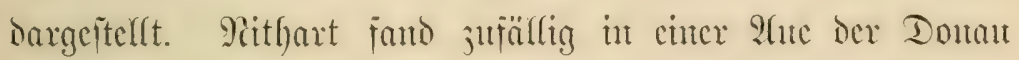

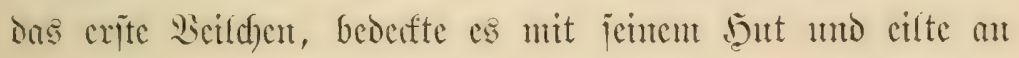




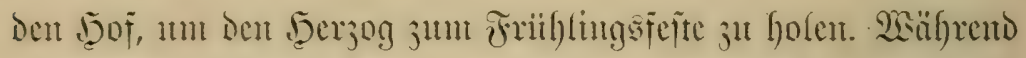

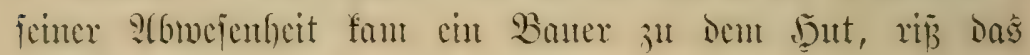
Beild)en ab

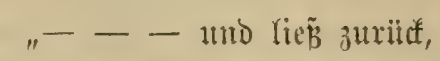

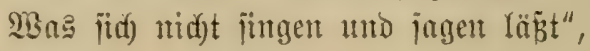

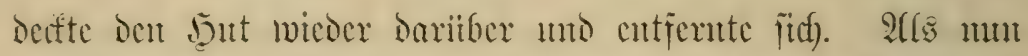
Stto mit Fement Gofintant an bie Stelle fant nuto ?ithart Deat but abgob, wax man von ber feltfamen Beffermug nidjt

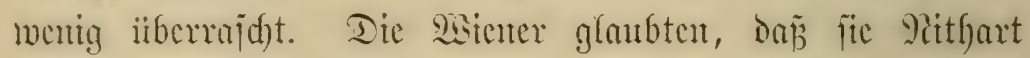

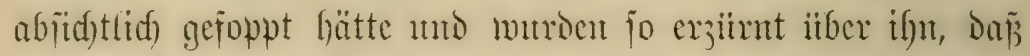

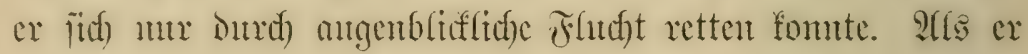
aber jein 2ieildyen anf einer Etange erblidte ntmo bie Bancu

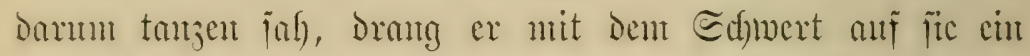

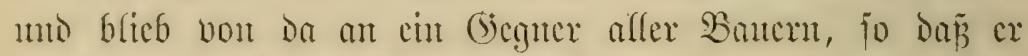

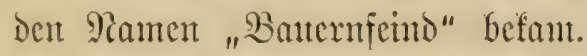

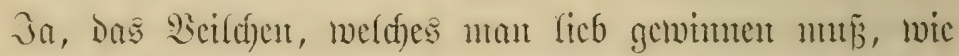

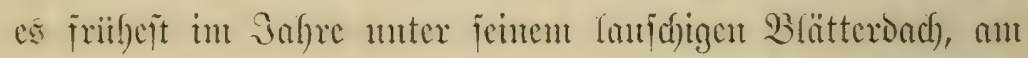

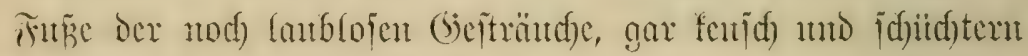
hervorb(iift und duffet, ijt and anderweitig borden in heifigen

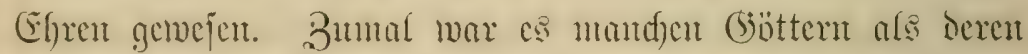

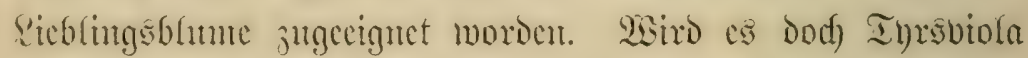
in ben alten Berthten geheipen, und jobn biejex Name weift

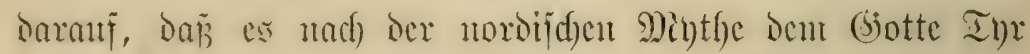
heilig war, den gewaltigiten ber (5ö̈tter, melder mit jeinem

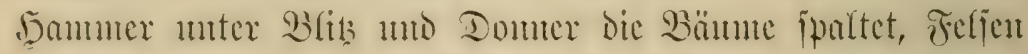

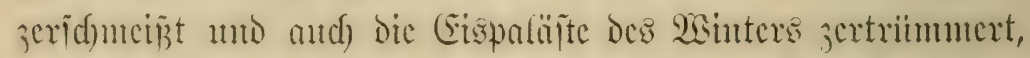

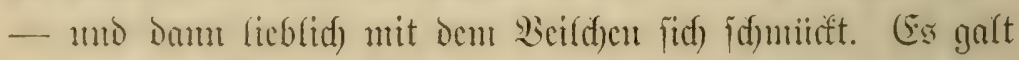
aber and im alfgemeinen Eimme als 2 sumberblume, co ber= mïge ctma verborgenc Ed)ätse anjujeigen, meld)e gebcimmi 


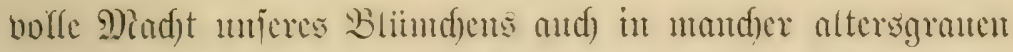

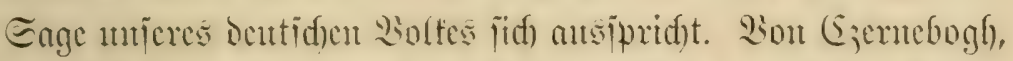

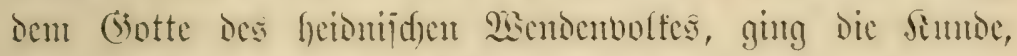

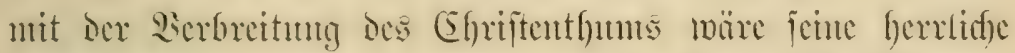

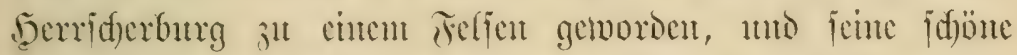
Iod)ter in cin Sicilden berwantodt; mex aber alfe funtoert

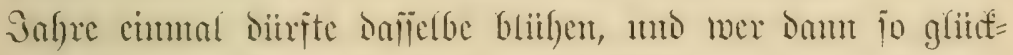

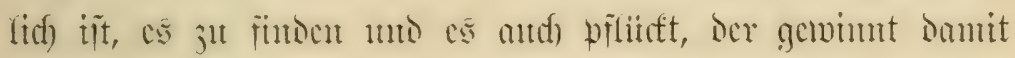
die Jutngfran janmut allen ifgren Edhäbent.

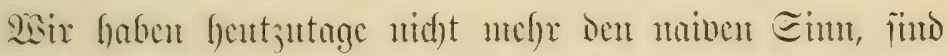

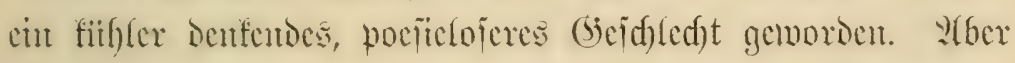

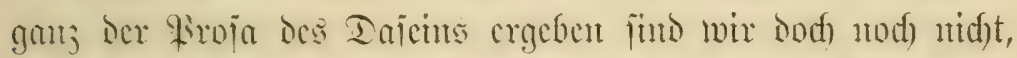

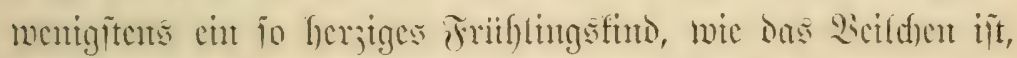

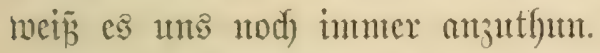

Tic alte Eitte bes Friblingsfuntens ift aflerbing ber=

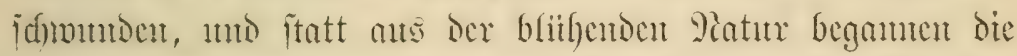

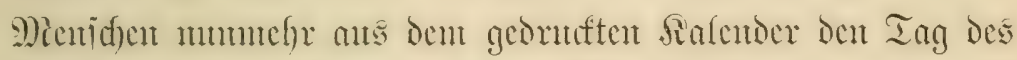

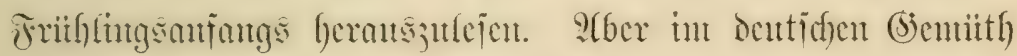

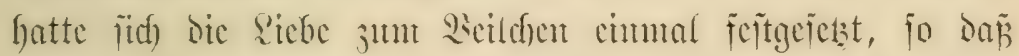

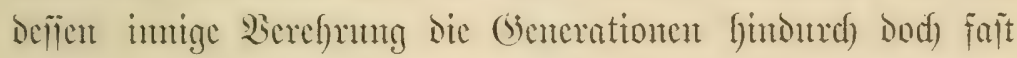
mbermindert weitergetragen wuroc. Itno mit jeoen Friiffing

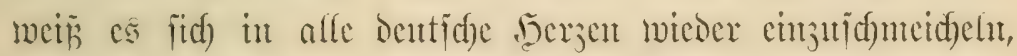

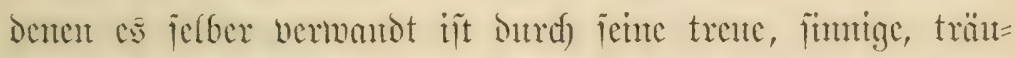

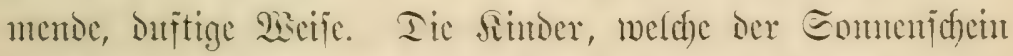

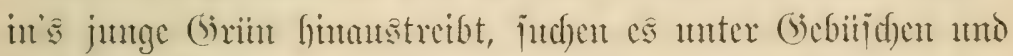

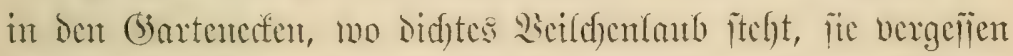

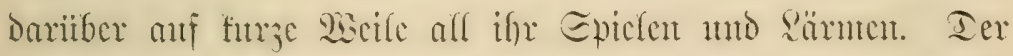

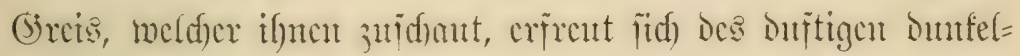

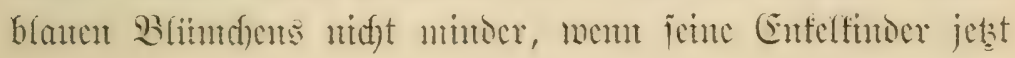




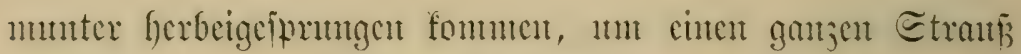

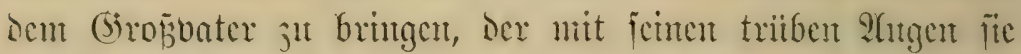

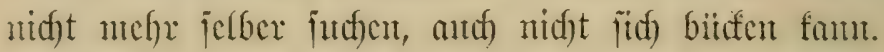

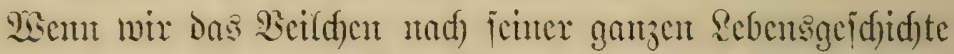

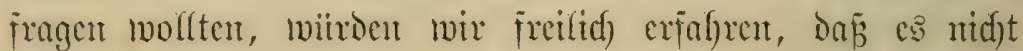

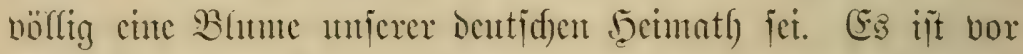

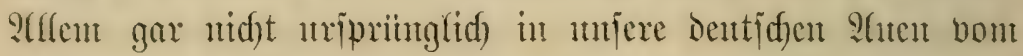

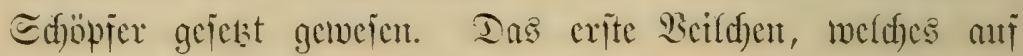

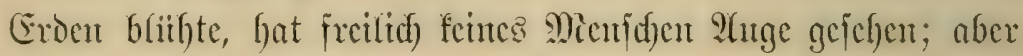

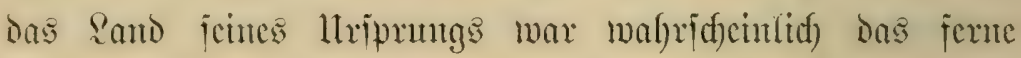

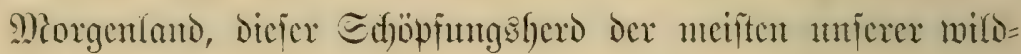

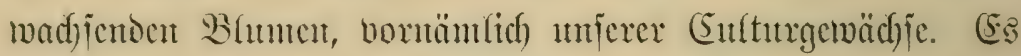
hat fid) and fernerfin nidit einjig als iebensitätte unjer Dentidges Baterland ansgetwäglt, jondern jeine Retje unt dic ganje Eroc angetreten und iiberall fitd traulids angefiedelt,

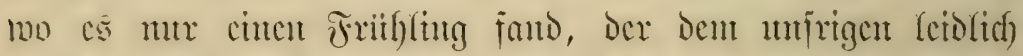
gleidft. Wenigitens in Der ganjen falten atno warmen ge=

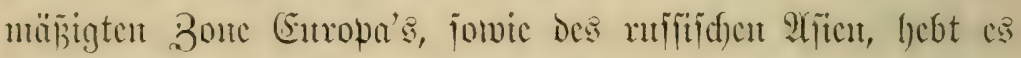

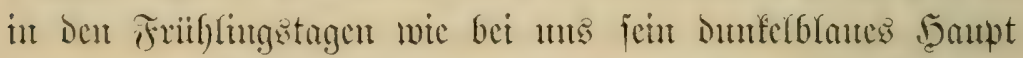

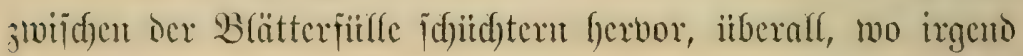

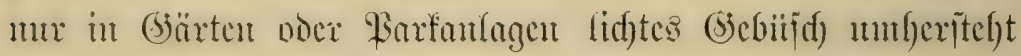

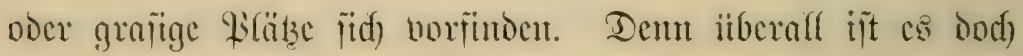

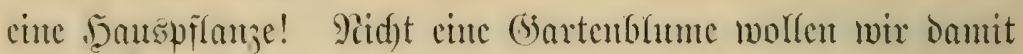

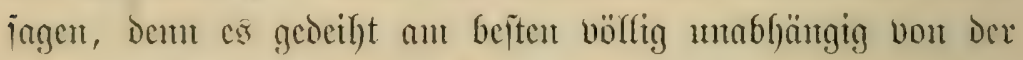

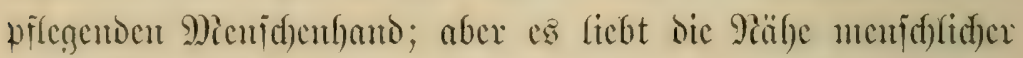

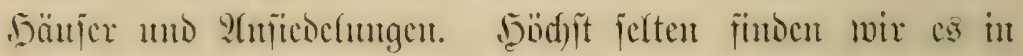

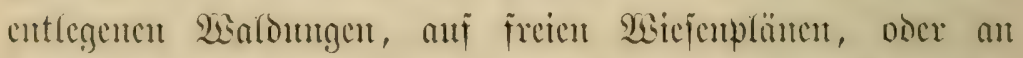

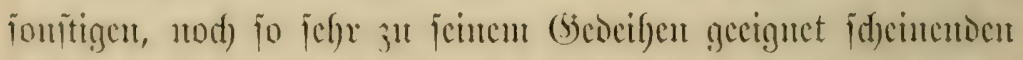

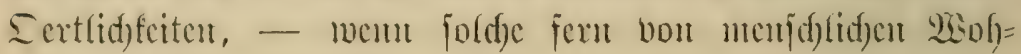




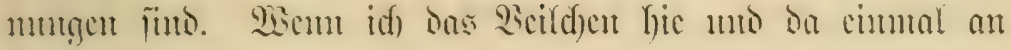

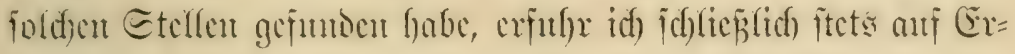

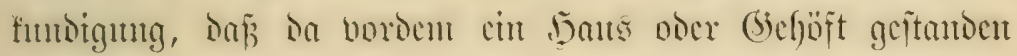
boube oocr ciuc alte Dorfintätte ici. Ecither crblite id in

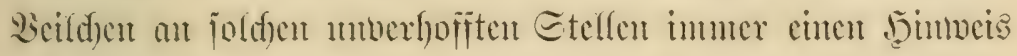
ani fritheres mentadlides

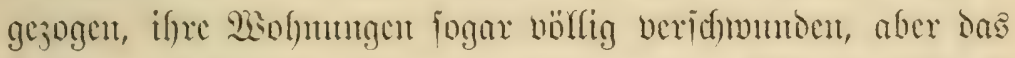

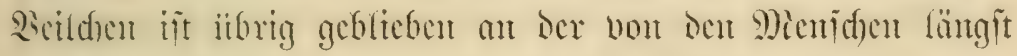
berlafienen Stätte.

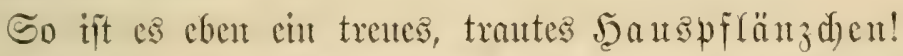

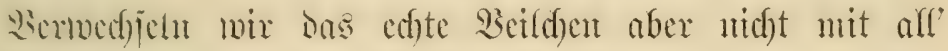

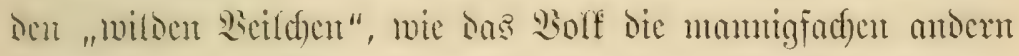

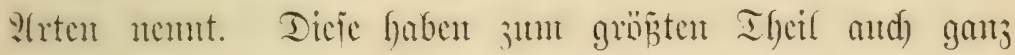

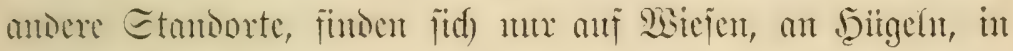

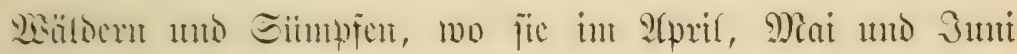

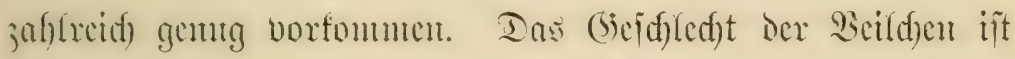
un ber Ifat cin gar grofes; ocr Botanifer jählt ctwa jefur

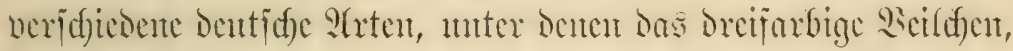
Dicis befante Etiefmitterd)en oner. Dreifartigfeitsblitudden, jo=

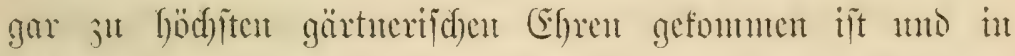

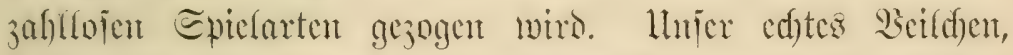

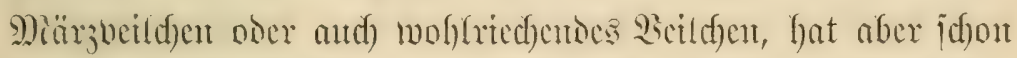

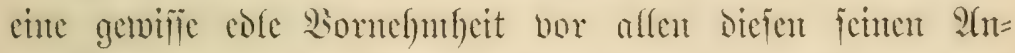

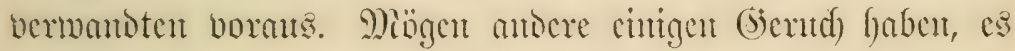

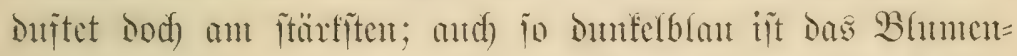

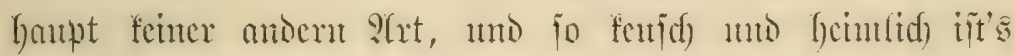

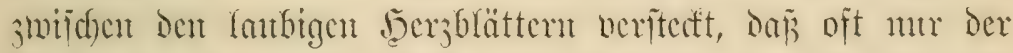

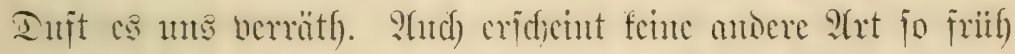

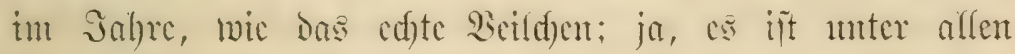


früblingsgeborenten Wiflanjen jiemlich dic exite, meldae; nadjoen

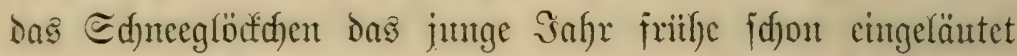

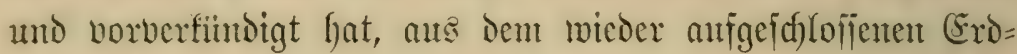
jd)oō herborb(itht. Darum bringt es nad) bent (5ilauben unjerer $\mathfrak{B}$ äter eben den Frühling jelber mit.

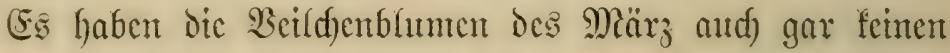
andern 3werf, als Der Mienjd)en Jeerjen ju erfreucn. Denn

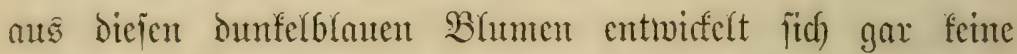
Frudfit, deren Eame dics cole Beildyengejd)(cd)t fort uno fort

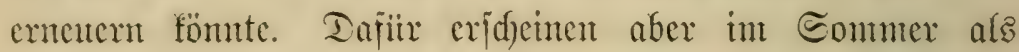
joeite $\mathfrak{B}$ (üthengeneration wicderum Beifdyenblumen, jedod) viel flemere, ja blumenblattloje. Sieje erjt fetsen famenteidfe Friid)te an, weld)e wir in Serbft als breifäd)erig aufipringente Sinpieht ïberall j̧wijd)en bem Beild)engeblätter wah)rnehmen. Freifid) wären aud) dicje faum nötbig getwejen; jede Meutter=

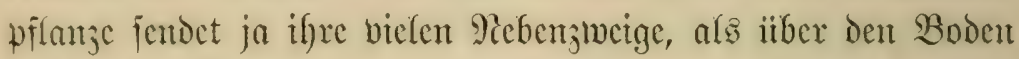

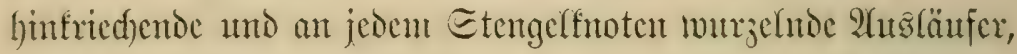
mad) allen Eciten weitfin ats, uto cin cinjiges Frib)(mgs= pflänzad)en ijt badurd) in Serbjt fdyon ju cinex ganjen (Solonie

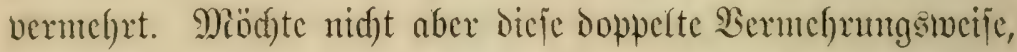
mittelït Camen und 2tusläufer, unfer Beildfen als cine Steblings̆blume dex Ratur jelber crmerīen! Wir wuntern

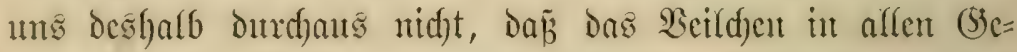

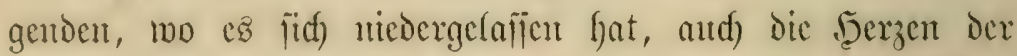
Menjaden croberte uno fie in ifm fo frentoevolf Den Fritgling

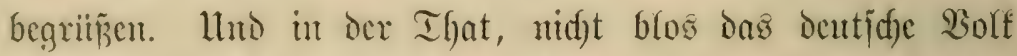
liebt es, mandye mocre Mation jumärmt gerabeju fïr dafjelbe, wic fiir faum cinc andere $\mathfrak{B}$ annc. In Franfucich ijt es jeit ocr

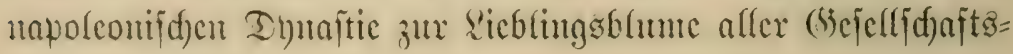


freife erfoben morton. Dic (särtucreicn non Liarts mo

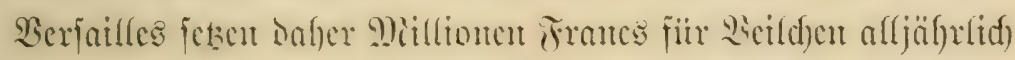
un, uno dic glänjenden Eoimen dajelbit fiffon lange bor

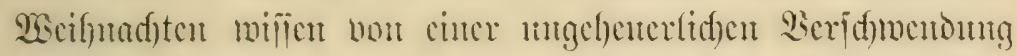

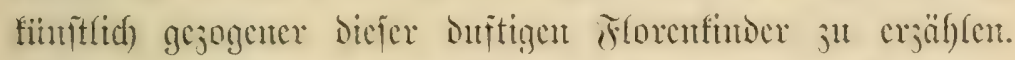
On mandfen (siegenten Frantreid)s, befontors in ber Frovente, merden fogar cholofe Feloffuren mte mit Beilden bepflanjt,

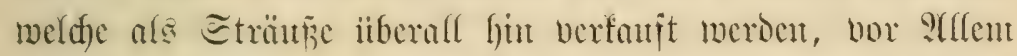
aber Den Deftillatenten jux (Sicwimnnty ätherifden Duftöles

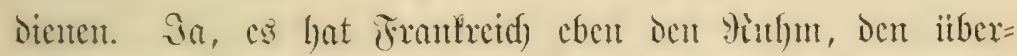

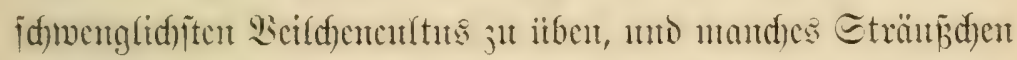
iff Dort fdyon thenerex bejahlt moroch, als wäre es durdyeneg von gediegenem Siolde gearbeitet getucjent.

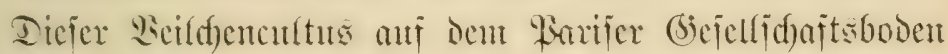

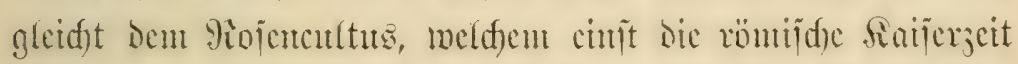

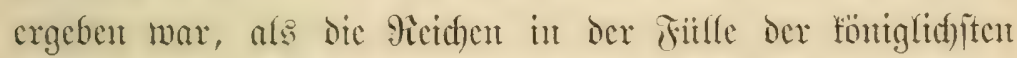

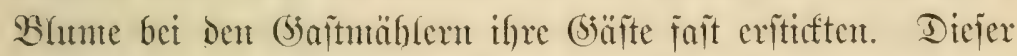
Majienconjun abcr, melde Serabwitroigung ber jartu Bfunct=

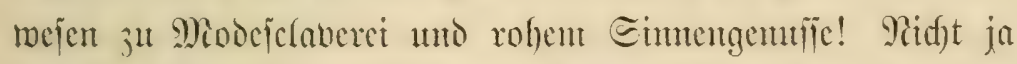
das bejdecion gejenfte blate fïpjd)en, weldes wic um

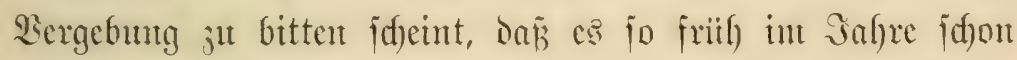
da ifit, bat cs dort jul Modeblume gemad)t; nem, vor ?trlem

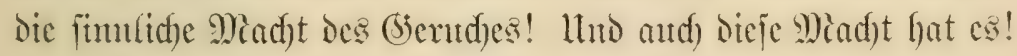

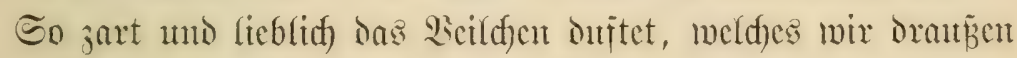

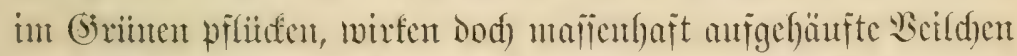

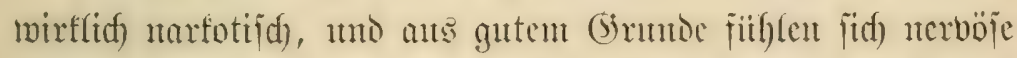

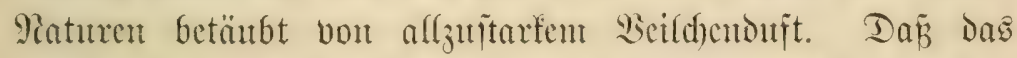

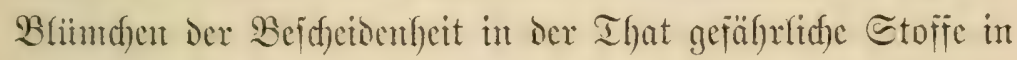
jeinen Bujen hege, hat and) bic (Shemic nad)getvicien. 
C.5 mill mixtlid) nidut in ben Galons glänjen, wo

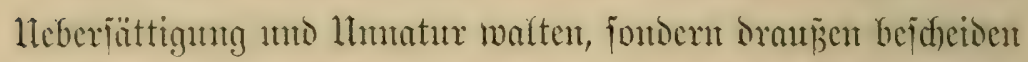

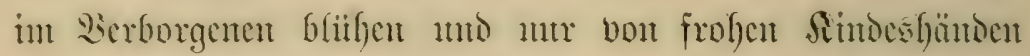
gepflitift und bout findliden berzen geliebt werdon als bas

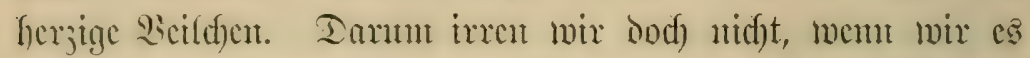

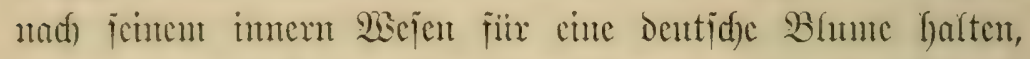

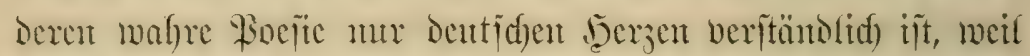

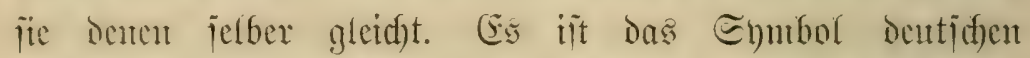

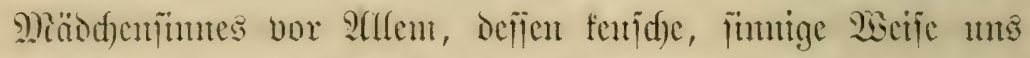
in weilden mit jedent Frithlum entgegenduptet. Darum haben anta) Deutidye Didhter wie femes andern Boltes Focten it iffen bejten Siedern boll jartefter Bejichntugen gejungen

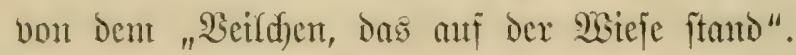

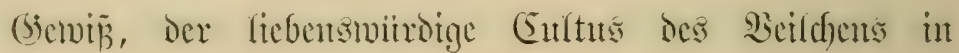

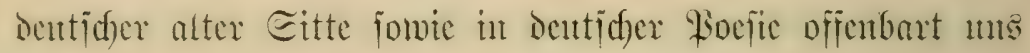

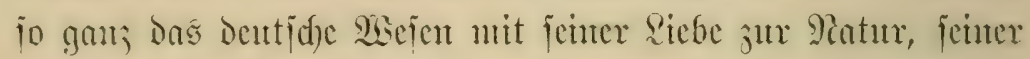

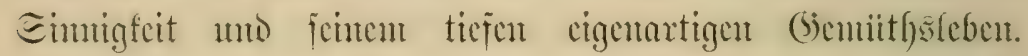

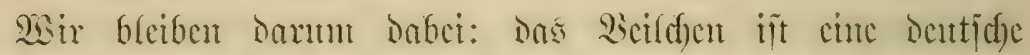

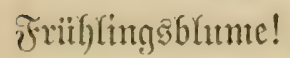

\section{3.}

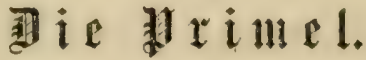

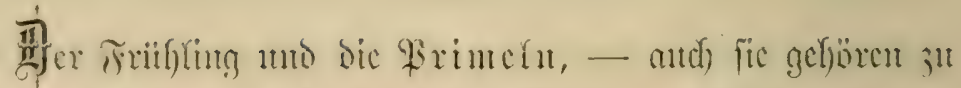

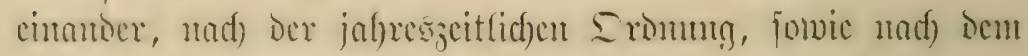

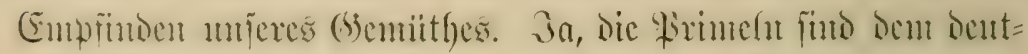

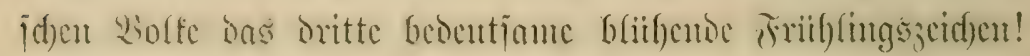




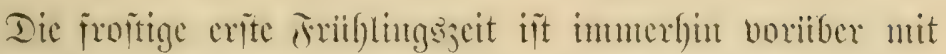

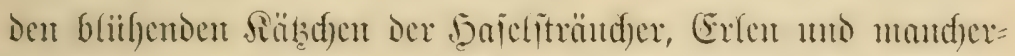

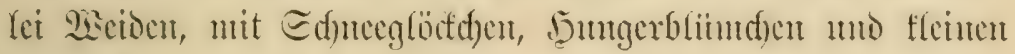

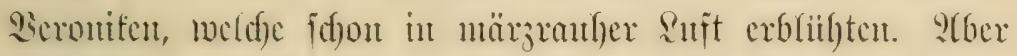

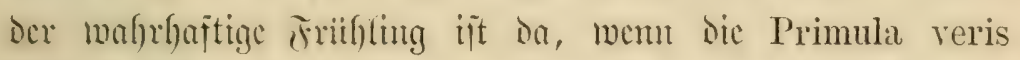

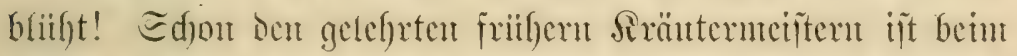

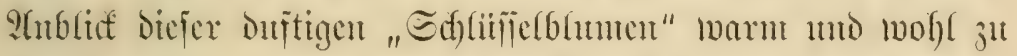
Mitth geworden; fie haben dicje criften Bfunten des Woune= iritflutgs nidft Prima, fondern mit licbfojenton Edyreidfel= morte Primula veris, flcinter Erytling Des Frifhlings, bentant. wie jene Botanifer voriger Sabrfunterte, jo jibertommt aber

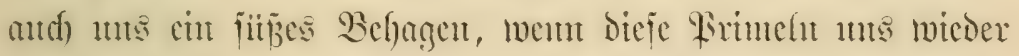

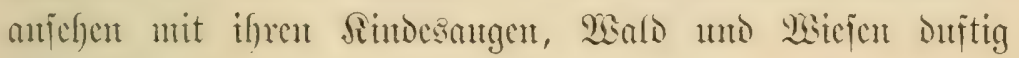
iiberbliibento.

Ghe Der Mat anbridgt, beweits in Der Mitte Des Shril,

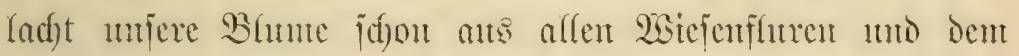
crmadten : Raube am Boden bredfen die gelogrinen, tweidfen, grop̈en Blätter hervor, ju Fiojetten bereinigt; ants Dem Edjooje ber=

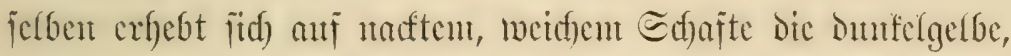
gejternte, duftige Blittgendoloc. Das ift Die Primula veris, Iweldye in Deutiffland weder int (5iebirge, nod) in Den Gbenten irgenomo feflt, dort wic fice in faft jedent santbualde, auf

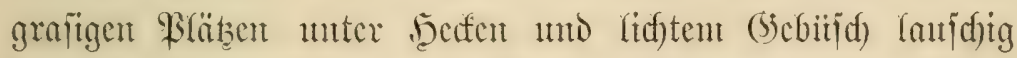
hervorbridnt uno ifre gologelben Edflifiefoolden fourt auf=

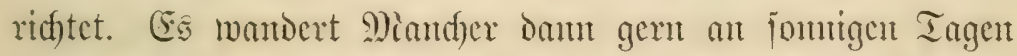

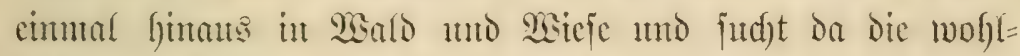
befamten Frimeliftelfen anf, pfliuft Eträtñe jujammen, in Denen man Den Frithling fetber mit geinmimnt. Tagelantg 
ftehen fie mun, bewabrt in (Slaje, an fonntgen Fenfter und es lad)t int ifnen der Walo, der Friflling unt die Wanderluft

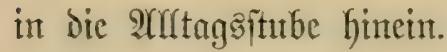

Das ijt die Baubermad)t, mit Der unjere BLume jelbjt Daş berdiuterte Sängerfer's cines Renau beriifurte, ber in alle welt uno ?adjwelt Ginausiang:

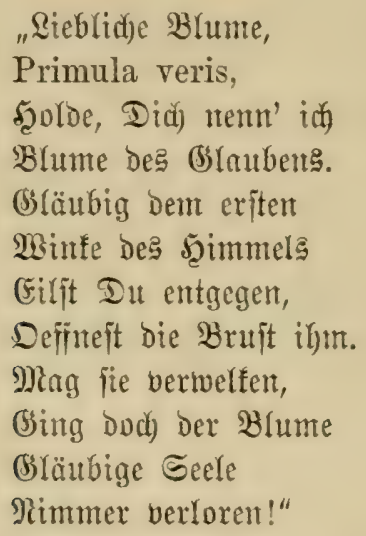

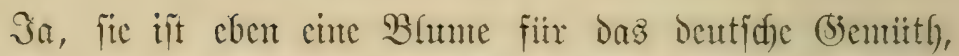

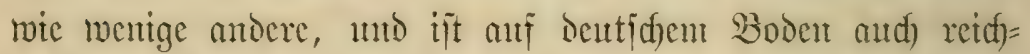

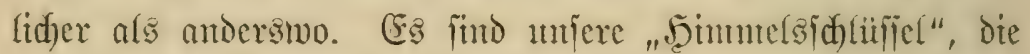

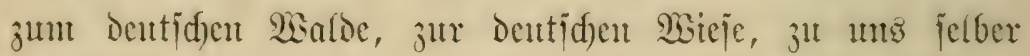
gehören.

Die dentide Sorgeit hat in ifnen jogar mandjes (Sichetut=

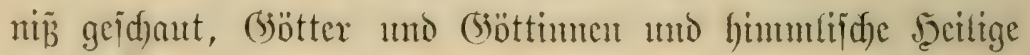

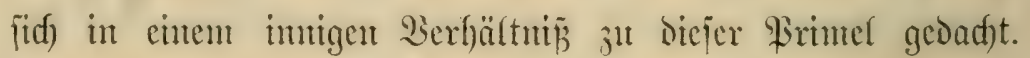

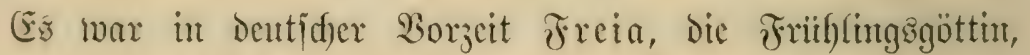
als die Echliufieljungiran gechrt, deshalb ftectte cin golduter Ed)liiliel in ifrer Sironte. Denten, weldfe Wrimeln pflititent,

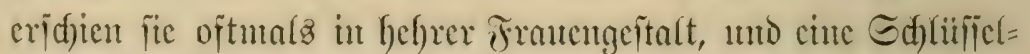
blume, die man in ifrer (begenmart brad), vertich bant dic 


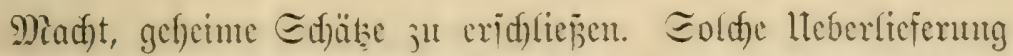

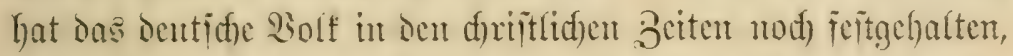
nur auf Die allerheiligite Intugirau Miaria Die themalige Duto Der Freta itbertrager. Eo flingt Der alte Gitaube ntod) alts mandfer fpätern Eage. Şon einem Edjüjor wiro crjählt,

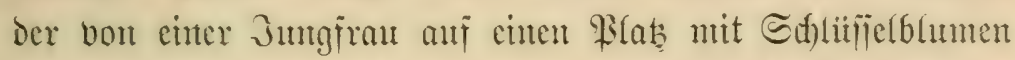

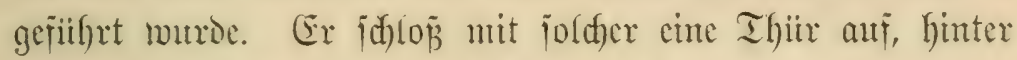

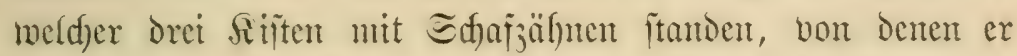

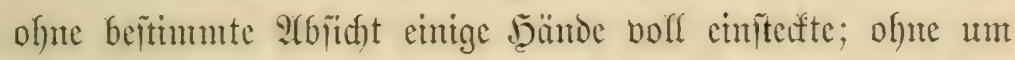

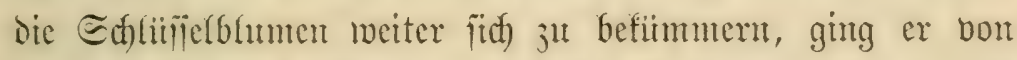

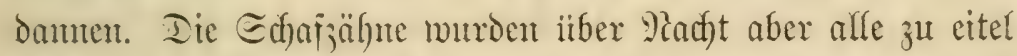

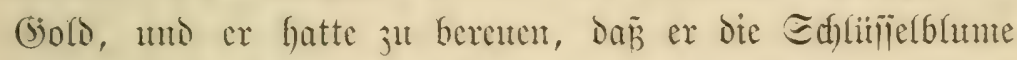

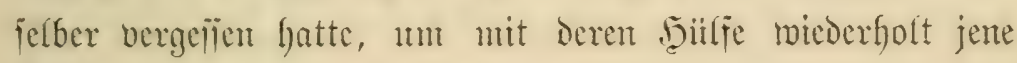

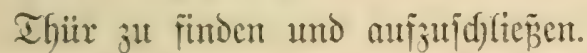

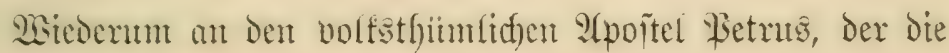

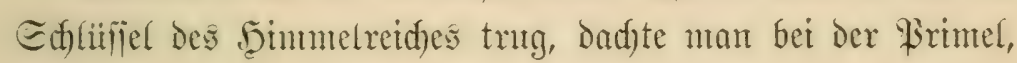

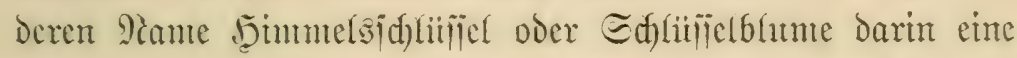
anid)aufidfere Ientung hat, als wern bie ntoderne gedoe mit

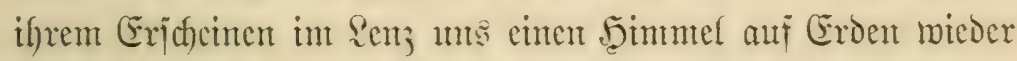

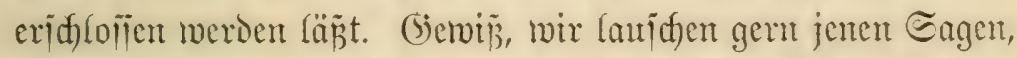

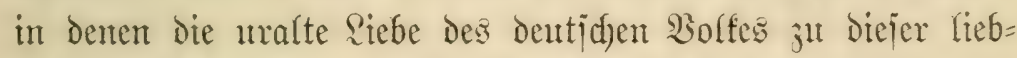

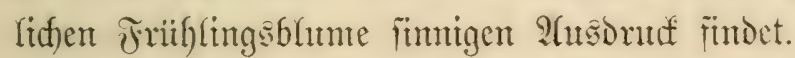

Itnjere beutīchen F(uen tragen indelien nifft cinjig die Primula reris, fondern neben ber edften, für duffenden

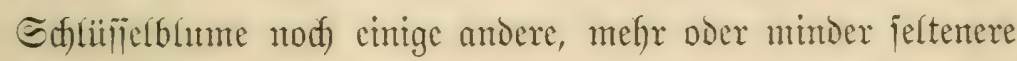

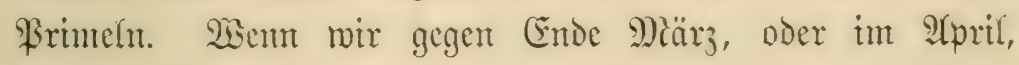
über feudte wisejen, bejonders Wartomiejen mandern, fönten wir die Ed)lanfprimel (Primula elatior) treffen, melde iiberall in Deutidglano jicmlid) Gäufig ijt unto mit ihren 


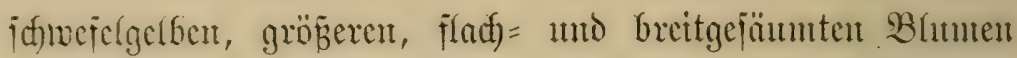

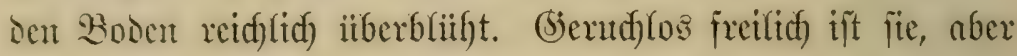
wir pffitifen bod) gern cinen Stran jujanmen, ja bejengen

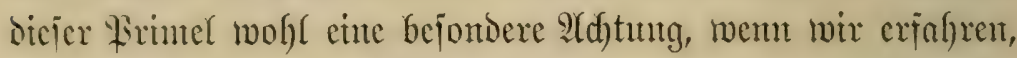

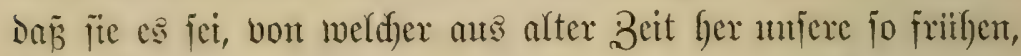

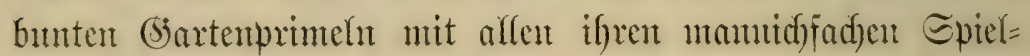
arten gejilidet finto.

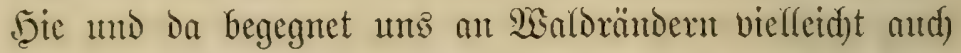

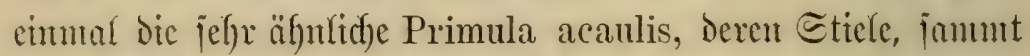
dem Siclde Iangjottig behaart, fajt ftets untr cine curzelue

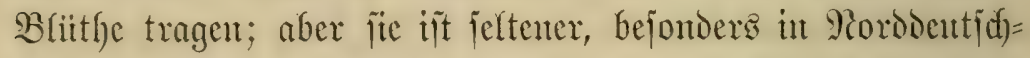
fand jefr jeritreut. Weit jeltener nod) jreilid) ijt bie röthfidf Gliitfende Mieflprimel (Primula farinosa), deren Bititfen=

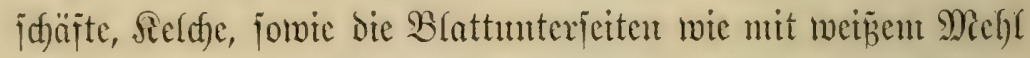

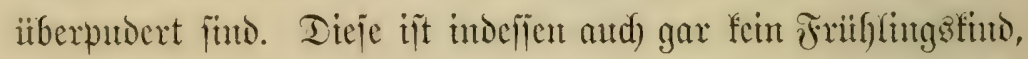

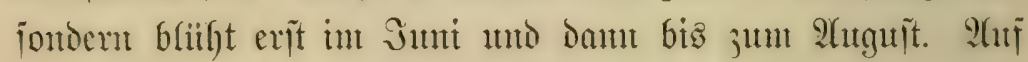

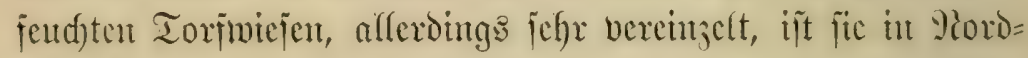

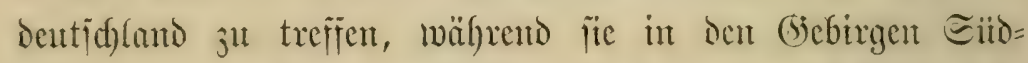

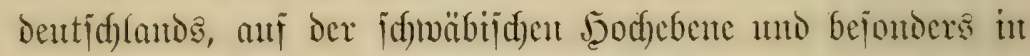
den At(pen inmerfin jiemlidg fäntrig vorfonmet. Lno utr

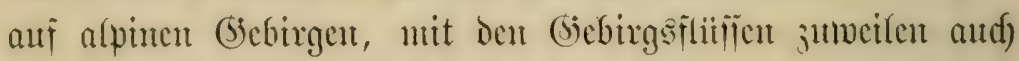

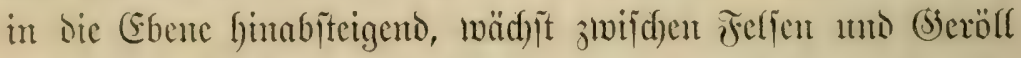

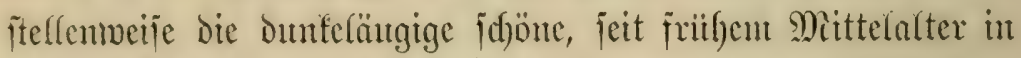
mnjeren (Särten heintijde IItrifel (Primula auricula). Sinto= (id) Die flemite, reizendite aller Brimeht, Die 3wergprimel

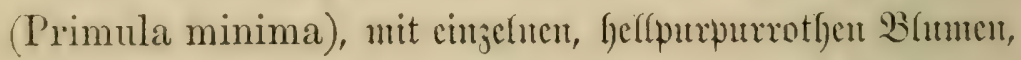

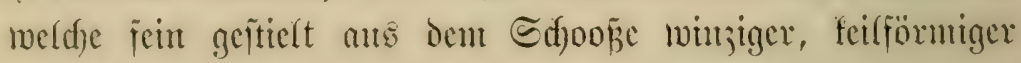

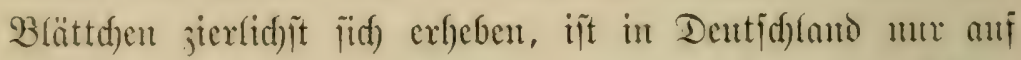

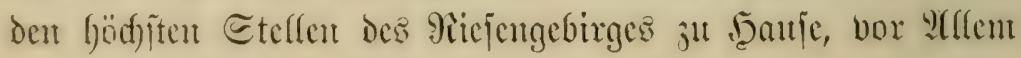




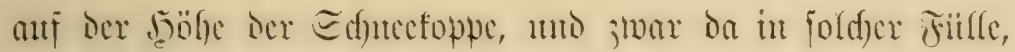

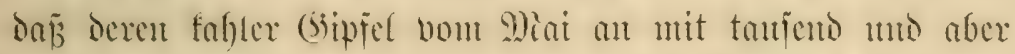

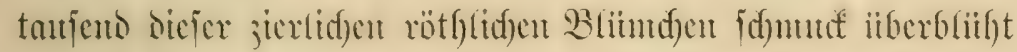
ift mo bant den ganjen Eommex iiber mit ifnen prangt. Cinjelue blithen hod Droben jogar bis in ben Ectober fintent,

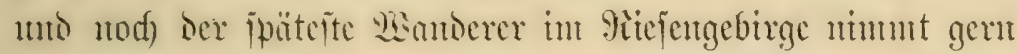
fold) Brimuden bon ser sioppe mit beim, als beren lebtes briiljentoes 2troctifent.

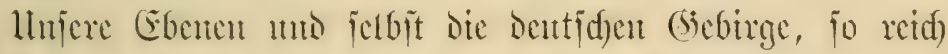

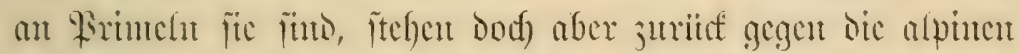

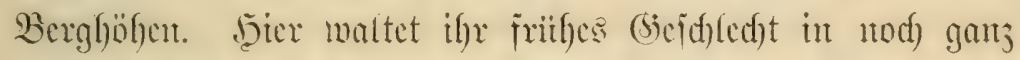

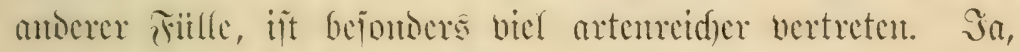

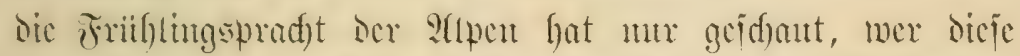
cimmal im Edmunt ifrex firmeln jah). In alle Farben getand)t, in blafictes (bicls bis jum inteniviten (Siolo anto Erange, von jartejten röth(id)en 2 (nthantd) bis jum bremtenditen

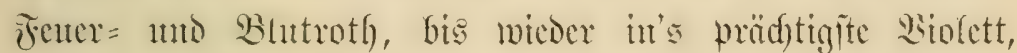

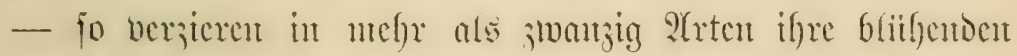

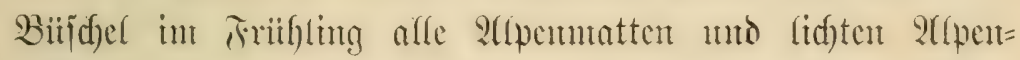

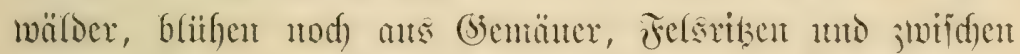
(Sieröll itppig uno mumbervoll herbor.

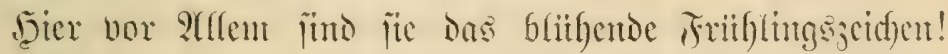





\section{II.

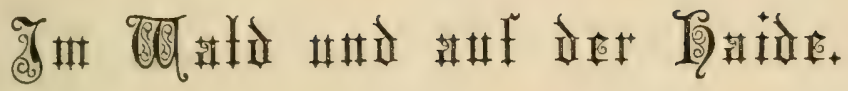

Wie wandert fidh's durch eitren wald fo traut, Wenn nur dic Wipfel nod von Some wiffer, 27ur nod 3uweilen eines Dogels saut Derhallt in ahnungsvollen finfterniffen. Das Juge Fann fein Chier des Wald's erfunden, Ein Eicḩorn nur erblict't idy in den jweigen, Es fam behend urd fill und ift verifwurtoen, Die Einfamteit des Waldes uns zu zeigen.

And doch hier lebt des Eebens welithe fülle! Ein ftumtmes Räthfiel, Jas fich thie verrathen, Die Pflanje ift fein Bild und feine Gülle, Uno allwärts grünen feire ftillen एhater.

Rertau. 
Die waiber find der Stol3 unferer Erde, und aud dic unter ihren fhattigen Wipfeln heimifhe Blumenwelt ift ftoljer, eller, vornehmer als die meiften andern Pflanjen. Die aus laujhigem Woldesidhoof in Exhabenheit aufiteigenden ธaubbäume, als der grofartige Gintergruno oer ioyllifhen fleinen Slumen, heben auferdem deren zarte Sđjönheit now bedeutjanter hervor; und

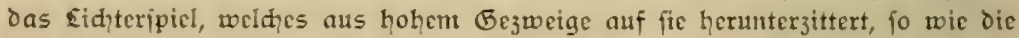
mäbige Dertheilung von Eiđht und 5 dhatten geben ben waldblumten all die behagliche Beleuchtung, weldic dent Bemuthe fo wohl thut. Der wald gerade überrajat jugleid Durh eine Mannidfaltigfeit feiner Blumenflor; es werden hier Jurch verịhiedenes Maaf ber Bejhattung, in folge davon aud ber feuhtigfeit, Durh die diđhtere obcr geringere Bobendede die verfhiedenten

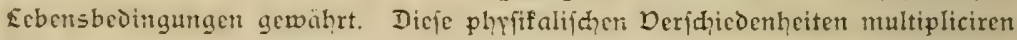
fich wicder mit jeder cigenartigen Bodenbejhaffenheit; Dent ein humusfandiger Walogrund hegt zum Cheil ganz andere pflanjen als der bruchige mo moor: croige, welcher wicoer bejondern Slumentharafter aufweift, uno oer falfigrundige Wald deutet uns jhon Durh jeinen auffülligen Reiḑthum an Orhideen eine wiederum cigenthümlithe Degetation an. Sollte dicje Dertheilung nicht auch nit

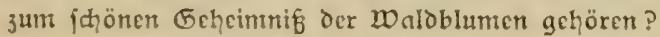

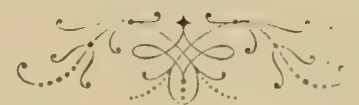




\section{1.

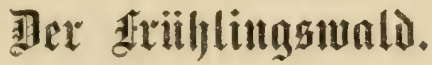

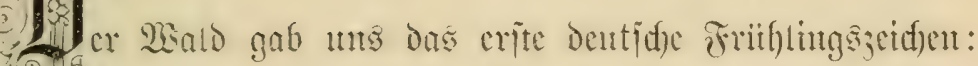

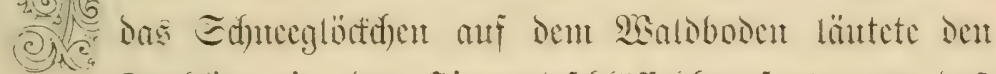

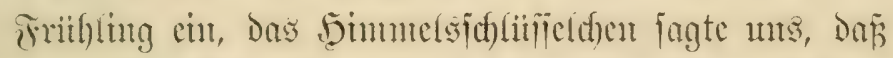

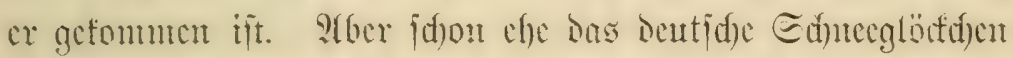

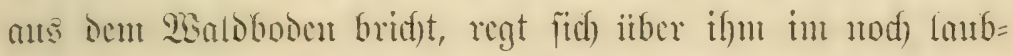

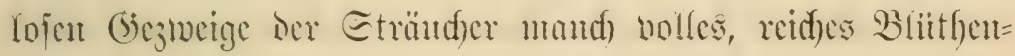
reben.

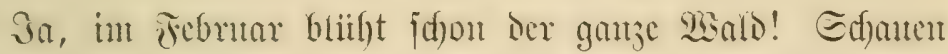

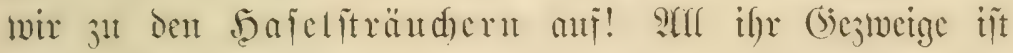
fdfon bolf goloftantiger Bliithengefünge, wont Deren ber= fdomenterifder lleberfitlle das Gebiifde bie und ba fait

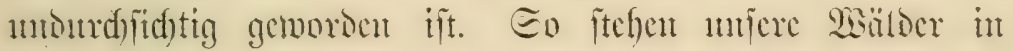

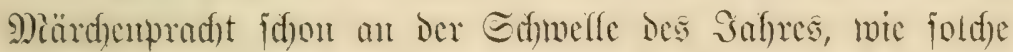
jelbît im vorgeid)rittenen Frithling mo Eommer faum cin

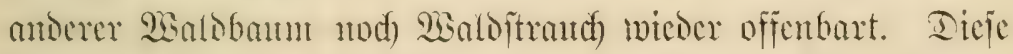

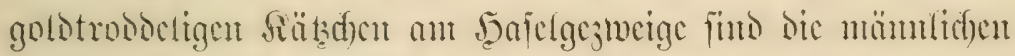

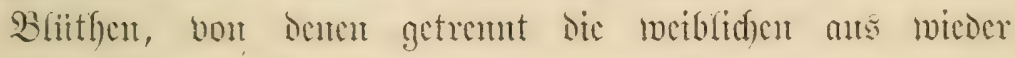

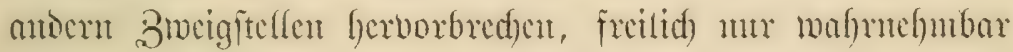

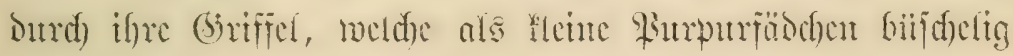
aus cinzelnen :sinterfnogpen fid) herborjtrecfen. 


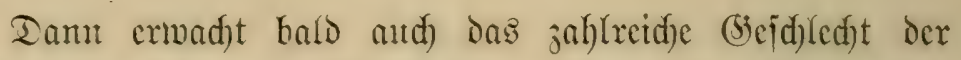

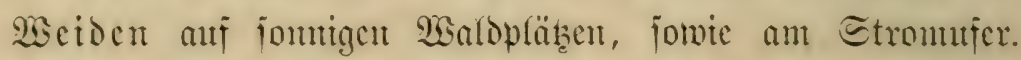

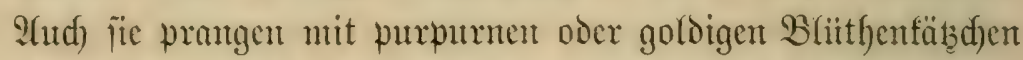

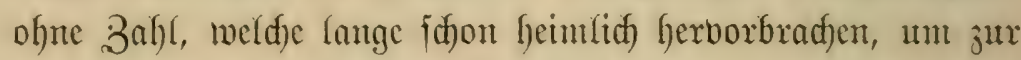

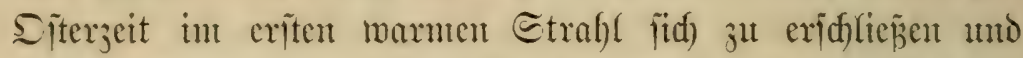

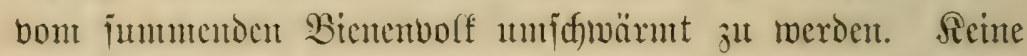

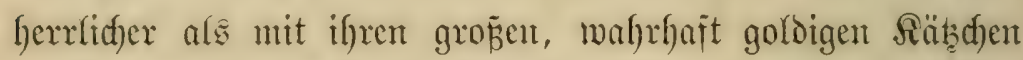

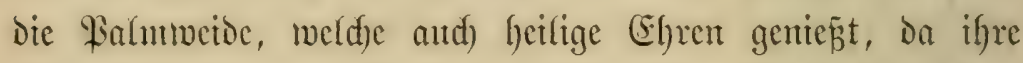

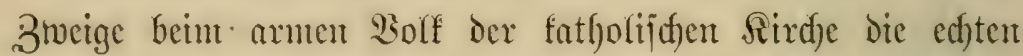
Bafmen cricken, mit Denen in Font am Fafmpontag bie

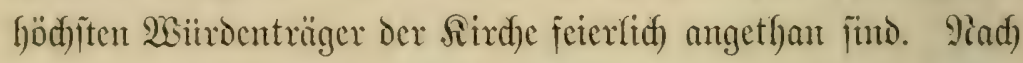

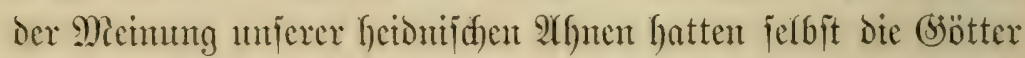

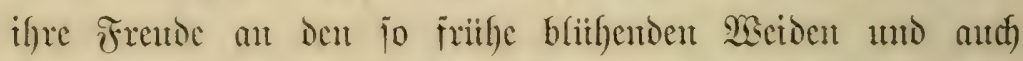
ifree Danto dabei in Epicte. Dom "wem im Frithfing bie

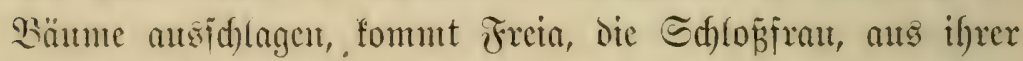

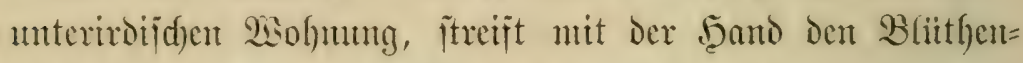

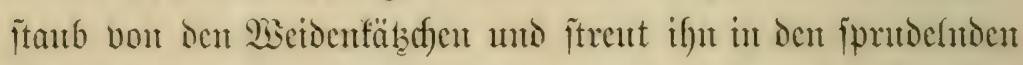

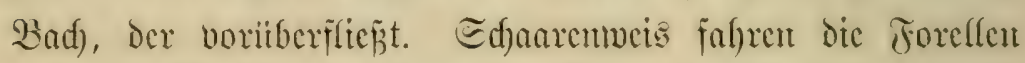

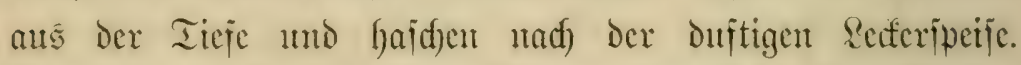

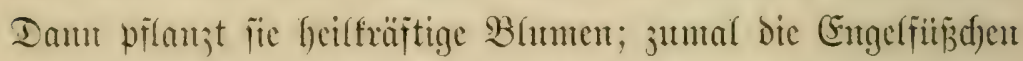

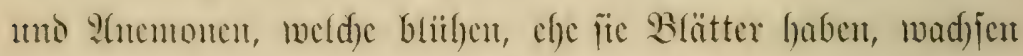
unter iffer gejegneten Danto."

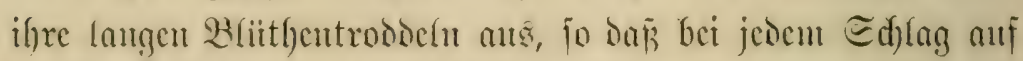

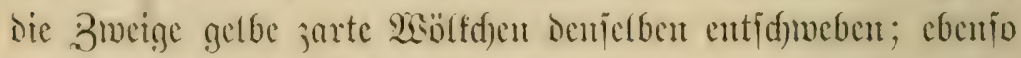
die berlibe oder seruteffirdfe (Cornus mascula), melde

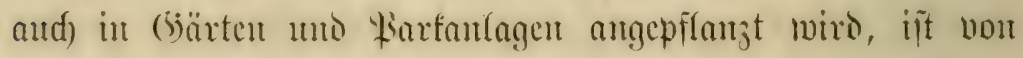
if)ren winjig itenbliitfigen gerben Dohben jest wie mit

*) Mannharot, Die bcutidje (s̈̈tterwert. 


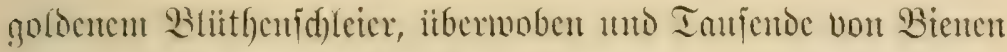
jummen uto brumment in if)rent (jojweige. - Lhto alle dicie

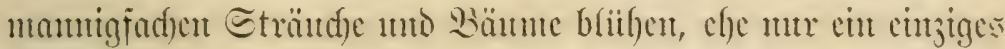

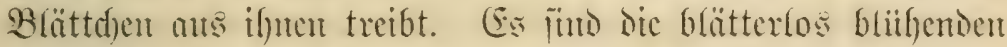
Etründfer Der crîten Frïlylungsjett, deren Blätter crit fonmen,

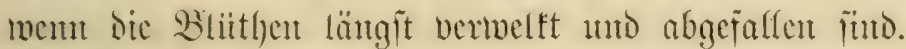

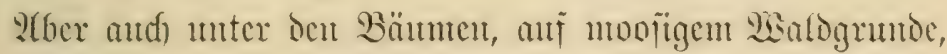
jwif̈den bent tooten gelbbraumen Sautbe des vorigen Safores

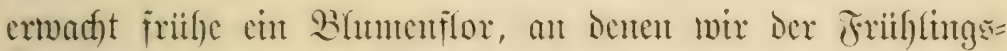
fieblidffeit uns frenten fömen. Freilid), nidft alle foldse iritfeiten Saloblumen wadjen in jeocm saloc, jie gefören jum Theil ju ben feltenten Piflanjen, aber bie che oder die andere wirt

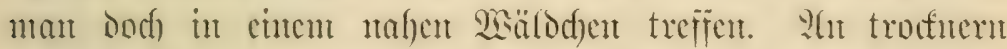
2ialditellen ïutgelt vielleid)t das blaubliutbige Miär; = oder

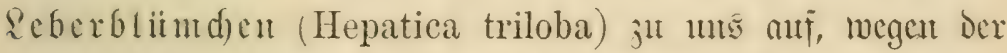

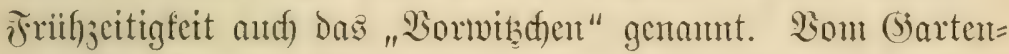
becte ber fenut cs Jeder, deffen autd) gefiullte und roth and meip fultivirte grop̈äugige Blämdyen ju reidjen Buifjeld)en beijanntentelen und in 9)

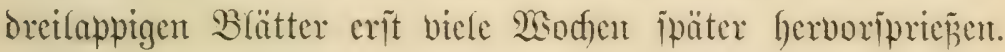

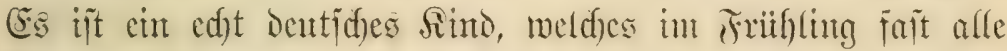

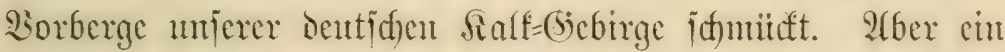

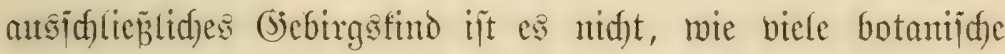

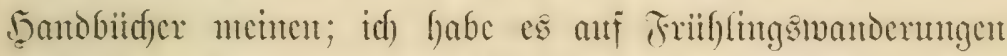
in Pord = und 9)

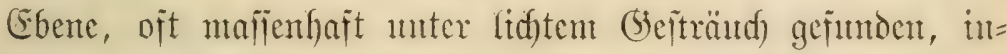

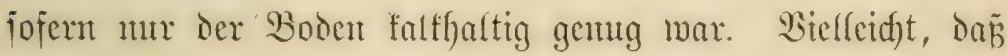

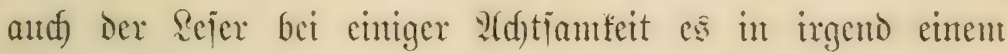

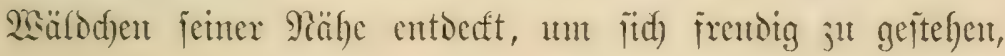




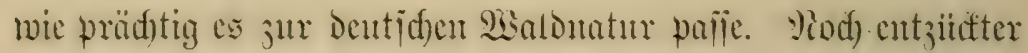

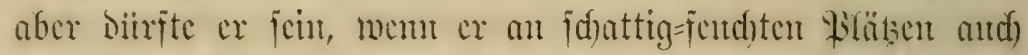

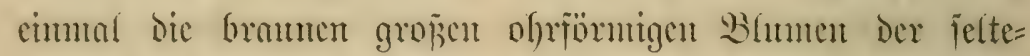

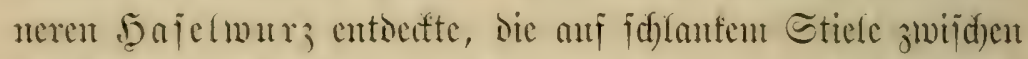

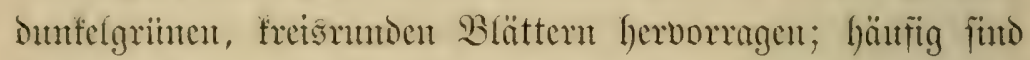
iie unter branum, abgejallenem Bammlanb verifteft und Daber

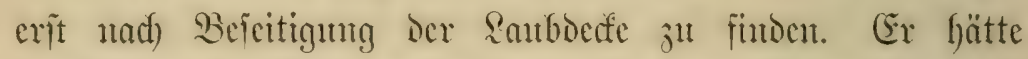
Dam die beften Wunder der criften Maldestlor jun Etranße beijammen.

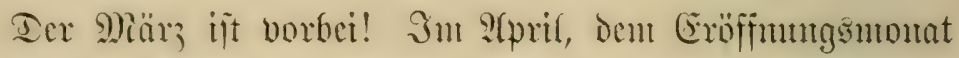

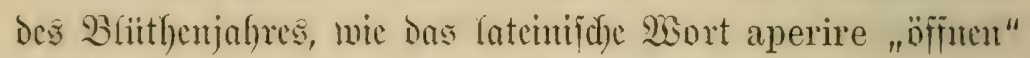

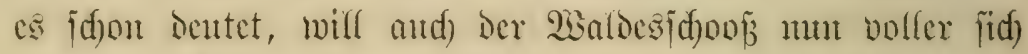

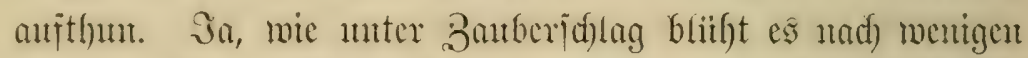
marmen Ingen alliiberall, wo bordem mu melfes Panb lag.

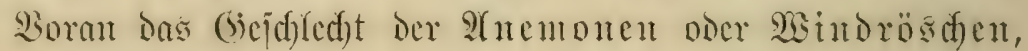
beĩonors dic Dainaumone (Anemone nemorosa), Deren

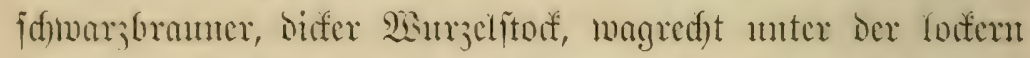

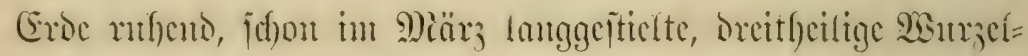

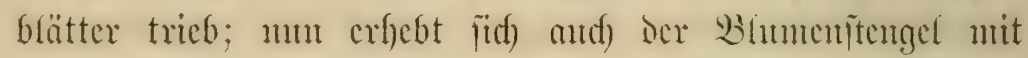

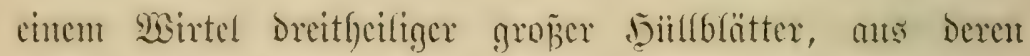

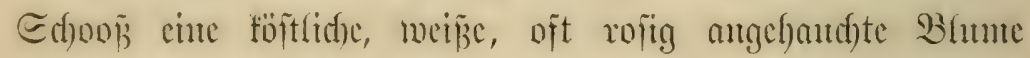

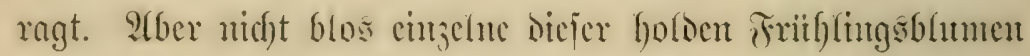
fonmen bic uns da herbor; jwangig bis humbert anto tanfento

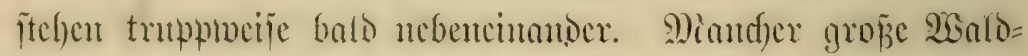

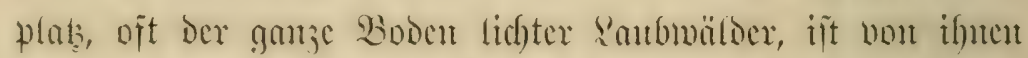

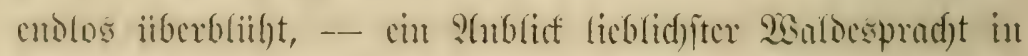

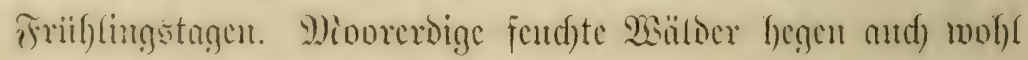

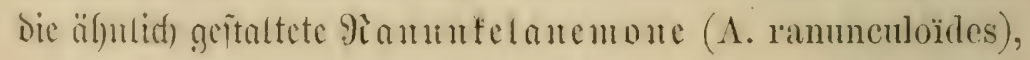

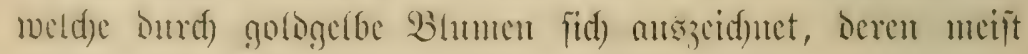




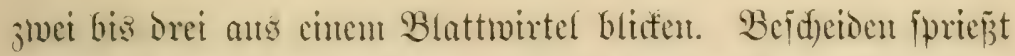
neben all befen weiñen ober getben :Hemtonen bas midjeintbare jarte Mioid)ustraut (Adloxa moschatellina), ocfien in Der Eroe rulfenoer fleijdiger, pertweip̃er, herjiftuppig gegliederter

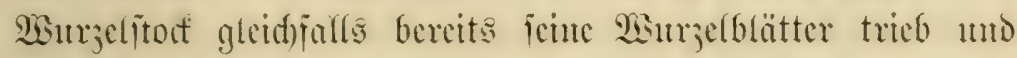

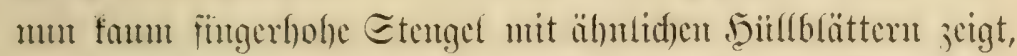

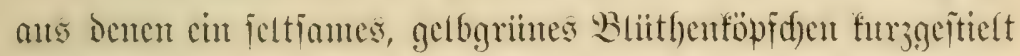
ragt; cuten umerfliden 9)ioidnusgerud) fömten wir an bent= felbent wabruelinen.

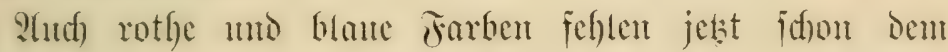

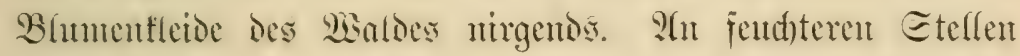
bietet jic Das \&ungentraut (Pulmonaria officinalis), Defien anjefnlidge blaurötblid)e Bliithentranben, anf ranthblättrigen

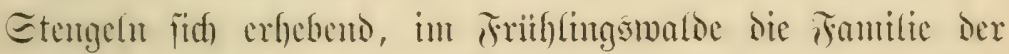

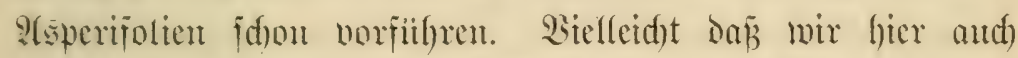

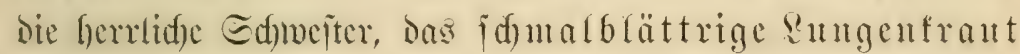
(P. angustifolia) mit ienten munerbar ajubfanen Błımen

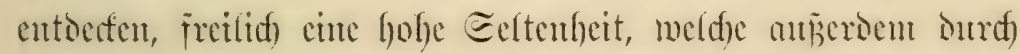

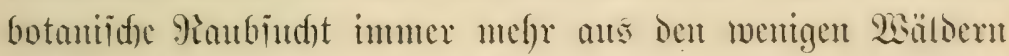

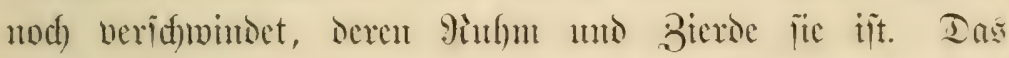

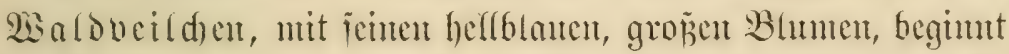
mud) allermärts mu bier ju bliihen; Die Trithliugswiofe (Orobus tuberosus), dicier erife Sdunctterfingsblithler Des

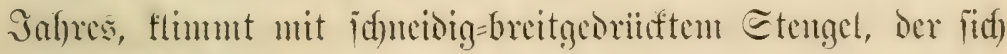

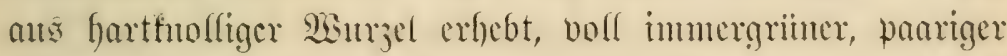

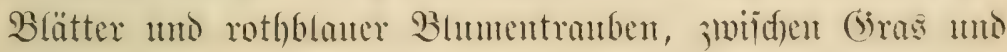

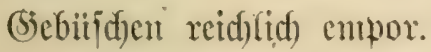

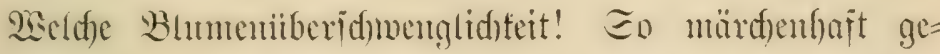

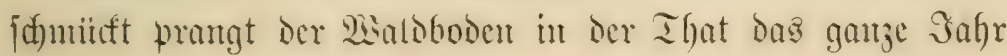




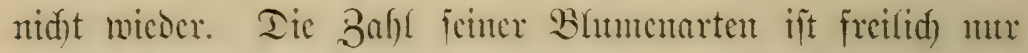
flem, afor in einer 2ffles itberblithenden Fitlle beffeiden bor

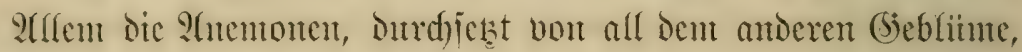
jeglide lidfte Etelfe miferer Sanbwälocr. Sa, der 2(prif ijt die Parndiefeszet der Waldgrimbe.

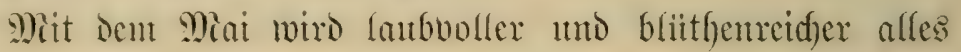

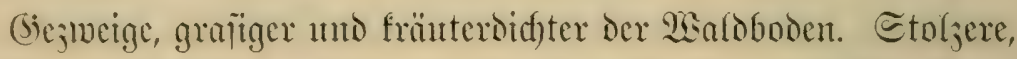

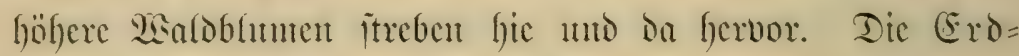

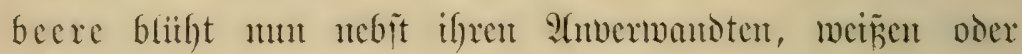
gelben fingerblätrigen Fotentillen. Die Miaiblunte unt Deren 2(nverwante, Das Buciblatt (Majanthenum bifolium) uno Dic Eicgelblunte (Polygonatum multiflorum), öffuen ihre Eilbertranben; die giftige Einbecre (Paris quadrifolia) uno Das neifelartige Bingelfraut (Mereurialis) treiben in

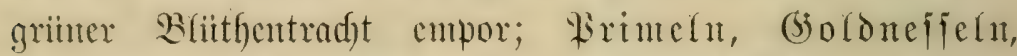

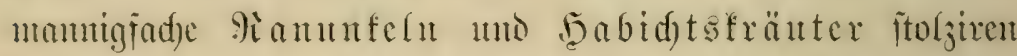
mit lidgt = oder Dunfelgelben Bhmen cinther. Dic feinen

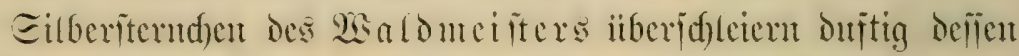
iipplge, wirtelblättrige Etengel. Alber an bie bliilfentoc

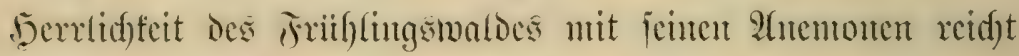
jelbit dieic mannigfaltige, cole slatflor nidft liman.

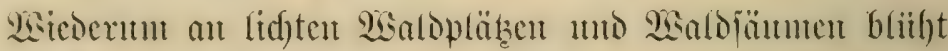

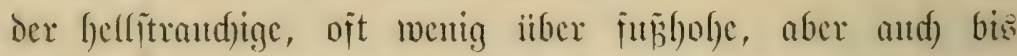
meterfohe Ecibelbajt oder ficllerbals (Daphne meze-

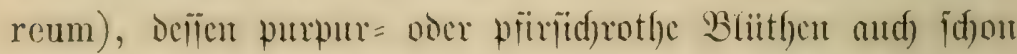

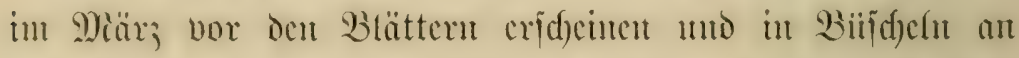
den Eettu der holjigen Etengel jiben. Freilich ijt dicie

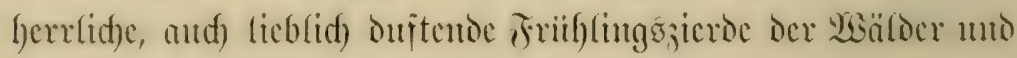

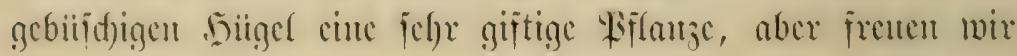




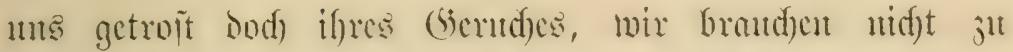

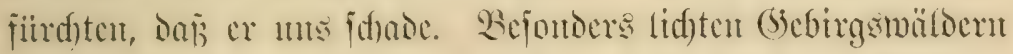

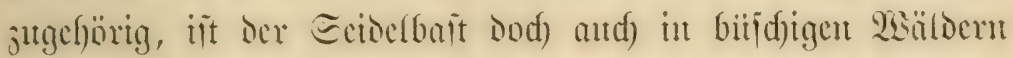

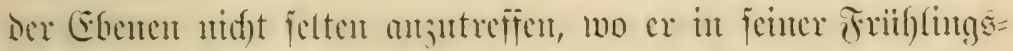
beitthe nidst itberfeben werden fant, aber wir werden ihn, aud) ment or abgebliibt ift, allerorten leid)t erfenten, intom ber Etengel Dan mit cinem enditütoigen Biifd)el lanjettfidjer

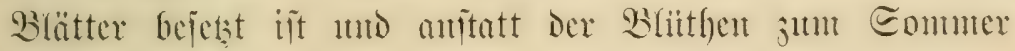
jd)arfadrothe, crbjengroge $\mathfrak{B e e r e n}$ ifn verzieren.

\section{昰er Sijlehourn.}

D)

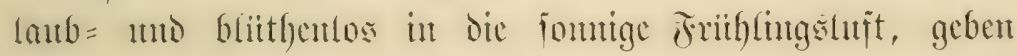
if)u nod) inmer jenc winterlidfe Dutrd)ïd)tigfeit; allmälig

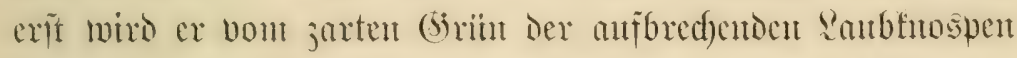

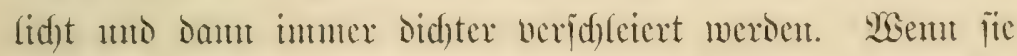

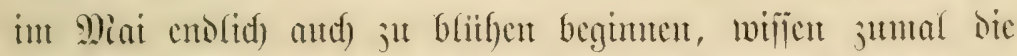

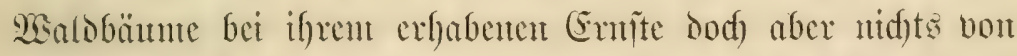

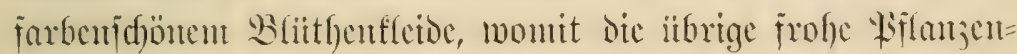

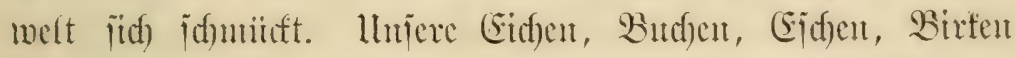

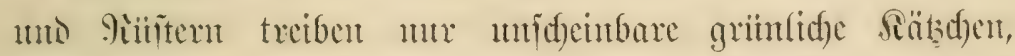

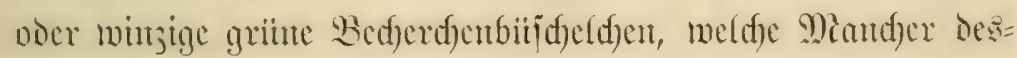
galb nod) niemals bendete. 


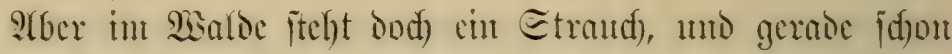

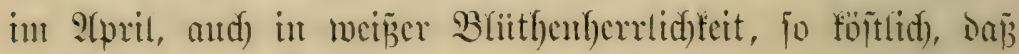
twir gern wohl eine Weile davor ftehen bletben.

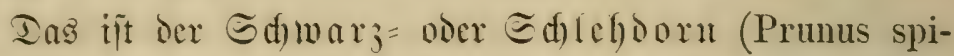
nosa). :(ts fömte $\mathfrak{c r}$ bic Beit gar nidft crmarten, fteht

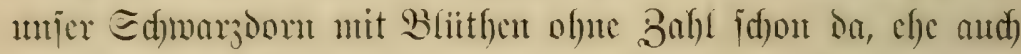
cr nod) grïuc Blätter getrieben hat, welde exft in cintgen

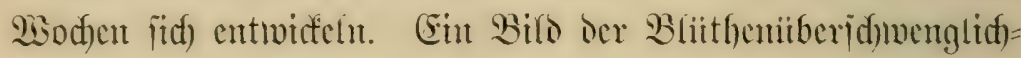

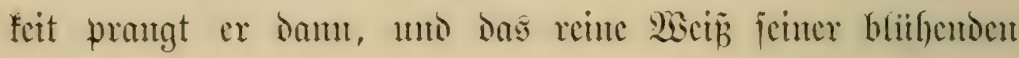

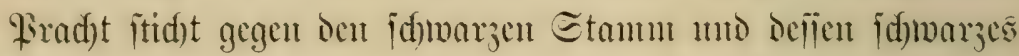

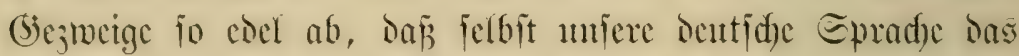

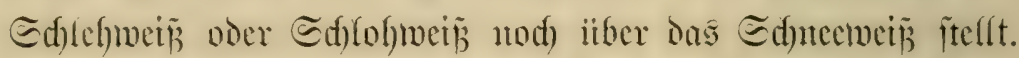

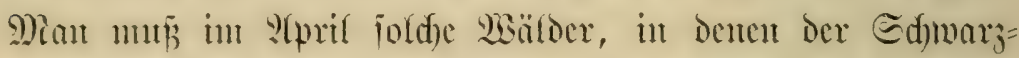
Dorn weite Etreaten iiberwäd)st, cimmal geidjant haben, Im

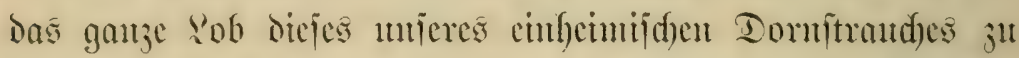

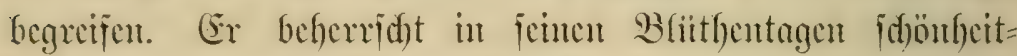

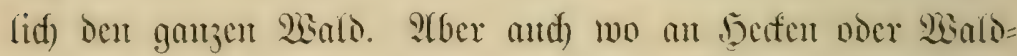

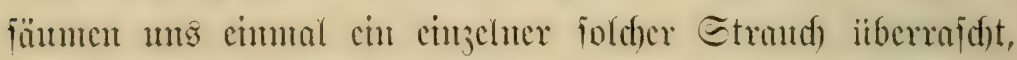

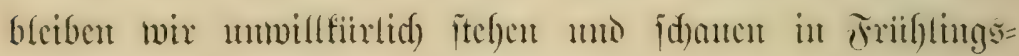

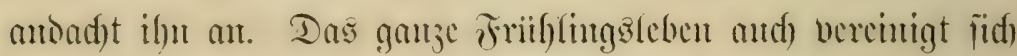

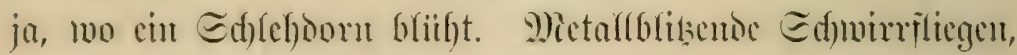

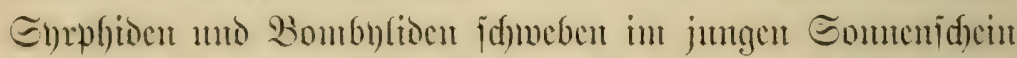

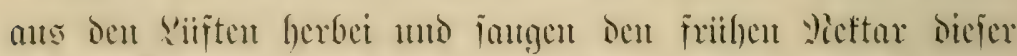

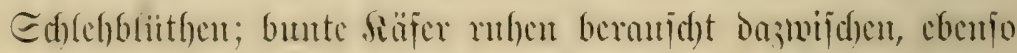

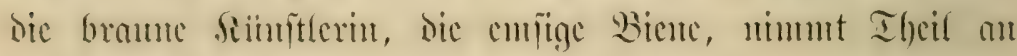

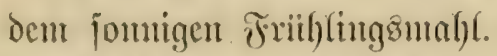

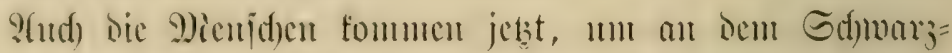

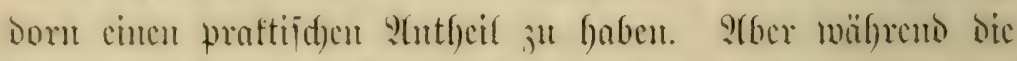
bejd)wingte Iljicrmelt jid) mit lcid)tem Siajdfen begniigt, ranfen 


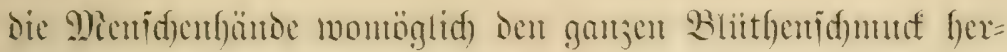

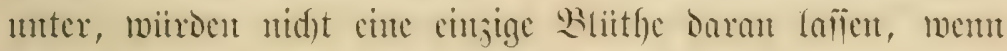

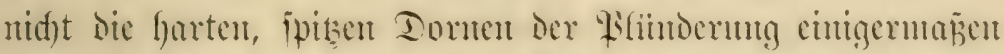

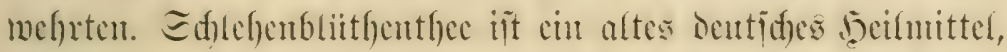

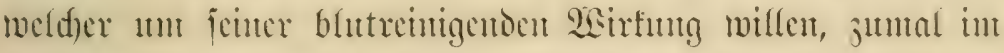
Irilgling gitruten, nod) heute in guten Ehren iteht, aber

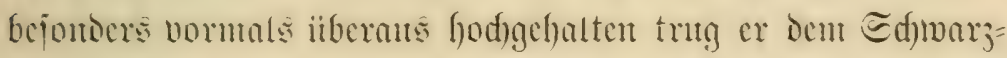

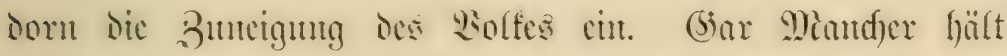

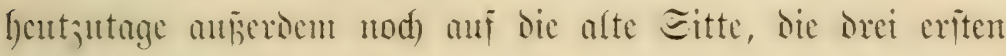

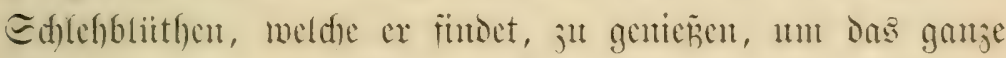
Salfr ïber gegen das Fieber gejtdyert ju fein.

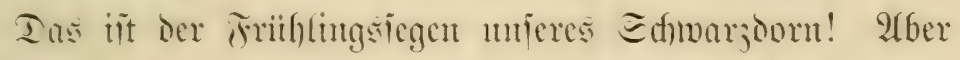
er reid)t uts and) cinen berbitiogen in jeinen futgelmuten,

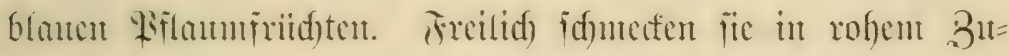

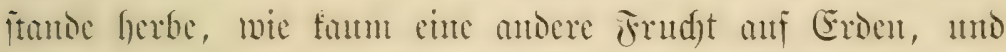

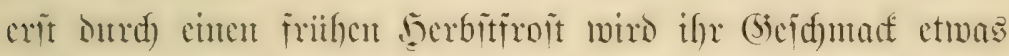

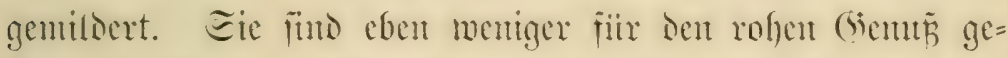

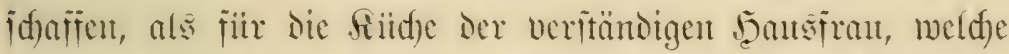

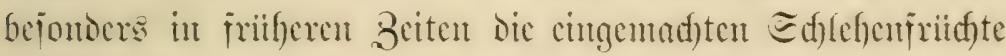

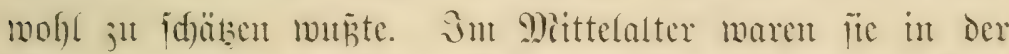
That cine iiberans belicbte Ielicatelie, oer in mandfen fulli=

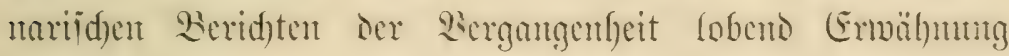
getbant wivo.

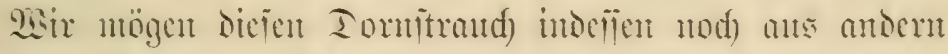

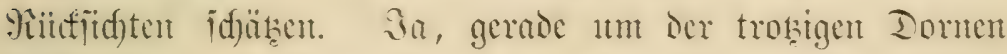

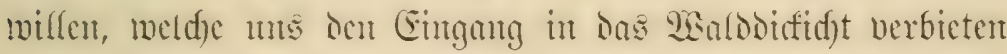

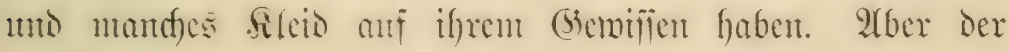

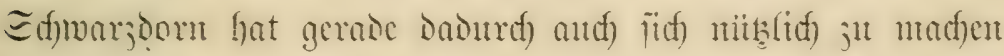

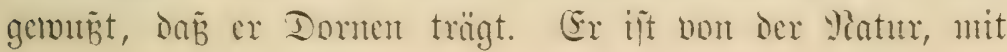




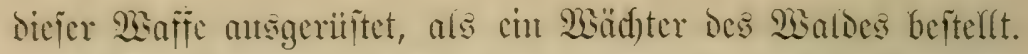

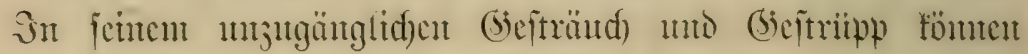

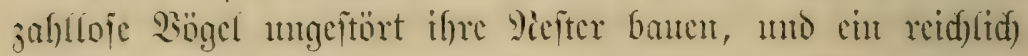

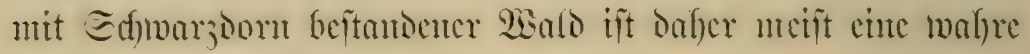
Bogelferberge, wo es fünt uno funtertert, ruft anto flötet ans jahllojon jangestitatigen fleinen fieblen. Sn mierer

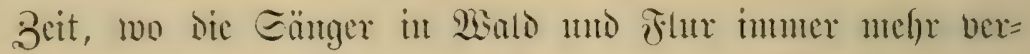

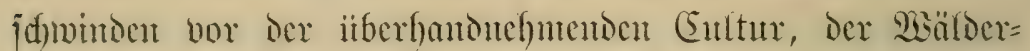

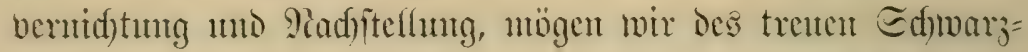

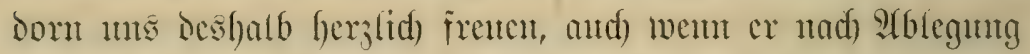

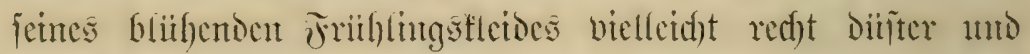

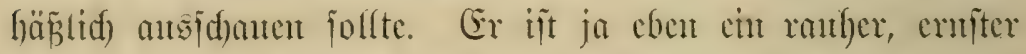

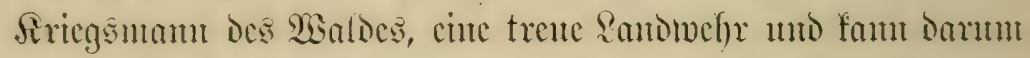

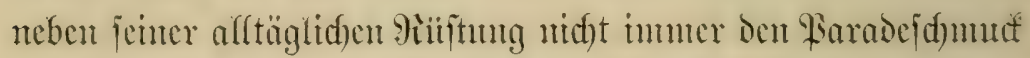
Des Blithengemandes tragen. Darnm wiro $\mathrm{cr}$ aud in mueren Felonarfen, Rarfanlagen ant Gärten als Gichege gerat ber=

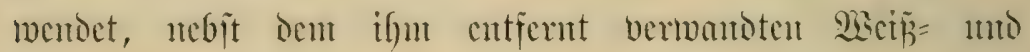

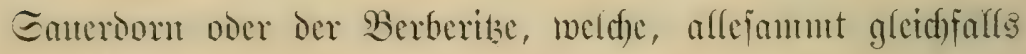
mit idfarfen Dornen oder Etad)h ansgeriiftet, fid als folide

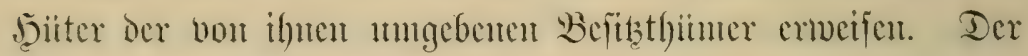

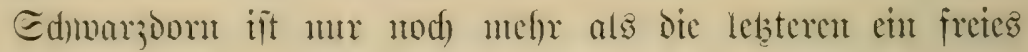
Waturfind, hat and robujteren (Sharafter, weshalb or wor allem añ Dem Sande als Umbegung beliebt ift, für bie (Särten Der Bantern als lebentige Mianter vermentet, forvie man ifyn and) gern un die Becidepläbe pflanjt, banit or bie Seeroen von den Felonarten abhalte.

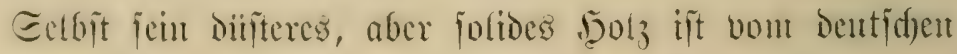
Wolfe uid)t verad)tet; ç Dient nad) altem நerfoumen Dazu,

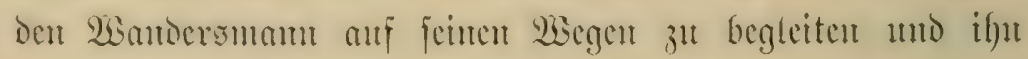




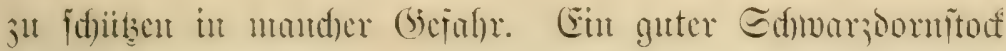

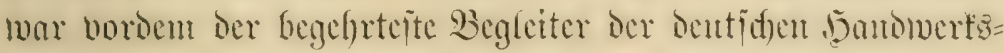

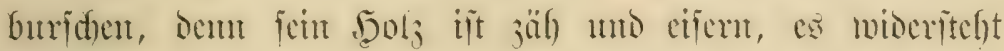

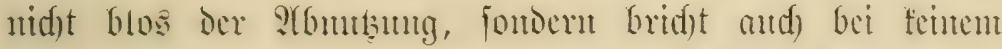

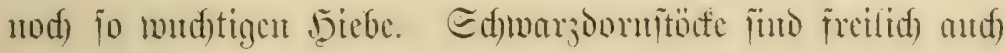
jeşt midft anper Sinrs getonmen umb werden nod) biel, jogar äuperị fauber gearbeitet. Dod) cin edfter folder, wie ifn

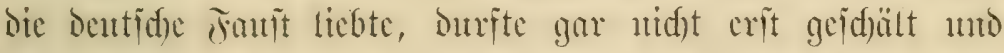
polint merocn, fondern wie sie Siatur ibn in meijt fingerdicfen

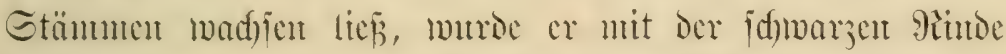
gentonnten; dic von sen abgeidnuttenten a(ejten fidftbaren Siarben und Sinoren mad)ten ifne mu atm fo idjöner uno

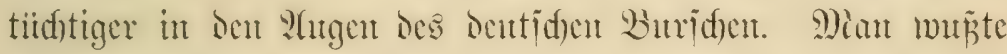

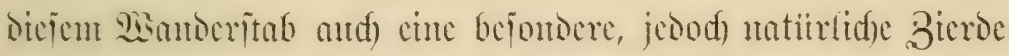

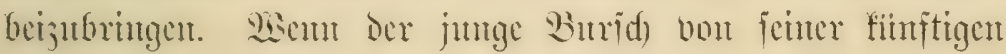

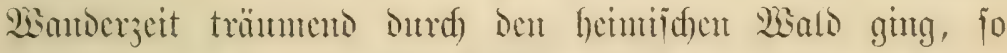

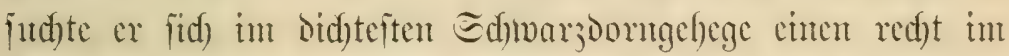

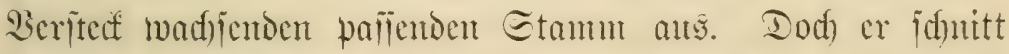

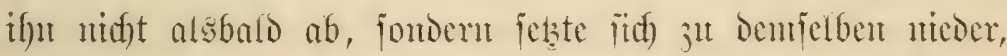
holte jein Iajdenmefier hervor und iffuitt in bie Rimos

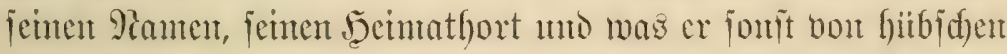
3eidjen und Beidumingen auf Dem timftigen Wanterifabe fid mïmidte. Ex verrieth Jientanden die Etefle; Der Etanm muths weiter, die Edyntte itbermallten langfant, aber fither, Into oftmals ging oer Burid) fin unto fah), we es gerathent. Went Damn nad) Jalfr tut Tag bie Beit getommen war,

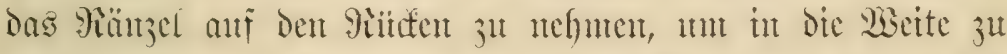

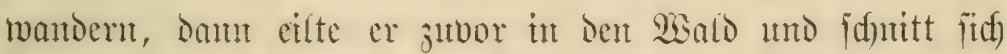
endlid) den antservählten Etamm Gerunter. Defien Ginde 


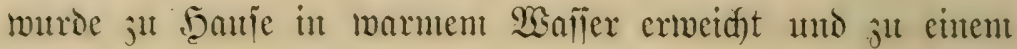
Srituffafen gebogen. Seşt war Der Wanderitab fertig, und mit trefffidyen 2arben itand darauf, was vorden bedentiam anf ilyn cingejdnitten wax. (5x wax feinem Befiker fortan

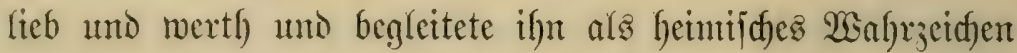
und trener (Sienofle mu in die ferne Frembe nit fintants.

Sa, ob es nidjt ein edler Dorn ijt, dent der deutiche Malo hegt! Miöge man ifn nidjt blos adjten, wenn ex in

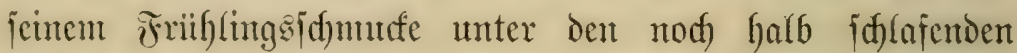

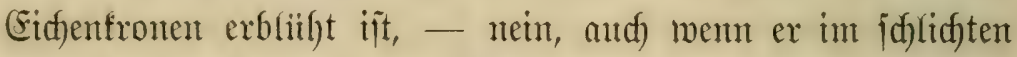
Dornenbarnif́, mit mur unanjefnlid)en Blättern begrïnt, uns im Walde entgegentritt!

\section{3. \\ Dir Mnaiblume.}

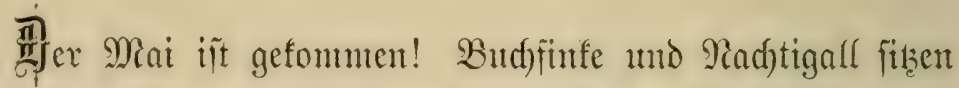

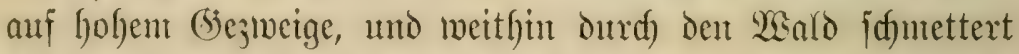
ify helles Morgenlied. WGas fie fingen, flingt wie Miaienluit. Ulles ijt Riebe, Freude, warnter Eonmenjacin!

Da idfallen audf Sinderitummen Durdf ben Wald: "Wier fint fic und fiex wieder welde!" Was anderes bem als

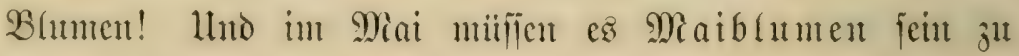

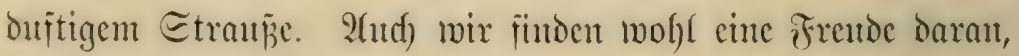

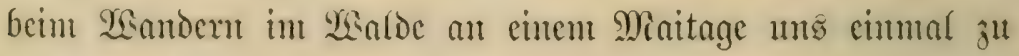
bürfen mad) Diejer coelften Duftigen Waloblume. 
Wählen wir ans ber reiden flor des Miamualdes fie

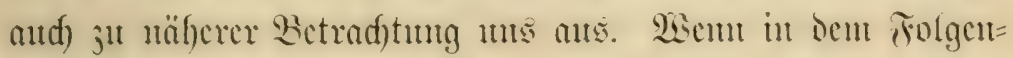

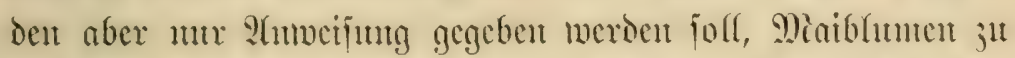

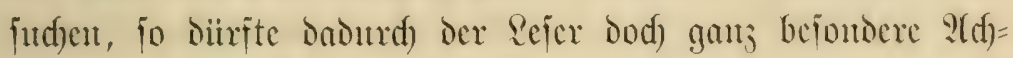

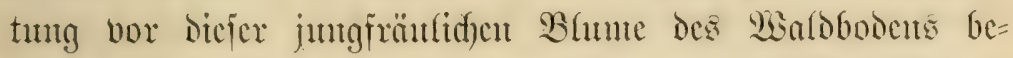

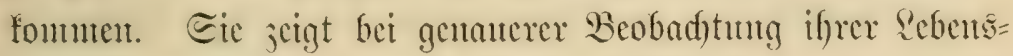
meife Eigenthiunlidffeiten, weldye uts ftifle Bemmocruntg $a b=$

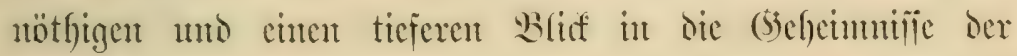

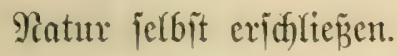

Die Miaiblume, jo allbefant mo allgepricien ifgr Yame ijt, möge vor 2 (lfent utd)t fiir cune afferorten in Deutid)lano

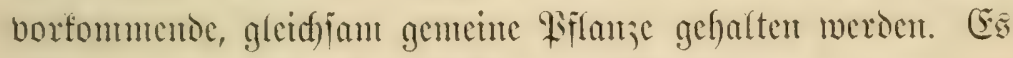

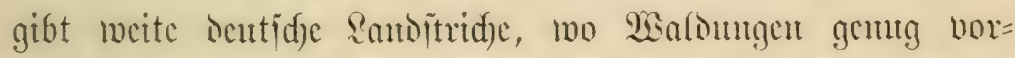
hamoen funs, aber durdjaus feine Maiblume vorfoumt. Das

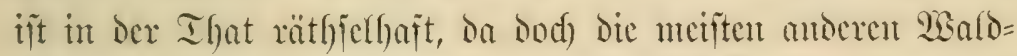

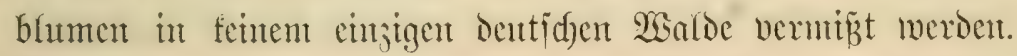
Wir möd)ten geradeju meciten, dẩ die Maiblume durdy dicjes

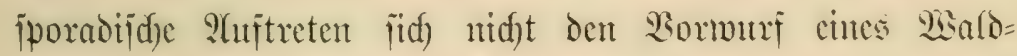
unfrautes maden lajien wolle. Wo jie mut cimmal crid)cint,

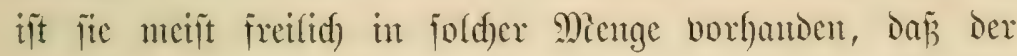
ganje Malsboden mit ifyen colen Sieblätter ïberwadjicn ijt.

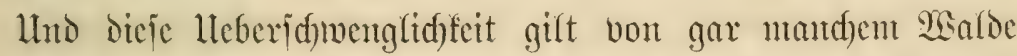
unjeres Dentidyen Baterlandes. 2tber der Preis des Miai=

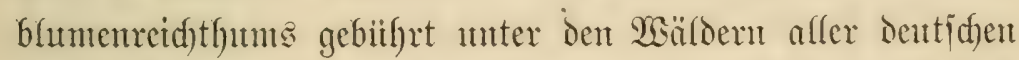

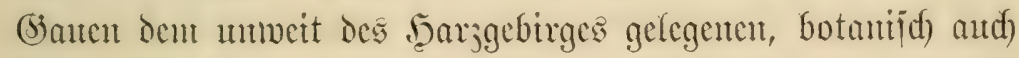

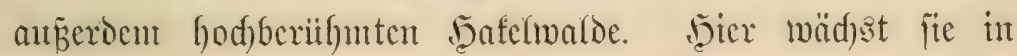

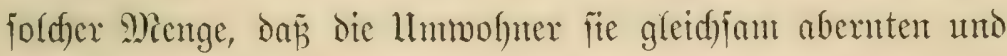
reiden Ermerb Davon haben. Ein armer Walobewohnex, welder nebjt feiner ans mefreren Findern bejtefenden Familie 


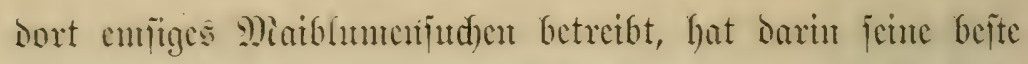

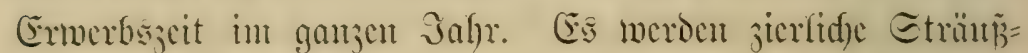
d)en gebunden umo jeden Iag fo viel dabour nad) Dex näd)

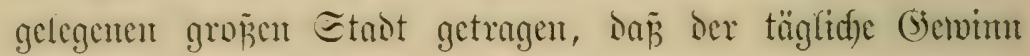
fiç) auf ctwa neut Marf beläuft.

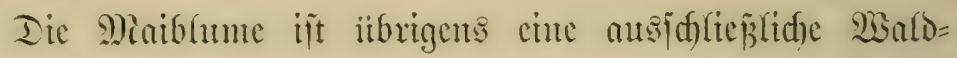
D. h. Edhattenuflanze; in birecten Eommentidhte fommt jie $a b=$ jolut nidgt fort, int fomigen Freicn angepflangt, geht fite baldigft

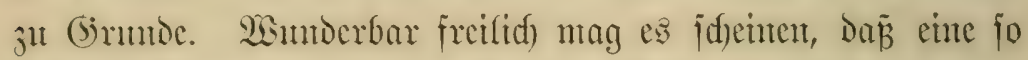
fräftige Siflanje ben frohen Giei; bes Sichtes nicht leiden mag,

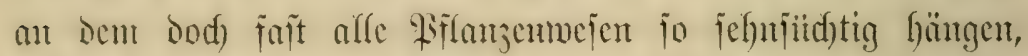

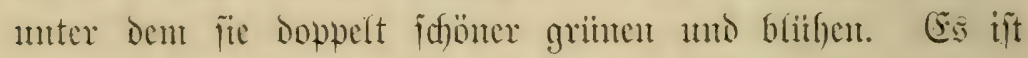

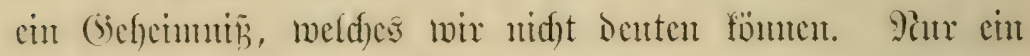

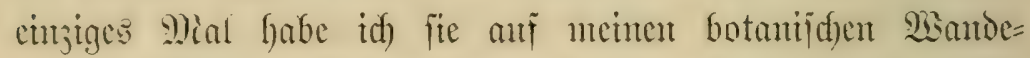
rumgen alferoings aud) an cinem foutigen Srte getroffen. Eis

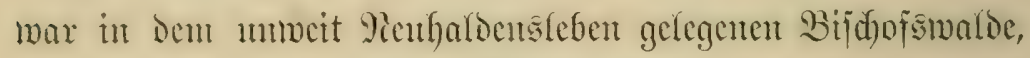

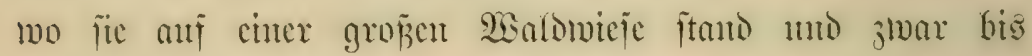

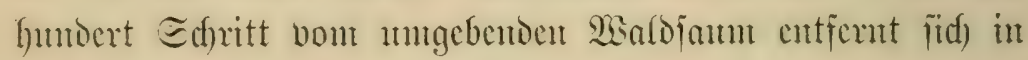

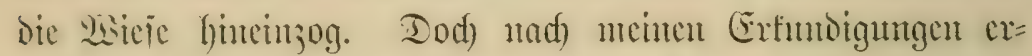
flärte fid) aud) Diejes auffällige Borfonmen feldr cinfad): jente witeje mar nod) vor ctwa drei Jalbren Wanto getwejen, ntmo jum genan bis ju der Etelle, wo dic \$llablumen in

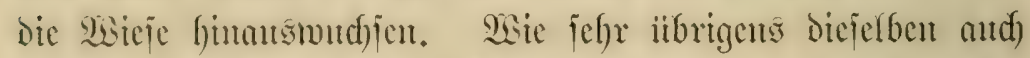

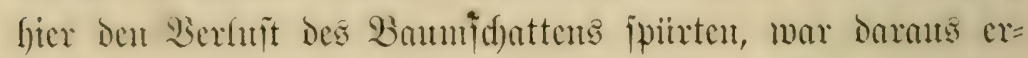

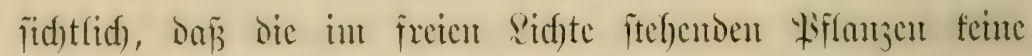

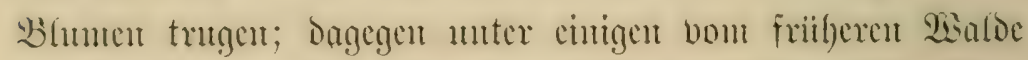

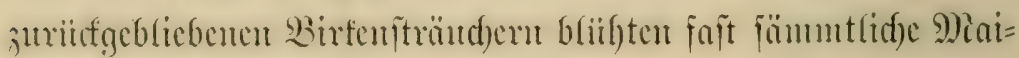
bumen. Ier Fan jeder dicjer Birten war wie mit cumm

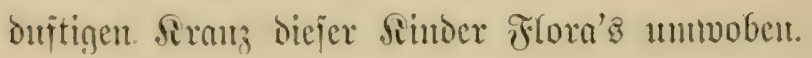




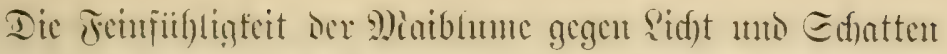

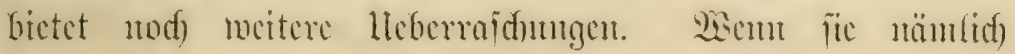

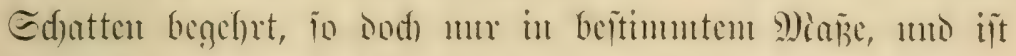

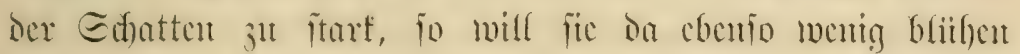

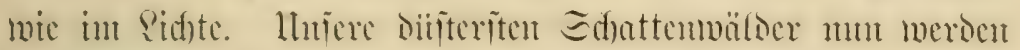

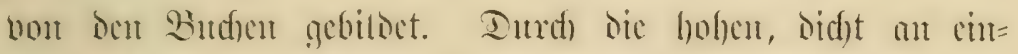

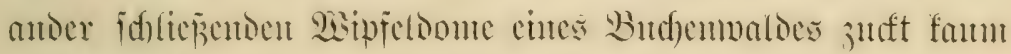

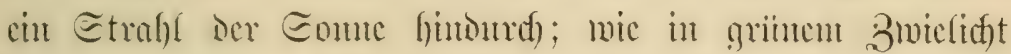

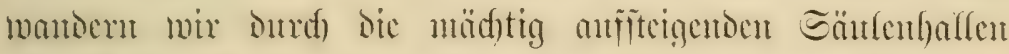

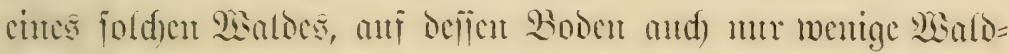

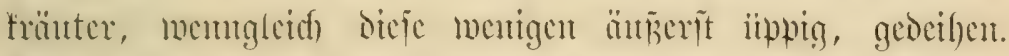

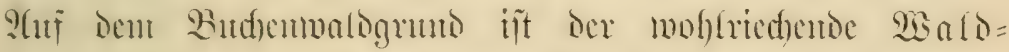

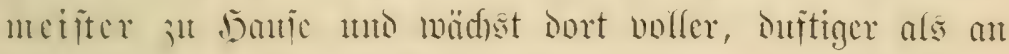
antocen Erten; mand)e (Siras nut cinge bunte bुhtuten Finto Dent grinten Ieppte) nod) cingewobent. Ja, and bie breiten Diaiblumenblätter fieft man biijd)lig da nuto bort, aber fie baben da untr geblitht, als bie Butuen uod) flem

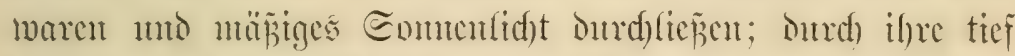

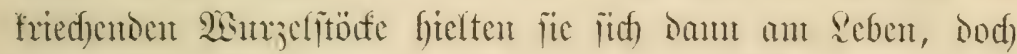

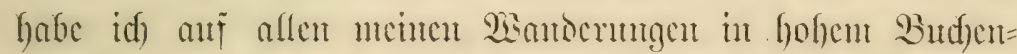

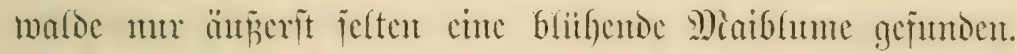

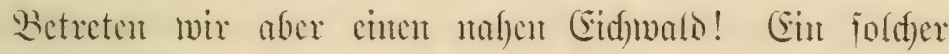
ijt feinem ganjen (Eharafter nad) ans jicntide meit ans cint=

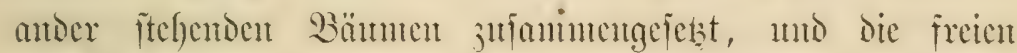

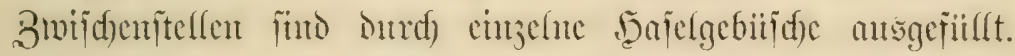

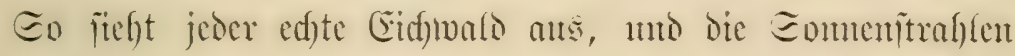

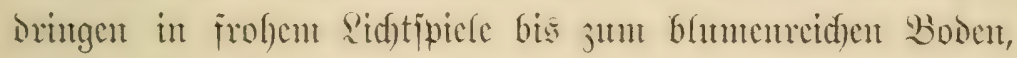

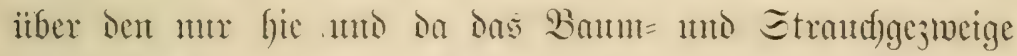
leidften $\Xi$ (f)atten wirft. 9au, hicr mögen wir Miaiblumen 


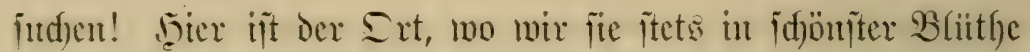

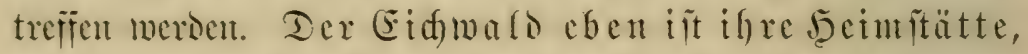

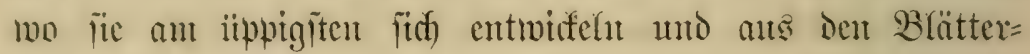

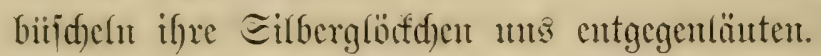

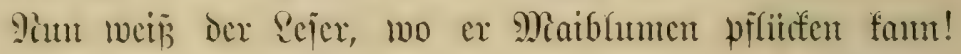

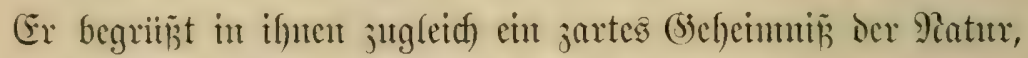
Die jedes ihrex Bummentuter anf eine bejtumte Etclle gemiejen hat. Fentes joll ja bas andere hindern, im Sampje um's Dajein foll nid)t die Miehrzahl von den Stärferen verorängt unto veruidfet werden.

\section{4.

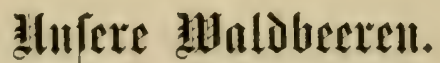

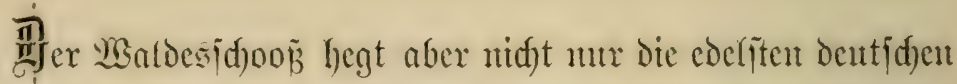
B(umen, and) alle unjere Becren bietet ex uns als erifrifdende föptlide 2 baldesgabe an.

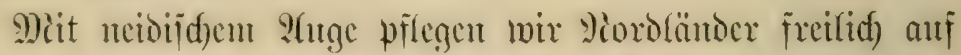

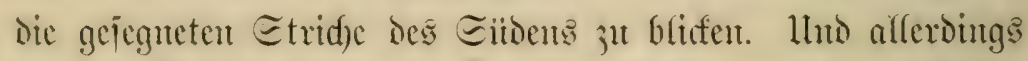

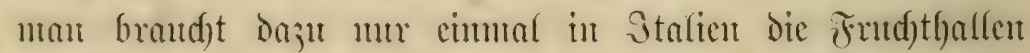

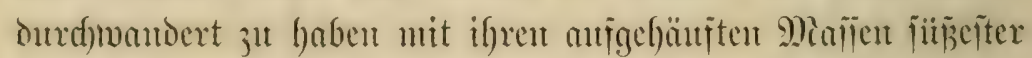

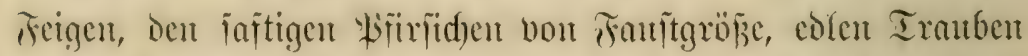

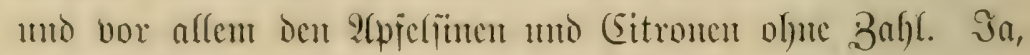

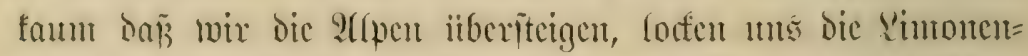

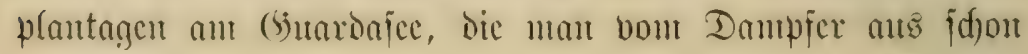




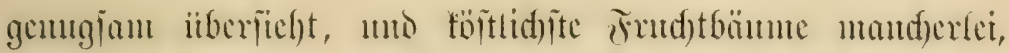

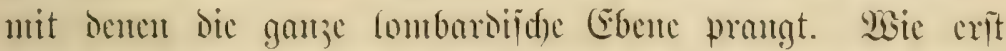
in tiefon Eitom! Die fentred)teren Eonnentrablen reifen alle die jafllojen, fo gan; berifficonartigen Wialmenfrieds)te,

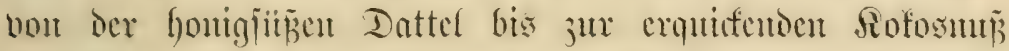

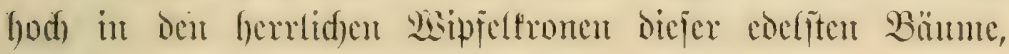
die Bantante, die Brobfuthtfänme.

Der Eiiou ijt bas ?no ber beiteren Sebensgeniilie. Wins

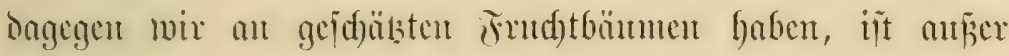

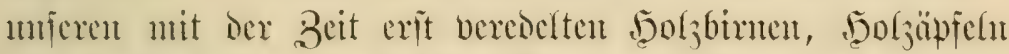

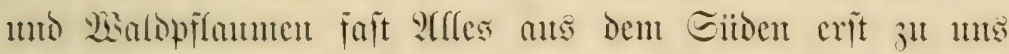
getomment, nermag barum aud) nod) immer mandfen miferer

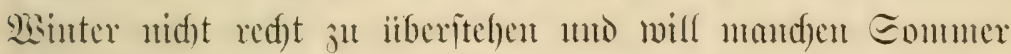

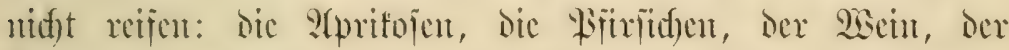

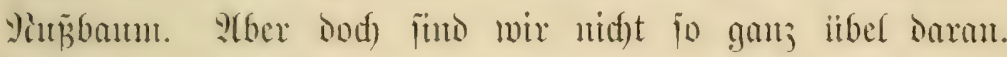

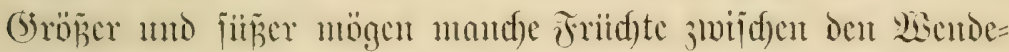

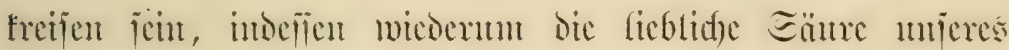

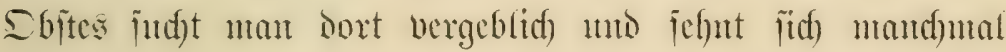

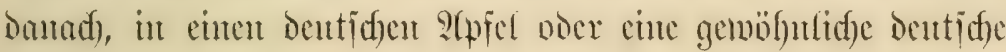
PSflaume zut betjen.

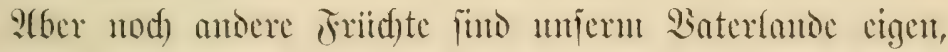

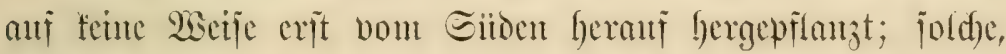
mit Denen die Miatur bis in den hofen polaren Piorden

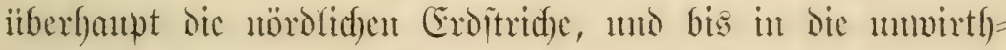

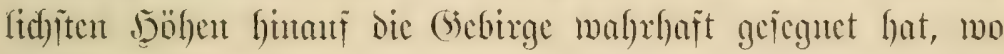

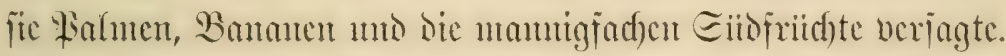

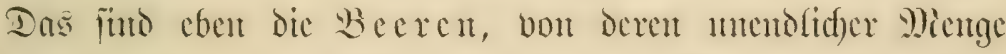
mo meiter geographijher ?tusbreitung Miander fene \&or=

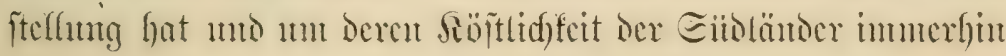


It

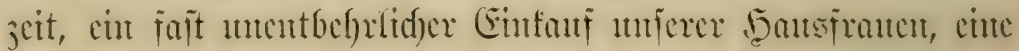

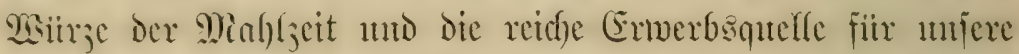

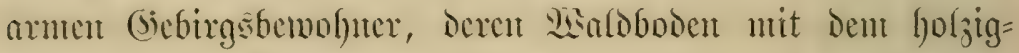
ftengfigen utno glänjenoblättrigen immergrinen (scjtriipp iiber=

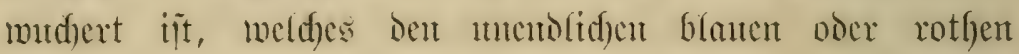

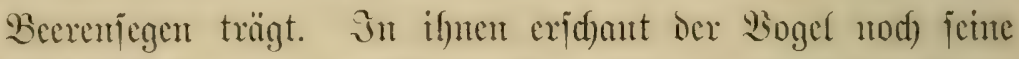

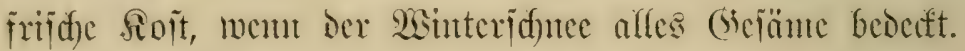

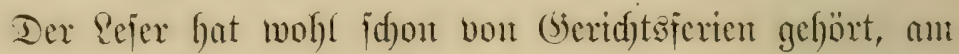

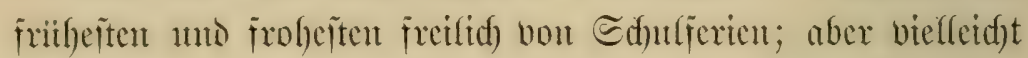
nod) nidst won beide(becrfericu! (5.s gibt Extidnften, in Denen dieje aber jo jelbjtberitündid) fints, wic Jerien iiberfant,

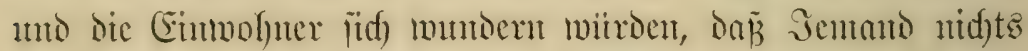

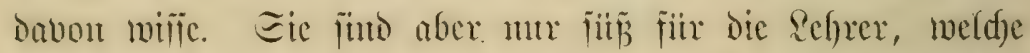
in bicjer 3eit nichts jut thut baben, als daranf jut denten, wie fic dic Beit hinbringen und Deshalb ab anto jut fidf jelber Der mitfjeligen Ferienarbeit ber ifnen jonit anvertranten.

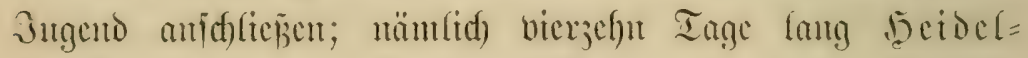

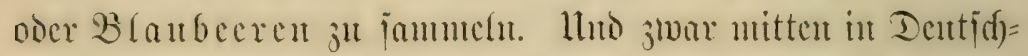
Land gibt cs jolde Ferien, 3. 23. In cintgen Drtjufaiten ber

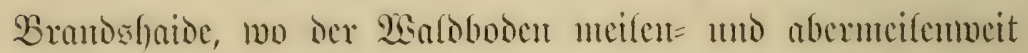

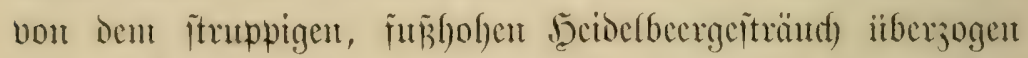

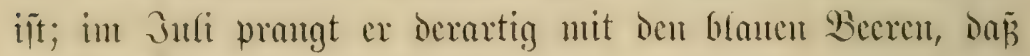

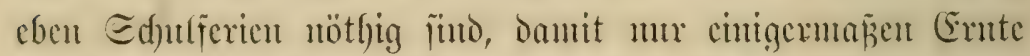
gebalten werden fann. Sung und sllt, Sater, Miutter uno

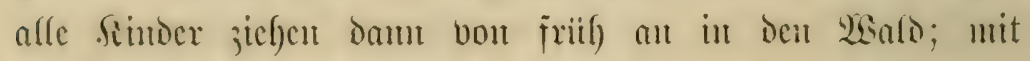
bö́zernen Sänmmen itreifen fie bie Seeren ab umb fammeht

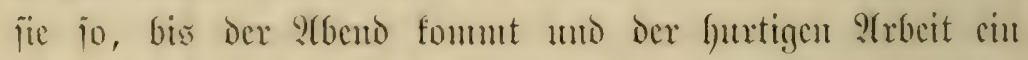

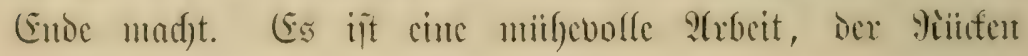




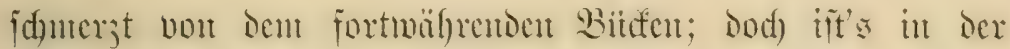

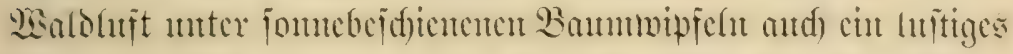

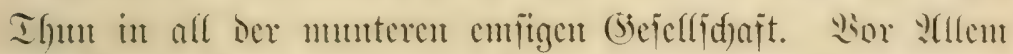

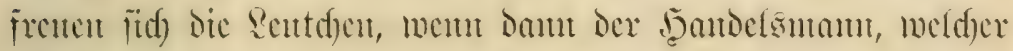

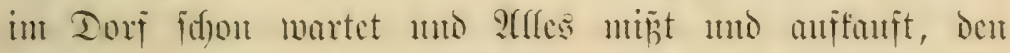
flutgenden ?ofn ifuen cutfündigt. Niandes fitul bort in

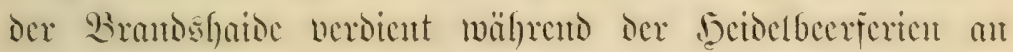

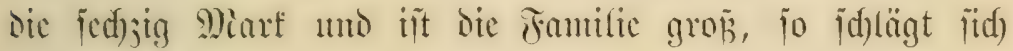

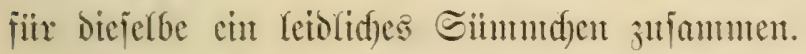

Darum liebt der dortige Dörfler das beciselbergeträud).

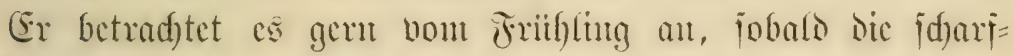

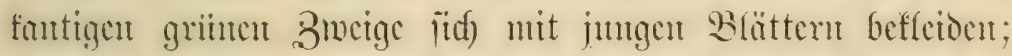

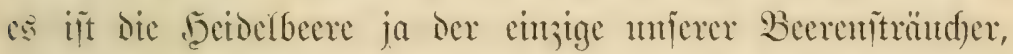

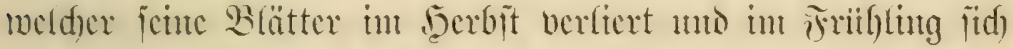

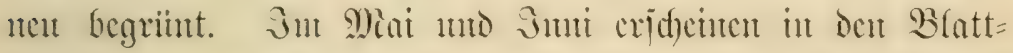

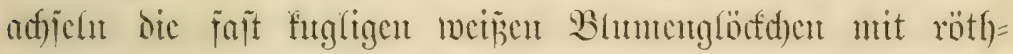
lid)en 2 (ntfand), weld)e sen feld)getrönten unteritündigen Futd)t=

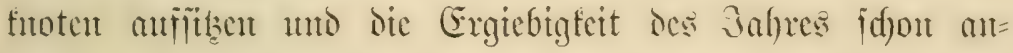

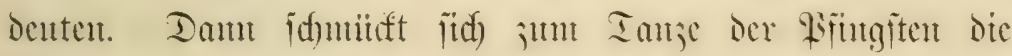

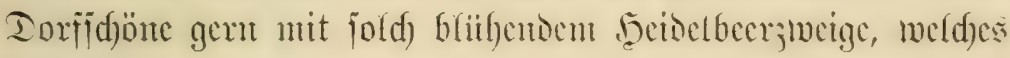
an Migrtent crintert, wic and) der latcinifide Piante Vac-

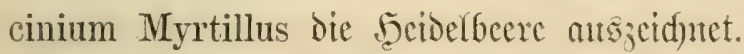

Dicjer Sultus fintot fidf freilid) nidft itforall; gax bic

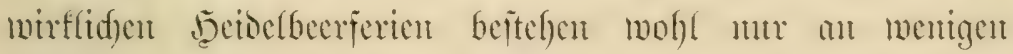

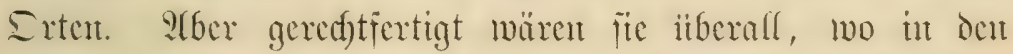

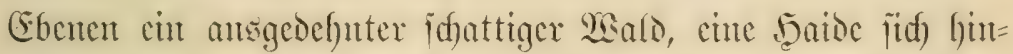

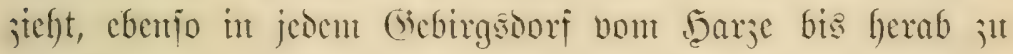

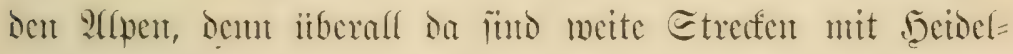

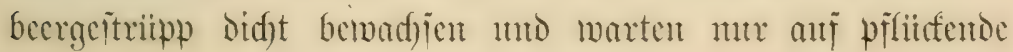




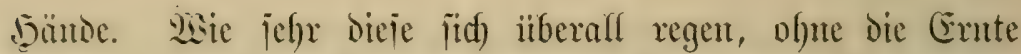
Dod) ganj ju bewältigen, Davon jagen แns Die Wagenladungen, weldye baum Ditref alle Stäbte fommen uno Den Bedarf ber

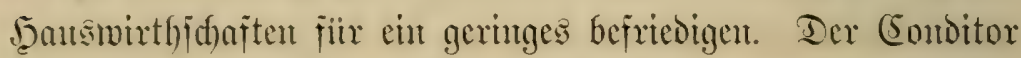
verbrandf)t ïe ju jement Sutdent Dic Jantsfan bereitet fie

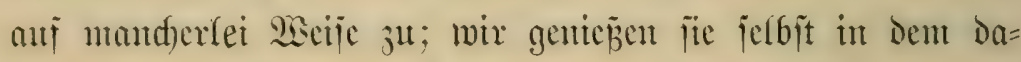
mit gefärbten Siothwein bei fröhlidfem Miahl. Man braucht

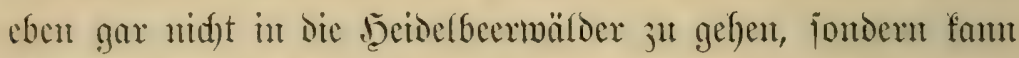
jid)on Durd) Berechumg dicjes (Sonjumes fid) eine Borjteffung machen von Den Mitliarden Beeren, welde jälyrlids allein in Ientidyland madjen. Der Eegen (Sottes iit aber aud wirt=

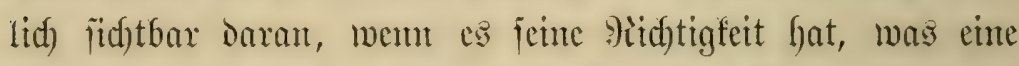
alte Eage berid)tet. Dem als (5ott Der Bater, heip̈t es,

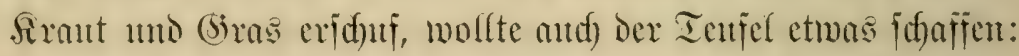
er bildete die blanjf)warjen beidelbecen anto fprad) jiber fie Den Ienfelszanber ans, Seder mitfie ifm verjallen feut, der

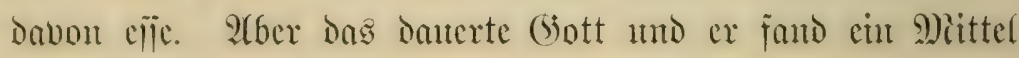
Dagegen; nümlid) ex jeţte jeder Beere cin nod) immer fid tht=

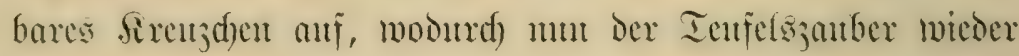
veruidjtet war.

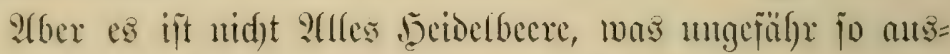

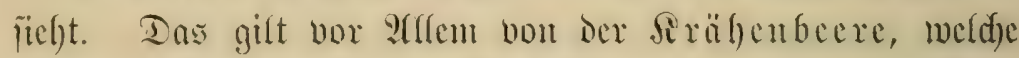
gleidyalls ïber den ganjen Shorden der alten mo nenen welt

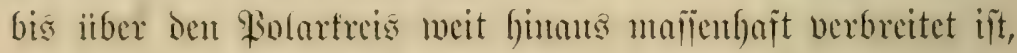

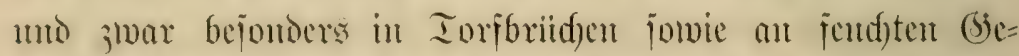
birgsorten ifren Etamourt hat. Deren Beren fömen alfer=

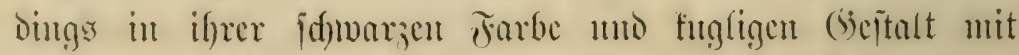

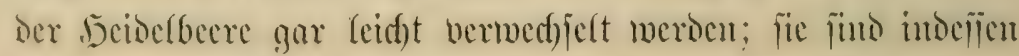

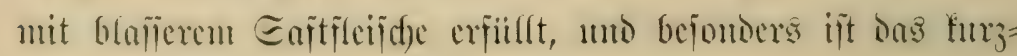




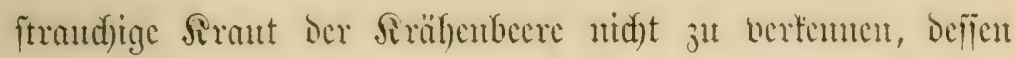

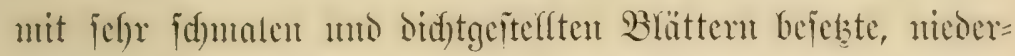

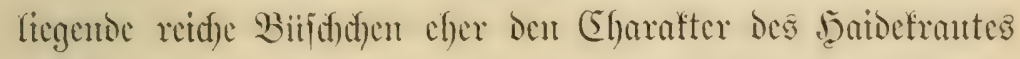

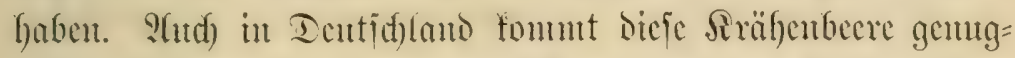
fam vor, mo jumar in torfigen baiben mierer (sebirge, aber

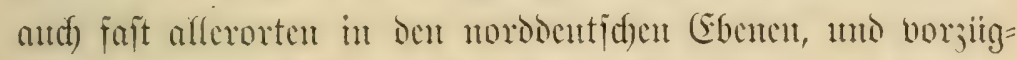

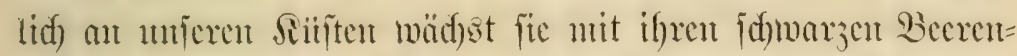
trantion ficllentucife gar majfenthaft. Ein Eegen fiir dic

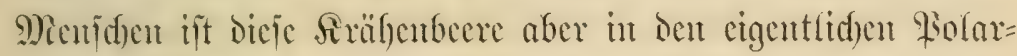

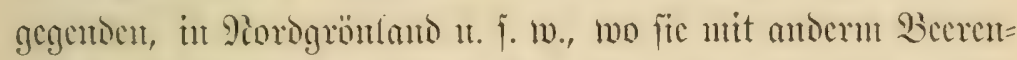
geitriipp, Decioclberen uns Brombecrent meijt untermitidt reichlidfit vorfomment. Eic geociht itppig oort ntod), wo fein Banm föher als fandhod wiro und an ben Baut weder ber

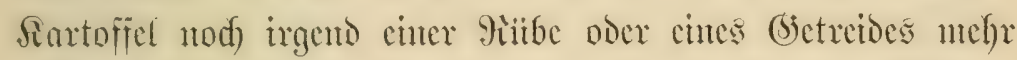
ju benfen ijt. Dic Siräbubecre reift bie arftijche Sonte

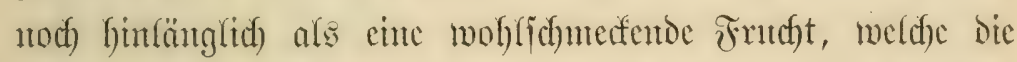
Pratur bort auf ben endojen Etrecten hervorbringt. Itno man weī fie ju juäben. Sion den Dortigen Bemofnern

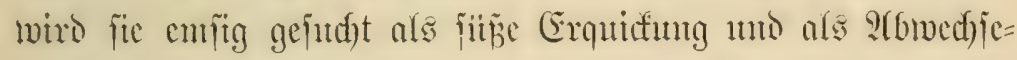

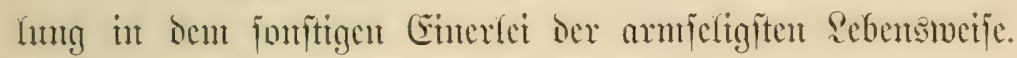

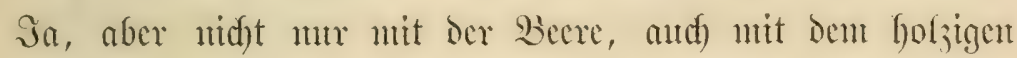
Seftriipp biejer immergrinten Siffanze mifjen dic polarifdent Bewohner jut redhuter; fic reffen cs hanfenmeife zufanmen und laffen es fid jutr Etreut ifres Sagers, box strem aber

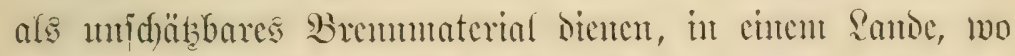

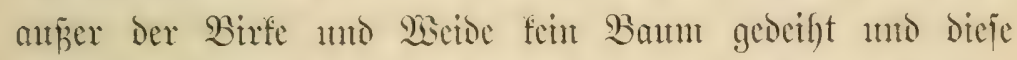

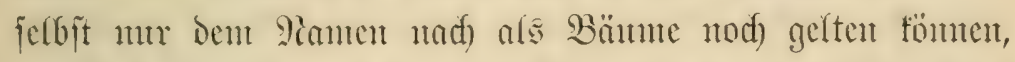

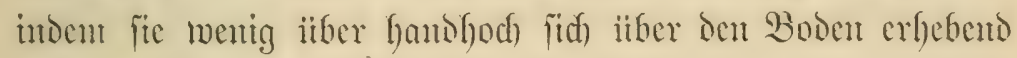
das Beerengeftritpp oft fanm iiberragent. 


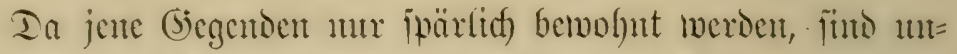
gchente Etrecfen dort mit den Beeren ïberfleidet, ofne Dä

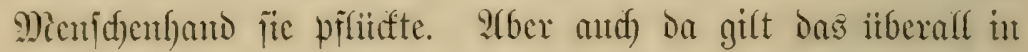

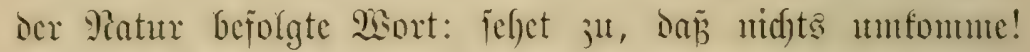

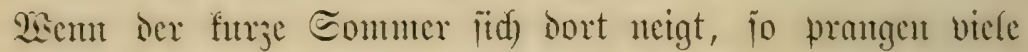
Sation mit bent vollen Becrenid)mute nod) fait unberithrt, Desu jun gropen Theil f̈mb autd) bie Bögel jobon weggejogen, den Eitoen jut. Dic Becren fömten Deshalb völlig ntšreifen; baben jie die lleberreje und Eirinigfeit crlangt, jo fällt aber

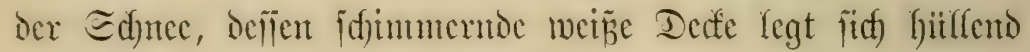

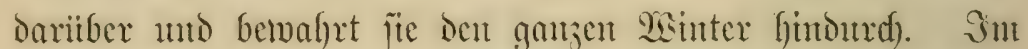

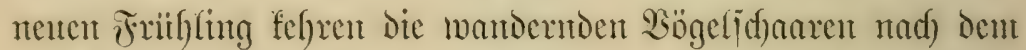
Porden juritif, und fiele, fie finden utter bent fdumeljenten

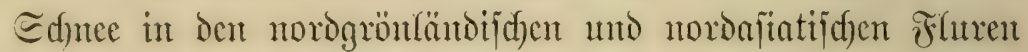
ifren Iijd mu reschlid) gedecft mo gierig fallen fie dariiber her. Die mentigen Mionate ifres polarijden Mfufenthaltes

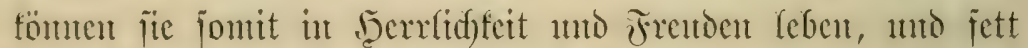
geworden bon diejer lecfern jiipen fioit jieflen fie cnslid)

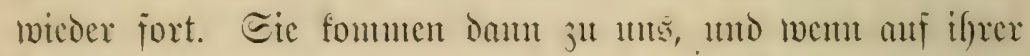

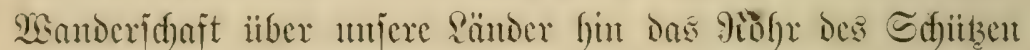
bicjen oder jencu Bogel crlegt unto cr jeffechlid) anf umjern

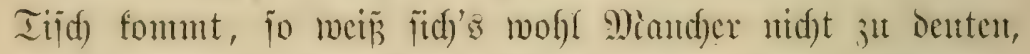

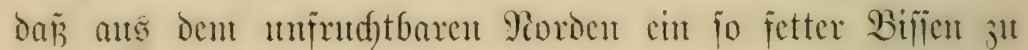

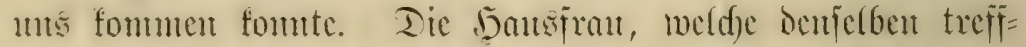
(id) jujubereiten weif;, bringt Daju als Eingemad)tes vielleid)t aud) dicjentigen föjtfidfen rothen Beceren iclber, meldse nidjt

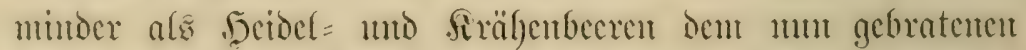
Boget cinte jo treffilid gemumbet baben. Das finto bie

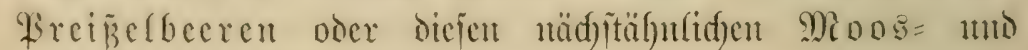




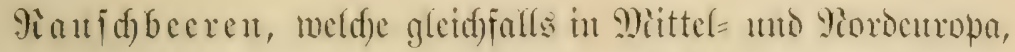

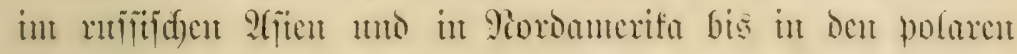

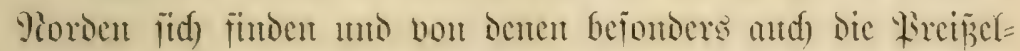
beere oft weite Etreffen itberjicht, bald fiir jids, bald mit

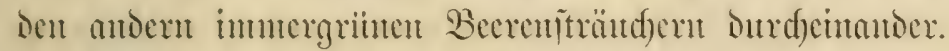

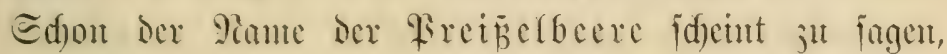

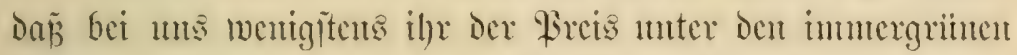
Becrengebiifden jujuerfenten jet. Woburef) ante) wollten wir

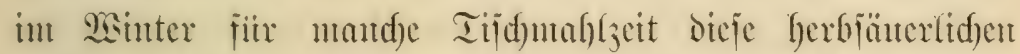
Becren erjeşen, ober weld)e exprifdendere Butfoit föm ten wir ben firanfen bieten? Sa, welde Frutht gicbt es, bie uns io fait boll entid)äbigte, went cinmal affe Banmobitiritidste

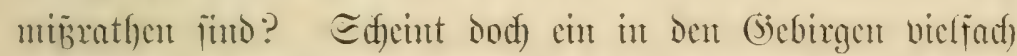

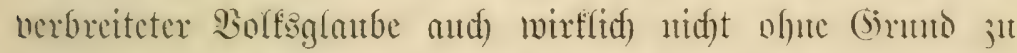

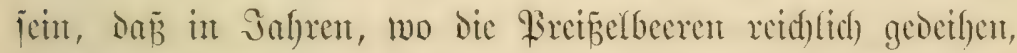
Das Ebĩ in ben Ifälern felle; ctwa das objtcere Jabr 1871 hatte einen gieidythum an jenen Beeren, saj fie folbjt trobs

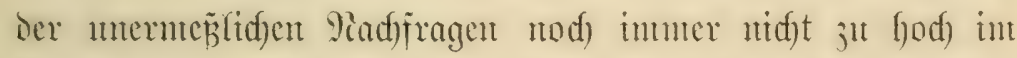
Wreije gingen. 2(6er um fie ju lieben, nod) beim lad)enten 2 hn=

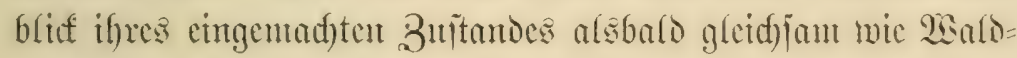

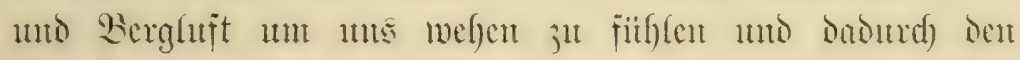

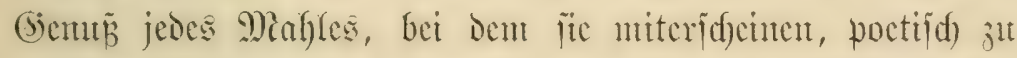

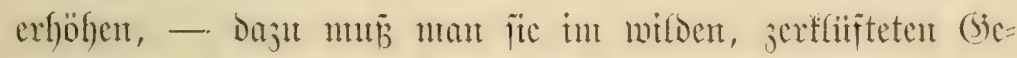

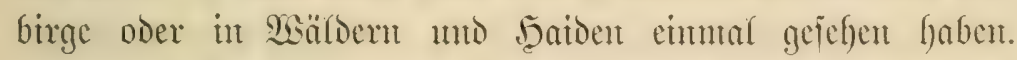

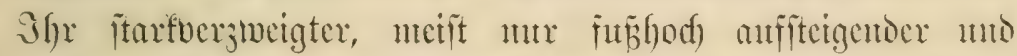

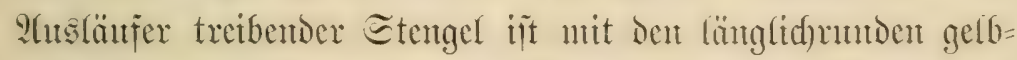

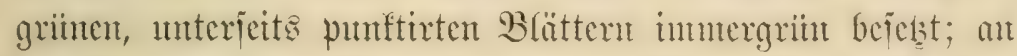

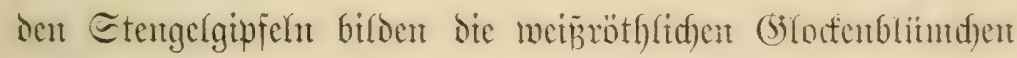

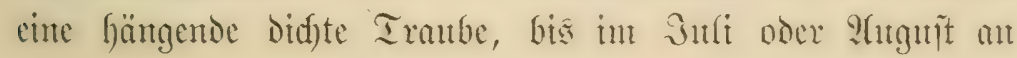




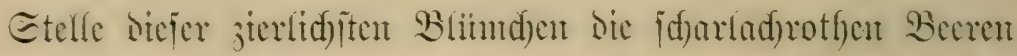
treten und un das ganje

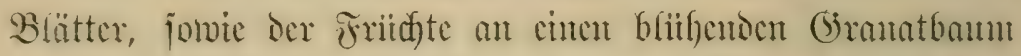

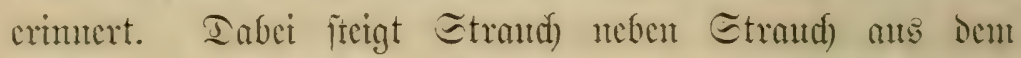

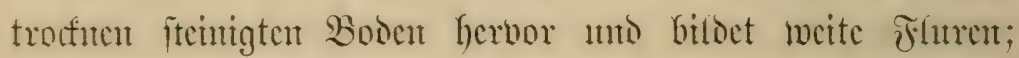

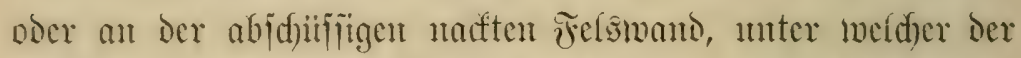

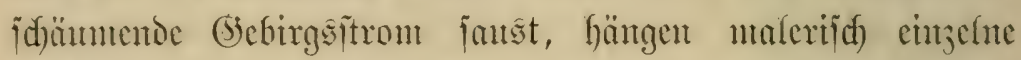

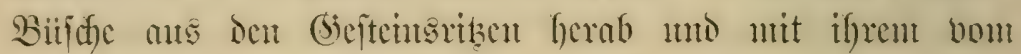

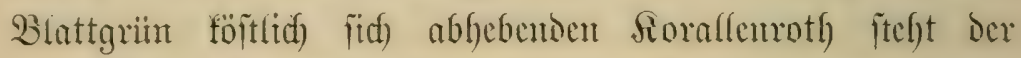

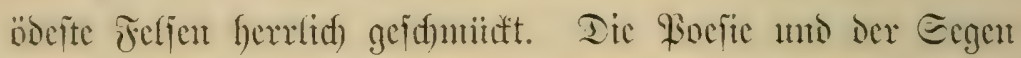

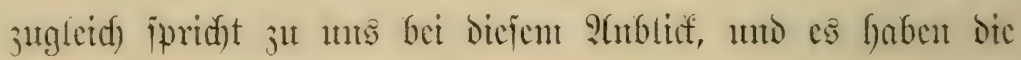

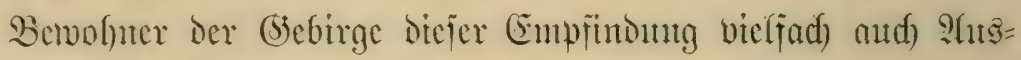

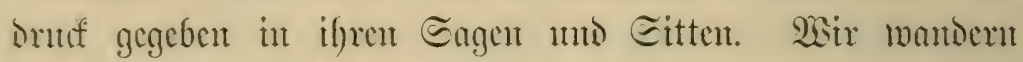

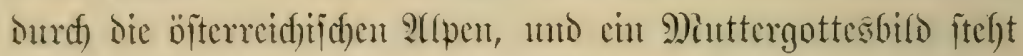
cimfan an bem vielbetretenen Felfenpfas; der fromme Ginn

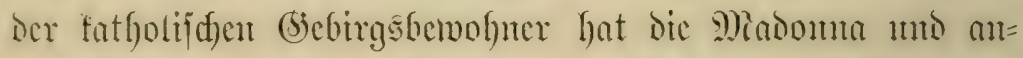
Dere Setlige am Wege fajt immer mit Sirünjen mo Etrüujen

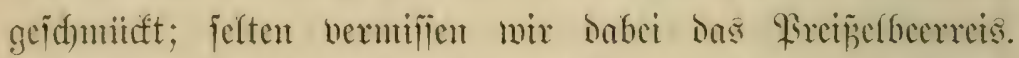

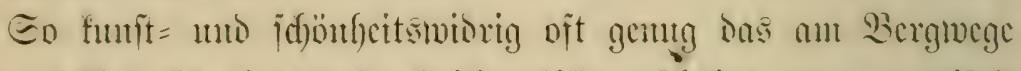

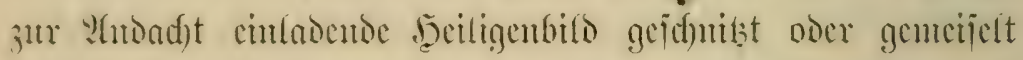

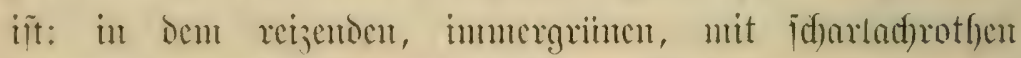

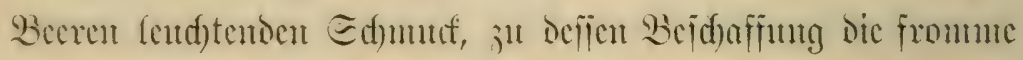

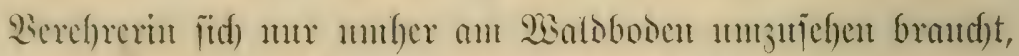

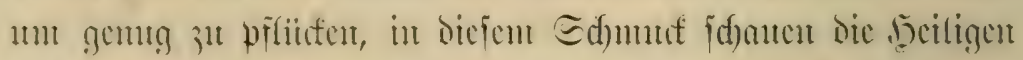

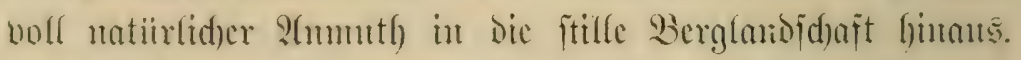

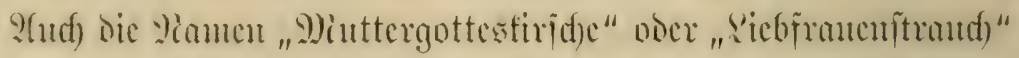

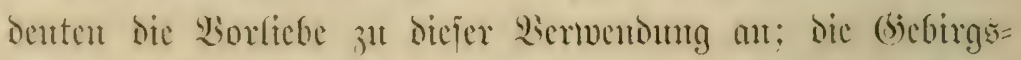

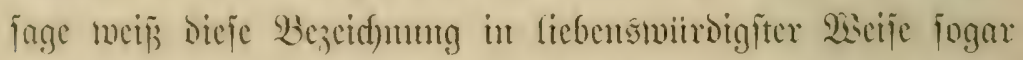

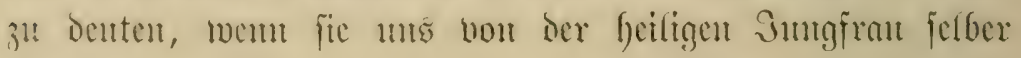




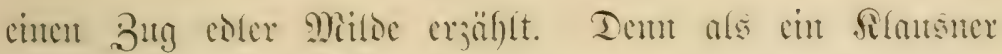

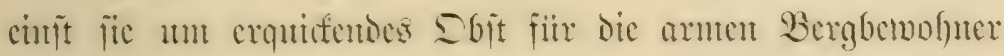

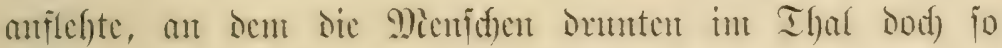

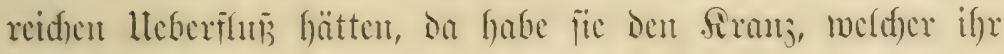

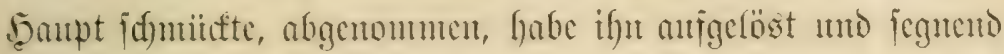

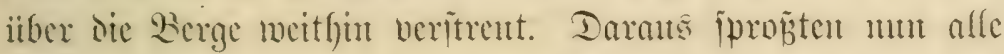

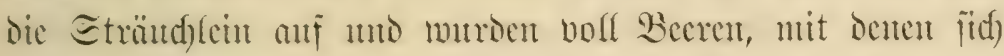

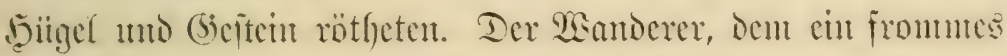

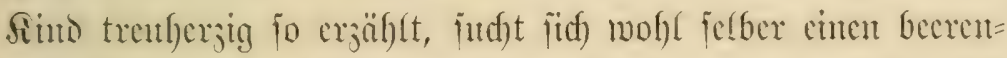

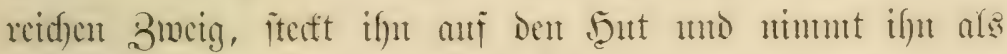
Bergesigabe mit heim, da in ber heimathlidgen Ebente die Breipelbecre fart nirgentos wolynt.

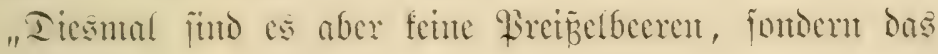

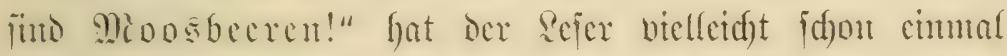
hören mitîjen, wem als (Eompot eingentad)te Beereat anf Den Tifich gebradet murben. Diejelbent haben die gleide rothe

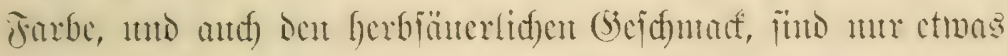

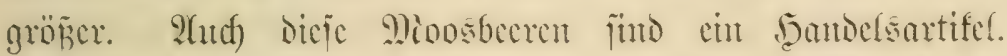

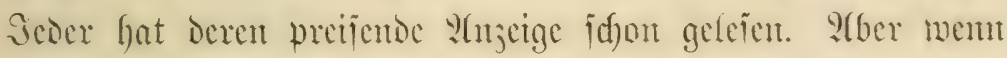

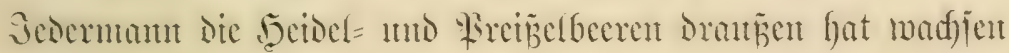
jehen: von der Mioosbecte fömen fïh) juther nutr Waenige

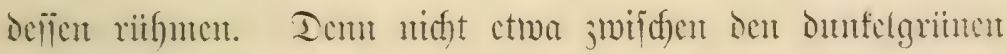

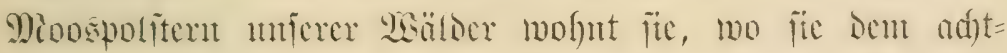
fanten Waldpajtergänger oder (Siebirgswmanderex nitht hätte

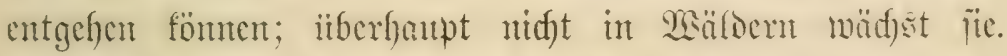
Jreilid) anf Moos wohl, mic ifre Name befagt, aber mut in

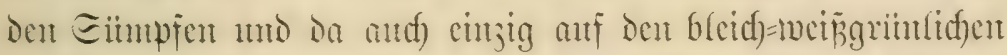

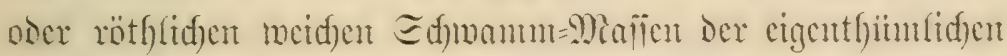
Iorfmuoje, weldye ïber den bebenden Exfamm noer Mioor 


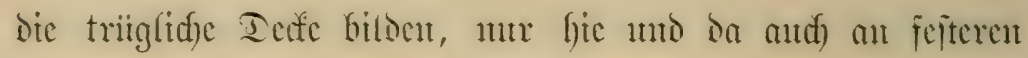

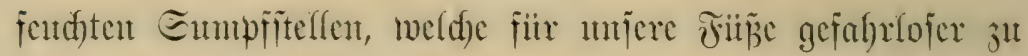
betreten find. Ia verirrt felten Semand fid) bin; aber cs

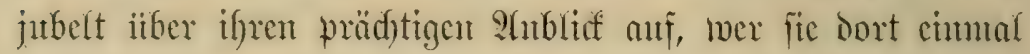

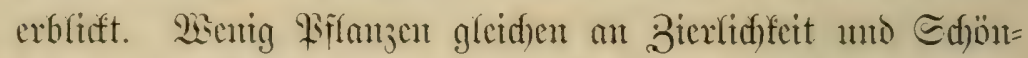
heit biejen Mroosbecrpflänzd)en. Shr fajt fabendiumer jäluer Etengel liegt langhin ansgeitreaft unto vielvergmeigt, wie 9)inrtenblätter unthen jeine dunfelgriunen, glänzenden, leocr=

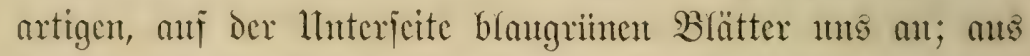

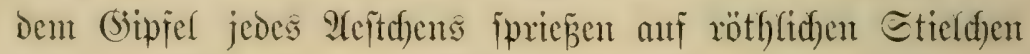

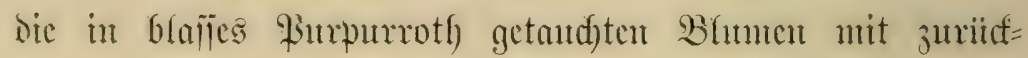
gebogenen Bipfefn hervor, melde uad) Farbe unt Bitoung

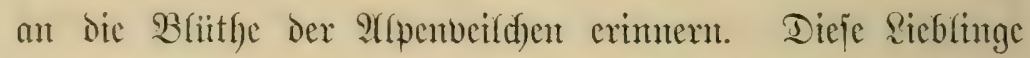
jedes 23otanifers funden jid aber anf fajt jedem gröpern Torf = moor uno da neift fo mafienhaft, da jes ein lohnentes (5ie= ichäft fïr die ammolynemben Dörffer ift, die etma jum Eep= tember reifenden grofen Beeren fïr den 2 Berfant

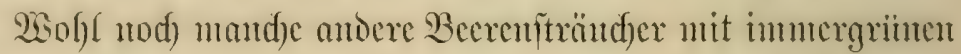

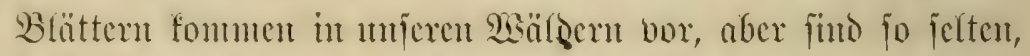

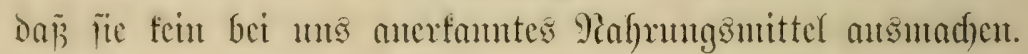
Wer fent ctwa die Bürentraube, weldye nad) Etengel, Raub,

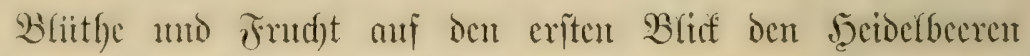

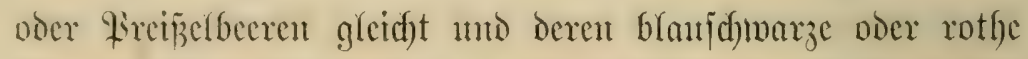
Beeren oft gem mo, swax ohute Edjaden mit ifnen cun= geianmelt weroen mögen. - Dex Deutjde Walo bat freifid) anperden dex Becten nod) gar mandfertei. Freilid) efphar find foldse nidyt alfe! 9atuf) unere Miaiblumen prantgen ja im Eommer mit fotharladrotben, grojen Becren. Der in

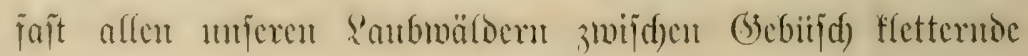




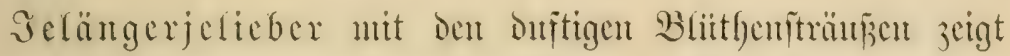

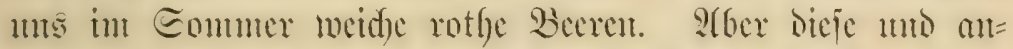

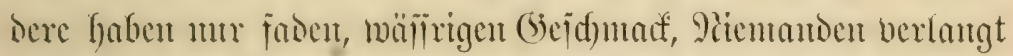

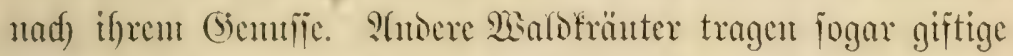
Becren; Das feltiane 23icrblatt (Paris quadrifolia) crirente

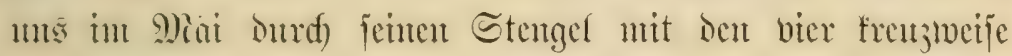

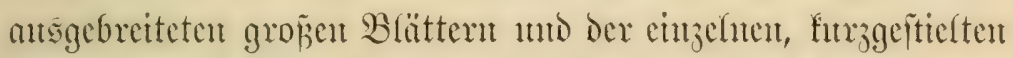

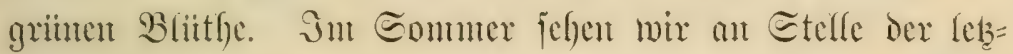

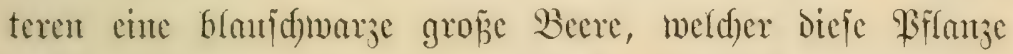

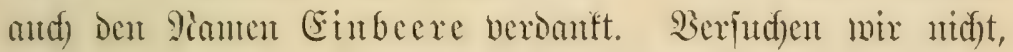

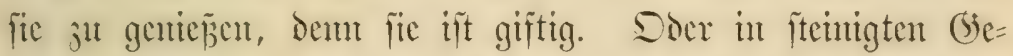

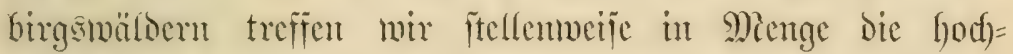
frantige Bellabonna, seren glänjendfdymazc, firịd)artige

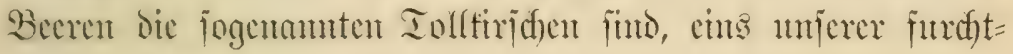
barfiten Biflanzengifte.

atber aud mandide edfe seere nod) reift in umperen

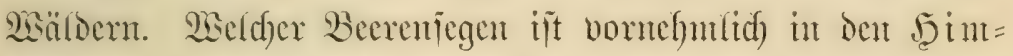
beeren, den Bromberen weit bis in don Plorben fin in allen Eanbwäldern ausgeídjittet; red)nen wix bazı bie

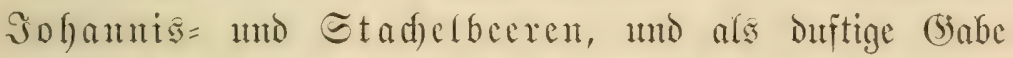

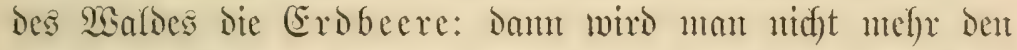

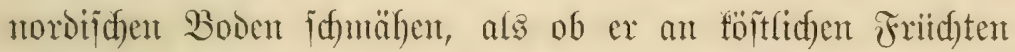
finter dent Sitoen ganj juritubleibe. Ltno wentgleid) der Rebitorf feine Becre, Diefe "Sututefienj bou Eomuengold

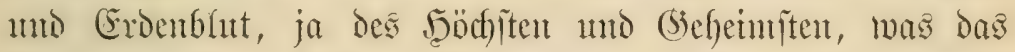

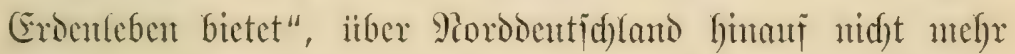
reift: io wei⿸j Dod) ans den genanten Beeren Der (5rön=

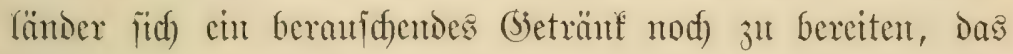
ifn wie der Eaft ber Bacdusbeere in feiner grinmigen 
Winterfälte erwärnt, mo wenn in feftfider Stunde der Becter cinmal reid)lidfer fretst, ifn and fein fitumterfidfes ¿oos auf furze Beit jelig vergefien läpt.

\section{5.

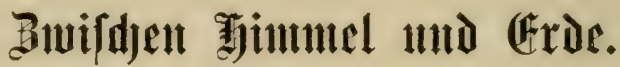

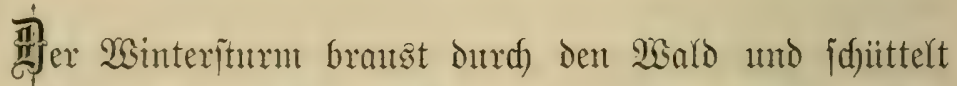
die Etänme der hohen Birfen umo malten Gspen. Bon Froit und Inwetter getrieben befiligeln wir mijere Edritte. Aber pröklich bleiben wir vermundert fitefen. Mit bent Geulenden Etıtm und Den ädjjenden $\mathcal{H}$ ejten un bie Wette ertönt hod) oben aus den Bammipfeln das (sejd)ret jahllofer Gin und her flatternder $\mathfrak{B}$ ögel; dieje f̈nt it enfiger $\mathfrak{B} e=$

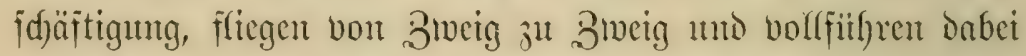
einen Särm, der nod) das Iojen bes llunetters itberiftumt un

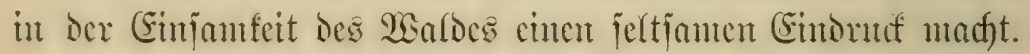

Ilm was ite fidd ftreiten, was fie jo beiffäftigt? Das Seäfte der laublojen Bämme benterfen wir ftellentweije vont

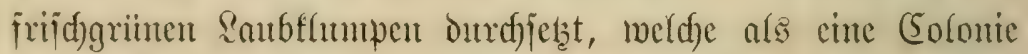

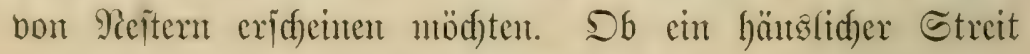
in biejer Rejtercolonie aug̊gebrodjen ift?

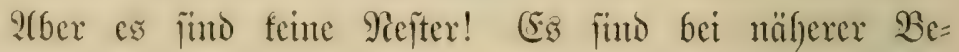
trad)tung fräftiggrime originelle Biifd)et oder Biifde, meldse an den laublojen 2feiten droben feftgemadjen bie anto da bie

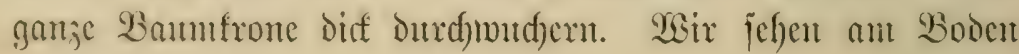




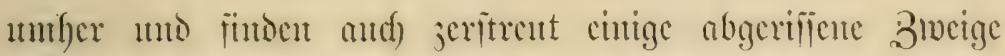

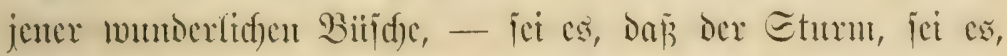

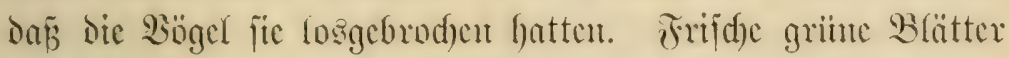

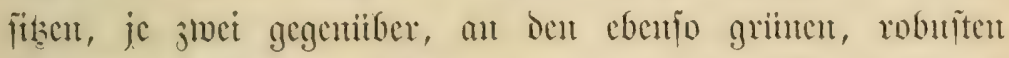
Stengelat, weld)e melgrfad) gablig berjweigt jüt. (Ët jeltjantes

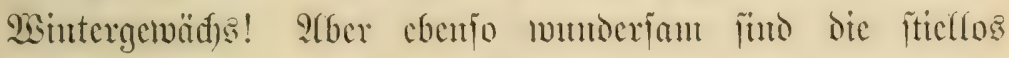

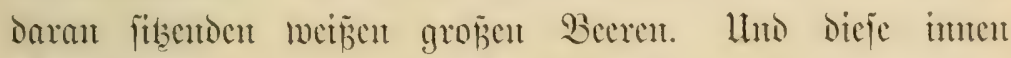

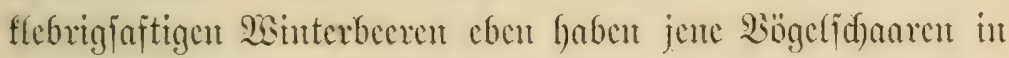

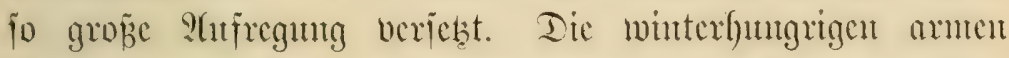

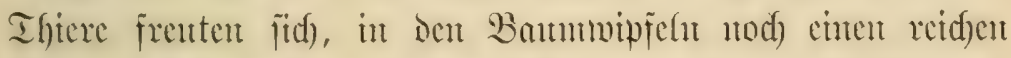

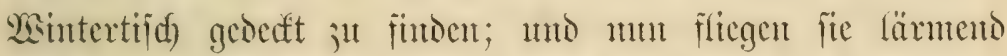
utmber, die Sojt jut verjebren, haten, piefent utto verjagen

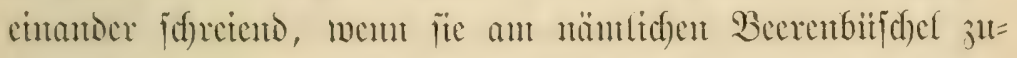

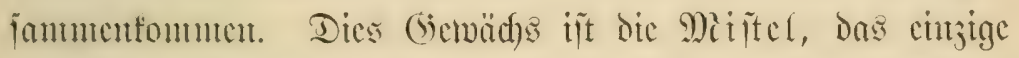

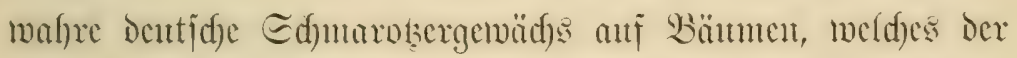
föher organifïrten Piflanjentuelt angefört, ein inmergrimes, winterlebendes (sietwäd).

Lleber menige andere \$iflanjen fint ans alter, mo jwar

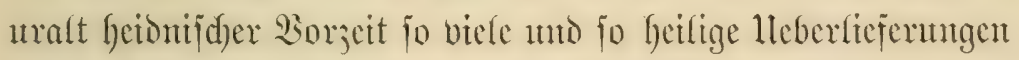
uns erhalten, als gerade iiber bic Mirtel. Da fie mit fenter Wumel die Erde berithrt, galt fie als oen (söttern beilig, wurde darum mit feifiger Edjeu geef)rt mo gead)tet. Wem

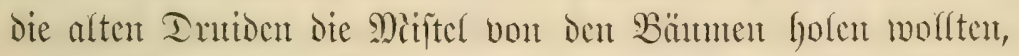

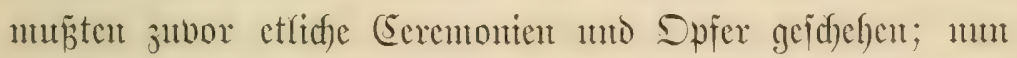
jticg Der Firicjter in weifjen Silcibern anf Den Bantm, jefuitt

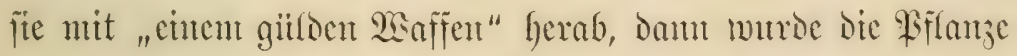
in einten meipen Miantel gejd)lagen, unto eş beganten wieder:

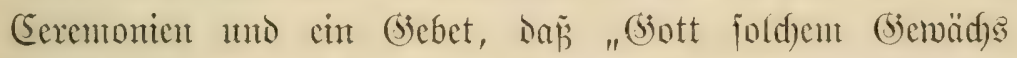
jein frafft wolte laflen". Gs muñte, wic amoere Bertate 


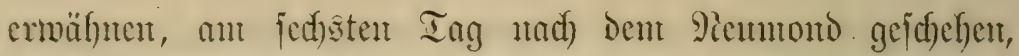

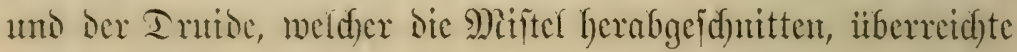

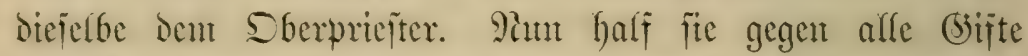
uno Srantheiten, bermod)te mfrudbtbare Ifjere fruddtbar ju

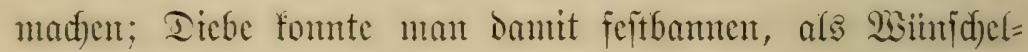
ruthe fidf ifrer bedienen. Ien Banm jelber, anf bent fie

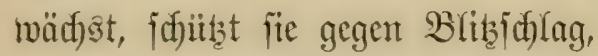

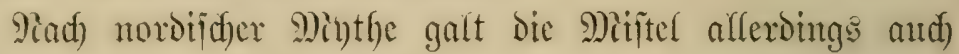

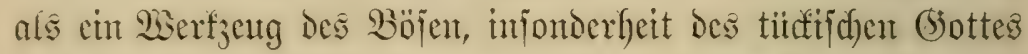
Soti, Int murde in biejem Einne ju Bauberfimiten benitizt. Päultad ber Sonnengott Balour war allen (Söttern fo werth),

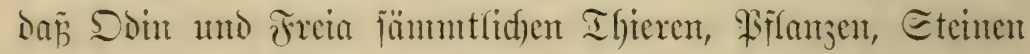
unto Elententen einen getwaltigen Gio abualun, fie mollten nimmer sen geliebten Baldur juaden. Sie leifteten dicien

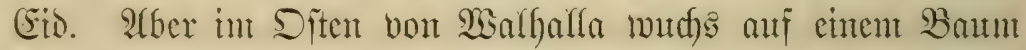

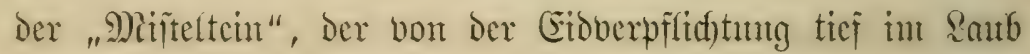
veriftect niousts vernommen hatte. Das muñte cin Jeino

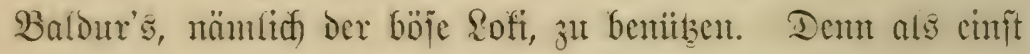
die (Sötter fid) in Epiel Damit belufitigten, auf Baaldur den unvertwundidacn Epeer zu werien, reid)te Soti sem blunden

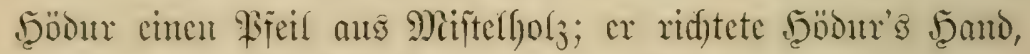
uno getrofien fanf Baldur mit ber Todesmunte nicoer. Was will bicie idjöne Sage wohl anders bedenten, als daf̧ die 9)iiftel immer nod) grïnt, wem die Eommerszcit, deren Gimbilo in Der nordijden Minthologie Bafour war, Dafint= geidutvumben ijt.

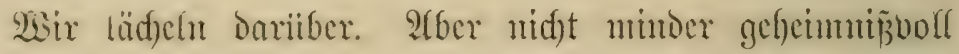

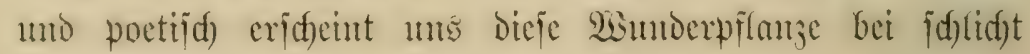
naturtvifienidaftitder Betrad)tung. 


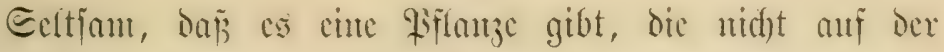

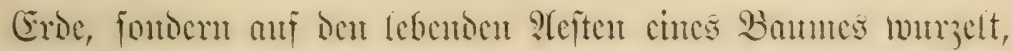

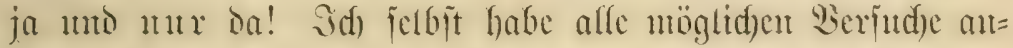

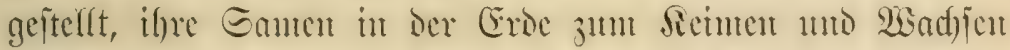
jlt bringen. Afber nergeblidf; fie femen da fo mentg, als

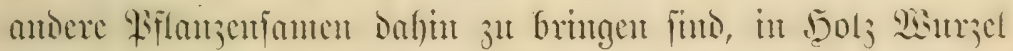

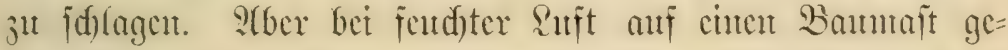
brad)t, tritt Das Seimmiirgelden ans dont Miiftelfamten balo

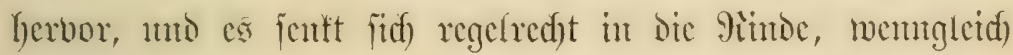

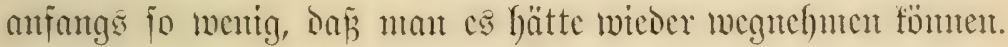

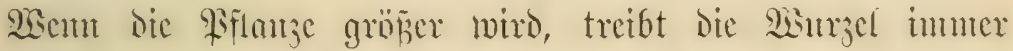

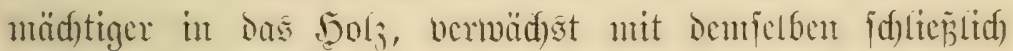
fo cijenfejt jufanmen, daj man dic \$iflanje abreipen famt,

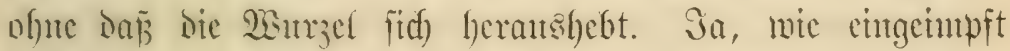

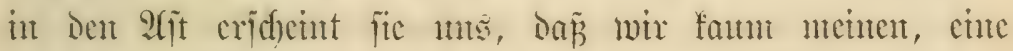

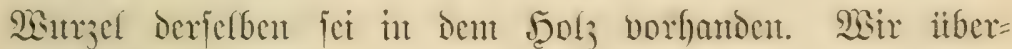

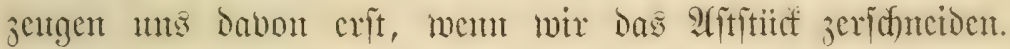

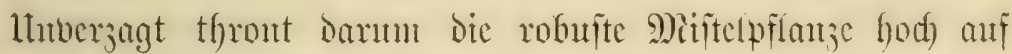
Den (Sczlweigen Des Banmes. Die Etiume jerzanfen ite jut= meilen und reipen Etengelftilde herunter; aber fic felber bleibt

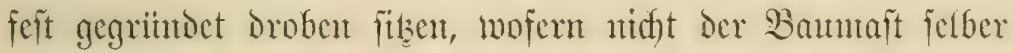

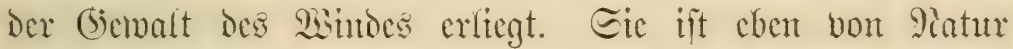

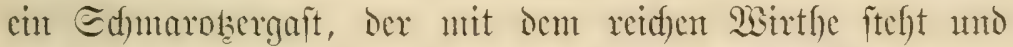
fäl(t. Dod) wie gelangt fite ju ifm? Dient Durd) Bere

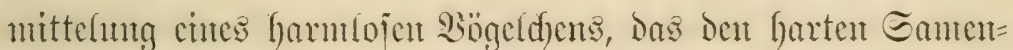
ferat ciner foldat Becre antwerdout amb antwerdorben mit feinem Soth wieder bout jidh gab; er bleibt mit demielbent an Dem Bweige haften, gleid) in fruthtbaren Duntg gefjullt. Der Eamte fenit, umb der Baum mag anfangs des fdumuten 


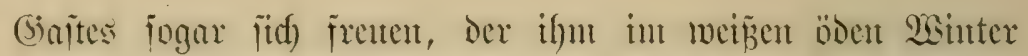
jein inmergrintes Saubwert leigt. Dad)te ex aber Daran,

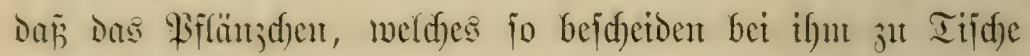

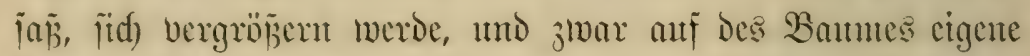

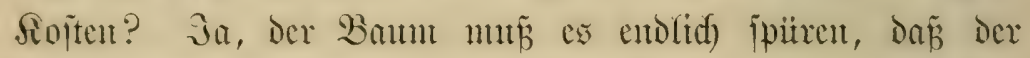
(bajt ïberutäd)tig gemorden ijt, Dem je lebenfrobender der

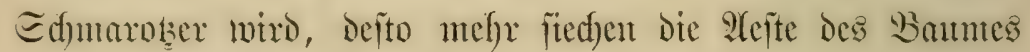

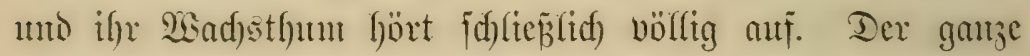
Baum jtirbt, jwar nid)t gleid), Denu jein Begetationsleben

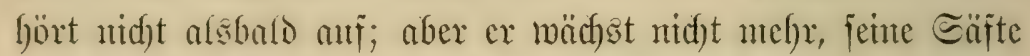
uno Siräfte faben cinjig nod) den ïppigen (siajt jut crnälurent.

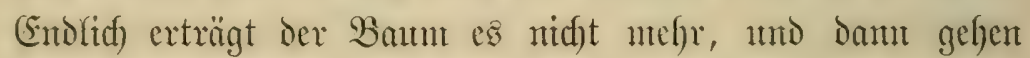
abjerbent 2 sirth uno (Sajt jugleid) ju (Sirunde.

Dex Durd) die Mijtelpflanze po angeridftete Edjaden ijt jwax jo bedeutento nididt, da fie fid gewöfnlich mux ab und

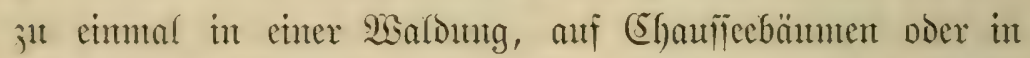
Sbitplantagen fintoet. 2(ber id) habe aud fdon ganze Warts= partien gefimben, in Denen fait Banum fïr Bantm bou Dem

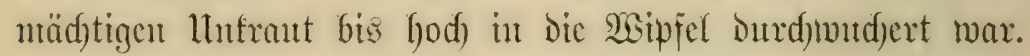
Wenn bic Mijtel anf colcru Softbäumen fid) cummal jeigt, jo fanu jie ïbrigens ju redfter Beit meift mit (cich)ter Maithe weggejdnitten werden.

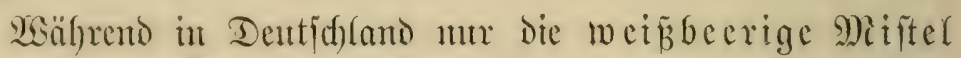
(Viscum album) vorfoumt, finbet fid fodon in Frantreid)

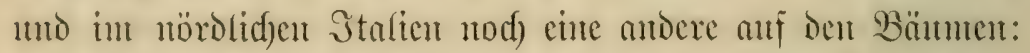

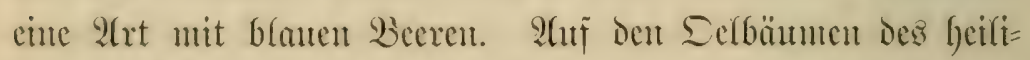
gen Sandes prangt die fireuz=9Xiftel mit rotben Becren. Ju den Wälocm 3amaifa's idmurobt auf mamigfadjen Bänmen mit fajrangelben Besen die Safranmifter, nto 


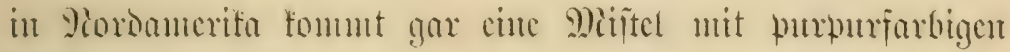

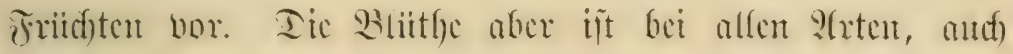

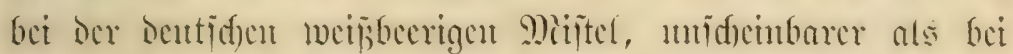

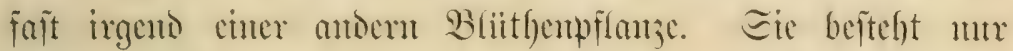

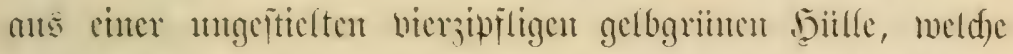

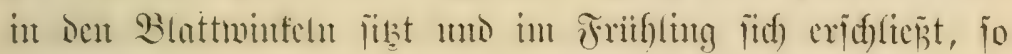

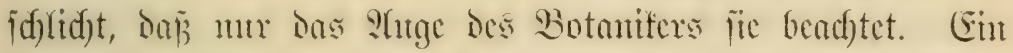
bejonterer Sharafter serielben ijt itbrigens, baj meiblide

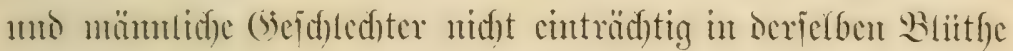

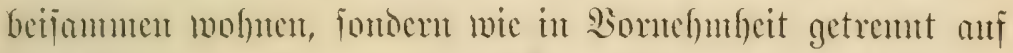

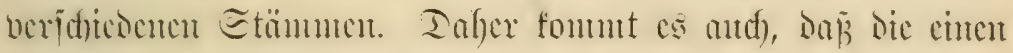

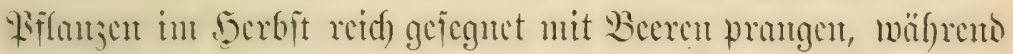

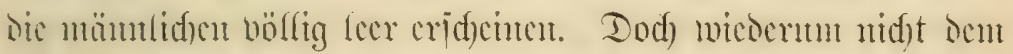

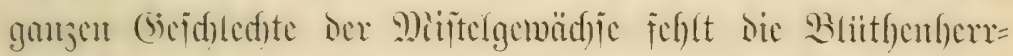

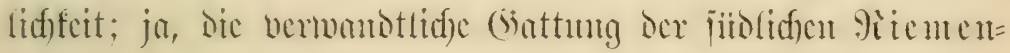

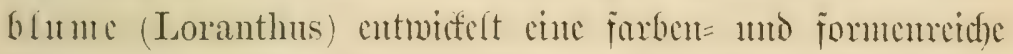

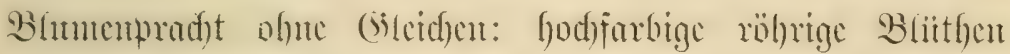

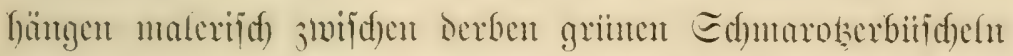

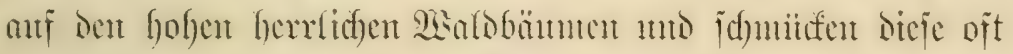
munterfar. Gine ât (Loranthus europaeus) mit ormunen=

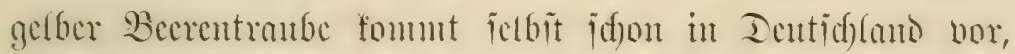

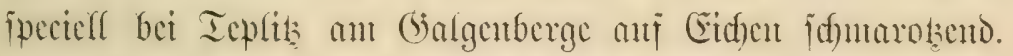
Die ïbrigen gebören alfe ben heiz̄en Etridsen anserer Erot=

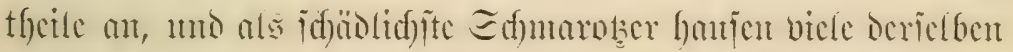

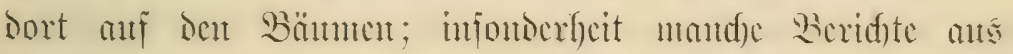

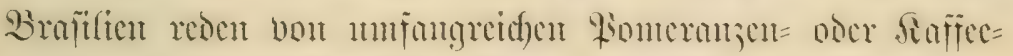
plantagen, bic oft Dured) bic Soranthutsmifted maheju bermiliftet

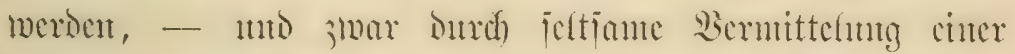

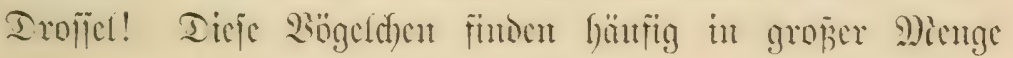




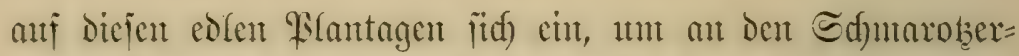

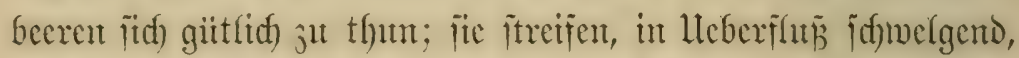
Die ifyren Ednabel läjtig anfartende fleberige Beere an dent

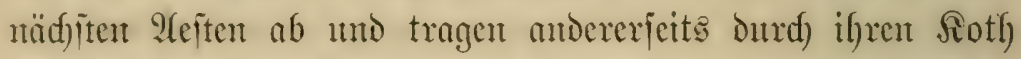

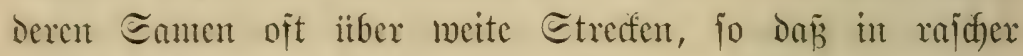
Beit die Raffee = und Pomeranjenbäunte nal) und fern mit Miijtelbiifdjen verfehen jein witrden, wenn nicht die Mentjuen= hano die Bänme wicoer befreite. Nad) Den Mittbeilungen

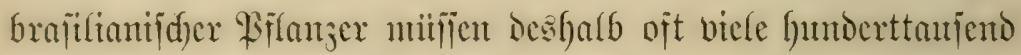
Bäume einjelı gereinigt merdent, wojern ntan nidft die ganjen Blantagen will verfonmen lafien.

We(d)e imige Bezicl)ung aber audf) ficbet jwiffyent den Zïgeln und der Miftelpfilanje! Sie füb anf einander ange= biefent nad) Dent Flane der Edjöpfung; Das Wohlfein der cinten fängt von der நृiffe ber antoen ab. 2(ut) von einer nod)

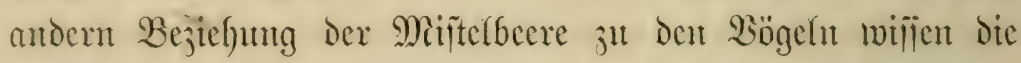

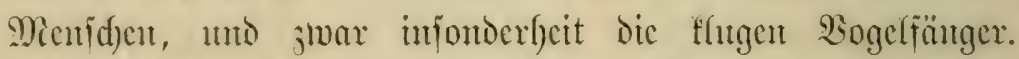

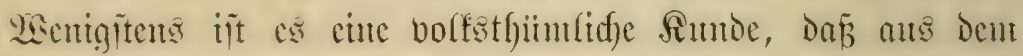
flebrigen Becrenfaft Der Bogelfem bereitet werde. Diefe

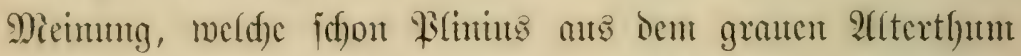

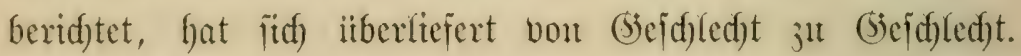

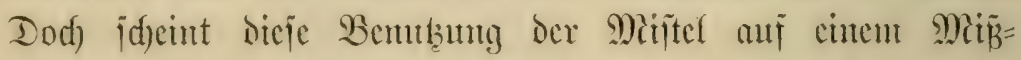

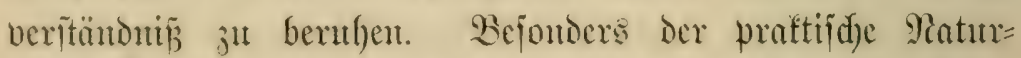

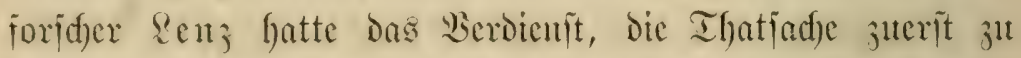
bejweipefn unt jut jagen: "wix wollen's dod) ciumal probiren!" Er hat anf alle möglidge Weife Den Mijtelfajt belfandoct, ifu

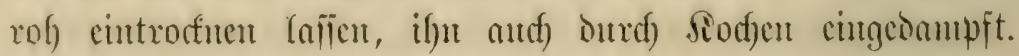
2tber inmer blieb derjelbe jebr fliififig, etwa wie cingefod)ter Ebitịnt, fo dañ an Damit bejtrid)enen Reimmetgen uidft cint= 


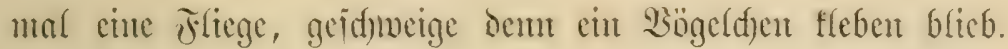

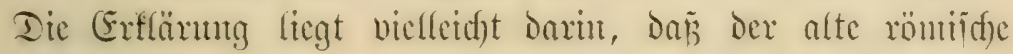

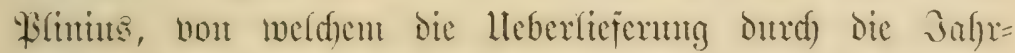
fymberte jidf lveiter nererbte, gar nidgt umjere weip̈berige Miiftel gentent hat, joubern ben bejonders in Stafien anf

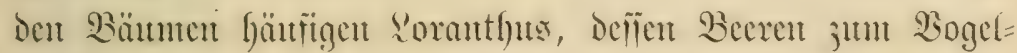
leint geeignteter jein ntögen.

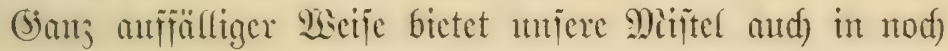

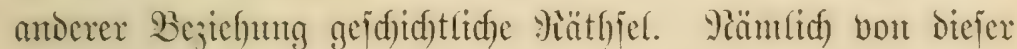
in Dentid)en 2(1tertfun vielfad) gentantent Fiflanje wiro in

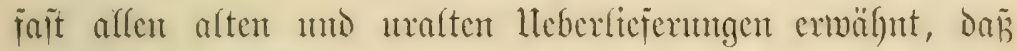
jie ipejicll anf Eidfen wadje. Tllerdings wird jie and als ali der llfme oder dem Birntbanm befindsida erwälnt, tmo jwax babe fie je nadjoen bejondere Eigenjafajten; ganj be= jonders ju bead)ten jecu auf நajelijträud)ern vorfonmende Mijtelpiflanjen, dent unter jold)ent Etrantde jet jtets cin Sdjats verborgen.

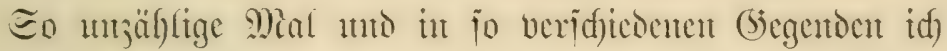
jelbit die Mititel getroffen habe, muth id) Dod) fiir meinen Theil befenten, fie nic an Eidgen entoceft ju haben. But=

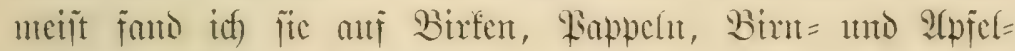

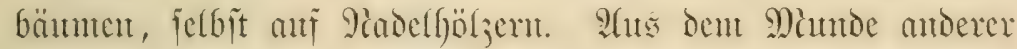

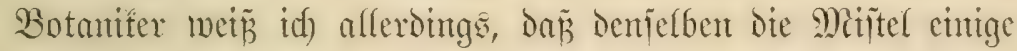
Miale autf) auj Eichen vorgefonmten jei. atber wie jelten

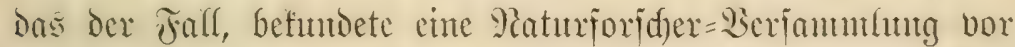
Suryem, in welder cin angeiehener Botanifer, der fid) fpejiell

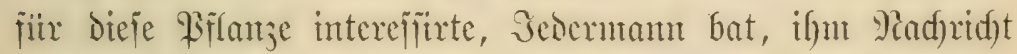
von allen ctiva $j u$ entocifenten Fumborten aut Fidjen $j u=$ fonmen ju lajien. Eelbĩt int Entglano, two jojöne alte Eidaent 


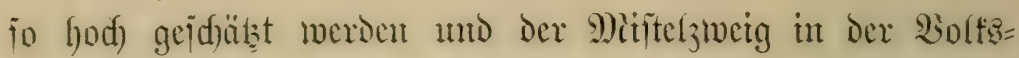
fitte cute fo beoutende giolfe iptert, Gatte der Botanifer

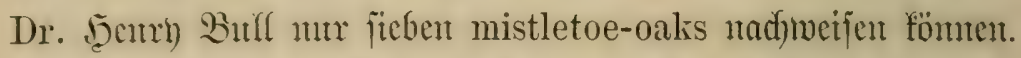

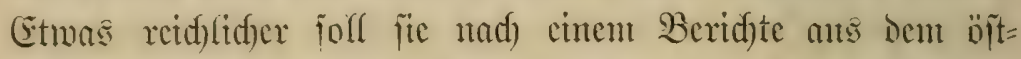
lidfen Folen Dajelbjt anf Eidjen vorfonment. Sb Die Borzeit

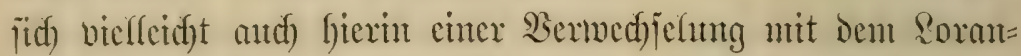
thus juhloig gentad)t hat? Sït diejer aber bod) (eid)t jut Interjecioent, indent er in Whiter jeine Blätter berliert.

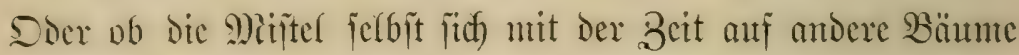

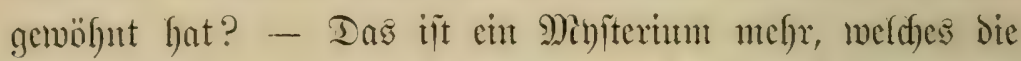

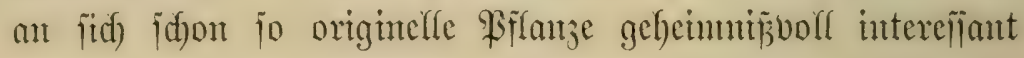
madit.

6.

\section{Streifereien in Illadshulde.}

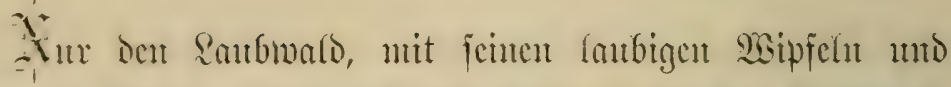

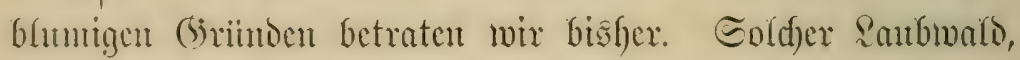

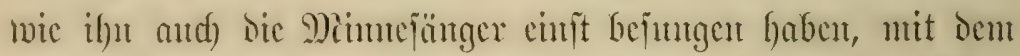

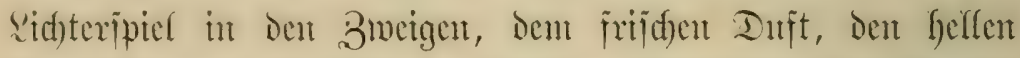

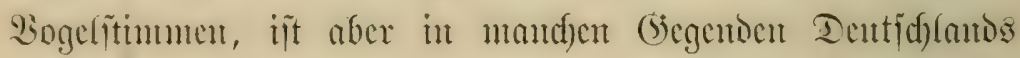
gar nidyt borbanden. In Der nordoentidjen Iicfebene, in

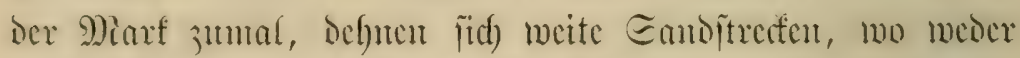

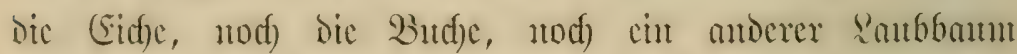

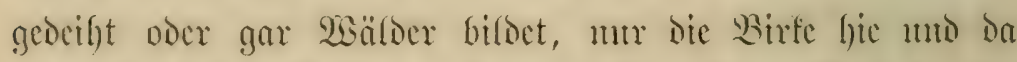

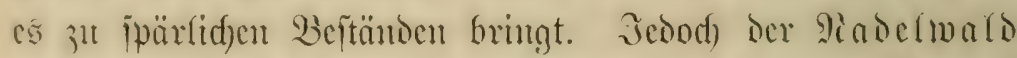

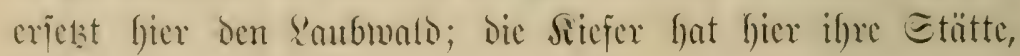




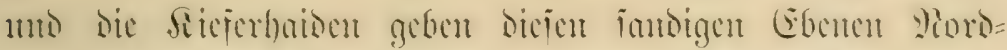

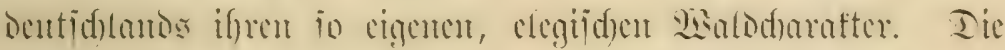

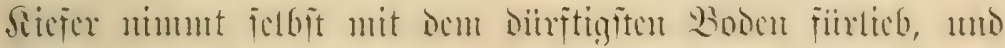

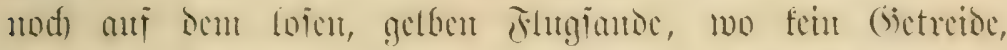

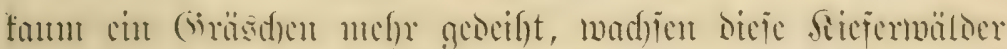

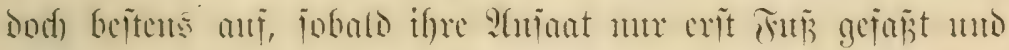

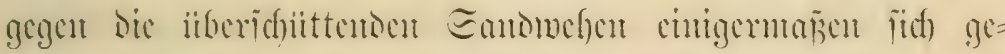

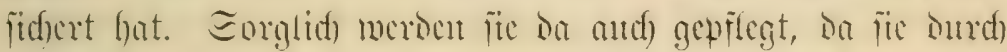

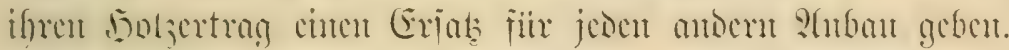

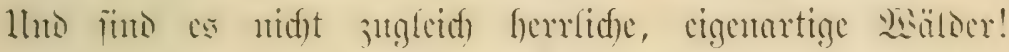
Etmmm an Etanm fteigen Die ferjengeraben, brannen Ë̈nten=

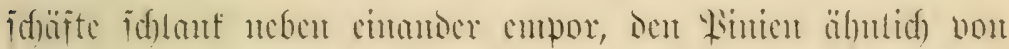

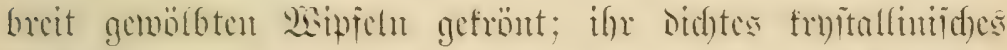

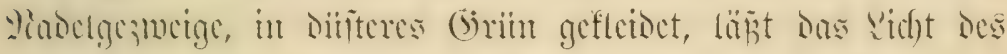

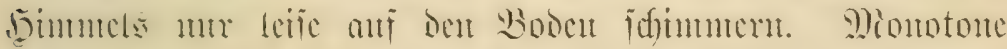

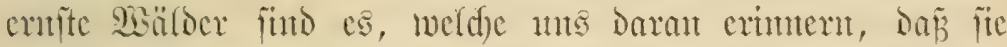

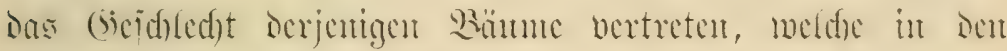

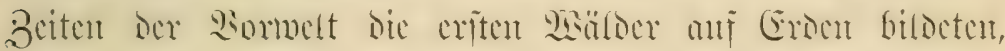

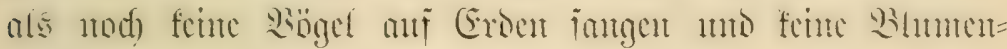

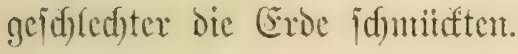

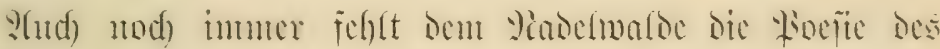

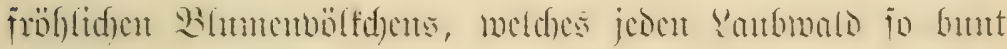

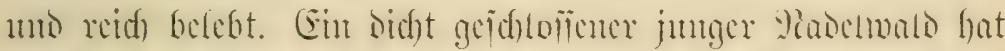

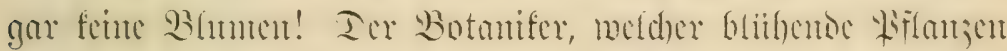

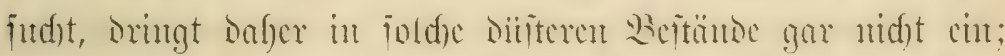

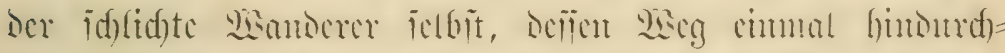

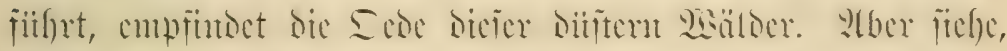

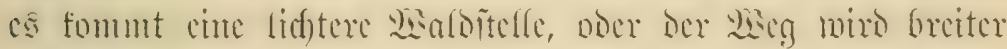

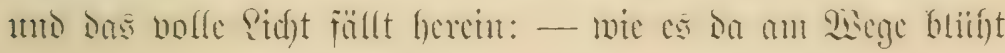




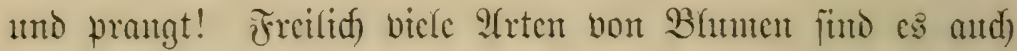
nicht, dic jidf Da mun geltend maden; aber dicje baben dafiur

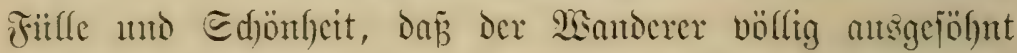
wird mit ber borigen Sede. Eolveit bas Ange reidt, ift Da oft alfer Booen itberjogen von blitfender Fradt, dic

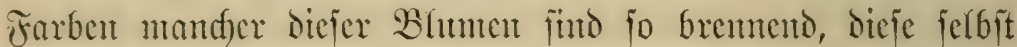

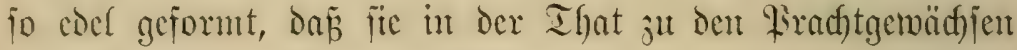
mijerer Deutīd)en நeeimath zä̆len.

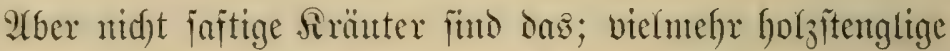

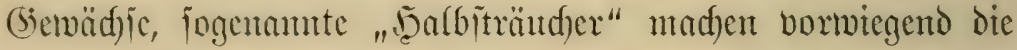

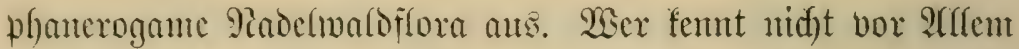

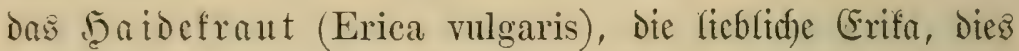
nadelblättrige, bis fubhobe @trüudflein mit den traubigen, föjtlid) rojenrothen Blïthenglöfdden, welde mit jarten Seident= glanje idjimmern. Dieje jdunnthaftejte Sharafterbume der

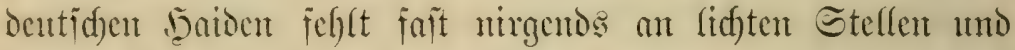

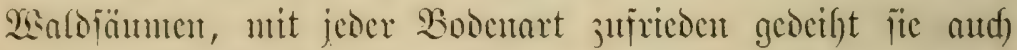
in ticjiten $\Xi$ ande mo itberjieft in älteren wie jinglten $\mathfrak{B} c=$

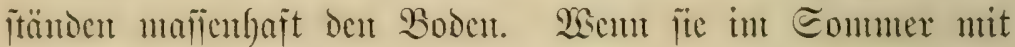

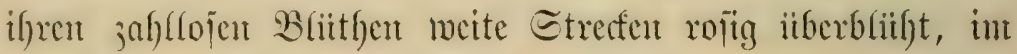

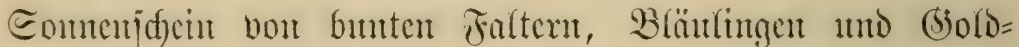
bögefn, fdanammeife itberflattert, bam bitrfte faum cine an= dere Blumenjtefle jolden Waloteppid) an 9icij ïberbietent. llno wieder cin anderer 3anber ift Daritber ergofjen, ment

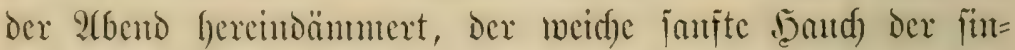
fenden Eome das Siojenroth nod) ticfer färbt, weit uno breit alles Seben dex Injectenwelt jum Єd)lummer cungelt, dic Edymetter(inge idjon miide an ocn B(iitfen hangen mo

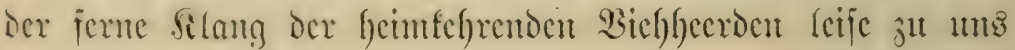




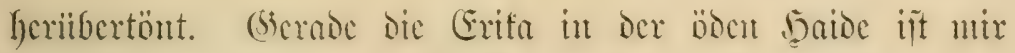

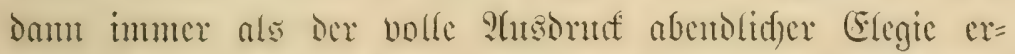

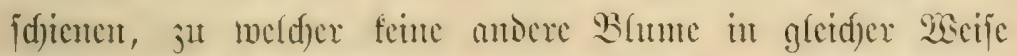
jtimmt. Eo jdmuituft jie aber and) nod) da überall bie Eroc, mo das Rebu fajt verflingt: wie fie bie boe saide jiert, fo fonme fie in ben höd)fen cinfanten Bergregionen nod) herr=

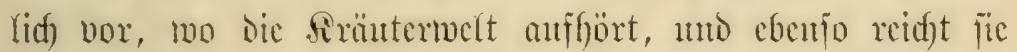

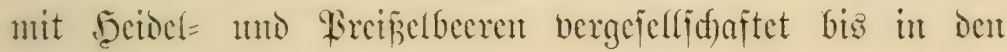
bohen Rorden hinauf.

In idjönfleitlidfer Bejicl)ung jteht ifyr in utuferen Radel=

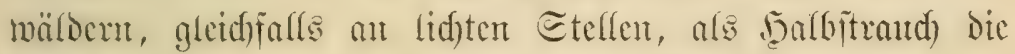

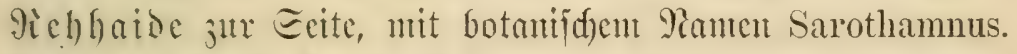
Deficu vierfantige! jelante, grime, aber jucintbar blattloje

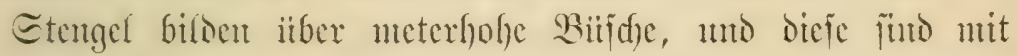

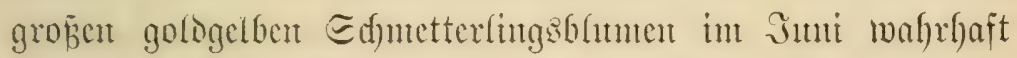

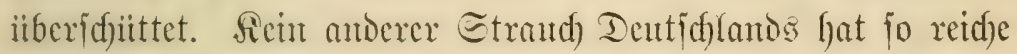

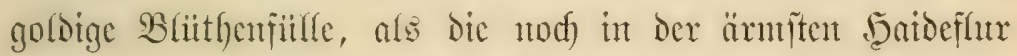

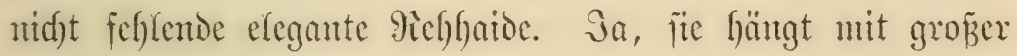
Imtigfeit ant Padelwalde, oder jutht dod) defien Miähe. Id)

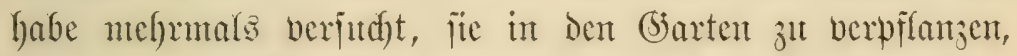
Dem fie in Der That ein föftfidger Sdumutitraud) märe. YYtber fie hat nidgt gewolft! CS ift nir weder gehmngen, fie dured)

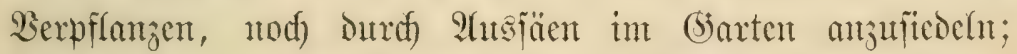
felbft ats id fuñtiefen Eand auf bie Beete bradte, gelang

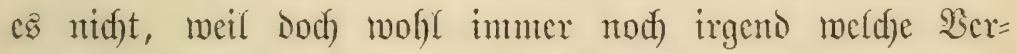

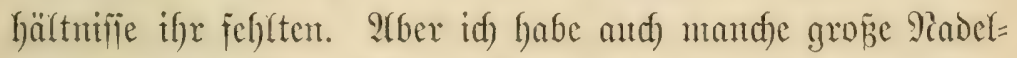

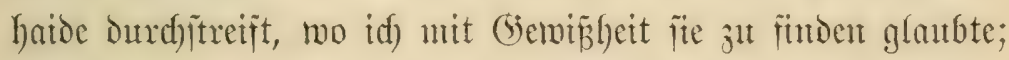
Dod) jie war nicht vorfanden, während off cin anjtofendes

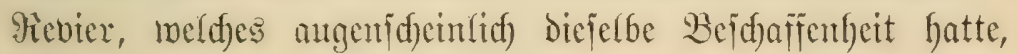




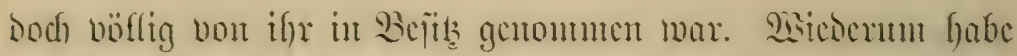
id) Viabelwäloer getroffen, ats benen jie jelbyt in bie Felder

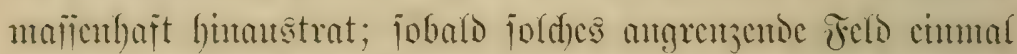

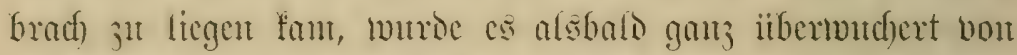

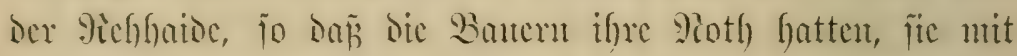

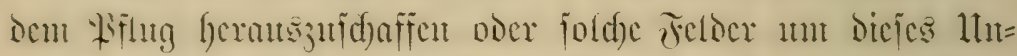

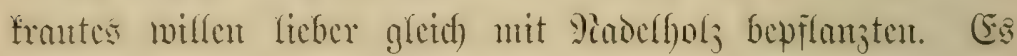

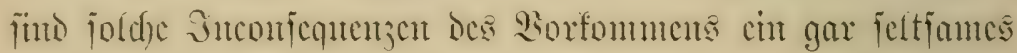

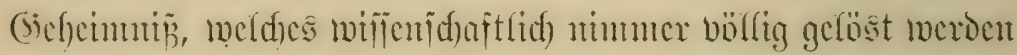
fant mo anf cine mijerm Foridgen verborgente intere 9 ht

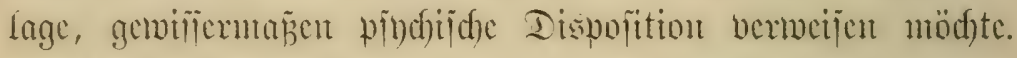

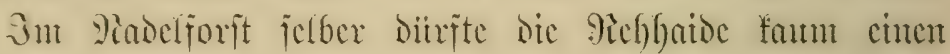

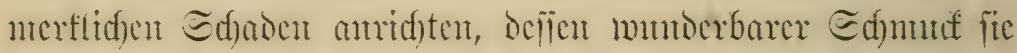
ijt unt gegen Defien Dunfles Dindelgrim das (5iolo ifgrex

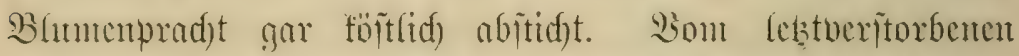

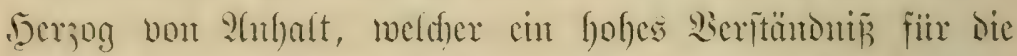

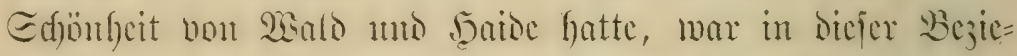

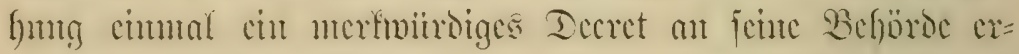

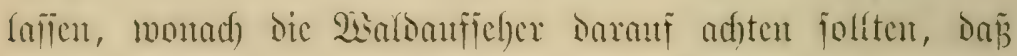

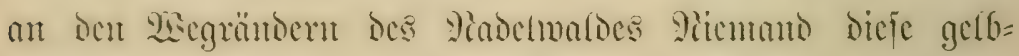

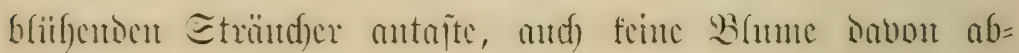

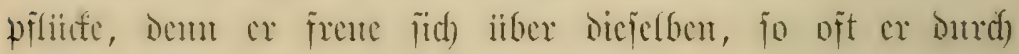

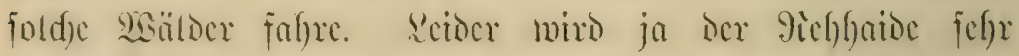

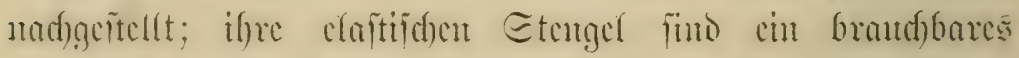

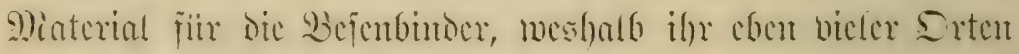

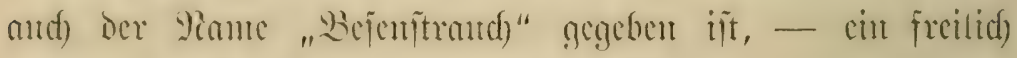

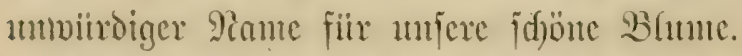

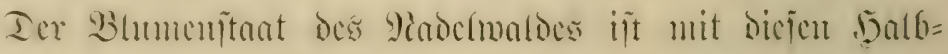

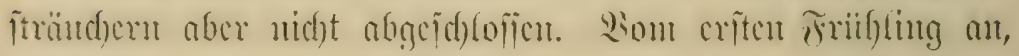




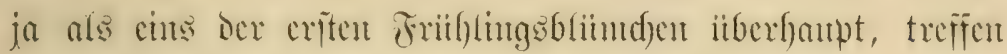
wir itberall gerabe in jambigiten Foriten cin jolstantes, funger=

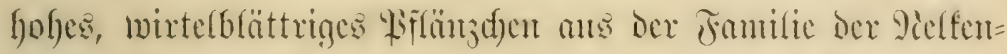

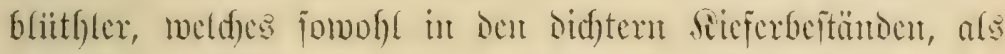

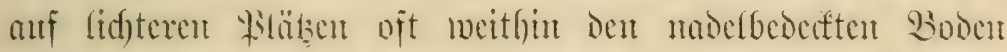

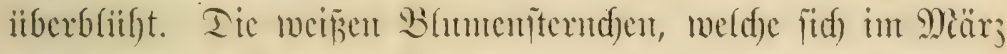

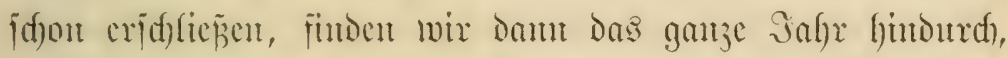

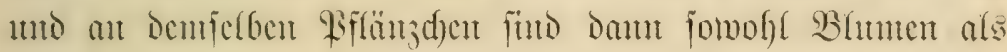

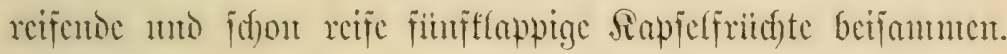

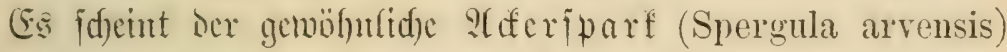

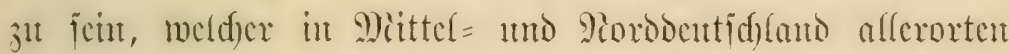
als Futterfrant reid)(id) angebant wiro, aber aud als lln=

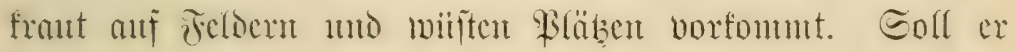
jidf) in ben $23 a$ ls un fincinberimt haben? Dod man betrad)te die fladjen Eamen, meldye bet nmjerm Piflänzd)en

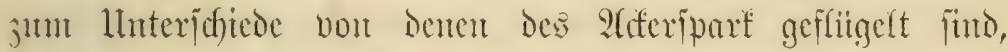

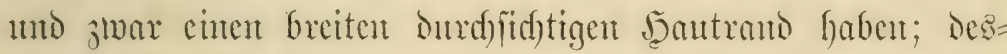
gleidfen find die Cintealen Mirtelblätter unterfeits obne dic

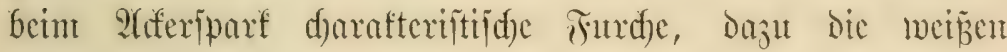
Bhlumenblättchen biel breiter. Es ift allerbiurgs cin Spart,

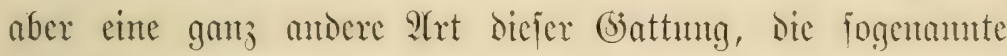
Spergula Morisonii, welde cben Durd)ans den fieferwalde angebört, aflenfalls aud) anf Eamobügeh borfonment, aber

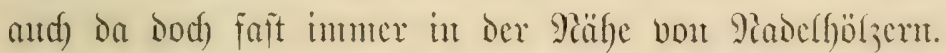

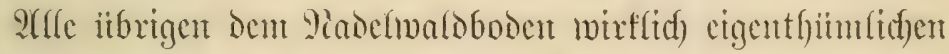

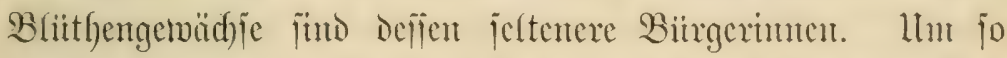

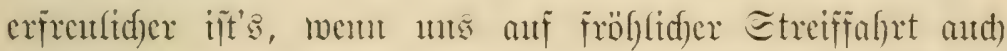

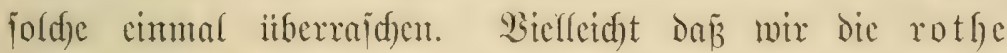
Bärentraube (Aretostaphlus uva ursi) mit antreffen, melde 


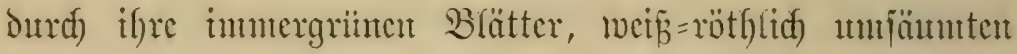
Błittfen und granatrothen Beeren ant dic nur int Gebirge borfonmente Preipelbere crimert. 9rod) cher fömten bir freilich) cimmal bas gleid)falls inmergrïn beblätterte munder= reijende Wintergritu, die Firolblumen füben; Deren

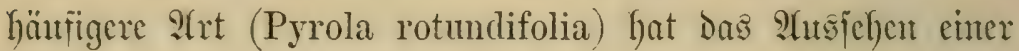

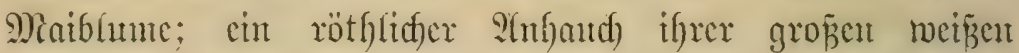

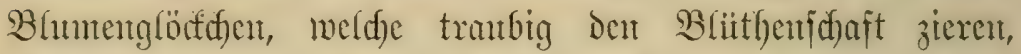

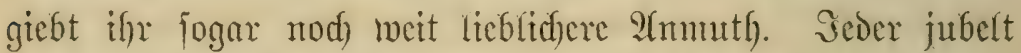
wohl auj, der bieje Pinrolblumen cinmal fintet, dieje alfer=

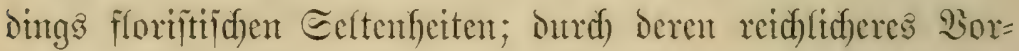
fommen hat manther Patelwals in Der. That botanife $\mathfrak{B C}=$

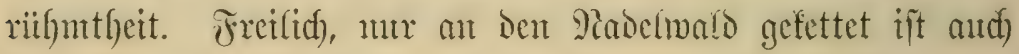
Diefe Piflanje nidft; bejonocrs dic P. secunda unt P. rotundifolia fant man and) in Ranbruälocrn juncifen treffen, wo= gegen dic $P$. uniflora tur in Tannenwälocru vorfonment. Man fintet ine alle bejontors an leidfidhattigen Stellen, an

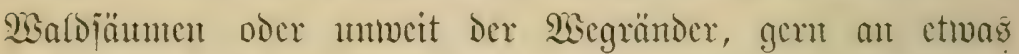

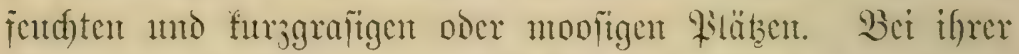

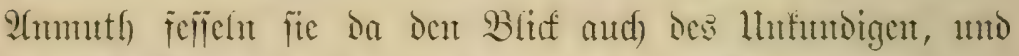

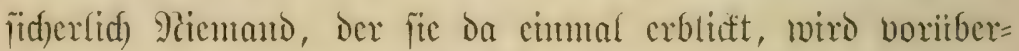

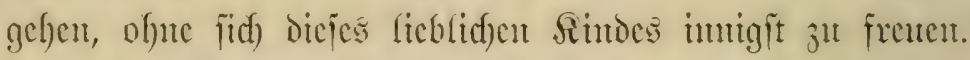

Ja, che Siflanje ijt nad) ben Siadeluald fogar benant

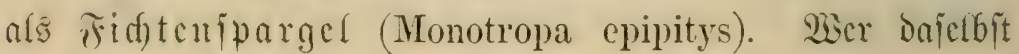
Dies böd)it originelfe, näntlid) Durd)weg bleidfgelb=rbabarber= farbige, blattloje, mu mit Edyuppen befleibete, feifte be=

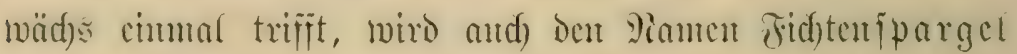
begreifen. :) der Eprise mit ährig=gereiften, glcidffarbigen,

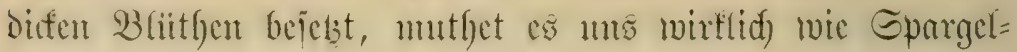




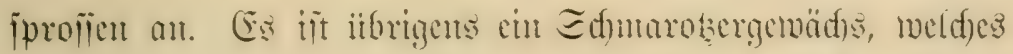

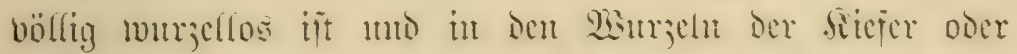

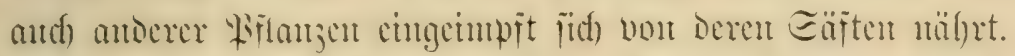

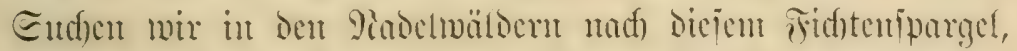
wir merben iffu ba fiffou cimmal treffen, wo er jwifiden dent

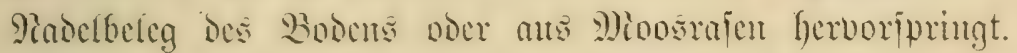

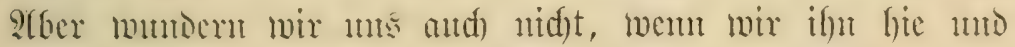

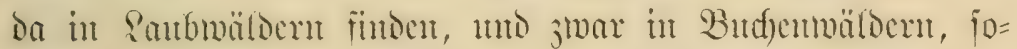

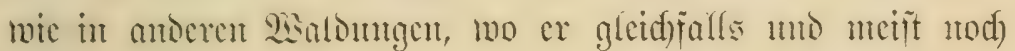
iippiger anto reidhlidfer gedeift; idf habe saidfoit idhon gegen fiunjig Exemplare trappartig Geijanmen gefimben, wäfrento

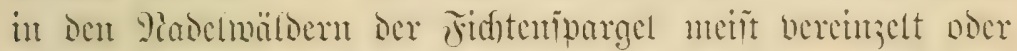
in febr geringer :

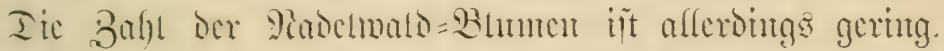

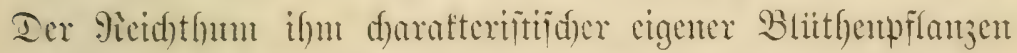

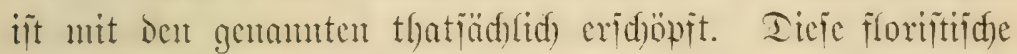

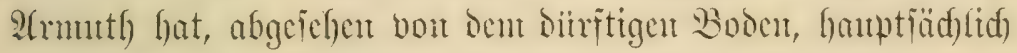

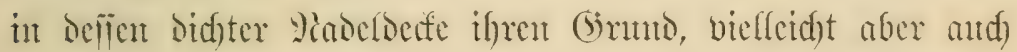

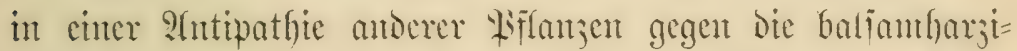

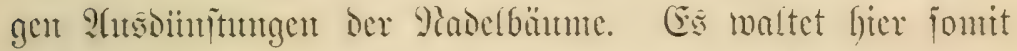
nod) immer ber bliithenlofe (Egarafter vor, welder ben cont=

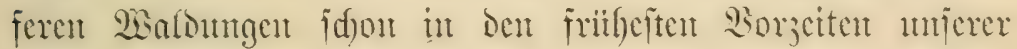
Erobe eigentfiimtid) war.

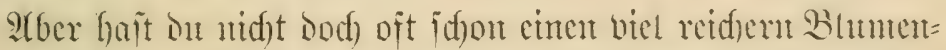

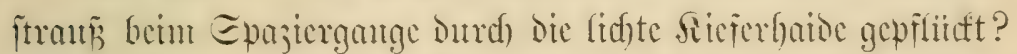

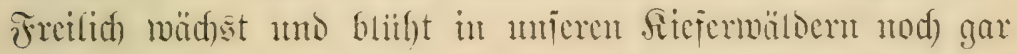
Manderlet; ja wir föment hie nto ba jogar nod chrte ganj

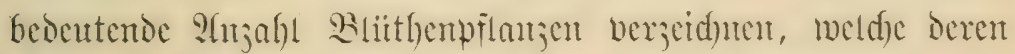

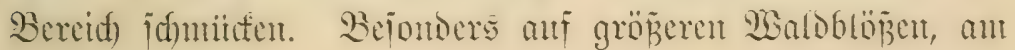




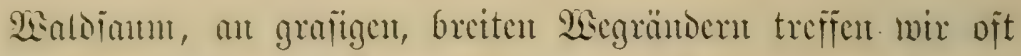

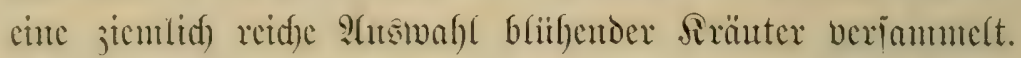
Seood) alfe sicje baben mu als (säjte, oder als Eutoring= linge ba ifren Einjug gebalten: es füb bas nux "Eand= pflanjen", bon Denten dic gelbe und rothe Jumnortelle,

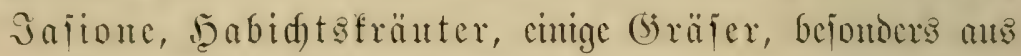
Der (Sattung Aira, jowie Riete jid) reid)lidyjt cinfinden unto oft ba and) jut präd)tigen Bhnmenbeeten gruppirt fino. כe

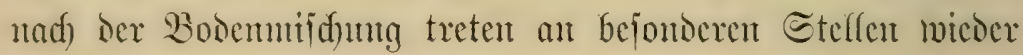

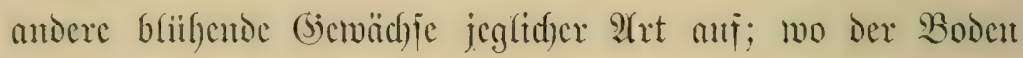
fendfter wiro, gar ein Bad) binplätjdert, fintet abermals eine neue ๔abar ifre Rebensbedingungen und fiedelt jidf lujtig an. 2rber Sharafterpffanjen Der Tadeflyaide find fie

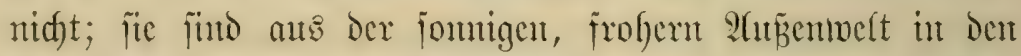
Radefivald unbernfen Gineingefommen, beffen botantipder Sharafter in ber. That die atrmuth an cigenen briifentom Sräutern î̃t.

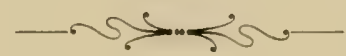




\section{III.

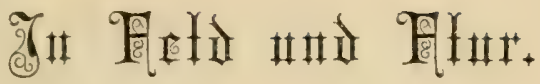

Roth' und blauc Blumen

Der mürrijhe Sd hitter verwirft euth als nutglos, Göljerne flegel 3erorciकen cud lÿhnend,

Sogar oer habloje wanderer,

Den cu'r 2lublict crgötyt und erquict t,

Schüttelt das 5aupt

Uno nennt cud fđönes Mnfraut.

2lber die ländlidge Jungfrau,

Die Kränjewinderin,

Derehrt eud und p flüft cud?

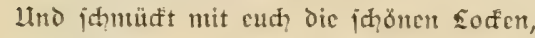

Ilno aljo gejiert, cilt jie jum एanzplat,

Wo Pfeifon uno Ecigen lieblid crtönen,

Woer jur ftillen Budfe,

Wo die Stinme Des Eiebîten nod licbliḑer tönt, 2lls Pfeifen und Eeigen.

f. f)eine. foud der ficoer. 
Das feld hat vor 2 lllem die 2 lufgabe, nüblich zu fein, denn es ift das cigentlidłc Culturland. 2lber es hat vom Sđ̆öpfer nod die andere 2lufgabe er= halten, Wolznjtätte auch einer wounderreichen Blumenwelt 3 fein, weldhe ander:

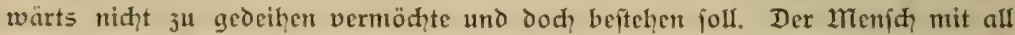
feiner Mtadht fämpft daher jẹt vergeblid gegen dies fogenannte Unfraut in feld und flur, ja er giebt gerade durd die Bodenverbefferung und die von feinem Straudh nod Baum aufgehaltene volle BeIcudtung demifelben immer von neuent die rechten sebensbedingungen. 2lber wohl uns, es gehen die meifen Cul=

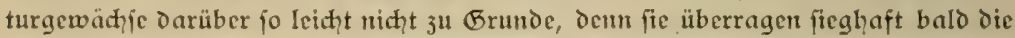
dod Jumeiff fleinen wilden Bluntenfihaaren, und unter dem Schatten des 2lehren= gewühlles mögen diefe darm fith beftens cntwiffelr. 27un, und find fie niḑt eine

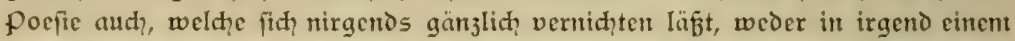

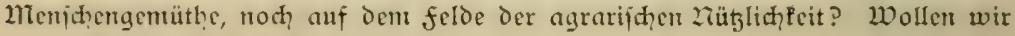

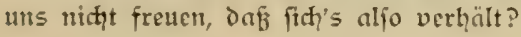

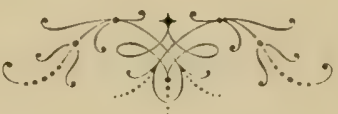




\section{1. \\ @intuturblumen.}

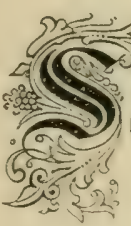

fröhlidy dic Serefe ant flarent Dinnmel idjont fungt,

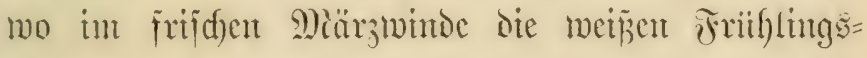

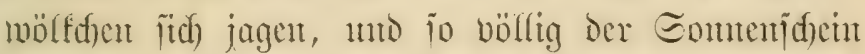

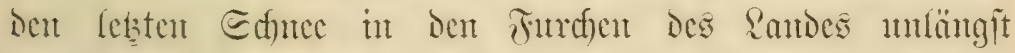
weggeid)molzen hat: Diejes felbjt fteht dod) den ganjen Miärj ïber nod) in vorjährigen Etoppelfteioe, dic Winteriant regt fith faum, 2Alles jodeint ntod) in tiefent $\approx$ d)laje jut liegent.

lind bod) fömtnen wir bamt jdyon nad) Butmen, gerade

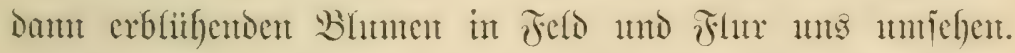
Gin erftes cigenartiges, jierlides Feloblumengejd)led)t ijt

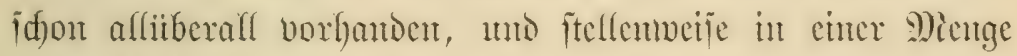

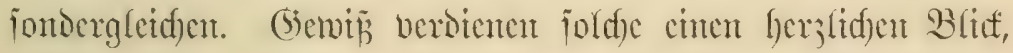
- dic eriten Feldblumen des Jahres! 2tllerdings Blïmd)en,

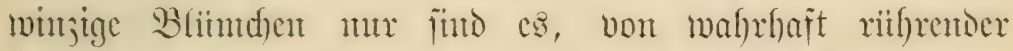

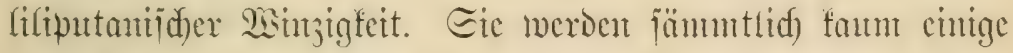
Boll hod), jo ba bezeidjuen fömen.

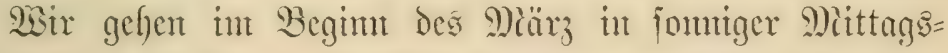

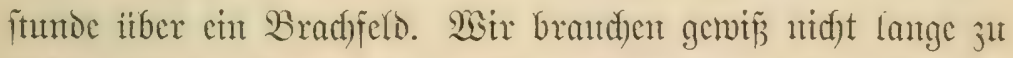
judchet, jo getwalyren wir cint faum fütgerlanges fintgelagertes 


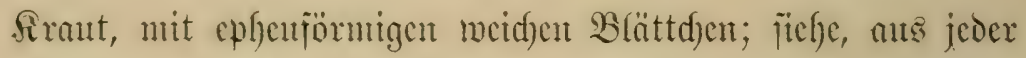

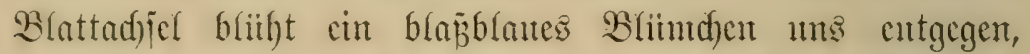

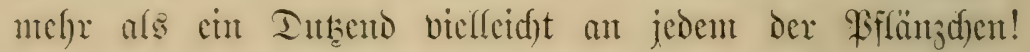
3u cuten criten Frithlungsträu仿en legen wix fic frendig jufammen, Das wix mituchmen, un ç dabent in's fontige Feniter ju ftellent und uns der jo jarten (Sabe nod) länger ju frencn. 2res den epheublättrigen Ghrenpreis (Veronica hederifolia) bat cs die Piflanjentunde benannt; ja, unto wix

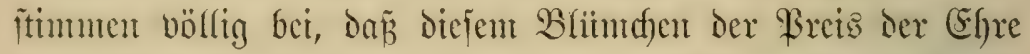
gebiifrt, da ç nit fenten farbengarten Bamenfröndent auf= jubliifen wagt, che nod) Der Friifling wirflid in's Sand gejogen fimt.

2tber wie murden wir betritbt, als wix daheim das Wiflänzd)en in cin Wajlergläsd)en ftellen wollten, dantit es aud in unferer

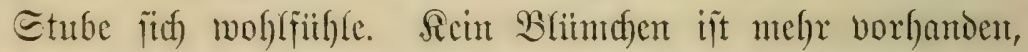

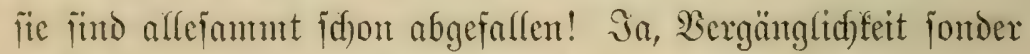

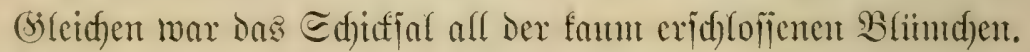
2tber warten wir den folgenton Morgen $a b$ !. Dant fintoen wir cs wieder iiberiät vout ueu crifflofienten bläulidben

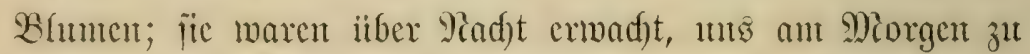
erfreuten. Eo verbfithen fite fitetig nut jeden 2 tbent, aber

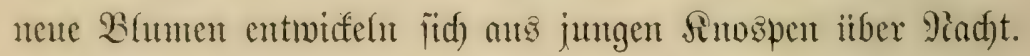
Eo geft es tagelang, wodjculang, bis wir andere bunte

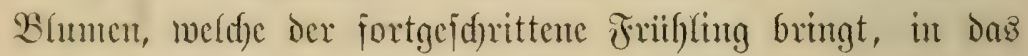
(S) fas jeben fömten.

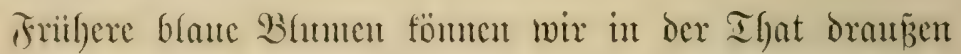

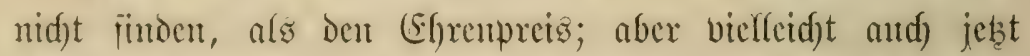
jd)on treffen wir nod) dieje und jene andere 2 (xt beflelben

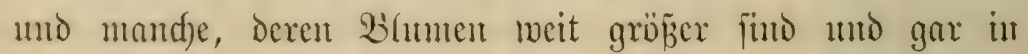




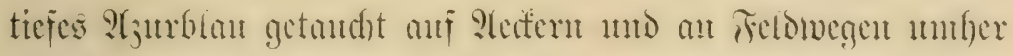

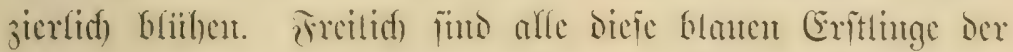
Flur glcidfalls gar winjig, oft um joll = nocr fiugerfobe

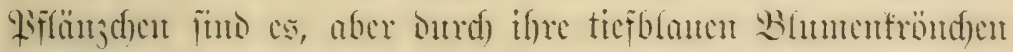

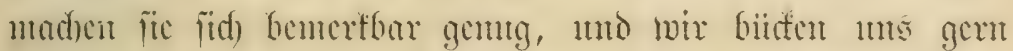

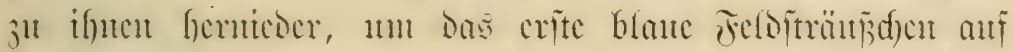

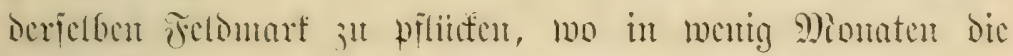

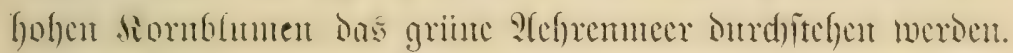

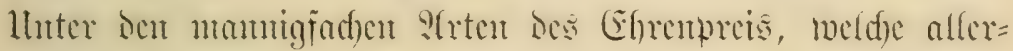

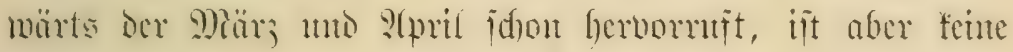
¡d)

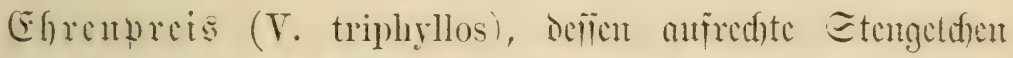
mit hanotheiligen suntelgrimen Blättem beickst mo mit ber=

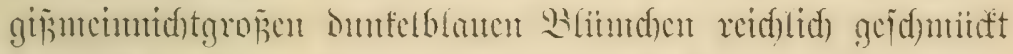

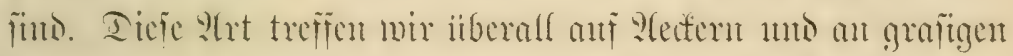

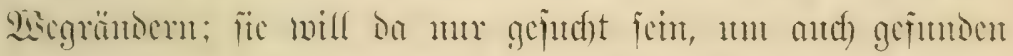

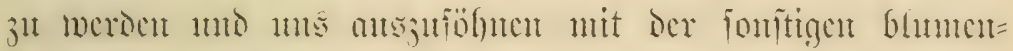

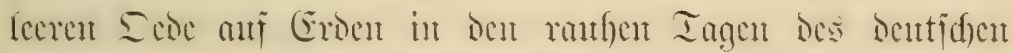

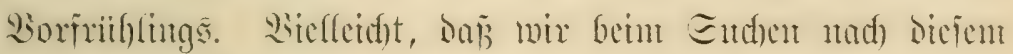

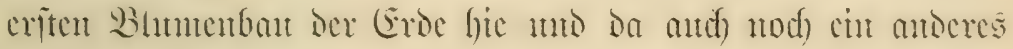

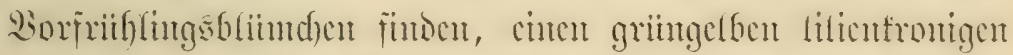

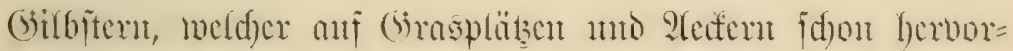

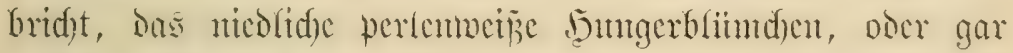
aud) cine bou borigen iafure her iberwinterte rothe Iatb=

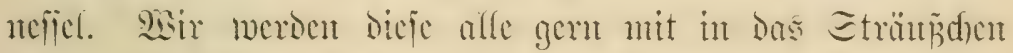

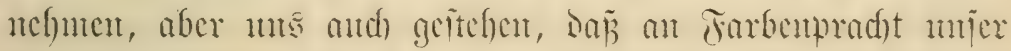
E.frempreis fic bod) alle itberitrabit.

Iarm babon bic 2)icniden bout jeber diciom blaten

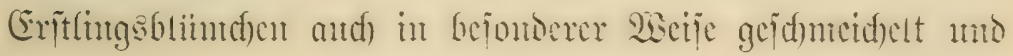


ifm cincu Pantu gegeben, wie ifn feine andere Bhlume bes

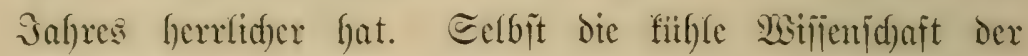
Botantif bat in wal)rbait poctijd)en : Beronica bejeidnut, und bas B(iind)en ift baburd) in Bejichung

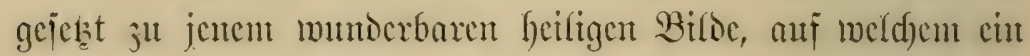

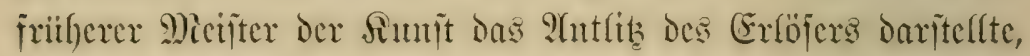

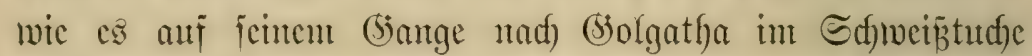

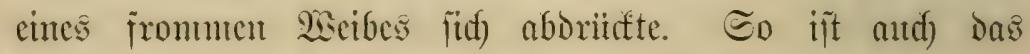

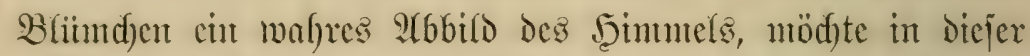
Deutung Der Pame Beronica bejagen. Derielbe läp̄t jedod)

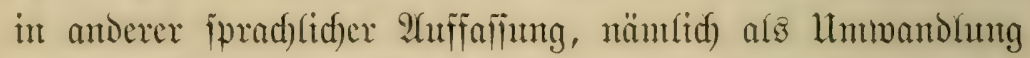

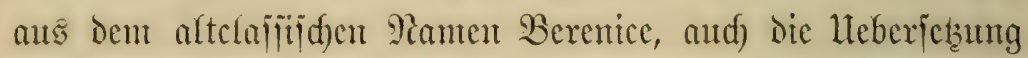

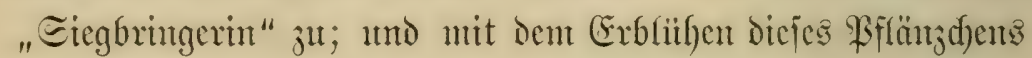
trägt der Friifling int Etreit mit Dem Winter in ber That Den baldigen vollen Sieg Davon.

Piod) andere chrenvolle Tamen fünon wir ats den mittel=

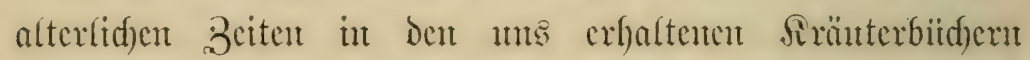

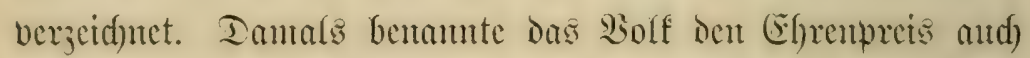

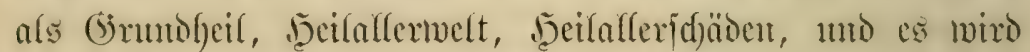

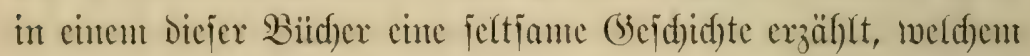

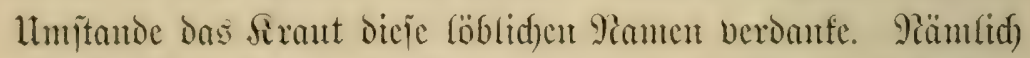

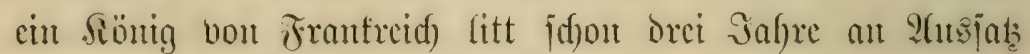
und Riemand fomte ifne heilen. Da fal) cinmal ein Dirt,

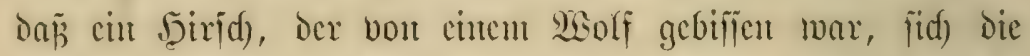

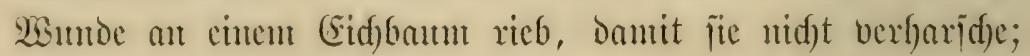

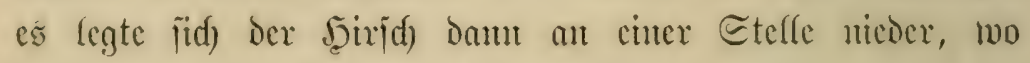

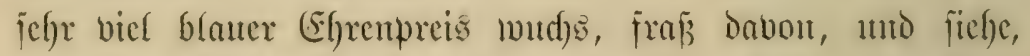
cr wax stad) adjt Iagen böllig weieder hergeftellt. Dex Deirt, welder boll Lermumbermug das bcobad)tet batte, bereitete 
mu cincu Eaft bou (E)renpreis; er ging domit jun Siönige,

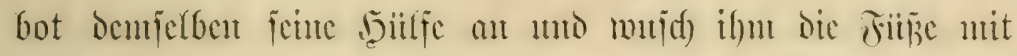
dicfen Eafte. In der Siad)t mun murde der Sïnig an der gewajd)entu Etelfe bon grö̈en Edunterjen bejallen; als jeinte

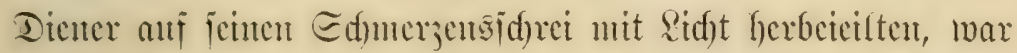

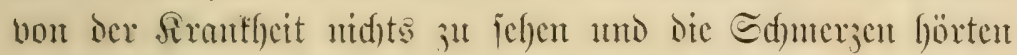
jekst auf. Surz, er war genejen Durd) diejes Sraut.

Sb Dicje Mismocrberid)te freifid) gerade vou unjerm fleinen Friil)(ingsel)renpreife gelten, möd)te fraglidf ien. Den ganjen

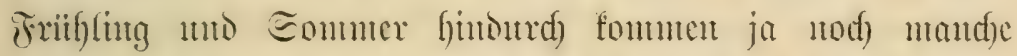

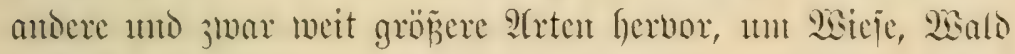

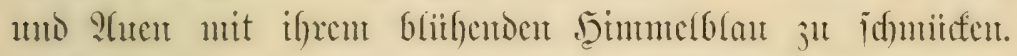

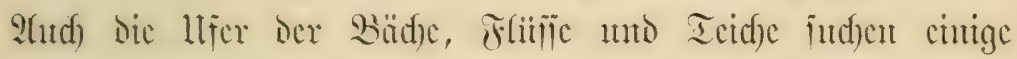

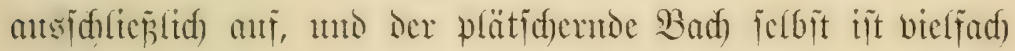
ganj crfitllt mit eincu Ehrentureis, defien jaftiges helfgrimes

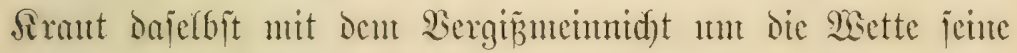

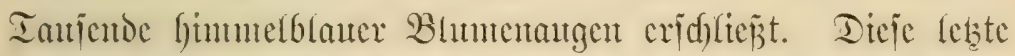

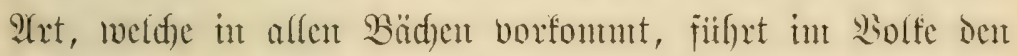

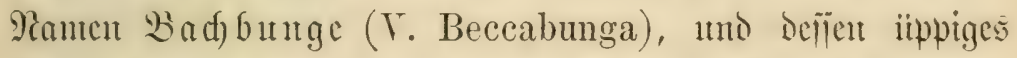

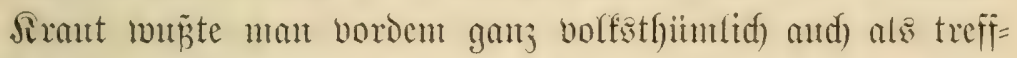

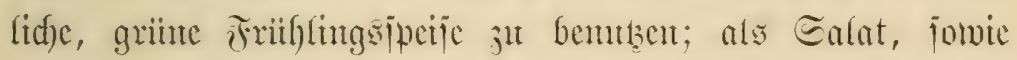
gefocht als (sicnitice, wurde es auf Den Familientijd) gebrad)t,

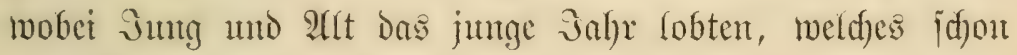
jo friil) edres (srün biete.

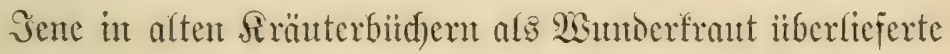

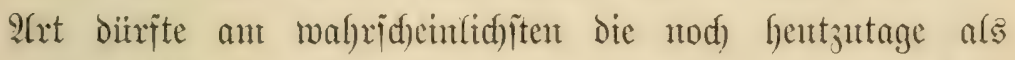
officineller Egrenpreis (V. officinalis) bejciducte fein,

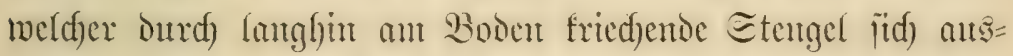

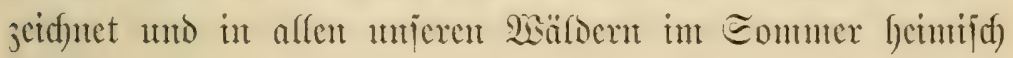




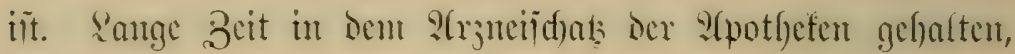
ijt cr crift mlängit baraus gejtridfen worben, weif man if)m

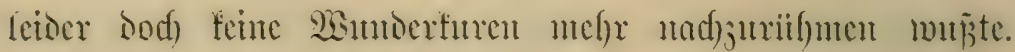

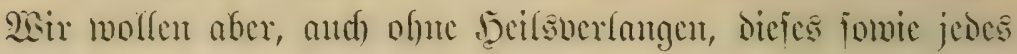

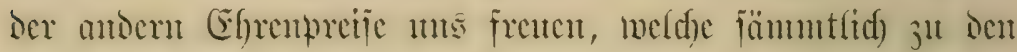

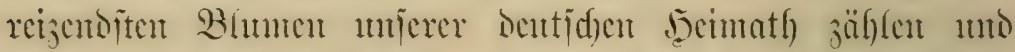
das ganze Sabr iiber Die borjiglidejten Siertreter des reinten

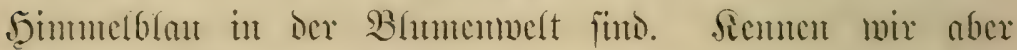

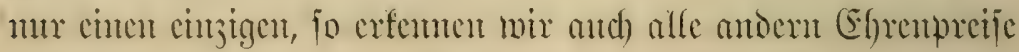

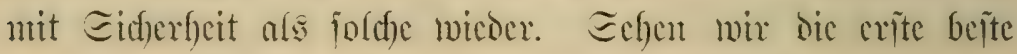
Sht cimmal an: wir gemahren, dañ bie in jmeiblättrigen

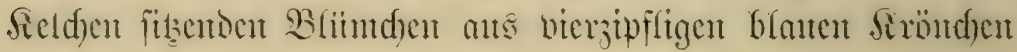

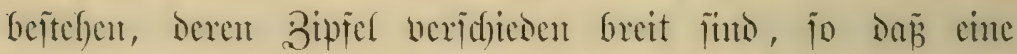

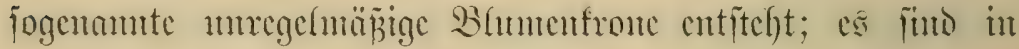

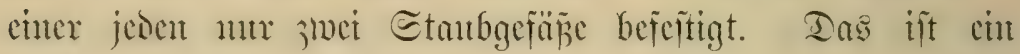

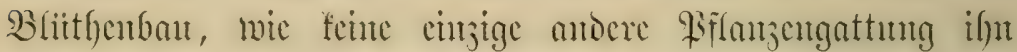
hat, aber ganz cbenjo fumb dic Blimtden all dor viclen

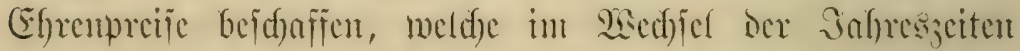

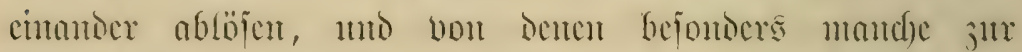

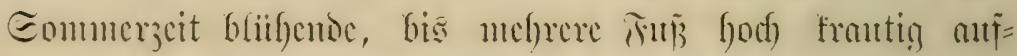

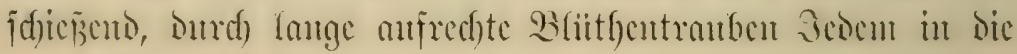
2utgen fallen.

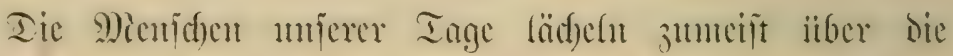

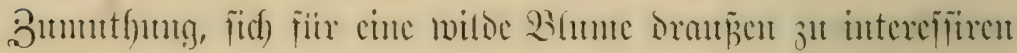

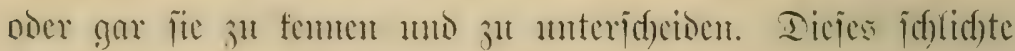

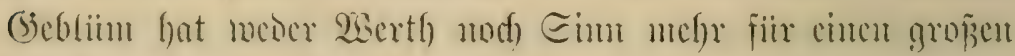

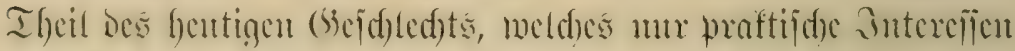

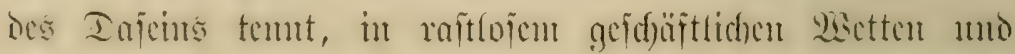

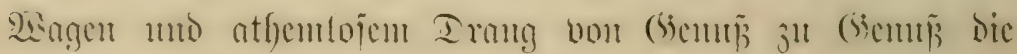




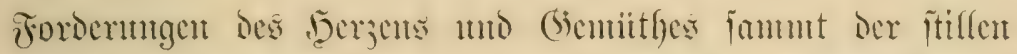
Epradie ser Matur berternt hat. 23as tam Eolden cine

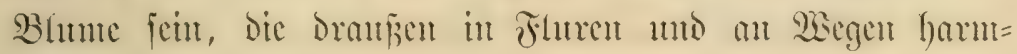
log bliillt?

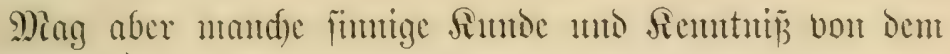

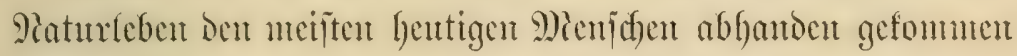

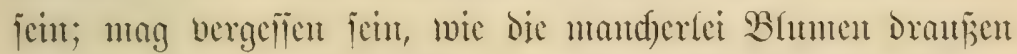
bormals mit ber Eprade, mit ben P(nj)anntagen mo

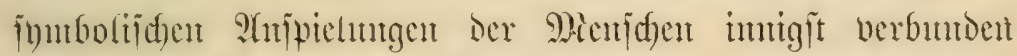

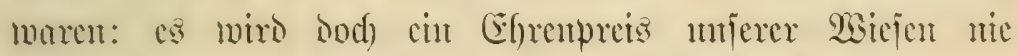

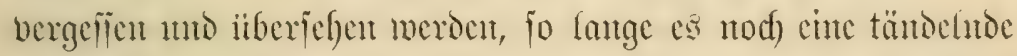
Jutgeno giebt, weld)e an anffärlfigen Eridyemungen in ber

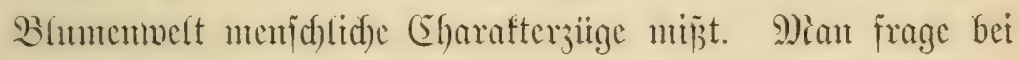

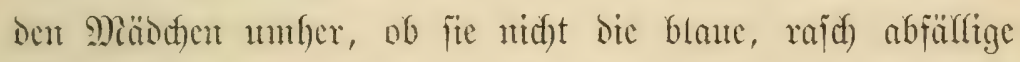

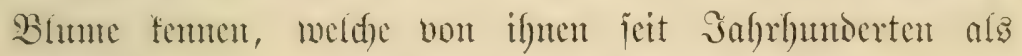
Miännertren (V. Chamaedrys) bentant ijt. Eie merden

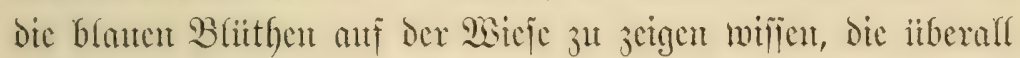
da ftolz emporblitfen. alber nur menige Beit brandfen wir

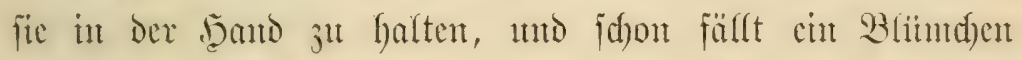
nad) bent andern ab. So - find dic Miänner! ruft bas

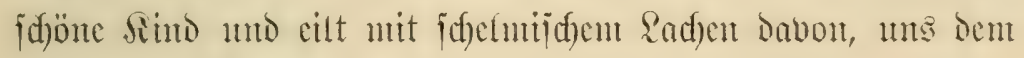
Tadodenten zut ïbertaffent.

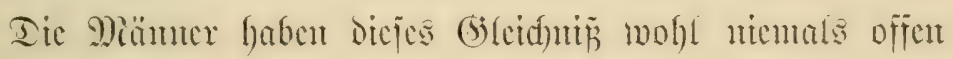
jugeftantou; fic haben viclntehr cine antore Siffanje (Eryngium

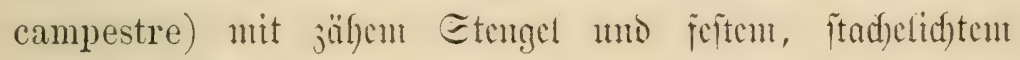

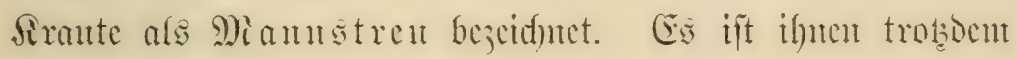

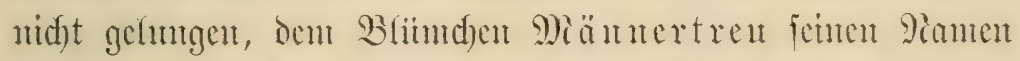

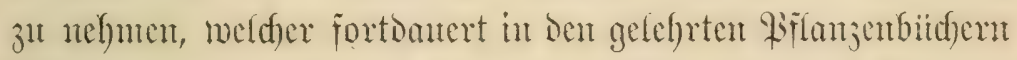

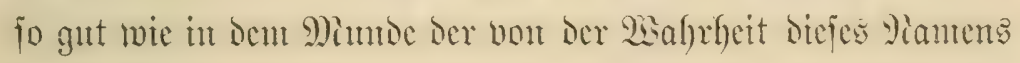




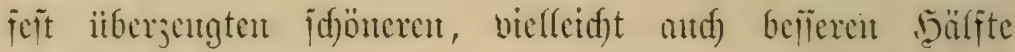
unjeres (sieid) (echts.

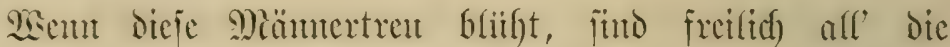

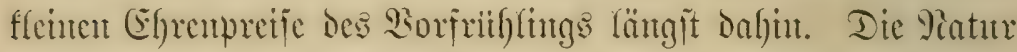

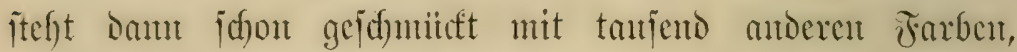
Bhlumen boll Frad)t mo Duft jicren dann die Erobe allitber= alf. Bis Dabin hat es aber nod) gute 2 beile in der frithen

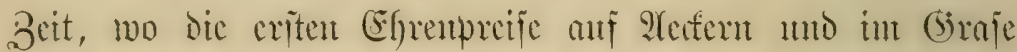

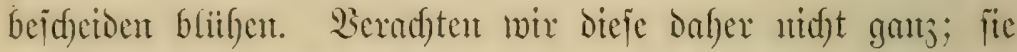
wollen uns ja and blos leije jagen, daz̃ jie die exjen blaten

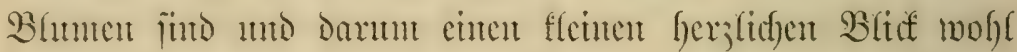
verdientent.

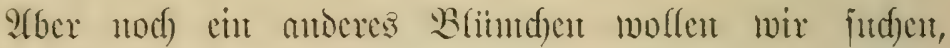

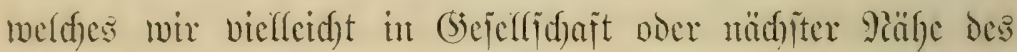

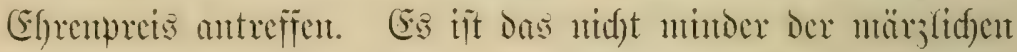

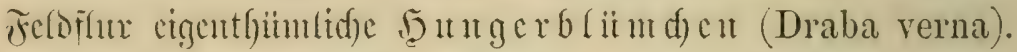

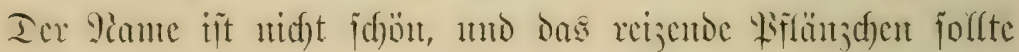
cuncu befieren baben; aber ex ift nidgt böfe gement, ocun cr

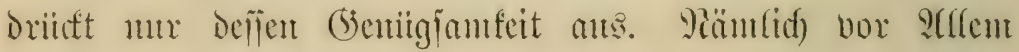

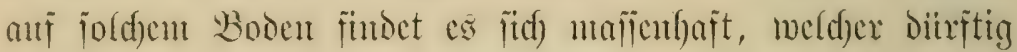

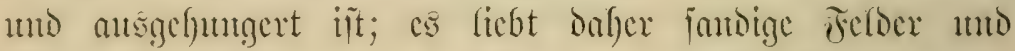
Iriften, and 9)iancen und Felien und wird da jelten vergeb=

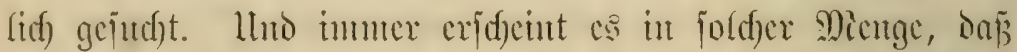

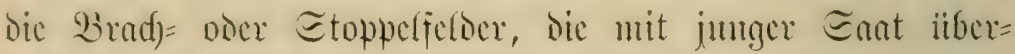
griunten Afeffer, Dic jomtigen Iriften mo furggrafigen baiged wic bou cincm weip̈lidfen, jart gewobenen Edf(cicr Durd) dic

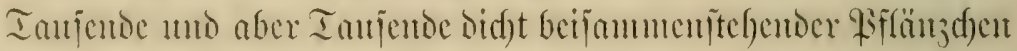

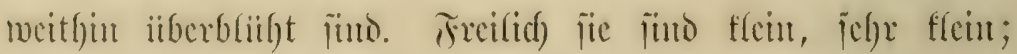

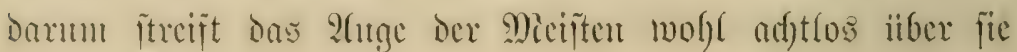




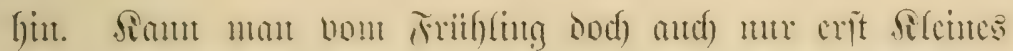

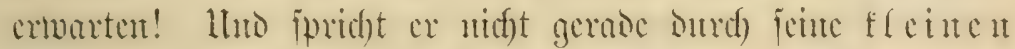

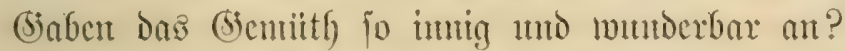

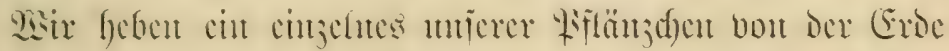

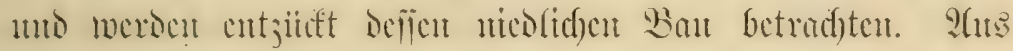

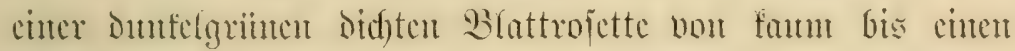

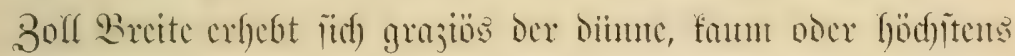

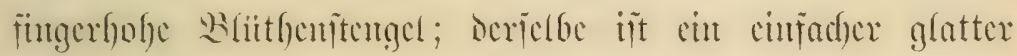

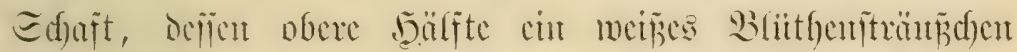

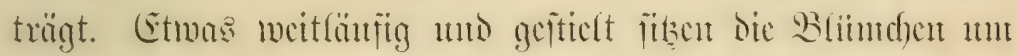

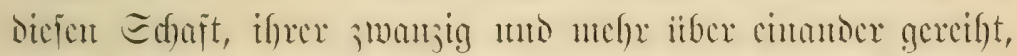

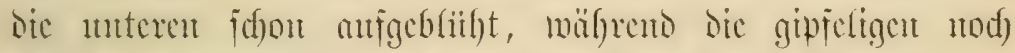

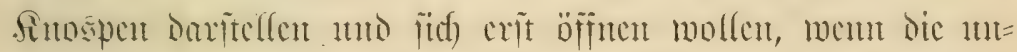

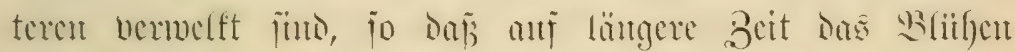

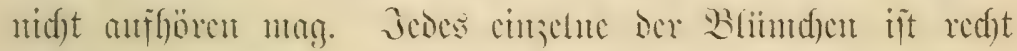

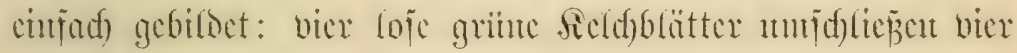

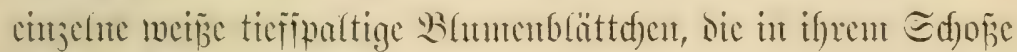
ject) gelbe Etutbgeräzic halten, in beren Ditte wicocrum dic

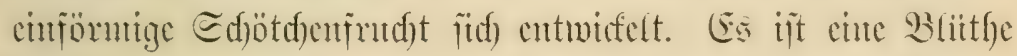

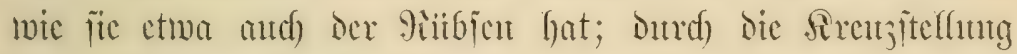

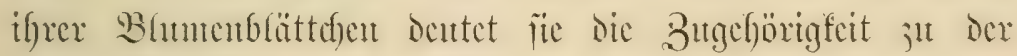

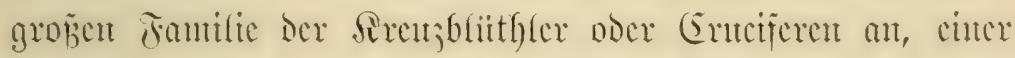

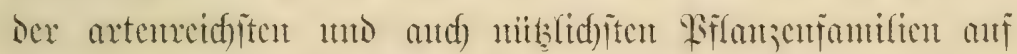

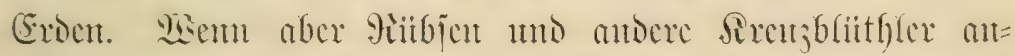

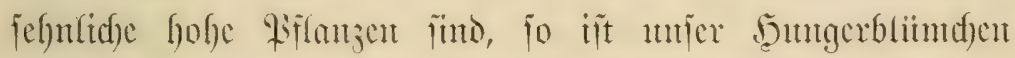

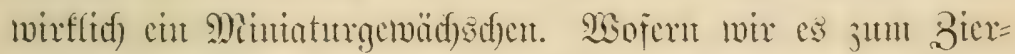
gewäd) exflebent wollten, witrde fïr bic filltur an fomtgent

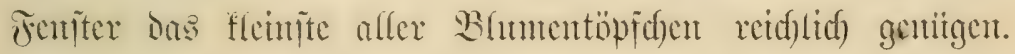

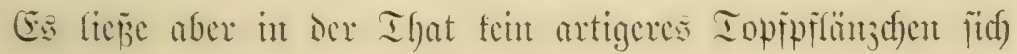




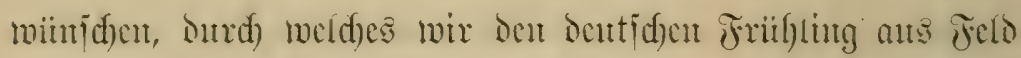
und ?tuc in die Etube jaubern und an cintent fenter woit= jigiten (sicbilde uns crirenen föm ten. Freilid), ntand) itoljen

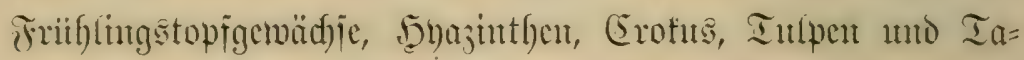

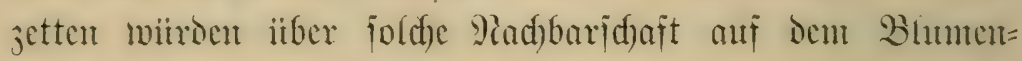

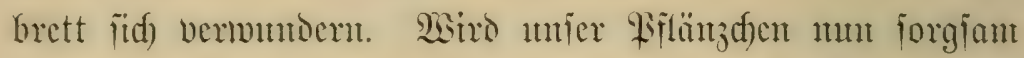
in cuncu Topf an Fenfer cingedflanjt, oder and) nur in cin (5)as

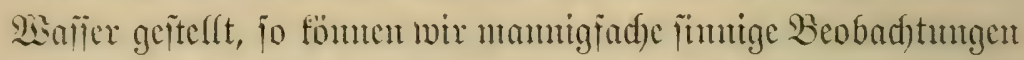

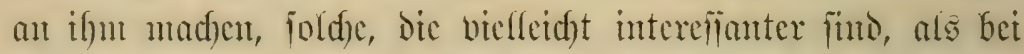

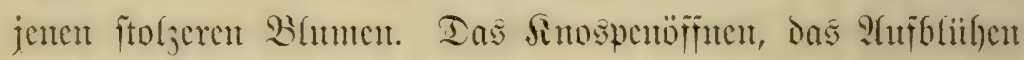

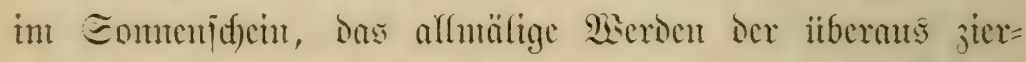

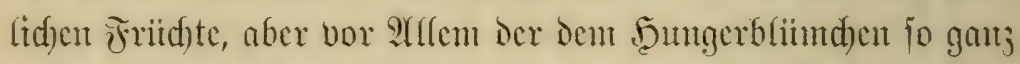

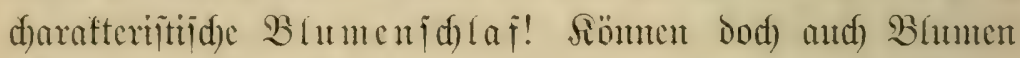
id)lajen, mo jwax meijt indout fie iffe Bluncufronen träı=

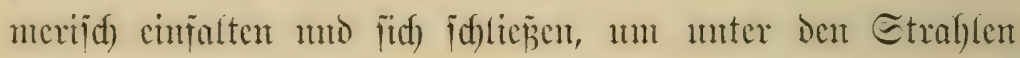

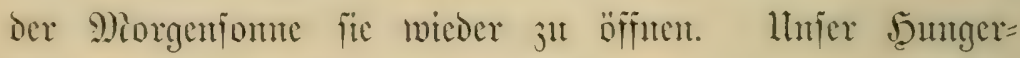

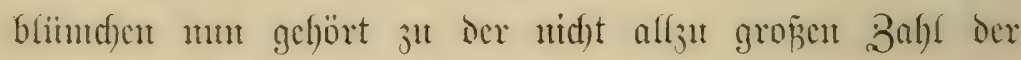

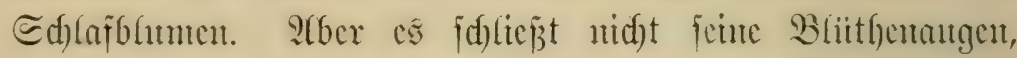
ment

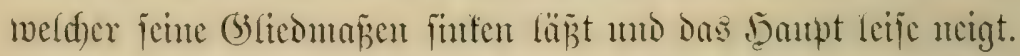

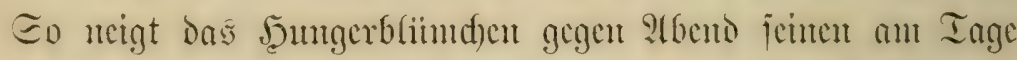
ferjengeraben anfred)ten Bliithenwipfel, ber Edhaft frimm fitd) Dabei in meitem Bogen alfmälig cin mo meigt fid

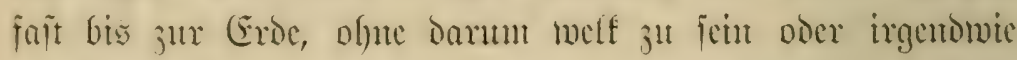
an Cla fiticität ju verlieren; cbonjo bengen fidf) bic an Iage

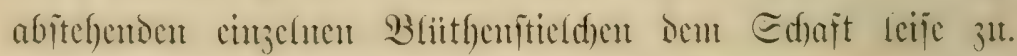

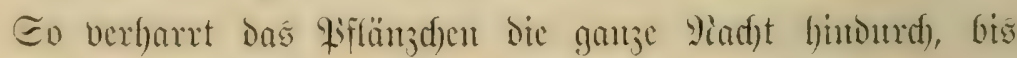
Der Diorgen fonmut un unter feinten warmen Etralslen fids)

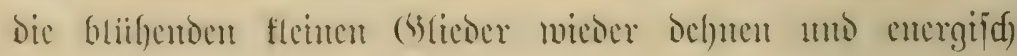




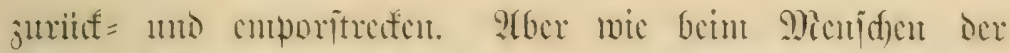

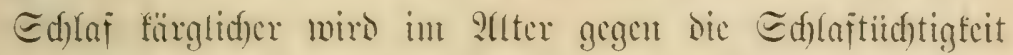

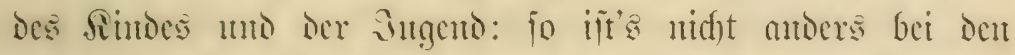

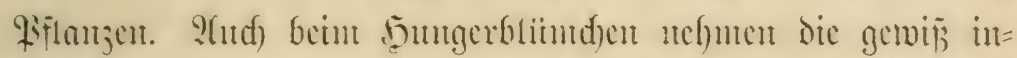

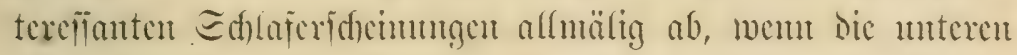

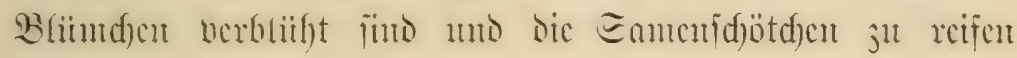
begintent.

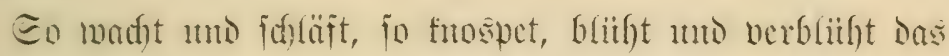

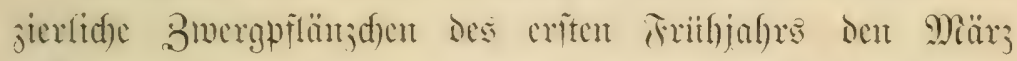

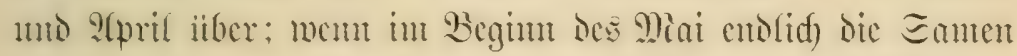

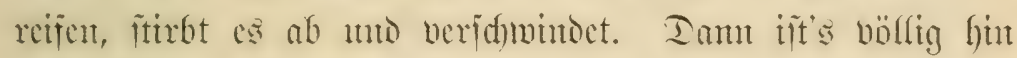
mo cridfeint ielbit in berbit, wo fo biele erite Frithlings

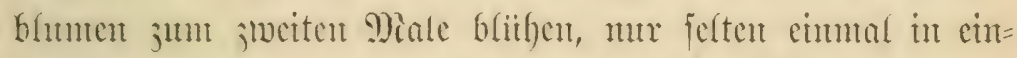
jelnen Exemplaren mieder. (Gi ijt Dod) eben cin cates trentes

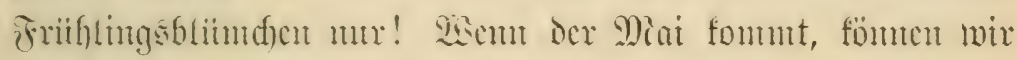
es aber aud entbeforen, Dem mogendes (betreione und buntes ïppiges BLumenipiel waltet bam anf den Etellen allen, wo

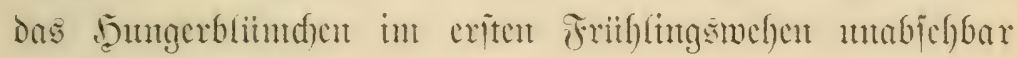

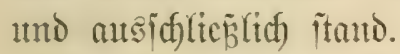

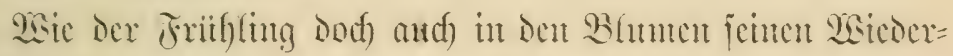

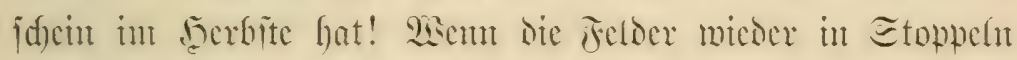

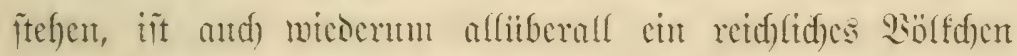
mix ganj anderex Dimiatndblïndfen erwad)t. Int bämmerigen

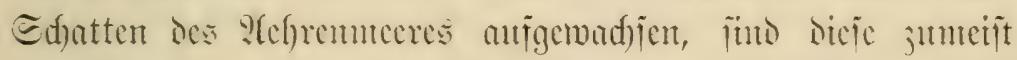
im Serbit crît jum B(ïhen gefomment. Waren es im

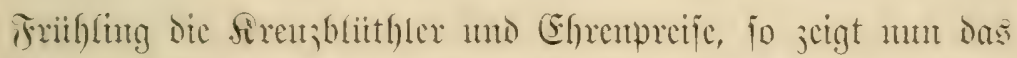

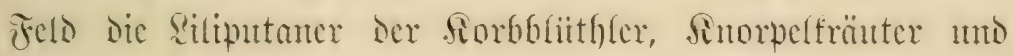

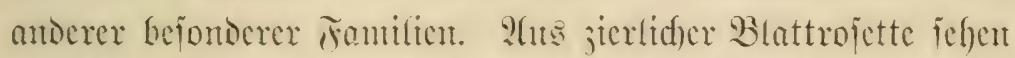

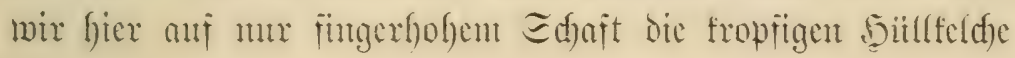




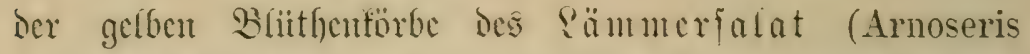

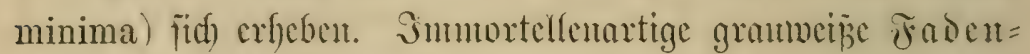
fräuter (Filago) ftefon fexjengerade, aber unt wentige 3oll

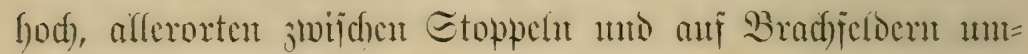

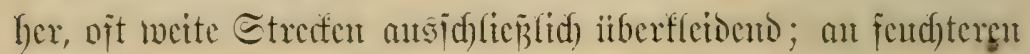
Etelfen werden fie nertricben Durd edfe Im mortellem

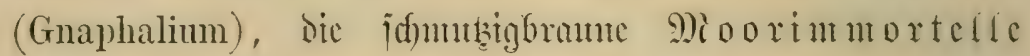
(G. uliginosum) and die gefbueipe 3 mmortelle $(G$. luteo-album), meldye allerdings fie and da weit jither funger= hod) werdon. Dit wimjigiten, aber zafllofen gelbitiden Stern=

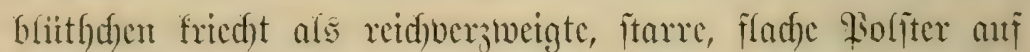

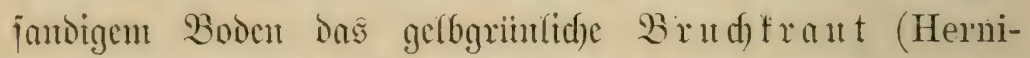
aria glabra); locferer gebitid)elt wäd)st viclfcid)t dancben Der

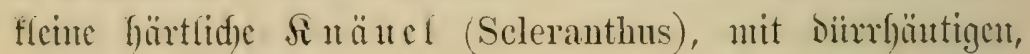

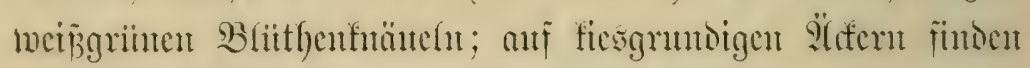
wir mohl aud bas jeltencre röthlidgriute, ftare finorpel= fraut (Polycnemum arvense), weldes gleidjalls fanm jiber fingerlyod) wiro. Das (bi)ps= oder Ed)leiertraut (Gypsophila muralis) trägt feine fenten, rojenröth(id)en gielfent= blitthdon anf reid) berjweigten, dimnten, blattarmen Etengel= d)en, welde off reizeno die Etoppelfeloer gleidjam iiberipinten.

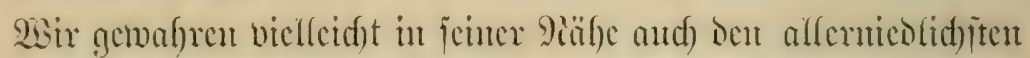
3wergflad) (Radiola millegrana), meld)er Durd) fente faum biš cunige Boll hohen, anfred)ten Etengel mit wohl humbert=

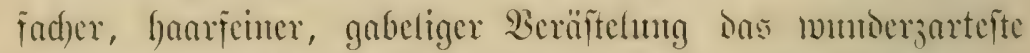

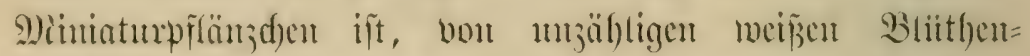

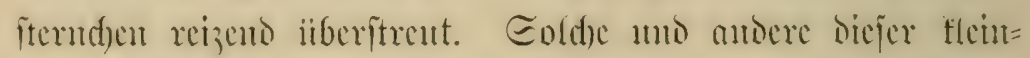
lidfiten Derbitrfor bes Felocs baben mir mit redst guten

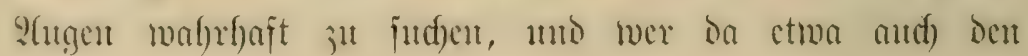


Sicinling (Centunculus minimus) cummal cutbette, bicjes

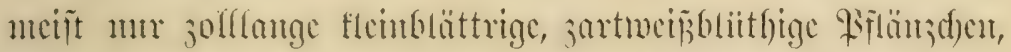

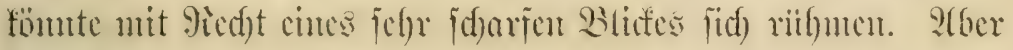

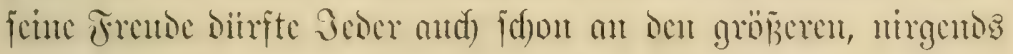

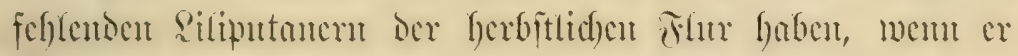

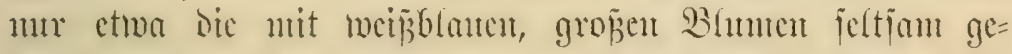
frönte fügerfobe 23 raut in Danaru (Nigella arvensis), baju 2) äแฐd)

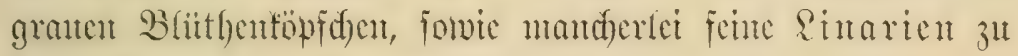
cinem lebten நerbftitranze pfliidft.

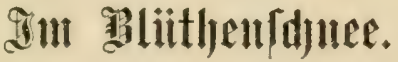

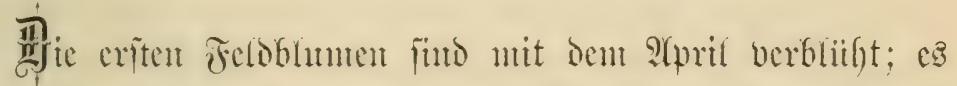

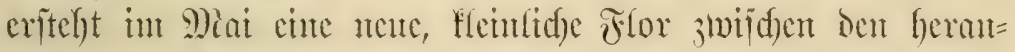
mad)jenton Eanten, Srenzblithler mandyerlet ât öffnen ifre weifen oder gelben Blitthentranben, und anderes geringes

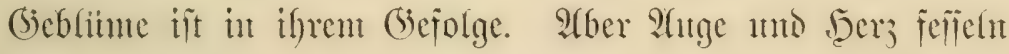

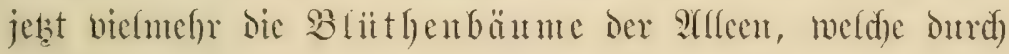

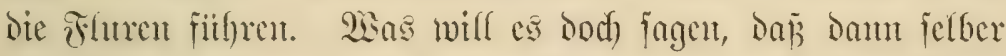

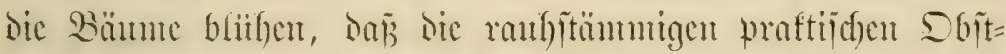
träger Mitliarben föjtlicher Blumen tragen, Deren Mienge

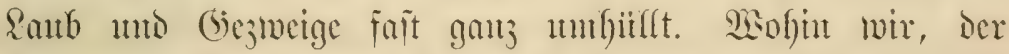

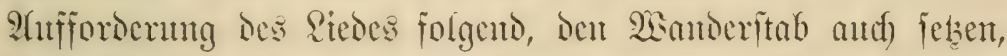

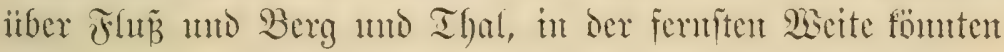




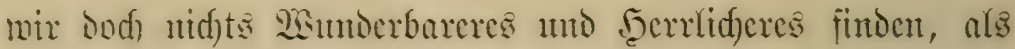

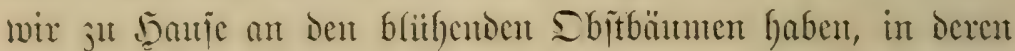

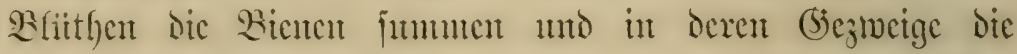

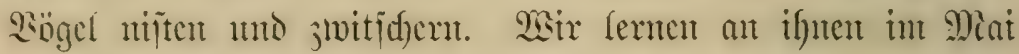
aud) unjere flemite Secimath adften und lieben!

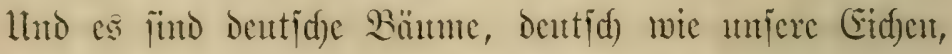

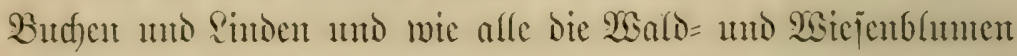

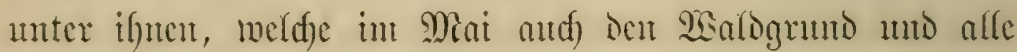
2uten jut eiten parabiejijd)en Erosengarten madyen. Eo wie heute haben jie in den Wäldom and Fluren unjeres deutid)en Baterlandes gebliift in unvorouffidjen 3eiten, uno es haben

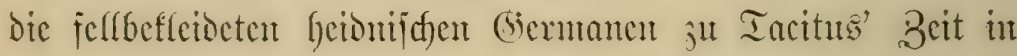

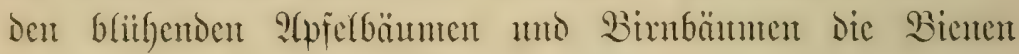

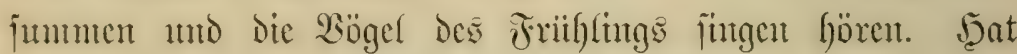

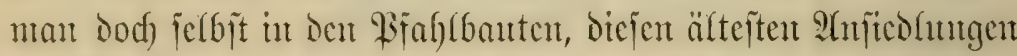

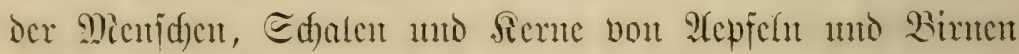

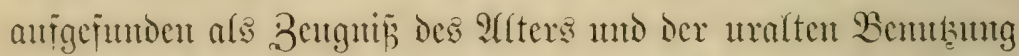

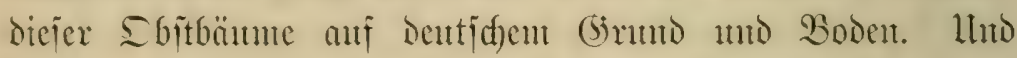

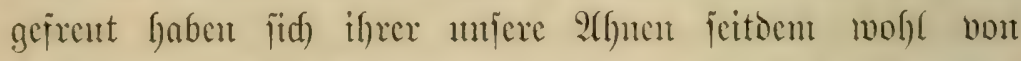

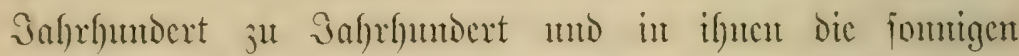
womigen Maientage gepriejen. Bmar bei Den Didftern ber

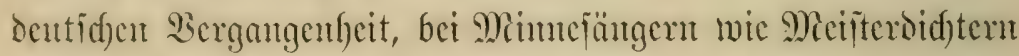

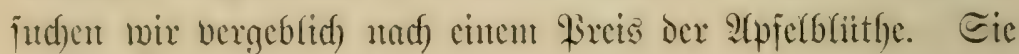

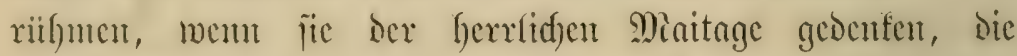

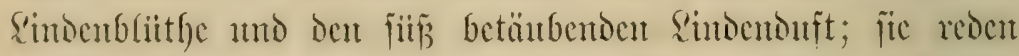

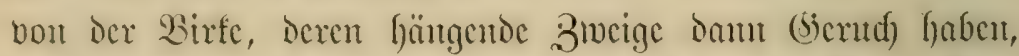

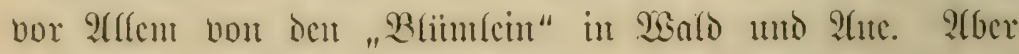

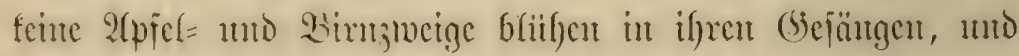

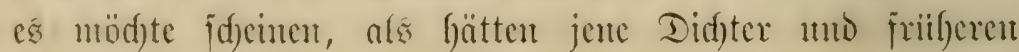




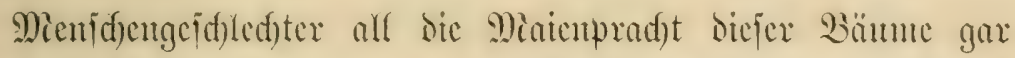

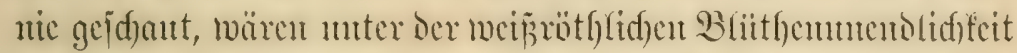

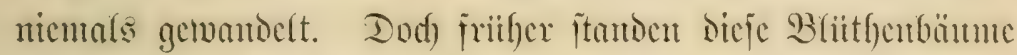

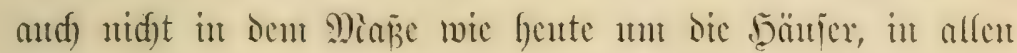

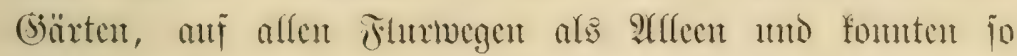

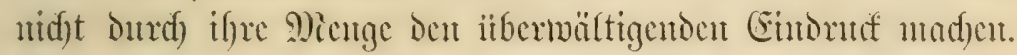

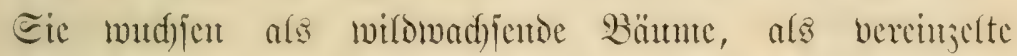

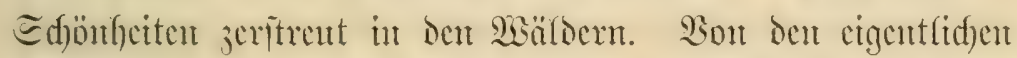

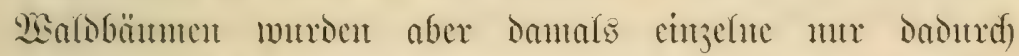

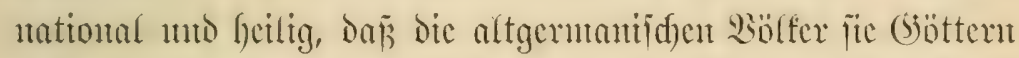

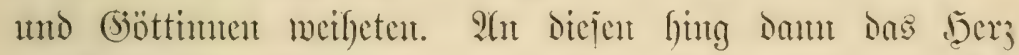

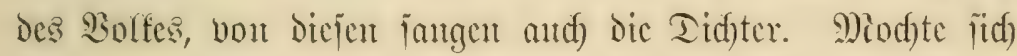

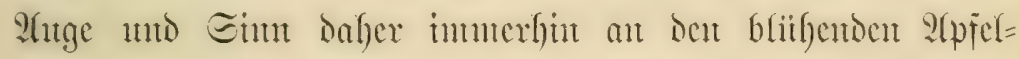

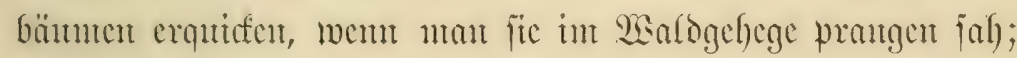

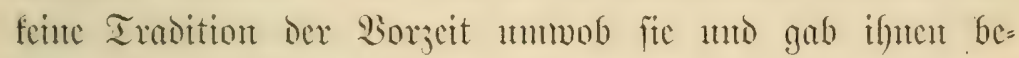

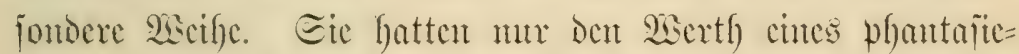
bollen Echnutes in waloc, aber nidyt cuncs jünigen frommen Siedanfents in der Matur.

(5änjlid) vergeffen waren fite barmm nidft in ber friiferu

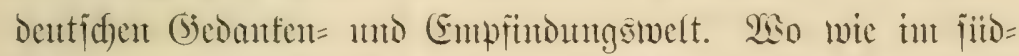

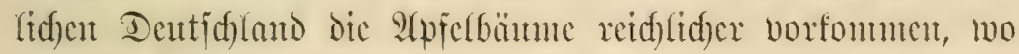
aud) jest noch) die Sbitbaumblitthe int Mai ergreifender ijt

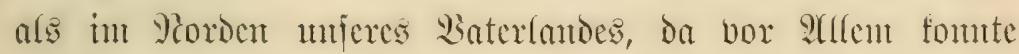

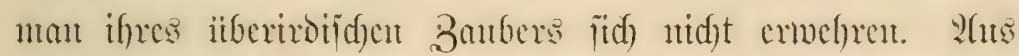

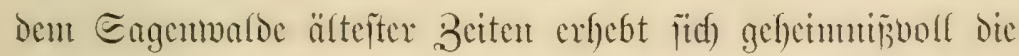
Simtic bout dent "Dentidfent Whutderbaum", welder io be= grabet und gottgejeguet jei, baj ex als gejdmitiftejter Baum

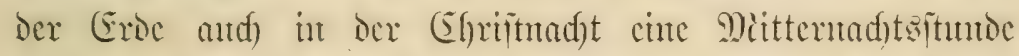

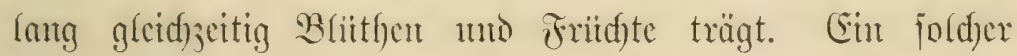




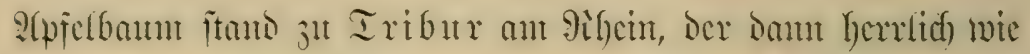

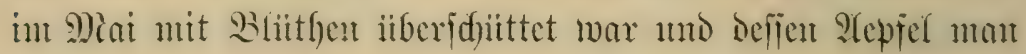

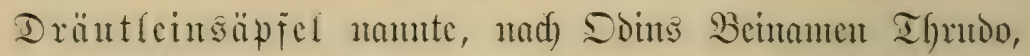

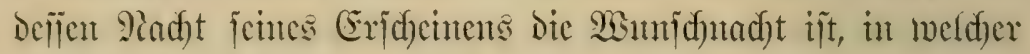

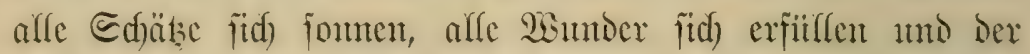

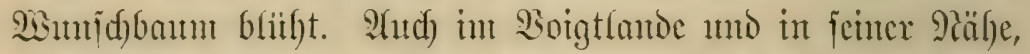

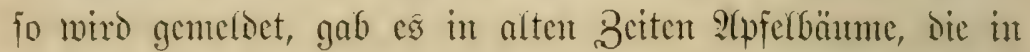

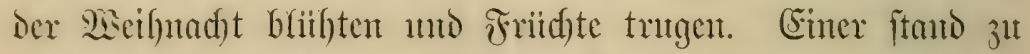

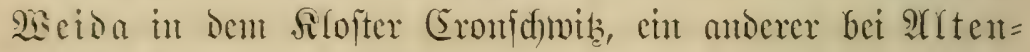
ftadt bet Baireuth. - Mitederum uad) einer Borftelfutg ber

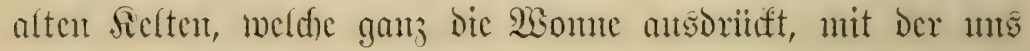

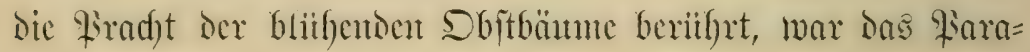

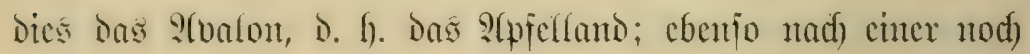

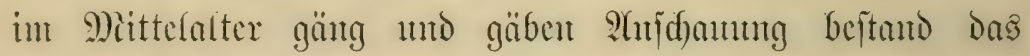
Harabies in einem grofen Sarten mit blithenden und fruddet=

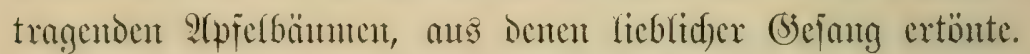
Sa wie bätte dentid)es (5icunith and fömen chedem völfig nuberiffert bleiben bou ber Sbitbaumberrlidfeit des Miai, wie cs feme jucite anj Erocn gibt! Mno wie ganj anters nod) witroc iffr Sireis bormats exflungen fein, wem mat unter Der bentigen B(iitfenumendidffeit gemandelt wäre!

Die Beit hat fid cben geändort umo dic Blithenbämme leije mit. Eeit bie gärtnerifge Dand ifge Sorten verebert

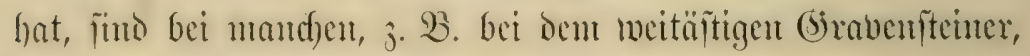
die 2Blithen viel gröper geworden. Sbitbä1me jefbit werdent

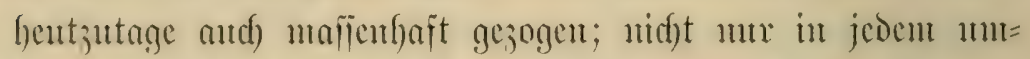

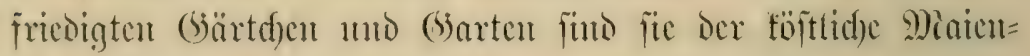
idfuntef, nidft un uveite Silantagen mad)en fie aus; ncut,

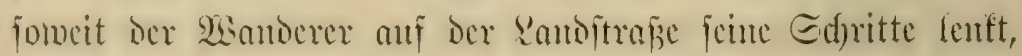




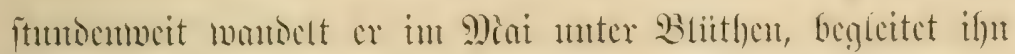

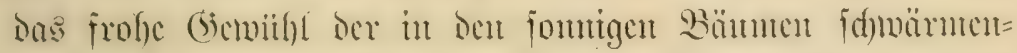

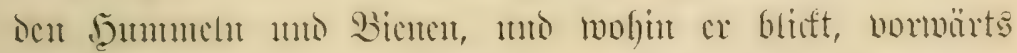

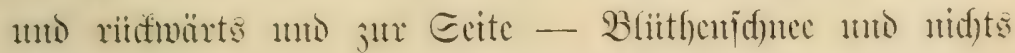

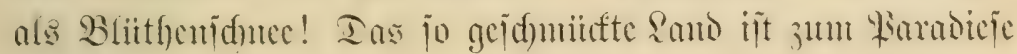
geworden.

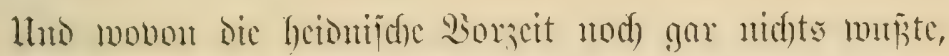

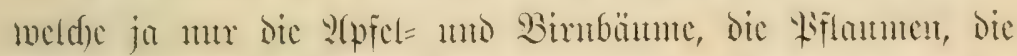

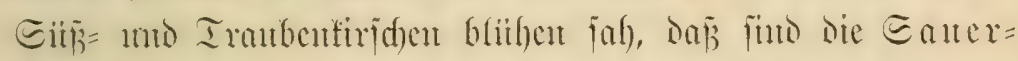

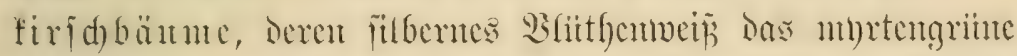

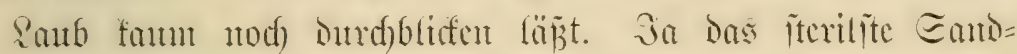

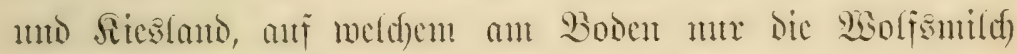
mo hartes Ednuclegras gedeiht, hat Dod, Sirajt gemtg, Den

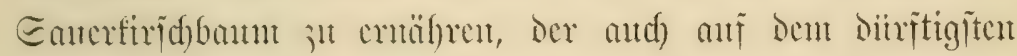

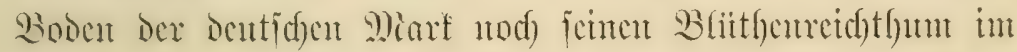
Slai mit ungebrodfener Edyönbeit entfaltet mo über bicie

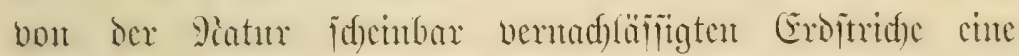
Banberanmth webt, Don Darin in biejen Tagen feir lluter=

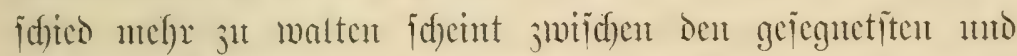

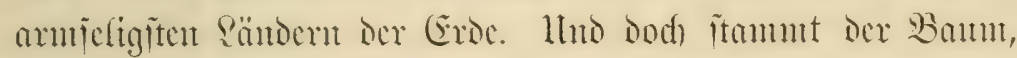
Dem jeşt dentidyer Eandboden genitght, ans ben hodigclobten

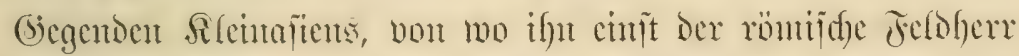

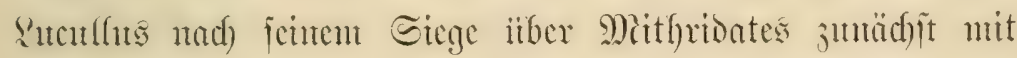

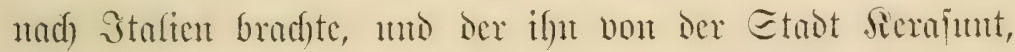

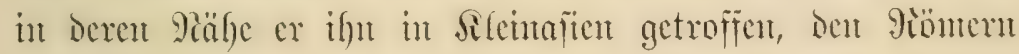

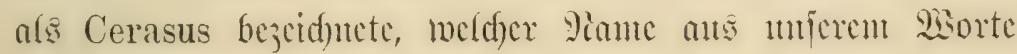
Siridge nod) beraustingt.

Bon do an hat ber cole Banm, ber in wilone Butumte

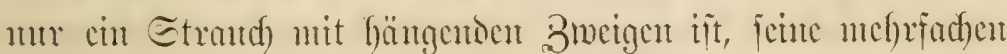




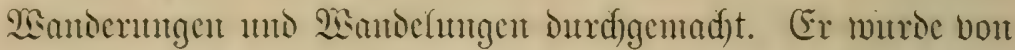

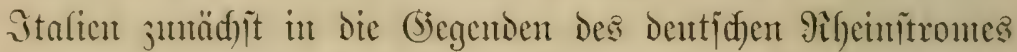
tmo vou ba alfutïlig itfor miler ganjes Baterfand verpflangt.

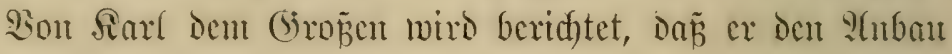

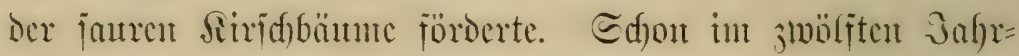
fundert waren fie in Ed)(ejien wirffid) cinthemitf) geworden.

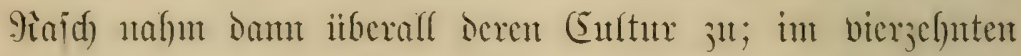
Salyrlfundert gab cs plantagenartige Sirfougärten, und in

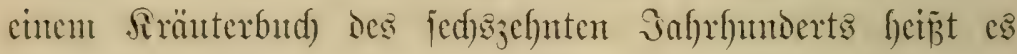
id)on, jie witdjen fajt an affen Sxten nut an ben Etrap̃en.

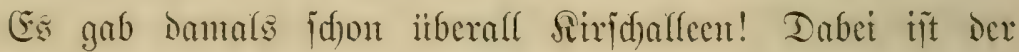
Baum aber nidgt böllig geblieben, twie cr wax. Die gärtre=

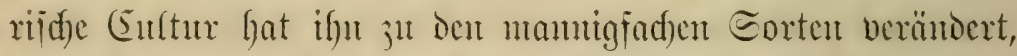

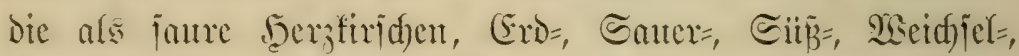

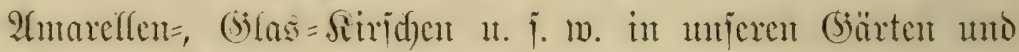

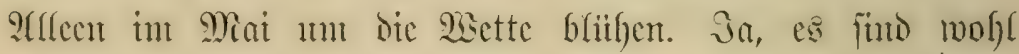

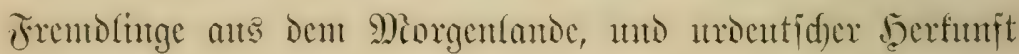

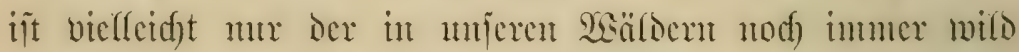
vorfommenoc Bogelfirjd)baum mit jeinen gleid)falls vicl= fad)en (Eulturjorten. P(ber ocr fdeöne frembling ijt dod)

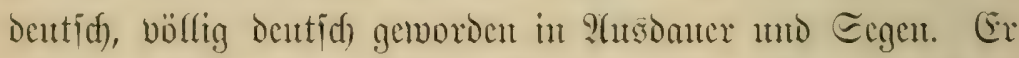

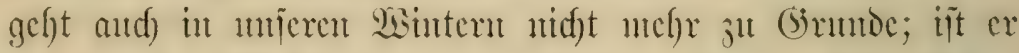
bod) aud) wirflid) artentormandt mit anferen uriprintglidfen

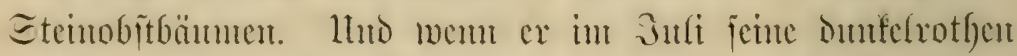

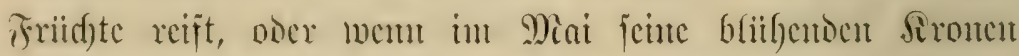

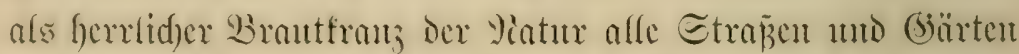
ocrjicren, won den Wergen bis in's That mo bis in bic

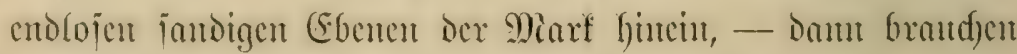
wix walyrlid) nidgt mefre in Die Ferne ju jiefon! 
Betrad)ten wir cin cinjelnes Blithd)en all otcjer Blittgen=

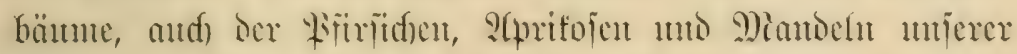

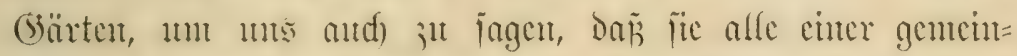

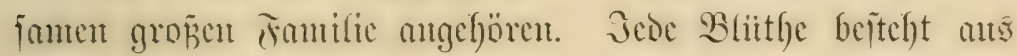

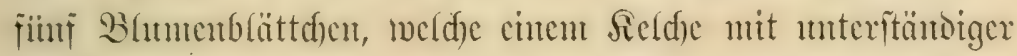

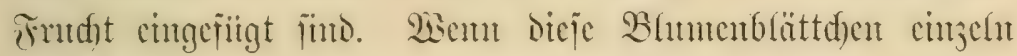

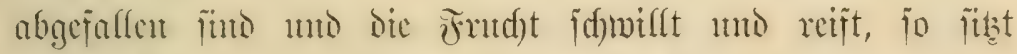

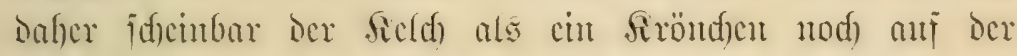

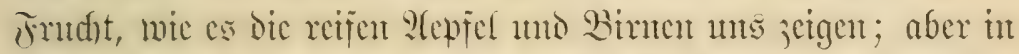

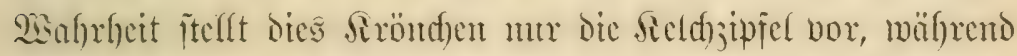

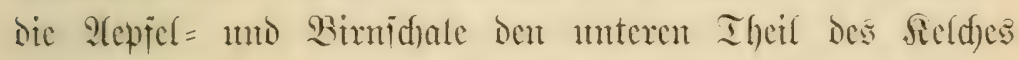

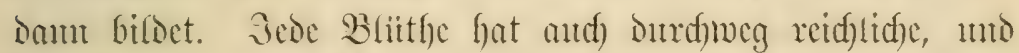

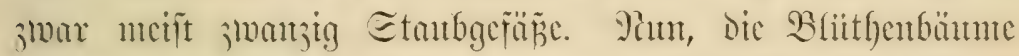

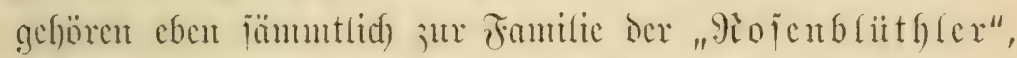
Deun and bic gioje, nad) der jomit dic ganje Fantile ge=

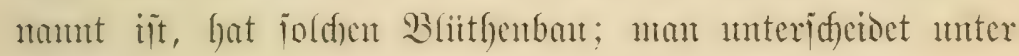

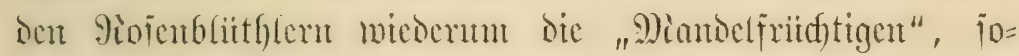

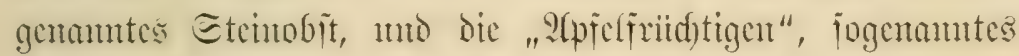
Sernobĩt, insbejondere die Alepfel uno Birnent.

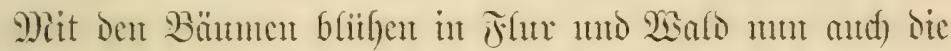
Eträudjer ofyne Bafl. Diantder Etrand) ijt gleid) Den Sbit= bäumen in entolojes Eilberwcip̄ gefletoct; bie auth) jutl (battung

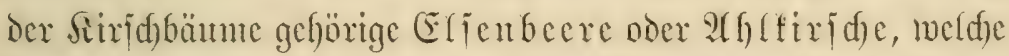

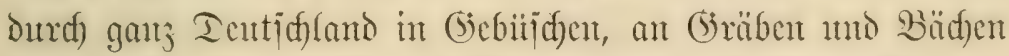

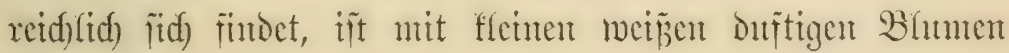

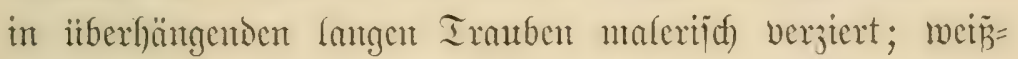

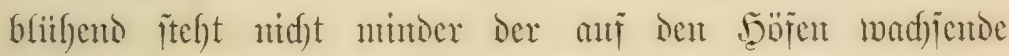
Theeflicder, die Ëberejde mijerer Mälocr und ber, wie

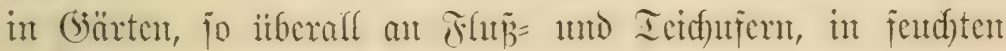




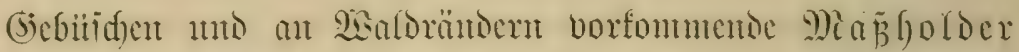
oder Eduecbalfitratd). 2(ber and) in amberen Farbent

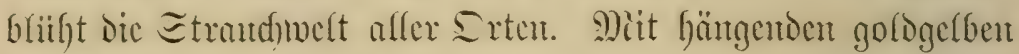

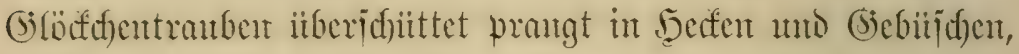

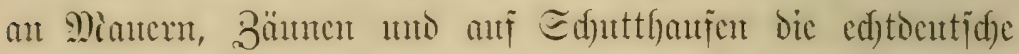
Berberije ober der Eauerdorn, defïen ftadeframbige

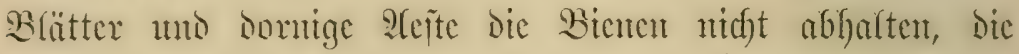
Berberijen in Miai fiir ifren Reblungsitrand) ju erflärest

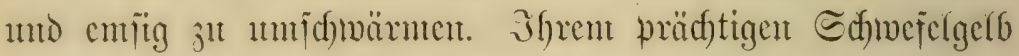
ober (5ologelb fonment mu bie id)metterlutgsblittfigen, weit gröperen Trantan des (5olderegens oder (5nthifus

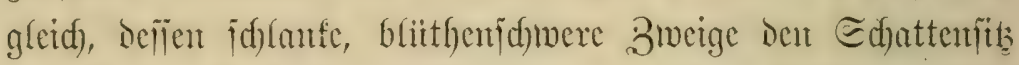
im (barten fod) übermölben, oder in den wäldom afler

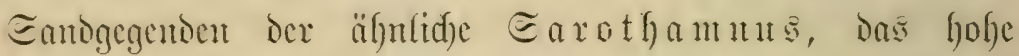

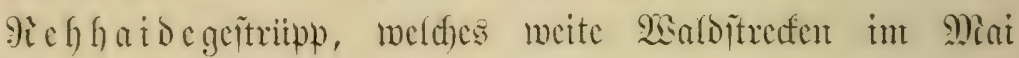

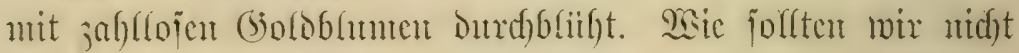

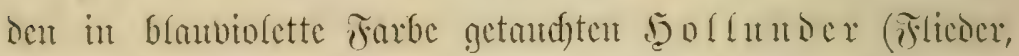

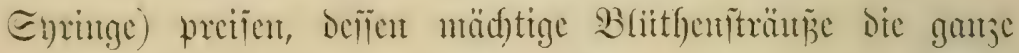
Sironc sicicr bohen Eträudfer bilden! Ilno dod) fanntent

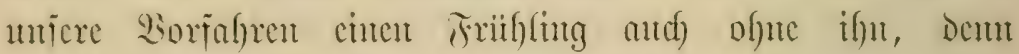

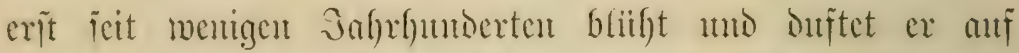

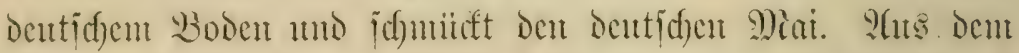
Eriente, wo ex bereits sillad) bies, muroe ex 1562 durd)

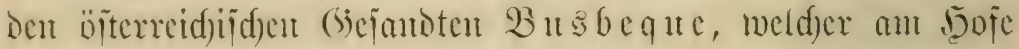

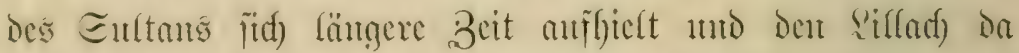
ferutcu (crute, uad) Emropa gebrad)t, unt jwar in cinten cint=

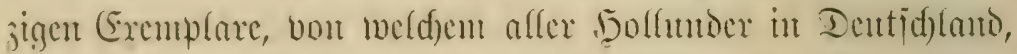
Frantreid) mo Befgien nad)ucistid) abjtanme. 3hum folgte im 3afore 1640 ans Perpien mad) Europa dex befmute per= 


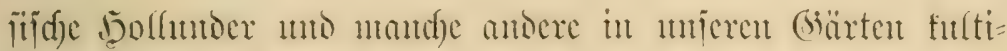
virte 2 rrt.

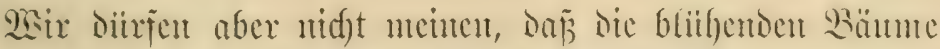
und Eträud)er mu uts Mienjoden bejeligen wolfen uno nidft

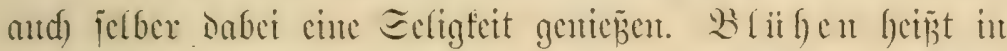

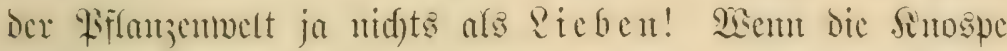

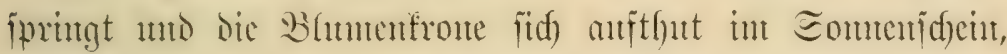

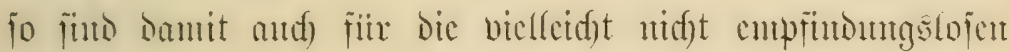
Wiflanjembejen die Tage ber Siebe antgegangen, melde un fo inniger ijt, je fitrjer jie wälgrt. Dic Etantbrätter mandfer

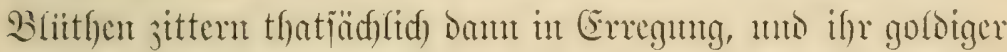

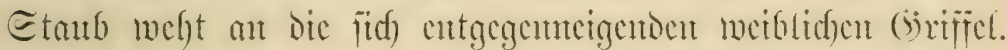

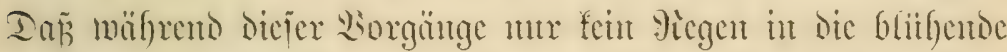
Brantffanmer falfe unto jente Beftüntbung itöre, sicje Besingung

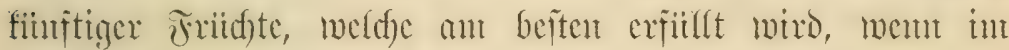

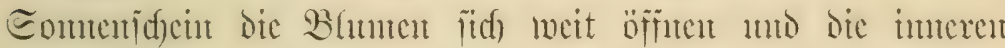

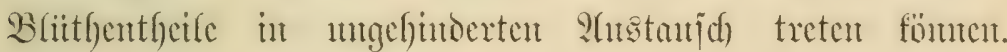

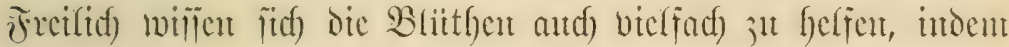

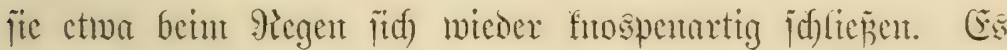

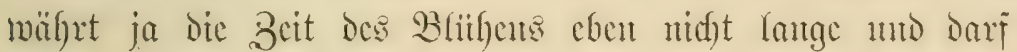
nidgt ungenilist verittecidyen.

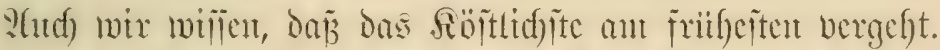
Siaun Daj wix jubeht: Der Mea ijt gefommen! - fo find jeme Iage faịt jofon voritber. Wistr itreiften an bie föjtlide Sber= fläd)e, obue jemals ganz gejättigt ju jein. 


\section{3.

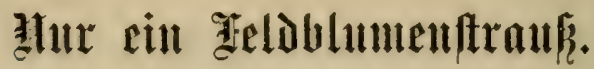

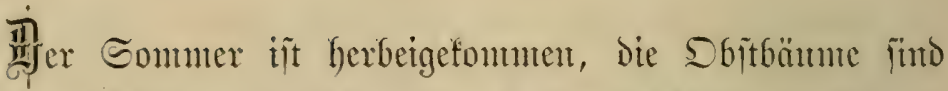

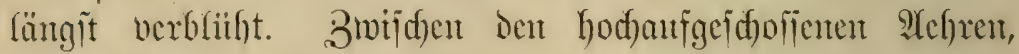

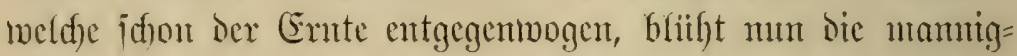

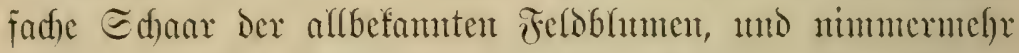
möd)ten wir ifgre Focite, Dieje poetijgen Simoer unjerer Fhtren entbefren.

Finen Fefobfumenfrans winton bie Edjutterimen uno

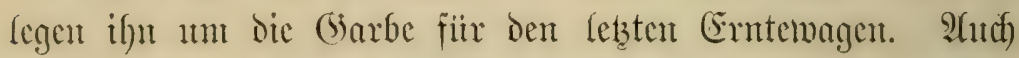
bie alte Eitte iit nod) nidgt itberall vergefien, nad) meldser

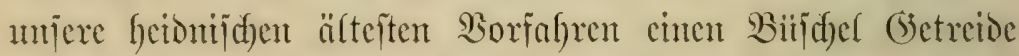

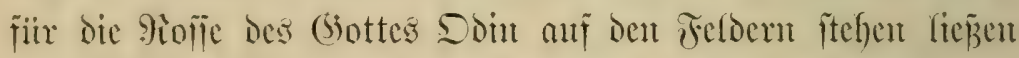

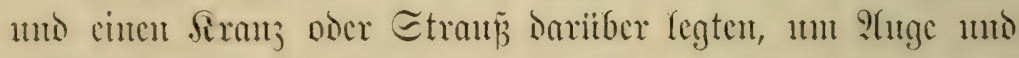

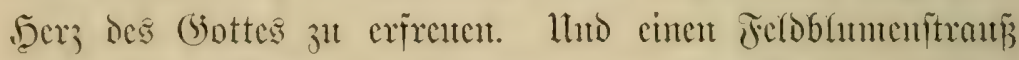
janmert Das finto, Die Sumgirne; Der Siingling futd) "Das

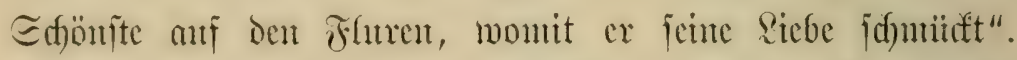
Die Mialer Gaben jeoerzeit oen Felobhumeniftran lieber gemalt,

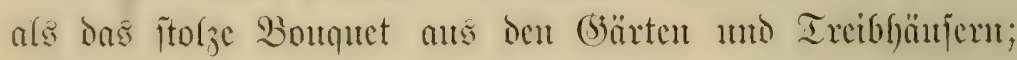
jelber der heilige Mimo des Bergpresigers hat iffu getweiht Durd) feinen ßreis ber Silten auf bent Feldoe.

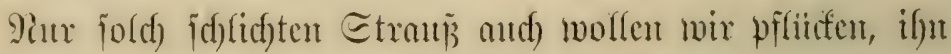

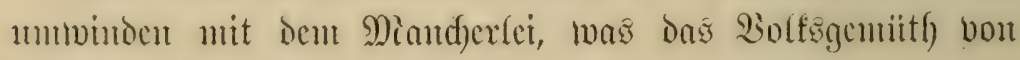

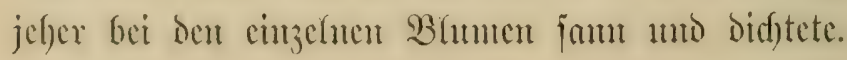


Dic Ifore der Staot liegen finter uns nit ifrem

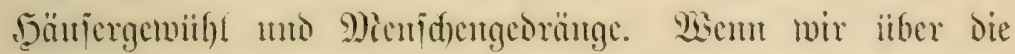

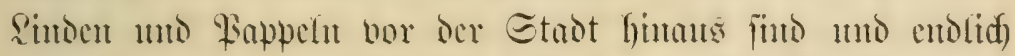
abjeits der ftanbigen ?antitrabe ofn breiten rajigen Iriftweg in's Fels cimfdatagen, weldfer ju ciner fernen antom Marf

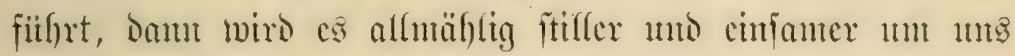
her. Son Weiten fehen wir bie Seänjer mo Ihitrme wohl

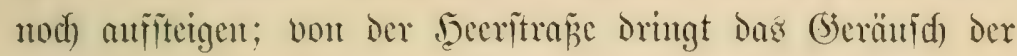

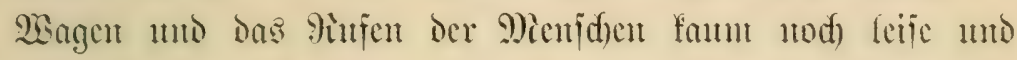
Dumpi ju uns heriber. S(ber um jo lebendiger redet das

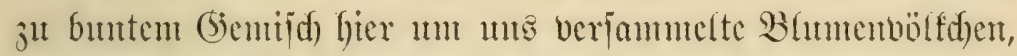
weldes jwoilden den (Stetreidebalmen hervor nengierig unz

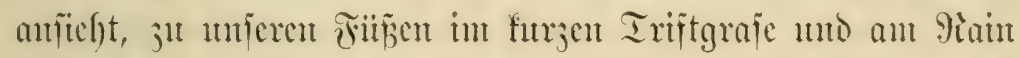
fid) ftrecft ober lagert, an cinjelnen balmen and) wohl feaf emporffettert.

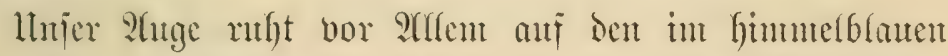
Sictide froljtrentoen Siornblumen. Bon ben Tagen Der

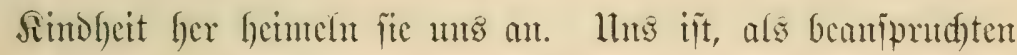

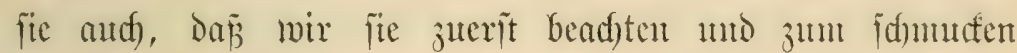

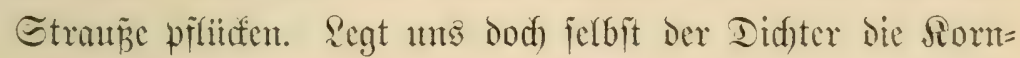
brume (Centaurea cyanus) an's Şerg:

"Mindet зum franze Die gotDenen Hehren, Fled)tet audi) blaue (5nanen binein."

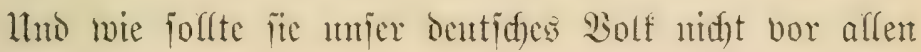

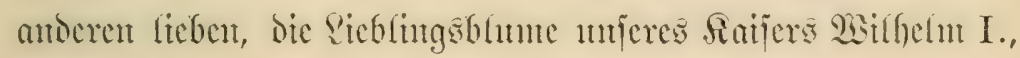

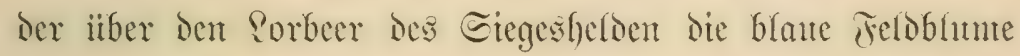

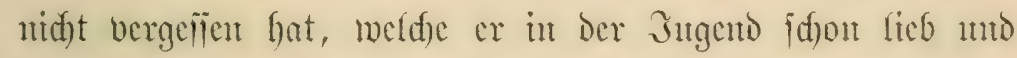
werth gewomen batte non femer hodjeligen Mintter her, ber 


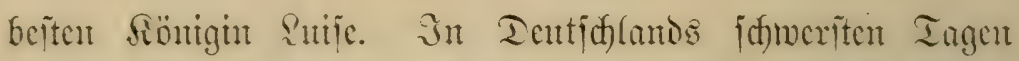

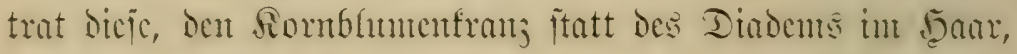

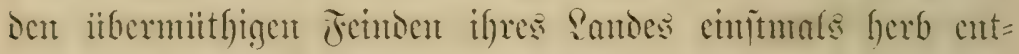
gegen und indete den ftoljen Epöttern dicicn firanj: bas

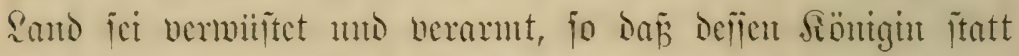

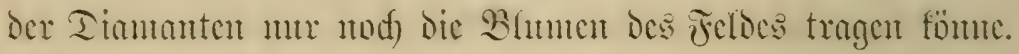

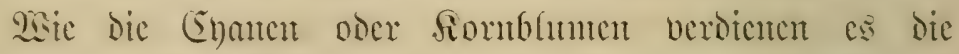
Siatidrofen mo giaden aber faum minter. Cino Dod

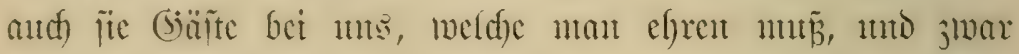
gleid) den formblumen fint and) fite, fantunt nod) eintgent

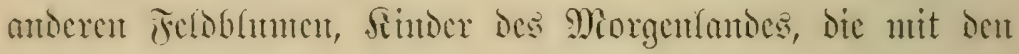

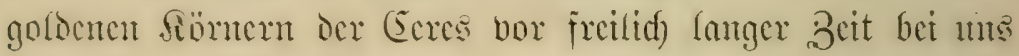

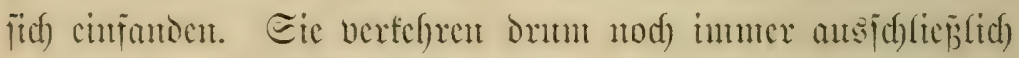

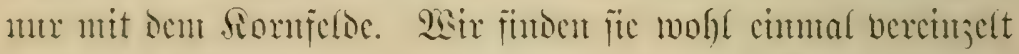

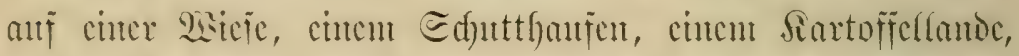

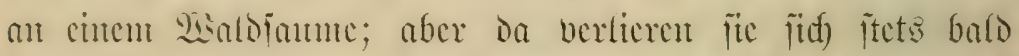

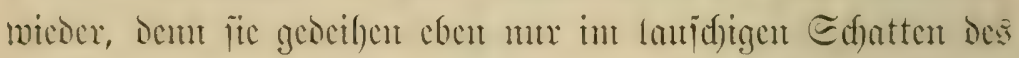

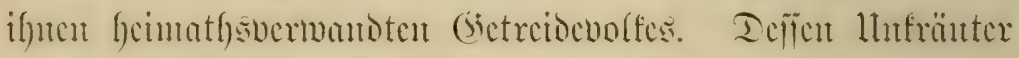

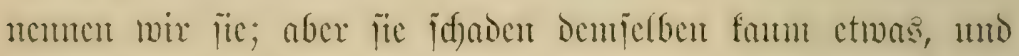

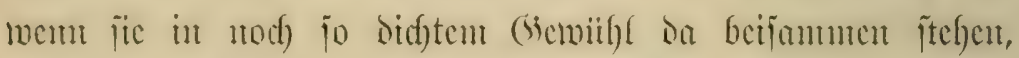

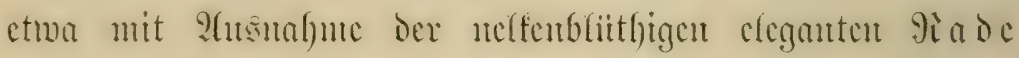

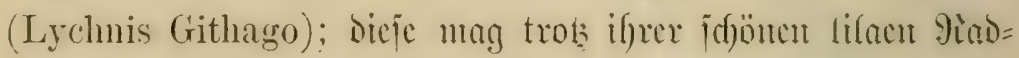

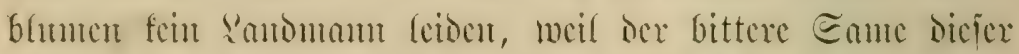

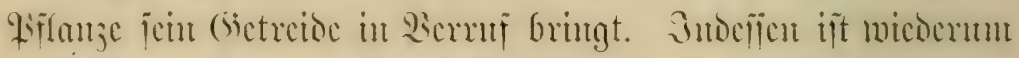

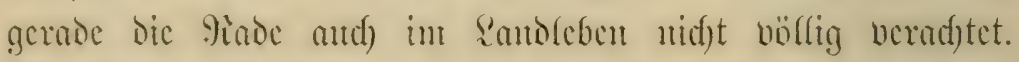

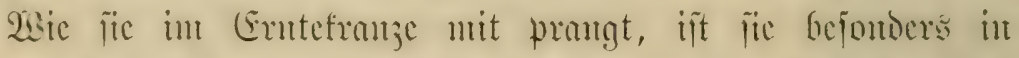

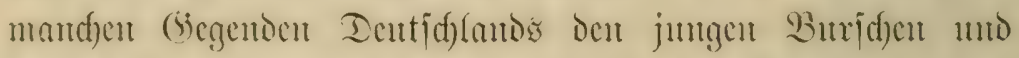

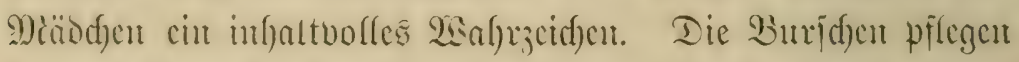
fic nad) alter Eitte Denjenigen Diädd)en, un weld)e fic freciu 


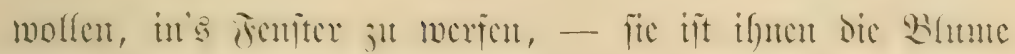
Des (Seftünduifies anfrid)tiger Riebe.

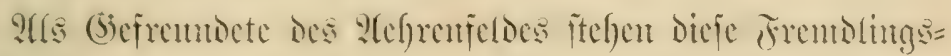
blumen bor Eage nad) abor and alle unter bour Edyuggeijt

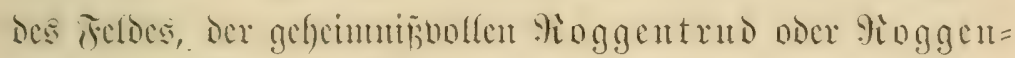

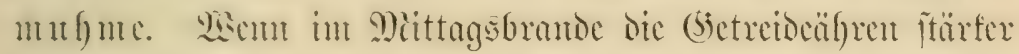

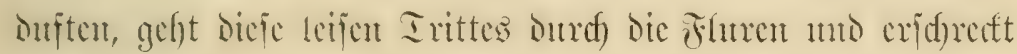

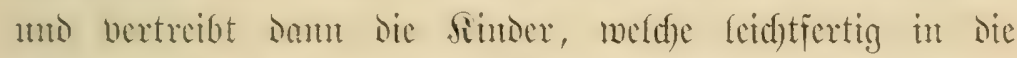

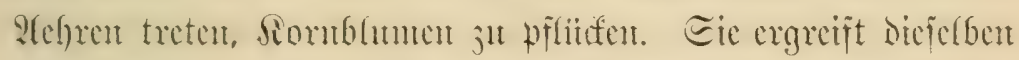
fogar mohl, fä̈ngt fic ant ifren id)marjen

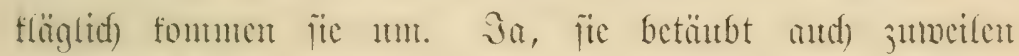

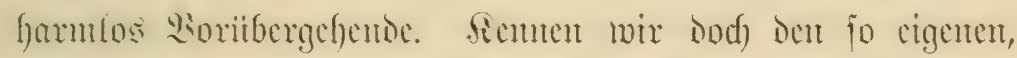

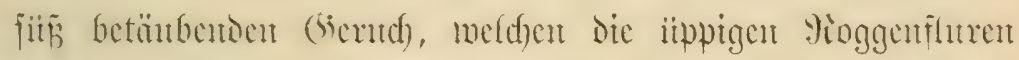

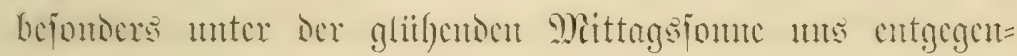
atbmen!

¿o bütten wir die bunteften Bierden der Sictreideffur jum Stranfe beifanment, Die blanten, rothen, lifacn Farben, Die Sornblume, Sinde umb Silatid)roje. Dicje founter wir

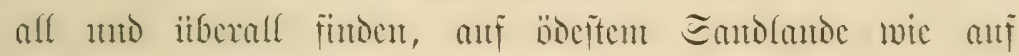

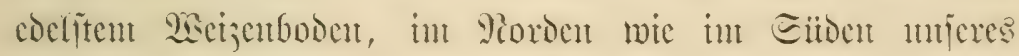
Baterfandes, in Den Fhuren der Ebenten fo gut wie anf Den

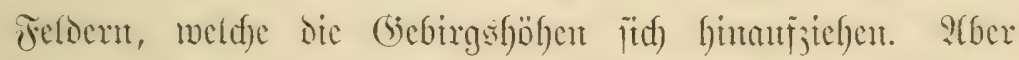

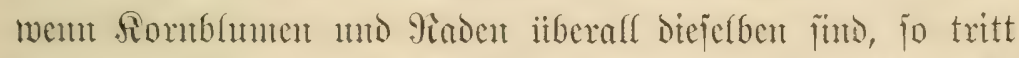

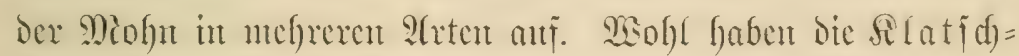
ut ohne (Papaver) alfe biefelben jd)arlad)enen oder fenterfarbenen

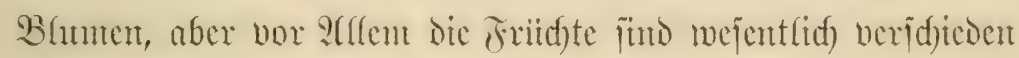

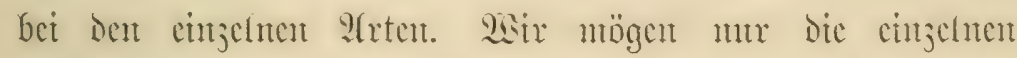

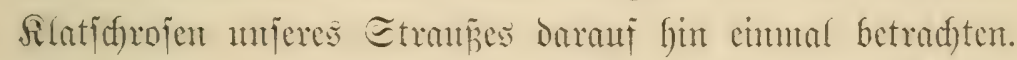
Siex pflitaten wix cincu biejex Miofne, Deflen ftemgetrönte 


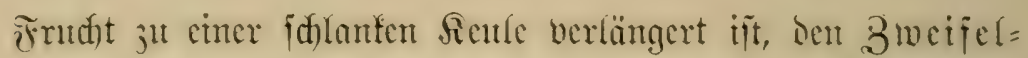
molnn (P. dubium); bei cuten andern ijt fie fajt cbento geftaltet, aber mit anfifebenton Borften bicht befleidet: es ijt Dex $\Xi a n d u b f u$ (P. Argemone), die flempte uno id)wäd)= lid)ite $2(r t ;$ bei nod) cuncr anderen, nänlid) Dent Fe(b) (P. Rhoeas), bifoct dic Frudf)t cin fdjönes Soal nut dic ganje Siflanje ijt jefre anjelunlida. Blifen mix jodan bie Blätter und ben ganjen 23 ted) biejer 9 treten an, jo merden

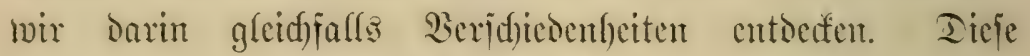

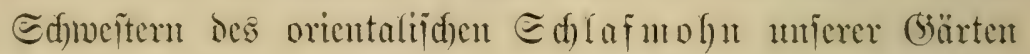

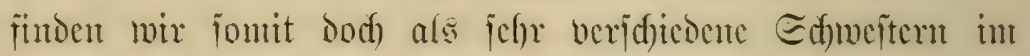
Sietreibefelde beijammen.

Der geefrte Sejer oder Sejerin, Deren Decumath Durdbueg

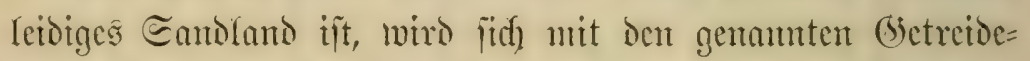
blumen fo jiemlid) begnitgen mitifen. Siut ocr idfwefelgelbe Jederid) (Raphanus Raphanistrum) oder der biejen jefrr äfnlid)e a( ferienf (Sinapis arvensis) madyen fidf) da nod) besentfam gettent unt itberjichen gange Fluren mit ifrem

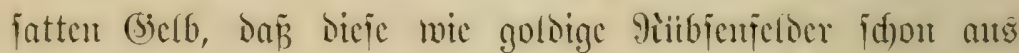

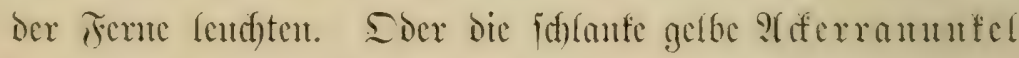
(Ranunculus arvensis) (ugt jwijd)en ben hohen ந̧aluen her= box. Die fiamilfe, cin edfes fimo des Gandes, deren

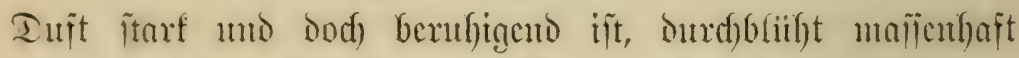

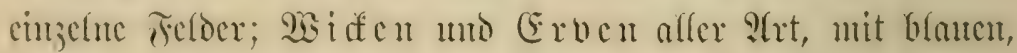

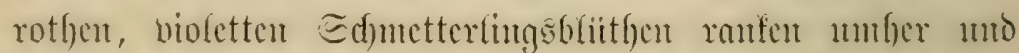

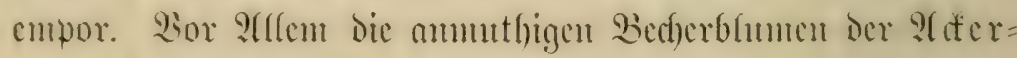
winde (Convolvulus arvensis) bermitien wir nirgends; if)re

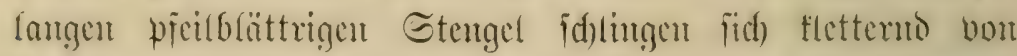
Dalm ju batm uno umbaljen in Serftritumg and andere 
Feloblumen. Dieje meiphamige Serwande der farbigen

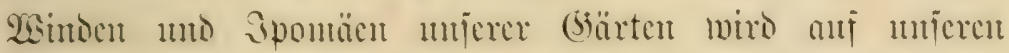
Feldern oftmals aber antef farbenjdön; in belleres oder

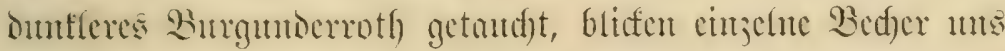

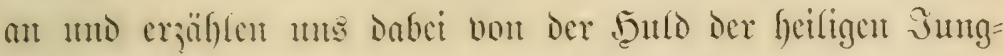
fran Miaria, ber jie bas ju nerdanten haben wollen. Wie

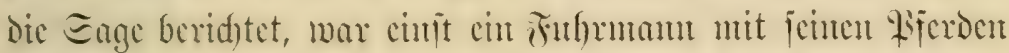

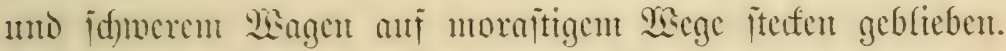
Ex hicb und trich, aber die Fiferde famen nidft bon ocr

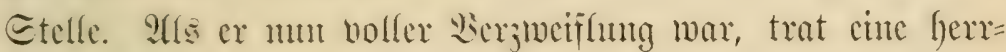

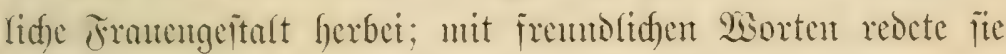

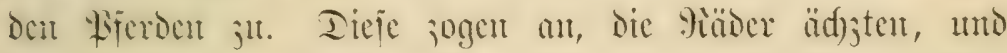

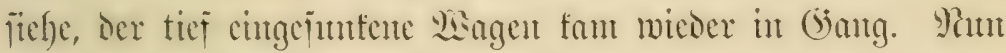
Golte Der Sunfbare Futhrman cinen Pabetrunt hervor, fïllte

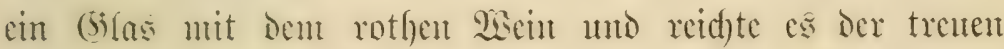

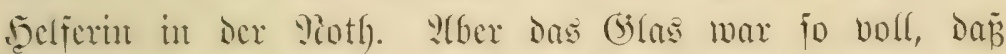

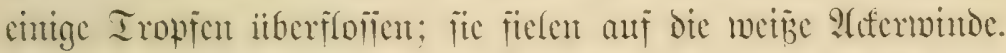
llno jux Erinnerung an Das Danfbare ber; Des Dannes trägt dicie Bume feitsen fort uno fort die Epuren des rothen Mcines.

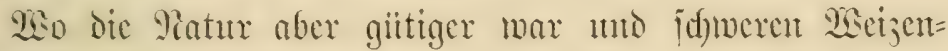
boden gab, ja wo alfuviales (sicbiet, womöglid) gediegenter

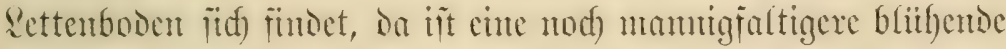

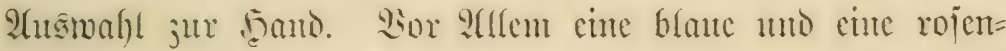

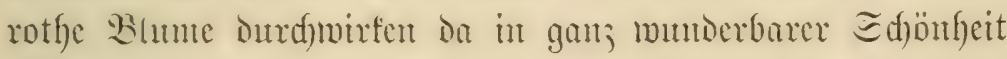
alfe Eantenffuren und wollen bem Sandmant Das bliiffende

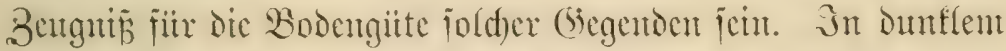
Cdjönblau erfebt fith Der Jelo=gitteriporn (Delphinium Consolida), welder den in unferen (5ïrten fultivirten fitter= 


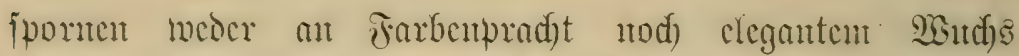

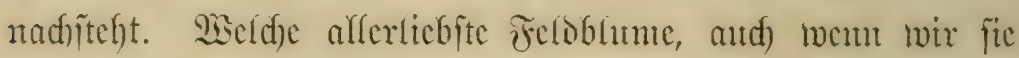
cimmal näher anjehen! Wisc cin Delphin ijt jede ocr cinjelnen

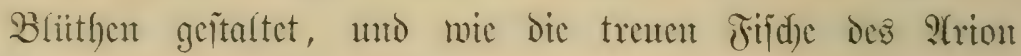
jdbummen ïe, bou hohen Etengeht getragen, in bent wohligent

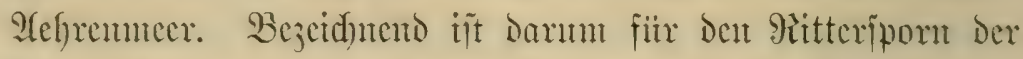

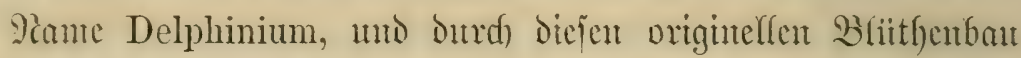
itteht bie fdjöne Feloblunte ganz eutzig oa unter allen anderen

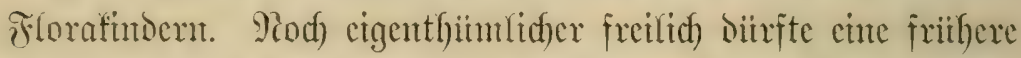

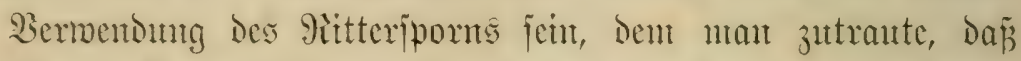

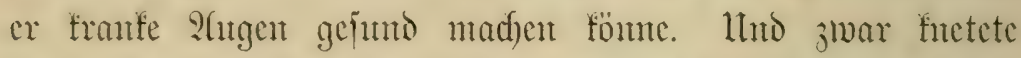

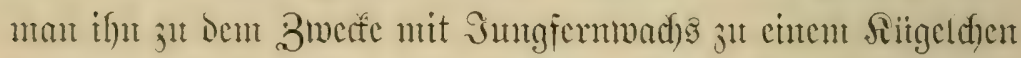

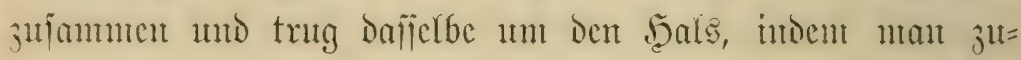
gleid) fiir bie beilige Sttilic, weld)e als :(ugentheilige galt,

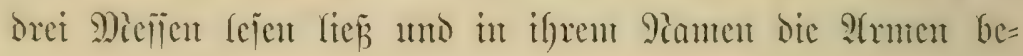
jojenfte.

Indem wir weiter den Felopfad cutlang foreten, wiro

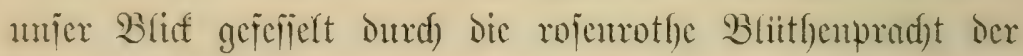
Flatterbie (Lathyrus tuberosus), welde ganze felder mit den 3anber ifrer Farbentoftlideit mo Buithenfiulle ïber=

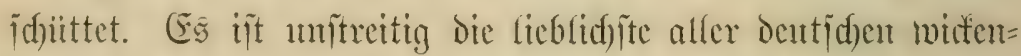
artigen B4umen, uno in Etaunen mo Entjitufen geräth der mur mit ber Fefoblumenflor von Eanogegenton Biertrante, went

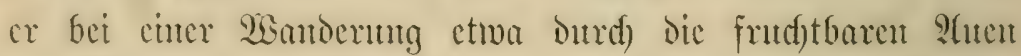
Der Miagoburger Böroe dicfe rofige Edjönheit der Felder

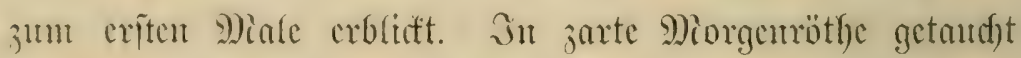

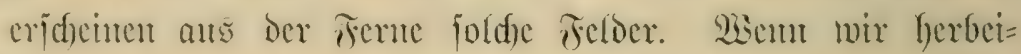

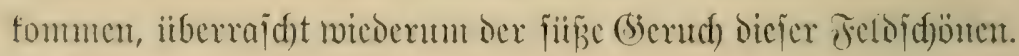

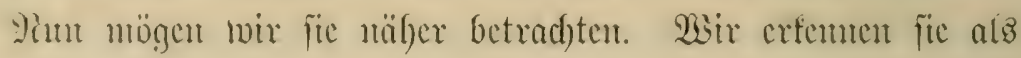




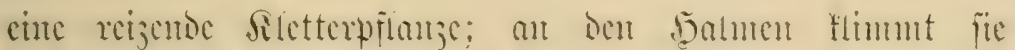

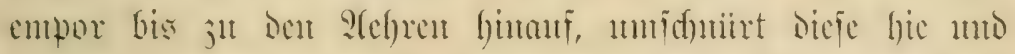
ba mit ifmen gianten, nob oben angefonmen cutwitelt fie

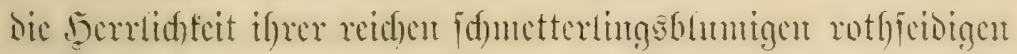

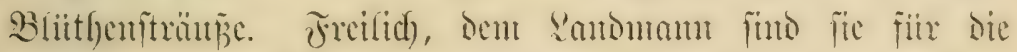
Enaten mur ftörende 9ianfonpflanjen; aber barum fönten

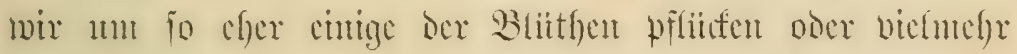

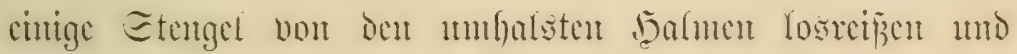
unjerm Etranje beitens beifïgen.

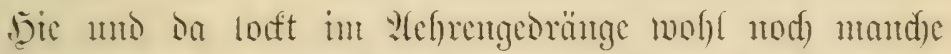
moere jelobhume, welde mix frendig begriijen. Jit bod) aud fajt Die gantje Ioilette Der (Enprifden (Söttin bier ju finden! Ior jicrlide S'enusfam (Scandix Pecten Veneris) orängt

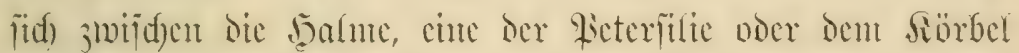

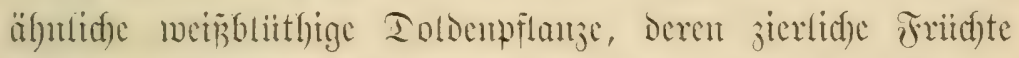

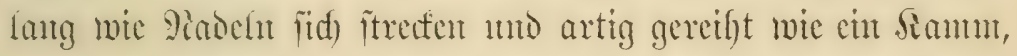

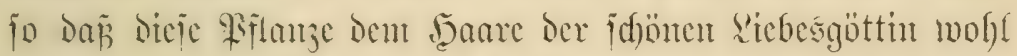

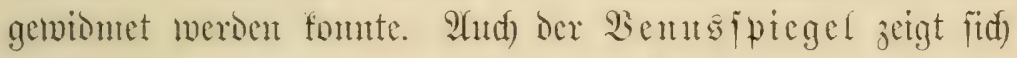

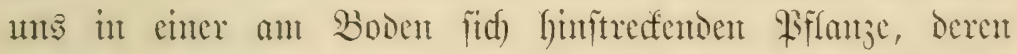

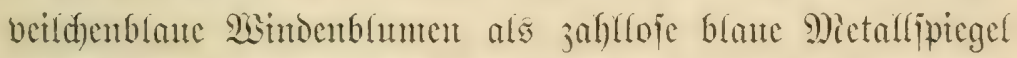

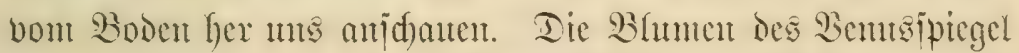
fint fo jübu, baj fie and) in (särten berpfangt funt, wo fic Die Beete malerifd itherjiehen, fowie mant jie aud in Toppfent

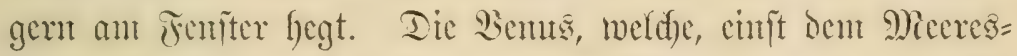

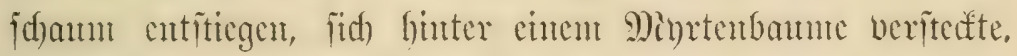
aber inmer bent Siece jutgethan blicb, - wir finden fie

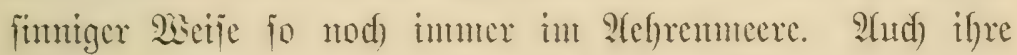

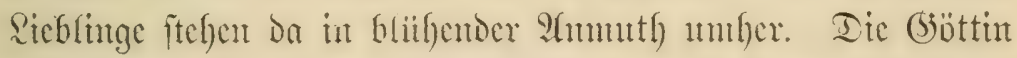

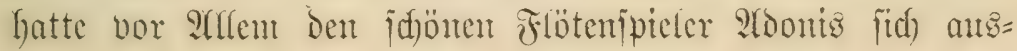


erwählt; cr martet nod) batte in der Nähe ber gennunten

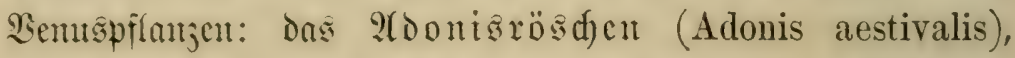

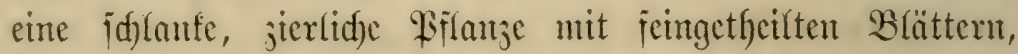

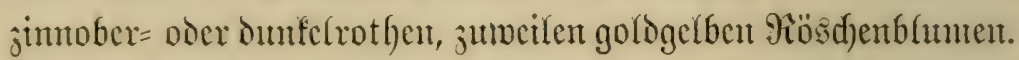

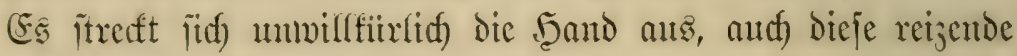
Alferzierde ju bred)en.

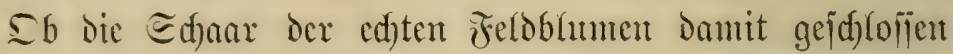

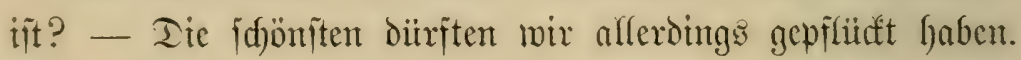

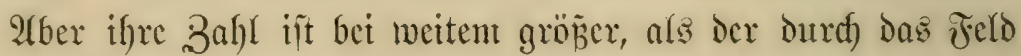
freifende Garmloje Wanderex meint. Whix treffen ab und ju, oit weite Ffuren völlig überblülyend und da dem Ranto=

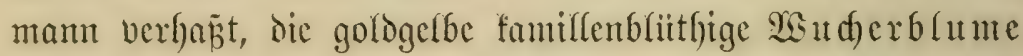
(Anthemis segetum). In (Sigenden, wo jite vorfommit, ijt iie ganj gentein verbreitet mo jedem Bewohner wohl be= fant, aber jie fommt eben nidft nfferorten bor. 2(ud) mande andere Blunten fömen wir mux in bejtummten Giegenden finden. In meiten Etridjen Ientjd)lands fudfen wir völfig vergeblid)

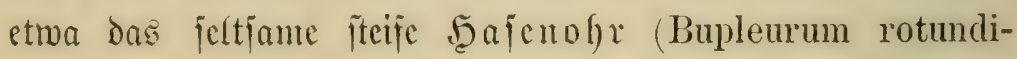
folium), dic mit grop̈en Dompriad)ten ftarrento baftbolde

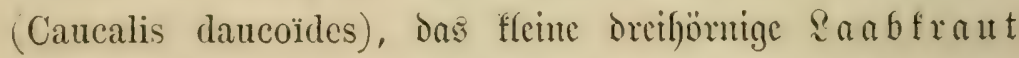
(Galium tricorne), die grop̈bumigen Edfirmbolden der Orlaya grandiflora, aber in Silfgegenden, bejontors in Den Sijtricten des Minjuelfalf, ̈̈nd dicje die Sharafterpflanjen allex F゙eldf̈uren.

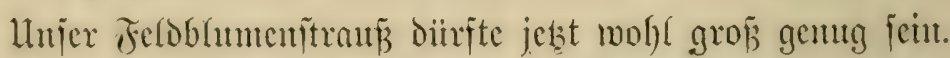
Iod) jeflt den Etrañze nod) die cigentlidje (Brajic, meldje erjt die jart gefiedertent bohen gïŝpenfabeife der (b) räjer berleifen fömen. Eine f̈mtige band wiro nic verjämmen, dicje dem bunten Etranpe cinzupingen mo jo demielben cine 


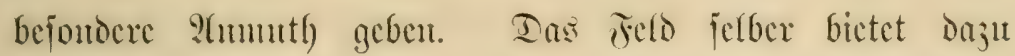

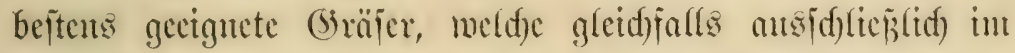
Betreide borfonmen. Bald werden wir bei mu cintger llut=

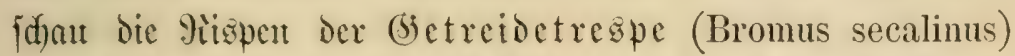

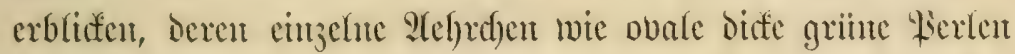
flatterig fängen, cin (Sras, das bormals im Minfe der (siftigfeit

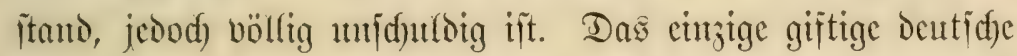
(biras ijt der idflante meizenäbrige Ianmellold) (Lolium temulentum), gleid)falls ein (Sictreioegras, wold)es vielfeidft

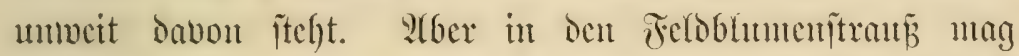
and) Dicjer ohne (bejaly aufgenonmen werben; ift ifun bod)

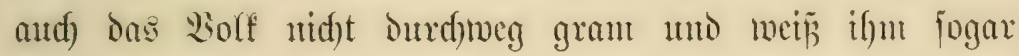

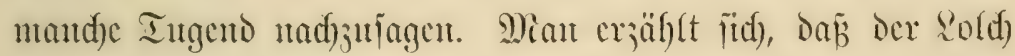

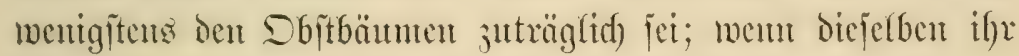
Sbit untreif ubfallen lafien, brantule man if)ren Etanm mux mit cincul firanj bon Sold) jut umgitrten, fo follen fie an Baume blciben und weiter reifen. - atfiiberall aber gewal)rent

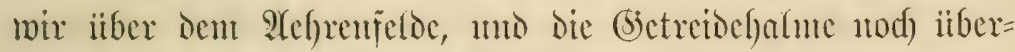
ragend, die wehenden blap̃grïnen gitspen des fetniftblitthigen,

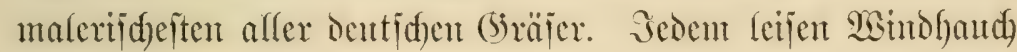
fingegebent wallen tmo neigen feine grofien volfen unt bod)

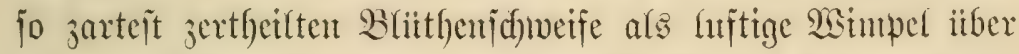

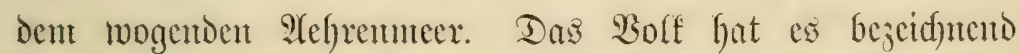
Den Windhalm (Apera spica venti) benaut, mo mierm

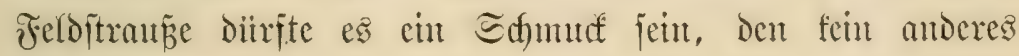
(3iras exjekt.

Win vergeffen aber nidft, als edelite (Sräjer aud) goldene

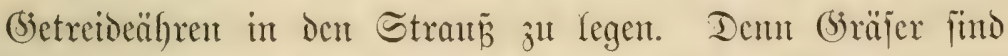
aud bieje! Das fint fie nad) ifrem botantijd)en Ban; mux 


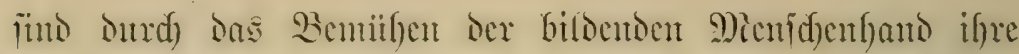

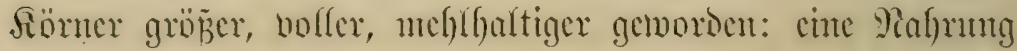
für Den 9)icnjuen, Der Eegen Des tügliduen Brodes! Eic fund Dic Sulturgräjer, mit deren ?tuban dor Entturjuftand Der Menjojen jelber begonnent hat.

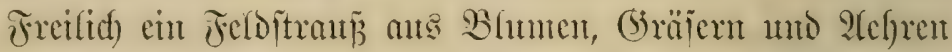
mux ift's, mas wo mu in ber Sant balten. Stber ob ex

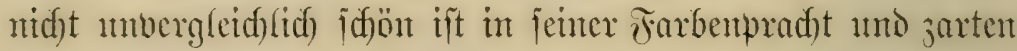

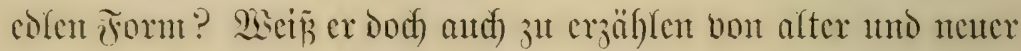

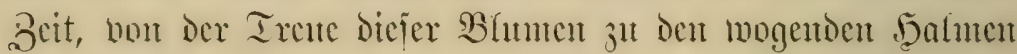

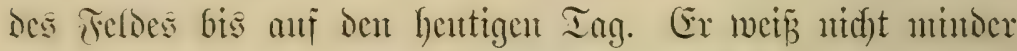

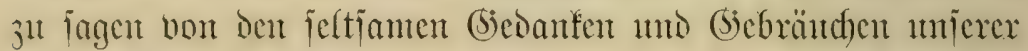
Sorfafren, deren (Semitth ticfer als bas stmjere fide in das

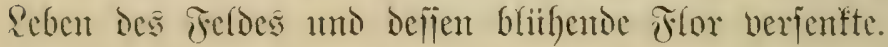

4.

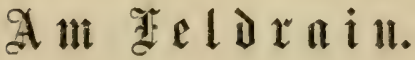

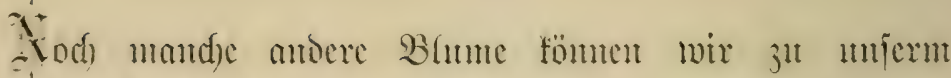

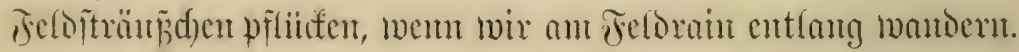
Ier Feforain ijt bon jefer dent Dentiden cin poetijefer

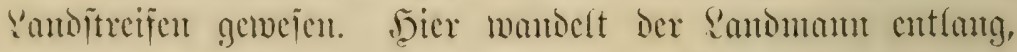
wem ex ant Eomutagnad)mittang feine Ffuren begeht, 1 m

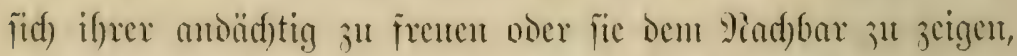

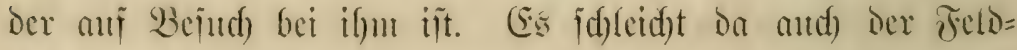

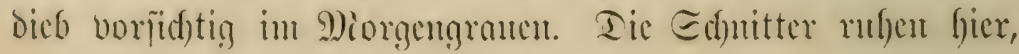




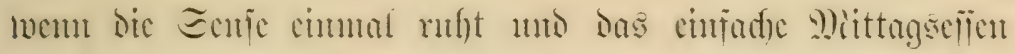
nergefort wird. bier liegen bic Sintor und fpicten umber,

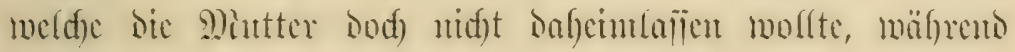
fie Dranpen in Felde die fleip̧igen Seände regt.

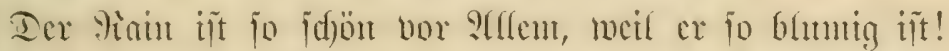

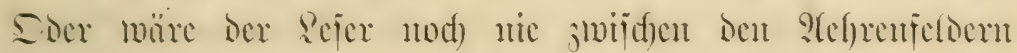

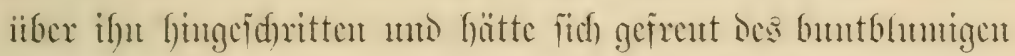

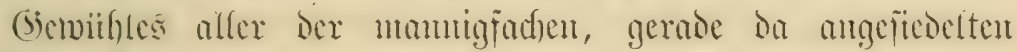
Srüuter und Den crquiffenden Duft Derjelben eingentfunet!

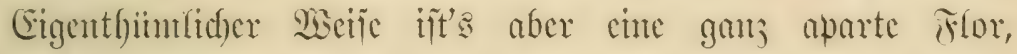

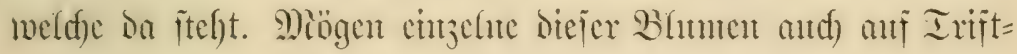

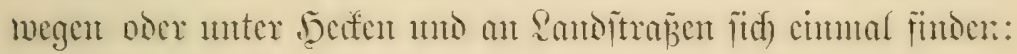
ifyr urcigeniter \$lak ift Dod) eben Der Feldrain!

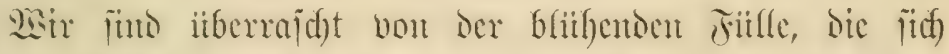

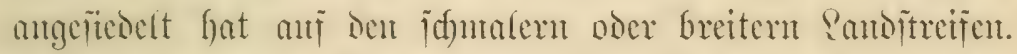

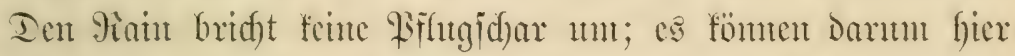

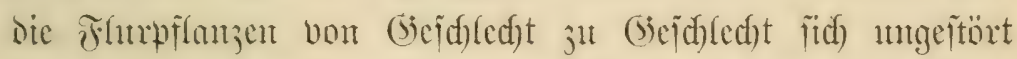
weiter entwiffeln, mo darum füno es dourafterijtijd) jum gröpten Theil autd) gant; antere, als metde in bidften ந̧atmen=

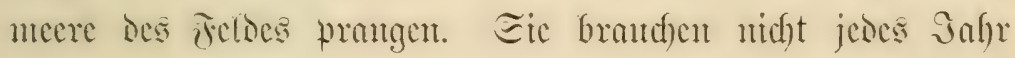

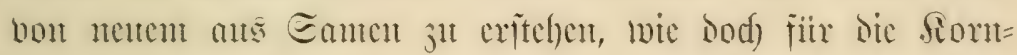

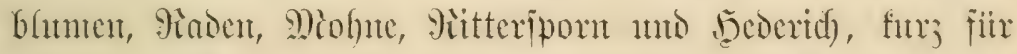
alle cigentlident Feloblumen nöthig ijt. Wir Gaben os baher bei ber gianff(or nidgt mit cinjälfrigen, fondern jumeijt nit

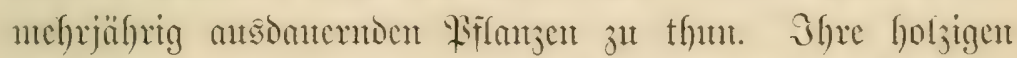

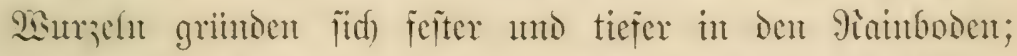
fic jelber aud futh meift holjige Siflanjen, seren vicle wir

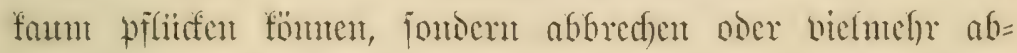
reī̄en miifijen. 


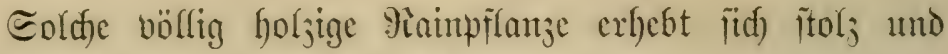
cocl dort ferjengerade mit dunfelgrimen fiederthetligen B̧lättern,

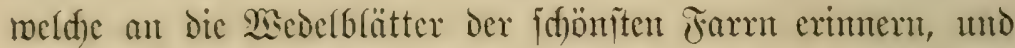
Der bohe Etengel ijt gefrönt mit einer Dolde Dunfelgelber

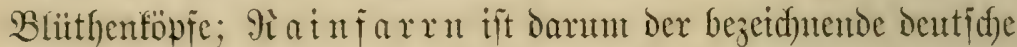

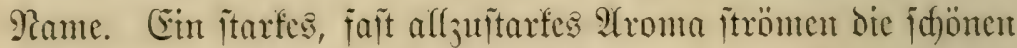

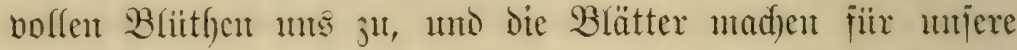

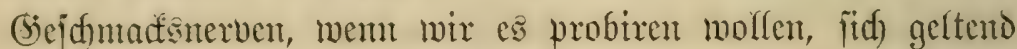
Durdh heftige Bitterfeit. Eollte mancher Sejer dicje Biflanze nicht femen, fo fent jie dod) jeder - Ed)lädter, wentigitents in Den meitten (Sicgenden Deutid)(ands: nad) altem Seerfonment holt und berwendet biejer die harten Etengel diejer Bifanje als Epciler jut icinen Siuriten. Somit hat aber Jedernmm

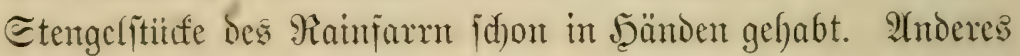

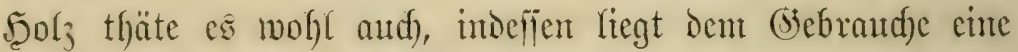

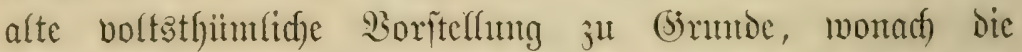
Wunte bod io an beiten berathen ijt. Der giamfarm itano

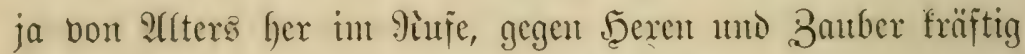
ju jein, und jomit war dic mit jold)em \$olje gejpeilte 2 surit

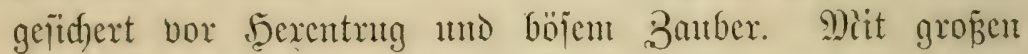

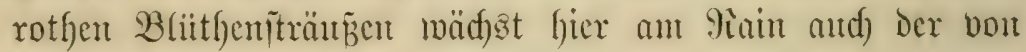
allen ltnboloen und llntboldimen ant meijten gemicoene Do ften, cin Sirant mit thynianartigen feinen Bfiithdyen un

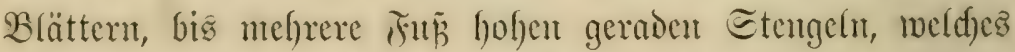

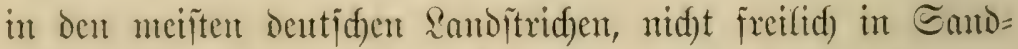
gegenden, an liebiten anf Sinlfboden, mafienthaft vorfommt.

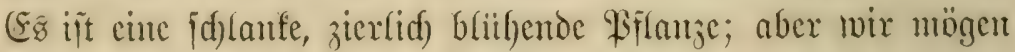

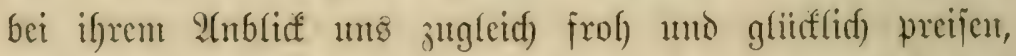
in einer 3cit zu leben, wo wir harmlog แกษ der Edjönbeit 
Dicier Błume froment fömten, ofute an Dexent mo Sauber jut

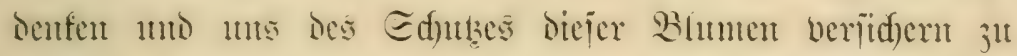

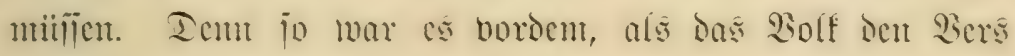
crọan:

\section{Dopten, Dorant und rothe Şaid Bringen den Seeren vieles \&eid.}

Ja, ocr Ioften mar cin mäd)tiges fraut gegen Alfes,

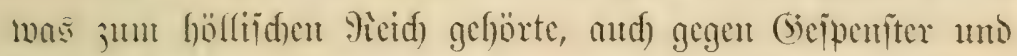
jeglide (Seijtermelt. Eo crifulfr os jene Frant, meldye, wie cine

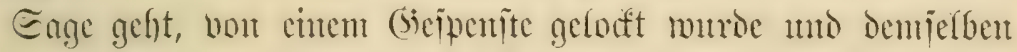
unvioeritellich folgen munte. Co gimg mit ifre durd) cinten

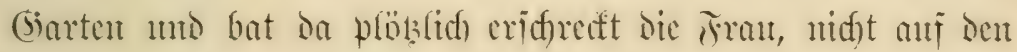

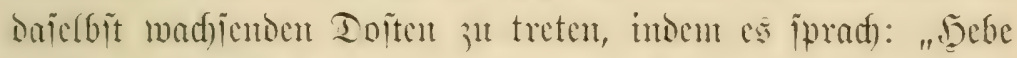

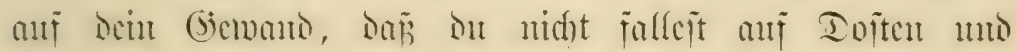
Iorant." Iies lestere Arant war nidft mintoer den ltn=

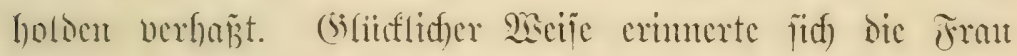
aber, chmmel bou dex firnjt Des Iojten gebört jul babent. ltut ficle, als jie fidf rajd) mu in bics firant legte, war

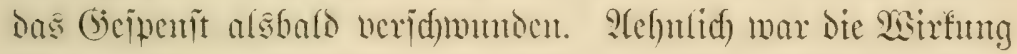

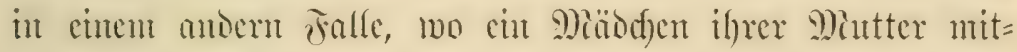

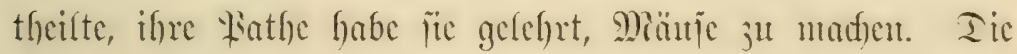

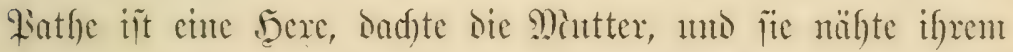
Sinte mun heimlid) Doiten unt Johamisfrant ins filcio.

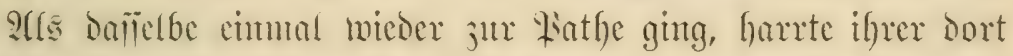

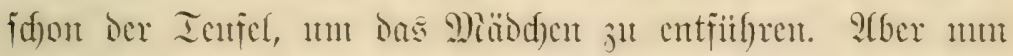
rod) er ben Ioiten, cutmidf jümmer(id), ntmo ricf ants: "Ioften unt Solgamisfrant berfithren mix meine Brant!" Eo mag

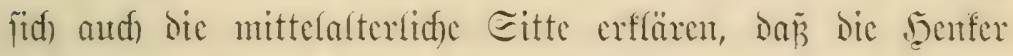
jene armen alo Seren gejolterten Diäbdoen und Frauen, ment 
Dieje untex Den Folterqualen ofumädytig wurdon, unt Dojten

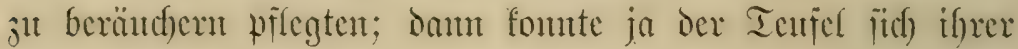

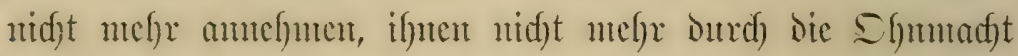

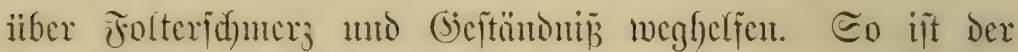

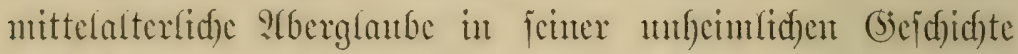

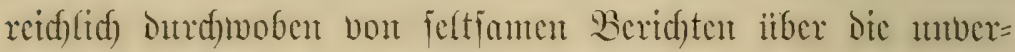

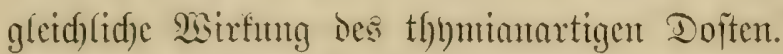

Heber die rotbe Jaabe, jiber den Duftigen Ifyutan treten wix fenter beim (Sange den Sain cutfantg. Sie blithen

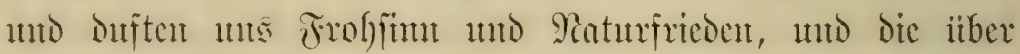
Den Thbuian fummenden, fumenten Bienen und Injeften

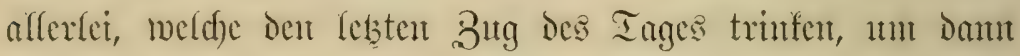

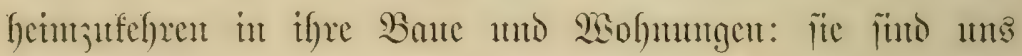

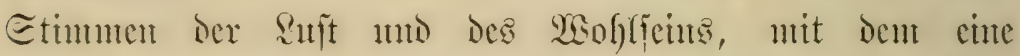

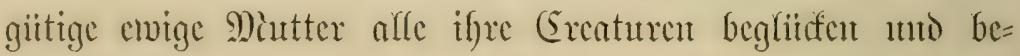
jreien ivill.

Sn rotbut Iönen beitht cos nod) reidflidfer nor unts ber

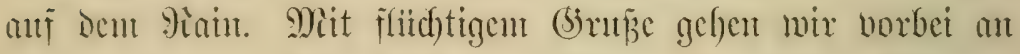
gar mandben in Dicje Farbe Der Wärme getanthten Blumen.

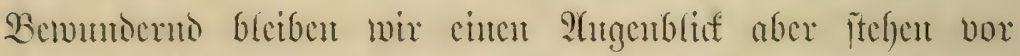

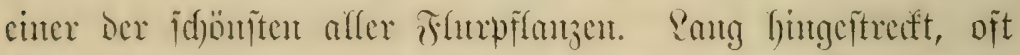

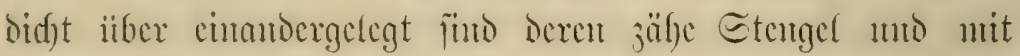
fiecrigen Blättern bejest; fo lagern fic mmber mo an iffem

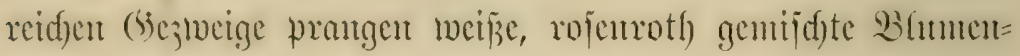

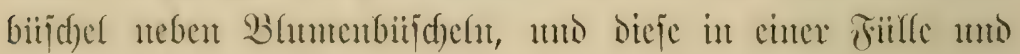

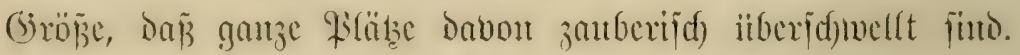

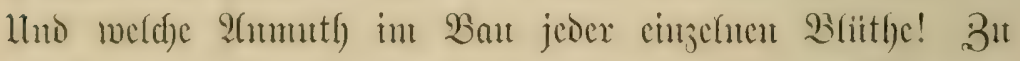

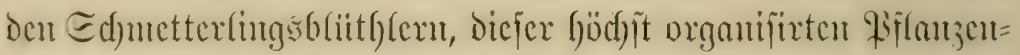

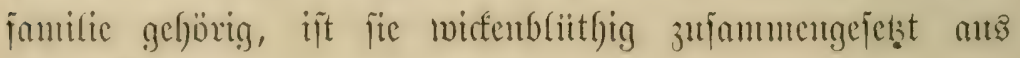




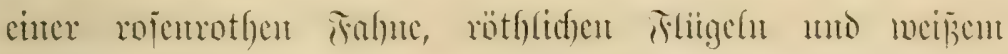

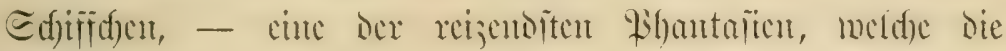

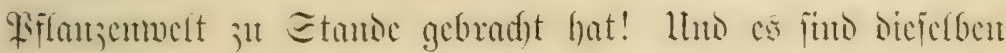

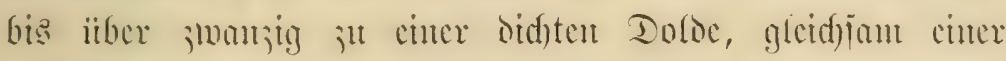

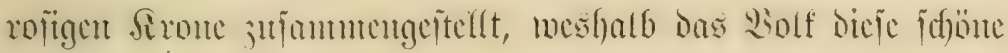

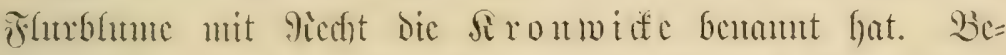

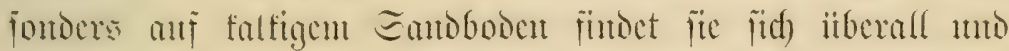

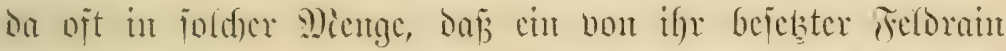

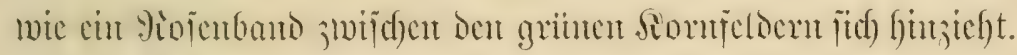

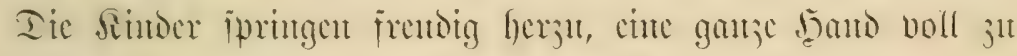
pfliaten, anto wir felfor ftefon in Entjiaten verloren bor Dicier ans öben, gelbem Eambbonen wie hervorgejanberten

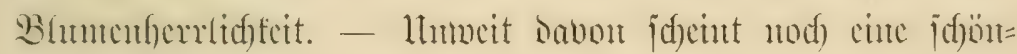

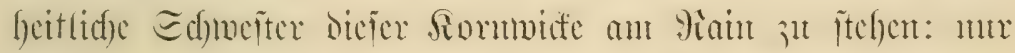
melse bereingelte mo nod) gröpere, in Form mo färbung

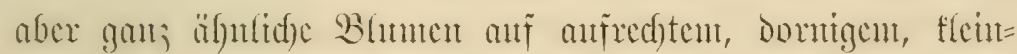

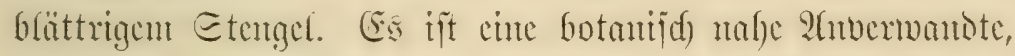
aber bod) cute gan; antore (Sattung: Dex Weiberfrieg,

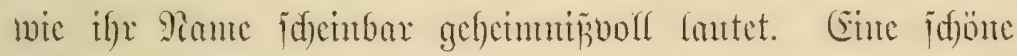

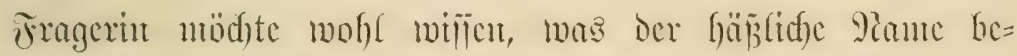
Dente. S(ber id) bitte jubor, chen der jüben Blweige abju=

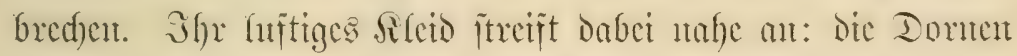

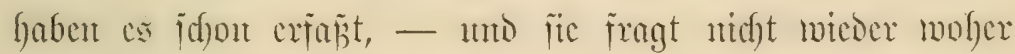
Der Эante Weiberfrieg rithre.

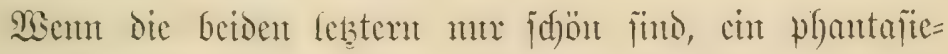

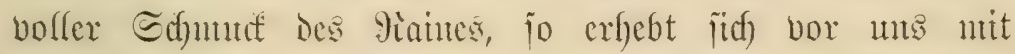

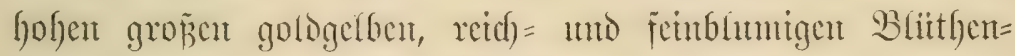

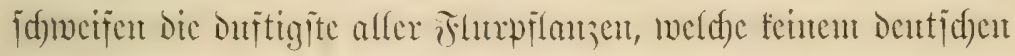
Pante febit und maficulbaft ba borfommt. Das gelbe $2 a a b=$ 


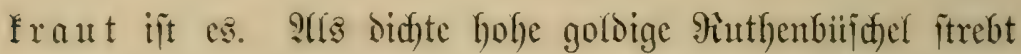

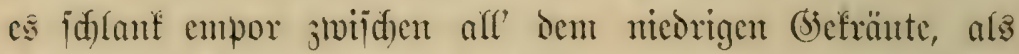
Defïen bornchntes es jidf nidft minder burd) cinen ftarfent, jeond) itberaus lieblidyen (Sientd) anszeidynet. Ecine vier= blättrigen feinen $2 \mathcal{B}$ (umenftenchen, Deren ein curiger Blitthen= id)weij vicle funtorte trägt, fowve die wirtelig geitelften

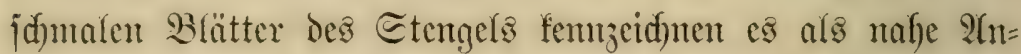

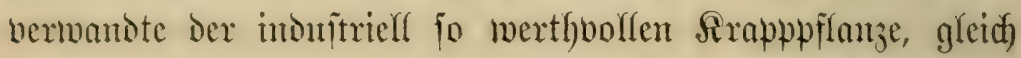
welder es ju der Familie der Etellaten oder Sinbiaccen ge= hört. Eo viel atrten Raabfrant in Mals uno Wicje, an llïern แnD Felijen fïr Den Botanifer aber wadjen mögen, - Das Bolf fennt dod) mu das gelbe Duftige Ranbfraut au

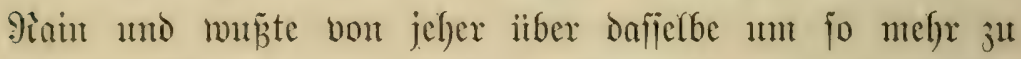
jagen. Wentgitens in frifherer 3eit; bie शreuzeit ijt ber grimen Natur ja fern getreten und hat vor :Uffent die Sagen,

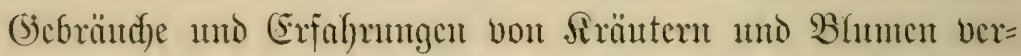

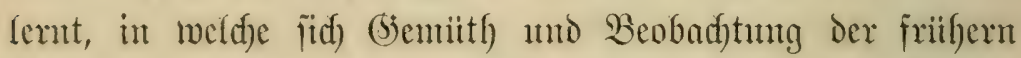
Beit jo gern und innig verjenfte. Selbit bis anf dic Iramen

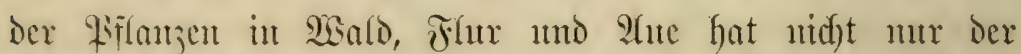
Etäbter, jonocm haben vielfad) jogar bie A(nnohner der

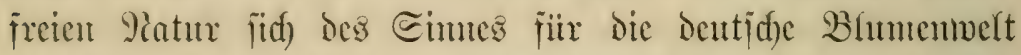

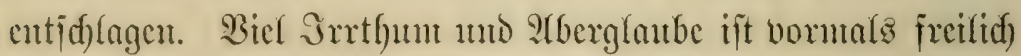

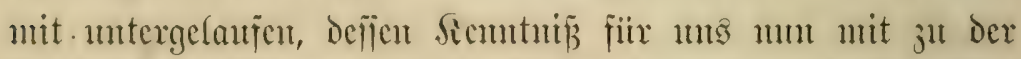

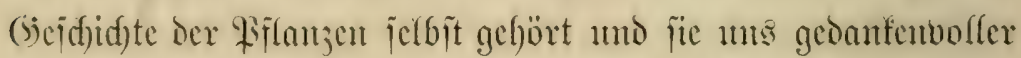

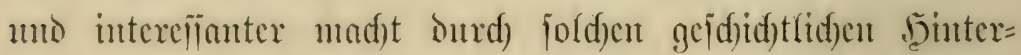

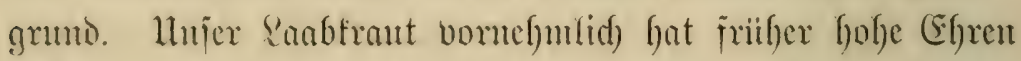

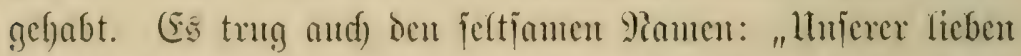

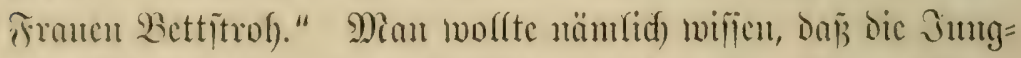

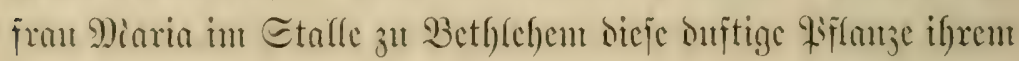




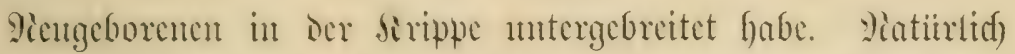
munte es in Folge dabon iettoem munorbare Eigenidaften

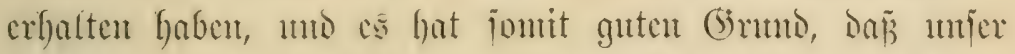

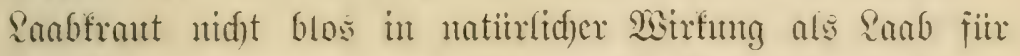

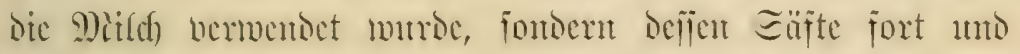

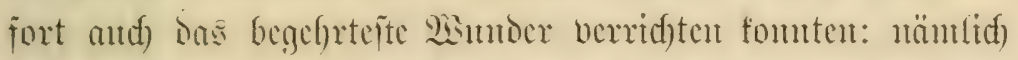
das Seben meit ïber das gemöhnlide Sian ju verlüngem.

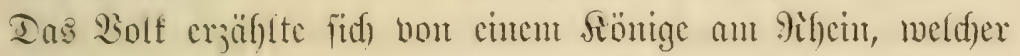

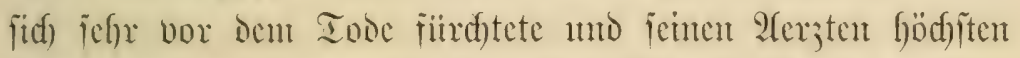

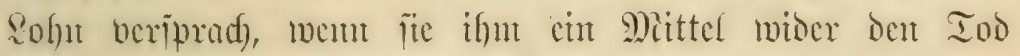

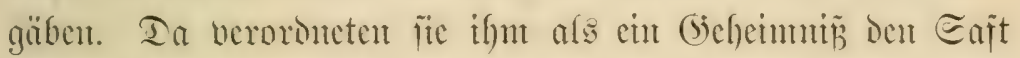

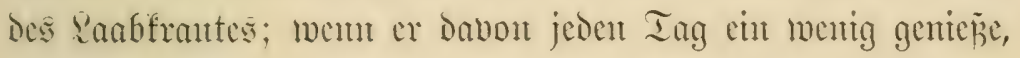

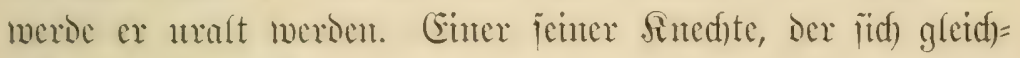

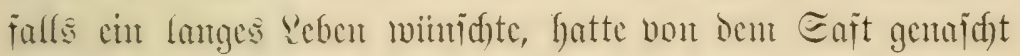

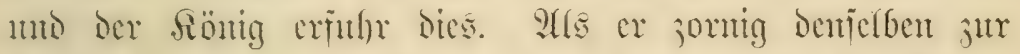

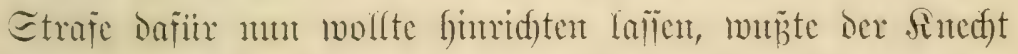

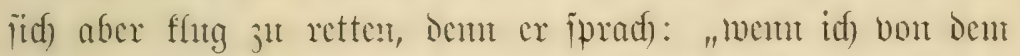
Eafte getrunfen unto Demtod) iterben nuth, jo bilit ja der Eaft nidft wioce ben Iob!" Ias madte ben fiönig be=

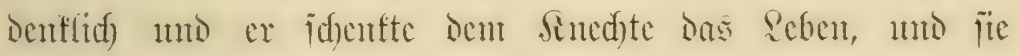

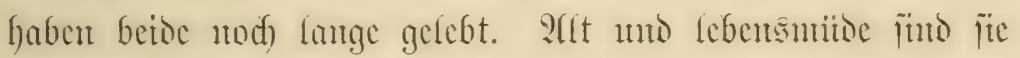

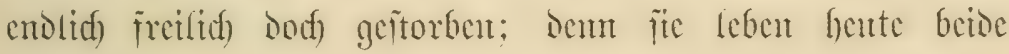
nidgt melyr.

Das Rabefrant bat in der That mintoctens aber cine pro=

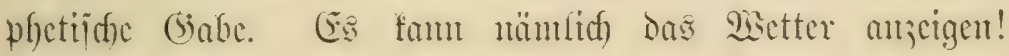

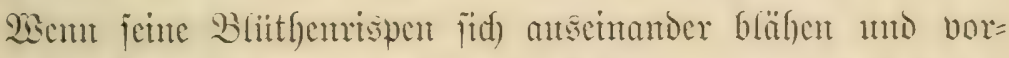

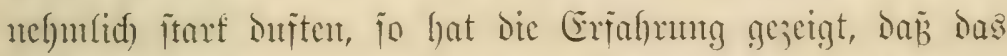

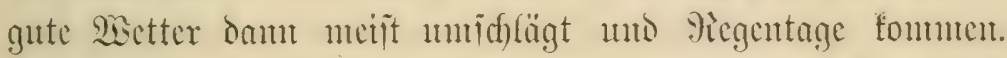

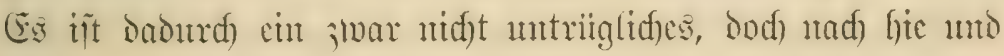


Da herrjdender Bolfsmeinung nidht ganj verüd)tliches brithenDes Baronteter.

Dexiclbe Pante "llujerer lieben Franen Bettitrol)" Gatte

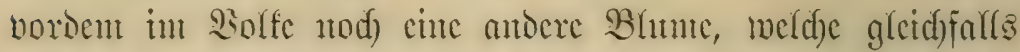

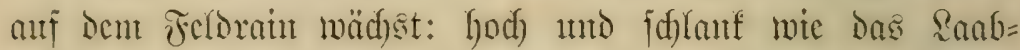
fraut, aber mit Duftlofen, weit grö̈peren gologelben, feibig

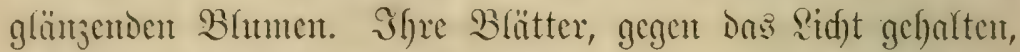

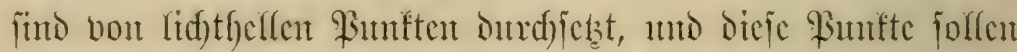

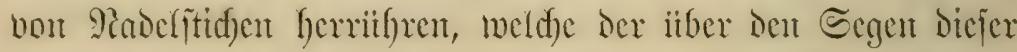

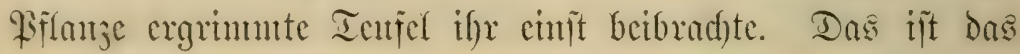

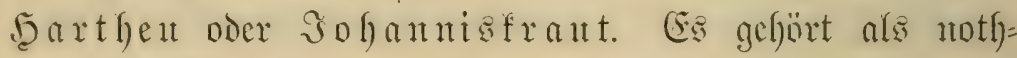

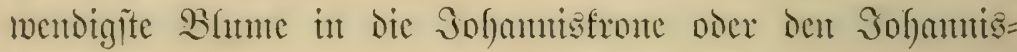

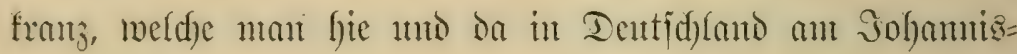

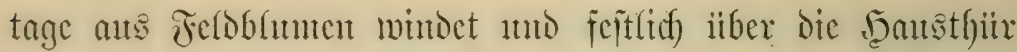

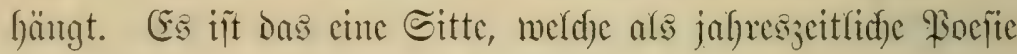
an bielen Srten, mix befant bejonders in vielen Stäbten und Dörfert an ber Mitloc und Gaale, bis anf ben hattigat

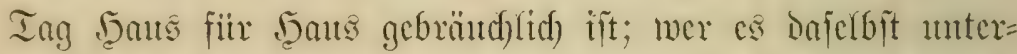

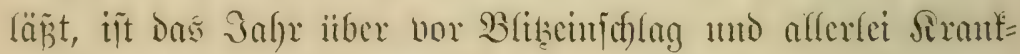

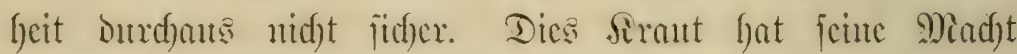

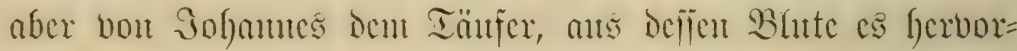

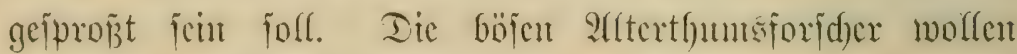

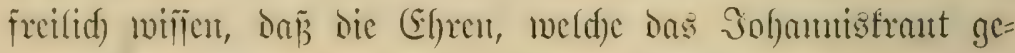

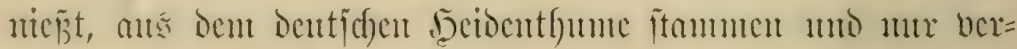

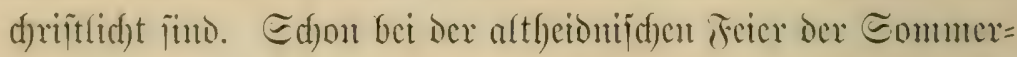
jomenuenoc, nuf Deren Iag in ber (Shriftenf)it Das Johamis=

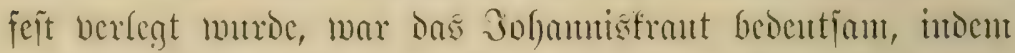

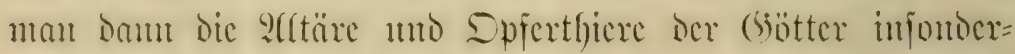

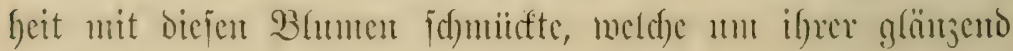


gelfen Farbe willen ocm Eommer mo dent Eomucugntte

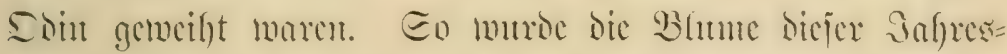

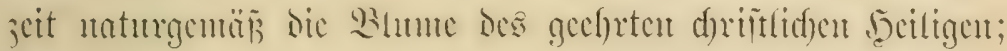

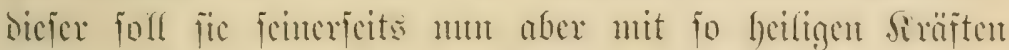

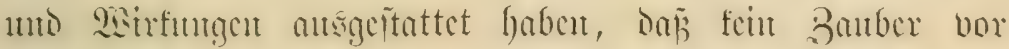

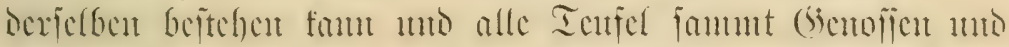

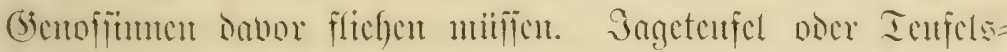

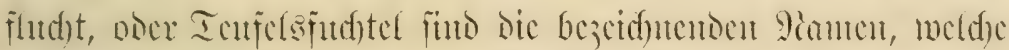

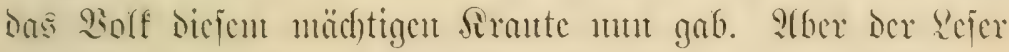

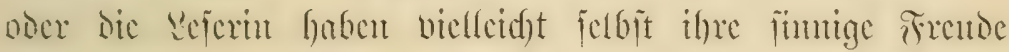

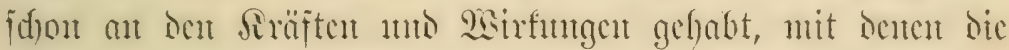

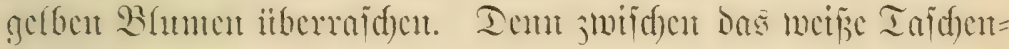

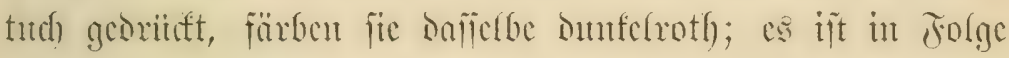

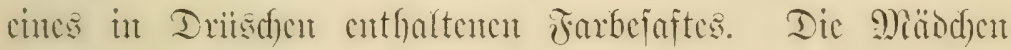

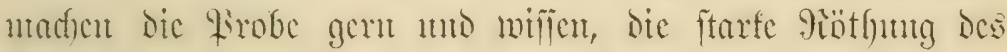
Indfes bedente bic llutucute bes fernen bieliebten, es blate

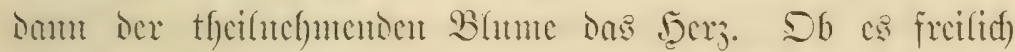
megen ber angegebenen natirrfidgen llwadge nidft immer blut= ten

Misolfen wir weiter den 9iant cutlang mantorn? Dic abentiche Eommerluft ruft fo ftiff und warm nod) ïfor Den

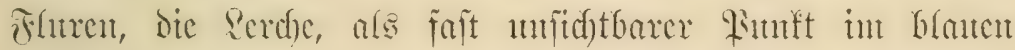

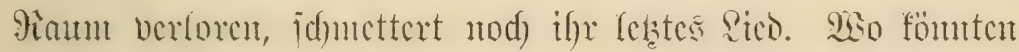

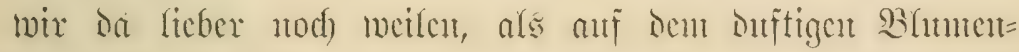

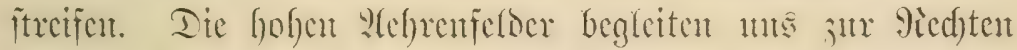

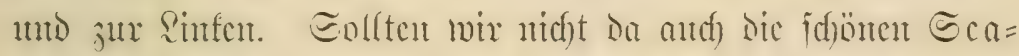

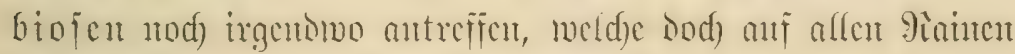

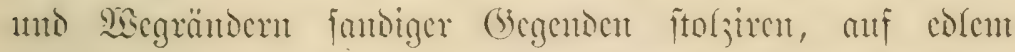

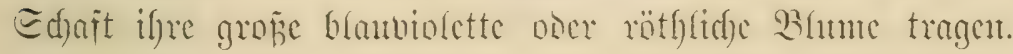




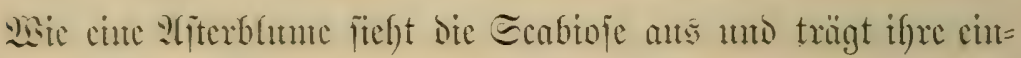

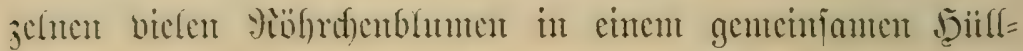

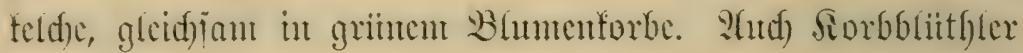

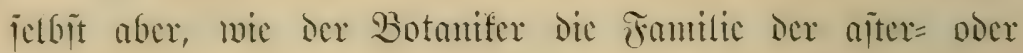

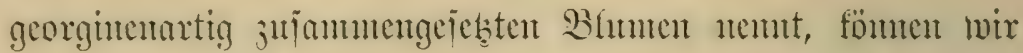
gar mande finton, went wix Iweiter ïber ben giant fint=

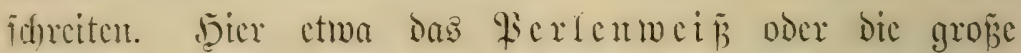

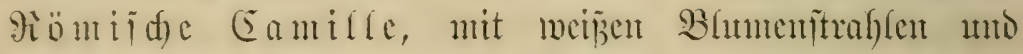
gulber Edheibe, ijt wie jut Bhunenteeten berentigt; Dort bie gelben ftrabligen Blithenföpfe des 3 aco befrantes, welde,

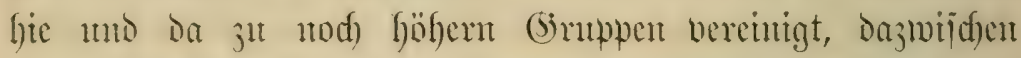

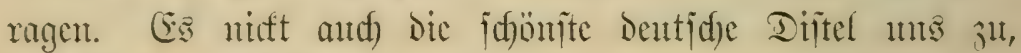

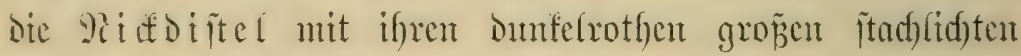

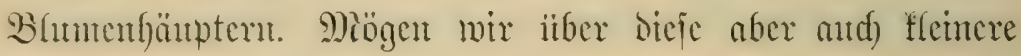
Siorbblïthler, die winzigen weipen Fabentränter, ben fropfigen gelben \&ämuterialat und anderes fleme (sicblüme

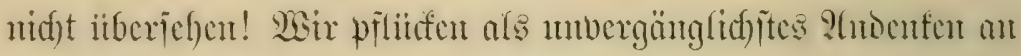

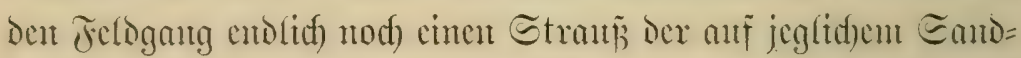
bodent weitfin verbreiteten getben $\Im$ mmortef (en oder Etroh)=

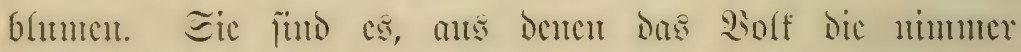

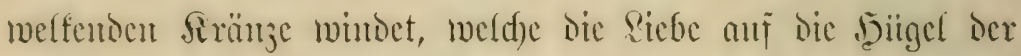
Deimgegangenen antö̈d)tig legt, oser mit benen wir dabcim

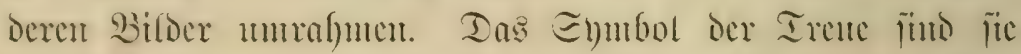
vou jefer gencient, und an mandfen Exten joffen nod) jelst

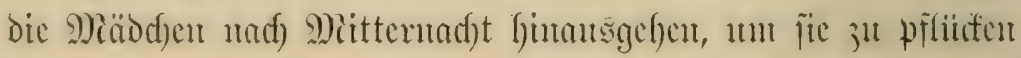

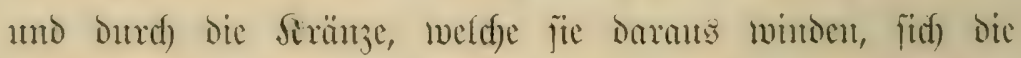
Irente ifres Rebjten ju jichera. Dex ftarfe Duft mo das Gologelb der vielen Blïtgentöpfe anf ben grangrian beblätterten

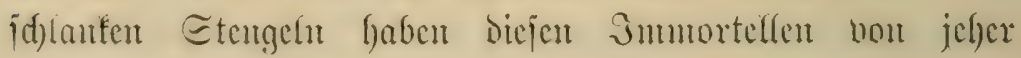




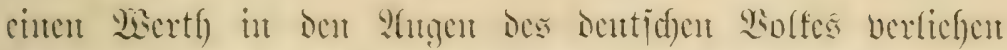

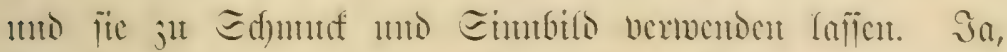

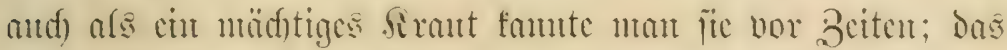

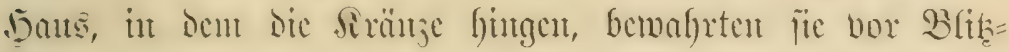

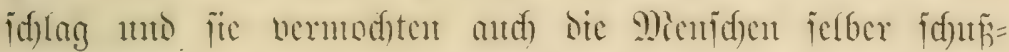

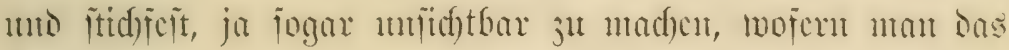
Sirmit unter Echweigen au cuten Eomutage ausgrub, anf

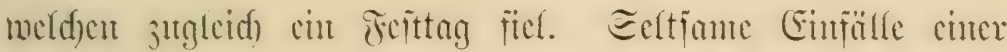

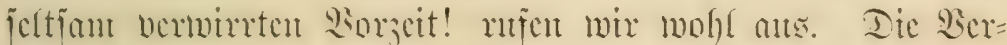

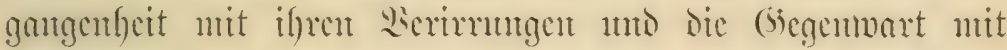

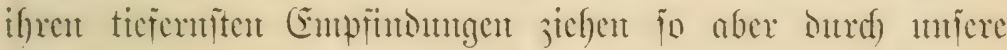

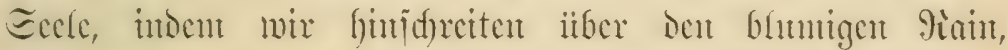

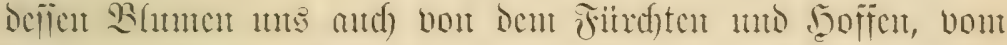

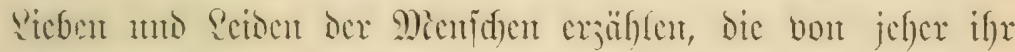

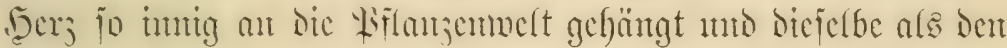

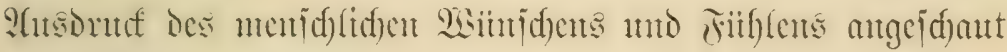
Gabent. Eolfon mir mit miferm flareren (sicife, als ifyt dic

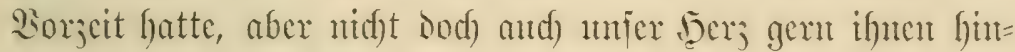

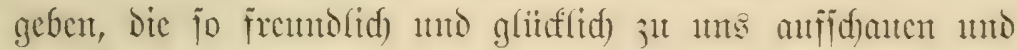
Durd) ifgr Dajen bas unjerige bejäntigen mo crantefen wollen!

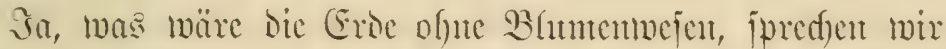

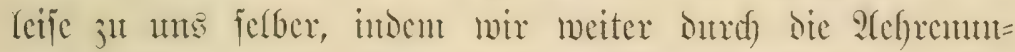

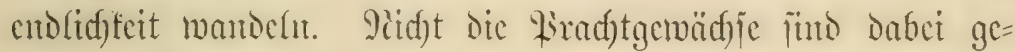
meint, weldye bic fintît als Bicrrath amjerer (bärten jicht,

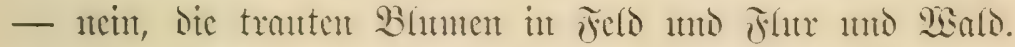

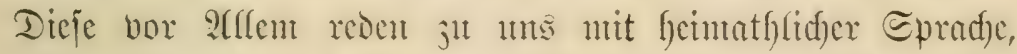

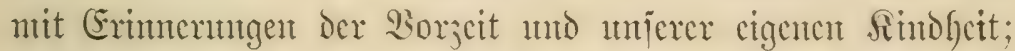

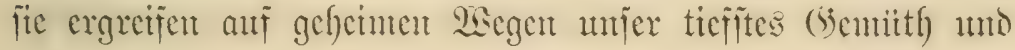




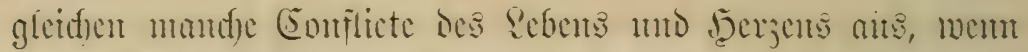

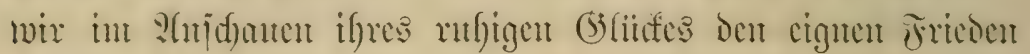
int)ent.

Ser Felorain bejombers aber ift iold) Geimlidfer Grocut=

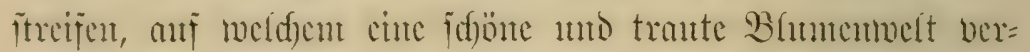

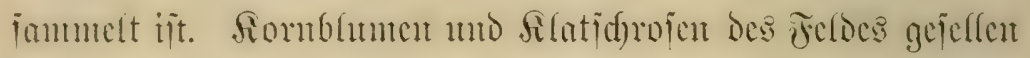

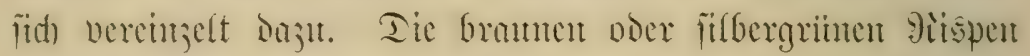

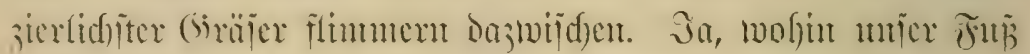
ficr mu tritt, fam ex nidft anders als iiber Bhamen mandelu.

\section{5.

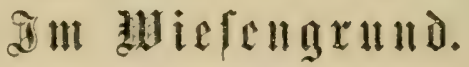

尊

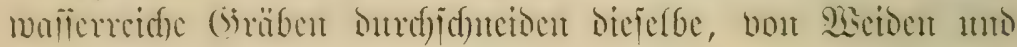

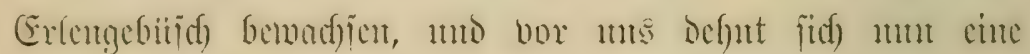

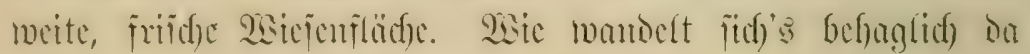

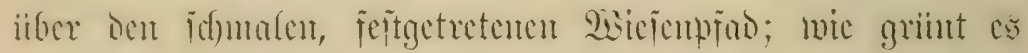
erintitem, io meit bas shage iffucift, und in ben joungen siiften gantedn bunte Eefunctterlinge, flimen sibellen mo

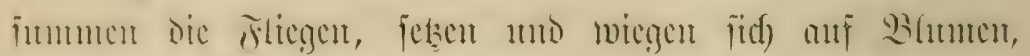

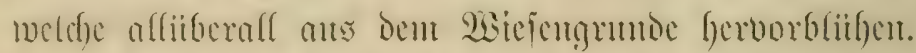

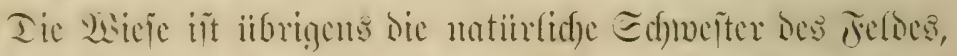

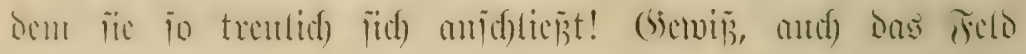

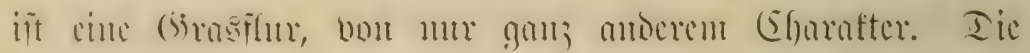

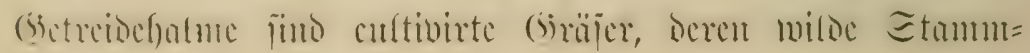




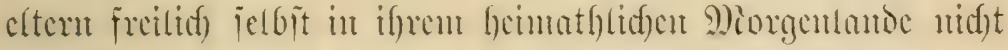

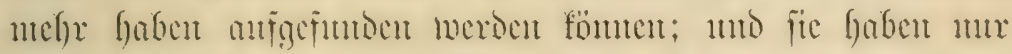

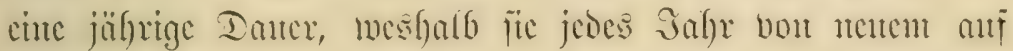
die Felder angejät werden, anf jesces Felo cine bejtumnte Eorte; der Sambunm läjt fic da bis jur Siörnerreife wadjen,

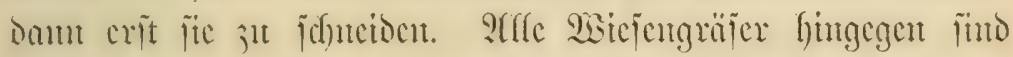

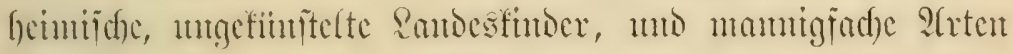

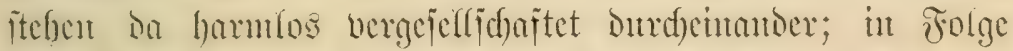

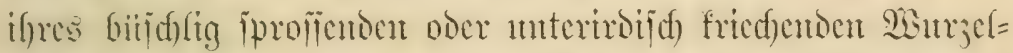

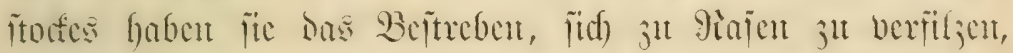

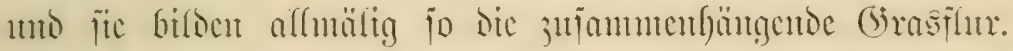

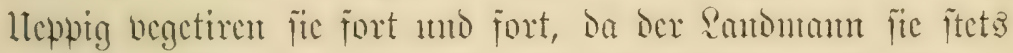

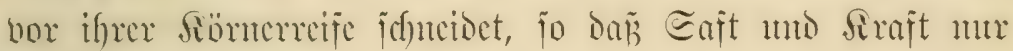

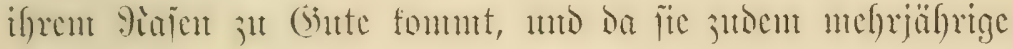

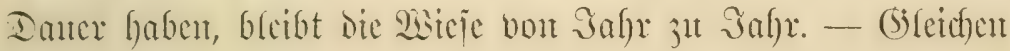

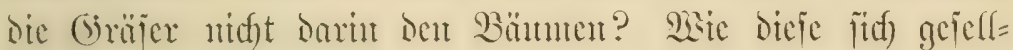

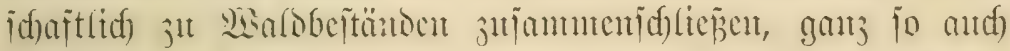

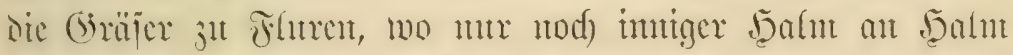

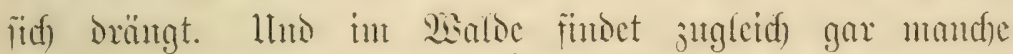

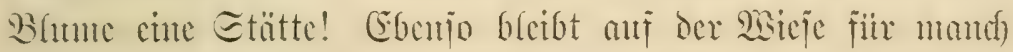

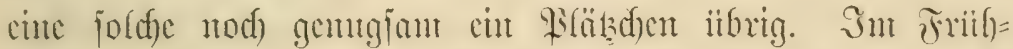

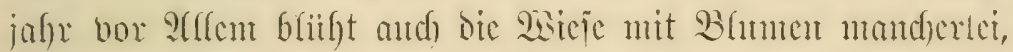

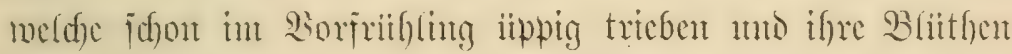
mut bereits offnen; bic (sirasuegetation fört jie ja nod) Dutrd)ates nidft.

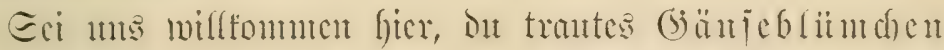

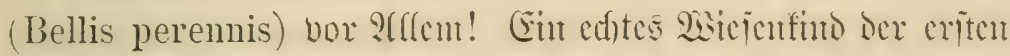

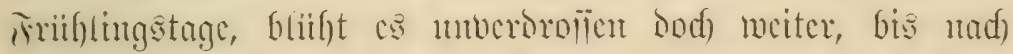

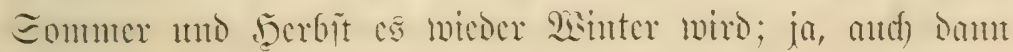




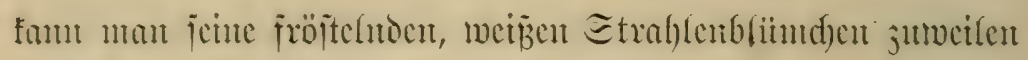

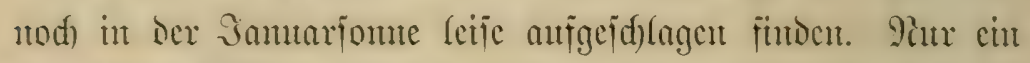
bitterfalter 23 inter töstet aud jeine Bhmen! Sa, wie unfer

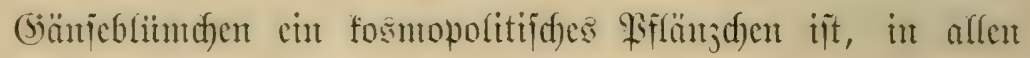
Erotheilen, untex allen Ränge= แmo aud) faft allen Breite= graden fich borfintet, fo ijt es jelbjt an feine Sabreșjeit ge=

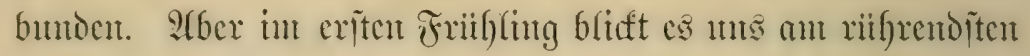
an, ment die itberwinterten, mu erit furzgciticlten, grinten

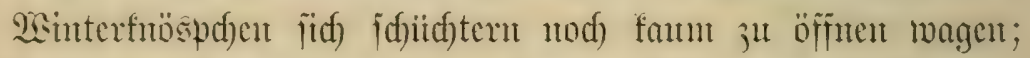

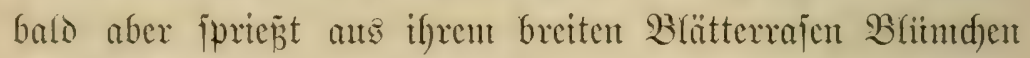
neben B(ïmd)en empor, unt cins nad) dem andern briift unnter mun aup. Die finder, welde nad) dent langen Winter jun crften Male in's fontige Jurcie finans durfiten,

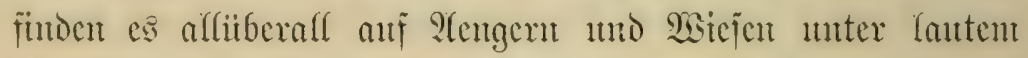

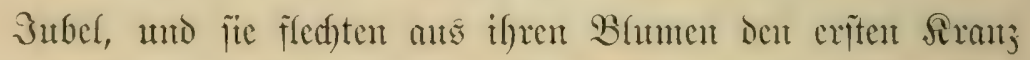

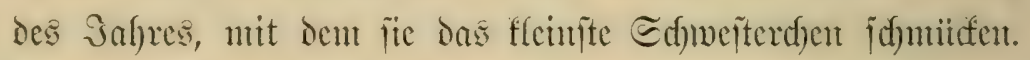
Aber der 2(beno finft oder bie Eome verjicht fitd); die Iveit=

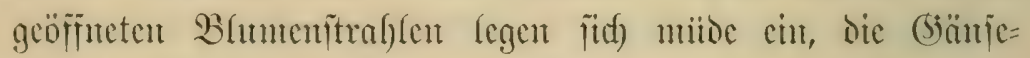

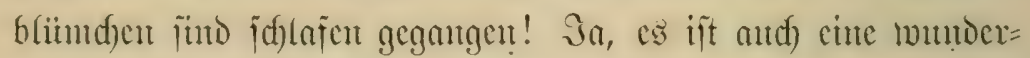

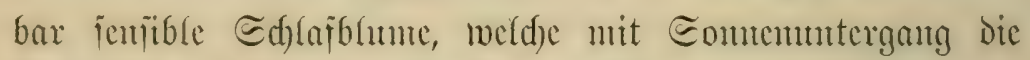

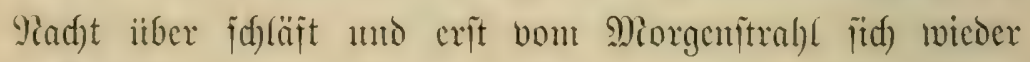
weçen läß̄t.

Sex frithe, grimte Inmmuclplas ijt die Wicje and)

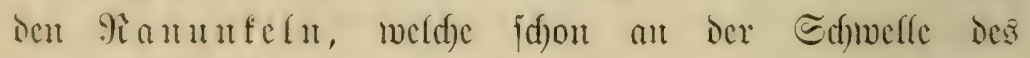

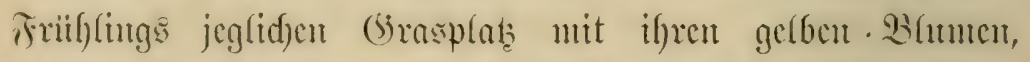

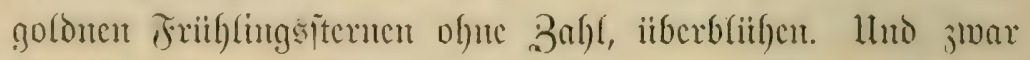

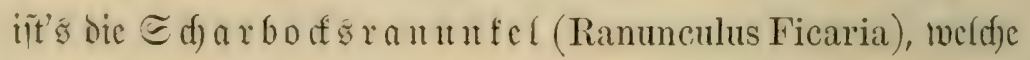
if)re inftigen Etengel, mit gläujenogrimen, nicrenförmigen

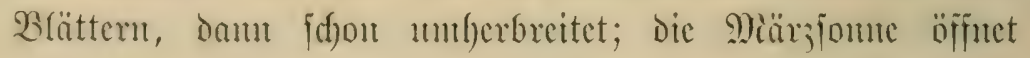




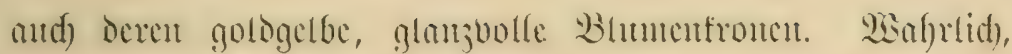

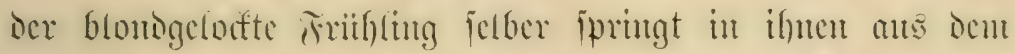

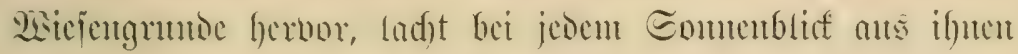

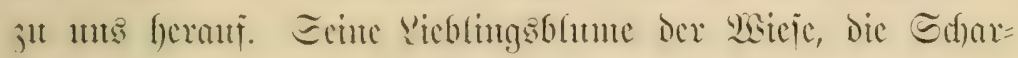

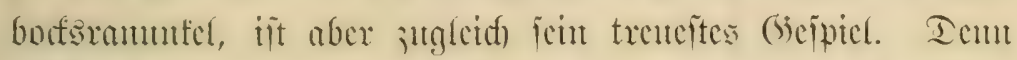

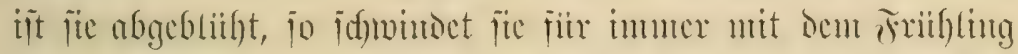

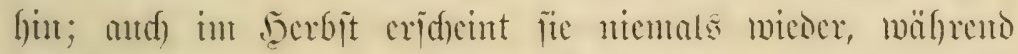

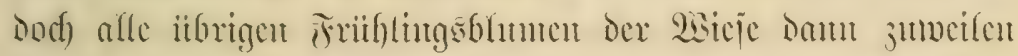

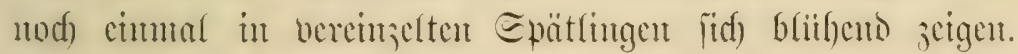

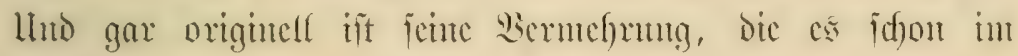

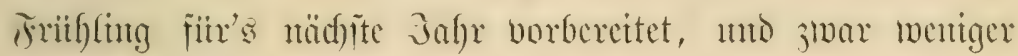

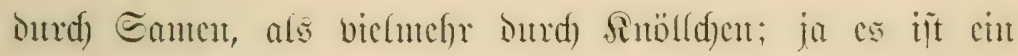

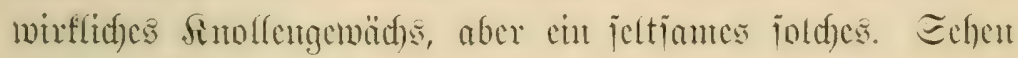

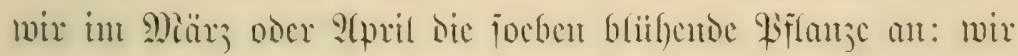
fintoen in ben Blattadjefn flène grïne, glatte Simöspdyen. Sicje cntmitchn jid) abcr nidjt jut Ecitenjmeigen, wic jut

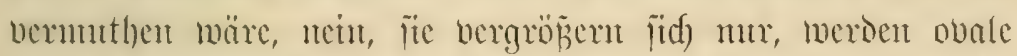
noer fenfenförmige, ipäter fid fortoffelartig brännende, itärefe=

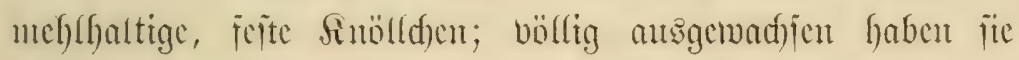

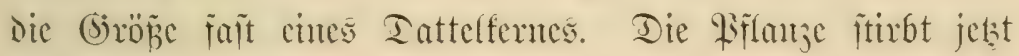
ab, legt jidc) jut Boden uno vertwest; jene Sinollen liegen ab= gelöst und jeriturut auf Der Erde umber. Bermundert $\mathrm{cr}=$

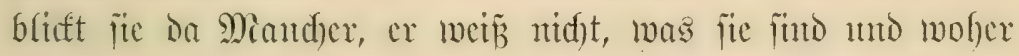
jie ftammen; als biumelsgerite bat jie vormals, wegen ifjer

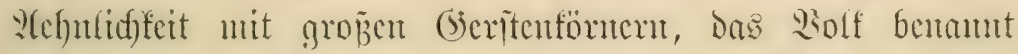
Into ermitlid) gencint, fie feicn bont bimmed berab geregutet. Eic warten untr, regututgšlos ant Boden liegent, Daj dor

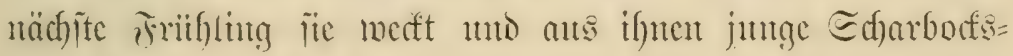
pfiängợen loçt. 


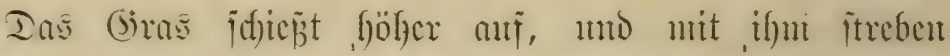

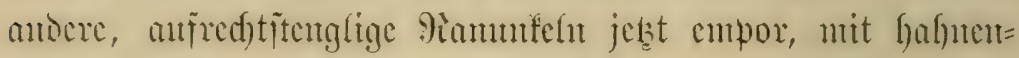

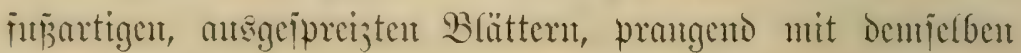
Gologelb jaftretder Bhament. Stfer wir beadjter jie bald

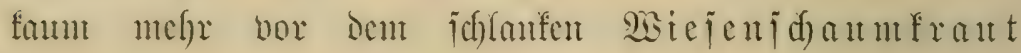
(Cardamine pratensis), Diejent erjter Sireujb(iitfler Der 2 iciejen,

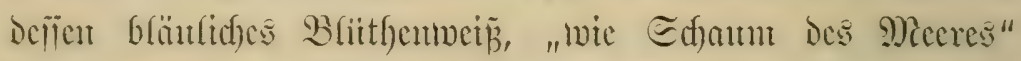

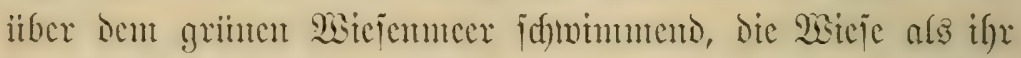

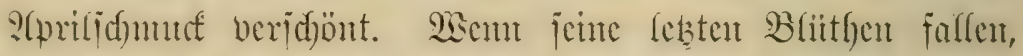

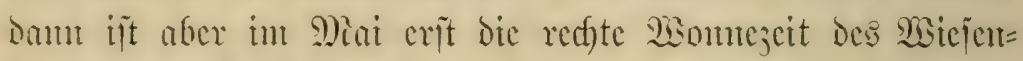
gruttoes gefomment.

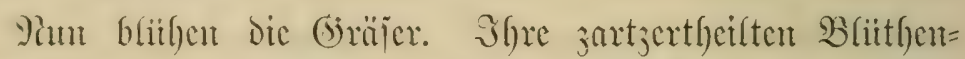

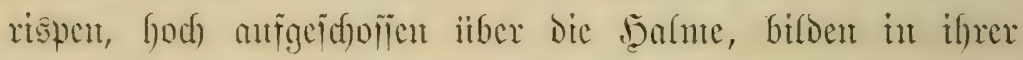

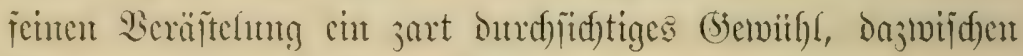
Fistegen mo bunte Siäfer ifre munteres Epiel jeşt treiben.

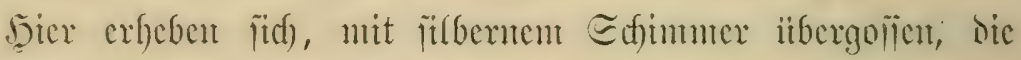

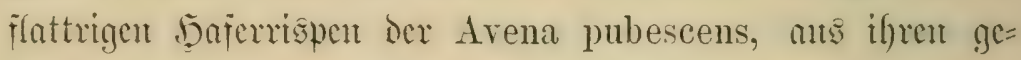

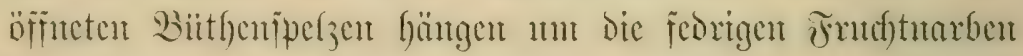

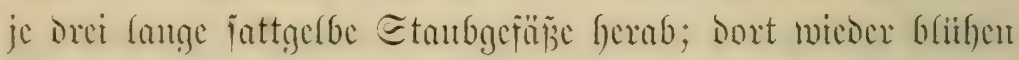

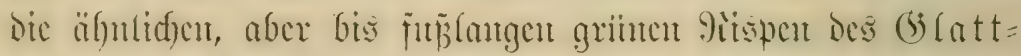
fafer (Arrhenaterum elatius), Deren futeförmig gebogent (Sirannen denjerfen cine grajiöic Iradgt verfeifent. Dort

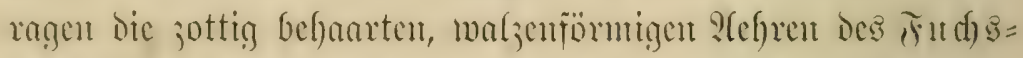

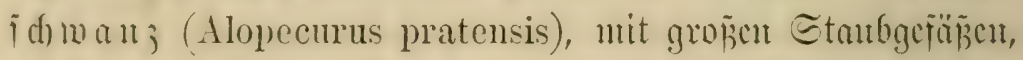

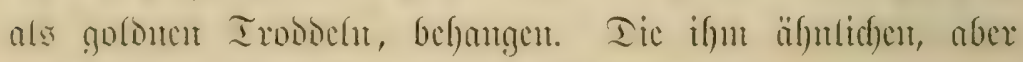

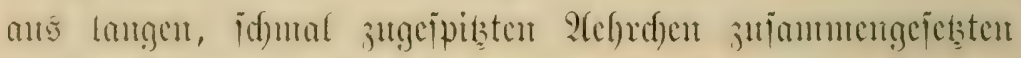

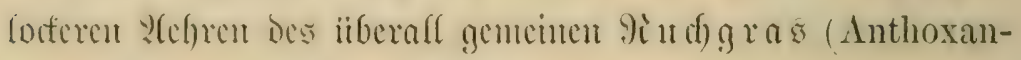
thum orloratum) wollen wir jogar ciumal abpflitten, andf)

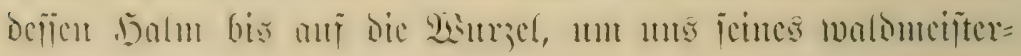




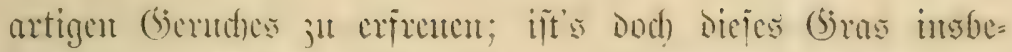
jondere, weldhes bem sen jeinten angendment Inft wer=

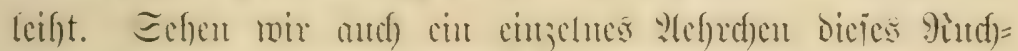
gras gentan cinmal an! Wiälgrend alle ïbrigen wicjent= mo

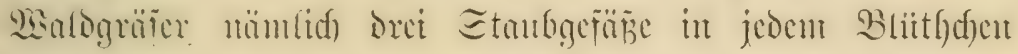

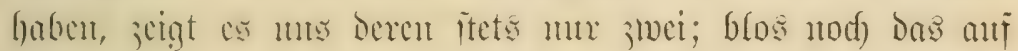
mooriandigen Iriften ibberalt gentente furje mo fteife, biijdecl=

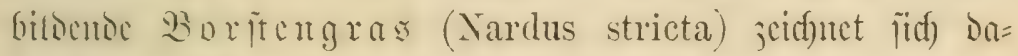

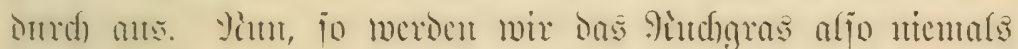

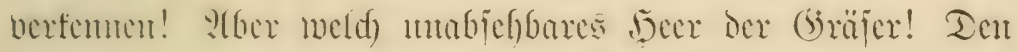

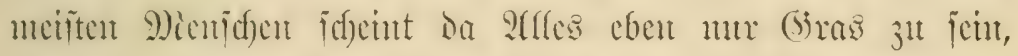

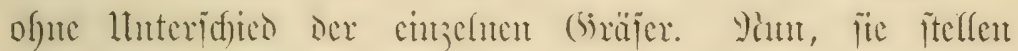

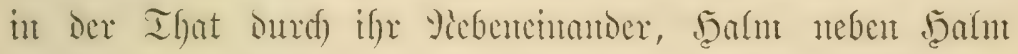

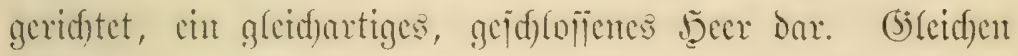

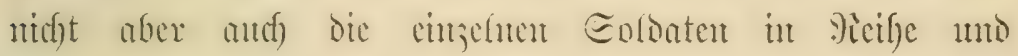
(5)lied unt in iffer Itriform cinander böllig? âber

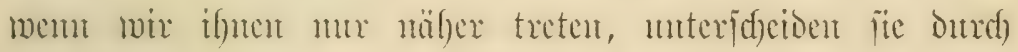

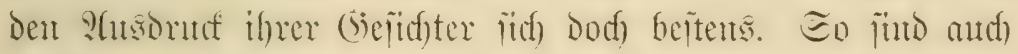

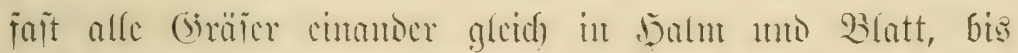

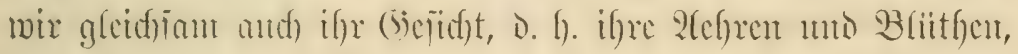

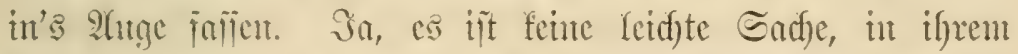

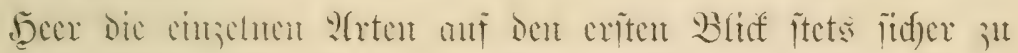

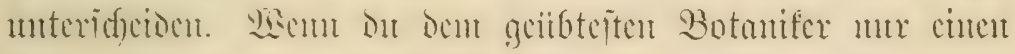

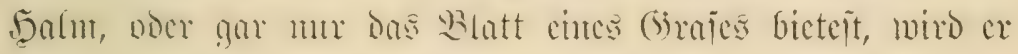
in Den meiften Fällen nidht im Etande jein, bix ju jagen,

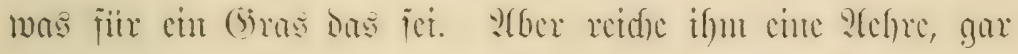

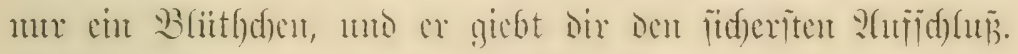

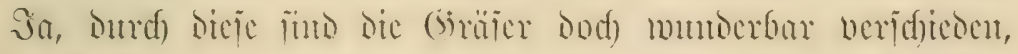

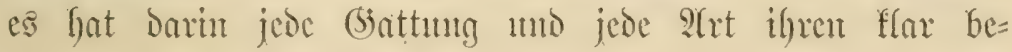




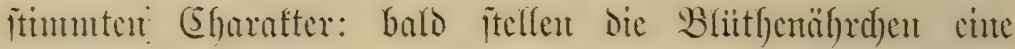
hajerartig flattrige grispe dax; bals finto jie ju cher waljen=

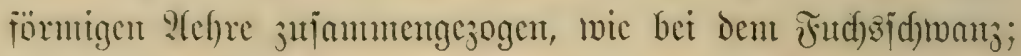
bals ijt bie gitspe untendfid) veräptelt mo mit ifrent feuten

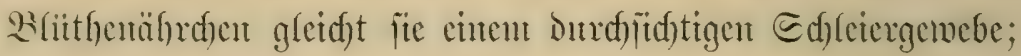
balo wicoer find fie gröser gejtaltet, cinzelu oder jut mefhreren an cinter Epintel gereigt, woie bei mieren (sictreidentent. Betrad)te anf entent Epajiergange ïbrigens cimntal nidjt blos

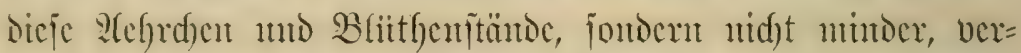
gleidento bei veridjedenten (Bräfert, cumnal and) die Bälge

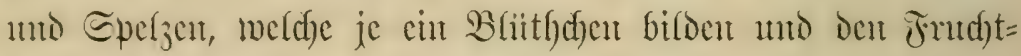

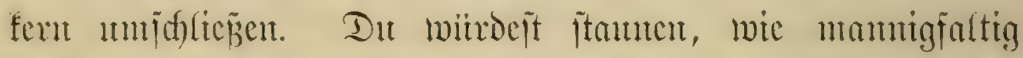
ıod) flente (Srajesbliutfen gejtaltet jein fömuen. Sa, anded)

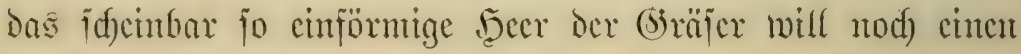

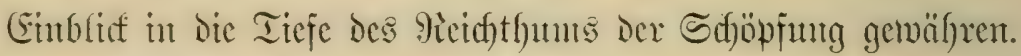
Wenn dic Gräjer blïben, ftebt aber aud dic farbenfrifde Bhlumenflor Der Wiejen in job̈ufter Entfaltung. Sn licblidjent (5amumoth feudften swijd)en all Den (Sirastipen, oft nod) fie

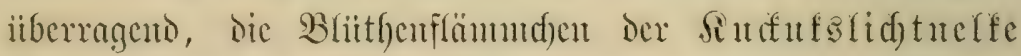
(Lychnis flos cuculi); ês läutet mit gro żen, Ginmelblaten (jilorfentumen dic Campanula Dured) all bas blitfento Frentoent= gerwithl; mandye asicjenord) is (Orchis latifolia, O. Morio, O.

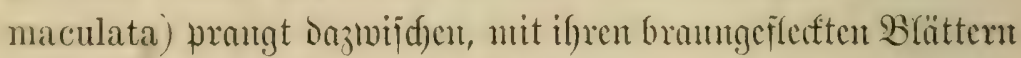

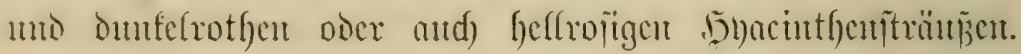

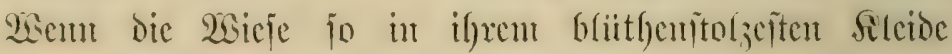

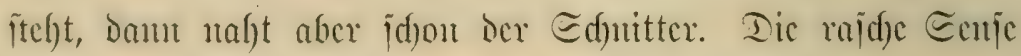

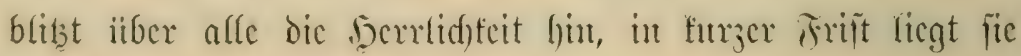

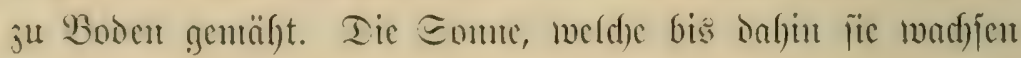

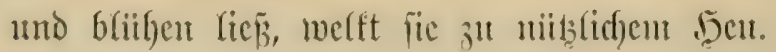


9)iandfe andore Bhtume crbebt jid) mit ber Beit wohl

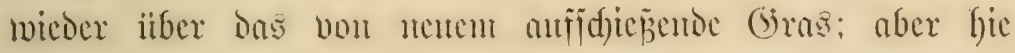

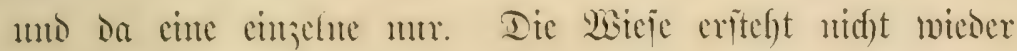

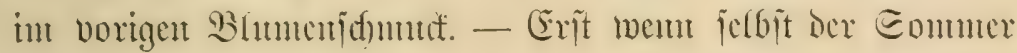

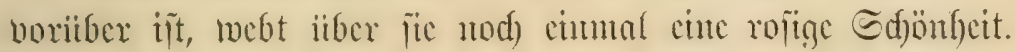

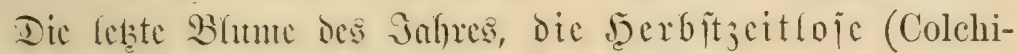
cum autumnale) b(ith)t ant! Freifid) nidbt iiberall ift ifor Etätte, indefien in manthen (segenden, bejonders anj den Worbergen des (Sebirges ijt fie itberreid) borbanten; cine iibrigens febr giftige, dent Sandmant mentg svilftonmente

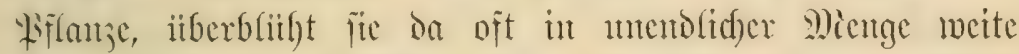

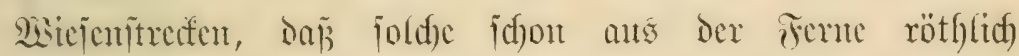
gläujert. 2lber böllig blattlos, jogar itengellos wächst fie da

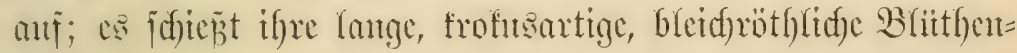

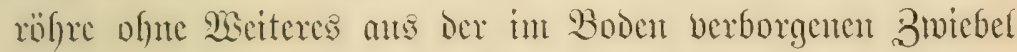

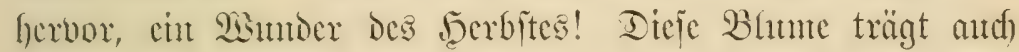

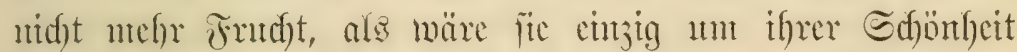
willen und) aus der Meige des Jahres da. stber fiehe, int

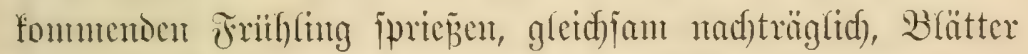

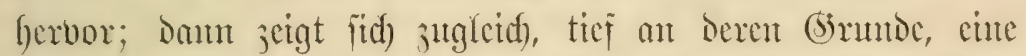
Diffe Futdffapiel. Den Winter über hatte dieje ifre Ent= wiffelung berjögert, unter Der Eome des Frithlutge crft be= gantu fie ju wadjen uno entolidf jut reffen; in der herbiftidgen Bhtme war fie aber freilid) idjout vorbereitet gewejer.

So reift in ber நeritjeitloje das ene Jabr Dent andern jeltian die 5ano! 



\section{IV.

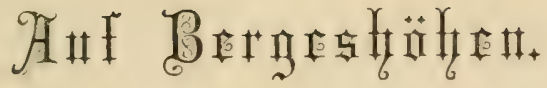

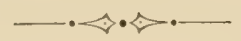

"Das war cin freuch, wenn cr wicder fam;

"Denu nientals fehrt er hecim, er bradht ench etwas, "War's cine fđ̆ötre Zllpcnblumte, war's

"Ein feltuer Dogel oder 2lmmonshorn,

"Wie es ber Wandrer fintoct auf' ben Bergen."

Sdiller's $\mathbb{T e l l}$. 
Eine wanderung über die 2lipen giebt 3 liffe anf cine $50 h e i t$ oer 2ratur, wic aแferden mur nody das unendihe Meer oder der gejtirnte Fimmel über uns. 2lud unjere nord = uno mitteldeutjhen Febirge erimnern hic und $\delta a$ an manthe foldhe gemaltige 2llpenicenerie, mo die Seele beugt fith vor der Majeftät Des Erịhaffenen. 2lber die hier vorwiegende malerifhe sieblidfeit der grïnen Thäler und fanften Eöhenzüge erquifft mehr Fierz und 2luge, es waltet hier vormelymlid? die gebirgslandihaftliche Benrentalerei; Der Blid Iabt fid an der

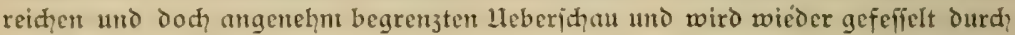
das Einzelne in mäditer zähe unther. So wie wir gejdzaut und empfunden, möđten wir dic Exinnerung überall voll und ganz uns dam nur bewahren föntr. Wohlan, dic Blumte, welche joeben freundid von einem felienlang

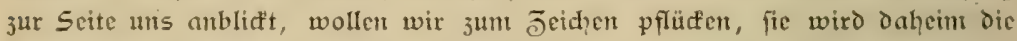
Erimerung lebendiger wieder wad rufen. 2lber $\partial \mathrm{cm}$ redten Bebirgswanderer find die bejonderen 3 lumen überall das Wahrzeidhen der Begend, und jo beachtet cr itets aud die fleine Blumenfitierei in dem reidin Bergfleide. Ind ob Soldher nidht dadurd viel getreuer das Bebirge würdigen lernt uno burd Beadituna Diejer blühenden Jierden ihm daffelbe reicher 'und reizvoller ift als dem mur landihaftlihen Enthufiaften! Wanderer, fo eile dent nid, ađtlos vorüber an der Bergblume! Mandhe ift fđön wic deine Bartenblunten, manḑe vielleiḑt jogar cine hier wildwahjende jold?e; cine andere ift wohl unjdieinbarer, aber Pönte dir dody mandyes feltjame Gehcimmís vom Dolf und Eamo der Berge erjählen, oder mandyerlei $\mathfrak{W}$ andergedanfen in bir anregen, ourd weldhe du das Gebirge aud in feinen naturwifferifhaftlidgen Bejiehungen dirfteft riḑtiger bes urtheilen lemen.

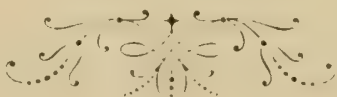




\section{1. \\ (Eil Brodkenttrülizdjen.}

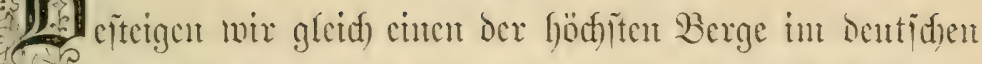
e.

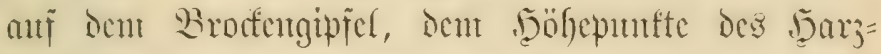
gebirges! Eci ntes gegritjt von fier oben, du fifjöne Waelt

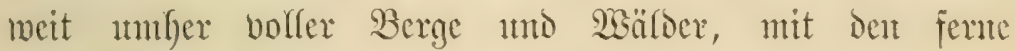

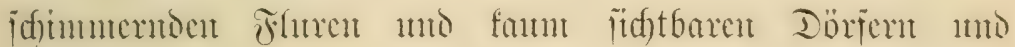

\section{Stäbten!}

Der crbabent Bstiaf von Der Broufentöble wäre idgon

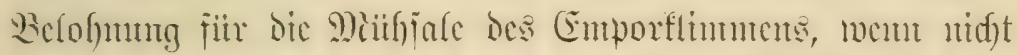

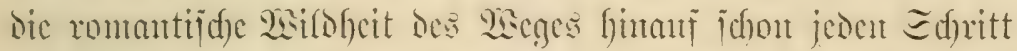

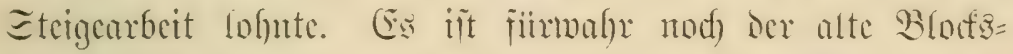

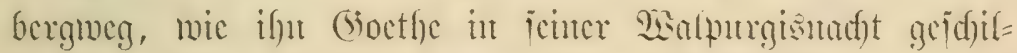

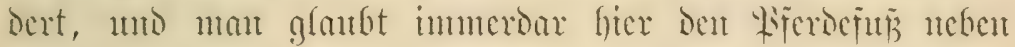

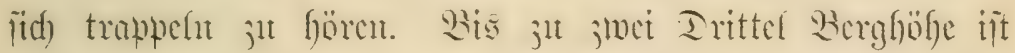

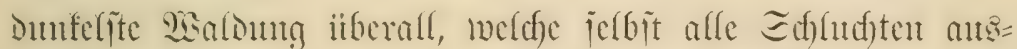

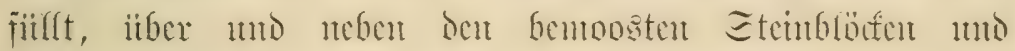

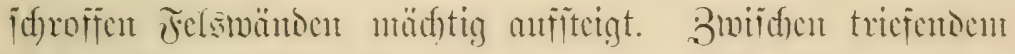

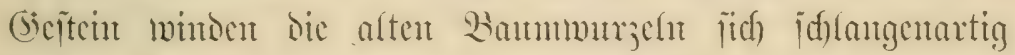
herbor, fhtorrig mo moosbetwadjen reiffen fie in bramtgrmotge 
Bäd)e fincin, weld)e unter Dent Etcingeröl heintid) Dabin= plätid)ern. Es mutumelt mo riejelt ïberall den Berg fintab;

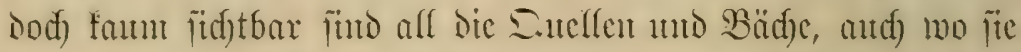

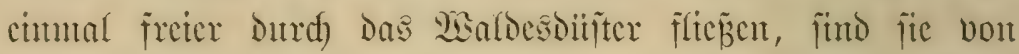
iippigen Farrentrüutern, Bärlappen nto Mionspolitern ïber=

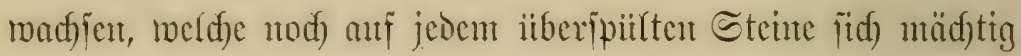

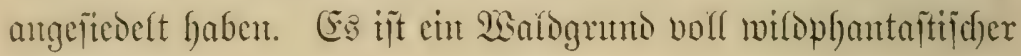
5eimlidy)feiten.

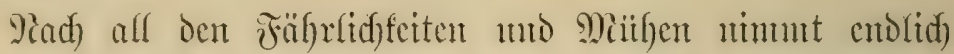

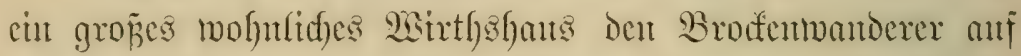

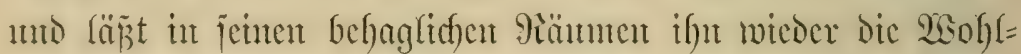
thaten des Sulturtebens curfintoen. Sa, bein eriten gicitautant

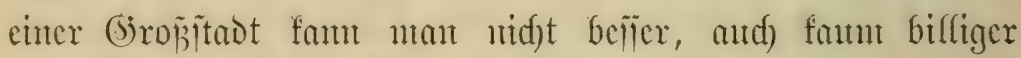
cijen und trinfen als fier Droben; Die feinen Sicijetoiletten ber bet der Table d'hôte fitbenden Damen verjebsen uns völlig in

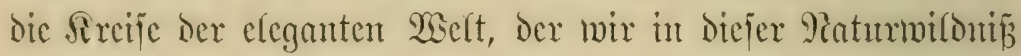

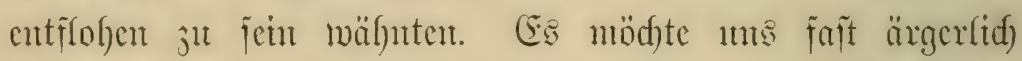

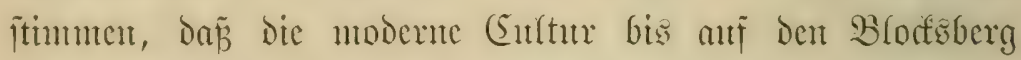
uns berfolgt, wo wir unt dic wilden Efentente der Tiatur herridfert glaubten. Qfber fie waltet blos drimen in cleganten Eaal, an bejelster Tafel. SAcun wix nten gejtürft berants= treten, (emen wix rafd) wicoer glantben an dic unbezäl)mbare ureigenc Yiatur des Broctengebinges. Es janten dic Etimute

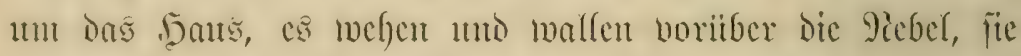
verfittlen anf fiirzere oder längere 3eit and den ganjen

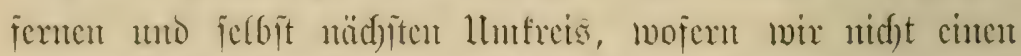
der jeltenen Iage baben, an Denen cumme die Eome flar bom blanten Dimmel Das alte Brocfenlyant bejd)cint mo alle ఇebel berid)eudft. 
Эa, will ift's anf ber wilben böbe! Darmm ift fic

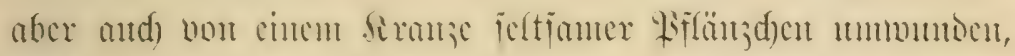

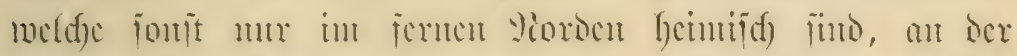

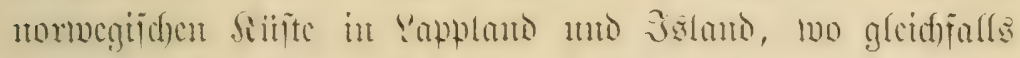

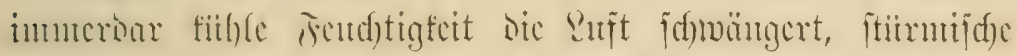

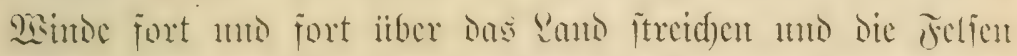

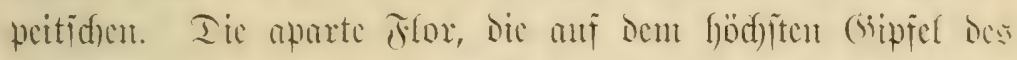
Broctems jidf) norfintoct, ijt in oce That cin (harafteriftijd

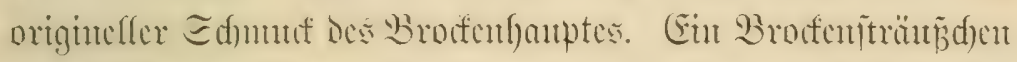

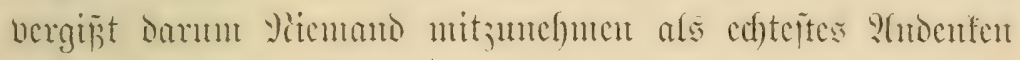

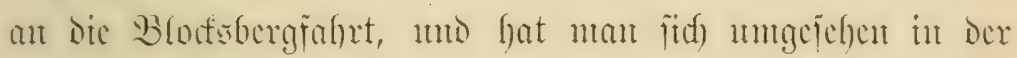

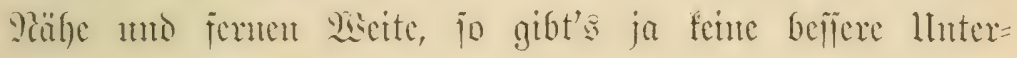

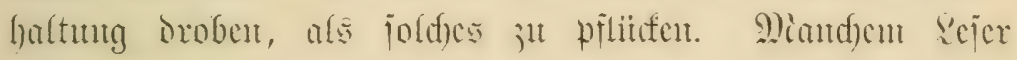
Dibrfte aber willfonmen fout, chmol ju crfab)ren, was fïr

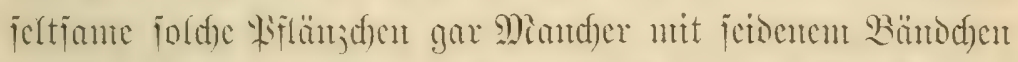

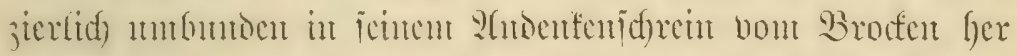

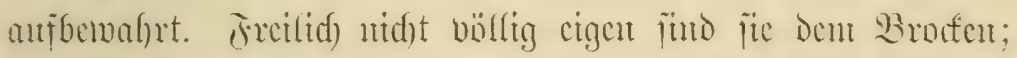
nein, alle Dentidfen (Sicbirgshlöhen, weldye in bie Viegion des

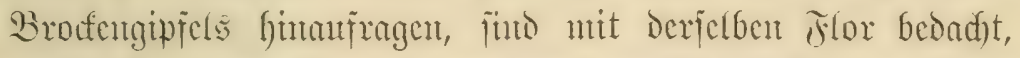

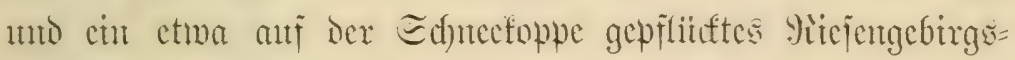

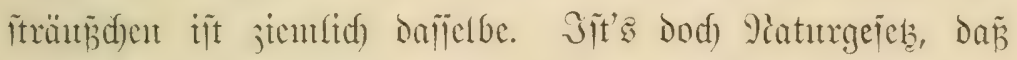

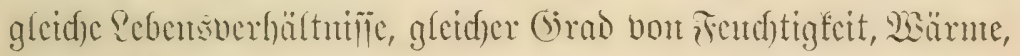

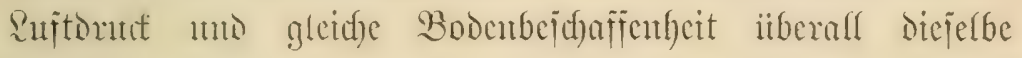
Begetation bedingen.

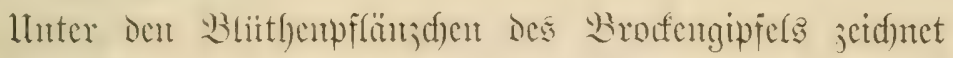

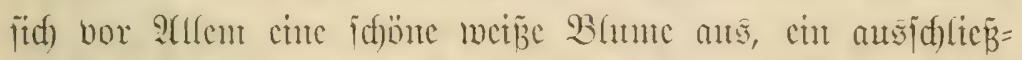

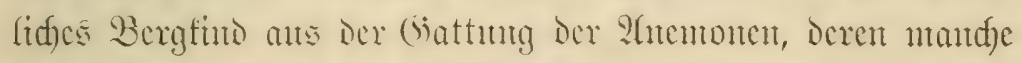

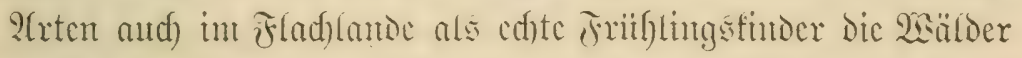

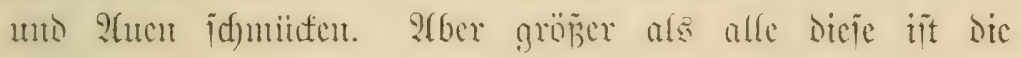




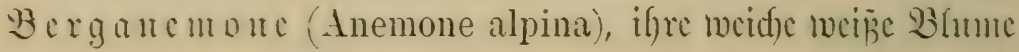

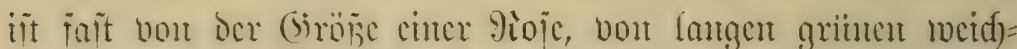

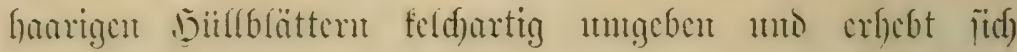

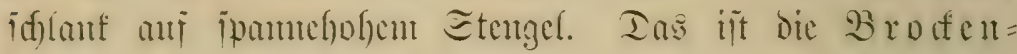

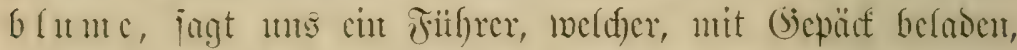

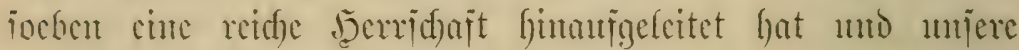

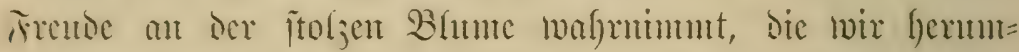

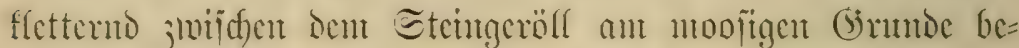

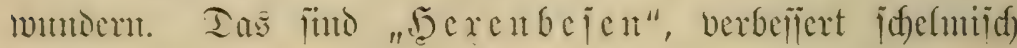

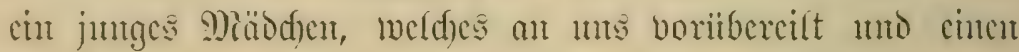

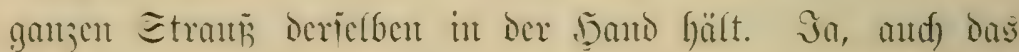

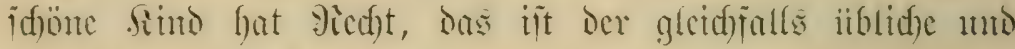
bielfeid)t ganj bejombers paffente Viane; den went die

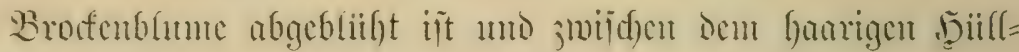

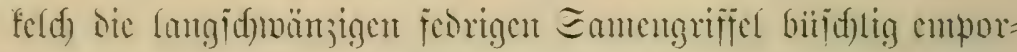
itchen, jo gelört wenig Whantajic daju, ntut an bicics mirth= id)aftlidfe 3nitmument crimert ju merosen, anj bem nad) alter

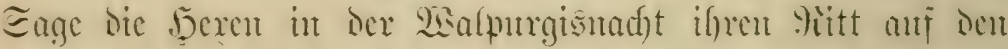

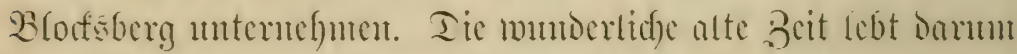

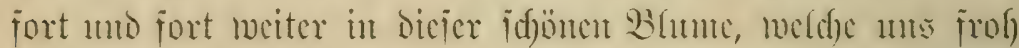

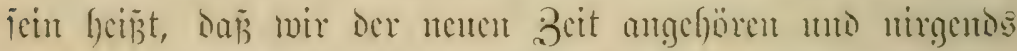

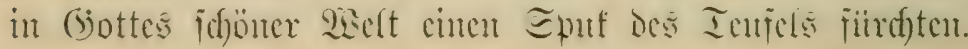

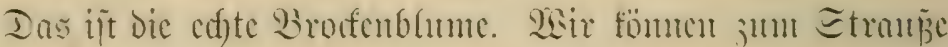

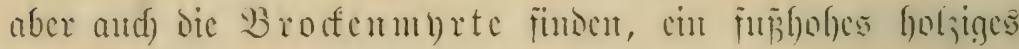

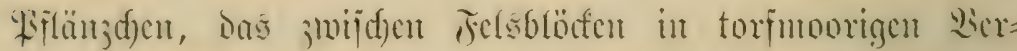

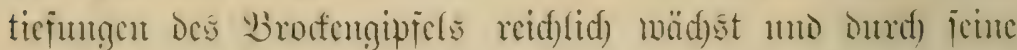

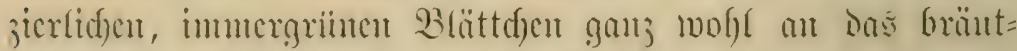

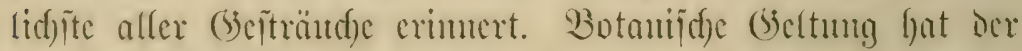
Piame Broffemmerte preifid) nidft, melder broben ani bem 


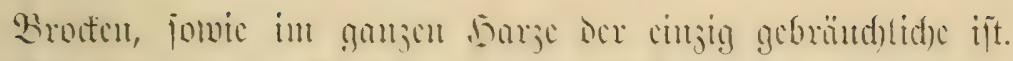

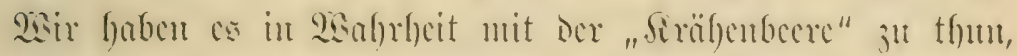

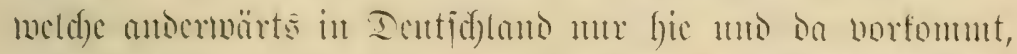

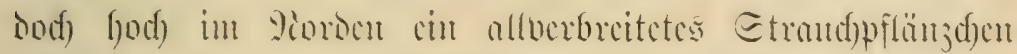

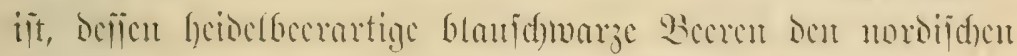

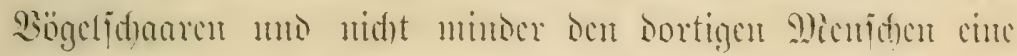
leafere Sioft ïns. Dort wo ctwa in (Siröntanto weite, öbe

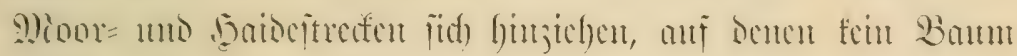

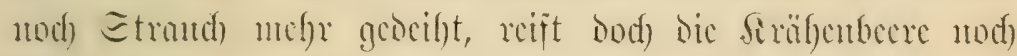

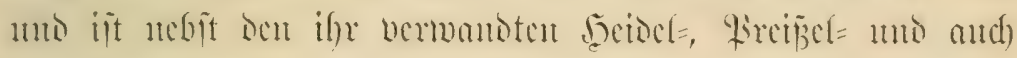
Den Bronberen, mit benen fie gern zujammen borfommt,

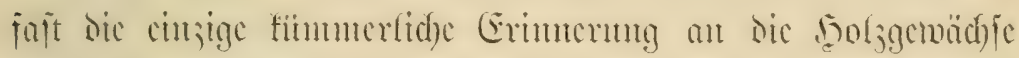

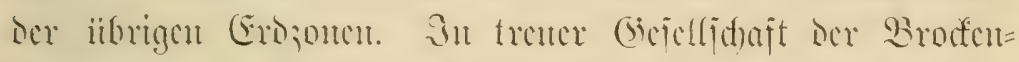

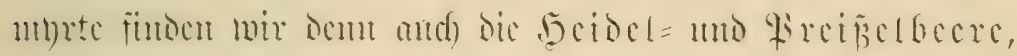

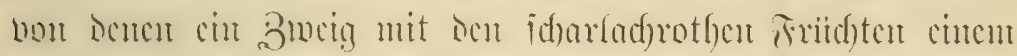

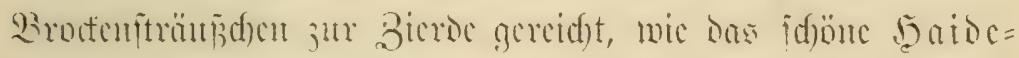

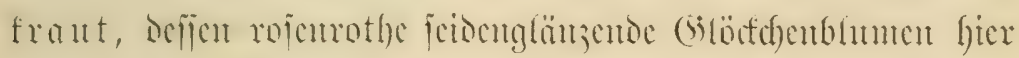

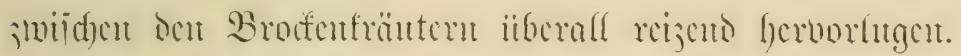

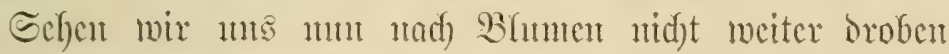

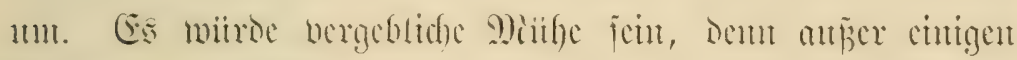

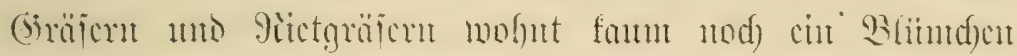

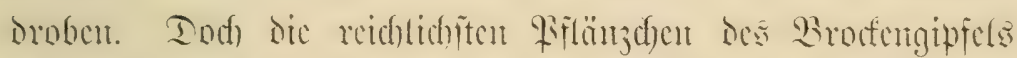

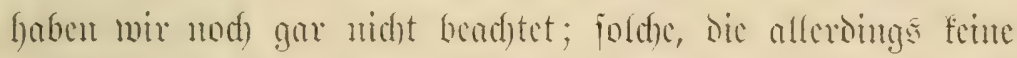

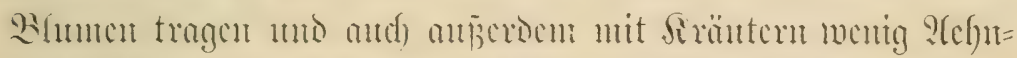

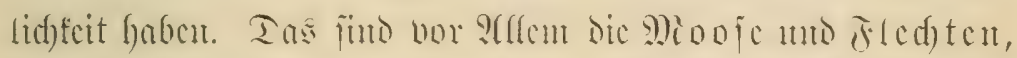
welde als granc, grime, brantuc arabestentörmige Silcutgebiloe

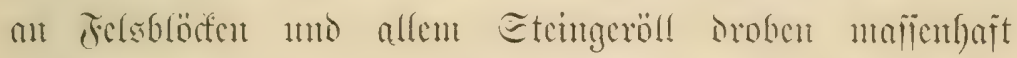

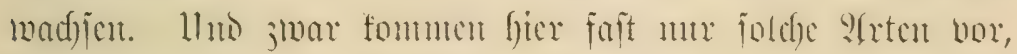

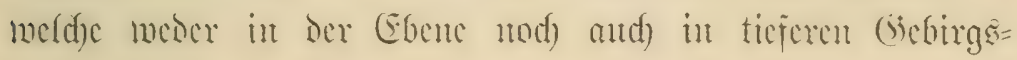


regimuen anjutreffen fints, nidet eimmal tiefer ben Brocten

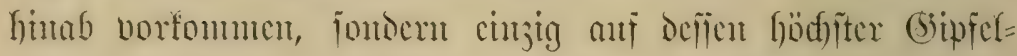

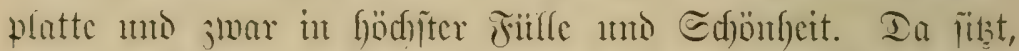

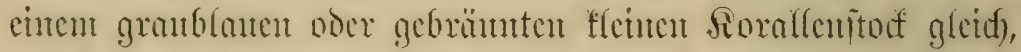

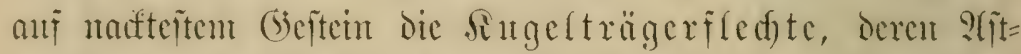

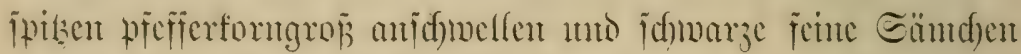
cuthalten; cs haften als granc Irancrupetten mit idswarjen Franjen miäum dic jogenamten Fefsblattfedeten, anf

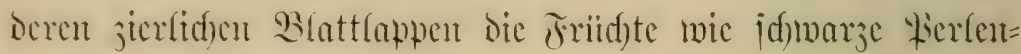

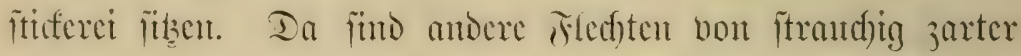

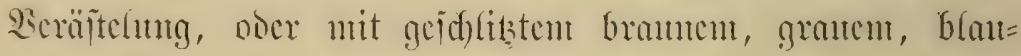

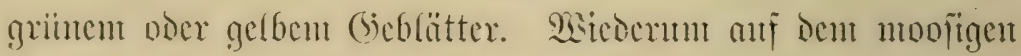

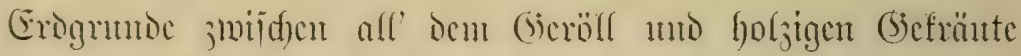

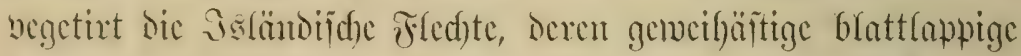

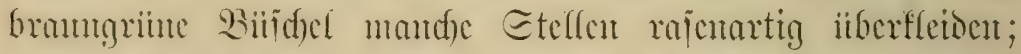

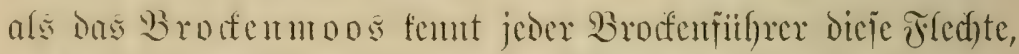

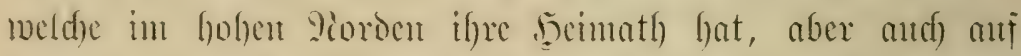

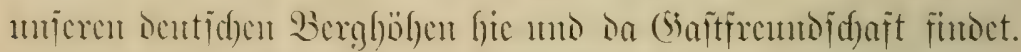

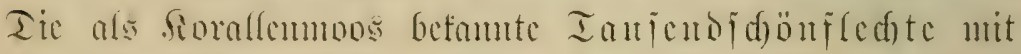

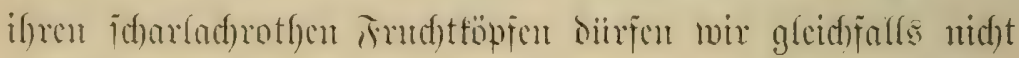
iiberiefent.

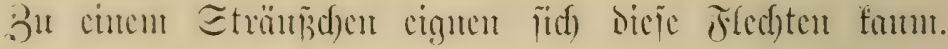

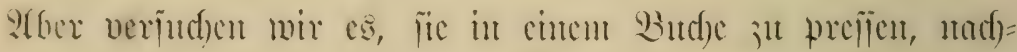

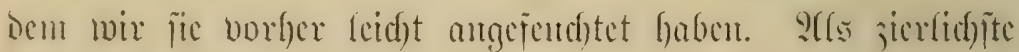

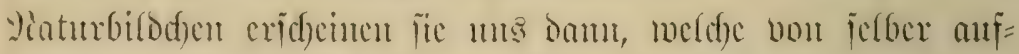

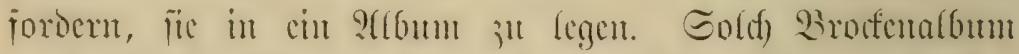

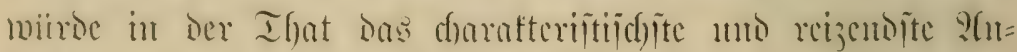

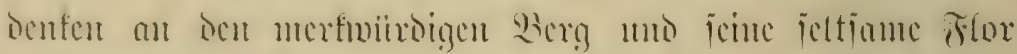

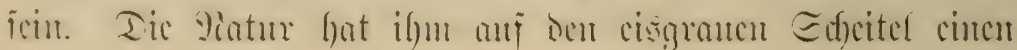




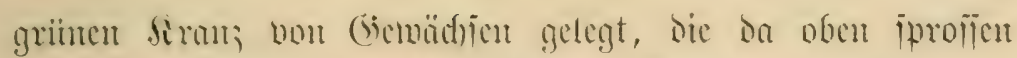

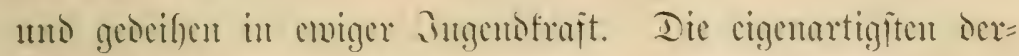
jelben bütten mix ans sicjem franje mumed) jufanmen= gedfliirft.

\section{Allerlei Gergkrüuter.}

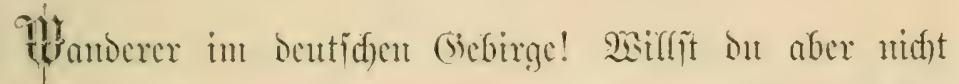

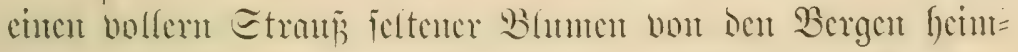

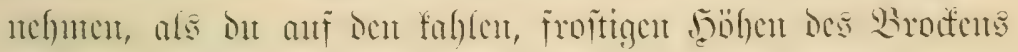

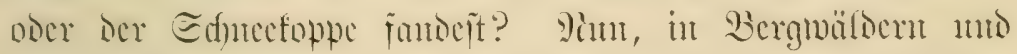

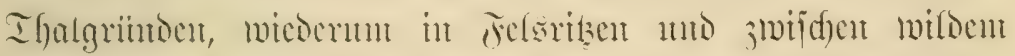

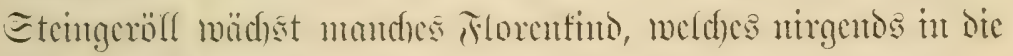

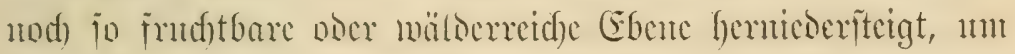

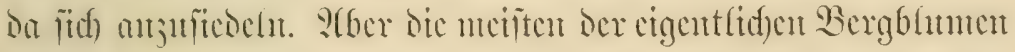

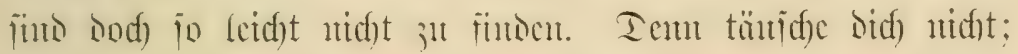

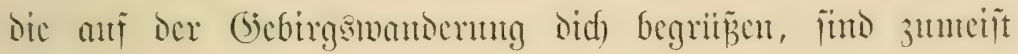

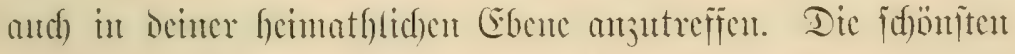

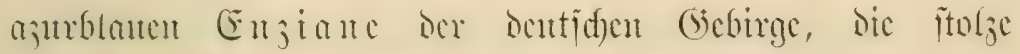
Liad) tuclfe (Dianthus superbus) Der Bergmalontugen, Dic

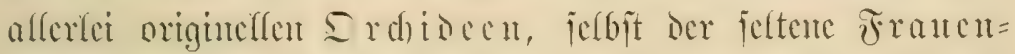
if) at h) (Cypripedium Calceolus), dicje föptlitfe Bicroc dor

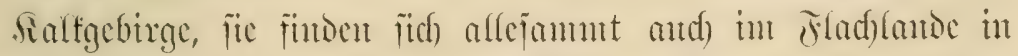

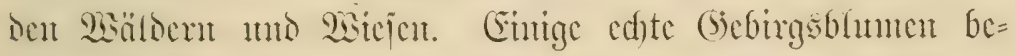

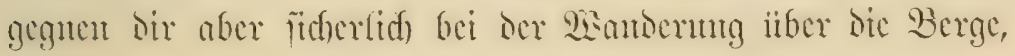


mande da jogar alferorten. Ilno das find gerade folde,

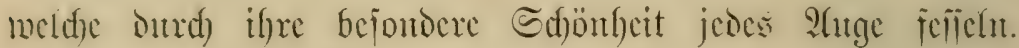

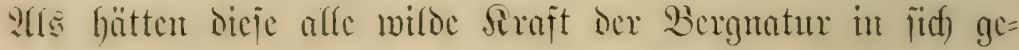
janmelt, fint fie aber zugletch ftarfe Giftblumen.

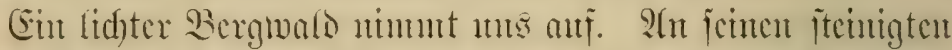

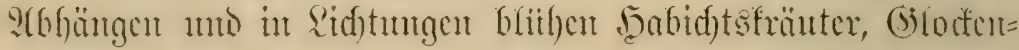

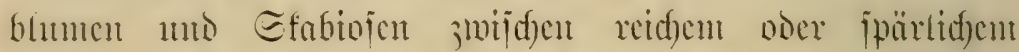

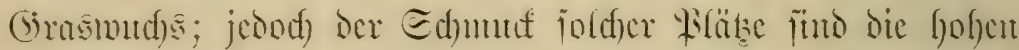
idhlauten Etengel des rothen Fingerfut (Digitalis purpurea)

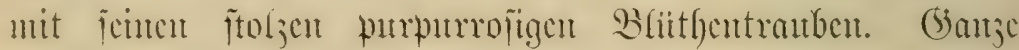

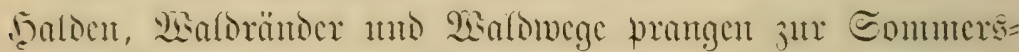

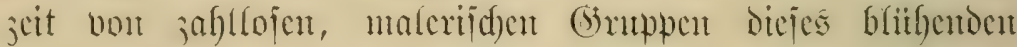

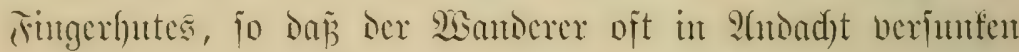
nor jolder itattlidfen rojigen Bhumentherrlidffeit ftelen bleibt.

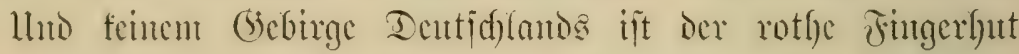

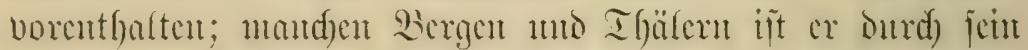
iiberrecdes Worfonmen wahrhaft dic Egarafterpflanje. 2fber

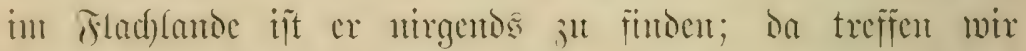
mohl in manden Sä́lom ben gefben Fingerfut (1). ambigua), melder ben (sibtrge, ats freilid) beflen

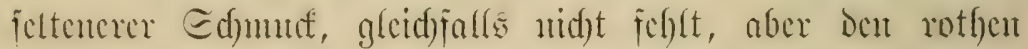

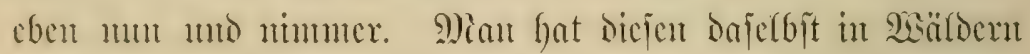

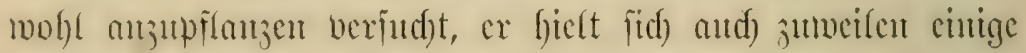

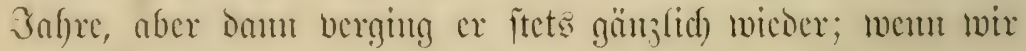

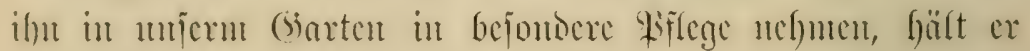
iid) alleroings, aber or nerändert fidf, jein giotf berblapt mit

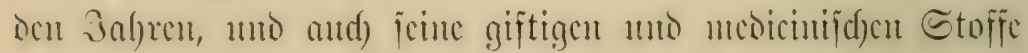

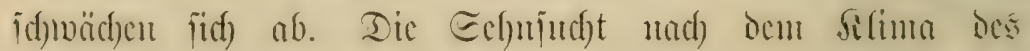

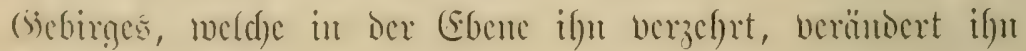




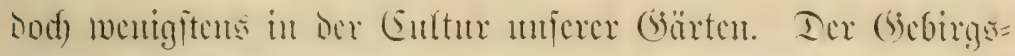

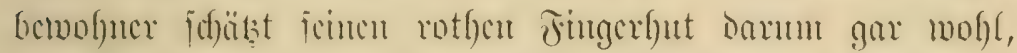
(x) jrent fid

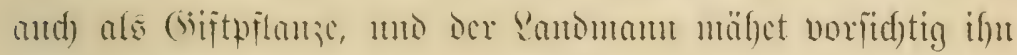

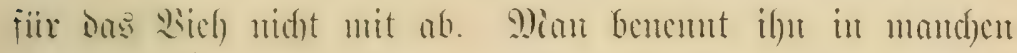

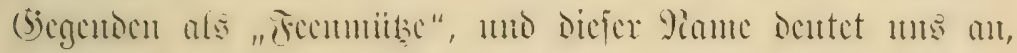

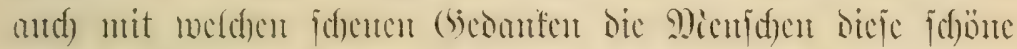
mo buch jo gijtige Whame betrad)tet haben. Die bösartigen Elfen trutgen nad) dem (blatben des vinlfers dic rothen

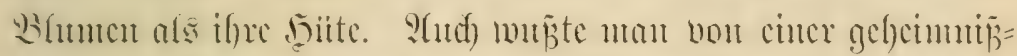

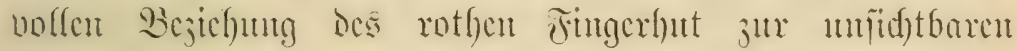

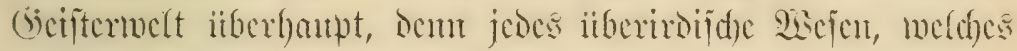
an ifgu boriibergeft, folfte er griijen, daj ban ber ganje Stengel jid) meigt.

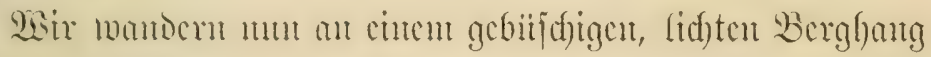

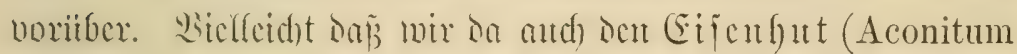
Tapellus), Dicic in Den neiften (Sicbirgen freilid) jicmlid)

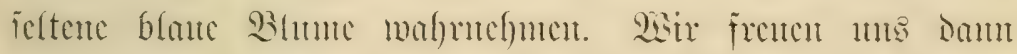

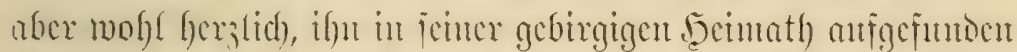
it haben, Den wix ans unferen Gärten bou Simbleit anf

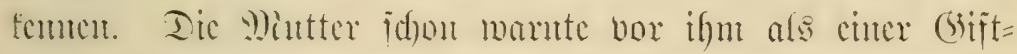

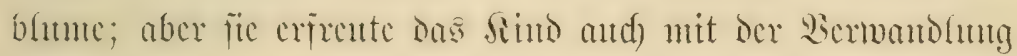

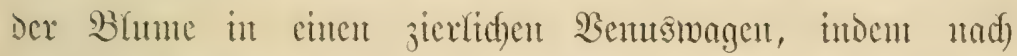

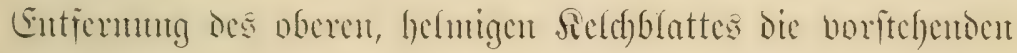

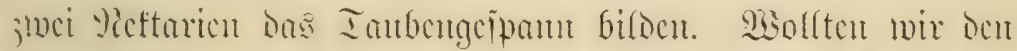

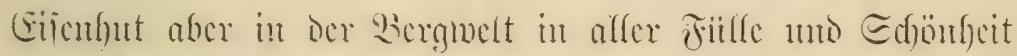

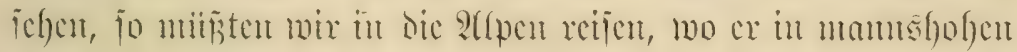
Biijden berrlid joljut, bejomocrs an dic Eembitten der

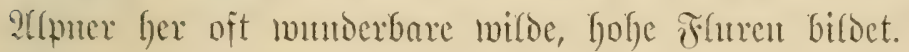




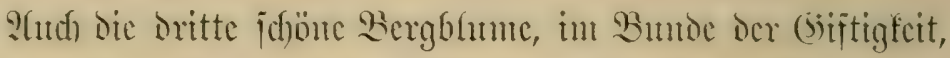

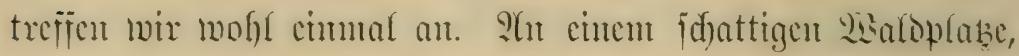

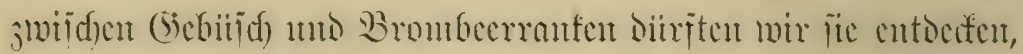

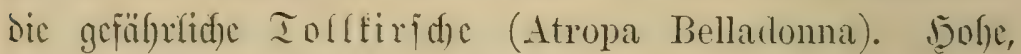
frantige Etengel ieben wir da in iipptig grinten B̉itidsen bei=

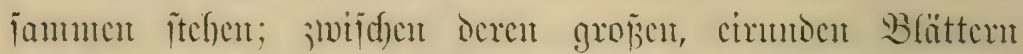

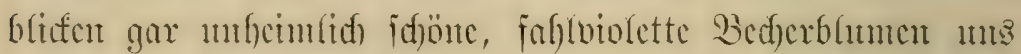

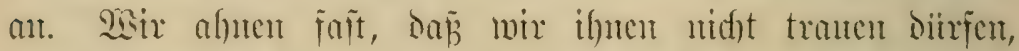

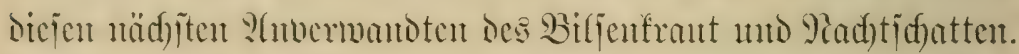

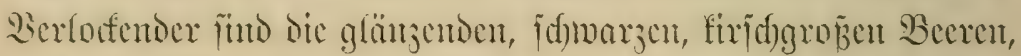
melde int Exätiommer reifen mo sonen dieje giftigite

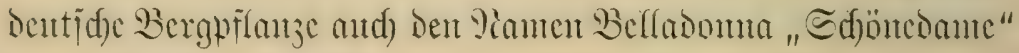

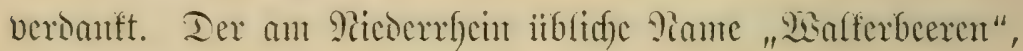

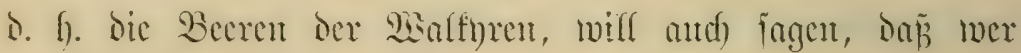

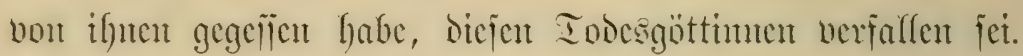

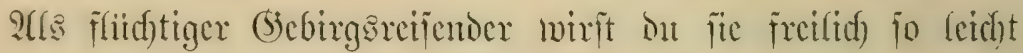
nidft finden, Denn fie wächst jelten an den Touriftenmegen,

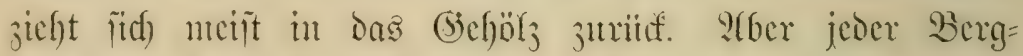
bemofnex fent ite mo fömte didf ju ifyen Etmoürtem

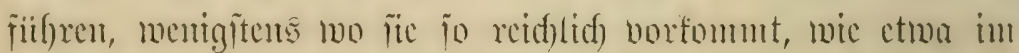

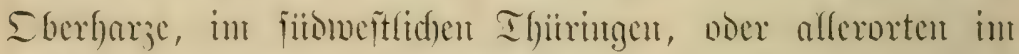

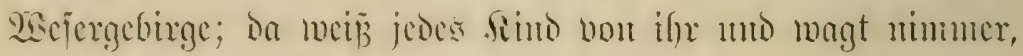
die verfilfurerifden Beeren mu anzurilgren.

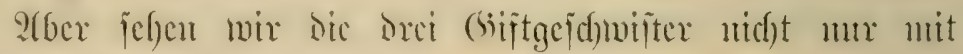

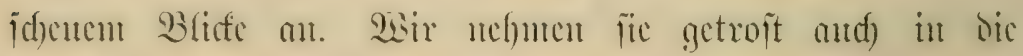

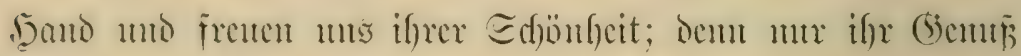

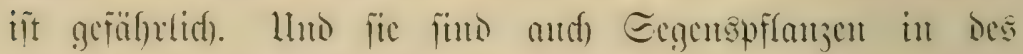

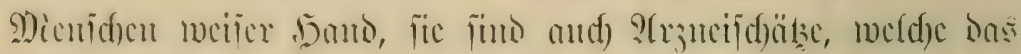

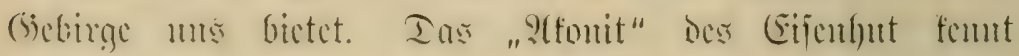


Jocrman ans Dex homöopathijd)en ?(pothefe, Das "Iigitalin"

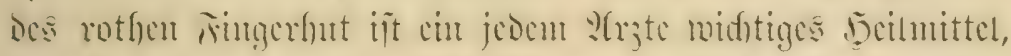

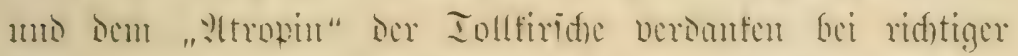

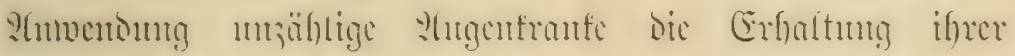
Eebfraft.

Sicber Pcier, millit sut mu mit mix mod) meitex mandern?

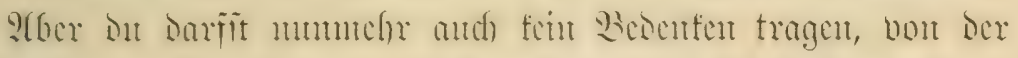

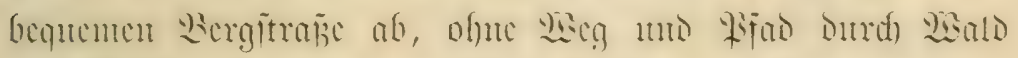

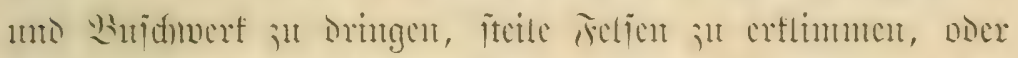

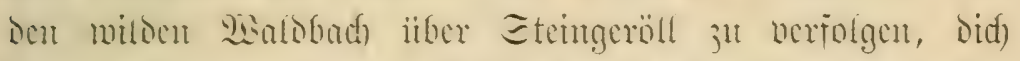

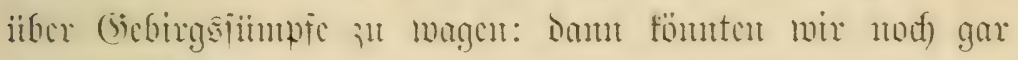

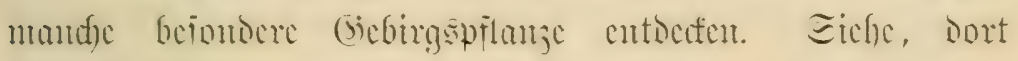

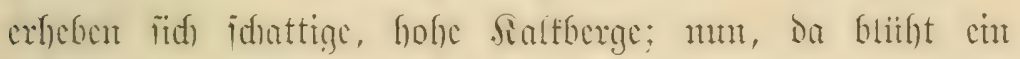

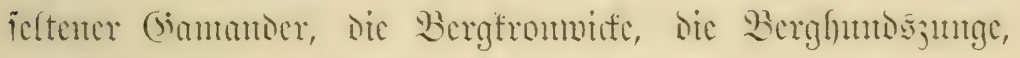

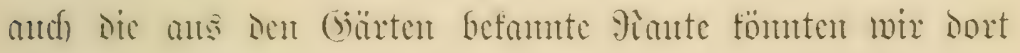

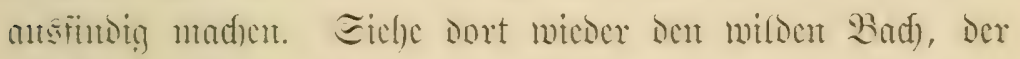

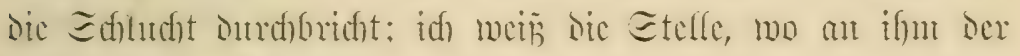

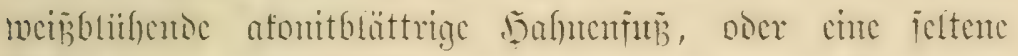

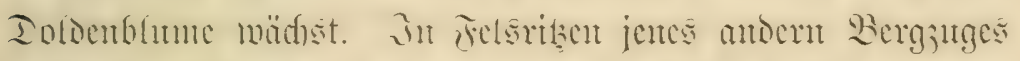

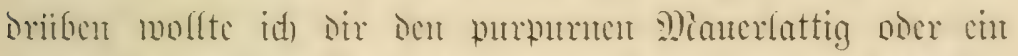

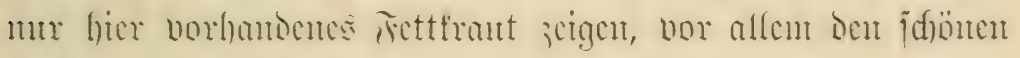

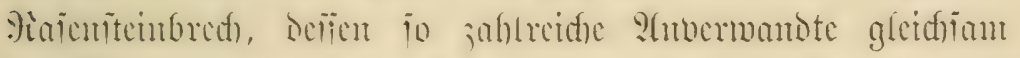

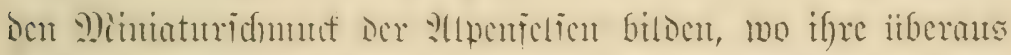

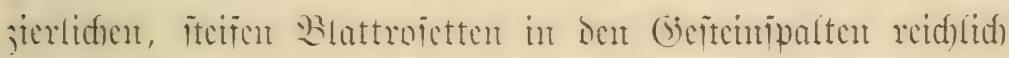

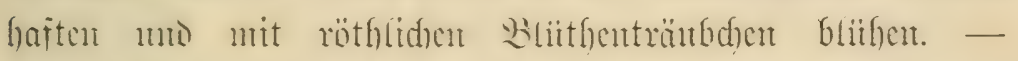

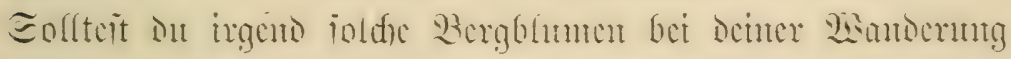
iclber cimmal fimben, io hele ite itbrigens forgiant ans, be=

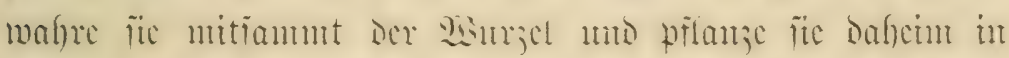
Demen (siarten, wo du bon Etcinen fimitlid) cinc fleme 
Jelspartic anfgebant haft. Eic werocn ba bantfar meiter

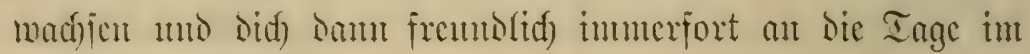
Gibirge crimern.

\section{3. \\ A $\mathfrak{x} \| \mathfrak{i} \mathfrak{n}$.}

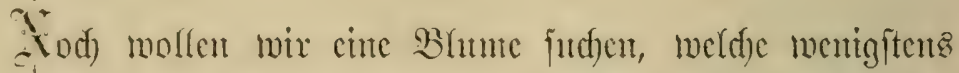

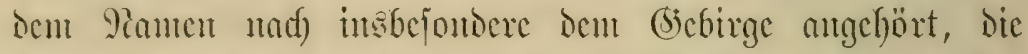
STruifa odcr Bergarnifa (Arnica montana), dics popurär bc=

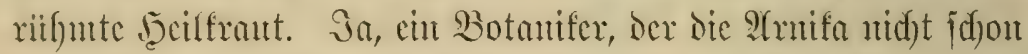

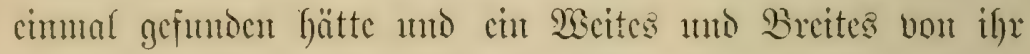

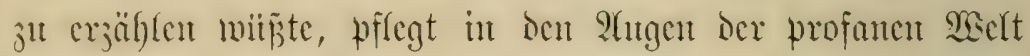

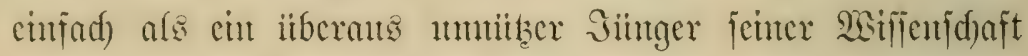

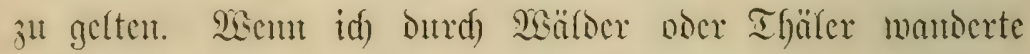

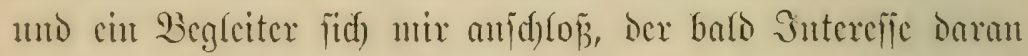

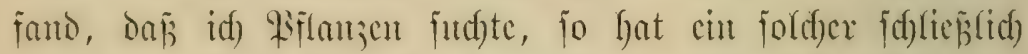

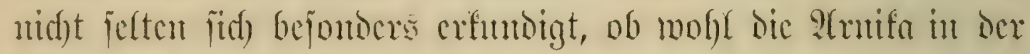

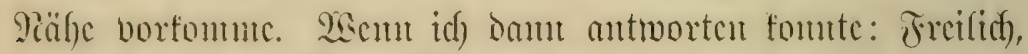
uncur sicber, da nocr bort wäd) st fie menigitens bercujelt, jo befan folder Begleiter gleid) cinen nenten Gicjpect bor feinem

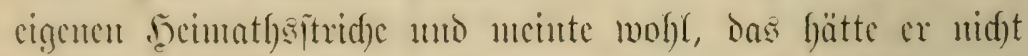

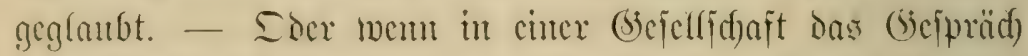

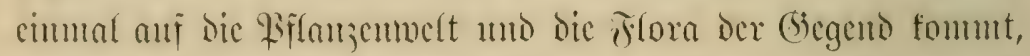
fo bin id) glcidffalls immer fidfer, bie Frage ju bören, ob

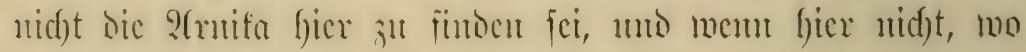
fie benn iiberlfanpt cigentlid) wadje. 
Da mandfer Sejer twohl aud) gern cimutal fören möd)te,

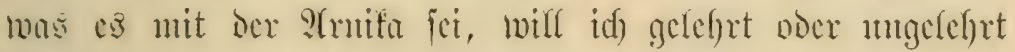
fier von derjelben einiges 2 seitere erzählen.

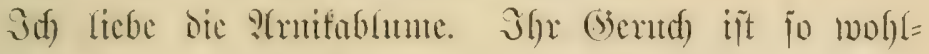
thueno ftart aromatij(s); ifre dunfergelben, meift itber thaler=

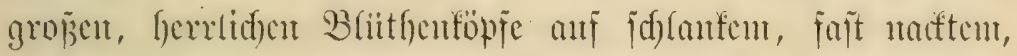

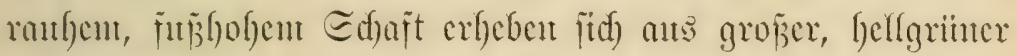

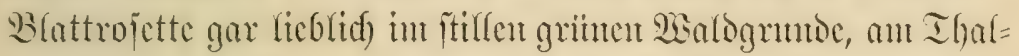
hauge ober anf lidfter Bergesharbe. Sie ijt aber cin inmerfin

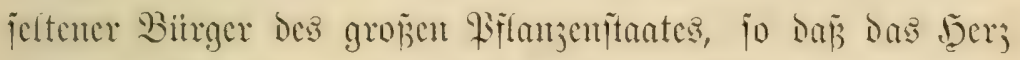

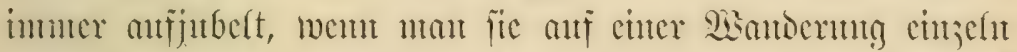

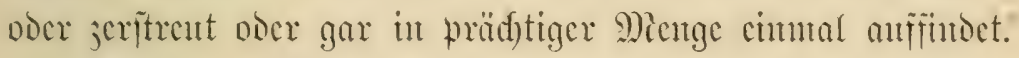

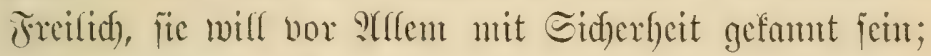

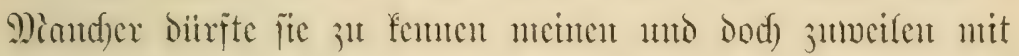

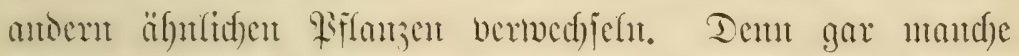

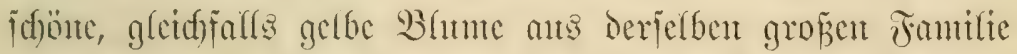

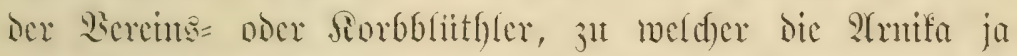

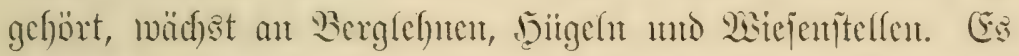

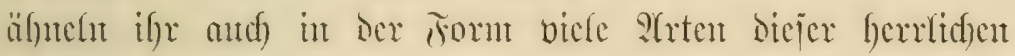

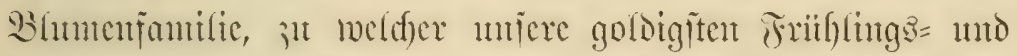

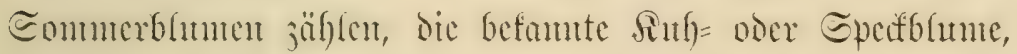

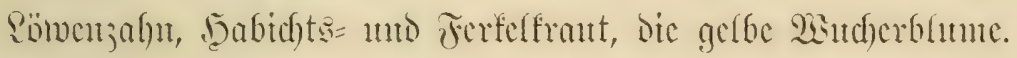

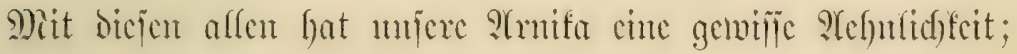

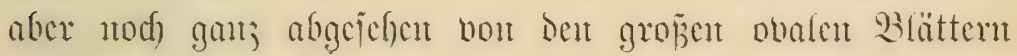

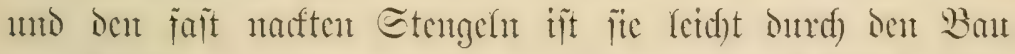

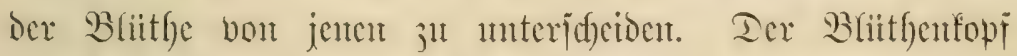

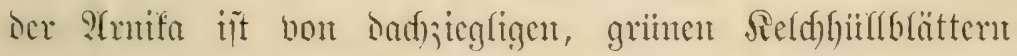

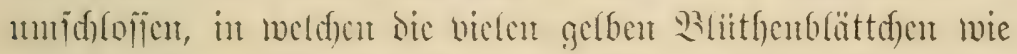

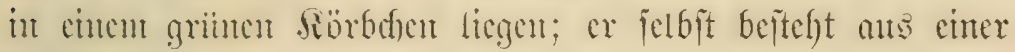




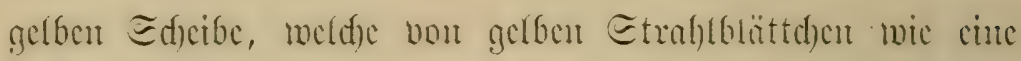

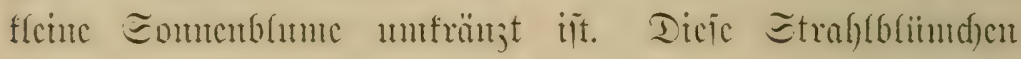

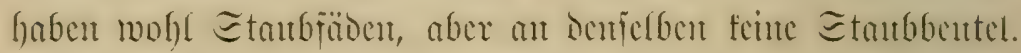

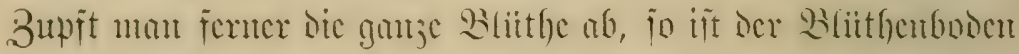

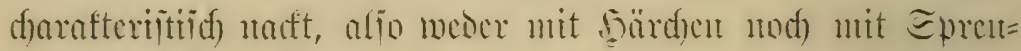

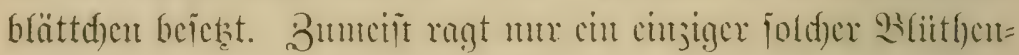

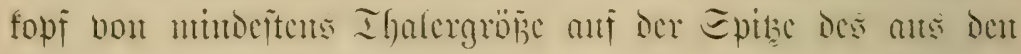

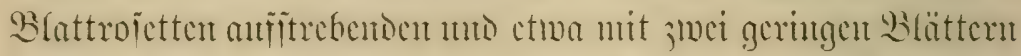

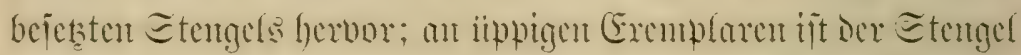
aber gablig getheilt, mo dicic licbenjtengel f̈nt ban mit

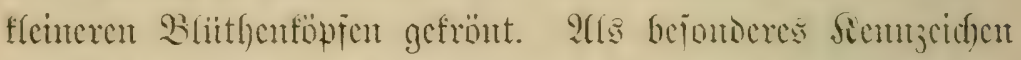

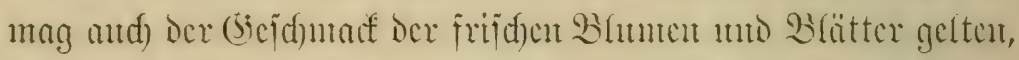

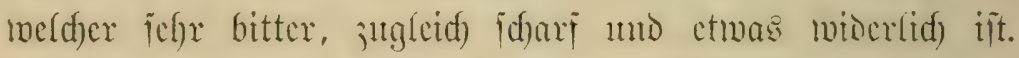

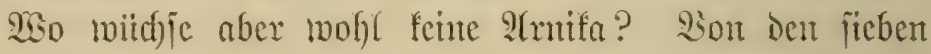

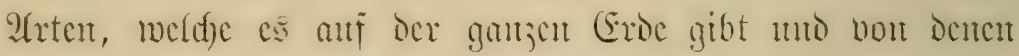

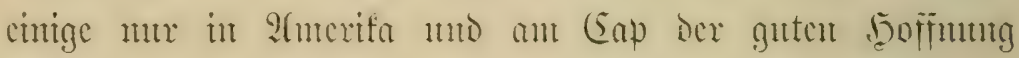
vorfommen, ijt dic Bergarutifa (A. montana) als dic einjige

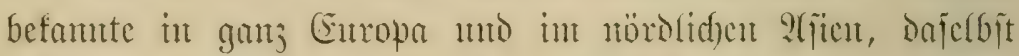

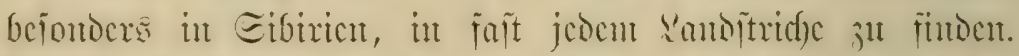

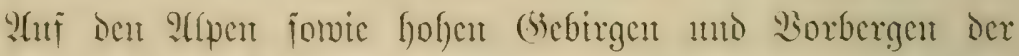

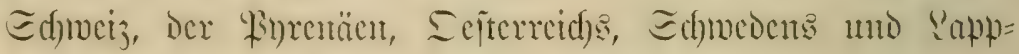

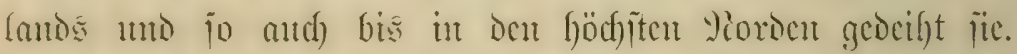

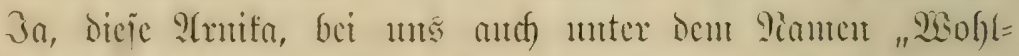

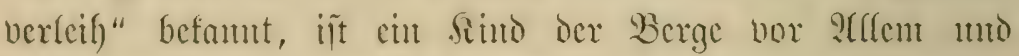

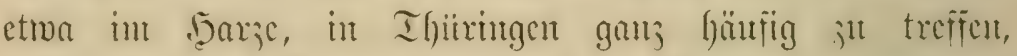

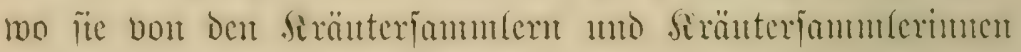

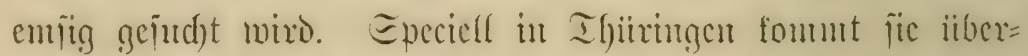

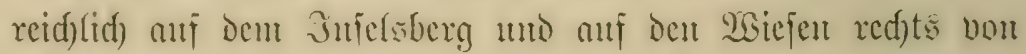
Brotterode vor. Eic mird Dajelbit bic Solgantisblume bentumt 


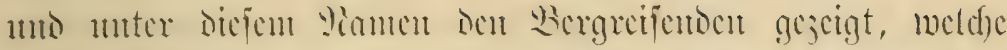

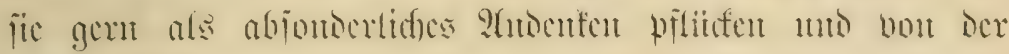

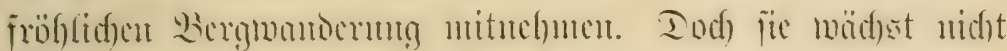

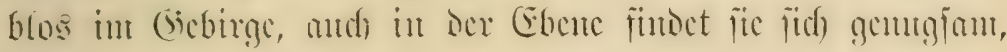

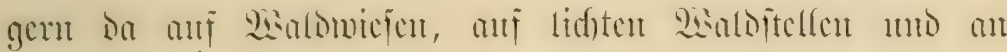

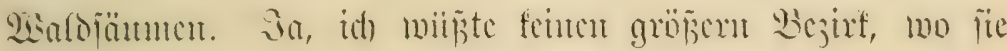
do nidgt menigitens vereingelt jut treffen wäre.

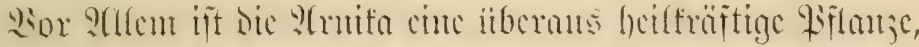
nad) ber mant, wie Das Solf bou jolden firäntern meint,

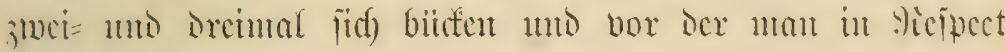

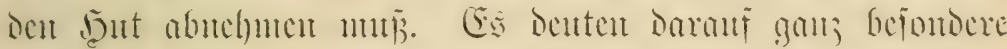

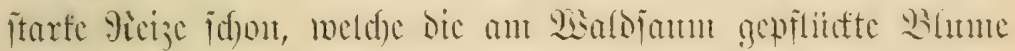

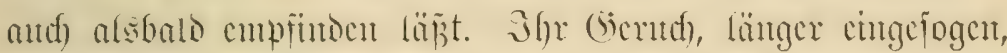
bervirft Ë̈ngenonmentheit des Sopfes und reijt jogar jutm

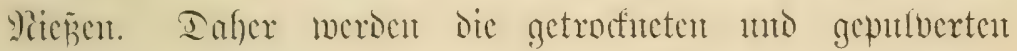

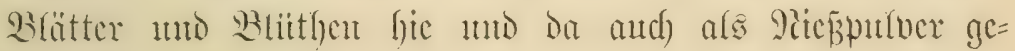

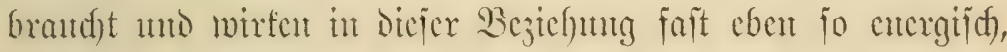

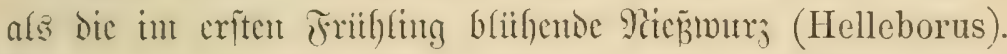

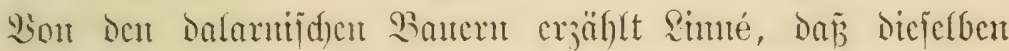

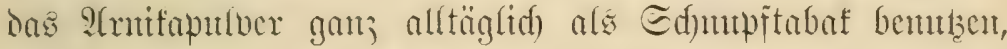

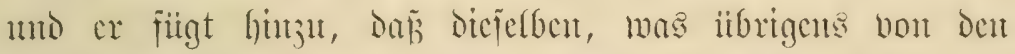

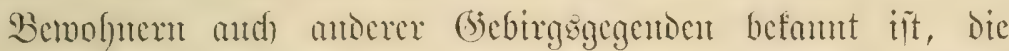
B̧ätter uno Bliitben gen troctucu und als ftarfen Inbaf

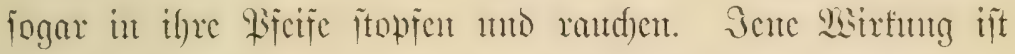
mun freilidy nidft immer gleid); cs fonment ant bic (sicgento ant,

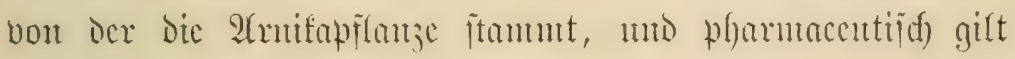
Diejentige fïr die wirfiamite, weld)e in Eadjfen in Dex (jiegeno

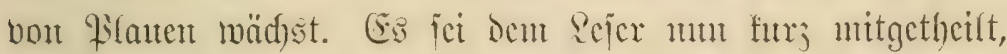
melde Ingenden fie befizen foll. Da mipere Weisheit iiber 


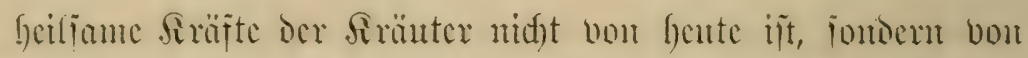

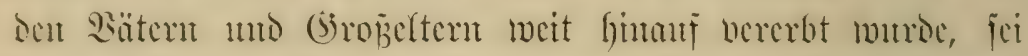

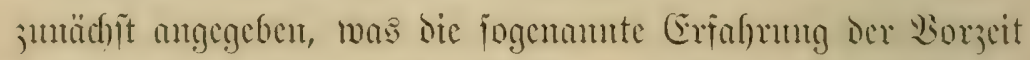

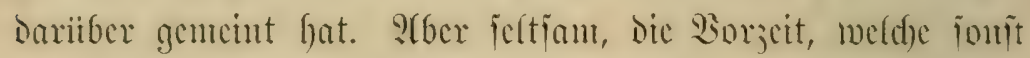

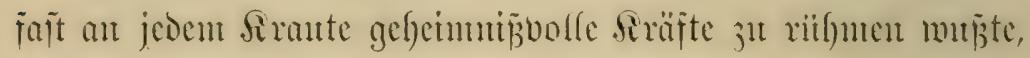

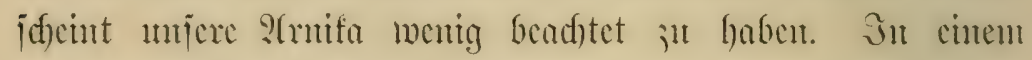

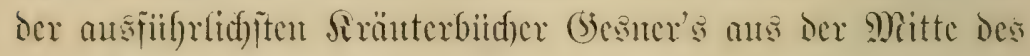

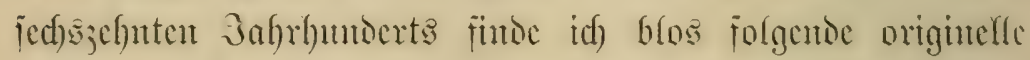

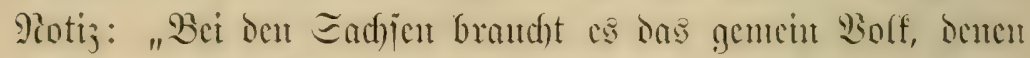
jo fimunter gefallen, ober fo fith etman mit ?frbeit werlest

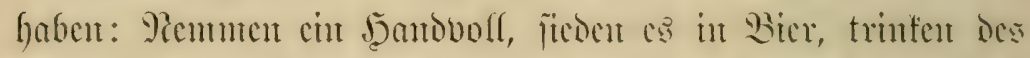

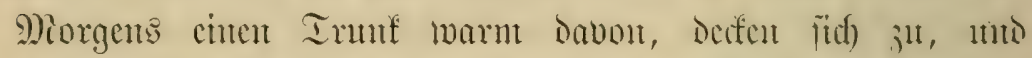

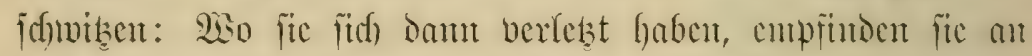

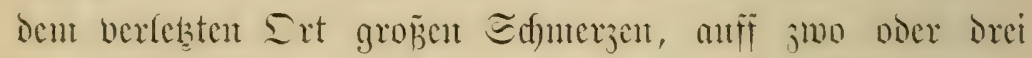

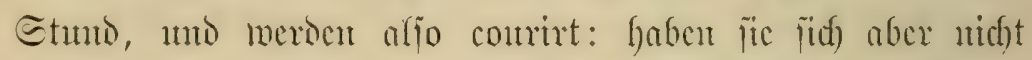

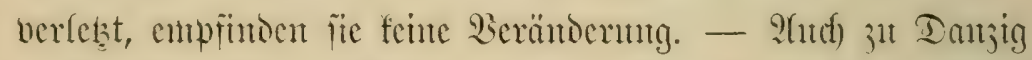

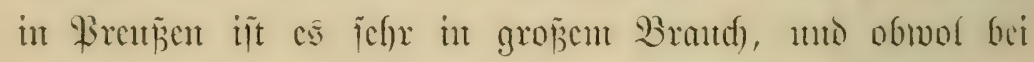

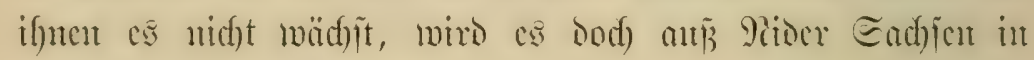
Fäfïern Dabin gebradht."

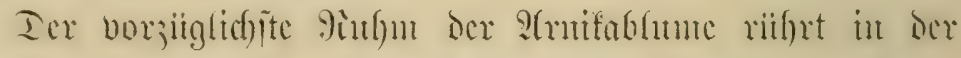

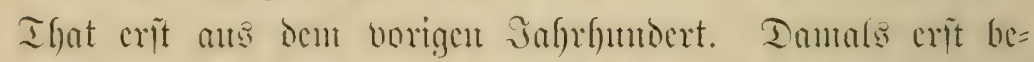

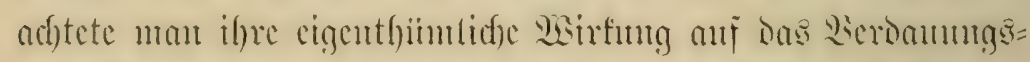

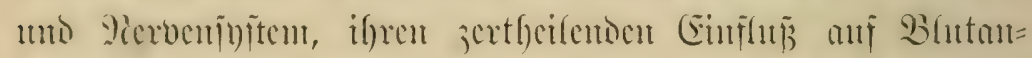

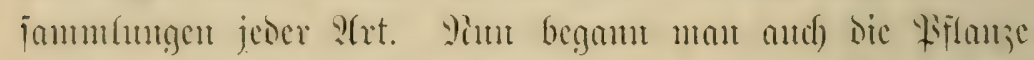

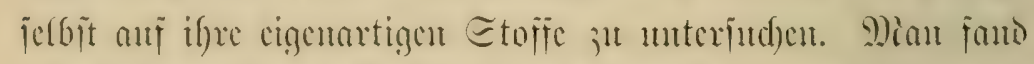

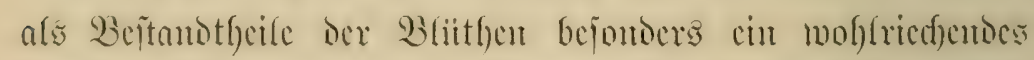

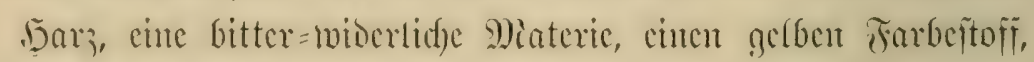

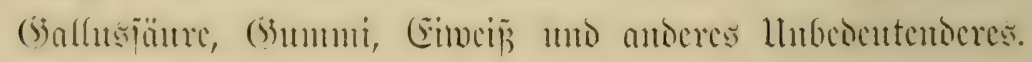

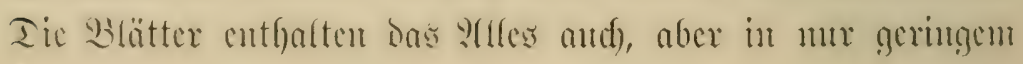




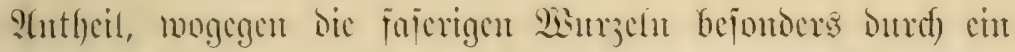

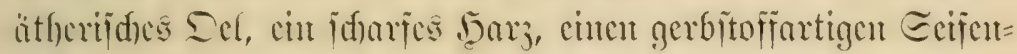

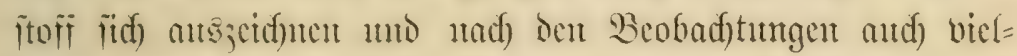

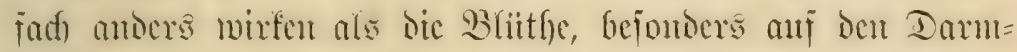

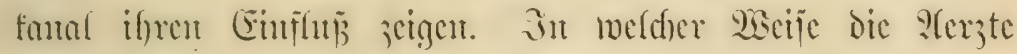

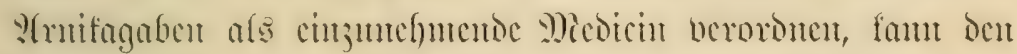

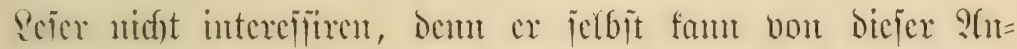
monoung feinen populären (5ibranth) mad)en, meil bicjelbe mu mit än

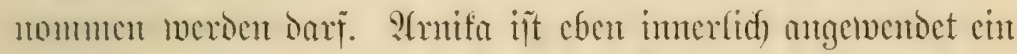

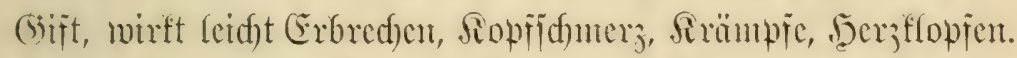

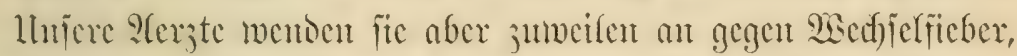

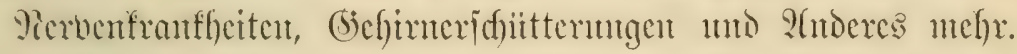

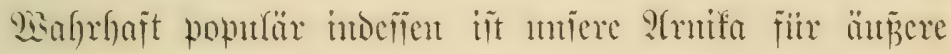
Edräben.

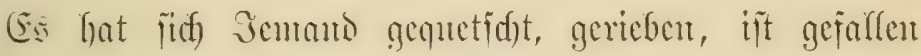

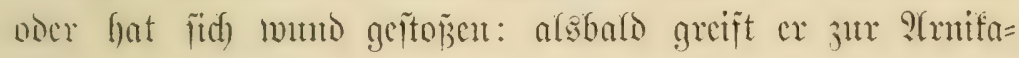

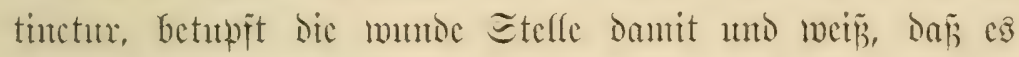

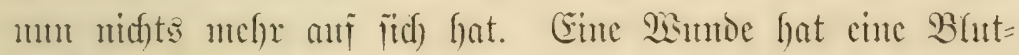
mfanm

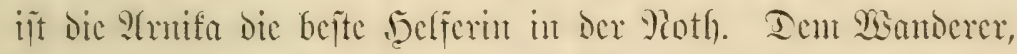

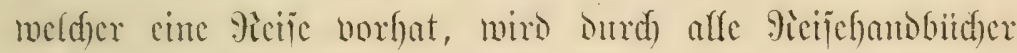

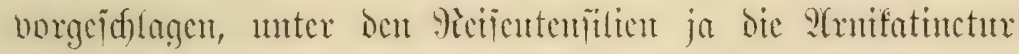

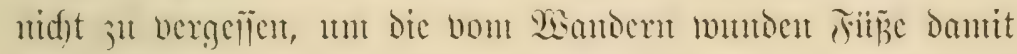

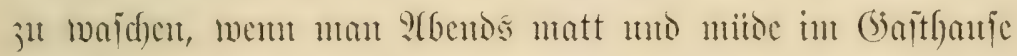

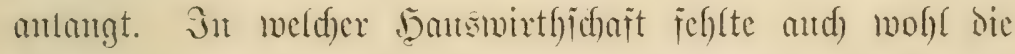

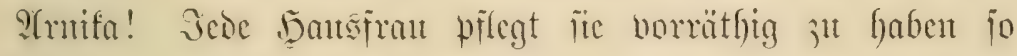

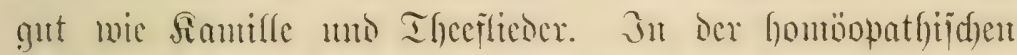

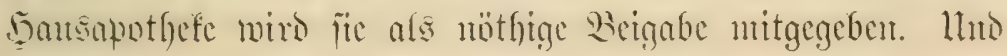




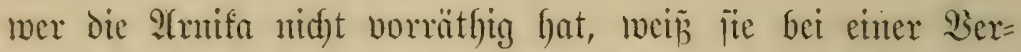

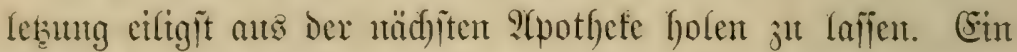

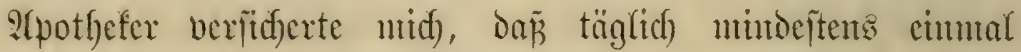
?Inifatiuctur bei ifm bou Seuten gefordert werde.

Uber uver jeinen guten (Stauben an die $\mathfrak{U}$ mifía bat, thut Dod) wohl, fic fitd) felbit ju bereiten, dent es werden wentige fräuter von den Eanmmlern fo vielfad) gefälfodt. Daritber flagte jofon Rimmé. Dex Ilpothefer, meld)er gejammelte und

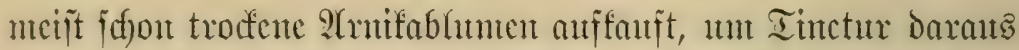

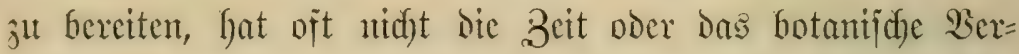

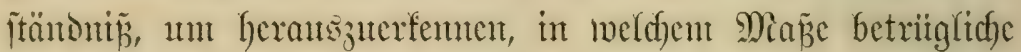

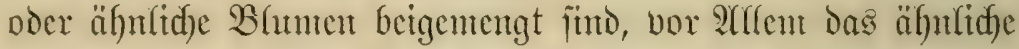

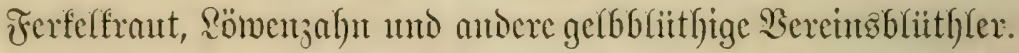

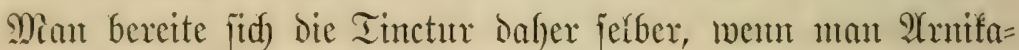

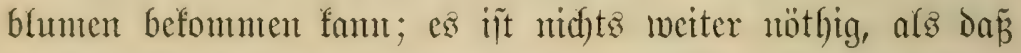
ntan diejelben in Secut oder in Seingeift legt und auşzichen

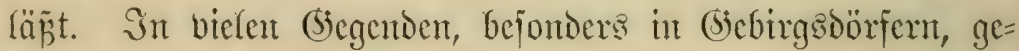

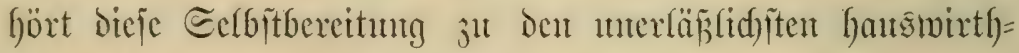
id)aftlid)en (sicid)äften, weld)e in Sult ooer altguft vorgentonmen

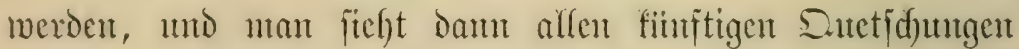

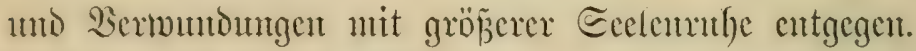

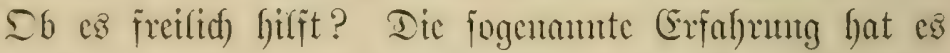

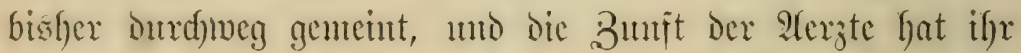

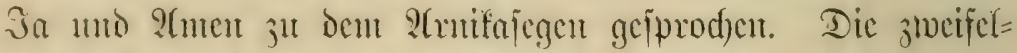
jiidstige (sicgentwart bat freifid) ifre Bedenten erfobent, uns

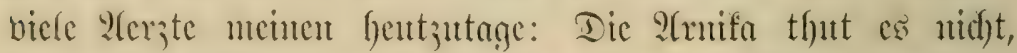

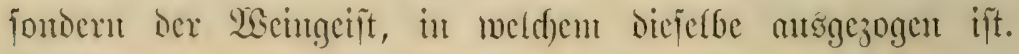

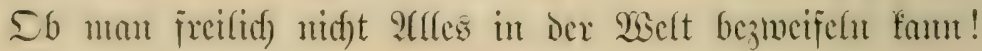

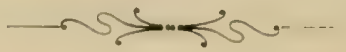




\section{V.

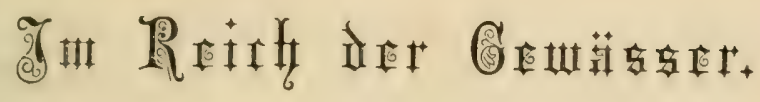

Wic mandeIt' idf fricolid oft als Kind

sängs ocm leife mumtinden Bah, Wo $\mathrm{cr}$, das ftille Wiesthal wäfferno,

Durch Sinfen und Dergiß̨meinniđt quillt.

๒઼rok, palntblätter. 
(1) Wne waffer fein Eeben! Filt das von jegliḑem organifinen Bebilde,

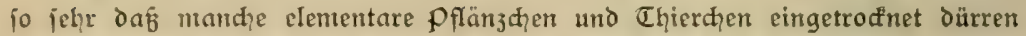
Mumien gleị̦ völlig leblos find, aber bei 2lnfeuchtung fich alsbald wicder regen

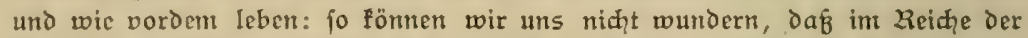
Gerouffer eine fflanzenfülle und Ueppigfeit des Seben= waltet, vout der befonders unjere ruhig flief̧enden Bräben und Bädhe, fowic di. ftagnirenden Teiḑe über:

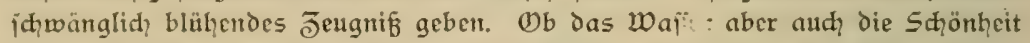
bedingt? Die zum gröften Theile allerhäblidfen đhicre oer Bewäffer möđten das Begentheil beweifen; aber in der pflanjenwelt ebendajelbit tritt wiederum eine farbenpraht oer 3lumen, eine Mannidfaltigfeit der formen und ein Blüthen= reiđhthum ohne Eleiḑen auf. Der Jauber des Wafferipiegels und das Behein:

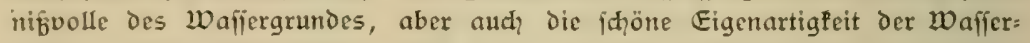
blunsen felbit verleiht diefent zudent eiren כ̄ug finmiger Melantholie, un oeffent= willen Sage und Diđtung ganz bejonocrs gern bei ihnen verweilen.

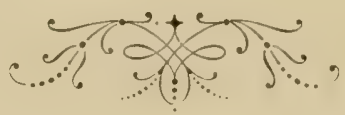




\section{1. \\ (9tulutenkrelle.}

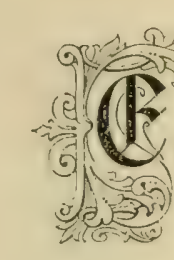

B wandert und prätidjert der Bad), von Eije frei, wieder duref jente llfer Dabut. Dit junge Eomme blibt uno glibert in Den ïber Siejelgrund fid

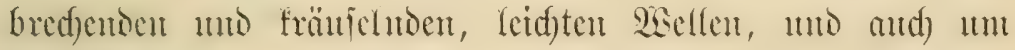

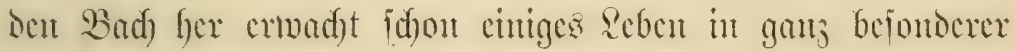

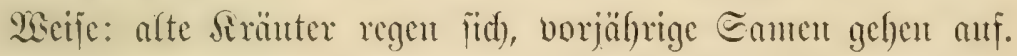
Eingelne Fliegen fomten fidh do anf hervorragenton Etcinen oder fitsen anf bitrem llfergeftritpl, und ither bem Spiegel

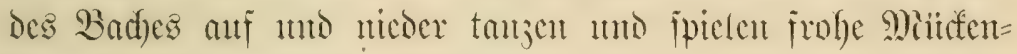
arten zu Sdjaaren verjanmelt.

Währent das Wiflanzenteben an lljex exjt ganj allutälig fid) cimfindet, - anf ben Badjesgrunde max in bürtefter Wintersat das Yeben nic ganj vergangen. Edjon ment bic Badfwellen nod) mit Dem flöp̈entoen oder bom llfer ftarrenden

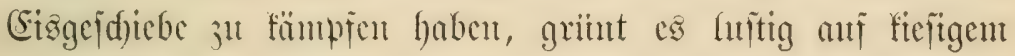

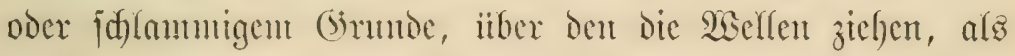
wäre da gax fen wisuter getweicu. Mafïenfafte Eonferven

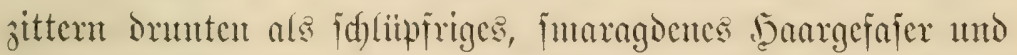

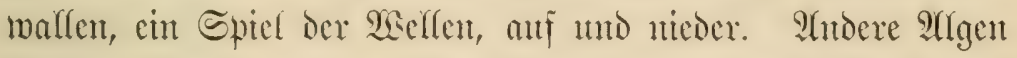

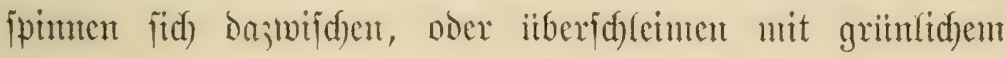




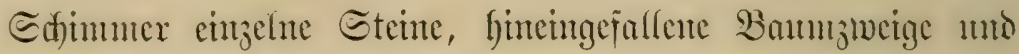

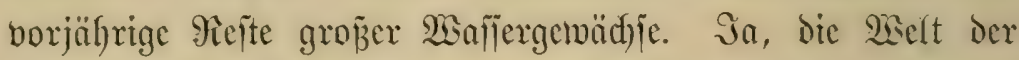

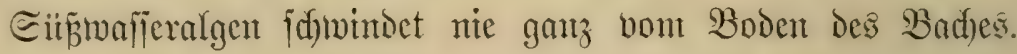

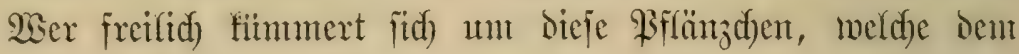

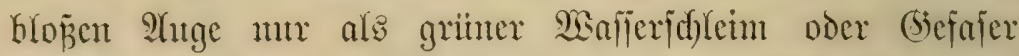
erfdeinen, wäl)rento fie alferoings unter Dem Mifrojfope cinc WGunderwelt fonder (5)eidfen offenbaren.

Mideft mintoer frithe, jefbit mitten in Wintertagen, ift

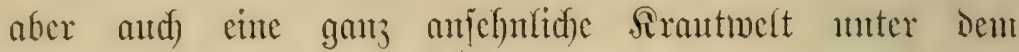

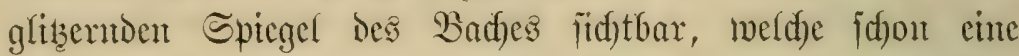
bejontere Begetationstraft jeigt. Wabarbaftige Rrüuter fino Das, mit diffen jaftigen Etengeln uno gefiederten Blätten,

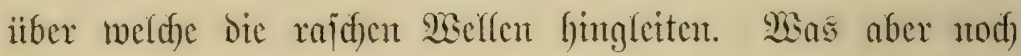

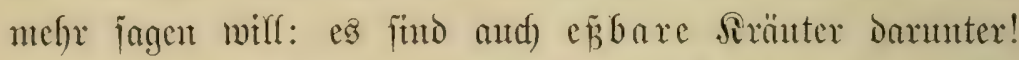

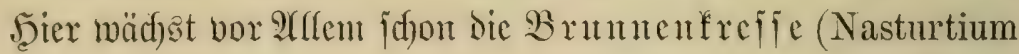
officinale), weldye gerade int eriten Frithfing, etwo bout

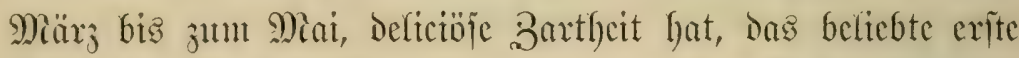

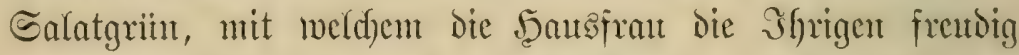
iiberrajdyt. San, ehe nod) Dex (Särtner die 9iapintzdent bon

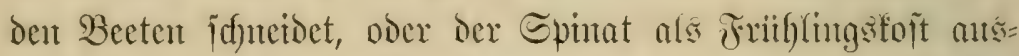
geboten wiro, hat Der Bad) feine Brumenfrefic fertig. A(ts iibcrall willfonmente (Sabe des Sabres mird fic mun zum

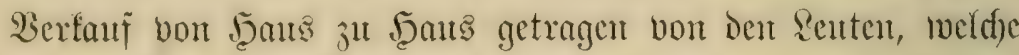

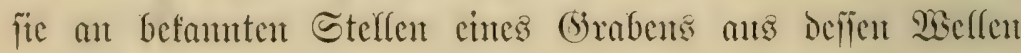

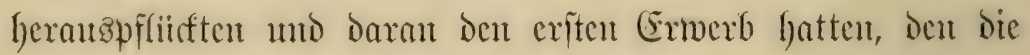
freie Patur ifnen bot. Foit alfer Drten ift fie jut finten,

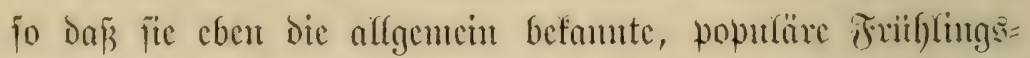

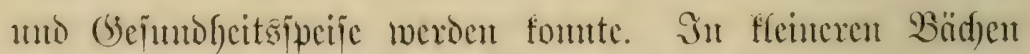
und an quelfigen Erten, befontoers mit ctwas moorigem 


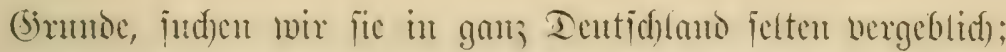
an itppigften wädjat fie da nuter den Wafjeripicgel, aber

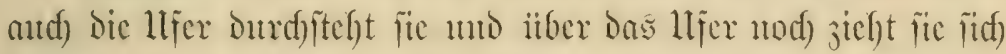

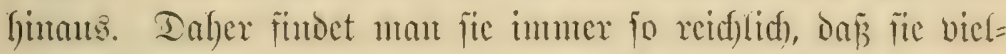
fad) fitc) gar nid)t ganj abcruten lä̈̈t und cin Sind in ciner

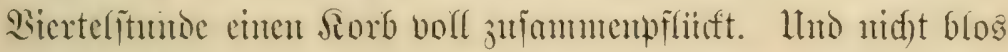

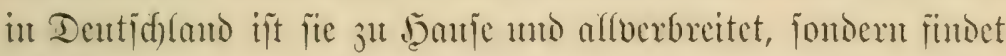

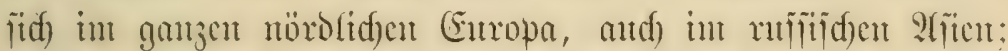
Felbit in 20ronmerifa hat ïe ïd) jestit itherall cingebiirgert.

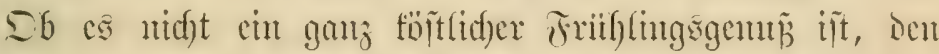
Bad) unt Suelle uns bietet! afls idjüd)ternte Eriftlugsgabe

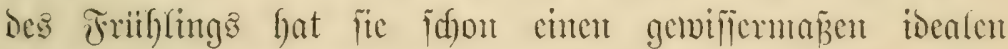

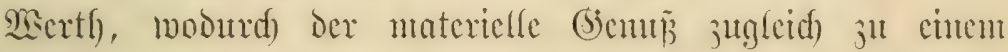

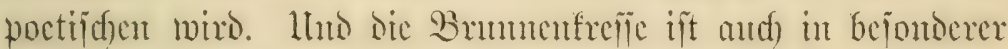

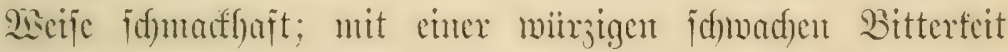
nerbintot jie cinen pifanten, etwas beizenden (bicidmarf. 9) it ctwas (Eijig frijd) zubercitet, gibt jie cinen Ealat, melder trefi =

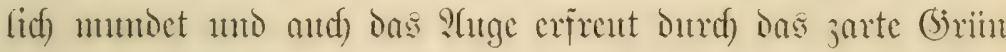

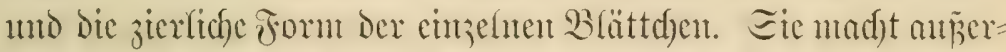
Dem, wie Das 2 olf jagt, "Scib mo Eecle flar und niidftern"; jofon cin alter medicmijder Botanifer bes 16. Jahrbutnorts

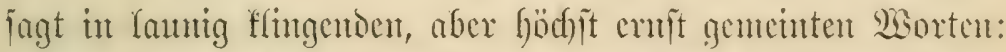
"Ias warfere Sirefientrant wird 1 me Tentiffen rohe ofuge= jotten mit Eilig in jenter Jugent anff ben Iiid) jïrgetragen,

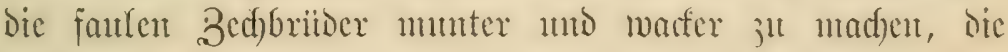

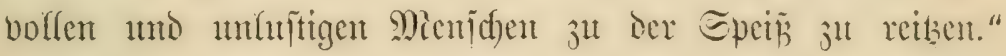

Die Erfalunumg beftütigt Das jum Iheil nod) immer. 2)it cintex vormals gäng mto gäben ?(uïtd)t hat es zmar

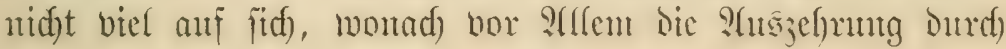




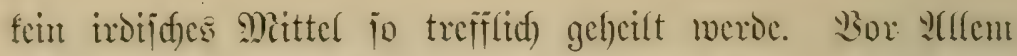
aber die nordijden Eecjabrer femen bie Brumentrejic als gejegnetes firaut, iic ijt ifynen cin bewäfretes Mittel gegen

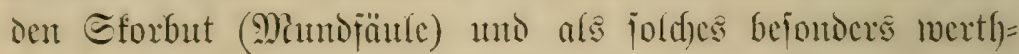
voll, weil man jie das ganje Safur itber mo allerorten im

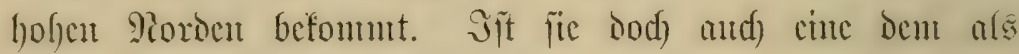

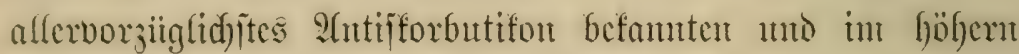
Torocn gleidffalls viclberbreiteten Röffelfraut (Cochlearia officinalis) botanifid) nädhituerwandte Fiflanje.

3isir fiix umjern Theil wollen jie nux als trefflidse

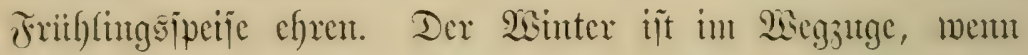
jic bor uns auf den Teffer gritht. 2fber wicderum der:

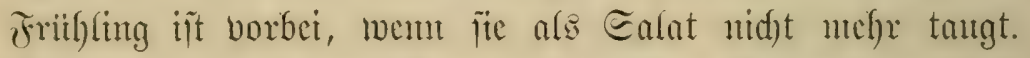

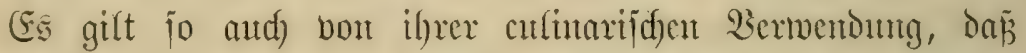

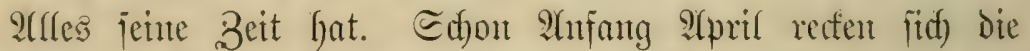
Etengel Der Brumtenfrefic höher, und int Niai bereitet j̈d)

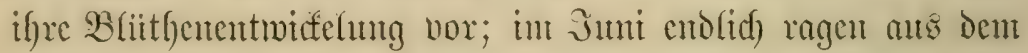

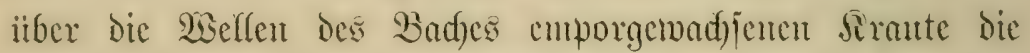

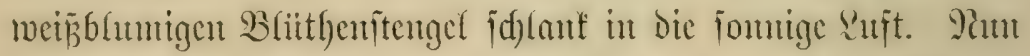
wird Eaft und Sraft ju den Blihthen und jur Fruthtbiloung

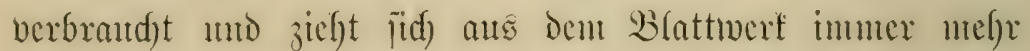
in die blumigen Gipjed empor! Das Brumentreflentrant will barum cben als Ealat unu uid)t melor jumeafen. Fort unto fort bliifht es Danu mit jeinten älyrentranbigen flenten

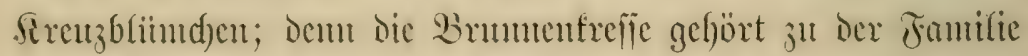

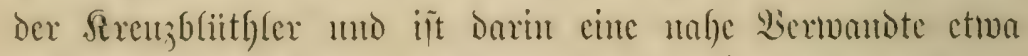

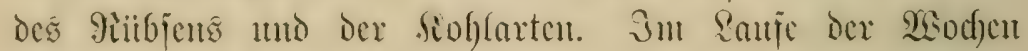

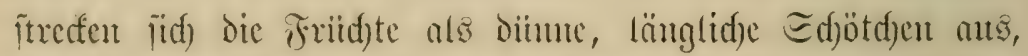
deren filappen balo anfipringen, jo dapj dic Eamen mâfallen. 


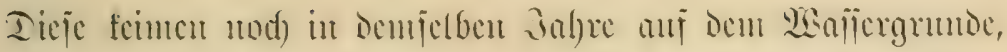

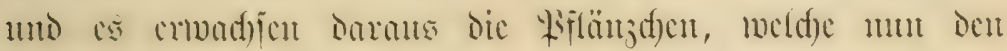

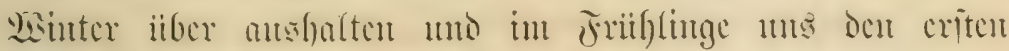
Salat licjern.

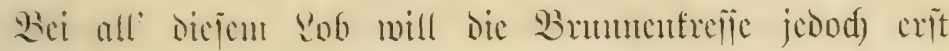

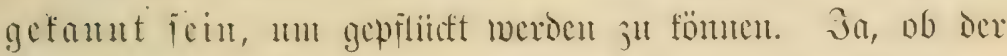

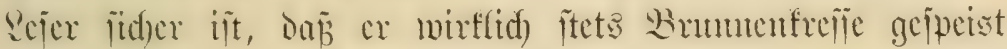
hat, wem man ifm jenen exíten Salat vorjebst? - 9 ut

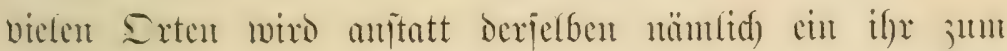

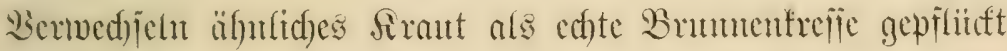

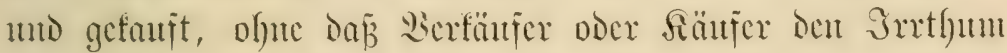

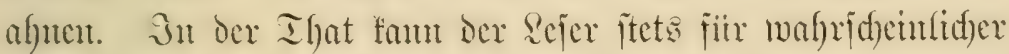

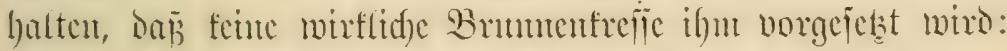
vichel)r Das Bitter=

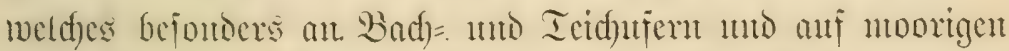
2isicien itberall reidflidyt wäd)st. Dies hat fajt denjelben (siejdunat, ganz ähnlide rundlid)e oder länglidbe, esfig gejälnute

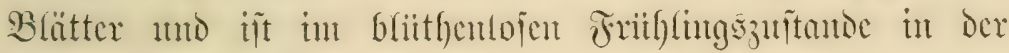

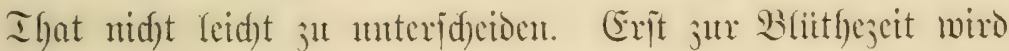

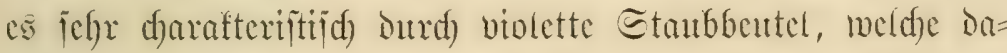

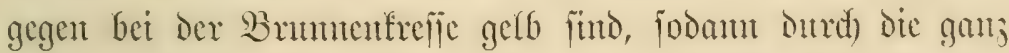

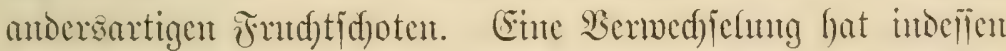

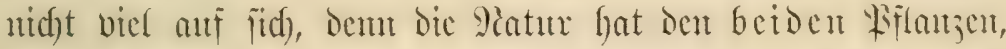

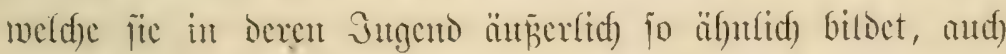
imerlich berwantent (Egarafter verfichen. Sim dap wir Dam

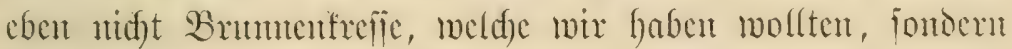

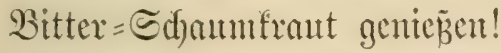

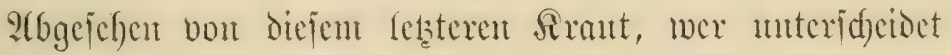

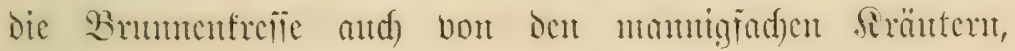




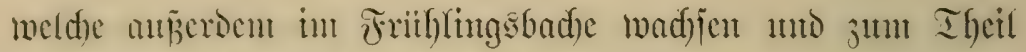

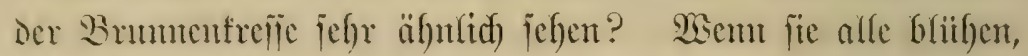

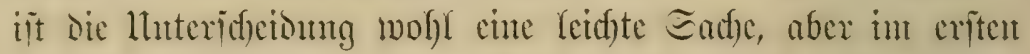

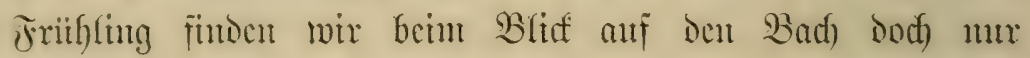
Blätter, weldse fajt alle ficoerig jertheilt, aber bod fo

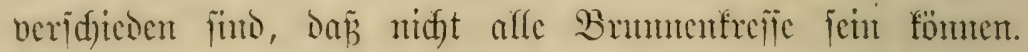

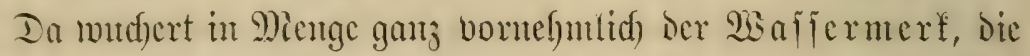

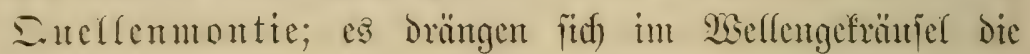
Badfbutgen. Wer foll Da fidser unteriduciden? - Der

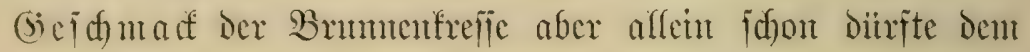
llnfundigen cut judferes Merfnal Derierben jein; hat man fie

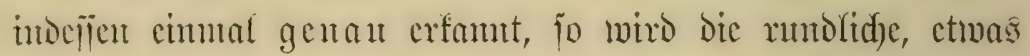
frante Form ifher Fiederblätter jie bod) and nie wieder

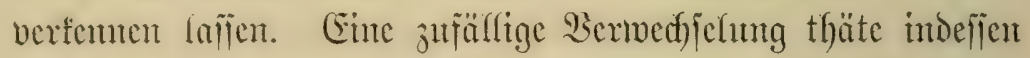
aud) nicht biel, dent es find wenigitens die felgr älunlidjen

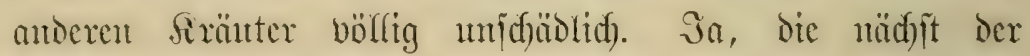

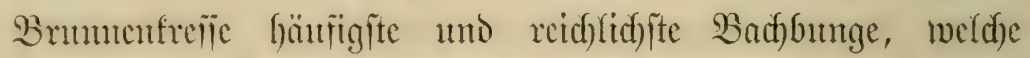

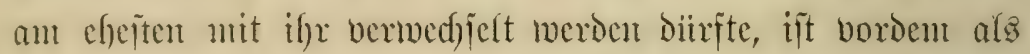

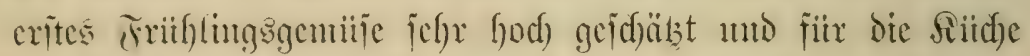
ganj bejouders gejudft worden. Sie geförte ju der Tieun=

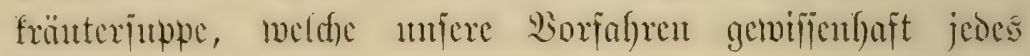
Friit)jalyr genoijen.

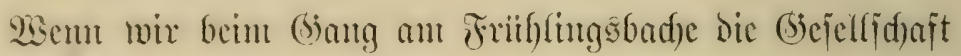

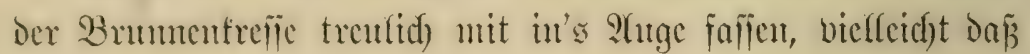

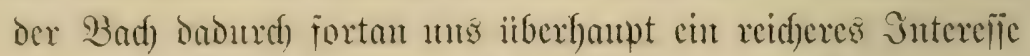

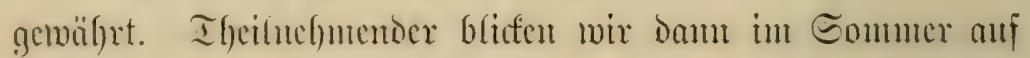

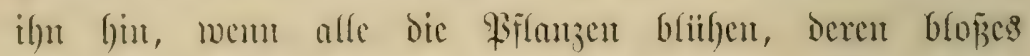

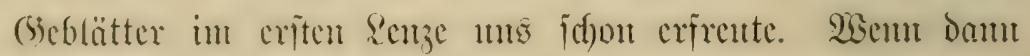

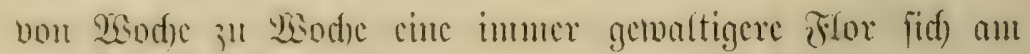


Llfer mo miter ben 2isellen entwifelt, mit fajt tropijacm

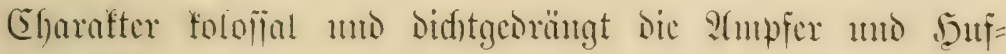

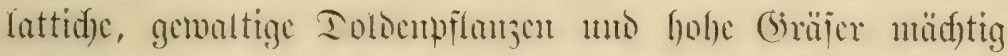

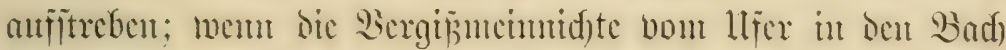

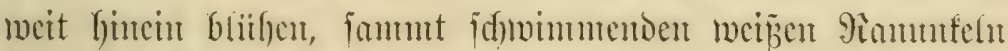

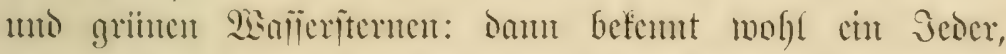

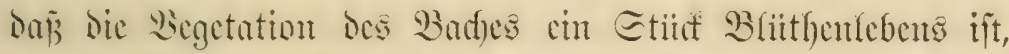
wie dic liatur as im

2.

\section{코s}

离

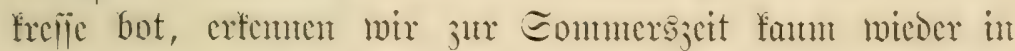

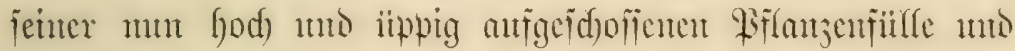

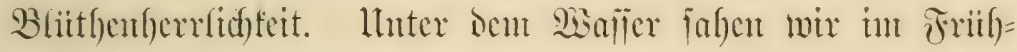

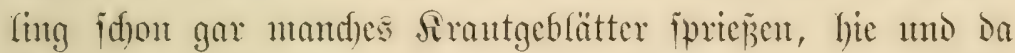

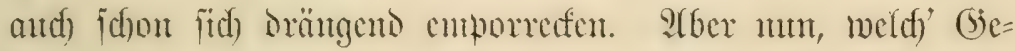

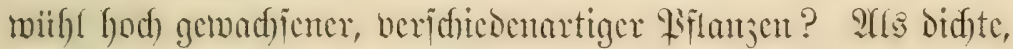

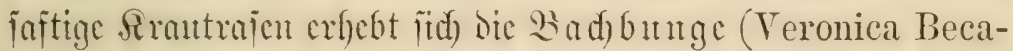
bunga) hanthoch itber sie 2 seffen, mit jartblanten Blumen= frönd)en reid) ïberblitht; andere 2 safjerefrenterife (be= fontors V. Anagallis) ragen baneben, mefrere Fur hohe,

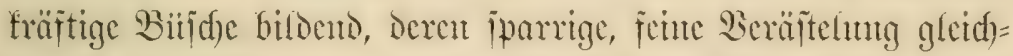

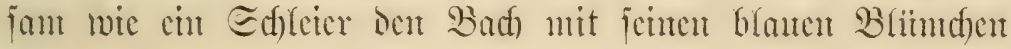


Durdjüfftig iiberfiillt. Ins J̈gelfraut (Sparganium ra-

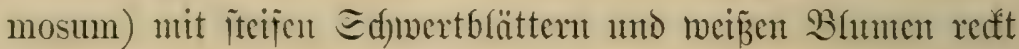

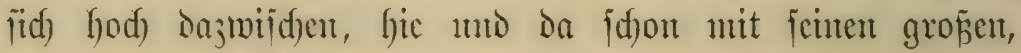

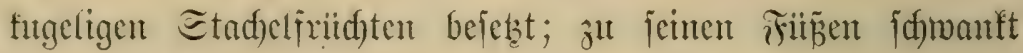

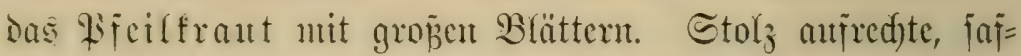
tige, berrliche Gruppen bildot wiederum die 2Bafferfeder (Hottonia palustris), auf bohen, fiederblättrigen Stengeln

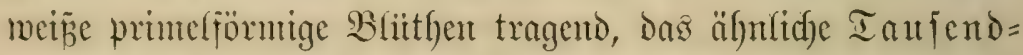
blatt (Myriophyllum spicatum), mit jemen grofien feder=

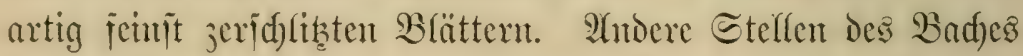
find völlig eingenommen von mädftigen Doldenpfflanjen, Deren bohe reid) beblätterte Stengel, bon wecijen colen Brithen=

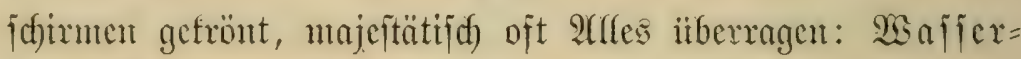

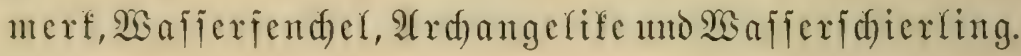

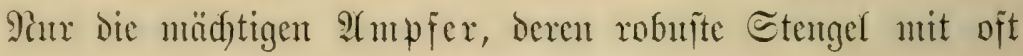
armlangen, weit über hanobreiten Blätten bejeb̧t finto, jomie

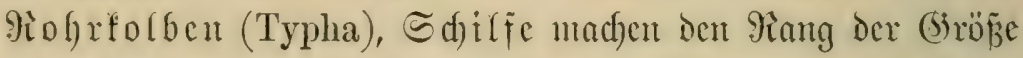

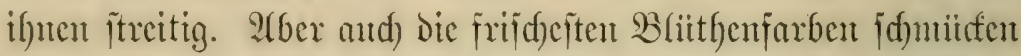

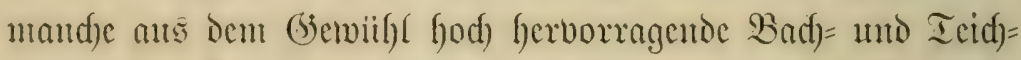

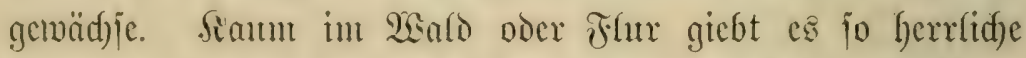

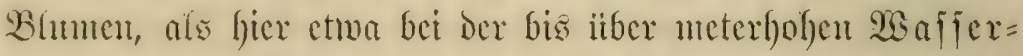
viofe (Butomus umbellatus), Dexen binfentartige Dalme mit veild)enblauen, großzbrittgigen Dorben getrönt fün, wälyend wicderum dex $\mathfrak{B}$ futweiderig (Lythrum Salicornia) mit

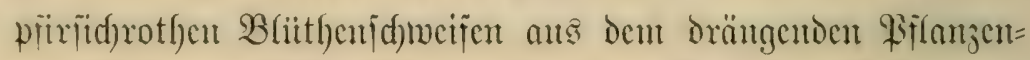

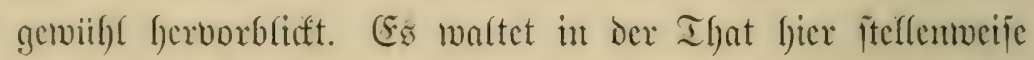

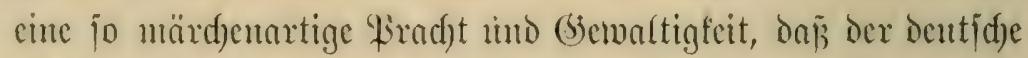

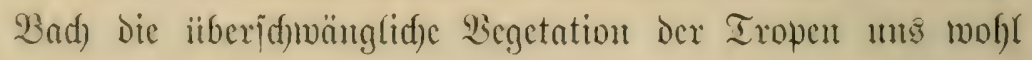
veramid)auliden faum. 
2(ber alf biejer mäd)tige Siflanzenmuths, mit jemen herr=

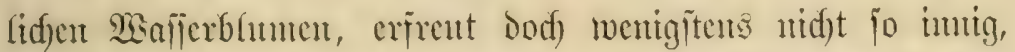

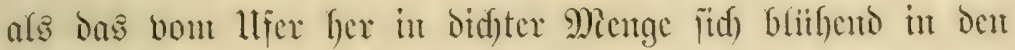

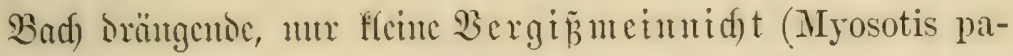
lustris). Ilnter al(en Bad)träıtern hat bas deutide Bolfos=

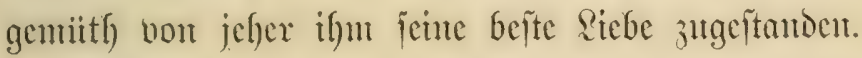

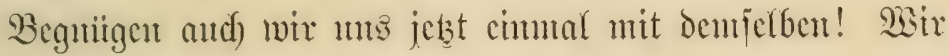

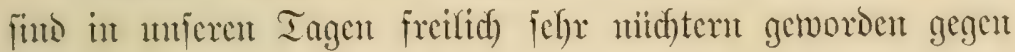
bie fritfere Beit. Demtod) Fehen wir in Dent Bergifmenumidt

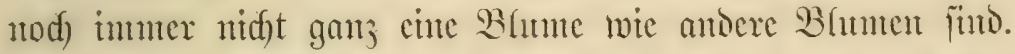
Scr Sïngling pflüteft fie am Badye nod) immer jeiner (Se=

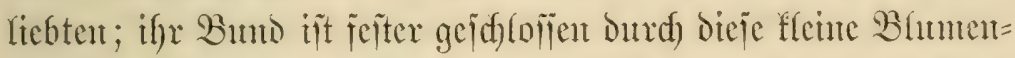

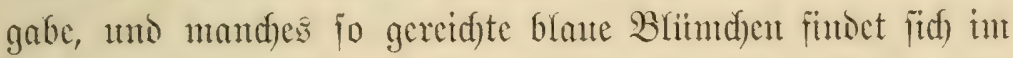

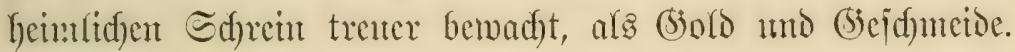
Den Sinde fodjon jeigt die Mittter bein (Sang ins Freie

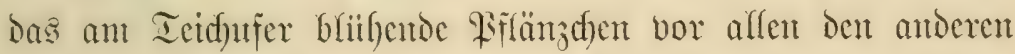

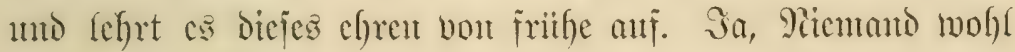

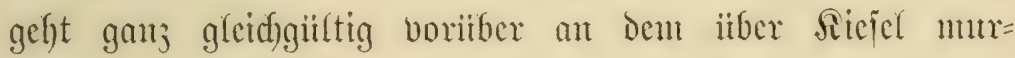

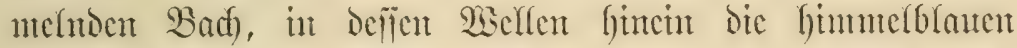

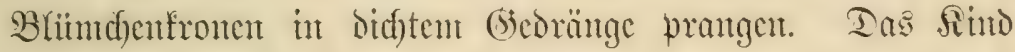

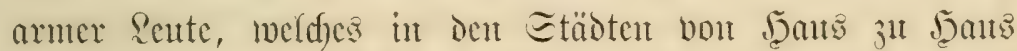
Bergiñmeimudatfränje feilbetet, fundet baber jelten eine $a b=$

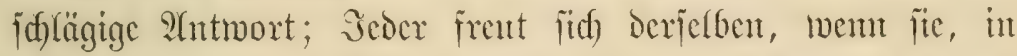
den wafiergefitlften Ielfer gelegt, ju nettent Seben fontmen,

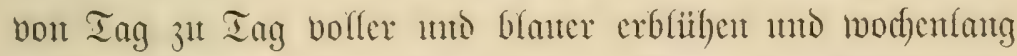

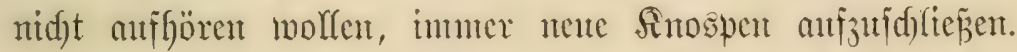

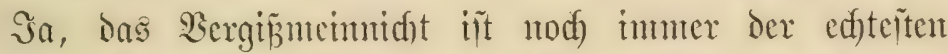

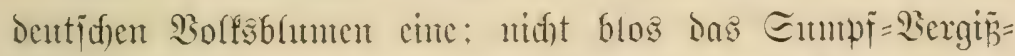

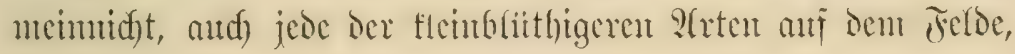




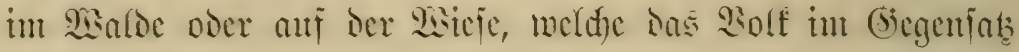
mu als "milbe" bejeidnet. Hnter bicjen ijt aber and cine

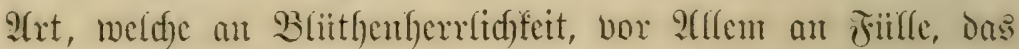

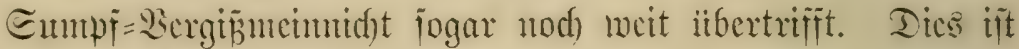

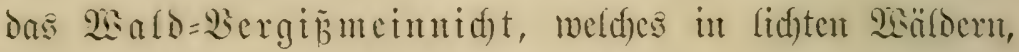

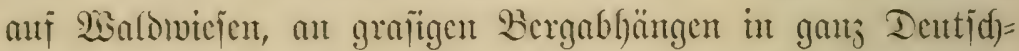
fand vorfonmt und von Mitte april bis Ende Frithling feinte

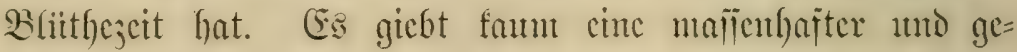

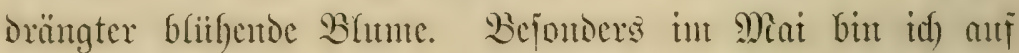

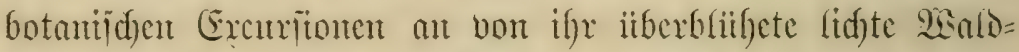

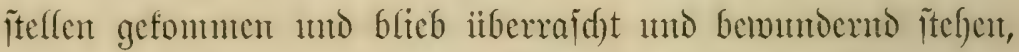
in Trithlingsandad)t bei dicjent 2htblicf verimfen. So weit das Ifutge jah), viele Miorgen weit bis an Das didfer wer= Dende 2 salogebiifd) breitete fidf) ein finntelblater Icppid),

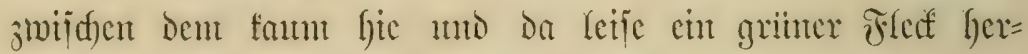
borial)! Socr cint filles, blates Nicer, in ber Ifat ein

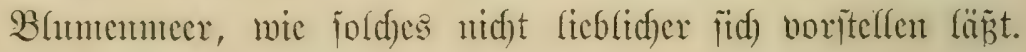

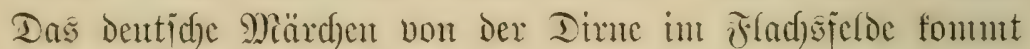
Dent Beidfanter in Den Eim, Die cin Bantorer wermirrt hatte,

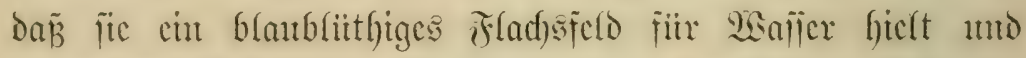

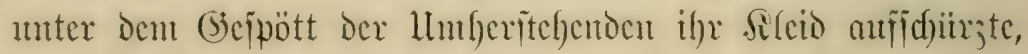

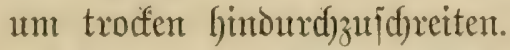

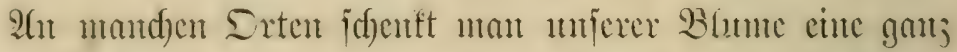

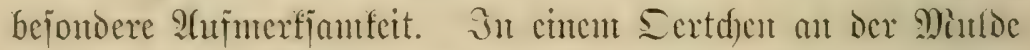
pflegt die liebe Edjuljugend mit ibrem Selyer itblidfer ascije

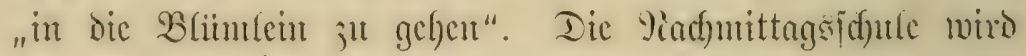
Frei gegeben, into im mildon Eommenfdecin mantert die muntere Ed)ar mit (Siciang in cincu naf)en Wald, wo bics

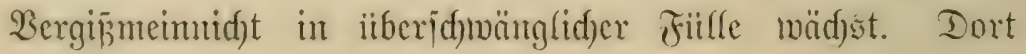


angetommen, bat mad) berfönmlicher Eitte crjt Jeser cintu

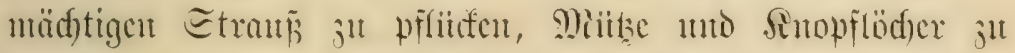

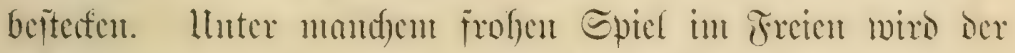
Trad)mittag berbrad)t un fommt Der a(bento locran, che man

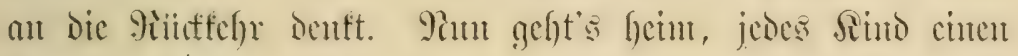

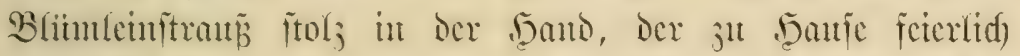
cincul Maficrglaje anvertrant wird. Ebenjo fudft dort fajt

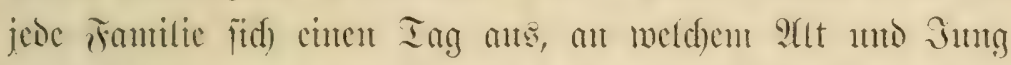

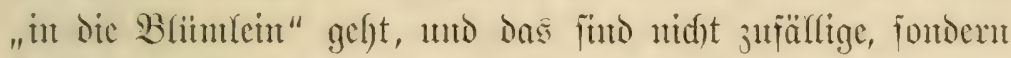

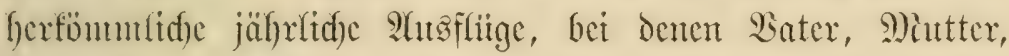

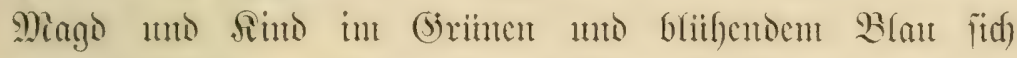

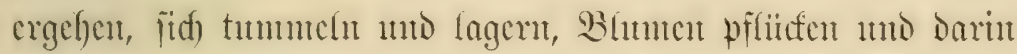

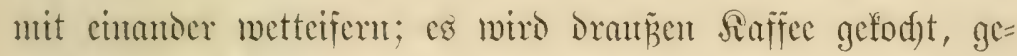
trumfeu, Suthen dajut gegefien und dic ganje (sicjellid)aft ijt Dabci cummal io fröhlid), wic Das ganje Jahr faum wieder. Man reinigh fid Da bon Den niedrigen itaubigen Eroenleben an Serz und Secle.

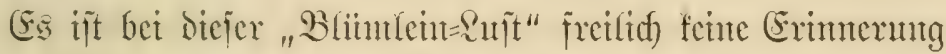

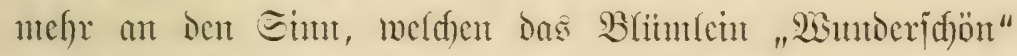
fiir unjere 2iorfafren hatte. Piur mas ca Den Didftern und Den Sécbenden borden mar, beocutet co nod) inmer; cs war mo ijt ja nod) beute Das lluterpfand der. Ireate unto die

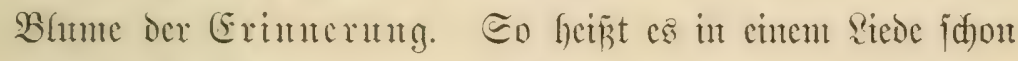

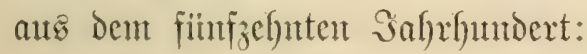

"Itno wero ir hören, wa ma mir ba lebite in?

$D a_{\mathfrak{d}}$ platwe plïmlitu, ba $a_{\mathfrak{j}}$ itat gar nadj babi;

Daว̉ platve Dütet ität;

Der füle wind hat mir Den meg verwät.

Daz plawe plümlin hoffet auf genad,

Und ftünd bie alfer jobnjt junffer bavor, 
Den swedjel welt idy nit triben,

Jad) welt bi minem plawen plimfin beliben."

Eo fteht and in ber altiberliejerten "Bedontung ocr

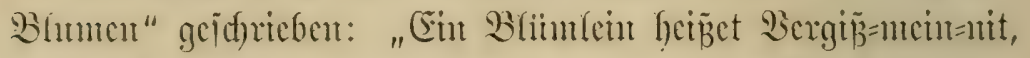
dem das emprohlen wird, der mag wohl fröhlidys Mintles

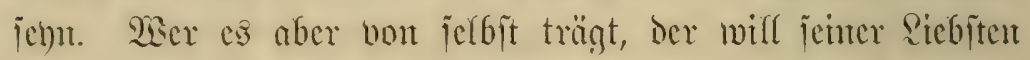
nid)t vergefien 3n feiner Beit."

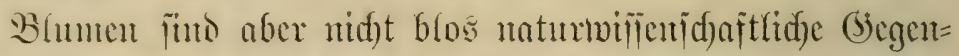

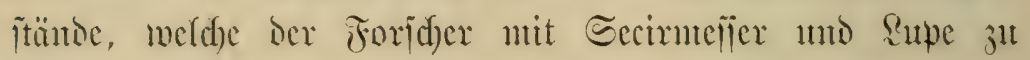
unterjudjen uns ju flaffifiziren hat, un gelebrte Bemerfungen

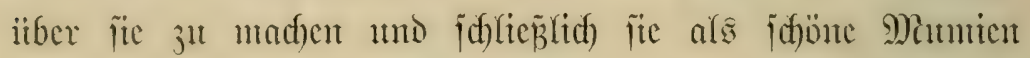
jantber geordut in cin 5erbarum jut legen. Cic fimb autd) Scilfräuter, unto das nidyt blos in officincllent Eiuute gegen Sciden mo Edjäben mijeres fiörpers, jondern and wie Sutituts ferner es meinte:

„Blumen, ach Blumen, bie heilen jeden Silymer

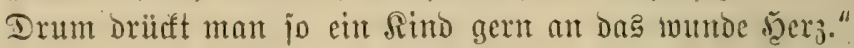

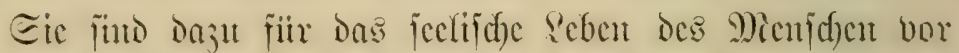
atfem ๔nmbole. Indo darim hat befonders die Simtigfeit

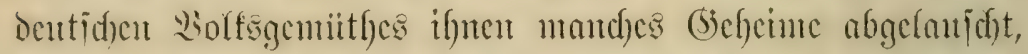

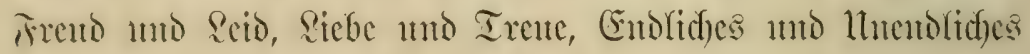

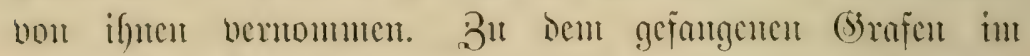

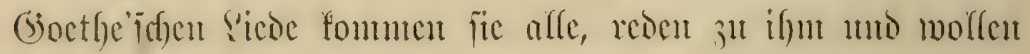

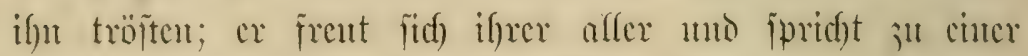

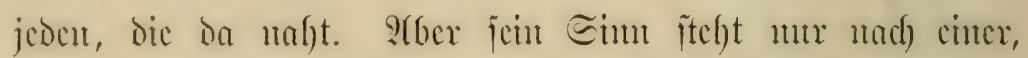

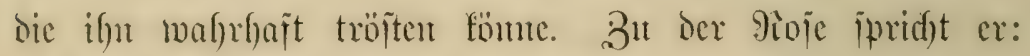

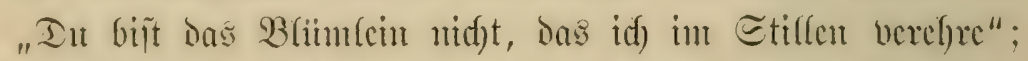

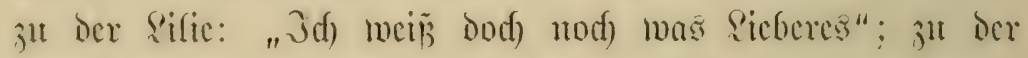

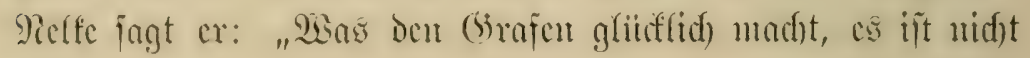




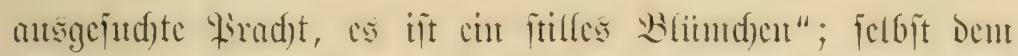

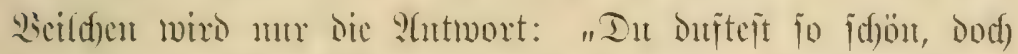

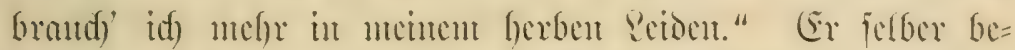

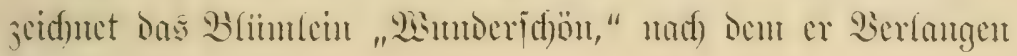
trägt:

"(Ẻ mandelt unten an Dem Bad

Das treuefte Weib Der (Erde, Und jeujzet leije mand)es $2(d)$,

Bis ide erföjet werde.

Wem fie ein blates Blümtein brid)t

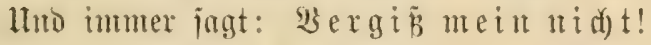

Eo fillfl' ide's it ber Ferme."

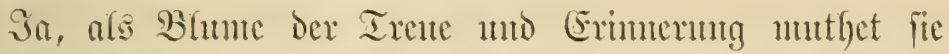

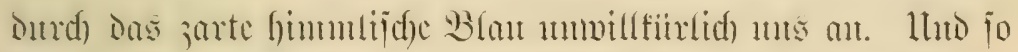

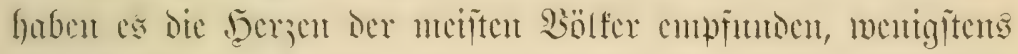

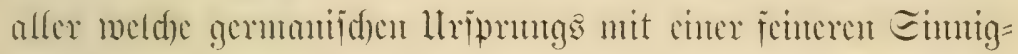

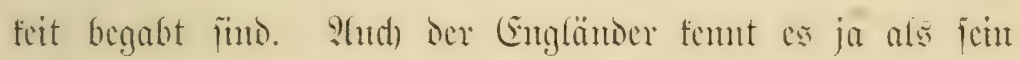

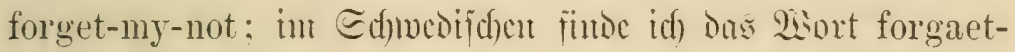
mig-ej. in Viormenijd) forglemm-mig-iklie, in Iänijd)n

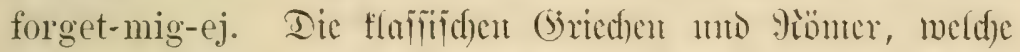

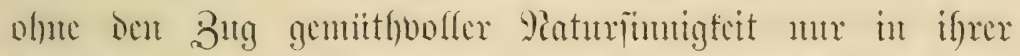

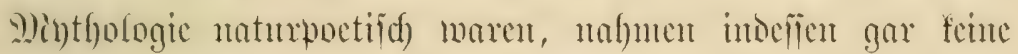

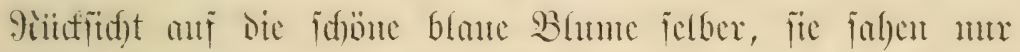

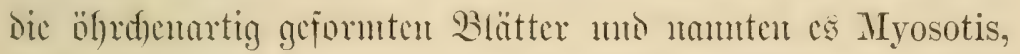

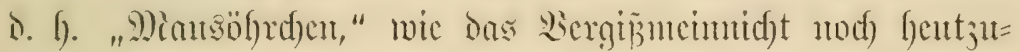
tage in der Botantf gentant wiro. P(ntd) Dic Franzojen

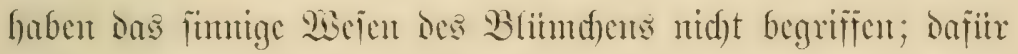

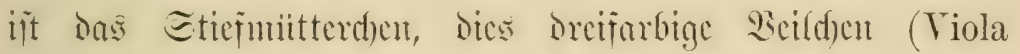

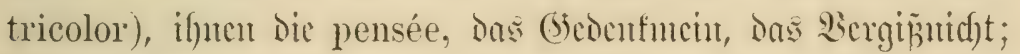

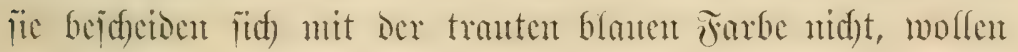


Fatte Frarben, präd)tige Beidfunntg, ftarfe 9icije haben, um an die Ireute ftart gentahnt jut wordent.

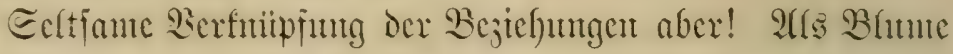

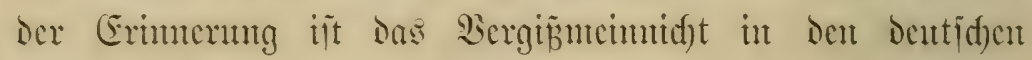
Eagen nod) in ganj antorex, fdjeinbar gantj entfernt licgender

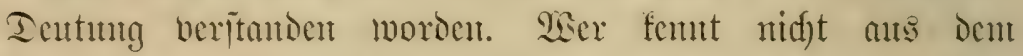

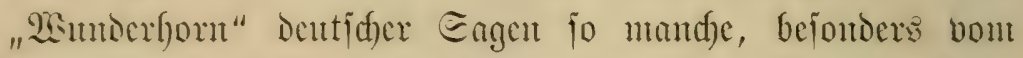

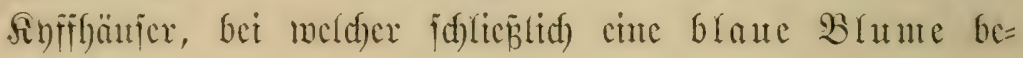
dentian wird!

Fin gutcr funbe, hcipht ç ba meijt, und armer Reute fint, lag an blumiger Berghalde, während dic ifm anvertrante Decroc des nafen Dorfes umber meidete. Da tritt cute Inugfrant jut ifne und redet ifnel licbecooll an; artig antwortet

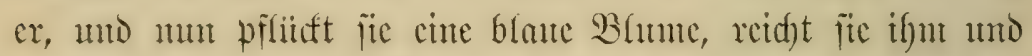

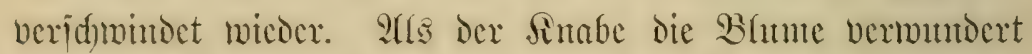

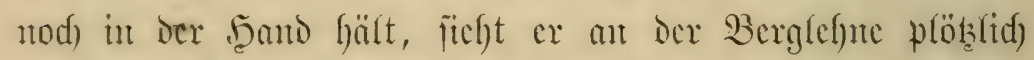
ciute Ifyit antgethan, dic ex bordom nimals bemerft hat. Ene Etimme rujt ifjm ju, oaj cr cintretc. Hub wie cr mu in bas folle Juntere bes Berges tritt, trant cr jeincu Augen faum, deum da briuncu rings umber funfelt und

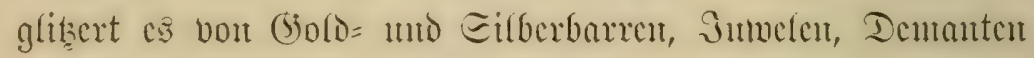
umo alfon fiöjtidffiten, wie er joldfe nic gejdant. Sr legt

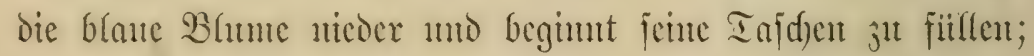
Danu will ex reid) beladen den Berg wicoer berlafien. Dod)

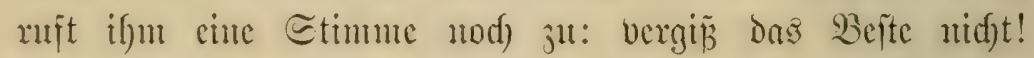

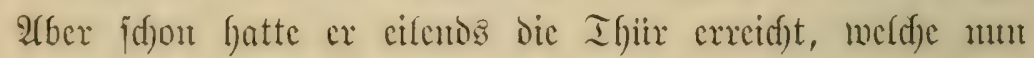

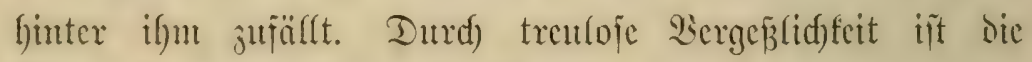
Batme drinten geblicbent, weld)e ifun immer bon nenent ben

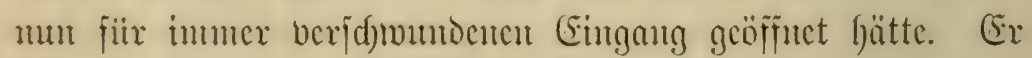




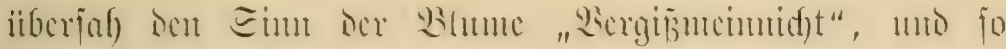

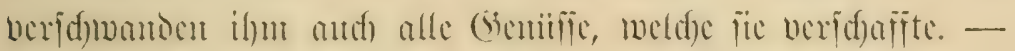

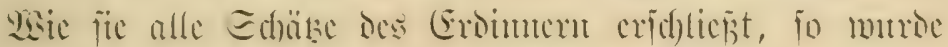

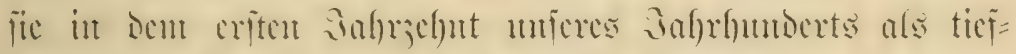

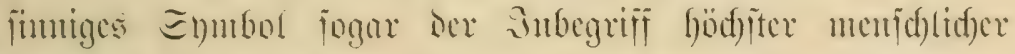

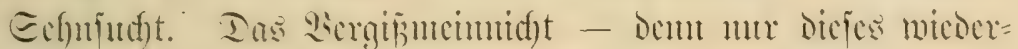

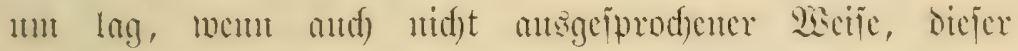

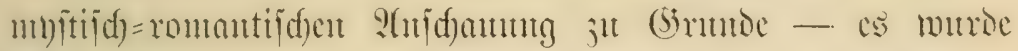
als Sultusigegentano idealiten Sharafters in cine feltfame

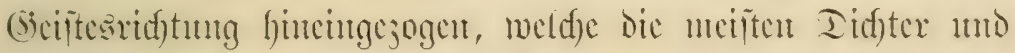

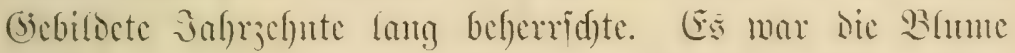

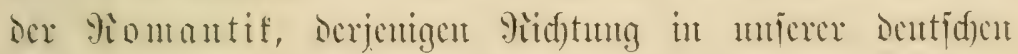

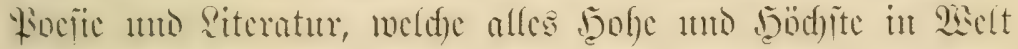

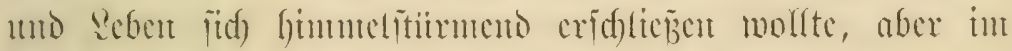

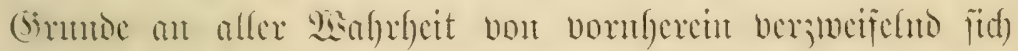

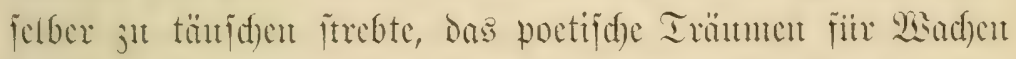

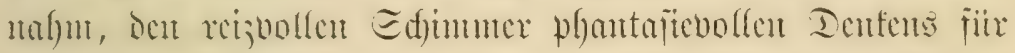

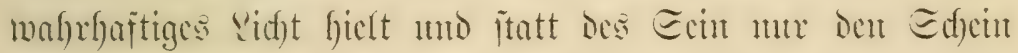

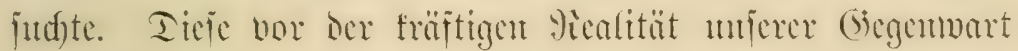

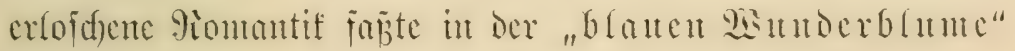
die Summe alfer ilyer Eelyund)t jufammen.

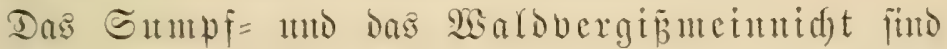

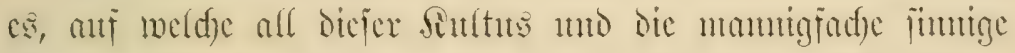

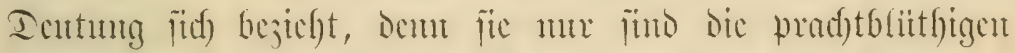

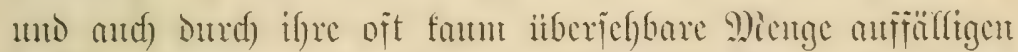

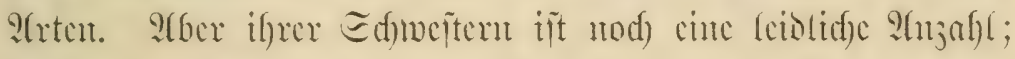

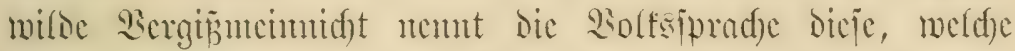

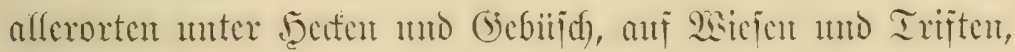

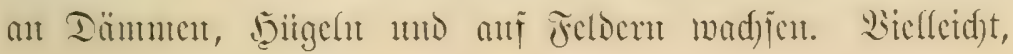




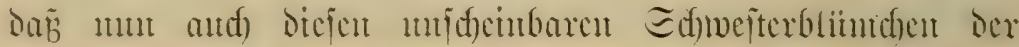

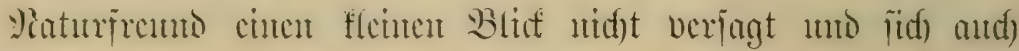

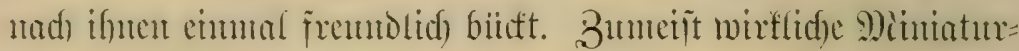

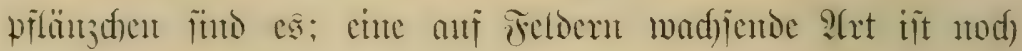

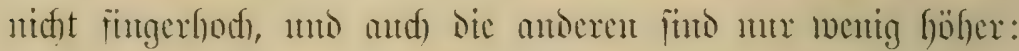
Das "raufe", Das "hanrige", Das "mentigb(iithige", Das "mittlere", das "bunte" - mo wie fie alle Geipen, die

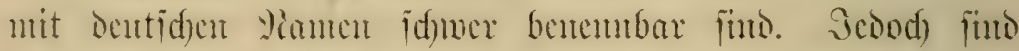

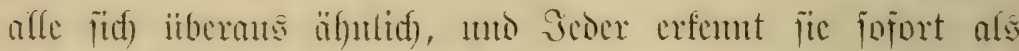

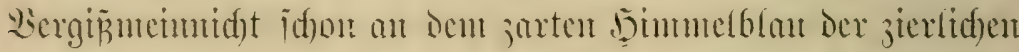

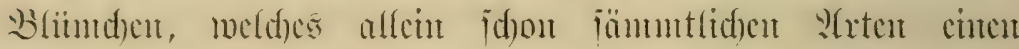

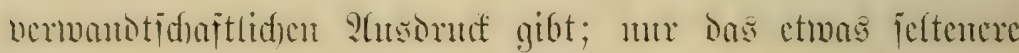

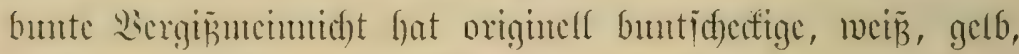

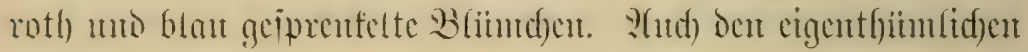

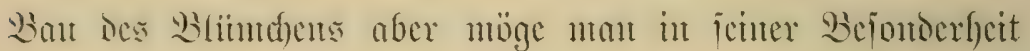
bead)ten, die prëjentirtellerïrmige blane Siroute, seren Butment=

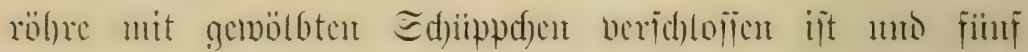

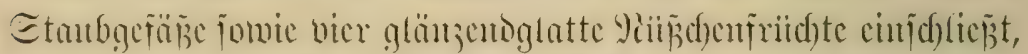

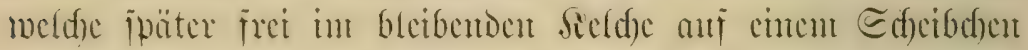

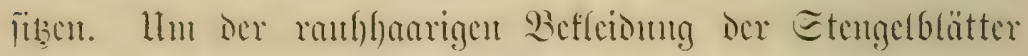

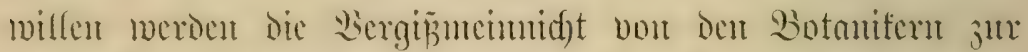

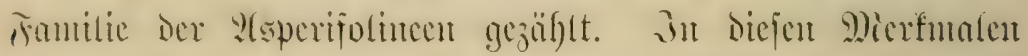
itimmen fie alle ibberem.

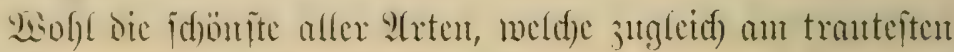

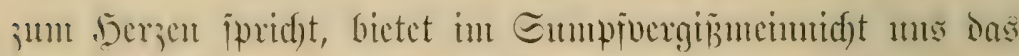

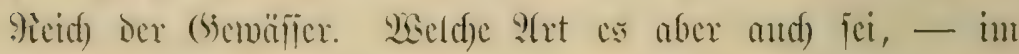

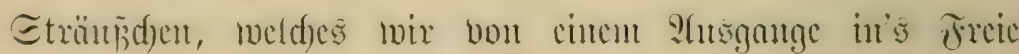

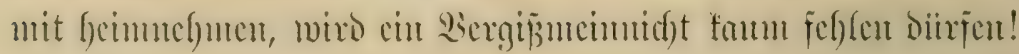


3.

\section{Die}

\section{ir}

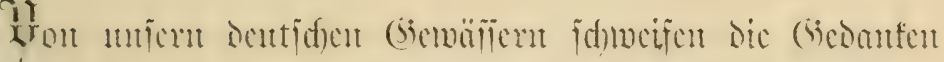

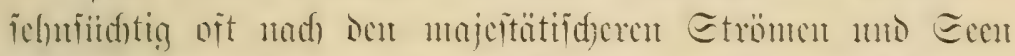

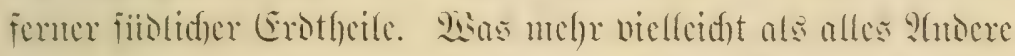

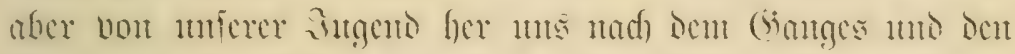

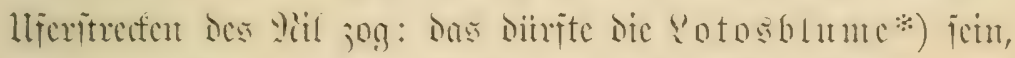

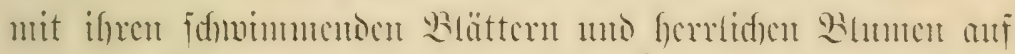

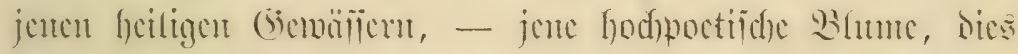

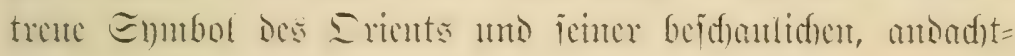
cruften Bölfer.

Siömen wir jic je(bit nidft idjanten, wic jie jid Yeife

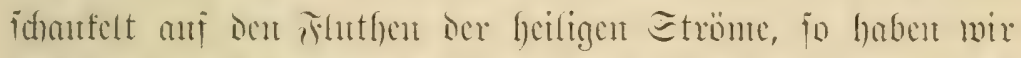

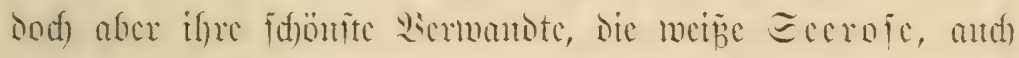

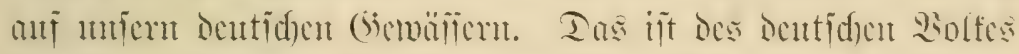

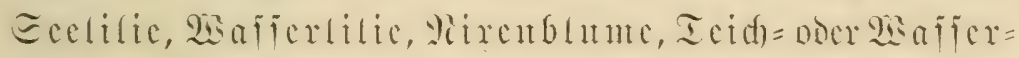

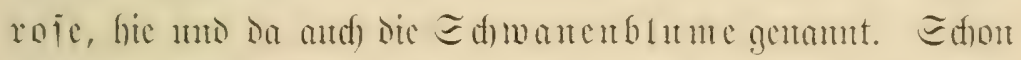

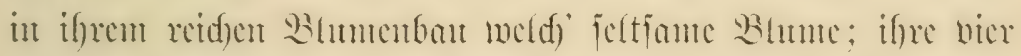

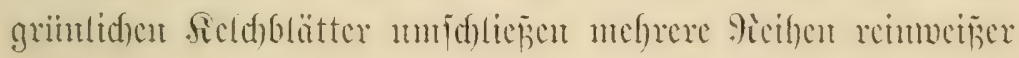

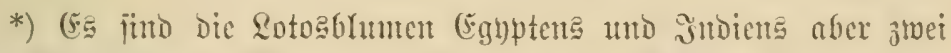

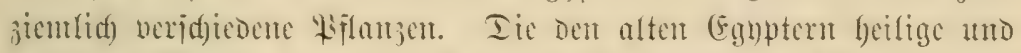

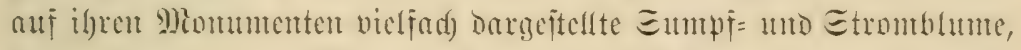

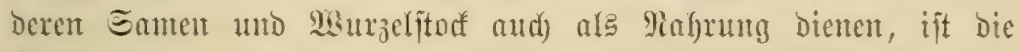
Nymphaea Lotos, L.. Dayegen Die in Die indijde (Gïtterlefre ver= wobente Gangesblume, mit bofnumartigem ç̧baren Enmen, ijt Nelumbium speciosum. 


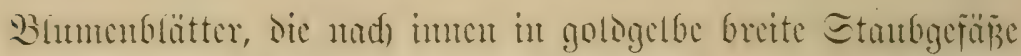

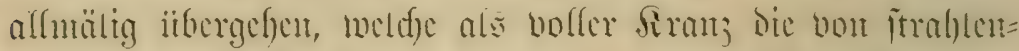

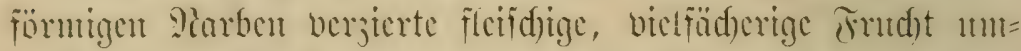

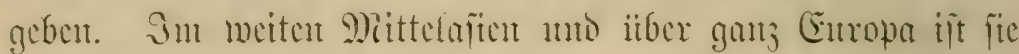

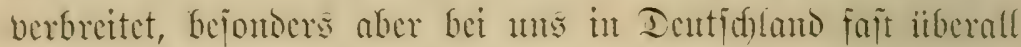

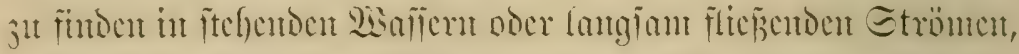

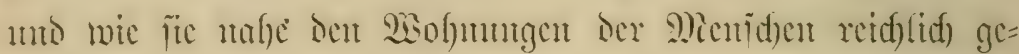
trofjen miro, fo nidit mintoer in sen grojen Edjiffeidsen,

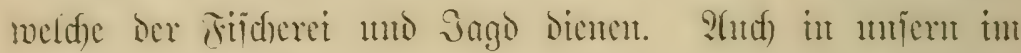

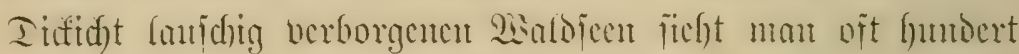
mo mebr ifre prad)twolfen Eilforfäunter itber son Disaffer=

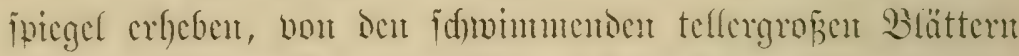

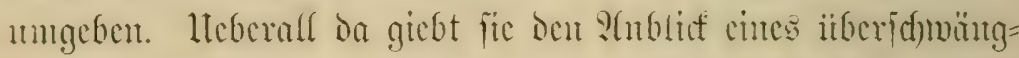

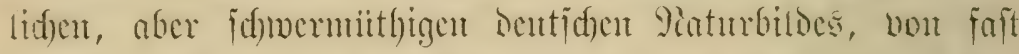

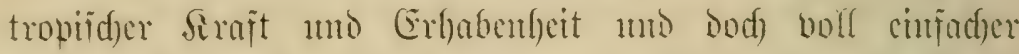

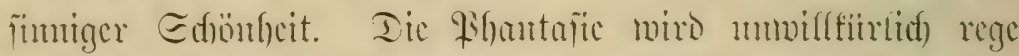

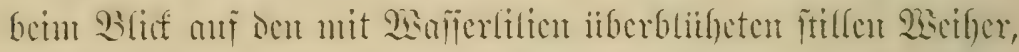

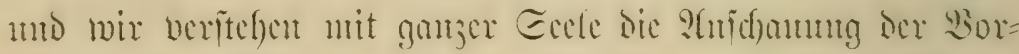

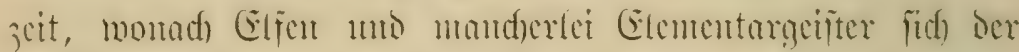

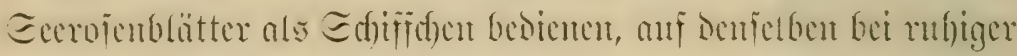

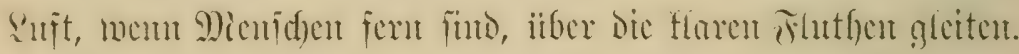

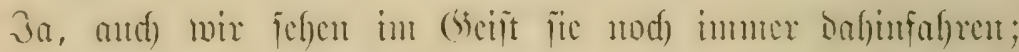

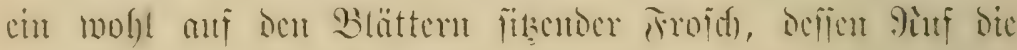
Etille ab und ;u ourdbormint, thut bicjer Etmmuntg ber

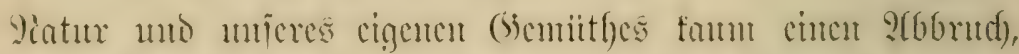
cr gefört mit daju, fanment alf don sibellen mo flimemom,

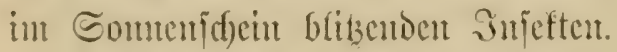

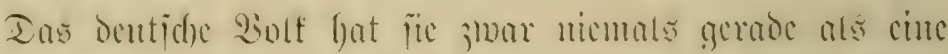

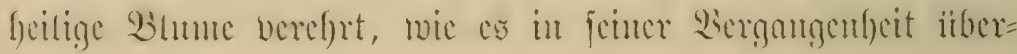




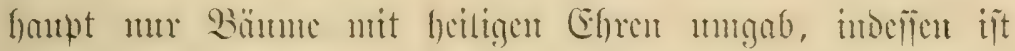

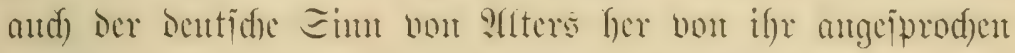
morben. Tiod) mantife mumberfante alte Eage weip jut $\mathrm{cr}=$

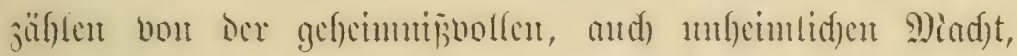
mit ber fie als Siönigin mitten in Sicid) der (berväiner maltet. 25so Diefe ticf funt, ift fie ja mur anjutreffent. Eeltent nähert

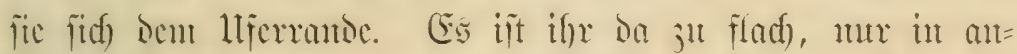

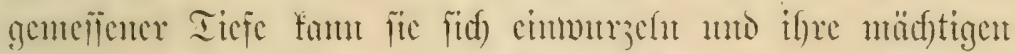

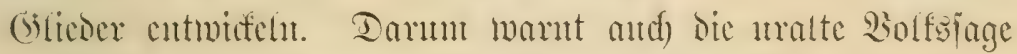

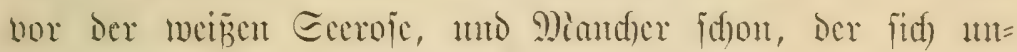
norfichtig ood) bis jte ifhe heramwagte, ift iffr als Spfer ver=

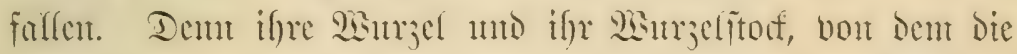

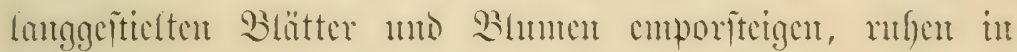

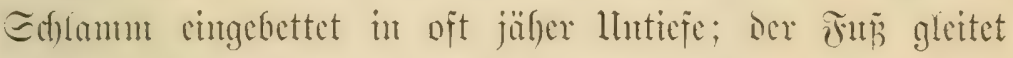

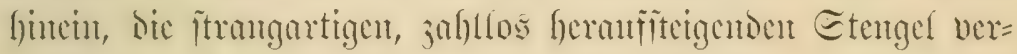

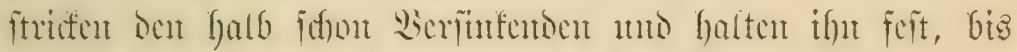

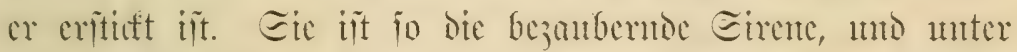
ifre mohnt das (branten und ber Tod. Siad) Dex Micmung

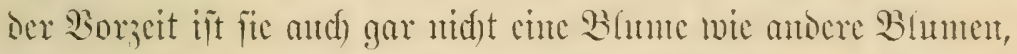
vielmelyr cine berwandelte Eecjutgfran, mo ment 9)itter=

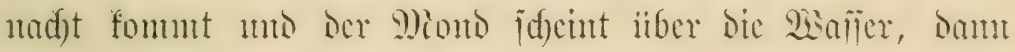

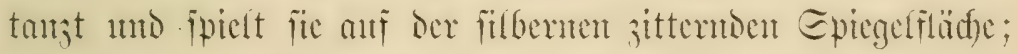

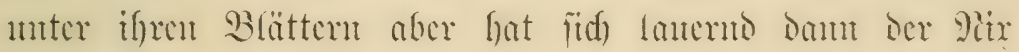

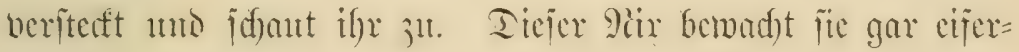

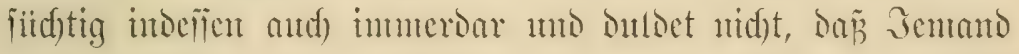

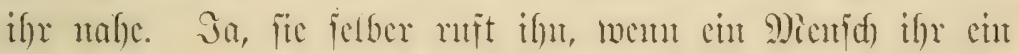

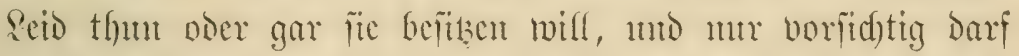
man ces barum anfangen, ment der Solfsglantbe, ifner hab=

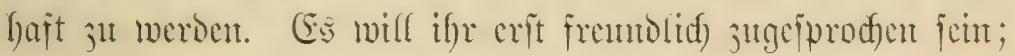




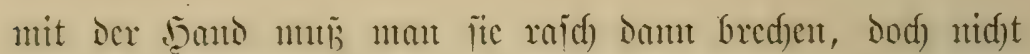

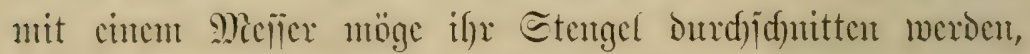

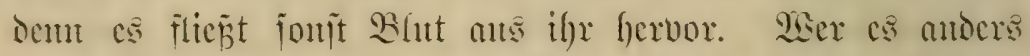

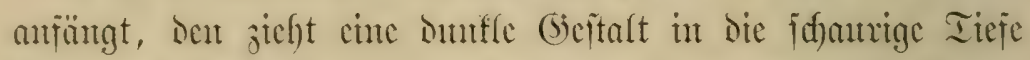

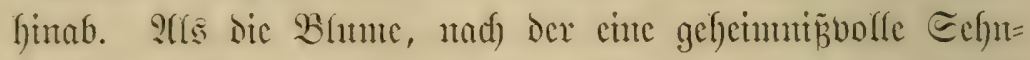
jutfit jedes :

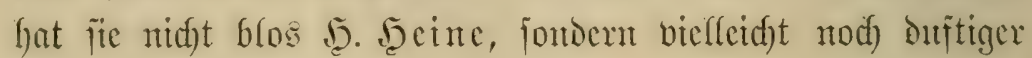
5.manuel bicibel bejungen:

Die itilfe Majīerroje

Steigt aus Dent blauen Eee,

Die B̊lätter flimmern uno blijen,

Der Reld) ift meip̈ wie Sdyne.

Da gieß̃t ber Miono vom f̧immel

2(Il' jeinen gold'nen Sd)ein,

(bieñt alfe jeine Etrahlent

int ihren Sdon hinein.

Jnt $\mathfrak{B a n ̃ e r}$ unt bie Blume

Sireijet ein weižer Sd)twan,

(Ex jingt jo jü $\tilde{b}$, jo leije,

Und jafaut bie Blume an.

(Er fingt jo jü̈s, jo leife,

Und will im Singen vergelyn -

D Blume, tweipe Blume,

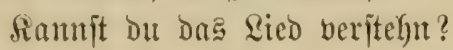

Gine ucije 2 Saljerelfe, cint jeclenbolles $26 \mathrm{cjent}$ - jo muthet

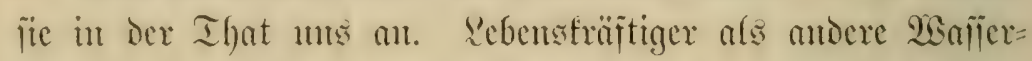
pflanjen mo wic jeclenbegabt idfuimmt jie ant Dent Epiegel,

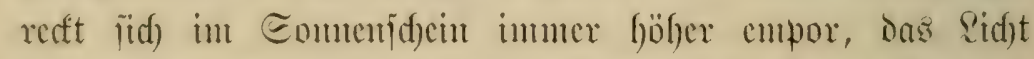

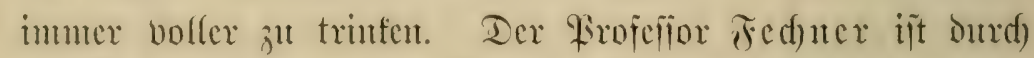

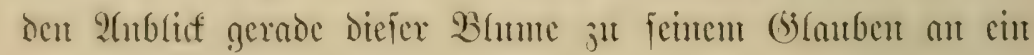


Ecelenteben ber

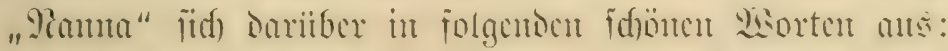

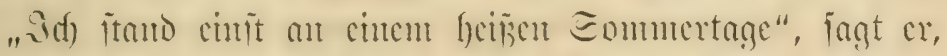

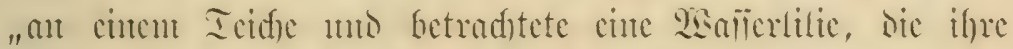

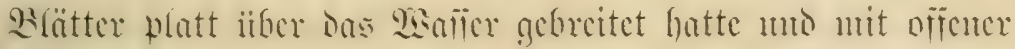

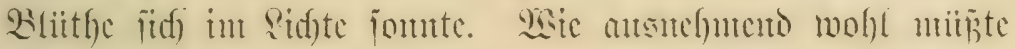

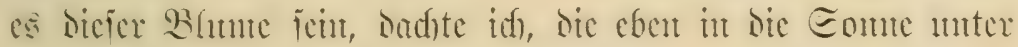

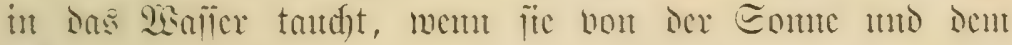

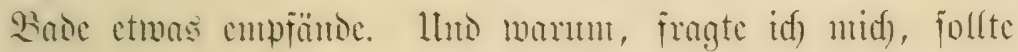

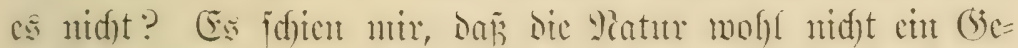

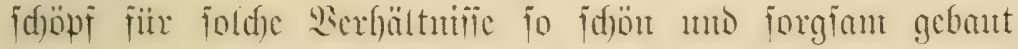

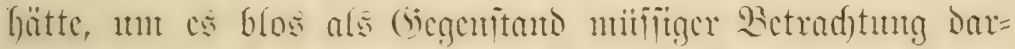

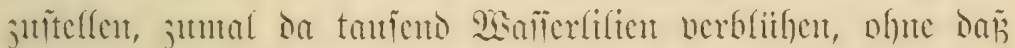

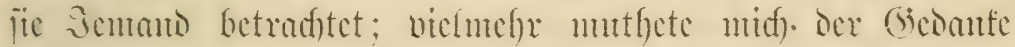

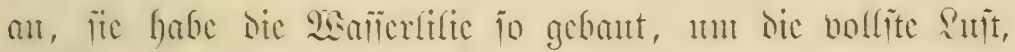

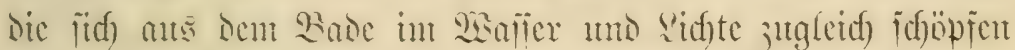

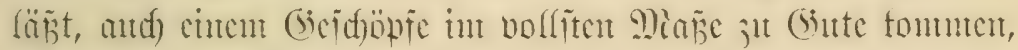

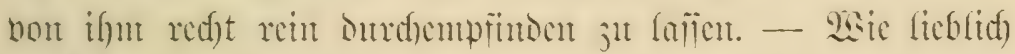

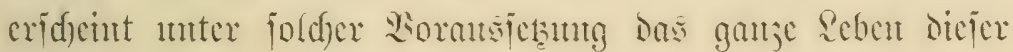
Bhtume."

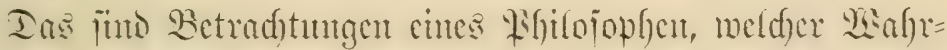
heit mo Sidftung vermed)felt! mödfte Der Seicr cimberfen.

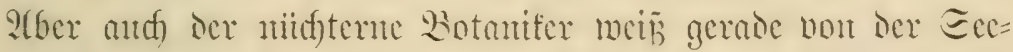

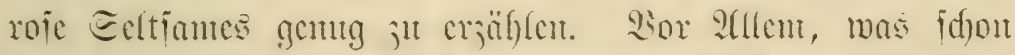

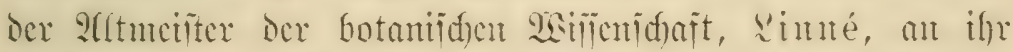

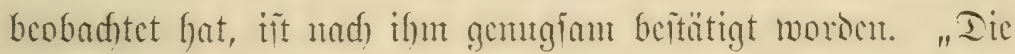
Nymphaea alba crheft jidf)", jagt er, "in ocr Miorgenfriife

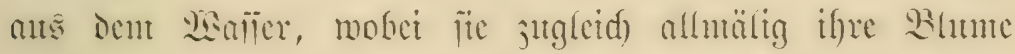

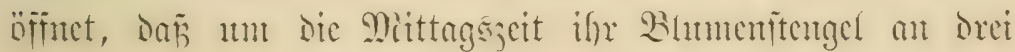




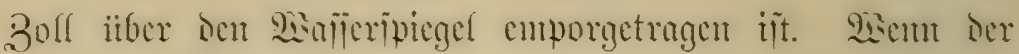

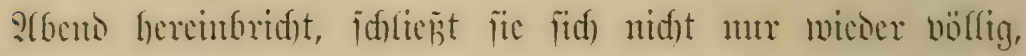

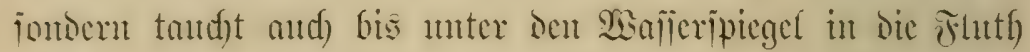

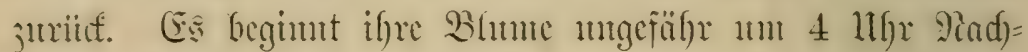

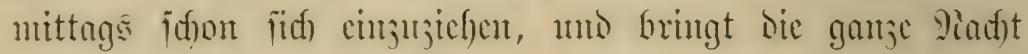
unter ben פăjer ju."

Iic Sotusblume Eguptens und Die indijde, Edfucitem minerer Eecroje, madjen es aber cbenjo. We(d)' mutuerjante,

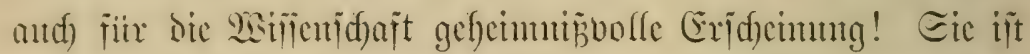

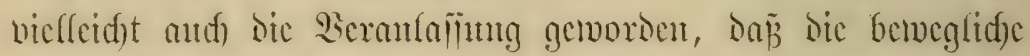

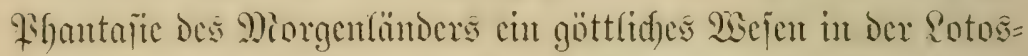

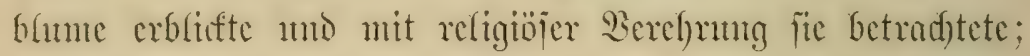

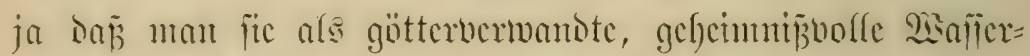
meien mutheftens unter dent bejonteren Eduts ber alfwal= tenocn (sïtter jaf), intom dic funtige Religion bes Inders

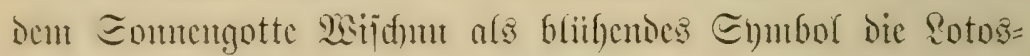

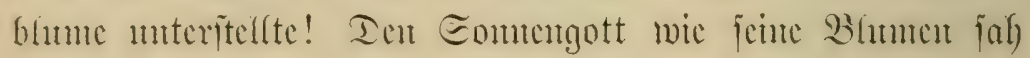

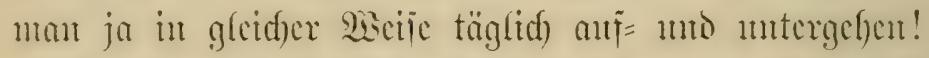

Ireiben wir in Geomfen mm aber cimmal mett hiniber

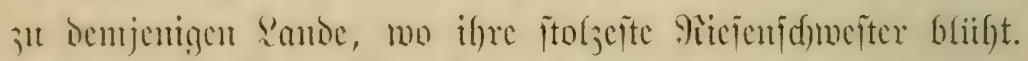

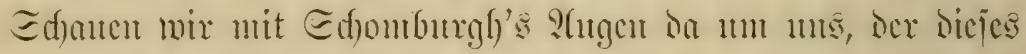

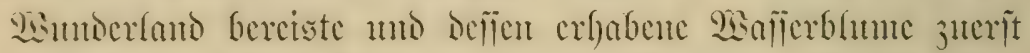

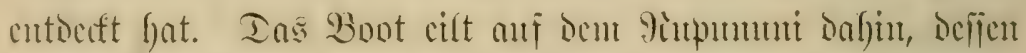

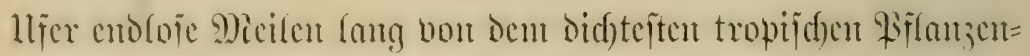

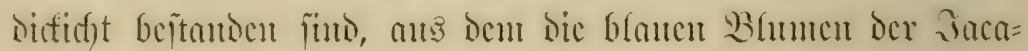

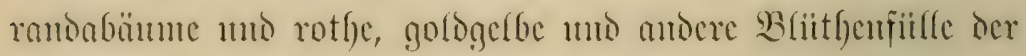

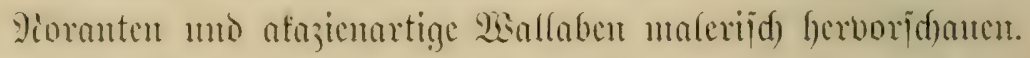

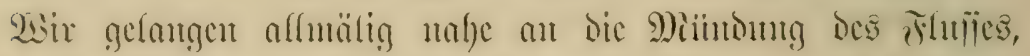
der da breiter mo rubiger biujicht. Iort mu follen wir 


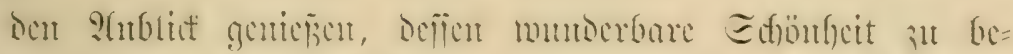

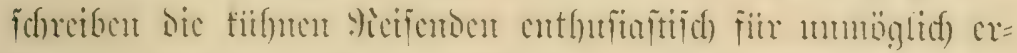

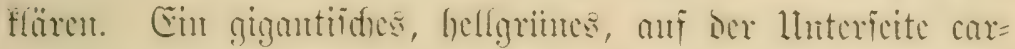

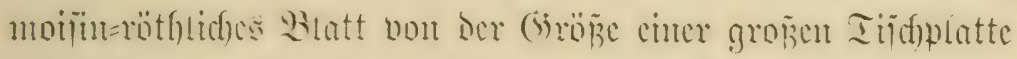

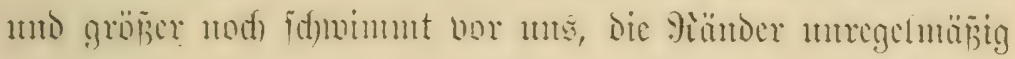

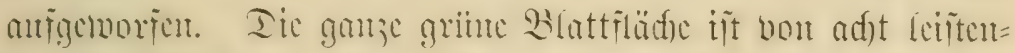
artig joll = bis hanthod) herborragenton röthlidfen sants =

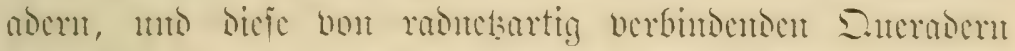

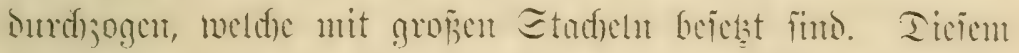

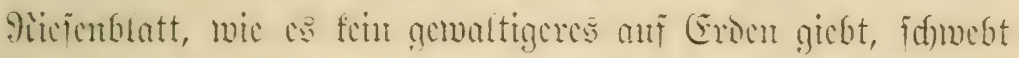
int Ecite anf fait ambifen, Dornigem Bummenticl menige

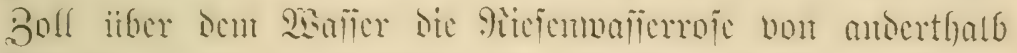

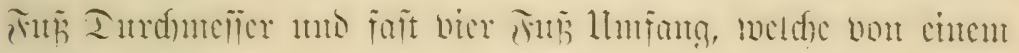

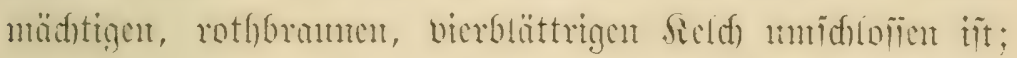

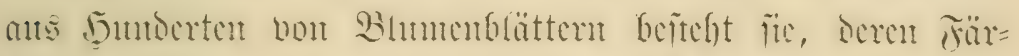

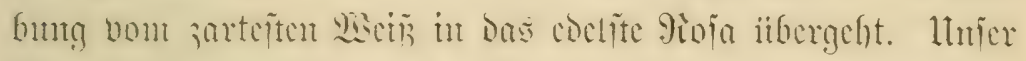

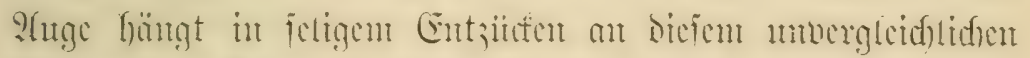

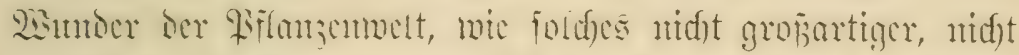

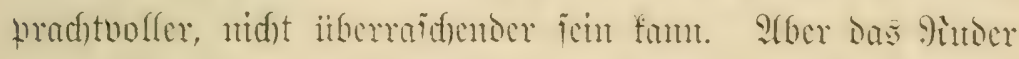
treibt mtien 9ad)en weiter atno weiter. Smmer neuen,

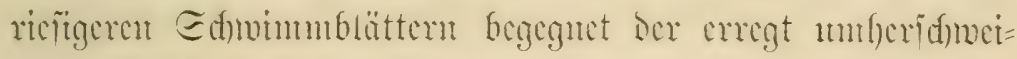

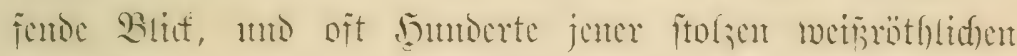

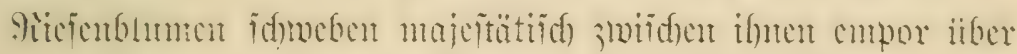

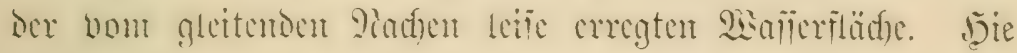

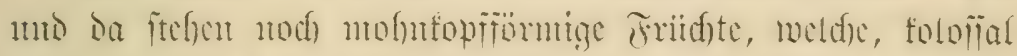

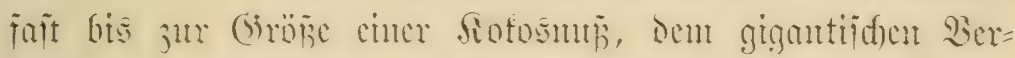

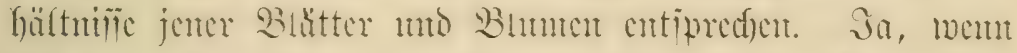

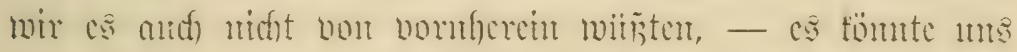

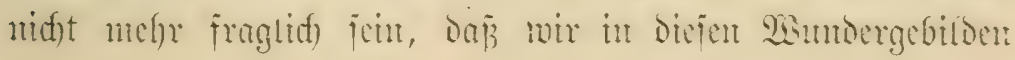


ber Riflanjenwelt bic gemaltigite mo hod)berithntejte fïnigin

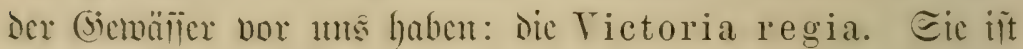

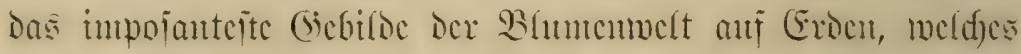

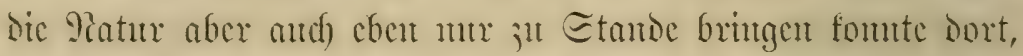

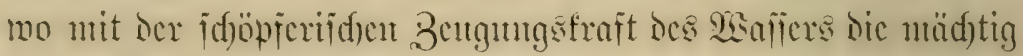

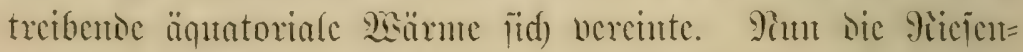
flume anf Erocu cimmal hervorgebradt wax, hat fie aber

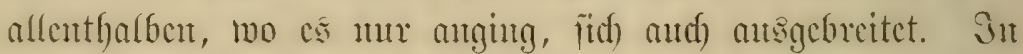
Den ganjen Etromgebicten Des äquntorialen 2 (merifa fom der

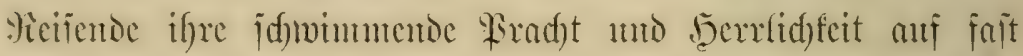

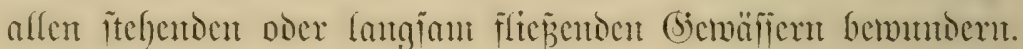

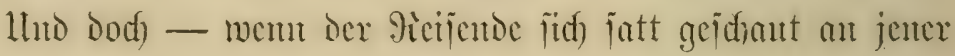

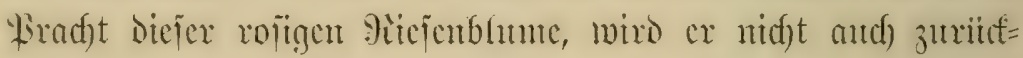
Denten miijien an ien tranten Dentjd)en Ievd), an dent dic Bumen leije ranjdyen unto wo bie fenid) Eecroje träunt. Das

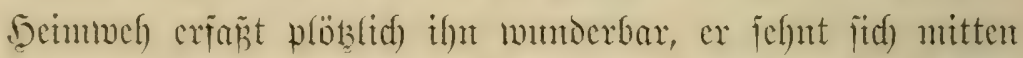

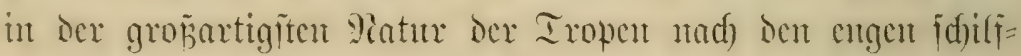

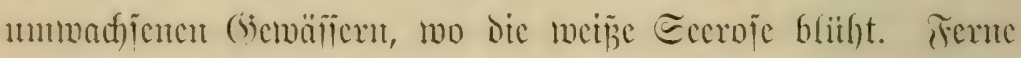

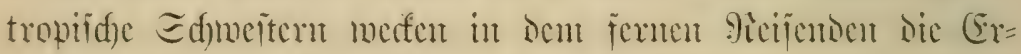
innerung der lange vergeffenen Deimath).

4.

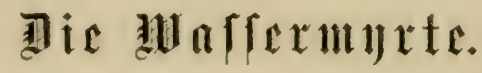

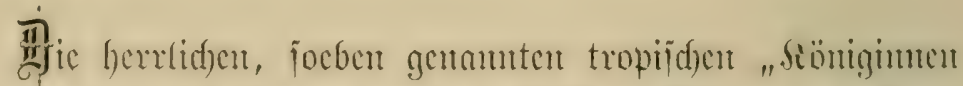

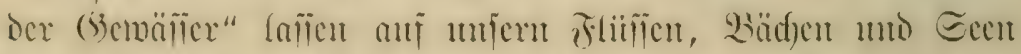

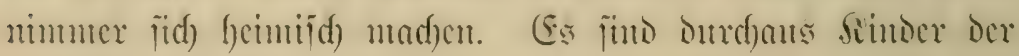




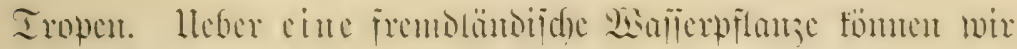

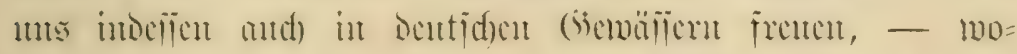

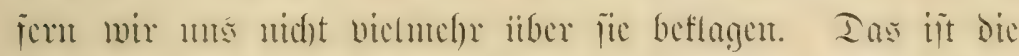

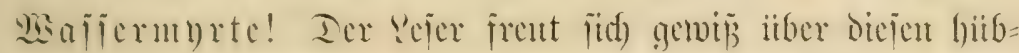

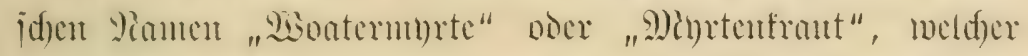

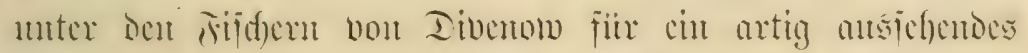

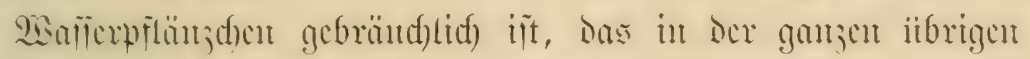

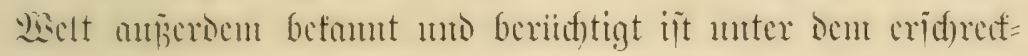

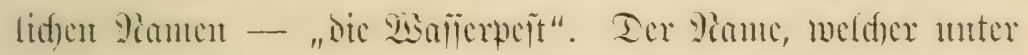

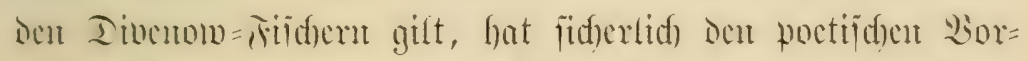

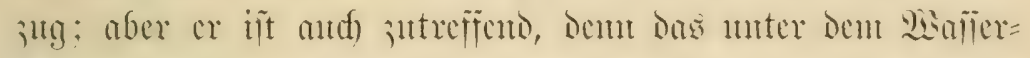

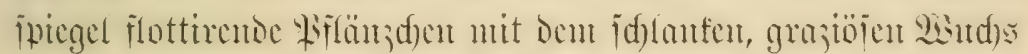

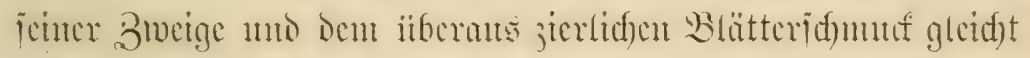

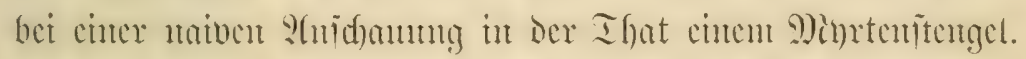

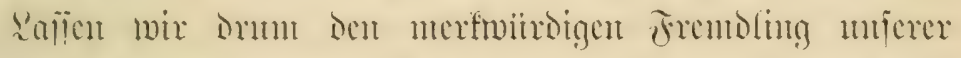

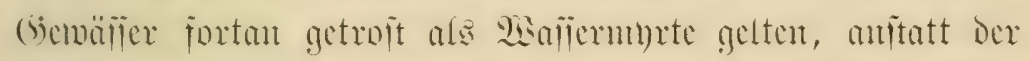

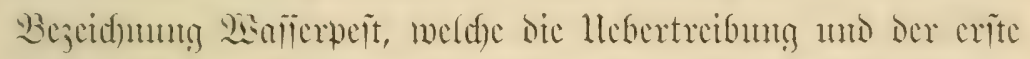

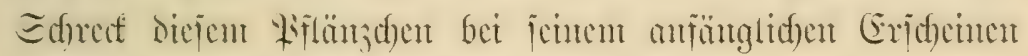

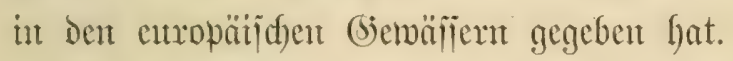

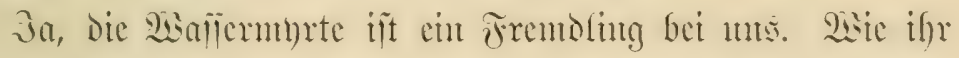
Vianc Eloclea canadensis beiagt, iit ite ans Prordancrifa,

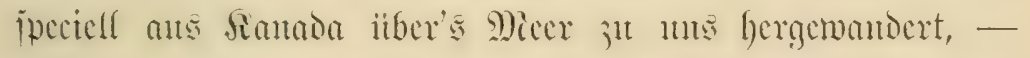

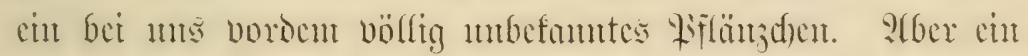

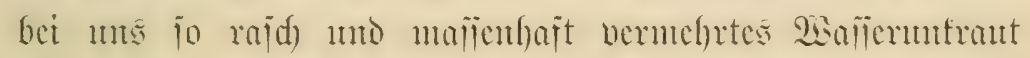

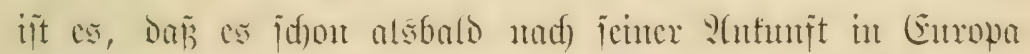

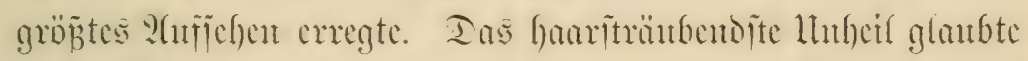

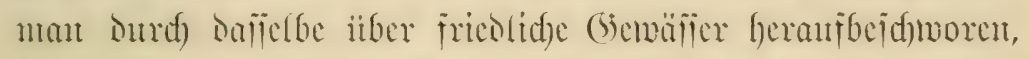

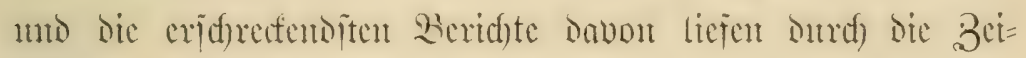
tımget. 


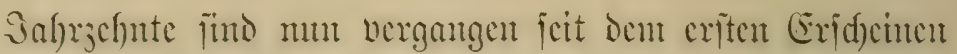

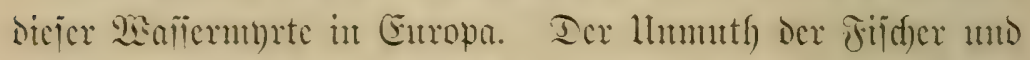
Ediffer iuber dicjelbe hat jidf menigitens ctwas gelegt, umo

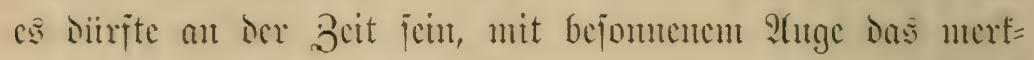

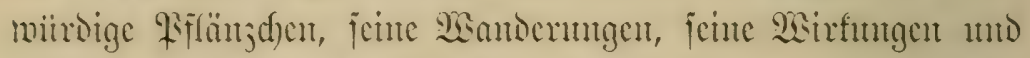
ichut ganjen (Sharafter ju betrad)ten. Mierfmiirdig wird Dic

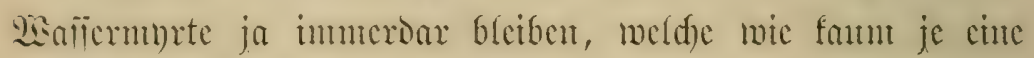
andore Fiflanje der Erose mit rapiser (Scid)mindigfeit anf cuten

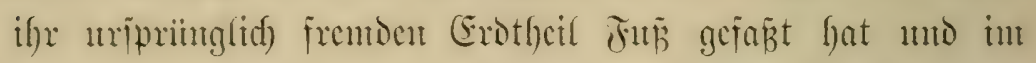
Simpi um's Dajeut jid) als unbejtrittenen Eieger itber mnere einfeimijden Hiflangen geltend madje.

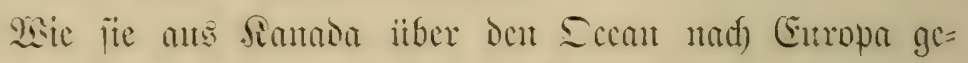

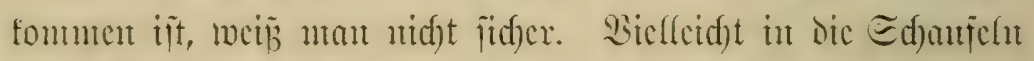

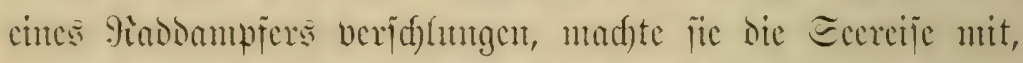

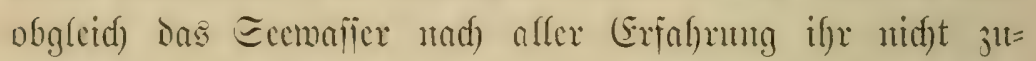

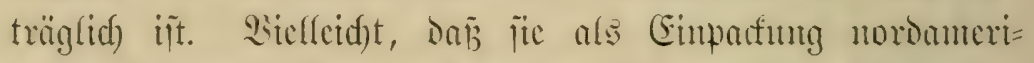

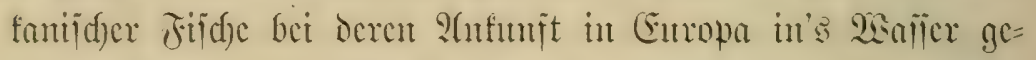
worjen murde. Ectian ift ifre lleberjicodung immerlju,

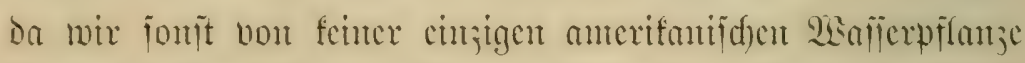

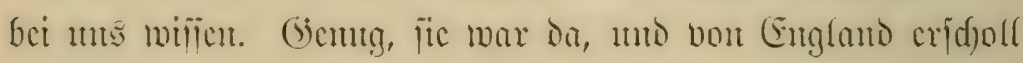

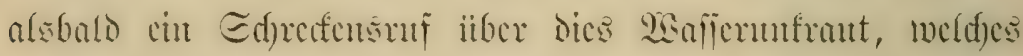
iid) mit cintem Diale in allen Budten Der. Themife, in allen Sinuälen wie in ben mit bicjen jujammenfängenten Ecen mo

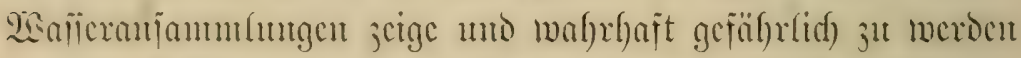

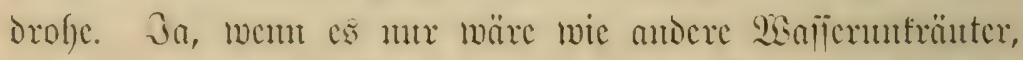

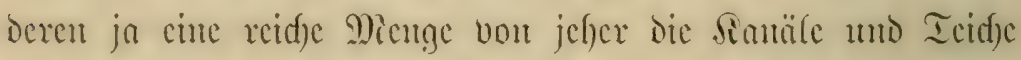

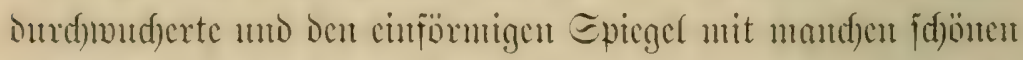

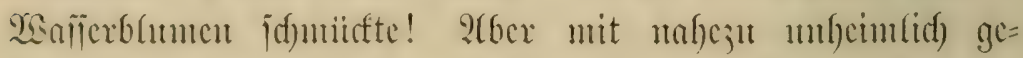

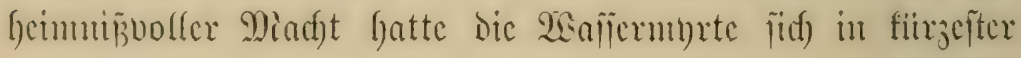




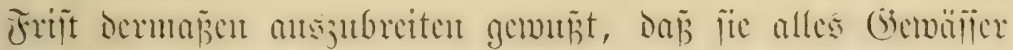

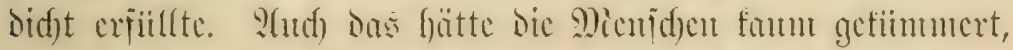

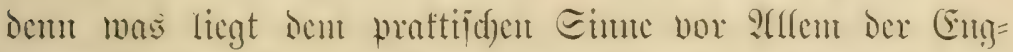

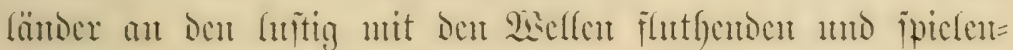

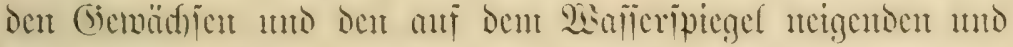

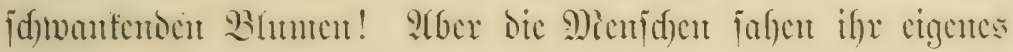

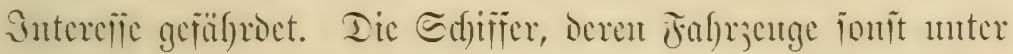

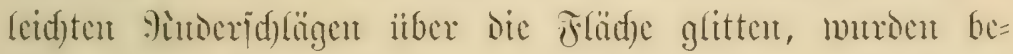

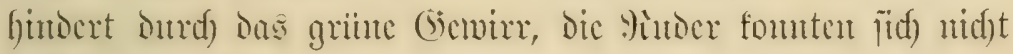

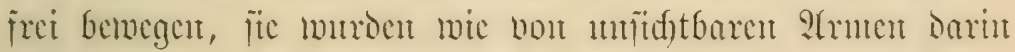

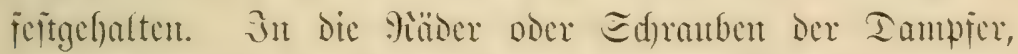
meldye die Santïle finab ifgre fendften sege jogen, wandent,

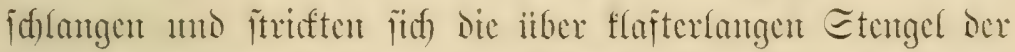

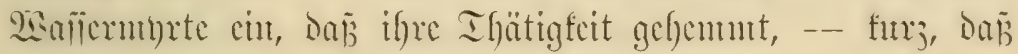
dic Sdifffahret beenträd)tigt wurde.

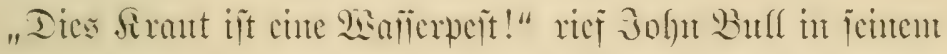

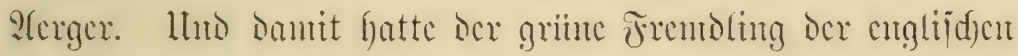

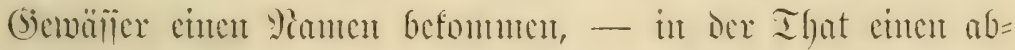

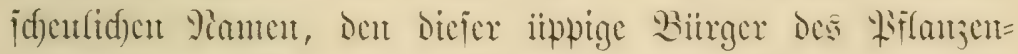
reidus jeiner (5olfijion mit den 3nterefien bex 9ianjden verdanfte.

Es hat, wie mit der Beit jid) heransiftel(te, bod) noer vid

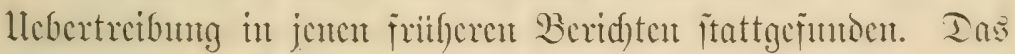

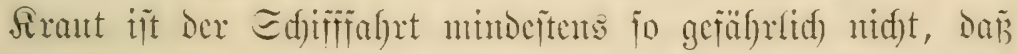
es, faum in Europa antgefonmen, mit joldyent ?lament be=

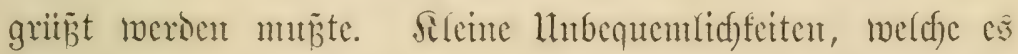

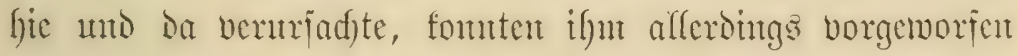
werdent. 2(ber fo find wir Nienfden! Witr bejtrafent dic Miüfe, weidfe uns jted)en will, alsbalo mit bem Tode umo 


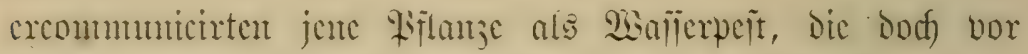

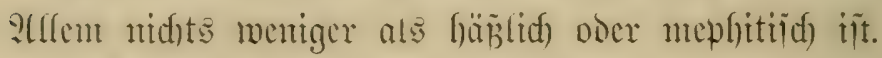

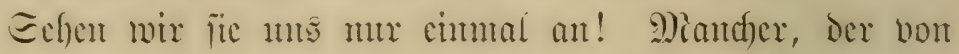

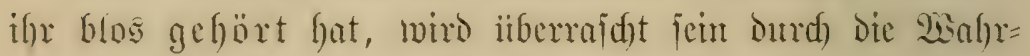

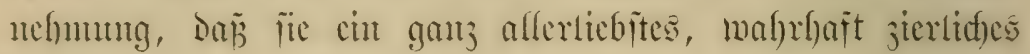

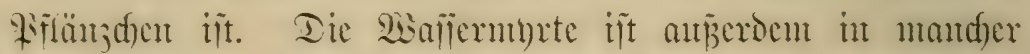

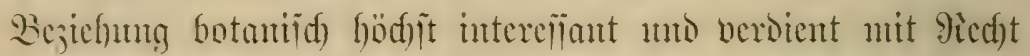

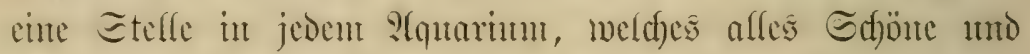

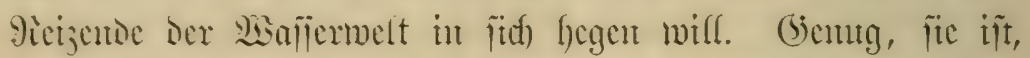
als Wiflanje jelbit betrad)tet, fiir cinten harntojen Bejuthanter

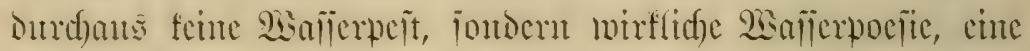

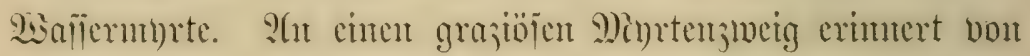

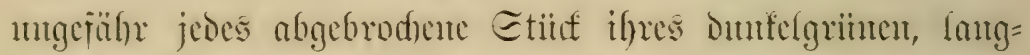

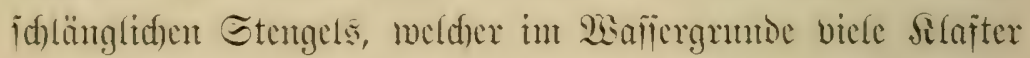
lang uns viclverjweigt fith îtrect mo fluthet. Dic Blätter

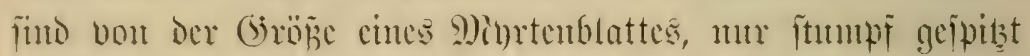

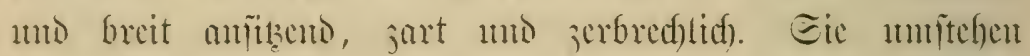
immer ju je srei wirtelig sen Etenged, mo jumar in bidfter

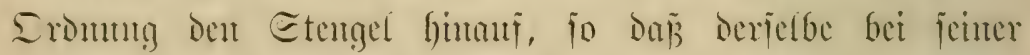

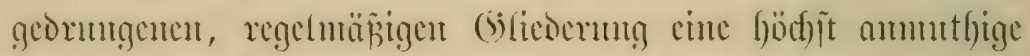

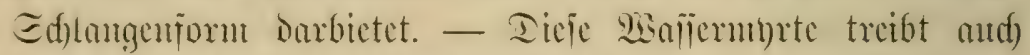

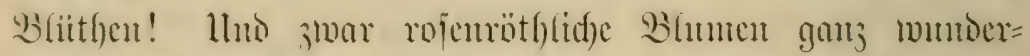

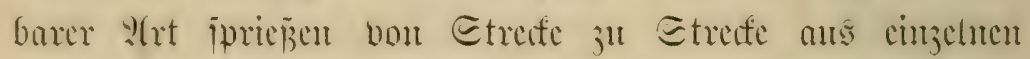
Whattadich berbor, ringen ans dent Wafier empor nad) Dem

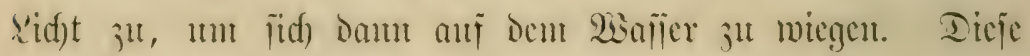

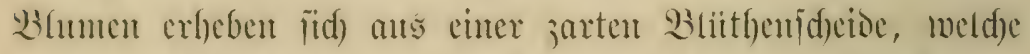

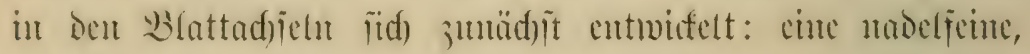

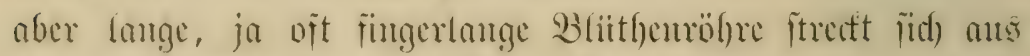

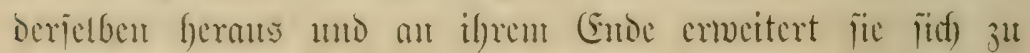




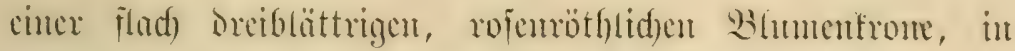

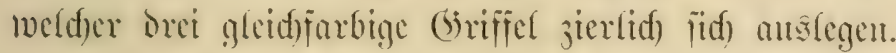

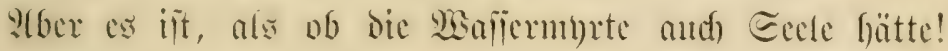

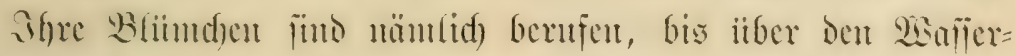

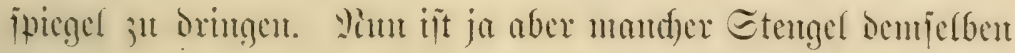

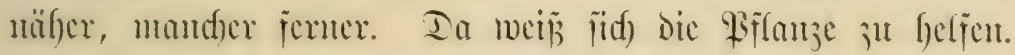

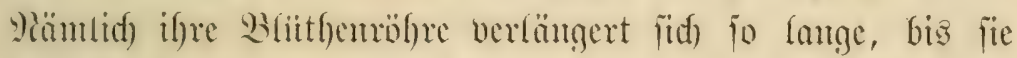

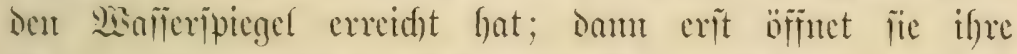

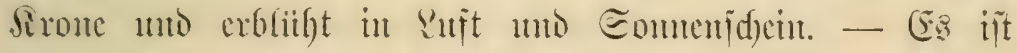

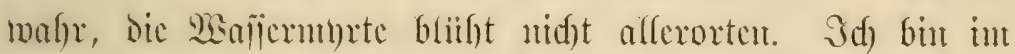

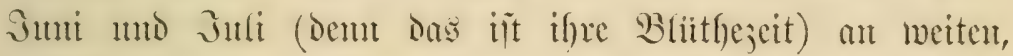

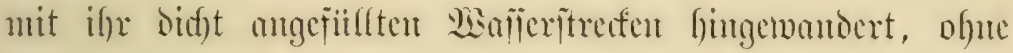

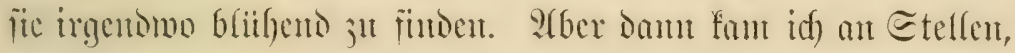

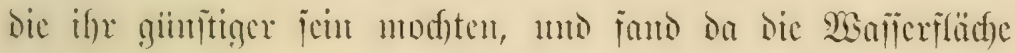

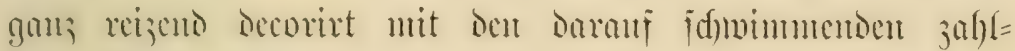

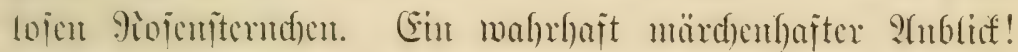

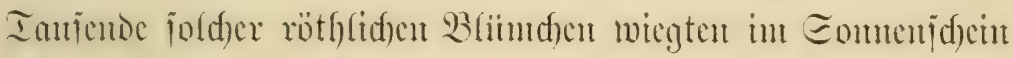
ïid) leiic ant dent flaren Eptegel, gebalten an Dem grium

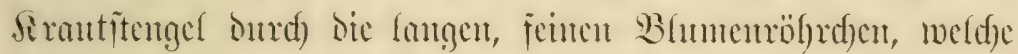

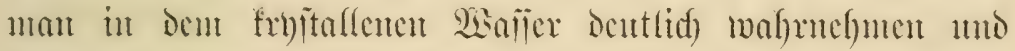
mit dent stuge bis añ das firant, ans dent jie iproźten,

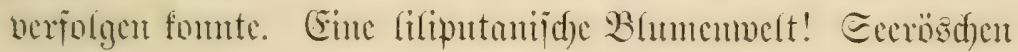

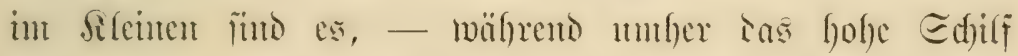

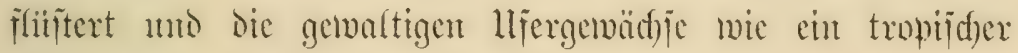
Hrtwald den majeitätijden Şintergumo bilden.

Tiur dic Siebe fehlt Der Wafferminte hicr in Europa!

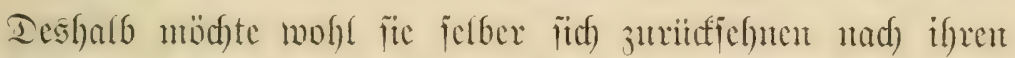
Geimiïd)en (bemäfïen jenfeit des Eccons. Eic gebört nämlid)

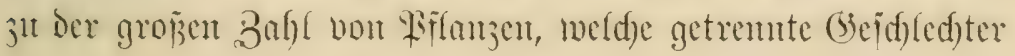




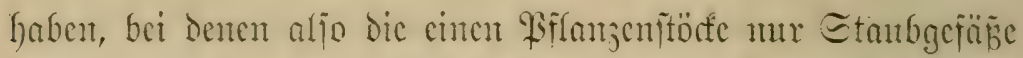

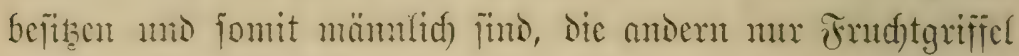

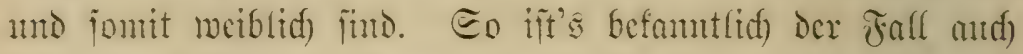
bei uniern Meiden, tiappeln, bei Sani und viclen andern minerer Bümme und Sränter. Das Editfial mu wollte,

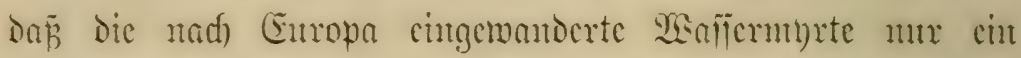
meiblid)es Exemplar wax. Plle unjere Maffermturten find Desfyalf micderum meiblicí) gemorden; nimgends nod) hat man

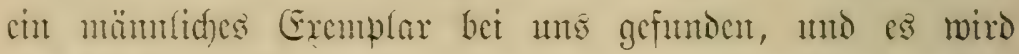

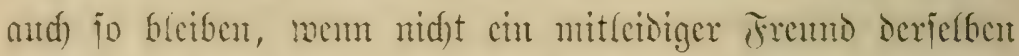

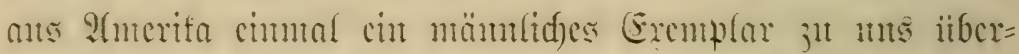
jufiif)ren unternimmt. Eo lange alfo fam fie von ber Sicbe

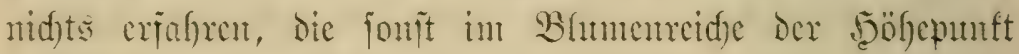
แnd das Bicl alles Rebents ît.

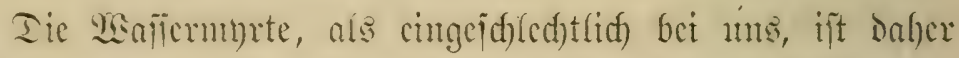

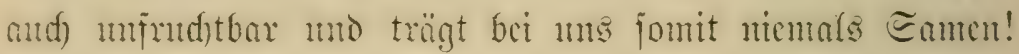

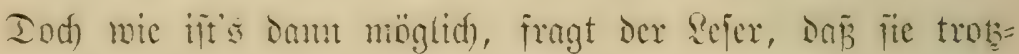

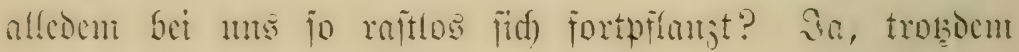
mantorte jie wie fein anderes nod) fo fantmeddes firant

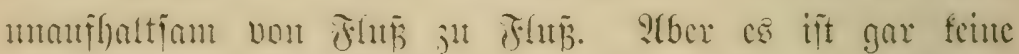

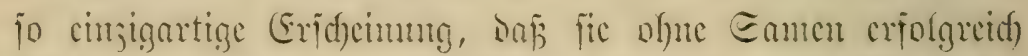

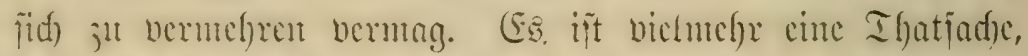

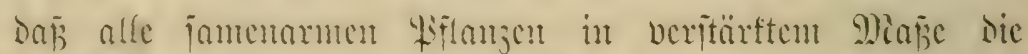
Fäbigfeit bnben, fid) surd) Eprofïmn, surd) Wurgeltriche 11. \%. 10. ju bermichen: Dic bort gejdonte Siraft fommit ber

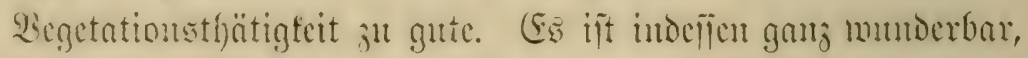

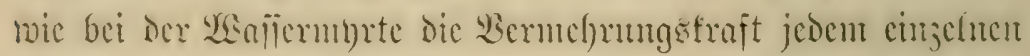

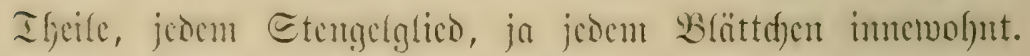
îcoes abgebrodjenc Etcugelftiiffden, mo ment ca nod) jo 


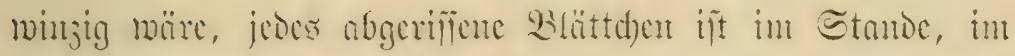

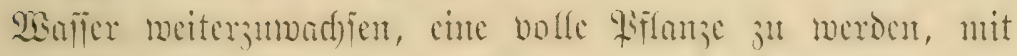

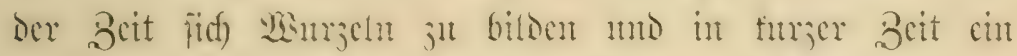

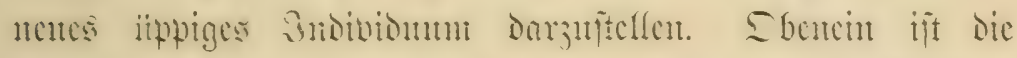

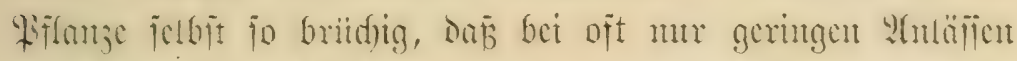

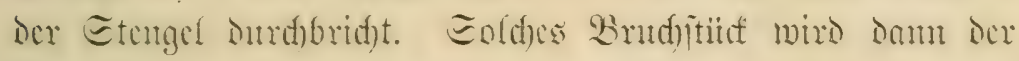

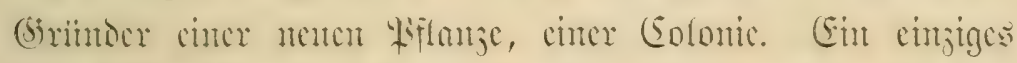

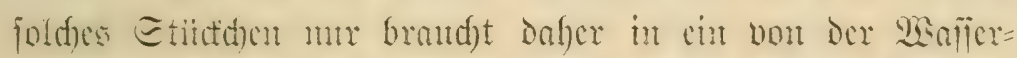

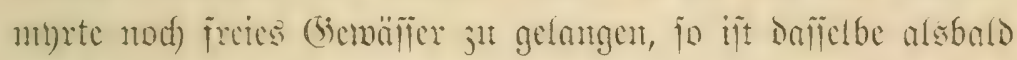
Deren \$erridhaft unterworfent.

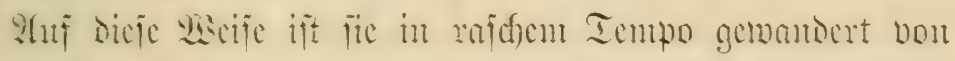
Eugland nad) Intidfland. Seicr find dic EDcr, dic Elbe mo Deren Etrongebicte alsoald mit ifgr benölfert worden.

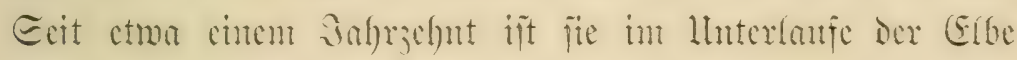
gefunden, aber ging do raid) immer weiter. Sn den fohth=

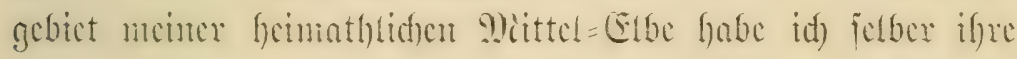

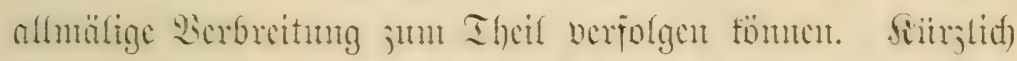
(1871) fand id) fic mänlid) andf) in Der (Brgent) Des Celb=

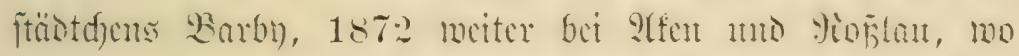

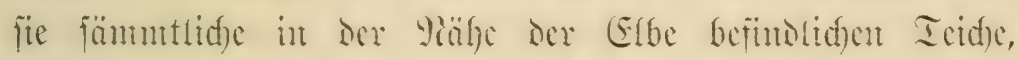

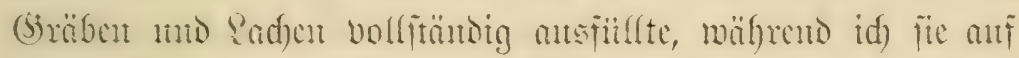

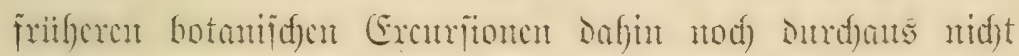

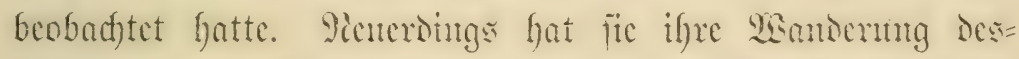
gleidyen in die Earle bincin angetreten mo fid bet Sialbe lnto auth ficjout bei Jalle gejeigt.

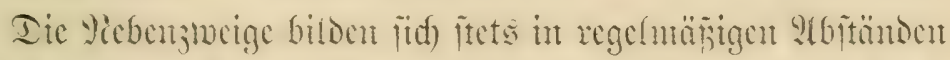

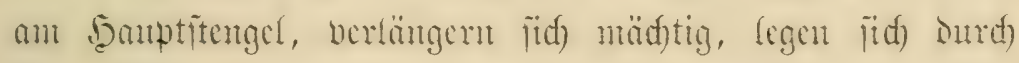

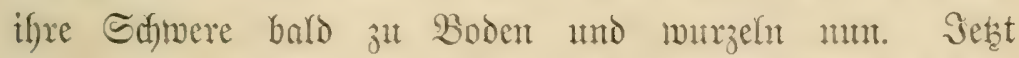

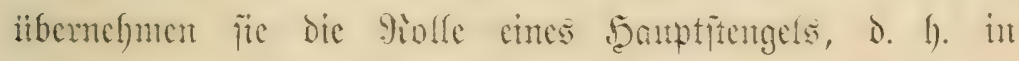




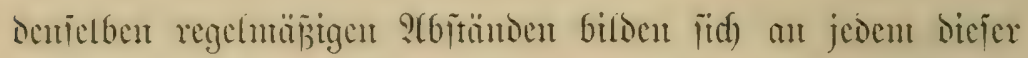
benurjelten Etengel wieder Mebenitengel, - währent aud) ber borige saniptitengel immerfort in ber \&änge weitemüd)st mo uidft mifgört, weitere Yebenjweige ju treiben. Eo gef)t

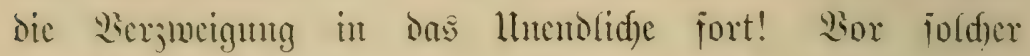
Heberid)mängltefeit mujten nothmendig fait afle andereu

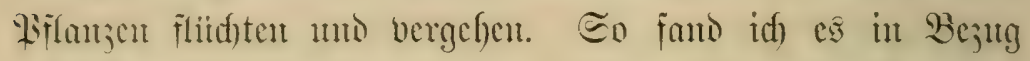

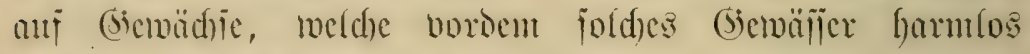
belebten: Saidffräıter uno Pinnthäen mit ifren malerijden

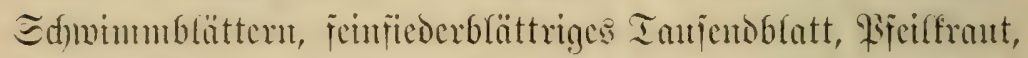

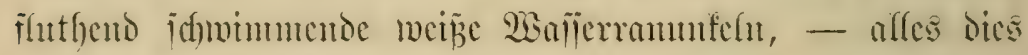

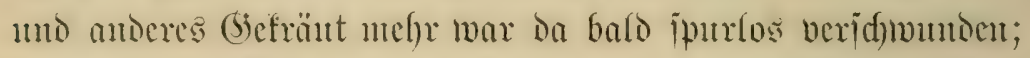

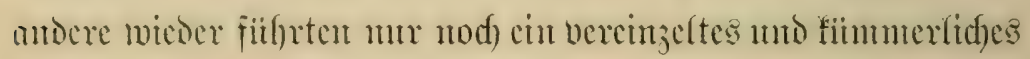

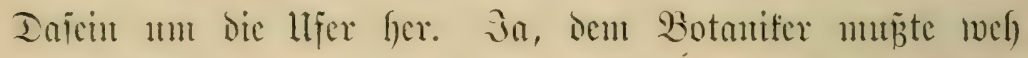
um's ber; merden, ment or io iaf), wie der fantadicte

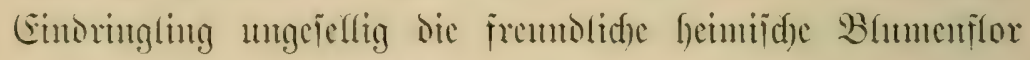

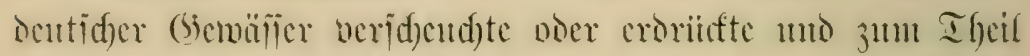
wöllig hatte berid)wintoen madyen.

Sic gemaltige, biejer Salferpflanje imemolnnende Sebens=

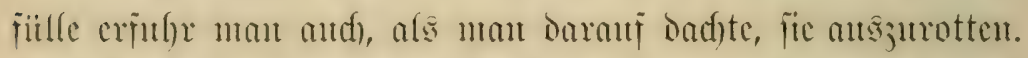
9)it Darfen jog man bie Diafien herans mo marf fic mif

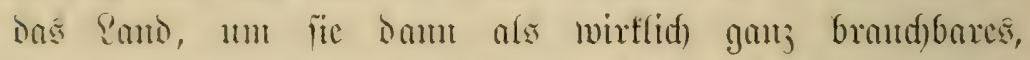

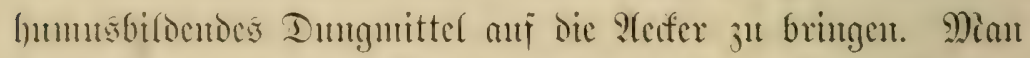

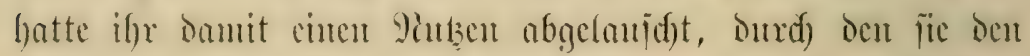

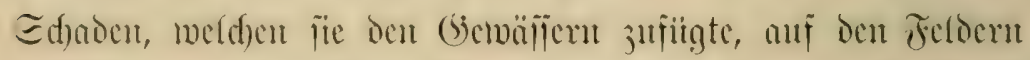
leiolid) wicder ansiglid). afber wenn mon gement batte, fic jelbit Dadurd) mit der Beit ju weruidfen noer bod) ani

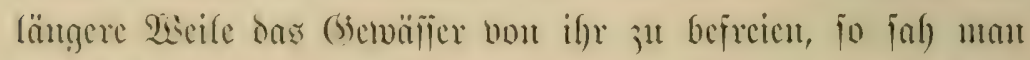
bas bald als ciuc citle Iäuíd)ung cin. Eelbỹt alfem jafbres= 


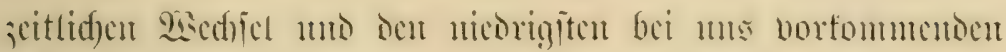
Iemperaturgraben bot jie unbermilitlid Irob; bis in den

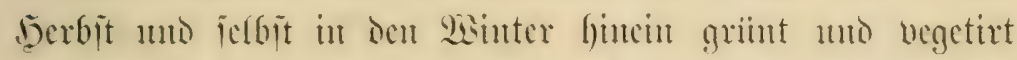

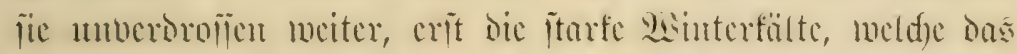

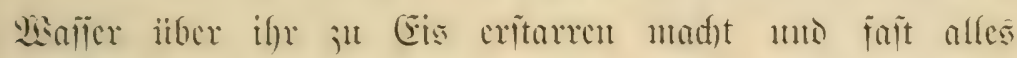
organifde ecben hemmt, ruft and) if)rem 2isad)sthum cin

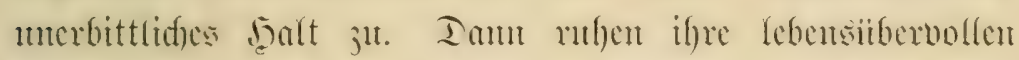

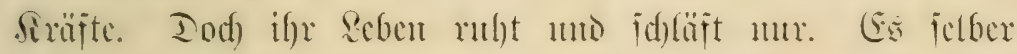
bergeft nidft, ja in irijdgriumen Buftante berbaret die

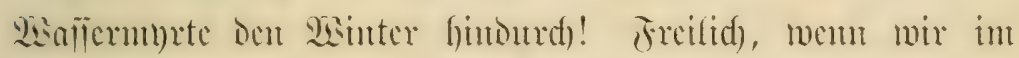

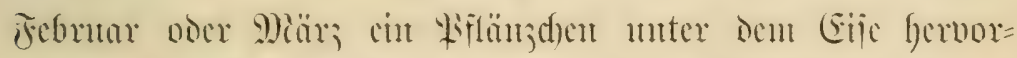

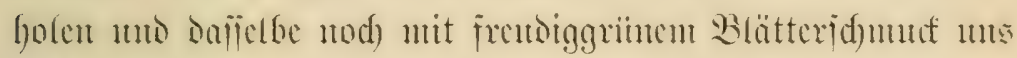
anidgant: jo ijt bas (briuleben menigitens ber Blätter bod) me

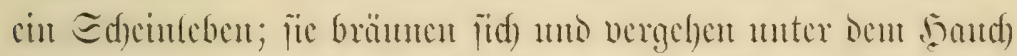

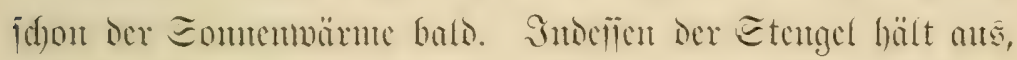

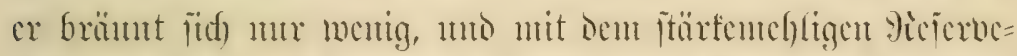

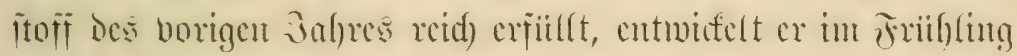

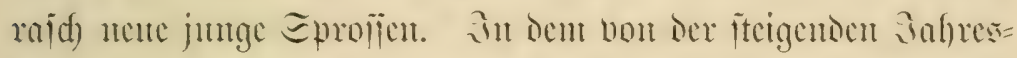

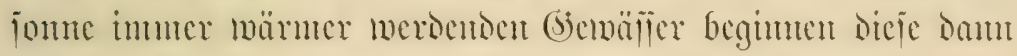

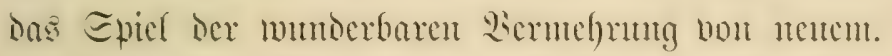

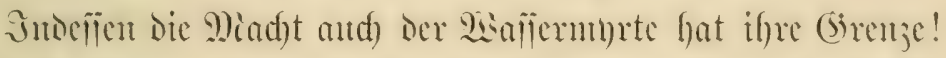

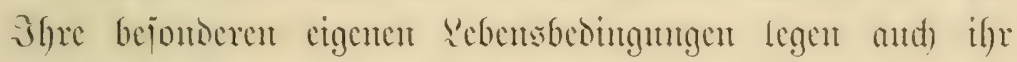

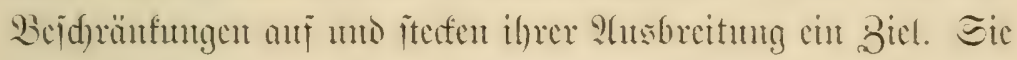
fann nünt (id) unr geocifen, ober menigitens fo iippig unt geocifen,

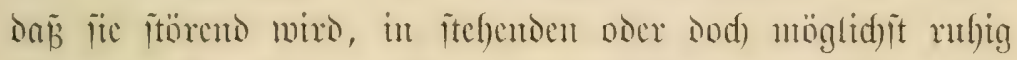

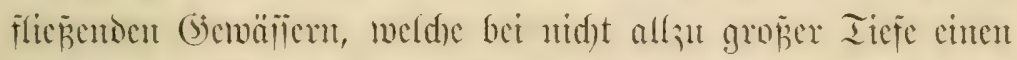
id)Lanntigen (brunto haben. Darum fïn die rutgigen fimäle,

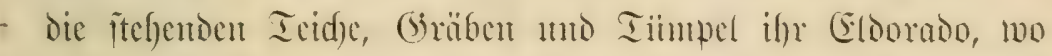

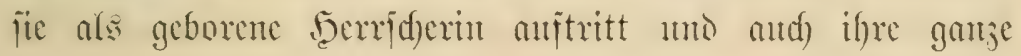




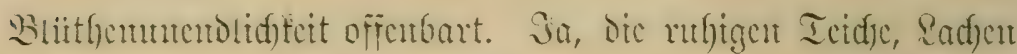

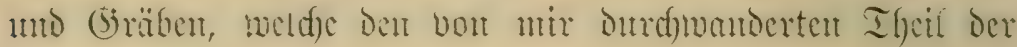

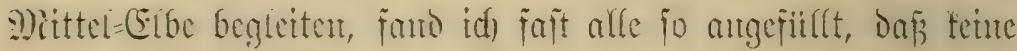

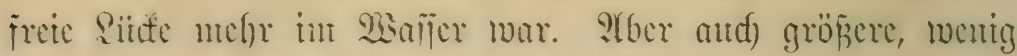

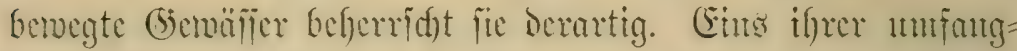

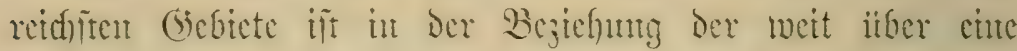

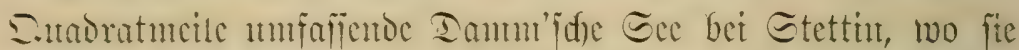
$1866^{\circ}$ juerft auftrat ut in rajdem Siegeszuge bald fid

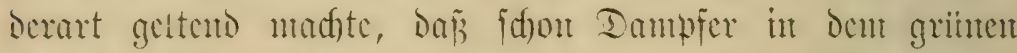

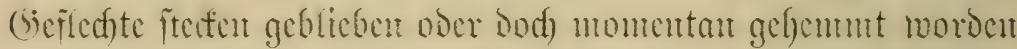

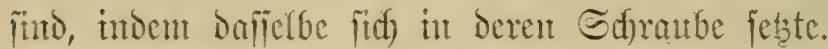

Shre Diad)t ift Dagegen gebrod)en, wo nut eimgermajen

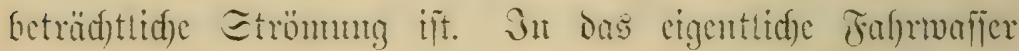

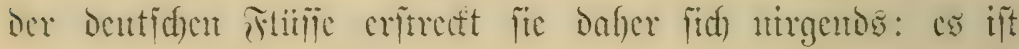

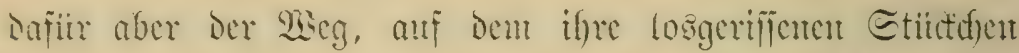

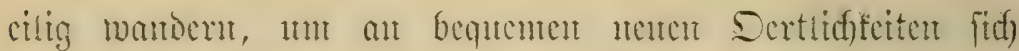

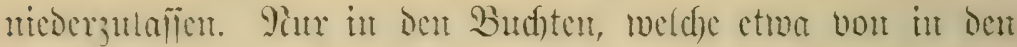

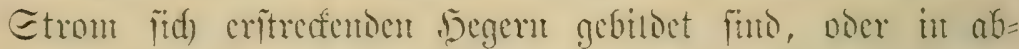

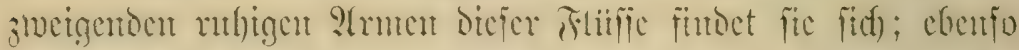

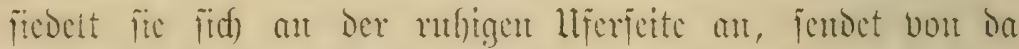

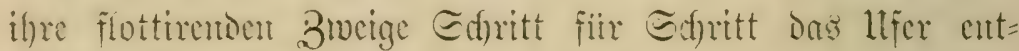

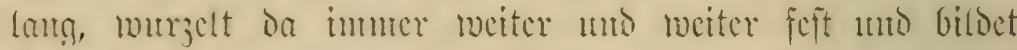

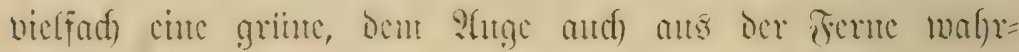

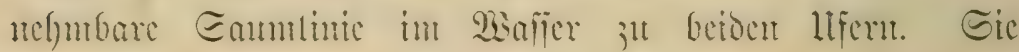
wandert mud) da gleidjan, bis fie anf eine biudgt trifft, wo

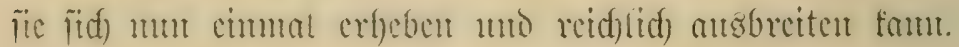

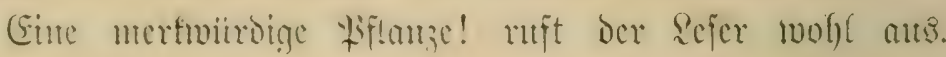

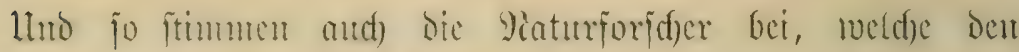

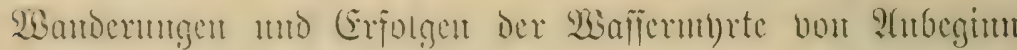


nad)gegangen fints, dic bor ctwa 40 Salmen in Europa $\mathrm{cr}=$

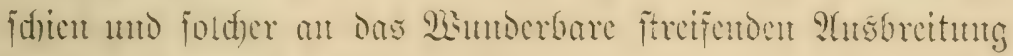
fidf) mut rilfment fantu.

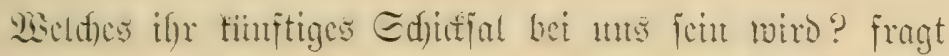

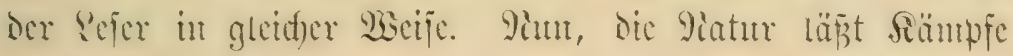

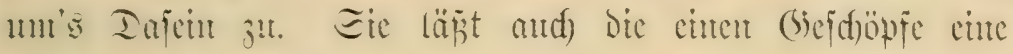
Beit lang cimmal gemaltig bonimimen. 2foer dann pflegt fic

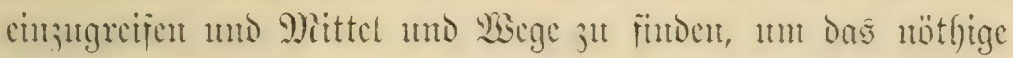

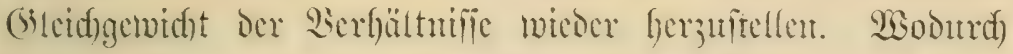

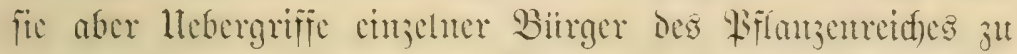

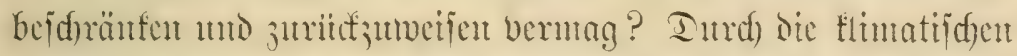
Buttünde cutestheils, Durd) bie Ihierwelt andentheils; Denu fait jede Siflanje bat and ihe befonderen Jufaffen, Wager

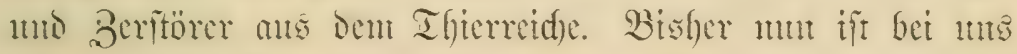
allerbings kem Ificr befant, weldes ber 23 afjermerte nadffelfe.

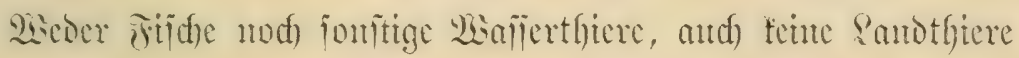

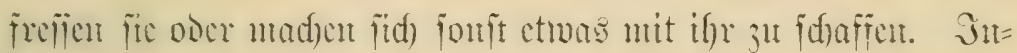
sellen ift die Frage jdon anfigemorfen, jedod) wohl nod) unbeat =

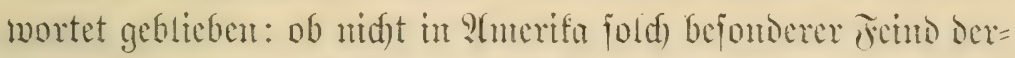

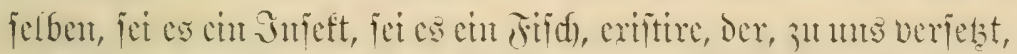

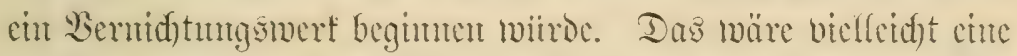

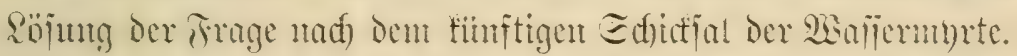

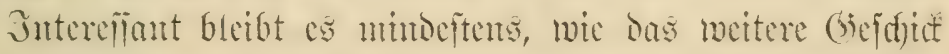

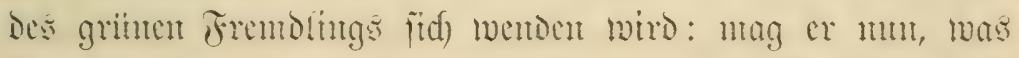
famm Dentbar, feinen Eiegeșug untgefdumälert bei uns fort= febent, oder mag or Durd) Imborbergejefent llmifümoc bas aud) it oer yatur geltento aite 2 bort an fid erfalyren: est modus in rebus! D. h. "Afrem iff eine (5renze geictst!" 


\section{5.

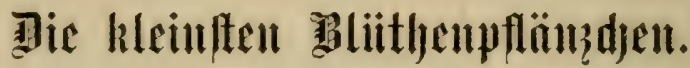

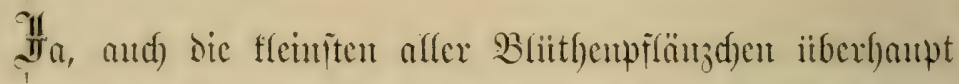

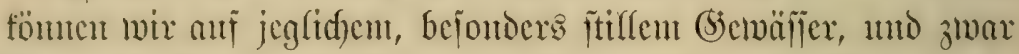

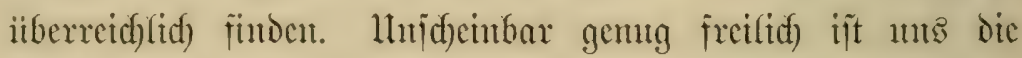
Wafjerlinje (Lemna), welde dannit gemeint fein foll.

(5icbt es fiir cin :Tquarium cinen unbeocutenderen pflanz=

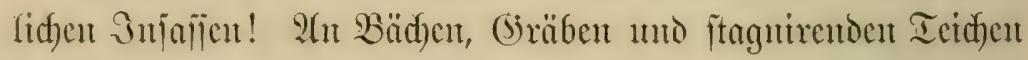

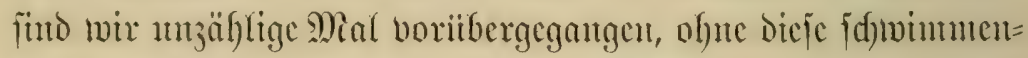

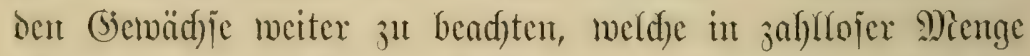
oft sen ganzen Waflerfipiegel iubergriunen. Entenfutter! Benfen

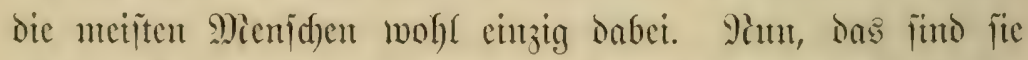

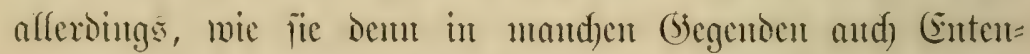
griun oder Entengriilze heipen, meil bie Enten gar gierin fic

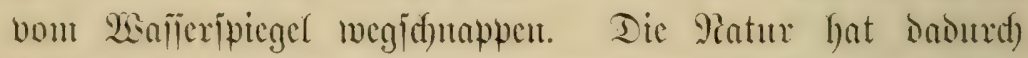

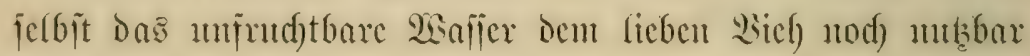
gemad)t.

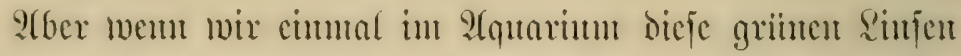

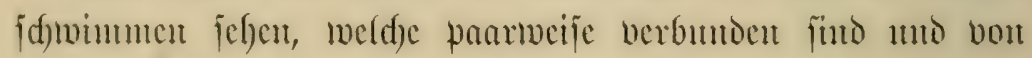

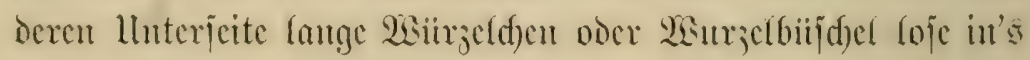
Sanjer fä̈rgen, ofjue irgentowic feitgemurzalt jut fein: fo $\mathrm{cr}=$

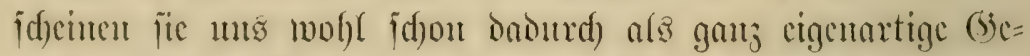

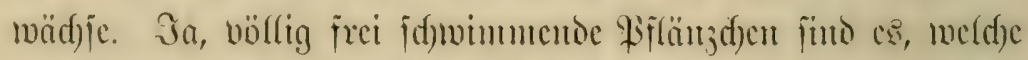

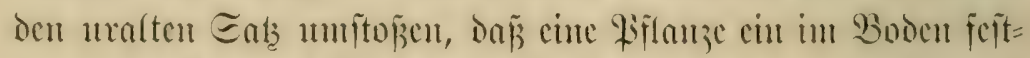

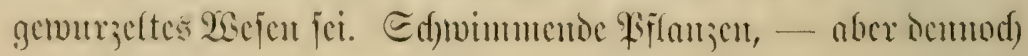




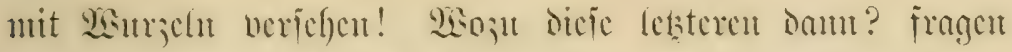

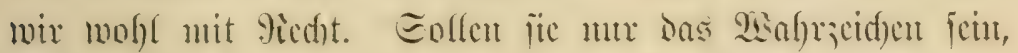

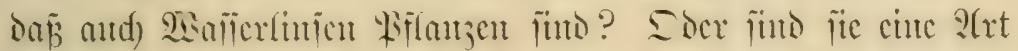

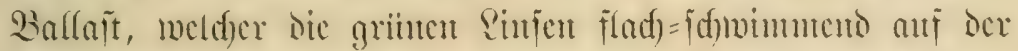

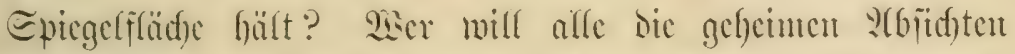
cruthen, welde in Der Platur Durdagchendos malten. Sijt Dod) felbit mandfes nod) meit flemere (bebiloc jo munterbar be=

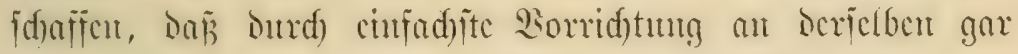

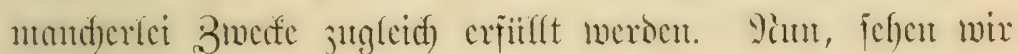

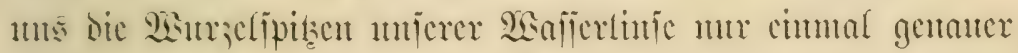

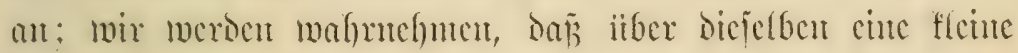

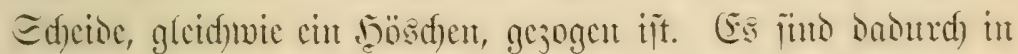

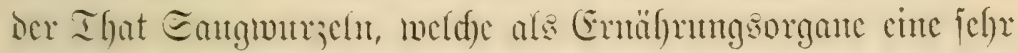

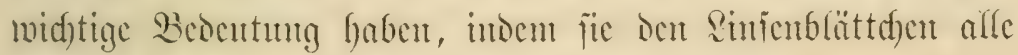

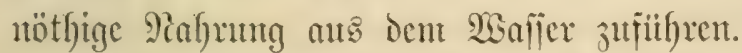

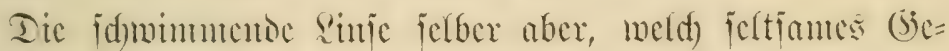

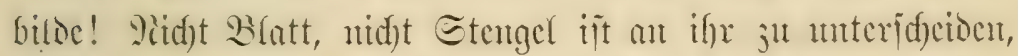

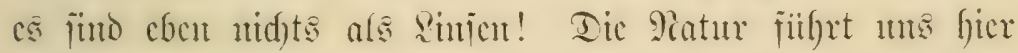

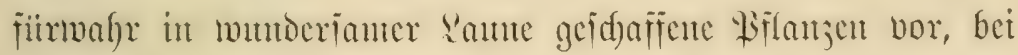

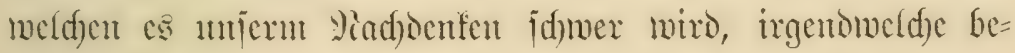

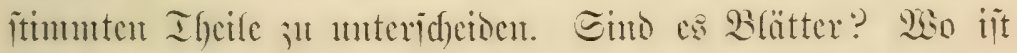
Deren Etengef? Dentod) ift Beiocs, wentgitens Der Stulage

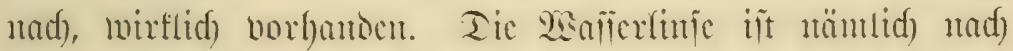

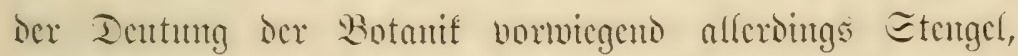
blattîlad)ex Etcngel; nid)t alïo Blatt, wic mir meincu möd)ten. Dent co ijt ocr Egarafter cunjig cines Etenge(s, an ocr Eptice

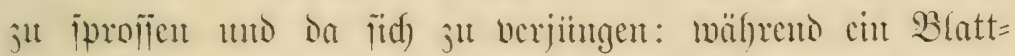
organ itets un an feinem (brunto mäd)st. Wacut wir aber

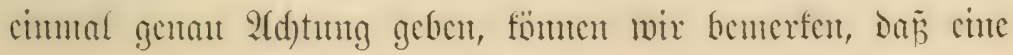




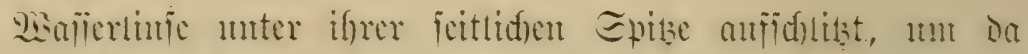

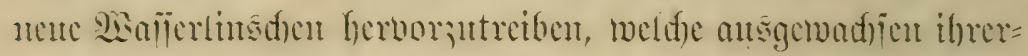

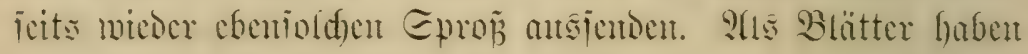
wir bagegen mu den ïber dem Edhlis befundidfen, fanm

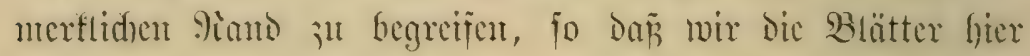
cben als faum worhanoen, wentgitens als börlig unentwitelt bezeid)nen miitīen.

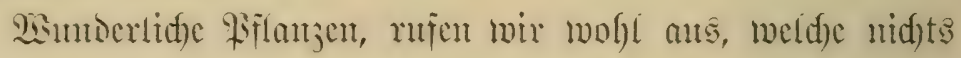

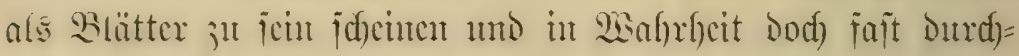
ats nidyts jund als Etengelgebiloc! ?tber unjere 23ajierlinje

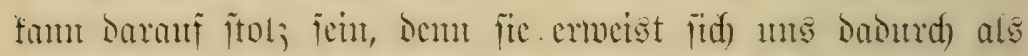
uahe afnuermante ciner hodjeden Biffanjenfantie. Itno jwar

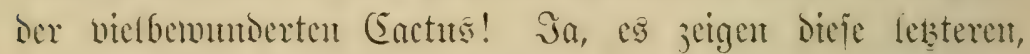

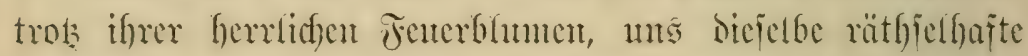
Thatiad)e Der Blattlojigfeit! Jn oer mumocrlidsen Etad)el=

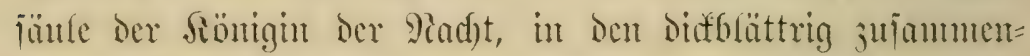

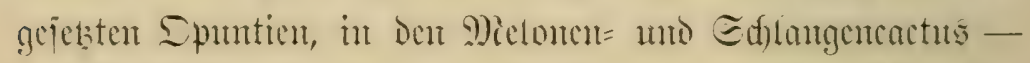
in alfen sicien io muntierlid) aufgebant abontenterliden (be= mäd)ien waltet cben dafjelbe (bebeinntib, als bei ber id)lidetent Waj̄erfinje mïerer Gräben and Tïmpel.

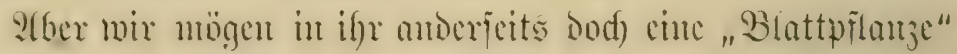
erfemen, ment wir darmuter cine foldse veriftehen, weldye nie

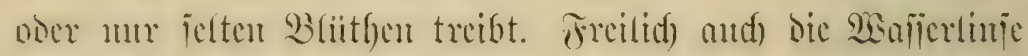
bliift, wie cin jeocs phanerogante (benüd)s, ijt in ber That

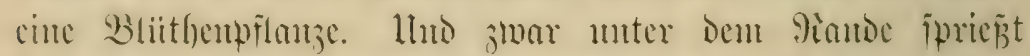

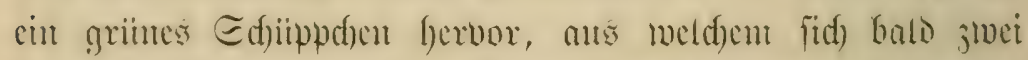

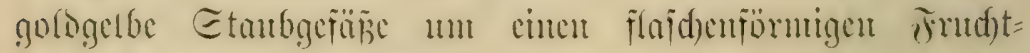

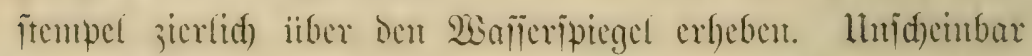

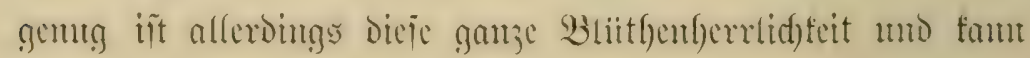




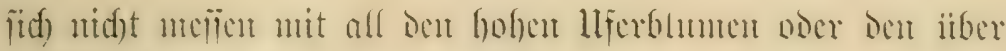

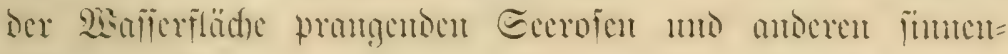

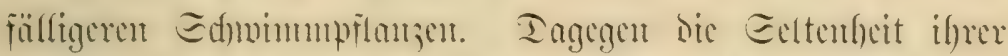

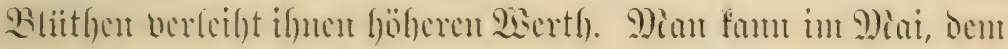

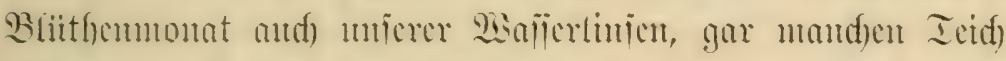

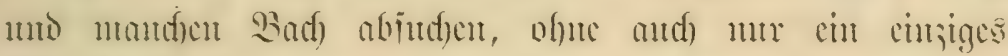
Blithd)a jut treffen. Eogar bick crfabrente Botanter be=

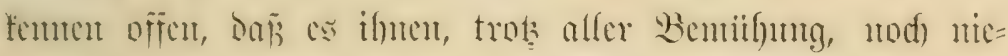

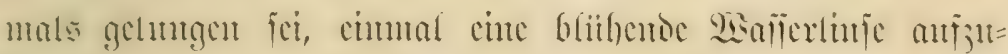

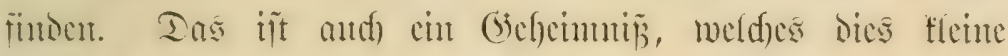

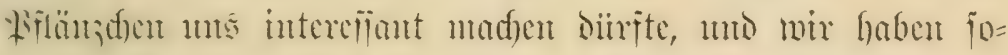

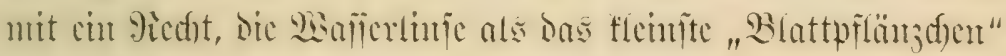

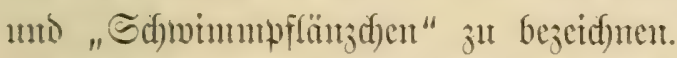

Edfon bie in allen trägen (bomäliem gemeinen 2 (rten Lemna polyrhiza uts L. minor) offenbaren uts fo mandje intereïante Ecite ihres flemen unbead)teten Sebents. Miande

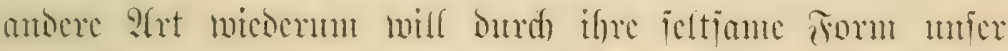
Etanten cregen. Sis giebt beren ja mefrere in unferen (biräfen anto Teid)en, dic mir alfejanmut als red)t origumele

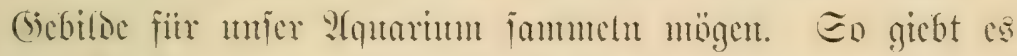

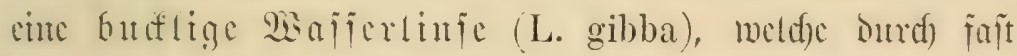

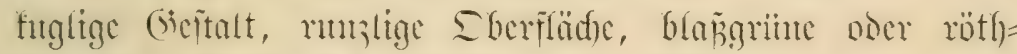

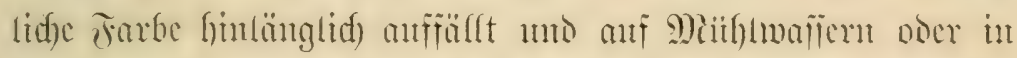

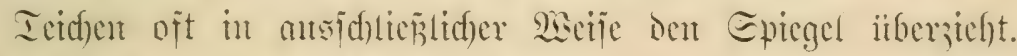

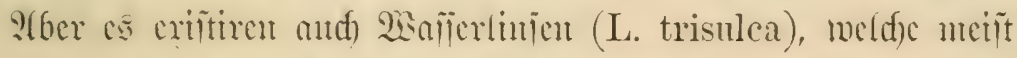

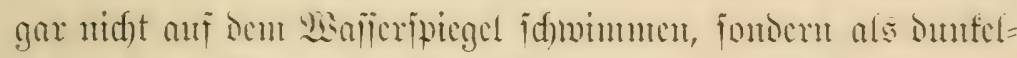

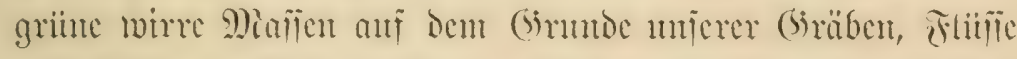

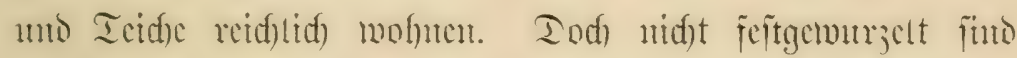

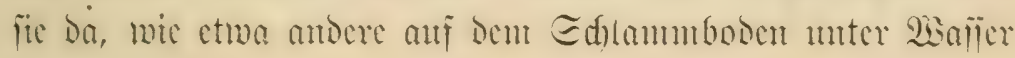




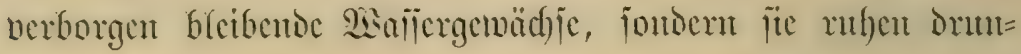

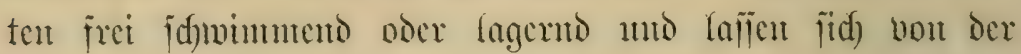
Etrönnmy belicbig treiben. Ilno eme gar originelle Form

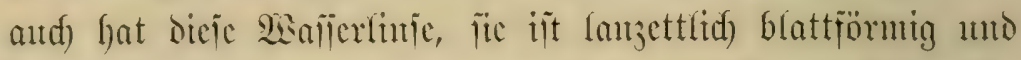

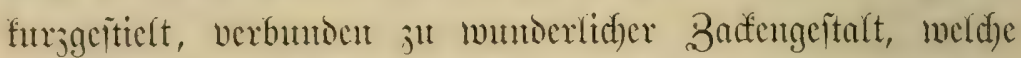

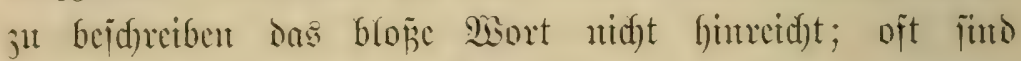

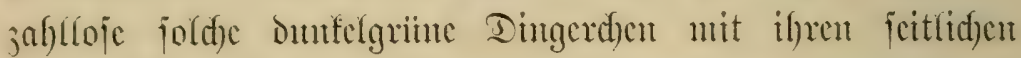
Eprofïntgen nerbumocn geblicben, füt mit Den Eptben fettenartig oder majdig, frews= oder antermeije verwadjen anto itelfen Daburd) abentenerlidfite Srdent uts Sterne dar, welde

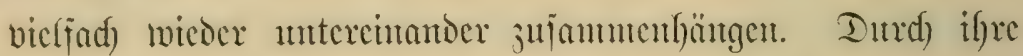
ganje Cigenthiumlidffeit legen fic ntt: wohl Die Frage unfe, ob wir es hier wirf(tid) nod) mit Siffanjen ju thut haben,

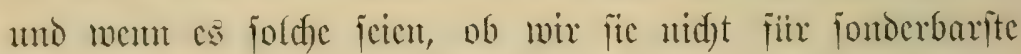
Âtgen Galten miitijen.

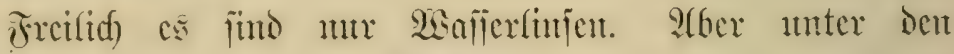
pflanjlidfen Wumbern ber Wafjermelt find fic das geringfte bod) nid)t! 


\section{VI.

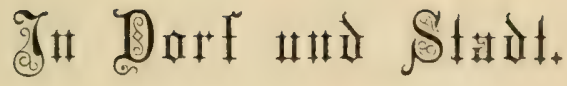

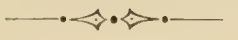

Uus der ferne fieht fichon das Zirthlein entpor, Und jwif̧hen Rüftern und Weidengeheg Derftedt fith das Dorf nod, bald blift es hervor IIno nimmt uns auf nach Dem itaubigen $\mathfrak{W}_{\mathrm{eg}}$.

p. fi. 
$\mathfrak{W}$

ald und Bergeshöhe lodf Manhen vergeblid in's freic ober gar in die jelnnjühtige blaue fertse. 2lber bis jum nähjten Dorfe mandert dod Jedcr

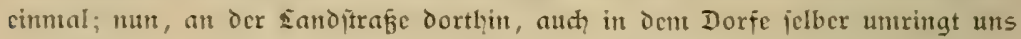
cinc Pflanjenwelt von io cigenartiger und reidher 2luswahl, dab wir uns fhon

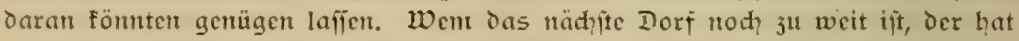
Dod aber vielleid̨t cin freundidies Färthen hinter bent Fauje; aud da blüht auf Den juberen Secten junt grof̧en Theil cine ocutjđe flor, die uns wenig= jtens int Geijt in dic Walder uno auf die Berge führt, von dencn dicje Blumen uriprünglid framtmen. Ja, nod in jedem Gofwinfel und in den Strafeneten haben fïh wildwadjicnde Blüthenpflanjen angefïdelt, fo dab wer in erigfte Ecbensverhältniffe cingcjhlofien ift, nod in nähjter 2iähe dod Jutritt hat 3 u

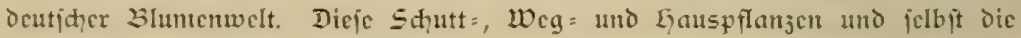
Gartenblunten mahen freilid unjere Srujt niht jo weit, wic dic Blumen in Walo und 2tue; aber bei näherer Zenntuif reden aud fie ju uns mit oer ciner jeden Pflange und jo aud thnen cigenen, fimtigen, gemüthpollen Spradį. Man leme dicje Epradic nur cinmal veritehen!

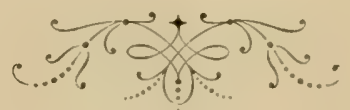




\section{1. \\ Dir (5etrenen des Dorfes.}

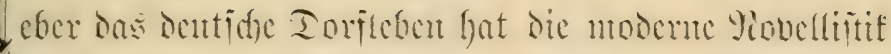

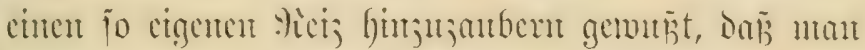
beint Sefen unferer trefflidyen Dorfgejdidaten bas

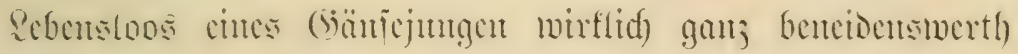

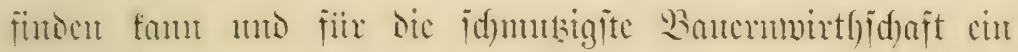

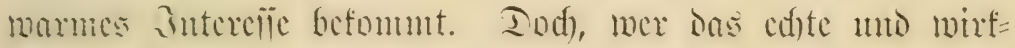

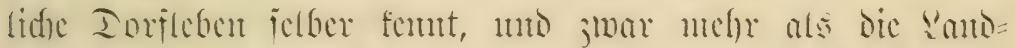
wirthidaft, melde sa getrichen mirs, sie lantomirthidaftlidgen

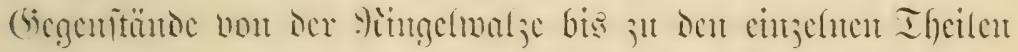

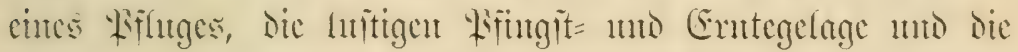

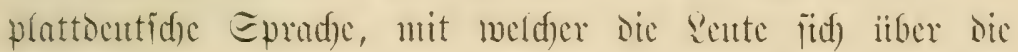

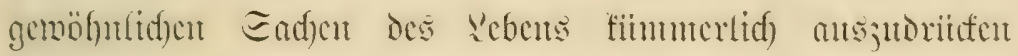

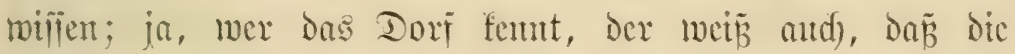

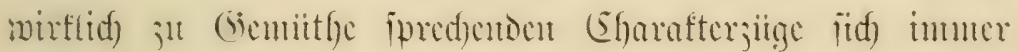

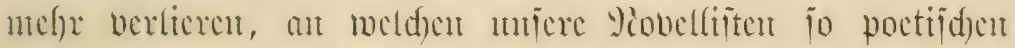

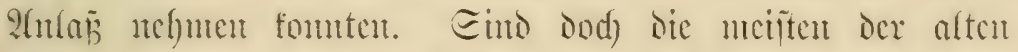

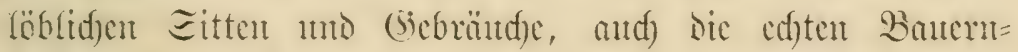

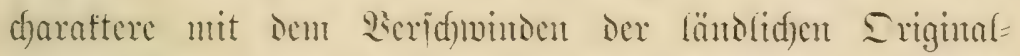

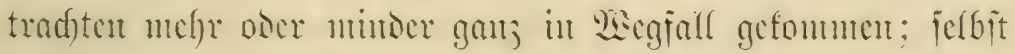

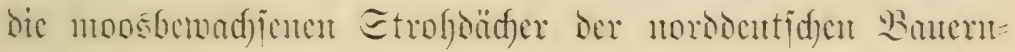


häujer merocn nach Folijciparagraphen cuergij(f) berboten,

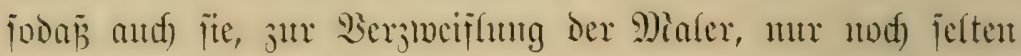

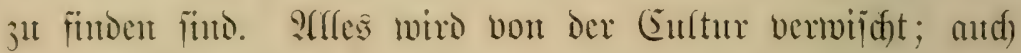

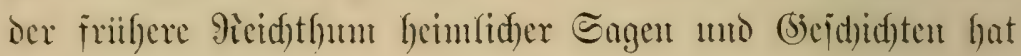

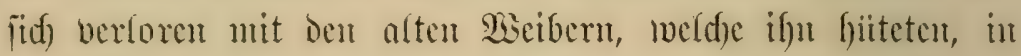
Don langen Sinterabento den anflyordyenton fintorn fie er=

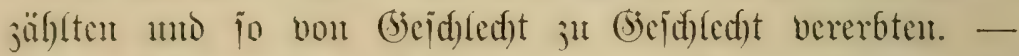
Heber alf biejem vedjefmoen Seben mo Treiben ber menjd)= liden Bewohner des Torfes nadht aber nod) cun anderes iidf geltens, Das Hein Dorfgejdidfter crmäfnt, mo meldes

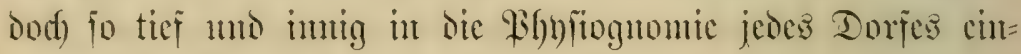

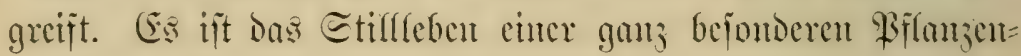
welt dajelbit.

Sa, es gibt cine Dorfirlora! Lho jwar ijt fie eine ganj chrenwerthe, an welder Das Epridfwort nod) feine 23 ablorbeit

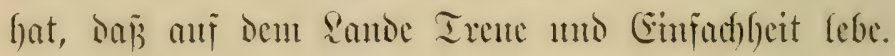

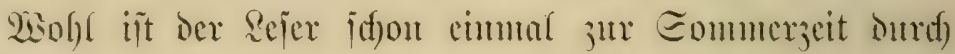

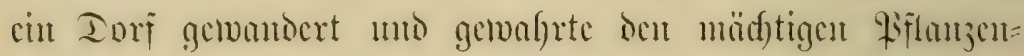

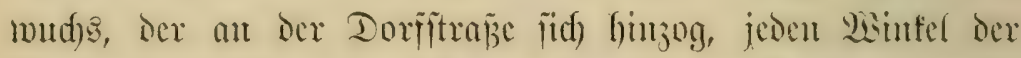

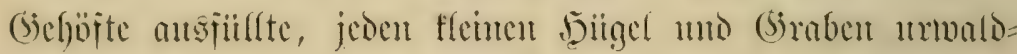
artig iibermuderte. (Sicualtiges llufrant war ca ifm, mo

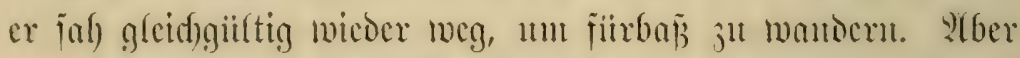

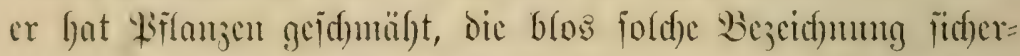

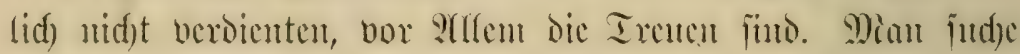

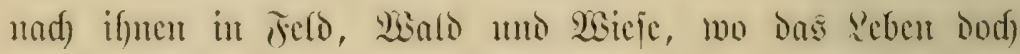

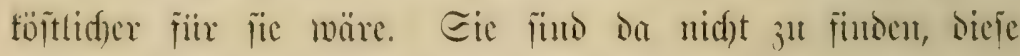

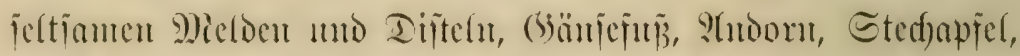

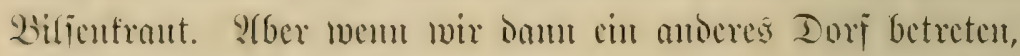

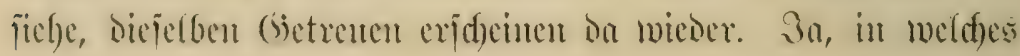




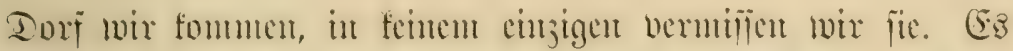

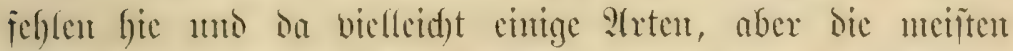

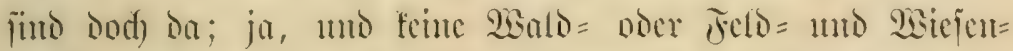

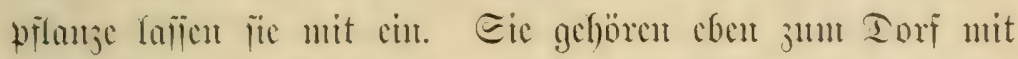
allen Fajern ifgres sebens, wolfen nurgends fourt cinc Etätte

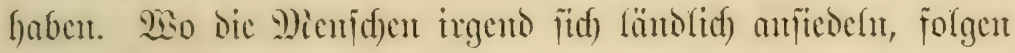
fie ifneu anf ber Ferje nad). Eelbit itber den Decan fint

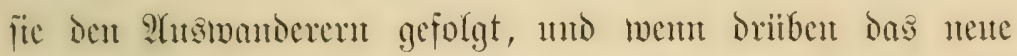

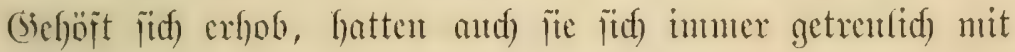

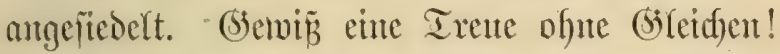

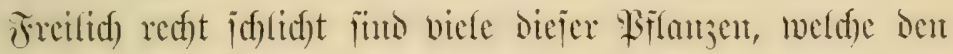
Torimeg ïppig, frantwalontig begleiten, dic meiften in mm grimer ooer grangriner B(iitgentrad)t. 2(ber d)arafterboll, junt

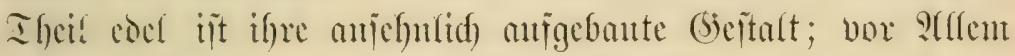
bafou je jajt jämmtlid) einen gemeinjamen, jun ganjen Wejen

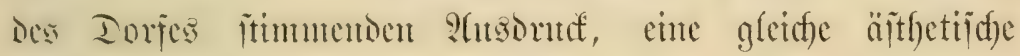

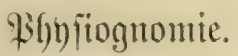

Eefoen wix fie mu eimmal an!

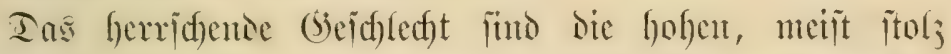

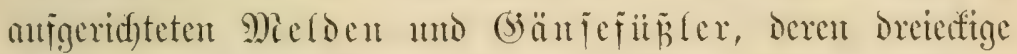
vocr rantenförmige, diffe Blätter anf ifrer lluterjeite mit

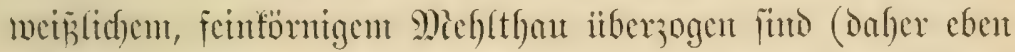

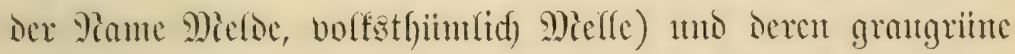

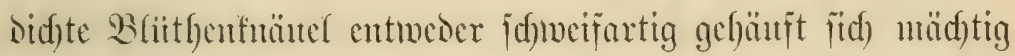

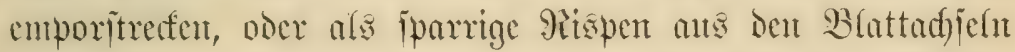

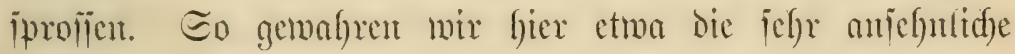

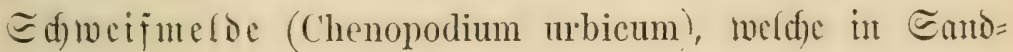
gegenton jelten in cincm Sorfe fehlt, mto mit ifren ju cintent

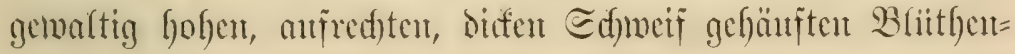




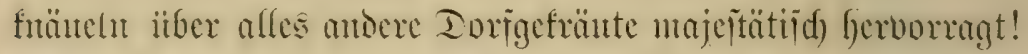

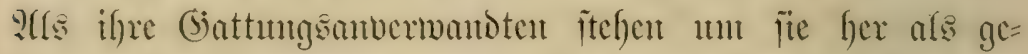

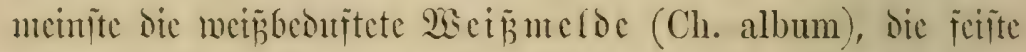
Dunfelgrime Dianerucloc (Ch. murale) mit breit ans=

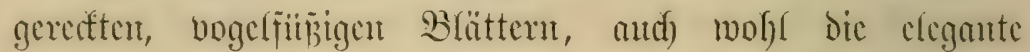
(5) ranmelde (Ch. glaneum) mit jicrlid) cidsentanbartigen, biffen Blättern, scren präd)tiges Eaftgritu vou ber granten

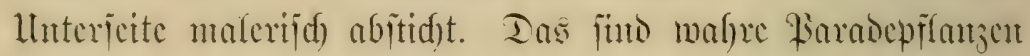

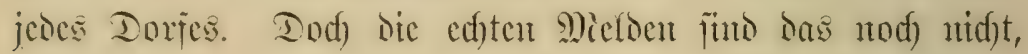

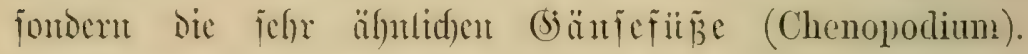

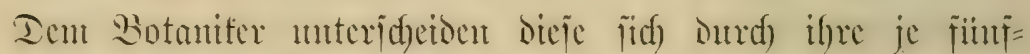

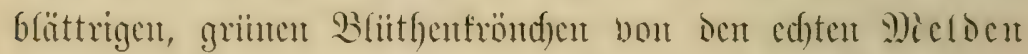

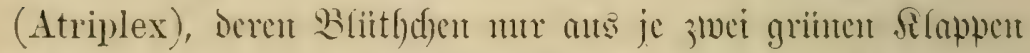
bejteber; Gejontors die präd)tige giofenucloce (A. rosea)

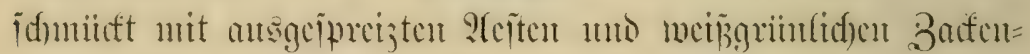

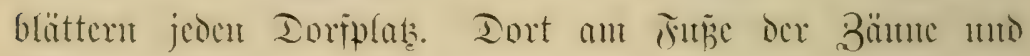

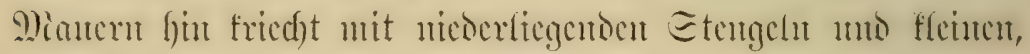

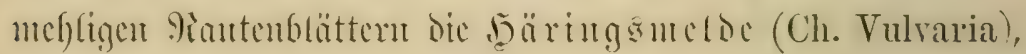

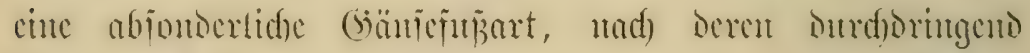

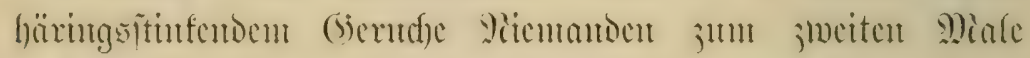

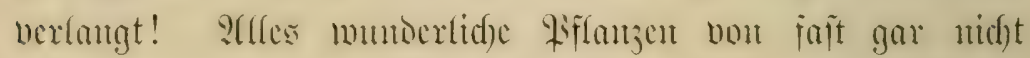

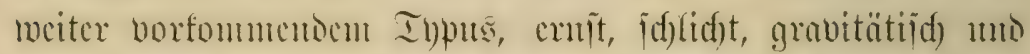

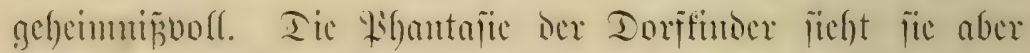

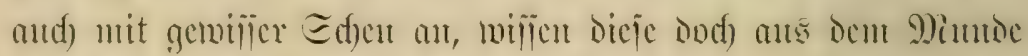

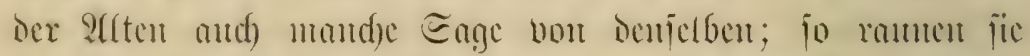

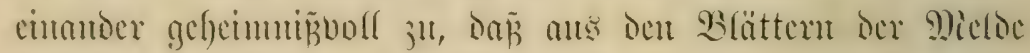

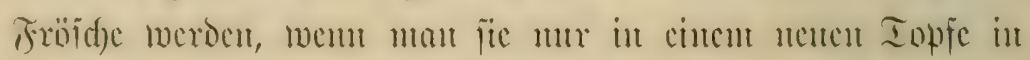

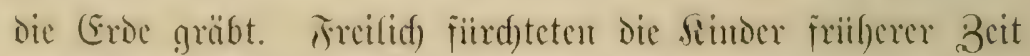

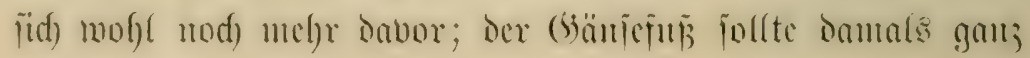




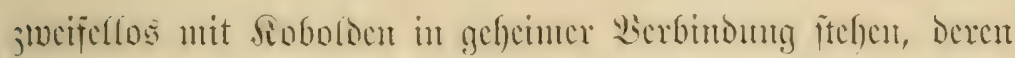

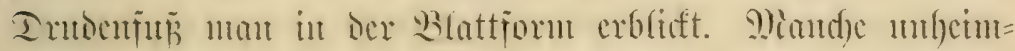

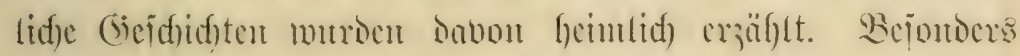

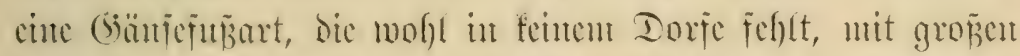

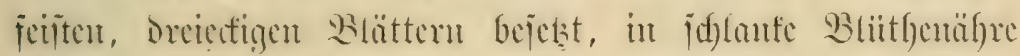

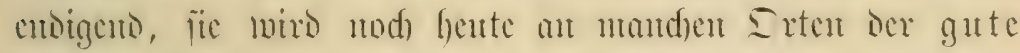

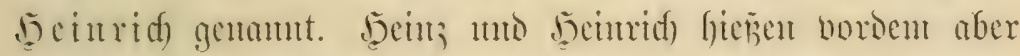
Die Siobolde, bielfad) ber Ienfel ictber, dest bie Eage ja and

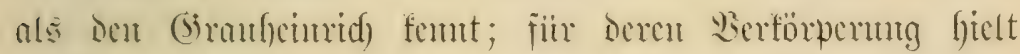

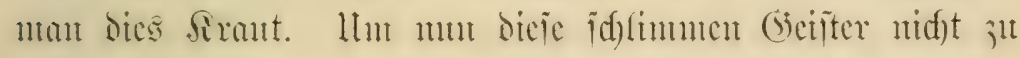

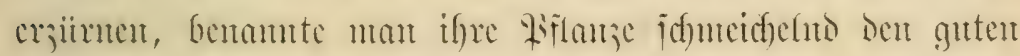

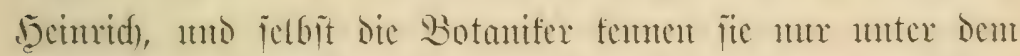

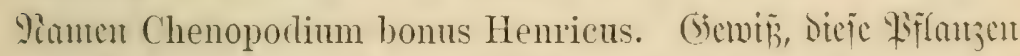

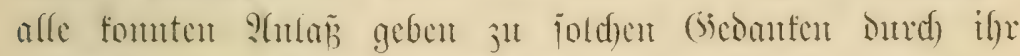

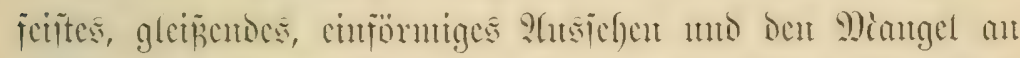

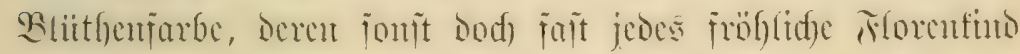

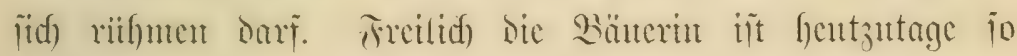

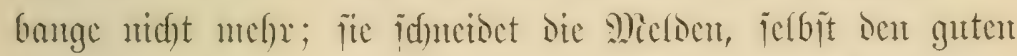
Seimrid), gant; wohlgentuth fiir bic Sildse nut fodt cinten

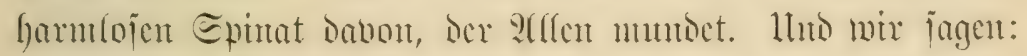

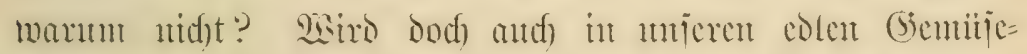

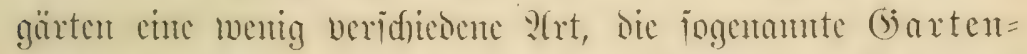

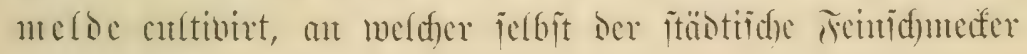
Wohlgệallen hat.

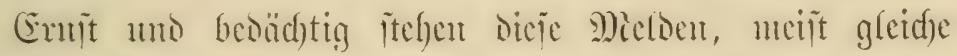

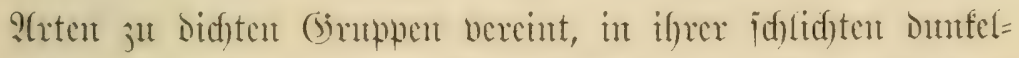
grïnen oder weingrienten (sictwandung da. 9ther als jöge

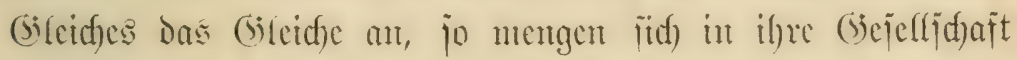
andersartige (Sattungen non derfelben Eimfad)heit; Dem jutr 
Slatie der "Sleracen", jut welder die Familie ber bänie=

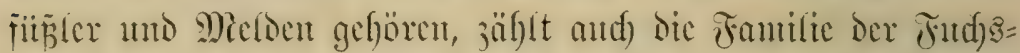
id)uäujler uno Sinöterid)e, ans beren (siattungen bier gleid)=

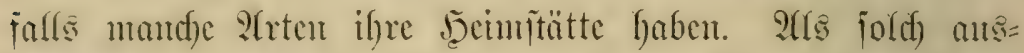

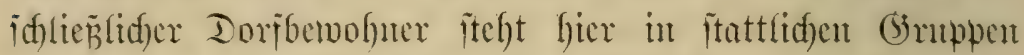
ber it umpfblättrige : mpjer (Rumex obtusifolius), weldfer

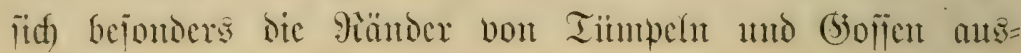

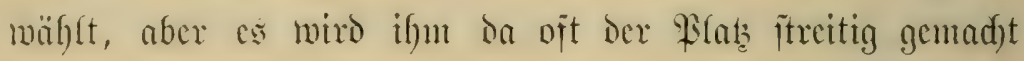
Durd) icuten Familientuerwanden, sen pirifid)blättrigen Rübterid) (Polygonum persicifolium); ants befien itppig da

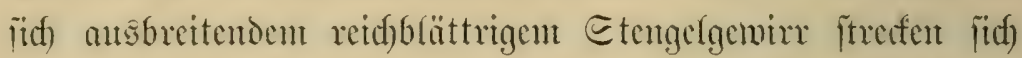

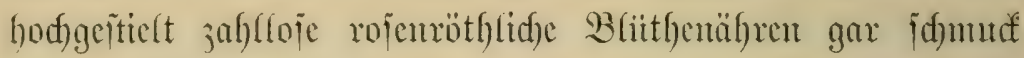

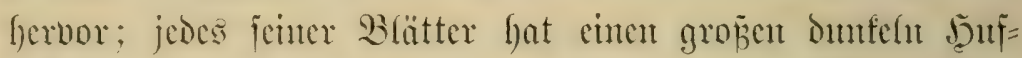
flect, ber, wie dic Eage geht, bon der Jumgirant Miaria her=

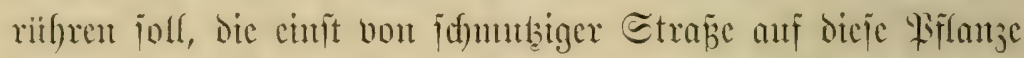

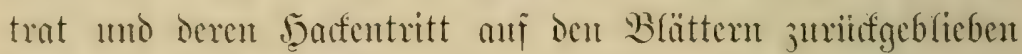

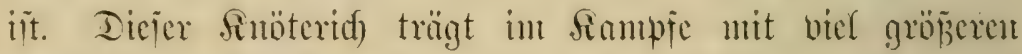

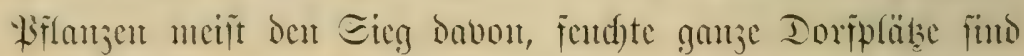

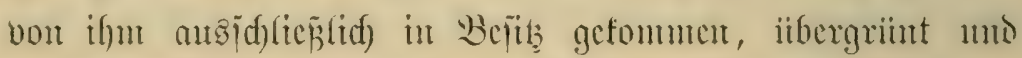

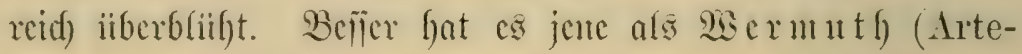

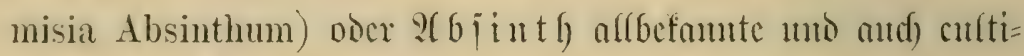
virte julante, Gohe Wiflanje, die in wohl fajt feintem Dorfe ieglt und obne bedentente (Encumen; ju firrd)ten fidf bic

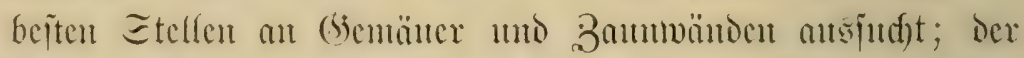

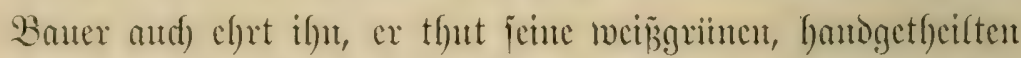

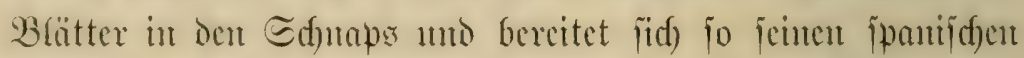
Bittern. Dagegen die Bä̈terin bült es mit bent douteben wadjenden, jebr älynlidfen, aber dunfelgrimen, aromatijacu Beifun (Artemisia vulgaris), den fie als (Selvitry fïr bic 


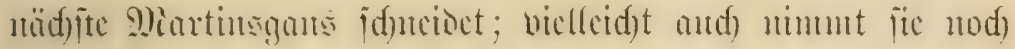

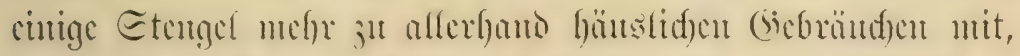

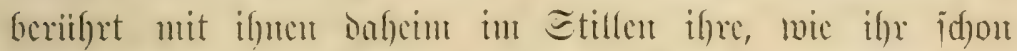

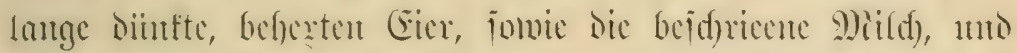

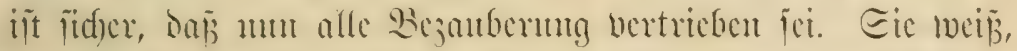

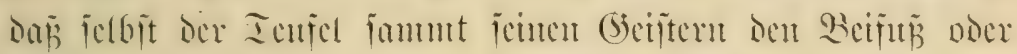

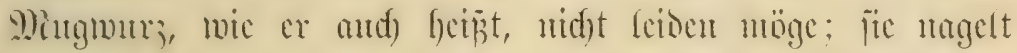

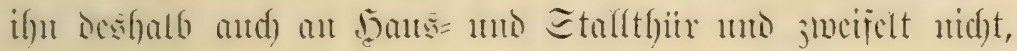

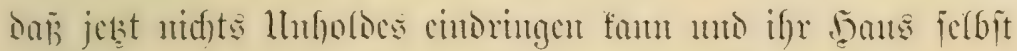

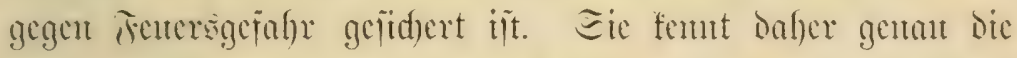

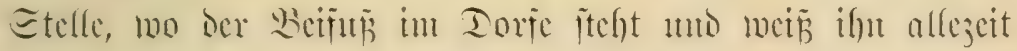

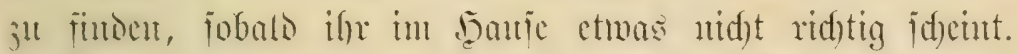

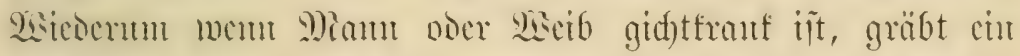

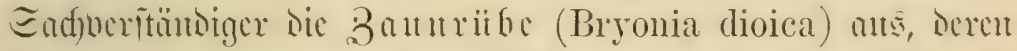

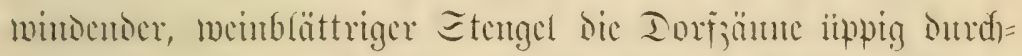

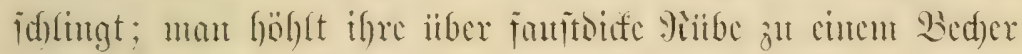

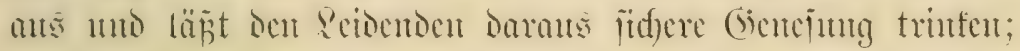

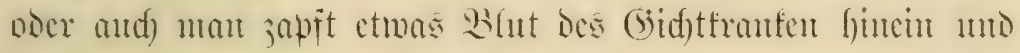

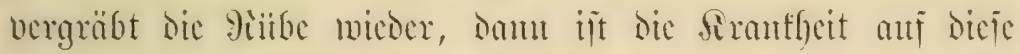
iibertraget. S(ber and) Dic Iorfidjönen temen in mand)en

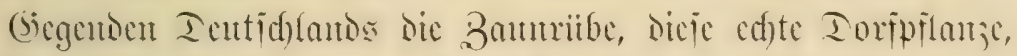

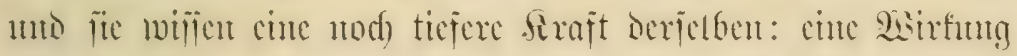

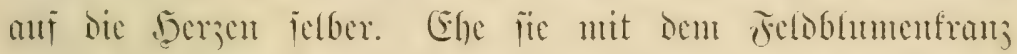

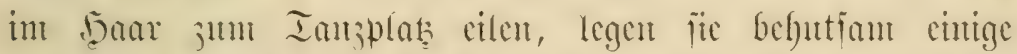

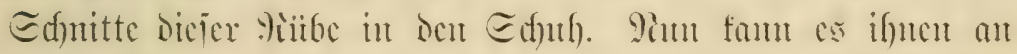

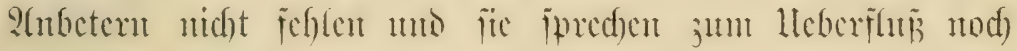
beimlicher Weije:

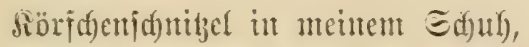

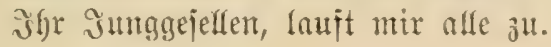




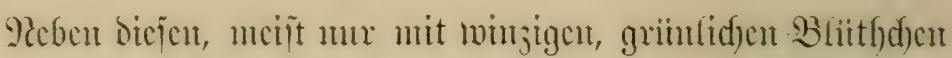

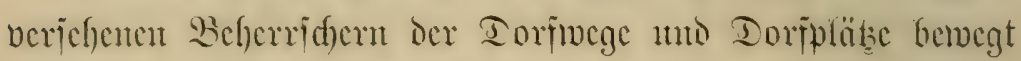
jid) nod) cine numterere Siflanjenidanar, ser cs an jiijem

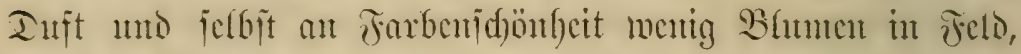

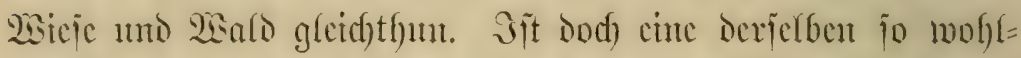

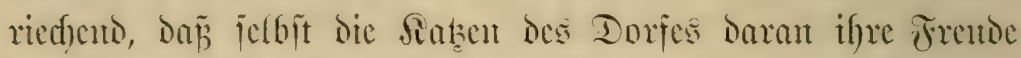
haben. Einto vieffecht ben Ecier bei ciner Dorfwanderntutg

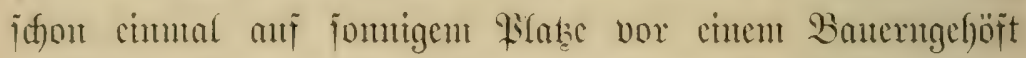

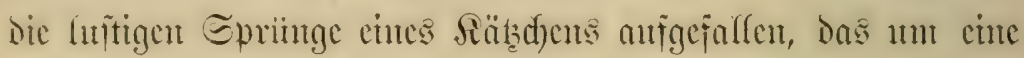
blithende Dorf̈blumengruppe orolligite (Sapriolen mad)te, Daran cutporfprang und vor Suft wic tolf itch geberdete? Ciut

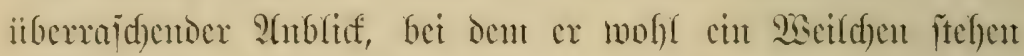
blich. Wemt ex das firant mu felber betradtet, wird ex

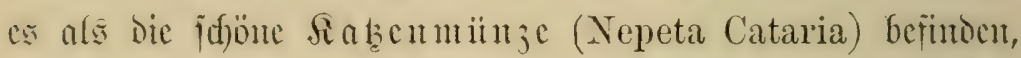
Deren hoher Etengel mit Wirteln meipröthlider Sippentblitthd)en

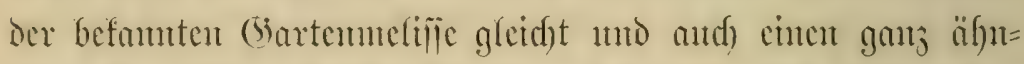

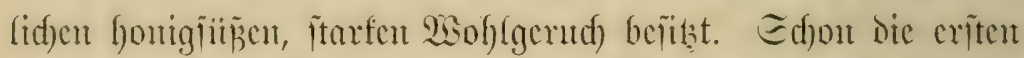

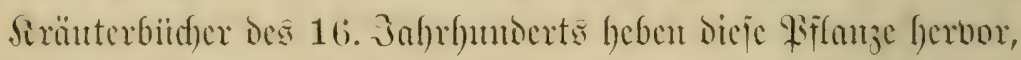
benterfen jugleidh and

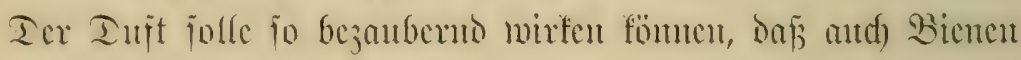

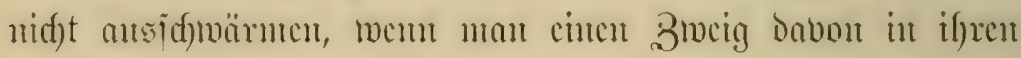

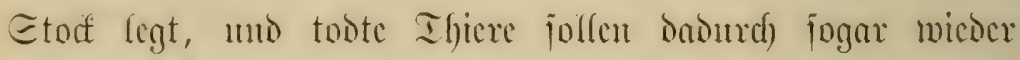
lebendig geworden jein. Eo munderbar ijt alferdings feinte

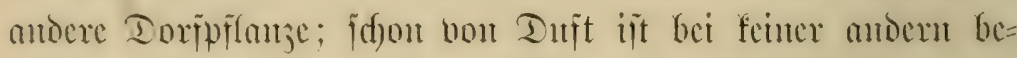
jonters ju reoen, anjer ctwa bei bent in fajt aflen Dörjem

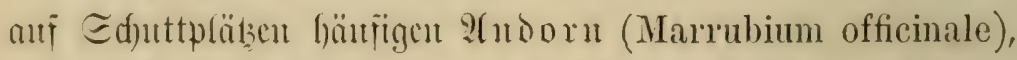

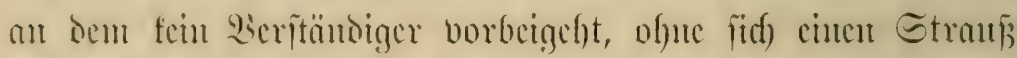

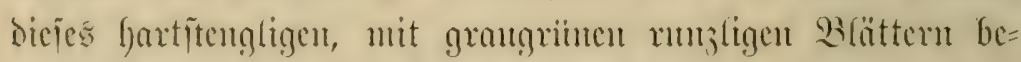

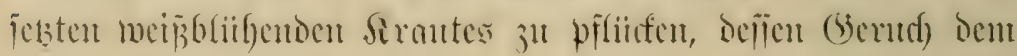




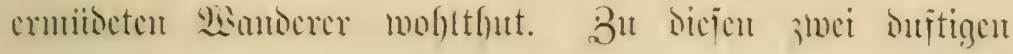

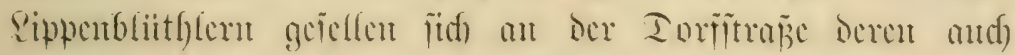
gerutchloie folde: meift an bic Bammono georängt ber ftcif mipredte Pömeniffmanj (Leonurus Cardiaca) mit lifaen

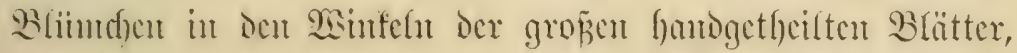

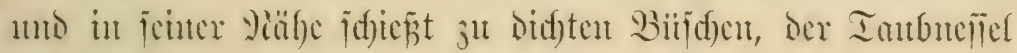
äbulidy), die Barlote (Ballota nigra) empor.

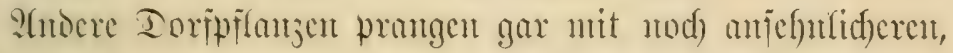

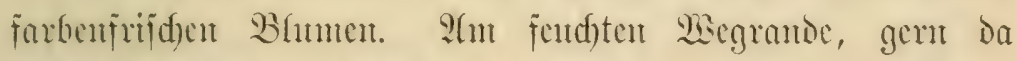

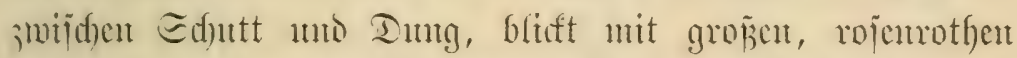

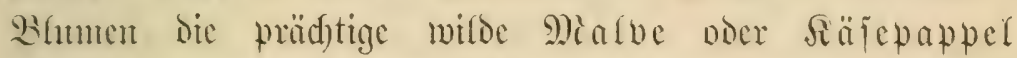
(Malva silvestris), oder dic flemtere blafiere Malva borealis

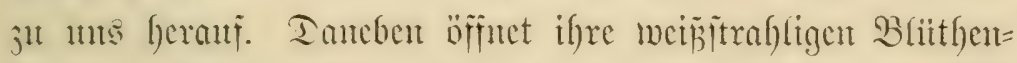

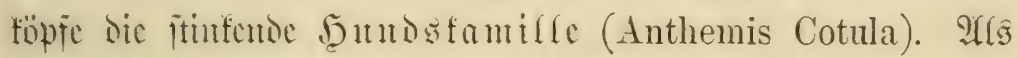

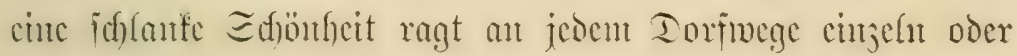
truppuncie dic biolettblate $2 \mathfrak{c}$ rbeute (Verbena officinalis),

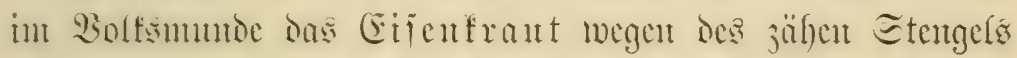
benaunt, bei unjeren Borfahren cin Siraut in allen Pö̈then

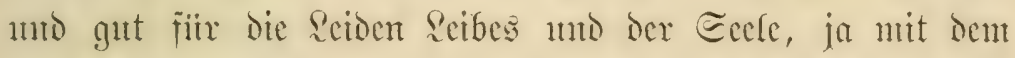

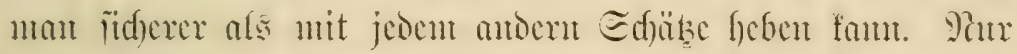

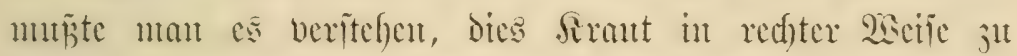

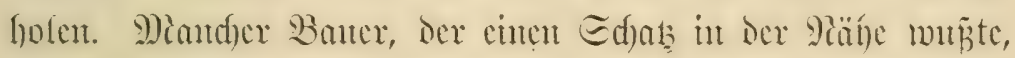

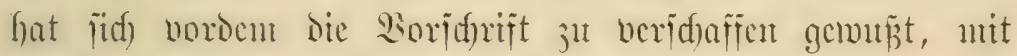

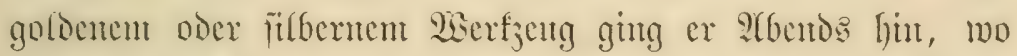

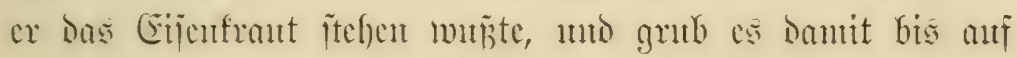

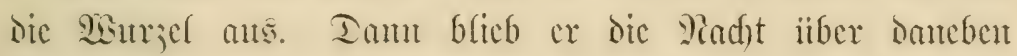

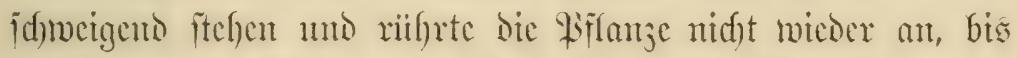
Dic Eome im Miorgenthan Daran blinte. Pim surfite $\mathrm{ex}$

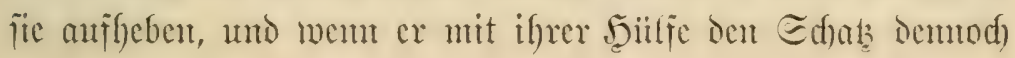




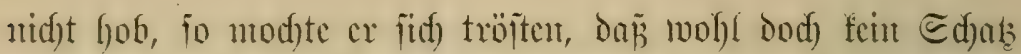

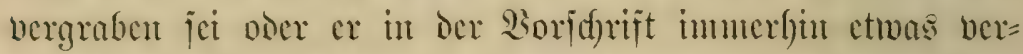
fäumt habc. Das Eijen= ober Sijenfrant gebört abcr fo treut

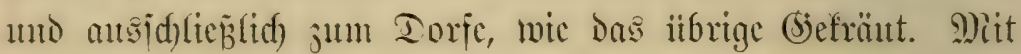
jaftigen (sirïn mijd)en j̈d gelbbrumige Fingerfräutex Dajtwijden uno anderes flemes buntes Gebliume, jlwijden dem

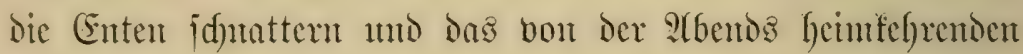
Sectoc als cekter Secferbifien des Iages abgerupt wird.

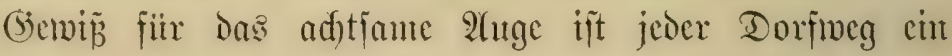

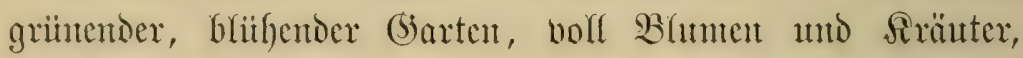
wie fie fonft uirgends fidh finden. Sied)nen wir baju cünge

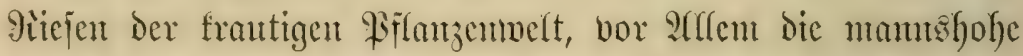

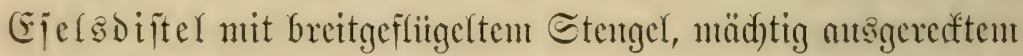

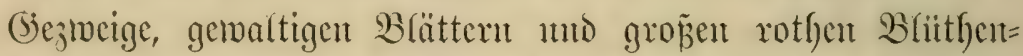
föpfen; ebenjo die nidft flemere blanbliithige $S_{5}$ r de: jo haben wir bartn (Sclväd) je, wie fie dic Iropentwiifte in ifyen Eactecn

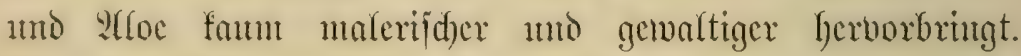

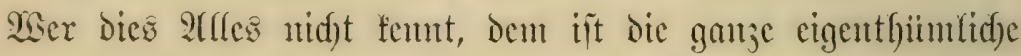

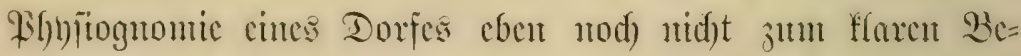
Imujticin gefoumen; Der femt midst Dic Bejonderfeiten, welde dic Sigantajic der Dorffinder jdyon erregen and ibr

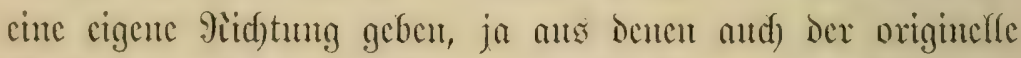

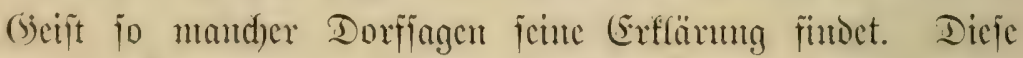
Fiflanjen gebören untrembar jum Dorfe, als beflen ureigentes Beimerf! R(ber aud) mand)es feltenc (Semäd) ift mu bort

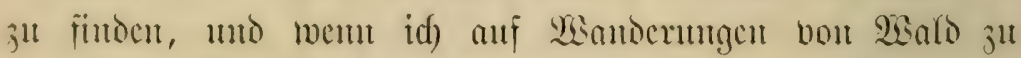
Wals, vou Srt ju Srt, ourd) Dörfer getommen biu, babe idf) mid) immer uidft unt geprent ïber dic gemölyulidfen Dorf=

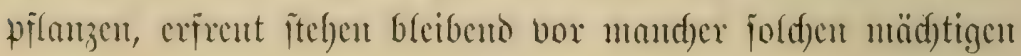




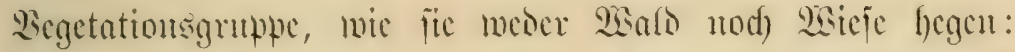

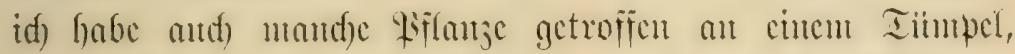

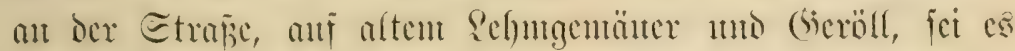

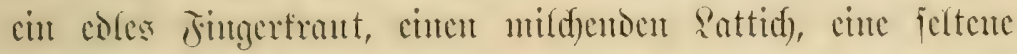

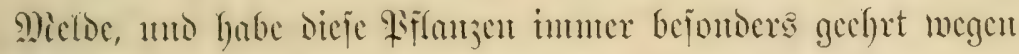

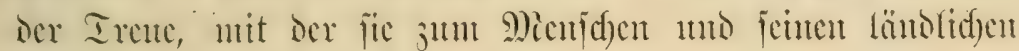
Solonitätten fid halten.

Einen cigenen Piciz hat dic Dorfflora fiir micum all

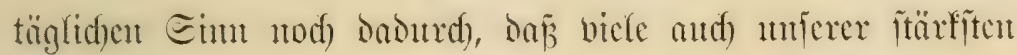

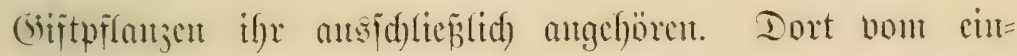

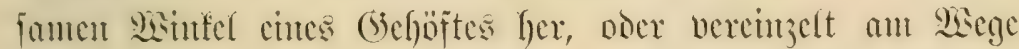

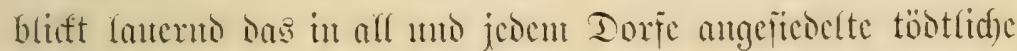

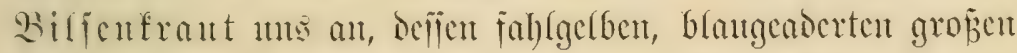

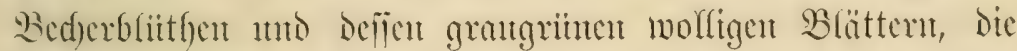

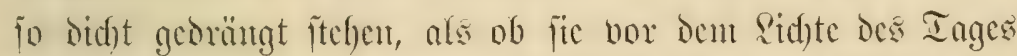
ïd) gegenjeitig werbergen wollten, man bie (siffigfeit fajt an=

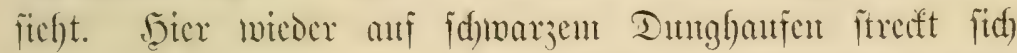
Das ïpplig berjweigte sutfelgriute Etcd)apielfrant mäd)tig curpor, ocficu fingerlange foncemeifie Irompetentulumen fo

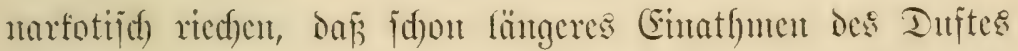

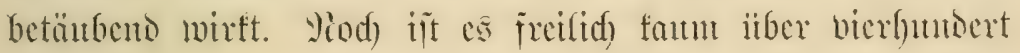

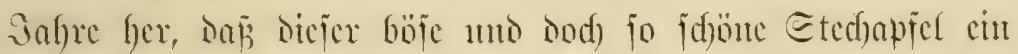

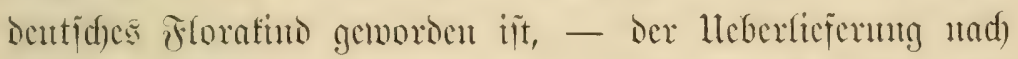

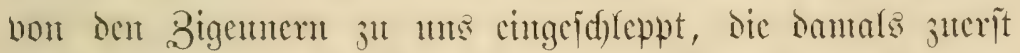

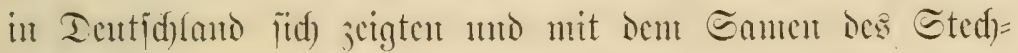

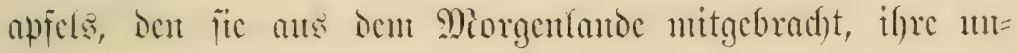

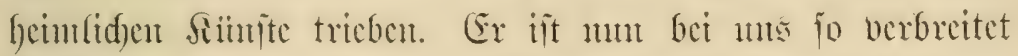

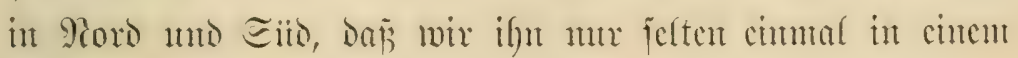
Dorfe vernifiten. 
P(ud) Dic hohen Etanom bes geflerten Edicrling begeguen un cinzig in ber llugebung des Dorfes, oder mitten

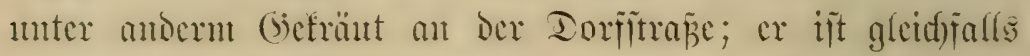

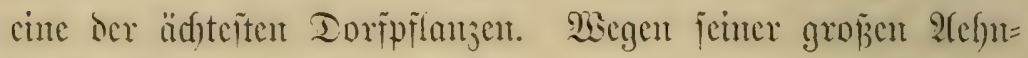

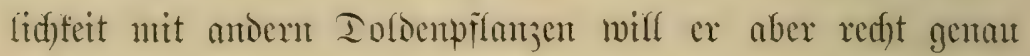

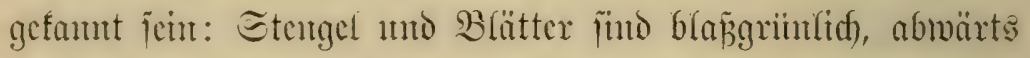

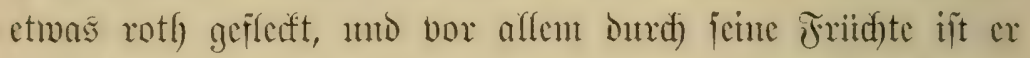

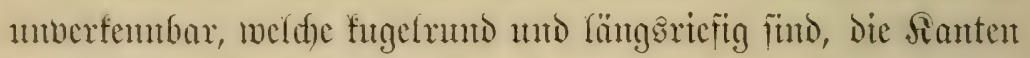

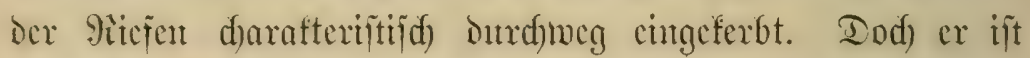

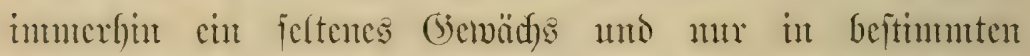
(begenden Deutidlands foum cr bäufiger bor, aber if and

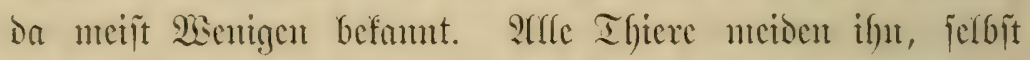

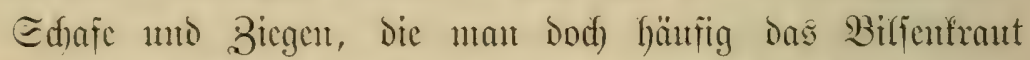
unbejdabet freficu fieft; Die Bänerit, weld)e ifnt feunt, warnt

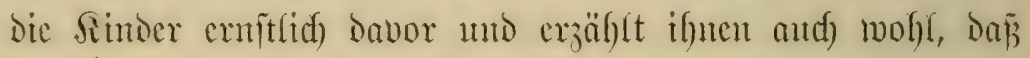

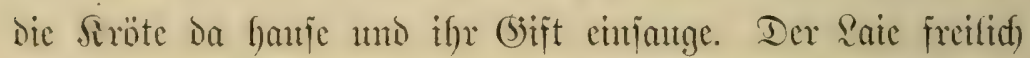
pflegt womröglid) alfe Dolocnpfínujen fïr Ecfierfing ju halten

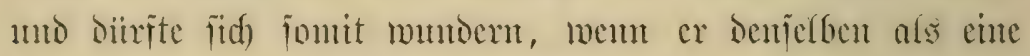
jeltene Iorfpfianze bejeidnut hört, an Dem cun Eofrates itarb

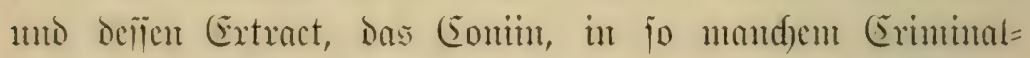

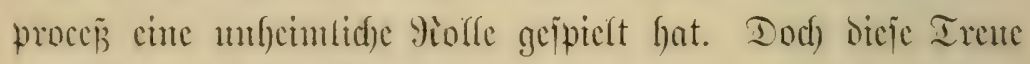

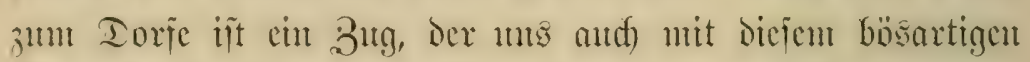

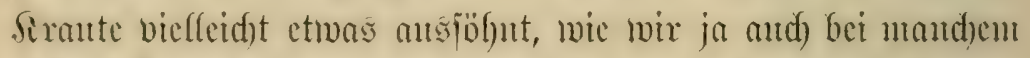
Edurfen nod) cinjelner guter Eciten nus frenten fömuten.

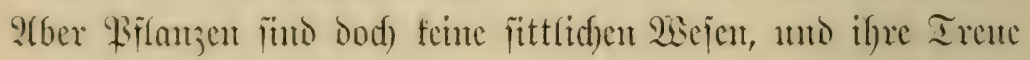

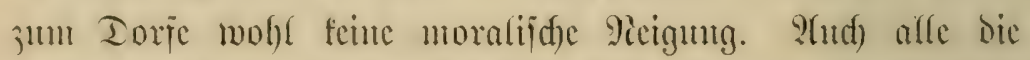
genamten folgen in ber That blos iffer shlage, welde fie ba geocifon beipt, wo fidf bic Beoingungen iffes Berdents แnd 


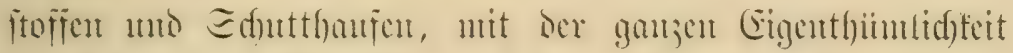

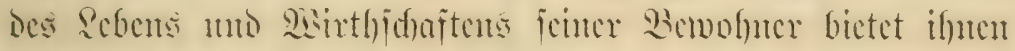
bicjelbe. Sic anmoniafolijden Enbitanjen, melde jo wie bei ocr Sanowirthideaft nirgents weiter geboten meroen, mögen

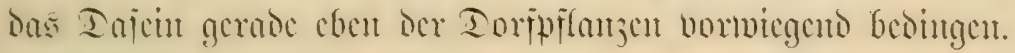

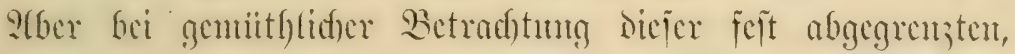
ganj cigenartigen Flora nergeffen mix ond gern alle bie

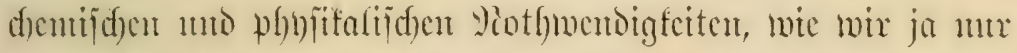

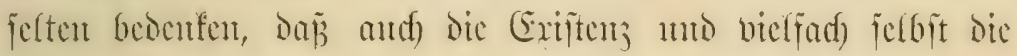

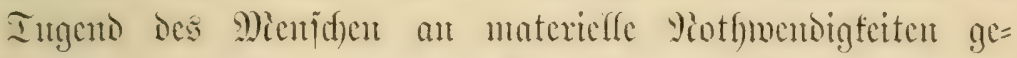

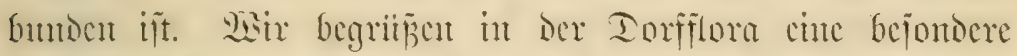

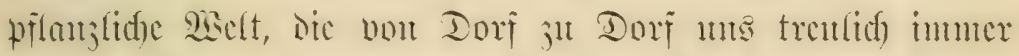

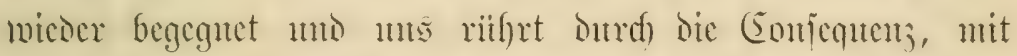
ber fie an ber Sonlle bes ?antebens fängt anto bicie mit

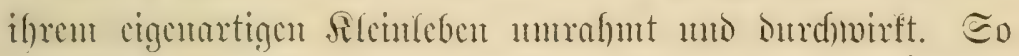
hängt aber and) jeincriets der Dörfler, mentigitens jeder ectute

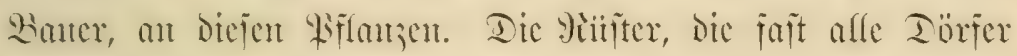

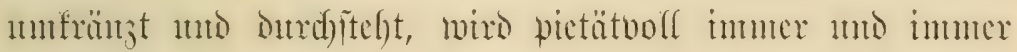
micder gepflanst; fic ift ber Gertömmlide Iorfban gemoroen,

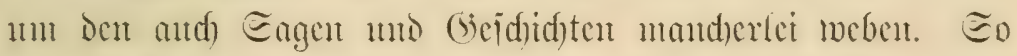

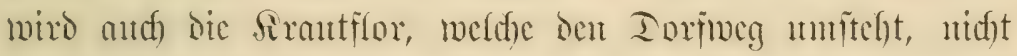

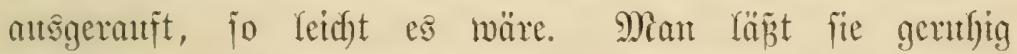
mo and ganj abjithtlids madjen, theils nod ans aber=

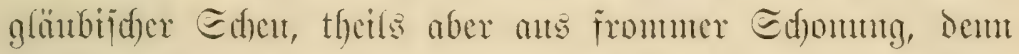
ber edfte Bance hat die Siflanjen nod) gern, an Denen or als Sinto jeine Frento hatte. Lhto gewiz, and bas Dorf wäre

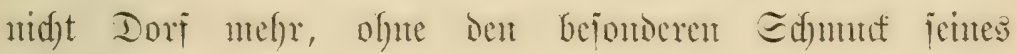
Piflanzenlebents. 


\section{2.}

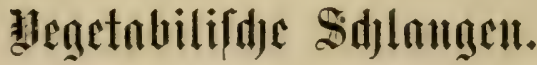

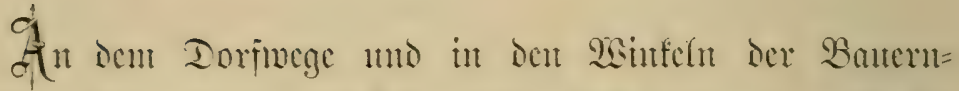

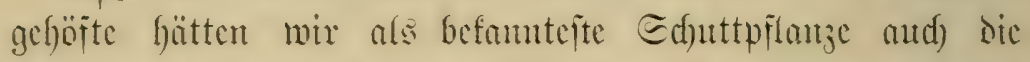

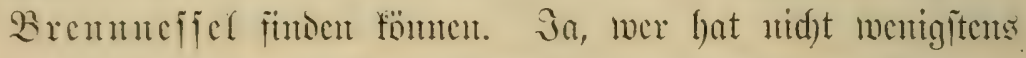

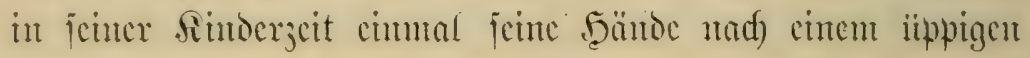

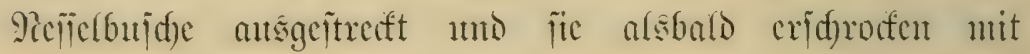
bremtenten Ed)uterje micoer juriitfgejogen? 3um juciten

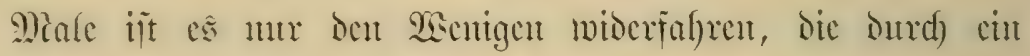

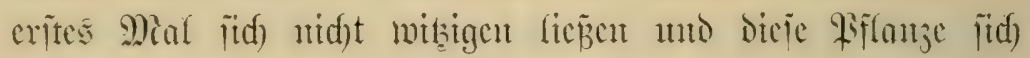

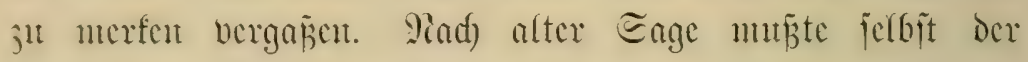
Ianjel iche llutbefamutidaft mit bicier Biflanje cmpfinton,

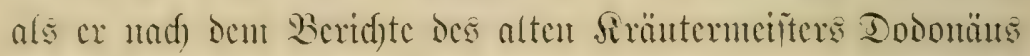
iprad): "Dat frut fem iif - fäd De Diivel, um fett ficf

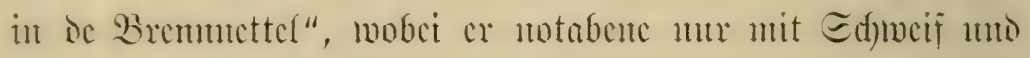
börnern beffeioet war.

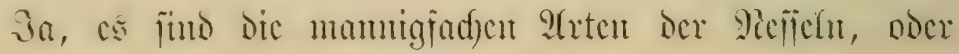

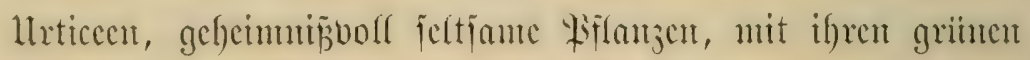

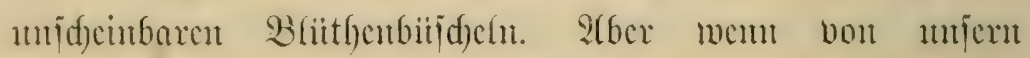

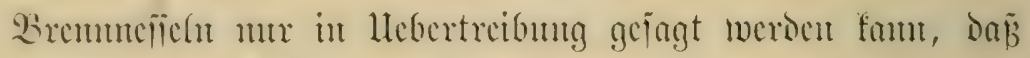

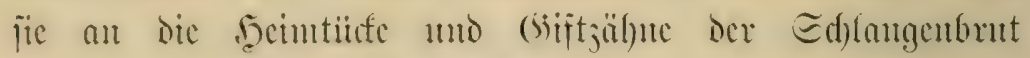

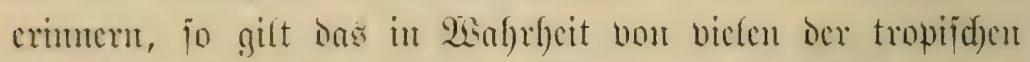

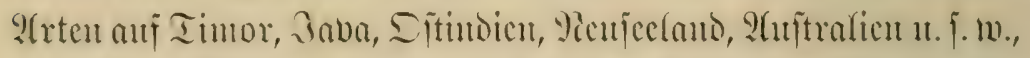

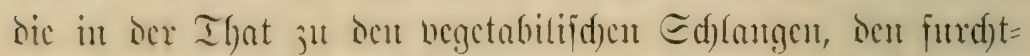

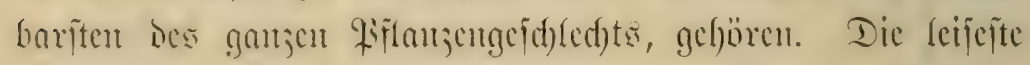




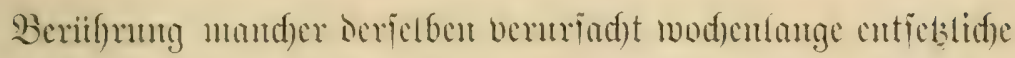
Edumerjen, madit ien ganjen Sïrper entjintoct uno an= geid)wollen; bei cince vou ben Cingebornen ant Imur die "Tcufclshand" genunten sert währen sie sciocn jogar

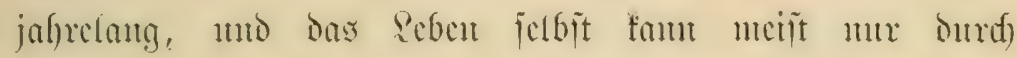

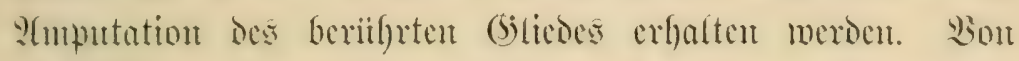

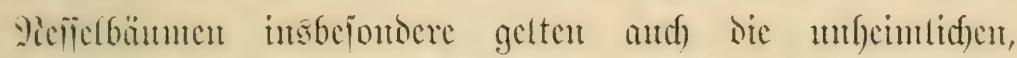
menteleid) iibertricbenen Berid)te vom Llpasthale ani Jaba

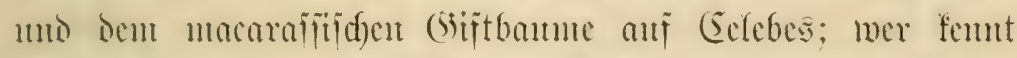

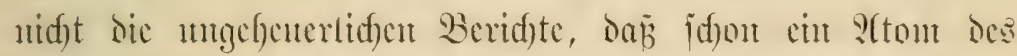

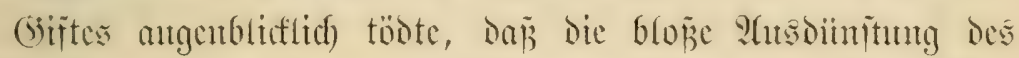
Banmes allent Sebonoigen in meitex llmgebung ben Tos

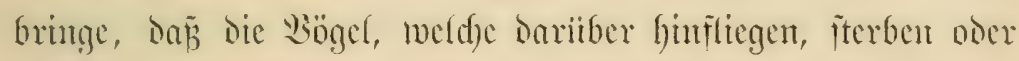

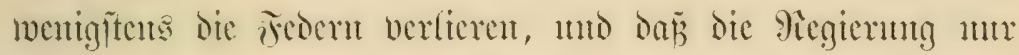

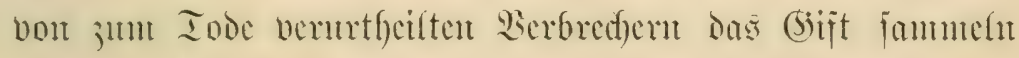

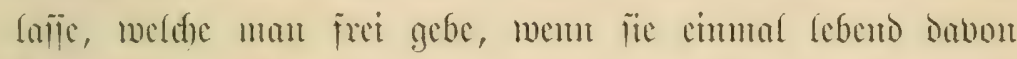

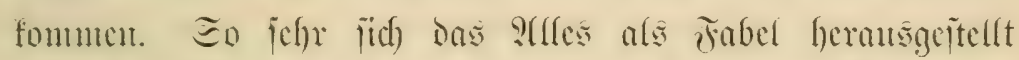

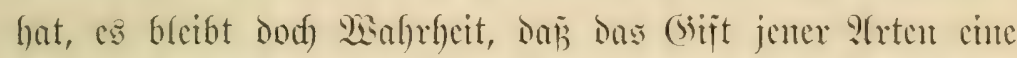

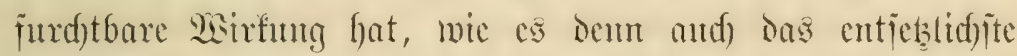

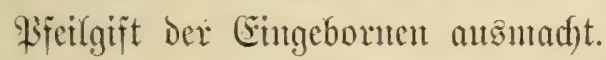

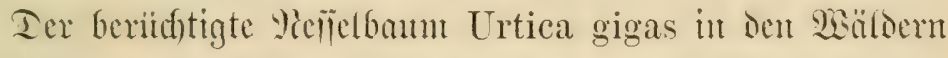

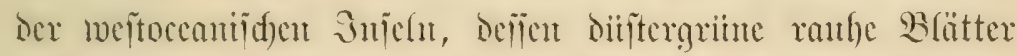
mit furdffiaren Etadjelu bemefort jumb, aber jmijd)en denen idfarlad)rothe Bibitfou prad)tooll hervor(endeten, töntet mit jeinen Blätteritad)efu bas allerītürtite beriilgrt unter Butfungen in tiirzejter Beit jujammenïnft.

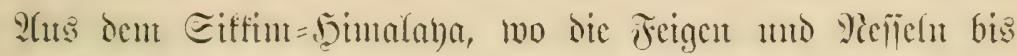
jul cuter Đöbe bou 10,000 Fun walsartig anfiteigen, be=

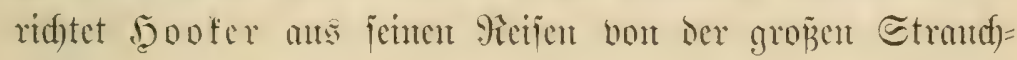




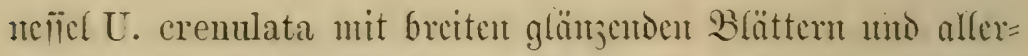

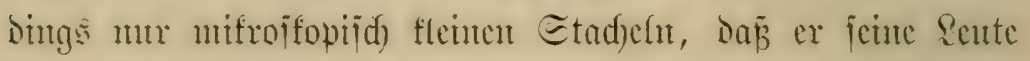
fanm baju bewegen founte, ifm ctwas vou biejen Peffeln,

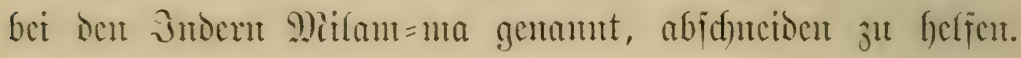

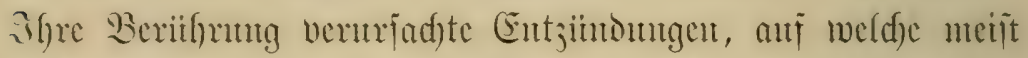

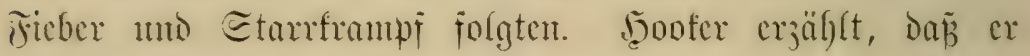

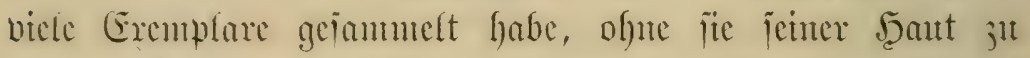
nabe ju bringen, aber jofon bie gerudfloje ?tusbinntumg war

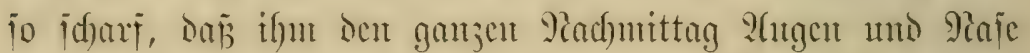

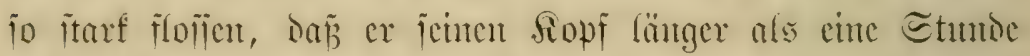
ïber cin Majdberfen halten muñte.

2isir atfuten oroentlid) anf, ment wir bou jouten tropi=

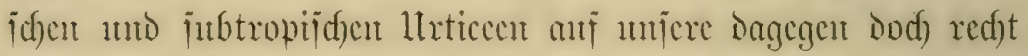

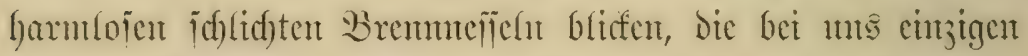
gïtigen sicpräjentanten dicjer Eippe. 29tber dest Fumilien= d)arafter berfengnen and ite nidft. Wise ifre Bermanten Dort, wo bie tropijdxe Eomme cin (sijt olme (silcid)en ans:

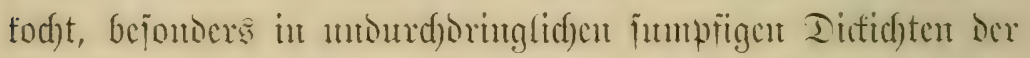
llrwäloer in lidfidfenter Buriefgejogentheit itchen, bou don

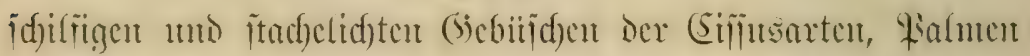

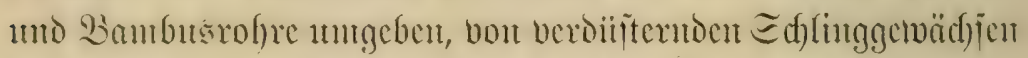

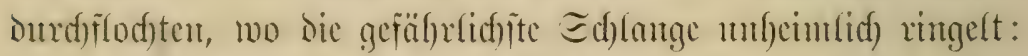

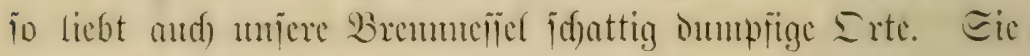

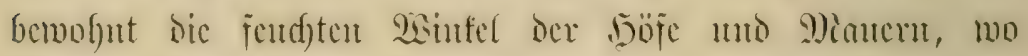

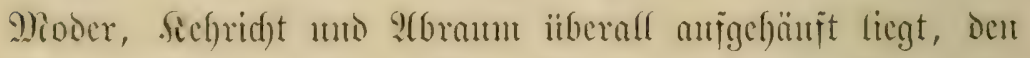

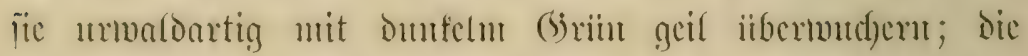

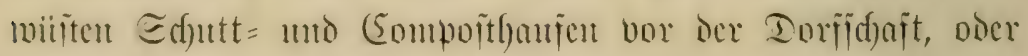
dic bour anderm llutrant jdyou ïbermiliserten fetten (siarten= 


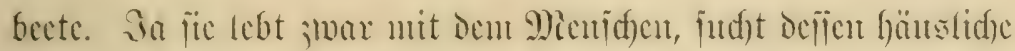

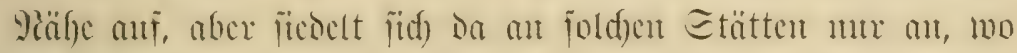

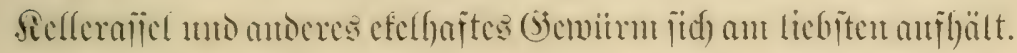

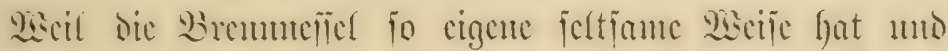

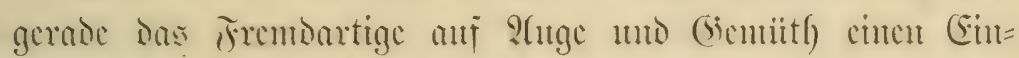

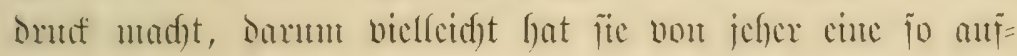

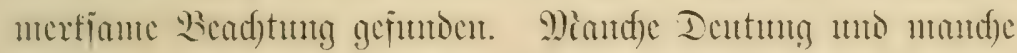

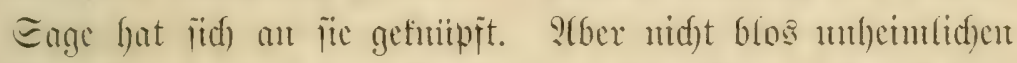

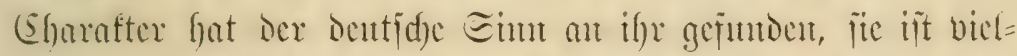
mel)r cin hodfgelobtes Sirant; jumal bie alten Botantifer

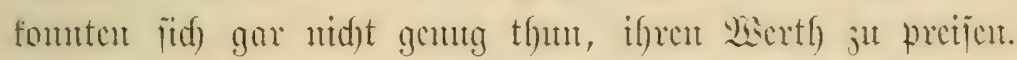

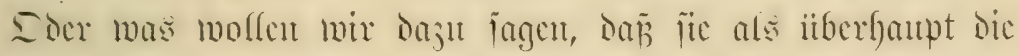

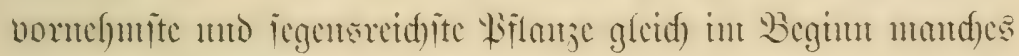
alten Liflanjenbuthes abgebiloct mo beiduricben itcht! Eo

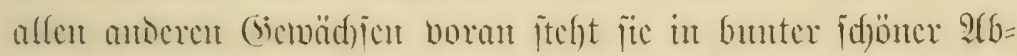

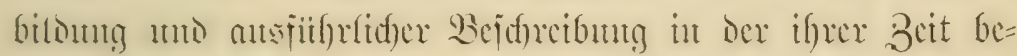

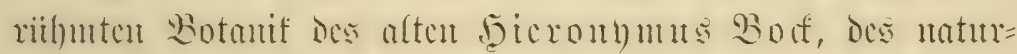
gelebrten Beitgentofien Dr. Martin Sutfer's. 3á babe in

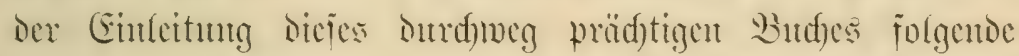

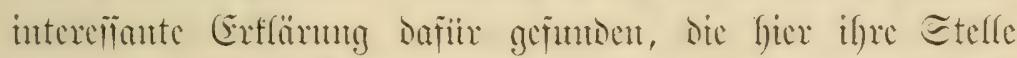

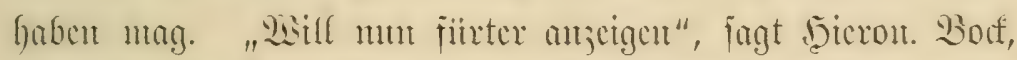

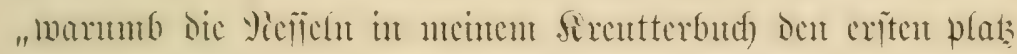
buben cingenommen. (Es irt ;

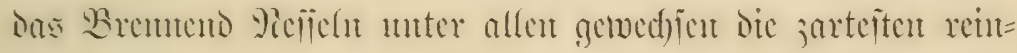

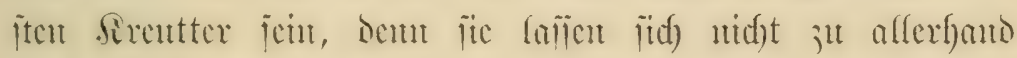
umbat wie andere sirentter gebrandyen, feis bor den ge=

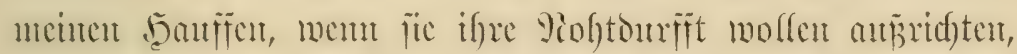

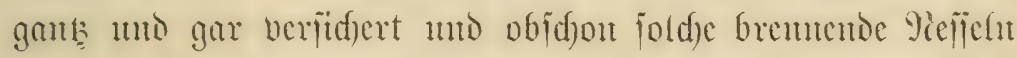

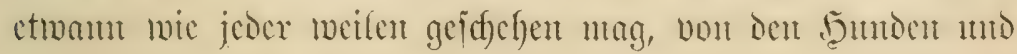




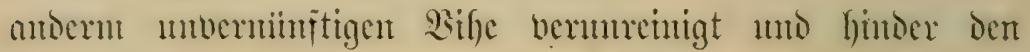
Bentuen, to ifre liebite wohmung ift, befleaft oder bejudelt

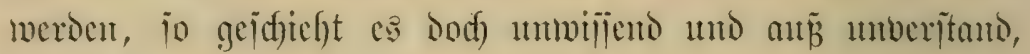

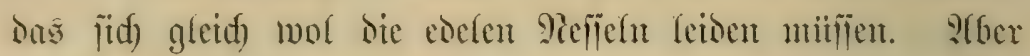
balo werden fie vout regent oder Jeimmelstan heraber, das

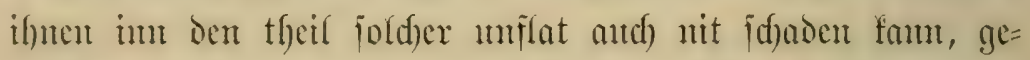
wajden und gejentert, barumb fie bam bislich fiir die remite

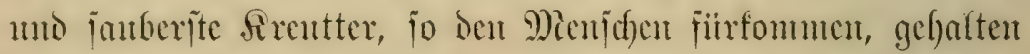

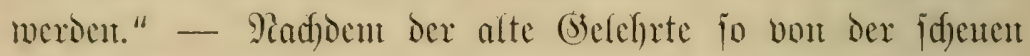

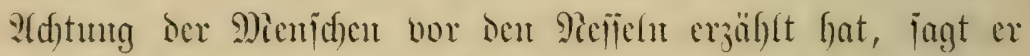

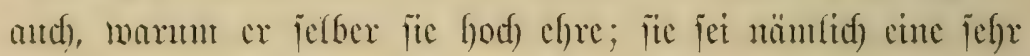

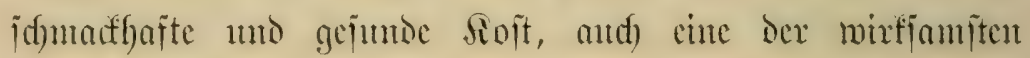

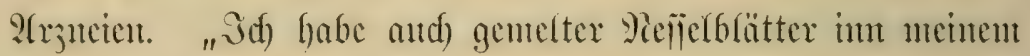
Eigut (D. 9. Wappon), dicmeil meine Sorältent int ifren

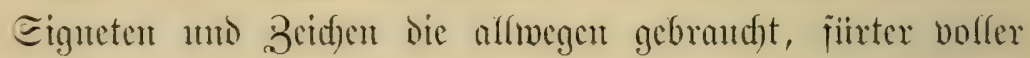
ef)rent amo befalten. Sarmub jolfent dic licbe reine brent=

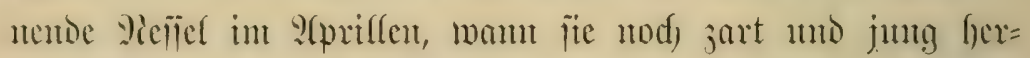

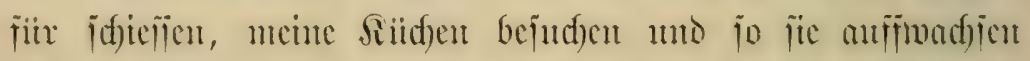

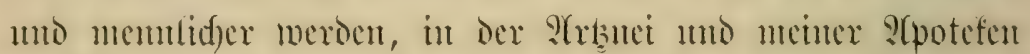

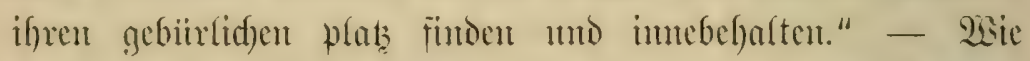

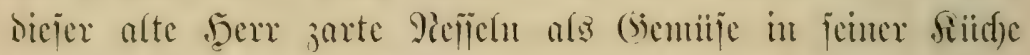
haben wollte, fo waren fie ibfrigens borbem ganj allgemein als

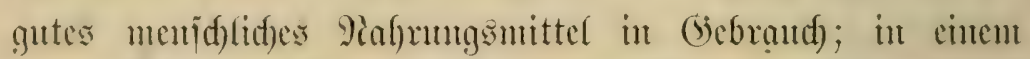

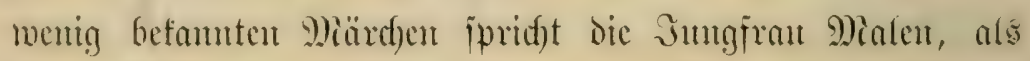

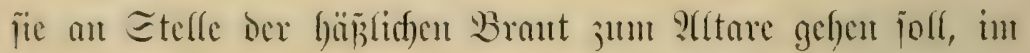
2orbeigeben zur গieflel an Wege:

Brenmuettelsbujd),

Brennnettelsbuja) fo fleene,

Wat iteift ou bier alleene? 
Jut bef die tut getveten

Da hef iaf on

Ilngejoben,

Itngebraden eter. -

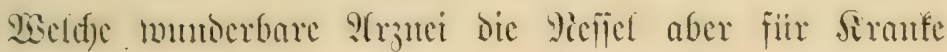

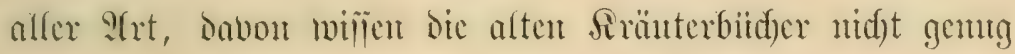

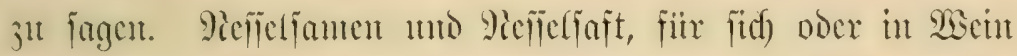

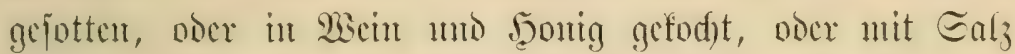

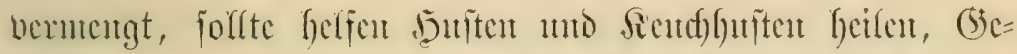
j(f)wiure jertfeiten, den 9)iagen gejumben, Sirebs mo Sungen=

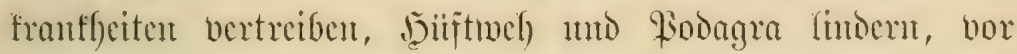

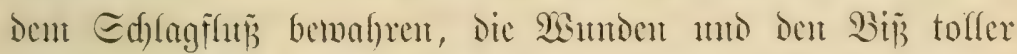

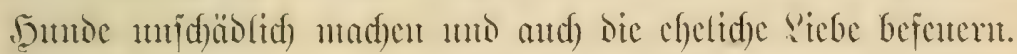
"כit cin Experiment", wiro da immer finjugejetst. Mian

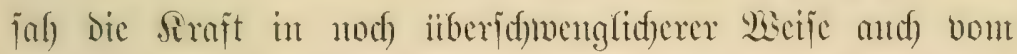

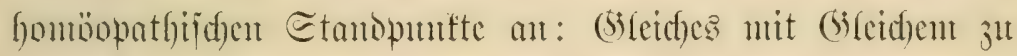

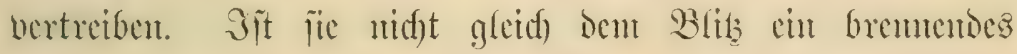

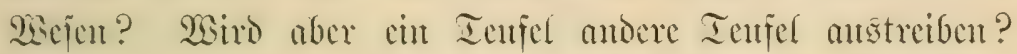

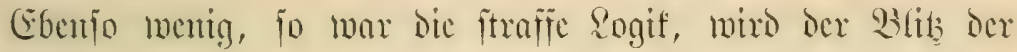

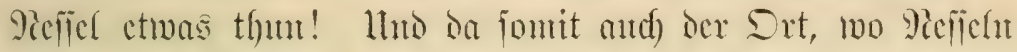

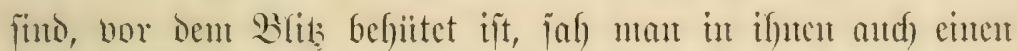

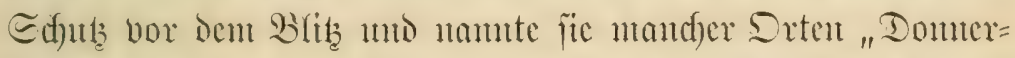

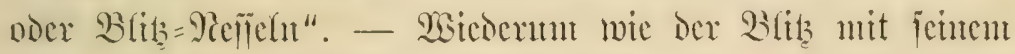

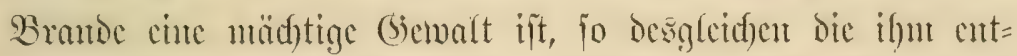

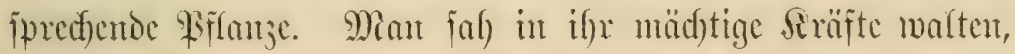

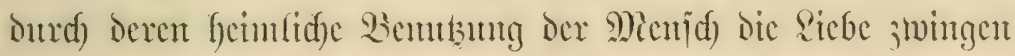

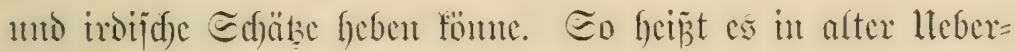
licferung: "Itt follt an cincm Freitag, Friih wom bic Eome

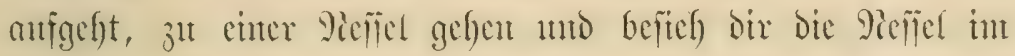




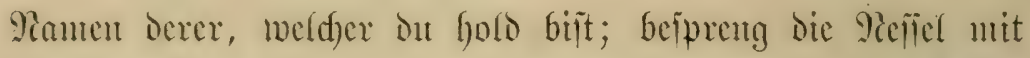

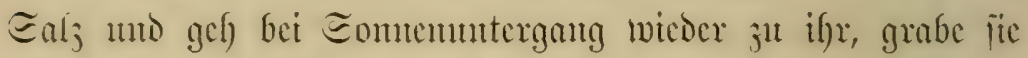

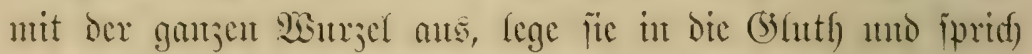

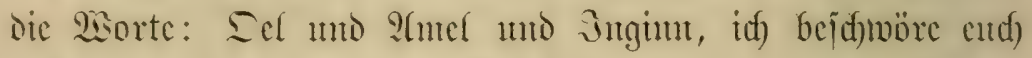

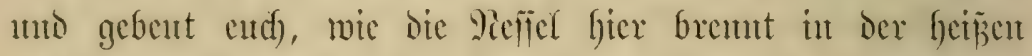

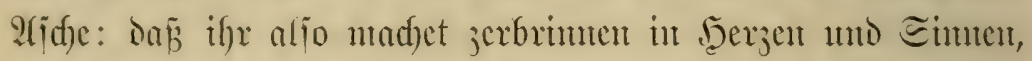

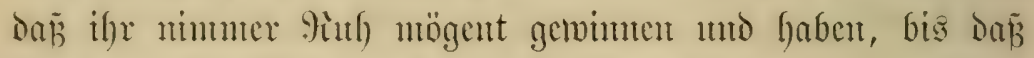

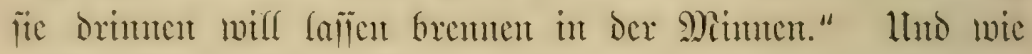

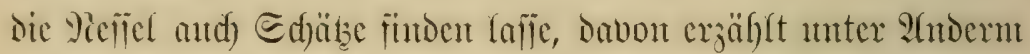

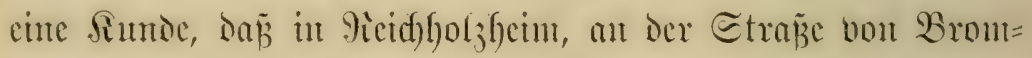

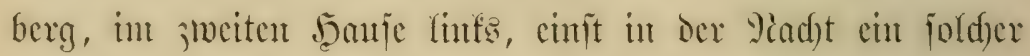

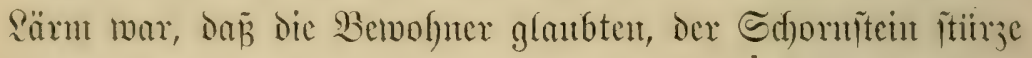

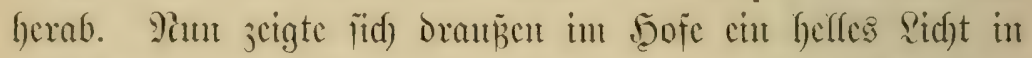
den Brentucfich. 9(ber mäbreno ífres Etreites, ob fic dou

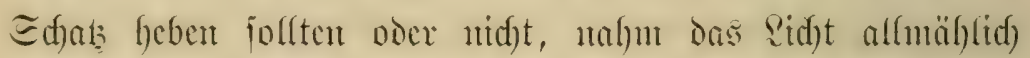
ab uno veridjwand cublid) gant.

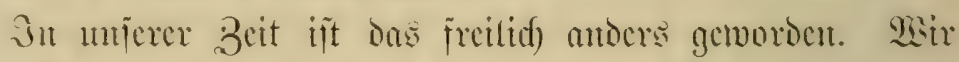
wilien, wo wir fie unt gewaf)r werben, blos jut fagent: cist

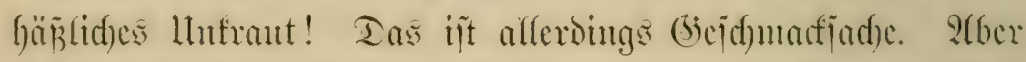

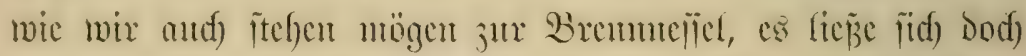

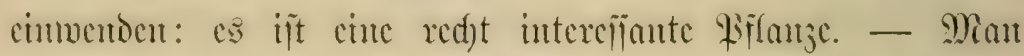

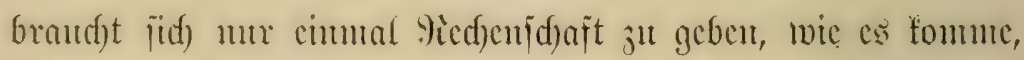

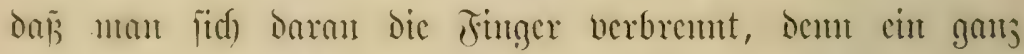

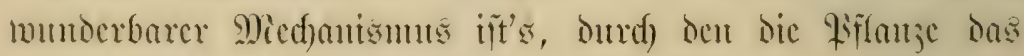
Fingerverbrement bewerfitelligt.

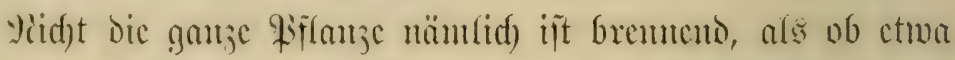

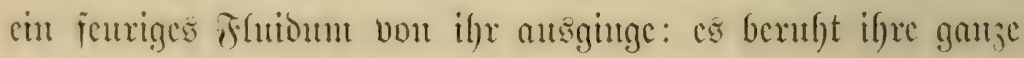

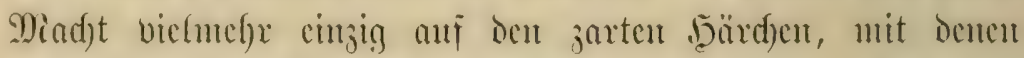

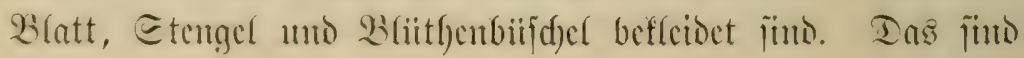




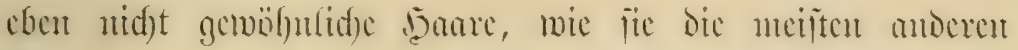

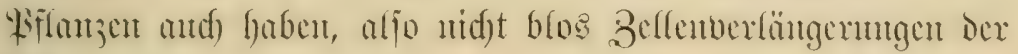

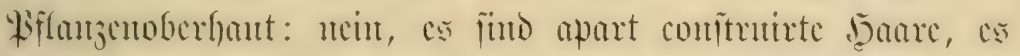

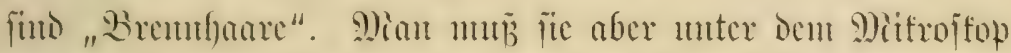

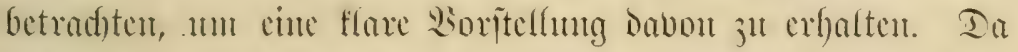

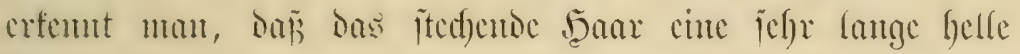

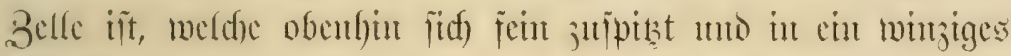

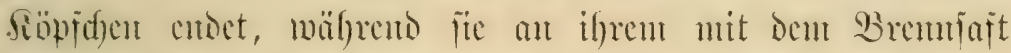

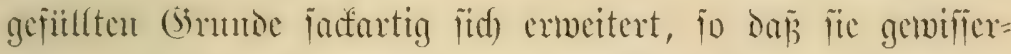

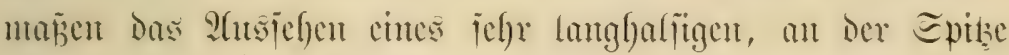

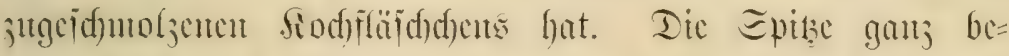
jontoers ift iteif, glasippröbe, bagegen der in bas fegelig ge=

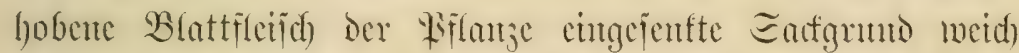

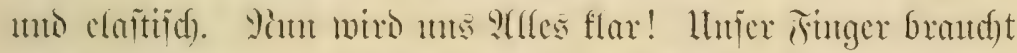

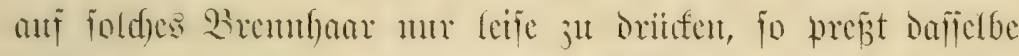

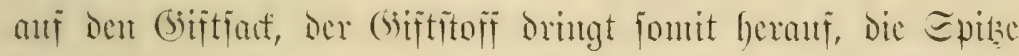

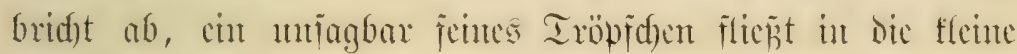
Etidumutoc cin mo verumadyt mu da bas fatale Jutefen mo

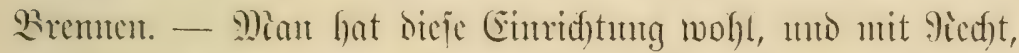

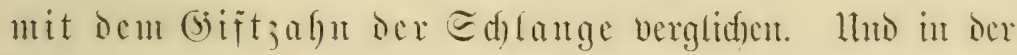

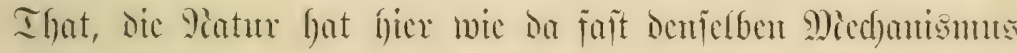
beticbt. 2tu ifrem Sberficfer bat bic Edylange betanntlid)

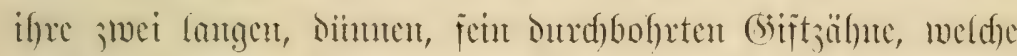

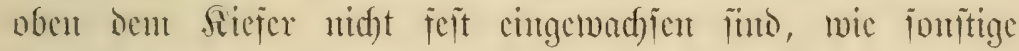

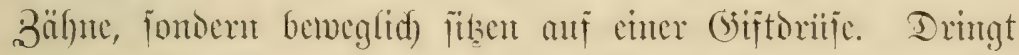

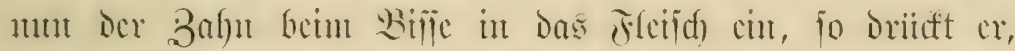

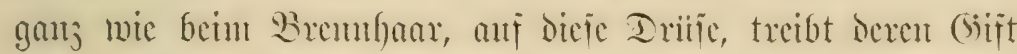

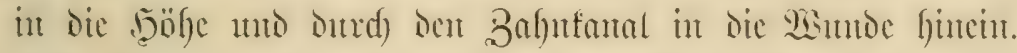
Ia 


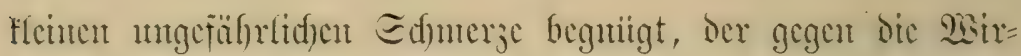

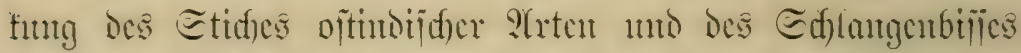
gan nidft in giede fommt, bas liegt an bent (sijt, weldes midft ganj Derjelbe furedftbare Etofi ijt, Den bie tropijd)en

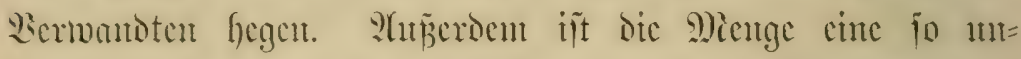

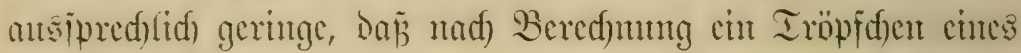
Bremthares nod) lange nidgt bas Demberttanfenditel cines (5ranmes antsmad)t.

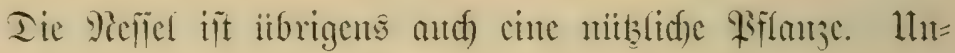

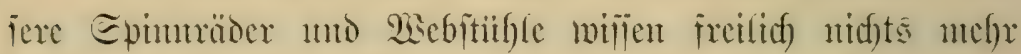

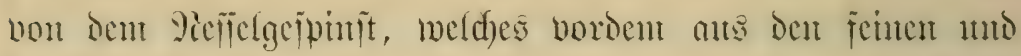

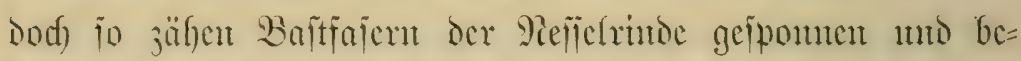

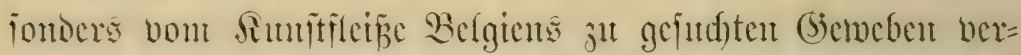

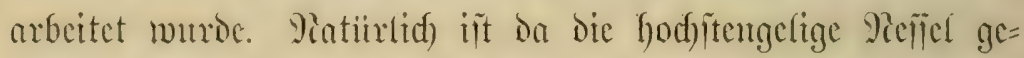

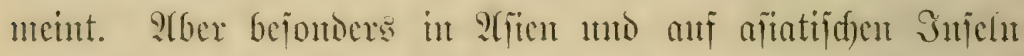

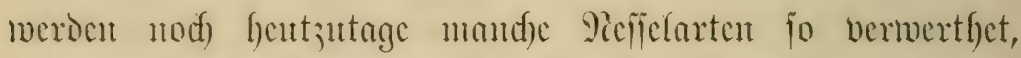
dent jeine ftarfen Baffafou fund cin Sharafter dicjer ganjen Wiflanjenfantic. Der Simpiffeip der Eutgeborenen bort fertigt

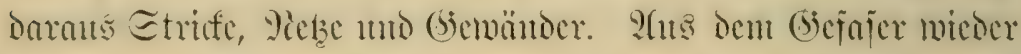

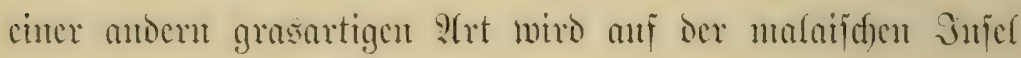

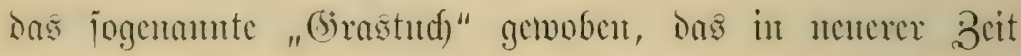
als Jemben bermendbar, and mad) Sngland berlyandelt wird.

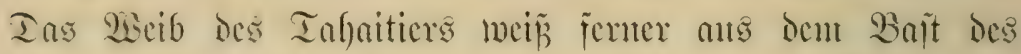
Hopiemuntberbanmes, Broussonatia papyrifera, ber and $31 t$

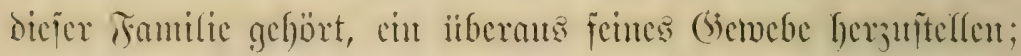

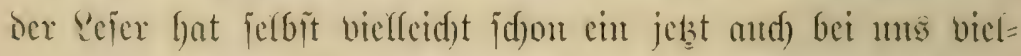
nerbreitetes (sicuebe bou den Fajern der Boehmeria nivea

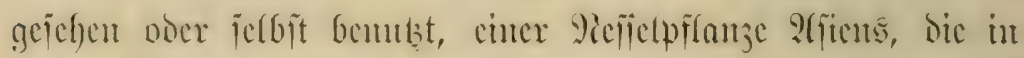

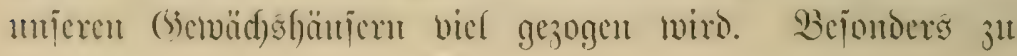




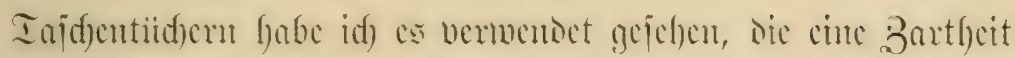

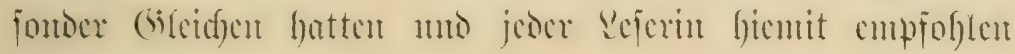
fein mögen.

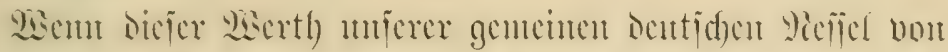

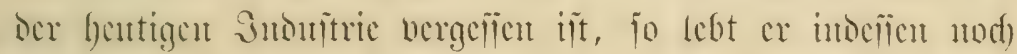

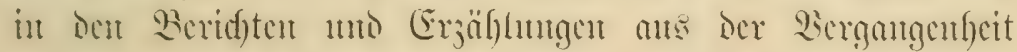

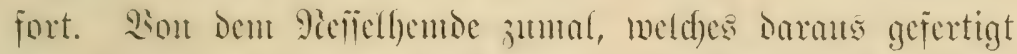

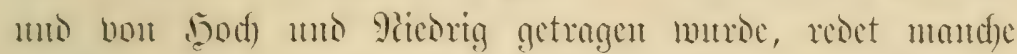

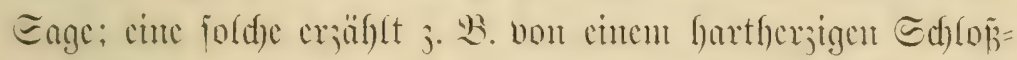
bogt, ber nidft jugeben mollte, dañ cine hitbidxe Dinte in

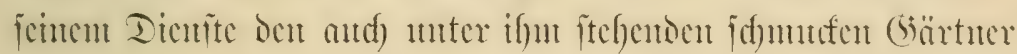

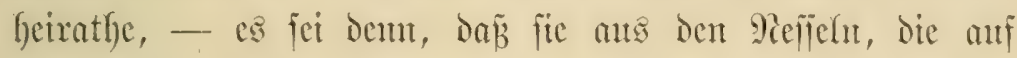

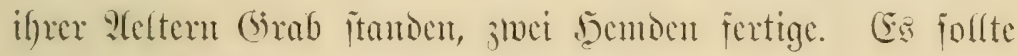

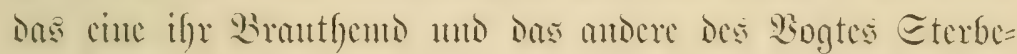

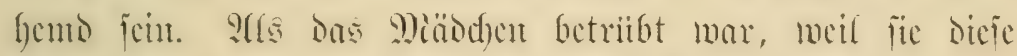

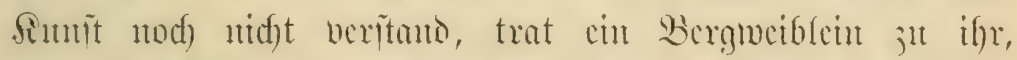

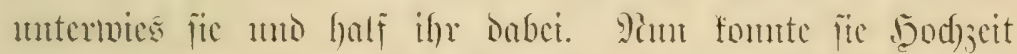

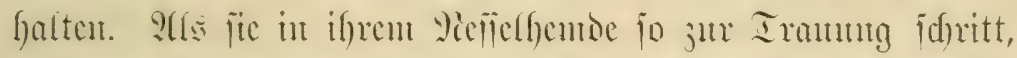

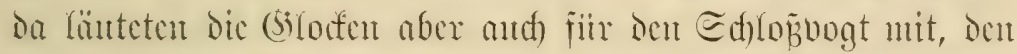

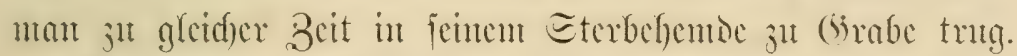

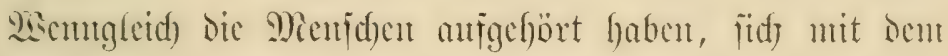

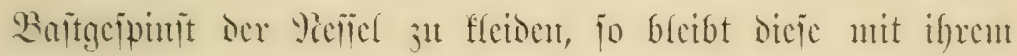

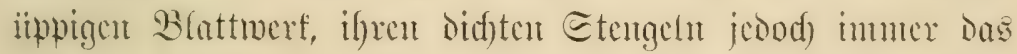

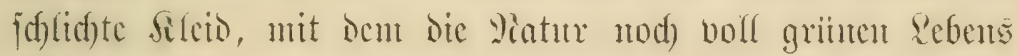

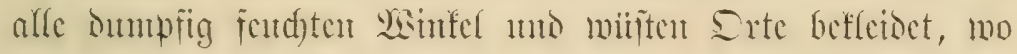

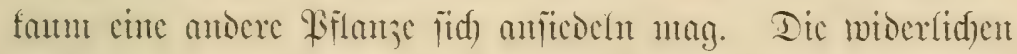

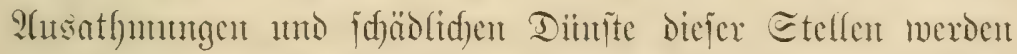

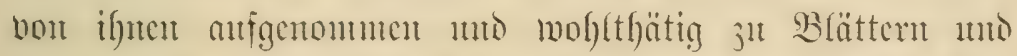
Etengeln verarbeitet. Dic Defind jelber, welde Dort fpriefit 


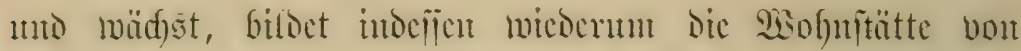

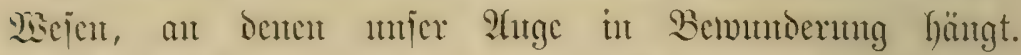

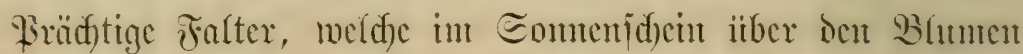
ınjerer (siärten flattern ınto gaufelut, sic Blanfante unb bas

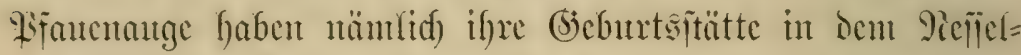
geitriipp. 3fre giautpen nagten an Defīen Blättern; fic putplen ïd) cin unto fprengten bie Buppe, Itum dam fortan jiber

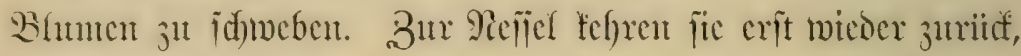

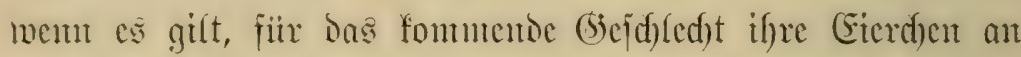

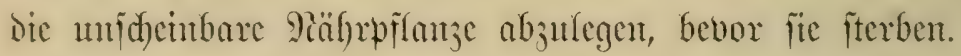

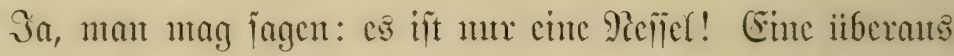

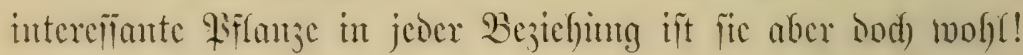

3.

\section{A III}

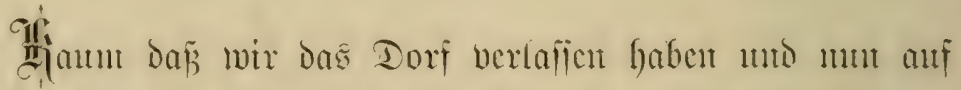

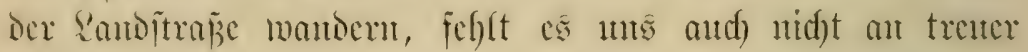
Begleitung. Wicocum ift's ein befonderes Blumenvöffden,

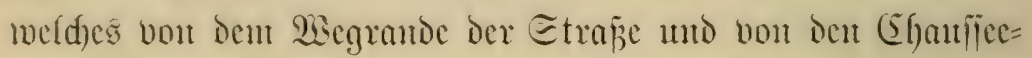

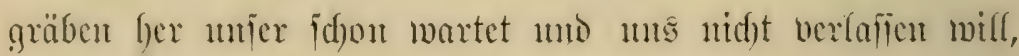
jo weit die Sanditrañe fich himjief)t.

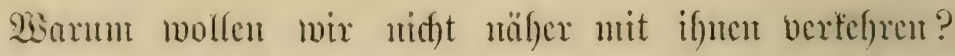

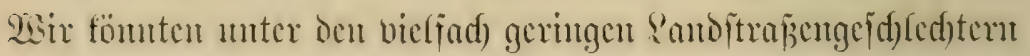

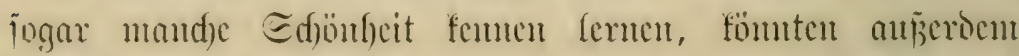

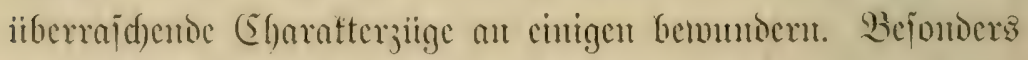
Trembidgaften werten wir wahruefunen, intem wo dic cine 


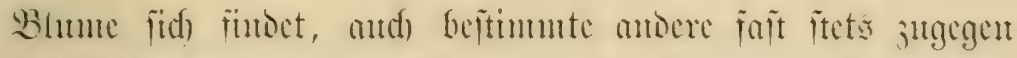

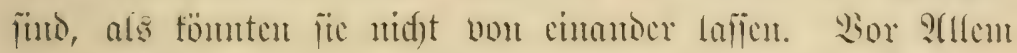

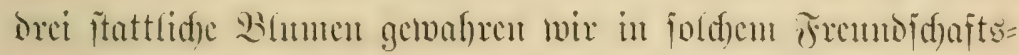

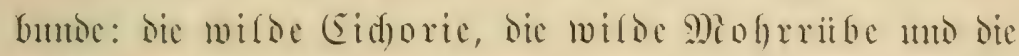
Bibernelfe. Eic mandern alferorten im dentid)en Siater=

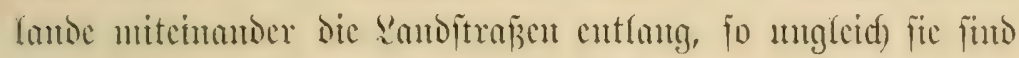

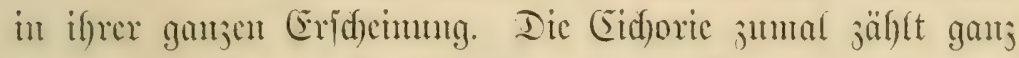

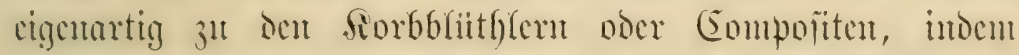

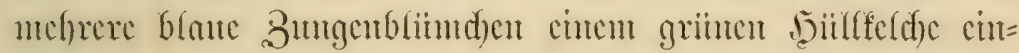

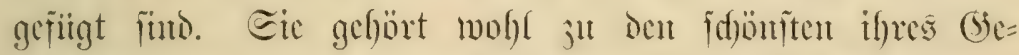

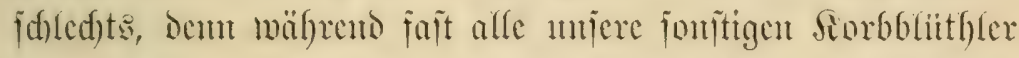

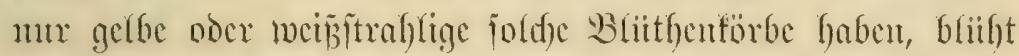

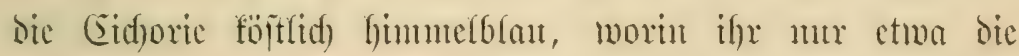

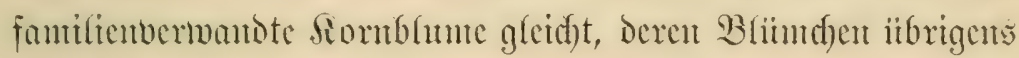
nid)t jungen=, fondern röfrod)en= oder fiiflfornartig gcjormt fint). Darmm hat die (Sid)oric trots if)rer grangriaten Blätter

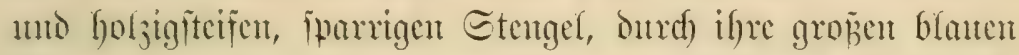

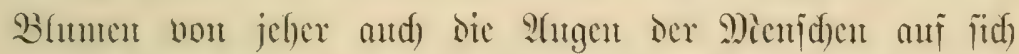

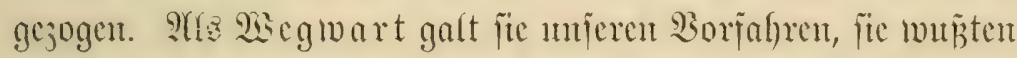

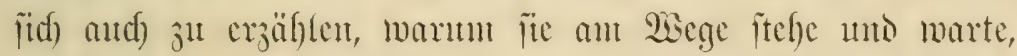
Deum fie ift cigentlid) cine Jumgfrant, dic in (siram um

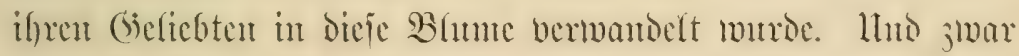

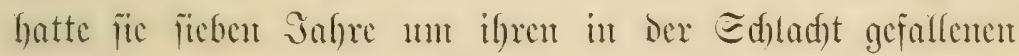
Geliebten geweint; als man fie bam bereden wollte, cinen andern Dian ju uchmen, da geidhah), was fie añ bieje $3 \mathfrak{u}=$ muthutg ervicderte:

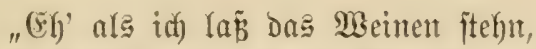

Wirf id lieber auj bie Wegidjeid gebn,

Eime Jeloblum' bort ju werden." 


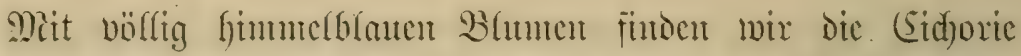

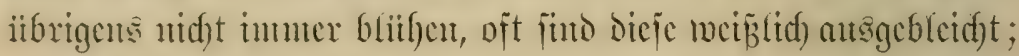

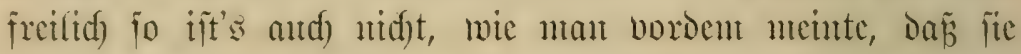

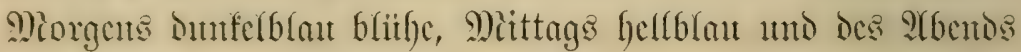

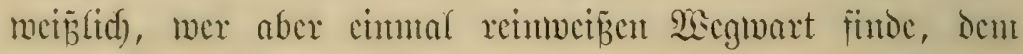
jolle bas ganj bejontores sthuif bedoutent. Die Eage ging aud), Daj affe Wegmarte verzanberte Micnidyen jeien, uno die viclen blanten feien böje Menjaden gemejen, die wenigen weipen bingegen gute. Faracelfus fdyrieb jogar, daj die waxjel

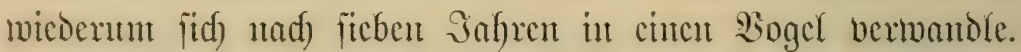

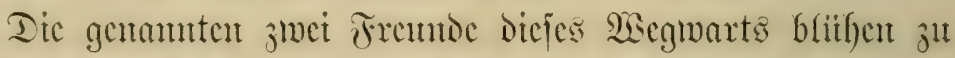

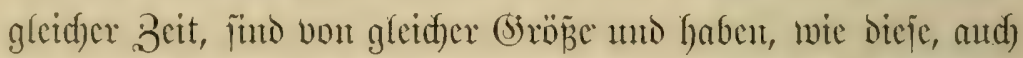

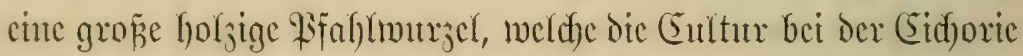

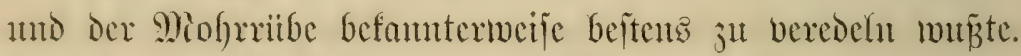

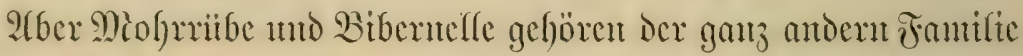
Der Doldonblitthler oder Edjumpflanjen an. Bejondors die

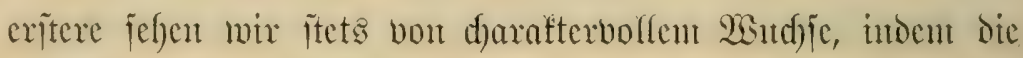

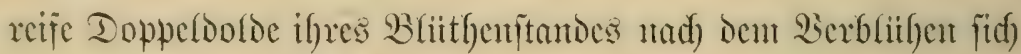

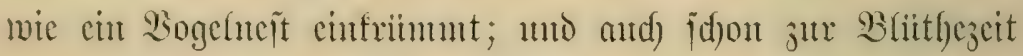

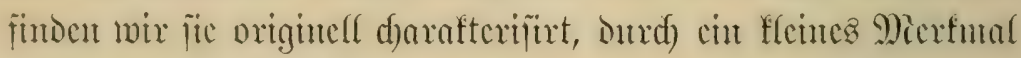

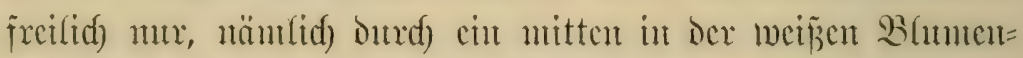
dolde befindidaes puxpunrotles Bumentrönd)en.

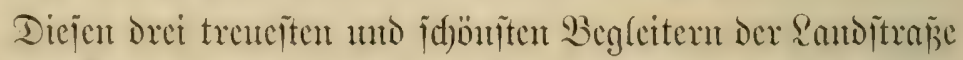

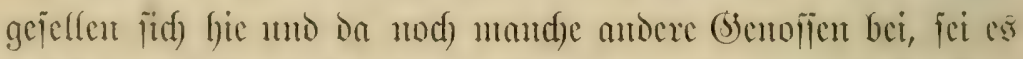

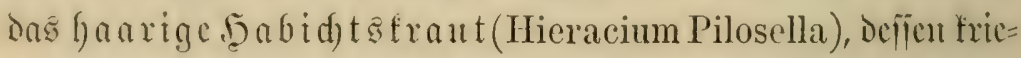

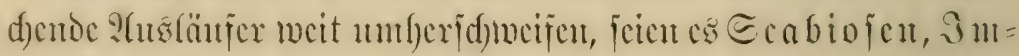

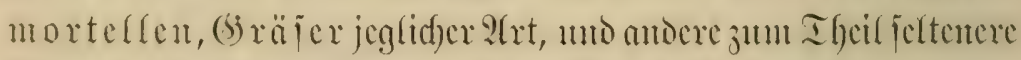

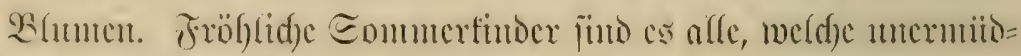

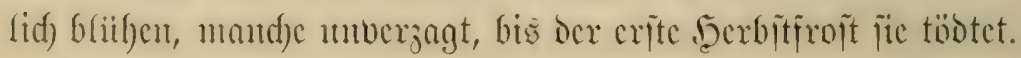


2(ber dic Randitraje, anf weld)er fie in bex Sommer=

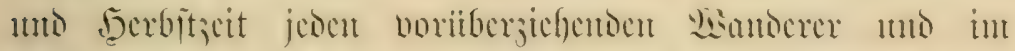

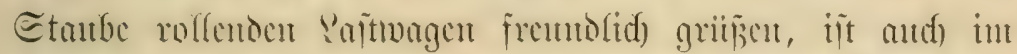

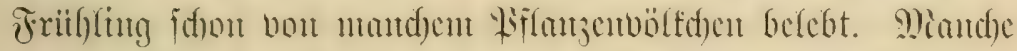

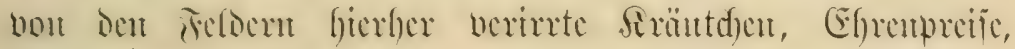

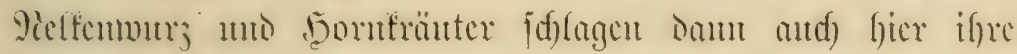

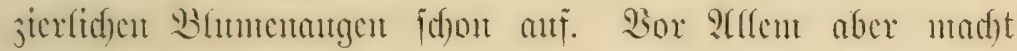

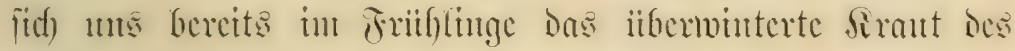
2isegebreit (Plantago) da benterfbar, Defien anjehnliche,

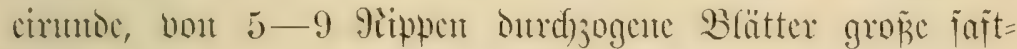

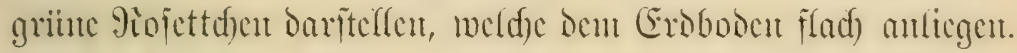

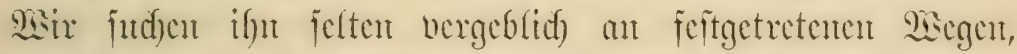

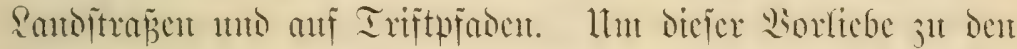

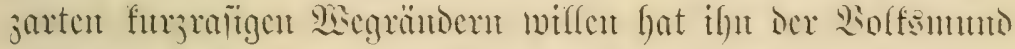

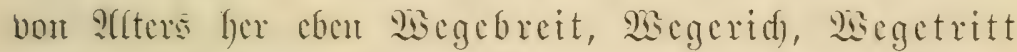

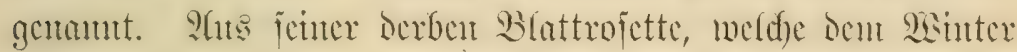

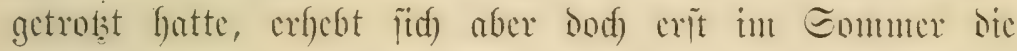

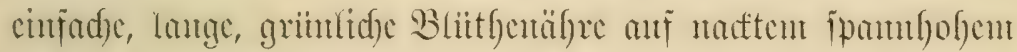
Edafte. Llujdeinbar gemtg ift je; jelbjt wem bic Stanb= färfden, je vicr in jedent ocr idslichten troctenfüutigen

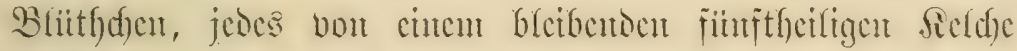

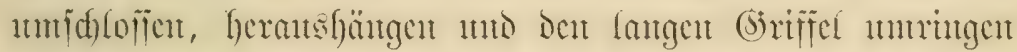

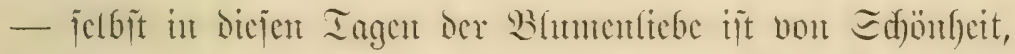

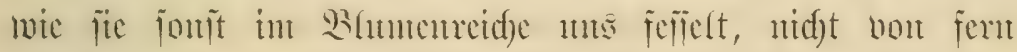
die Rede.

Dies ?fles gilt bejonters bon bem grofien $28 \mathrm{egcrid})$ (Plantago major); Dic beioen antoren: Der Lanjettlide mo der mittlere wegerid) (Plantago lanceolata nto media),

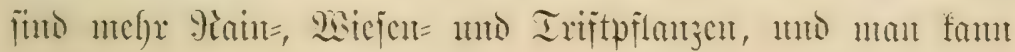


fic mux in fo fern als 2 segpflanjen betrad)ten, weif fic fitf)

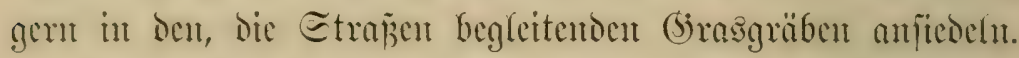

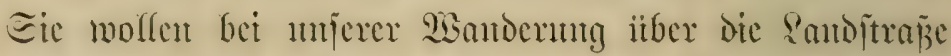

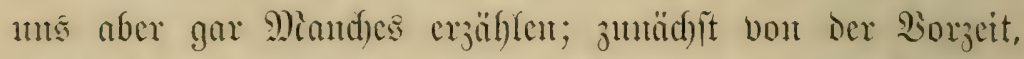

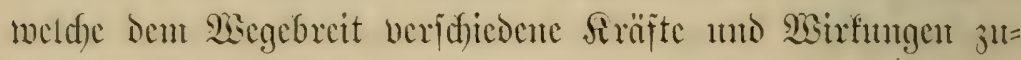
geidrieben hatte und ifu darum bejonders hod eforte. Es wax cin frant, sem man alfe nötbige faitlfe in menfd)lidben

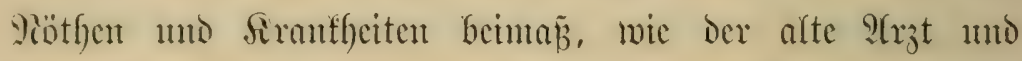

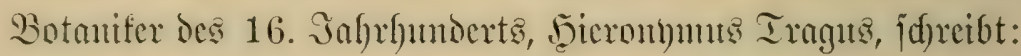

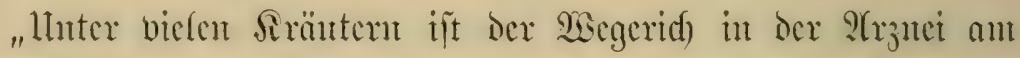

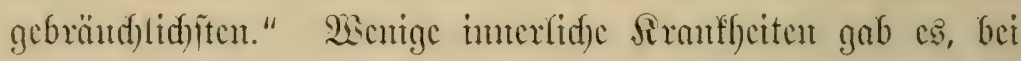

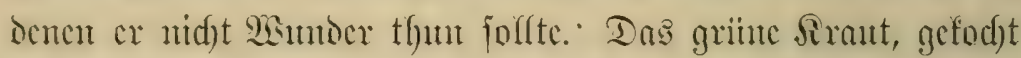

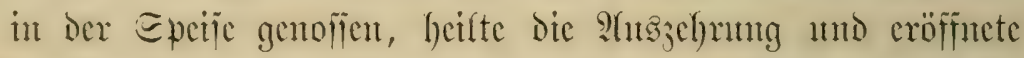

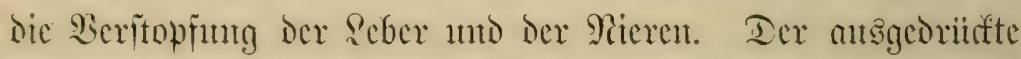

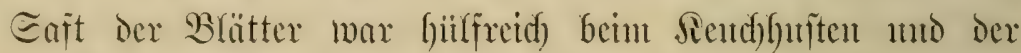

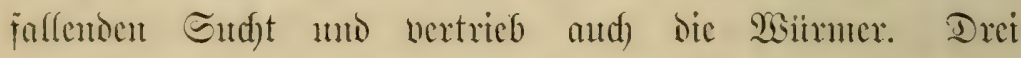

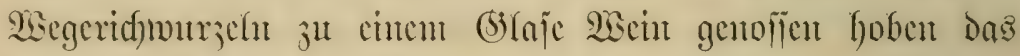
Ireitn̈gige Ficber, bier hingegen das viertägige Fieber. 9rber alle bicje imnerfide beille ift nod) Simberipiel gegen bie

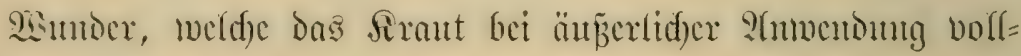

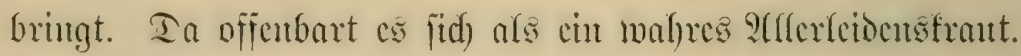

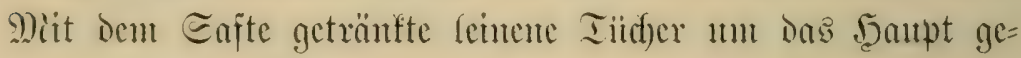

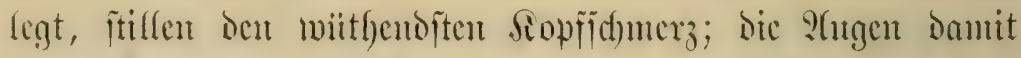
betmpfit werficren dic Entzimbung mo dic Sluren erbatten

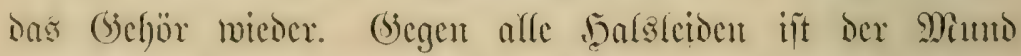

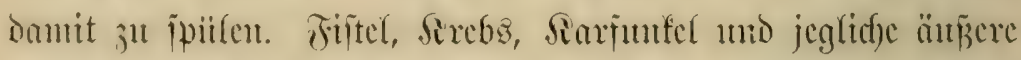

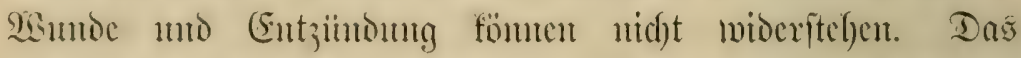

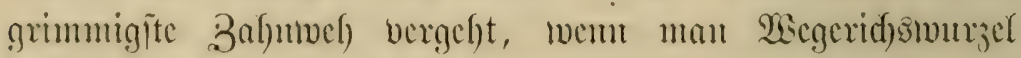

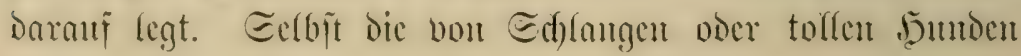




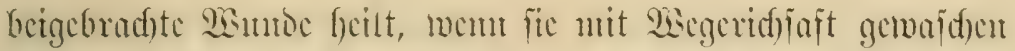

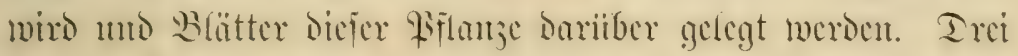

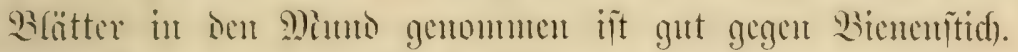

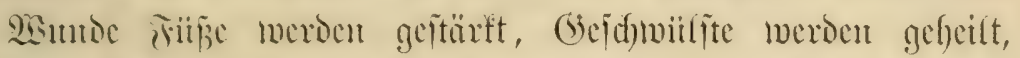

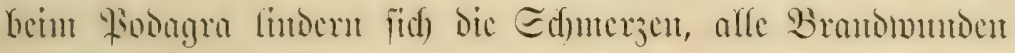

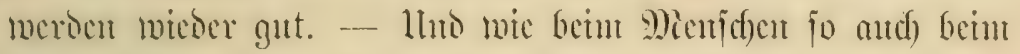

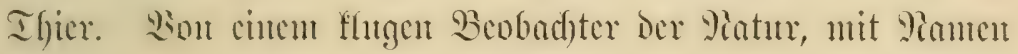
Irnsmms, boridsten dic alten firänterbildyer, cr babe bencrft,

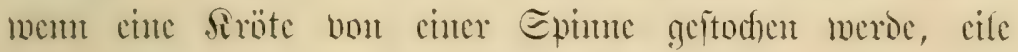
fic jutm Megerid and dantit werde ifjr geljolfen.

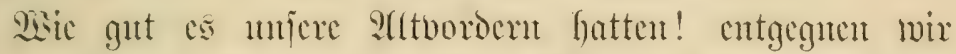

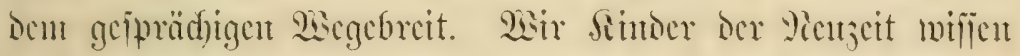

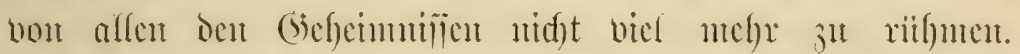

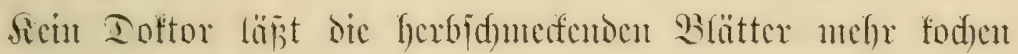

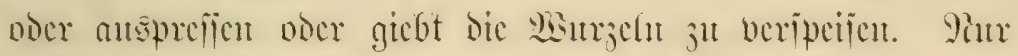

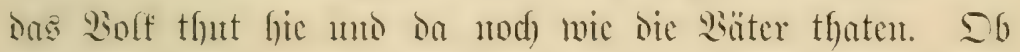

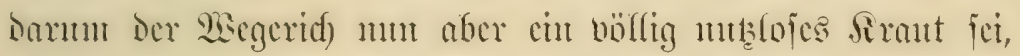

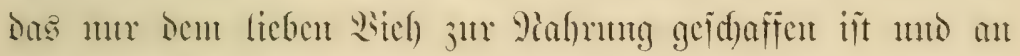

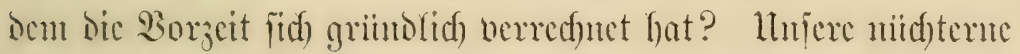
3eit bat rabifal mit allen lleberlicferungen gebrod)en, bejombers

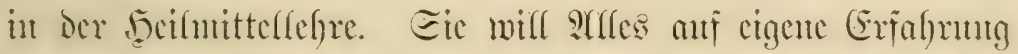

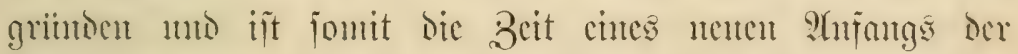

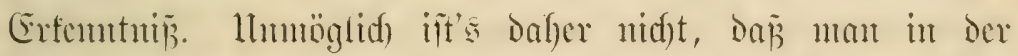

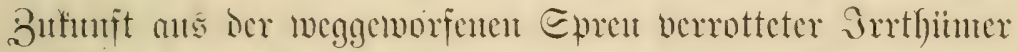

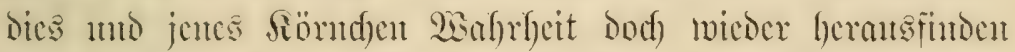

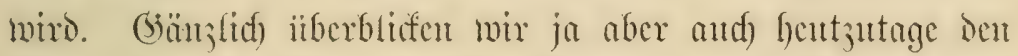

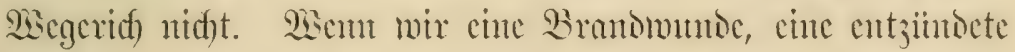

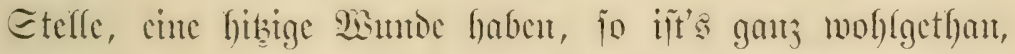

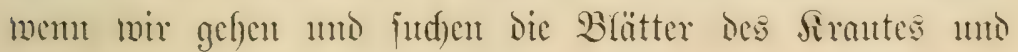




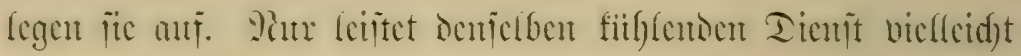
auth jedes andere faftreide Blatt.

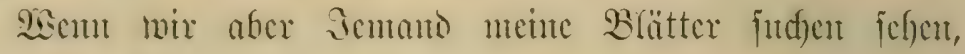

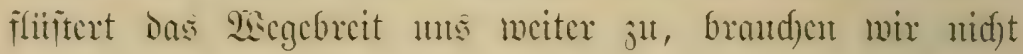

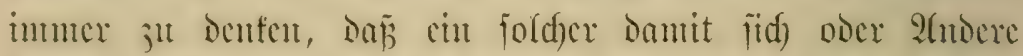

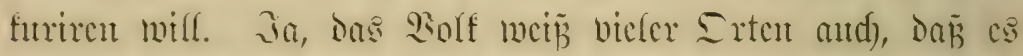

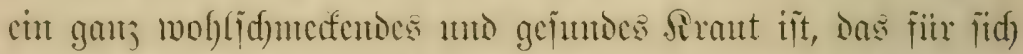

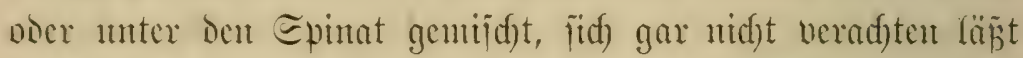

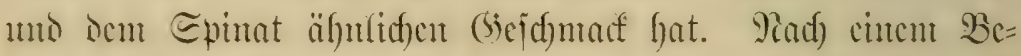

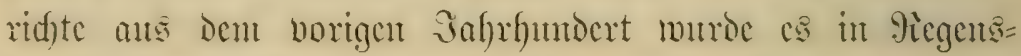

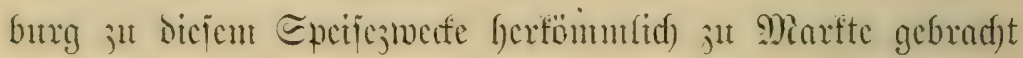

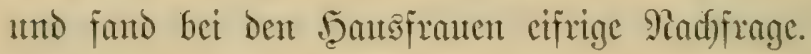

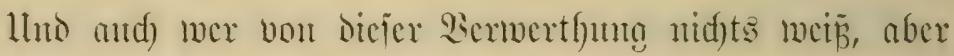

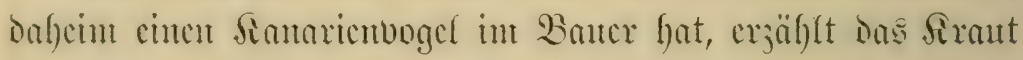

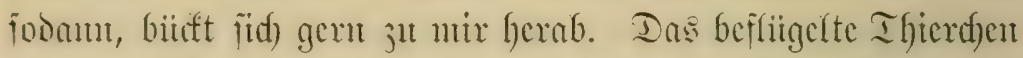
liebt mit Sciomichart das fene (Scjänte der reifen bruntge=

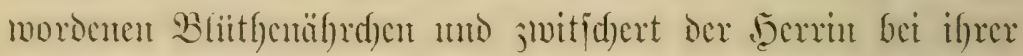
Desuntelgr Lntitiger entgegen, went jie cinten ganjen Bitifdel

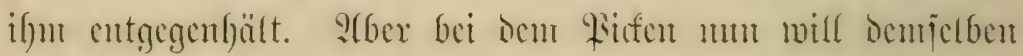

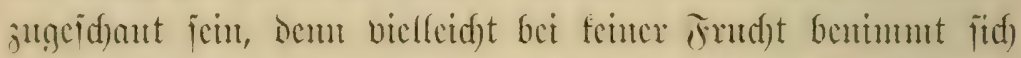

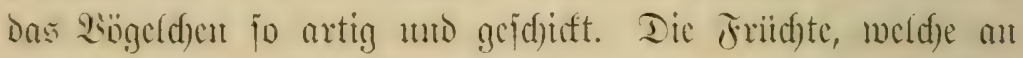

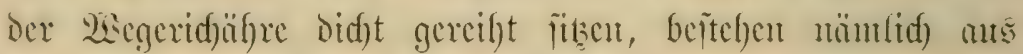

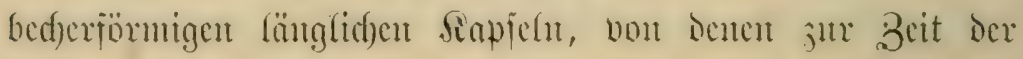

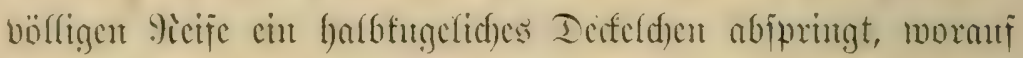

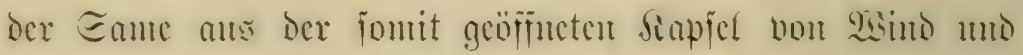

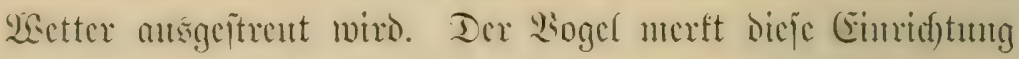

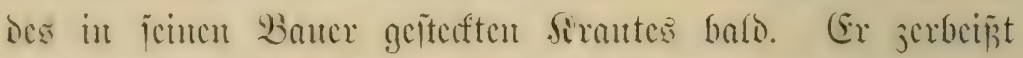

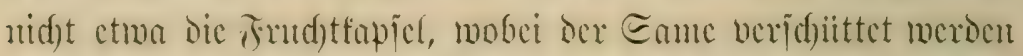

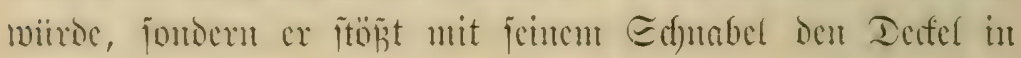




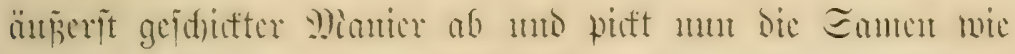

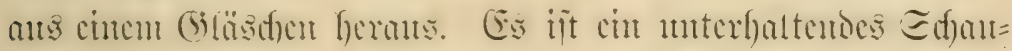
ipiel, an ocm wir uns nidft fatt jefen fömen mo bas anj

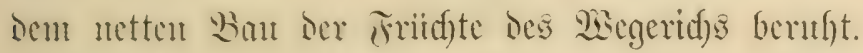

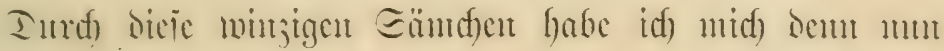

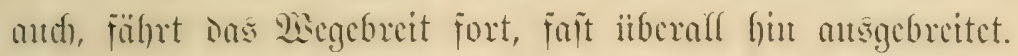

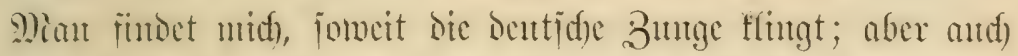

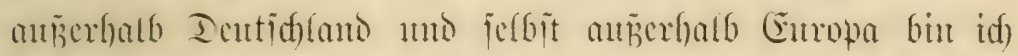

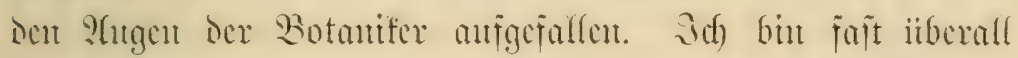

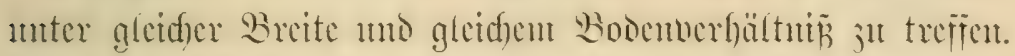

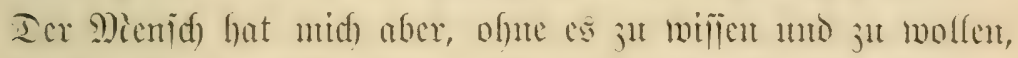

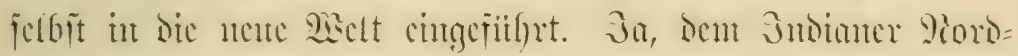

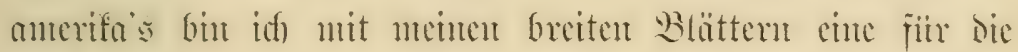
Wo!nutütten mo ganjen Difrufte Der Soloniften fo dourat=

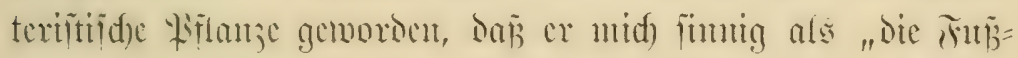

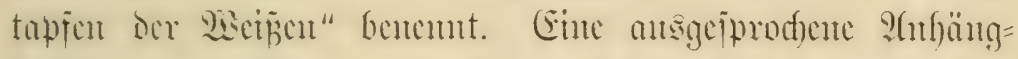

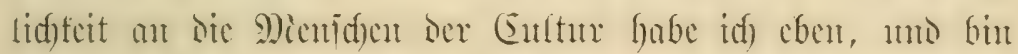
ifnent ïbor das ftille Dicer gejolgt, ganj wie bie Dantsthere

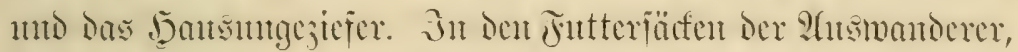
in ben mitgenommenen Eaatvorräthen uno anflaftent an alferlet antern Sram ît mein Eame in jeoe neut Seimath mit cinge= idfleppt morden, uno iff finde in ben bals eintretenten Eultur=

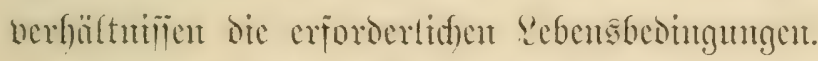

lluter all decient Geplanter bes belerjahrenen wegebreit

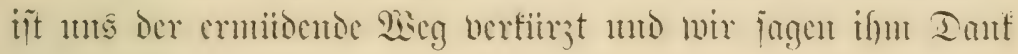
fiir jente 9)ittheifutgen. 


\section{4. \\ give Strakenflora.}

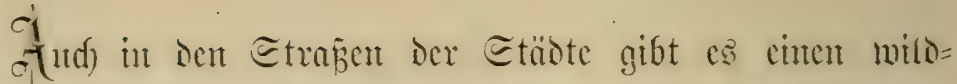

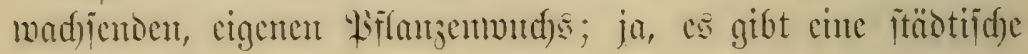
Straj̃enflora.

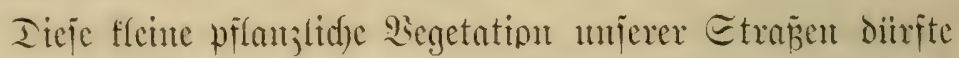

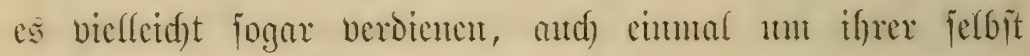
wilfen fremolids beadhtet ju werden. Mindoctens das auf

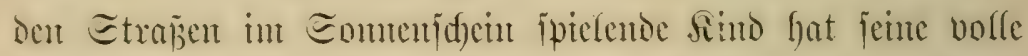

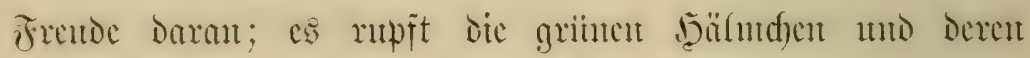

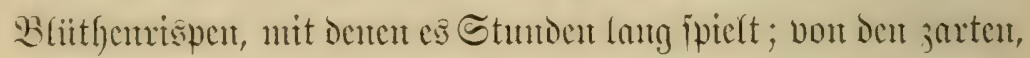

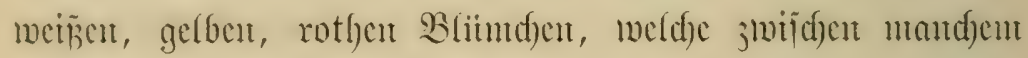
feinen Blattwerf Gervorlugen, madyt es fiir dic \$itppe allerliebite

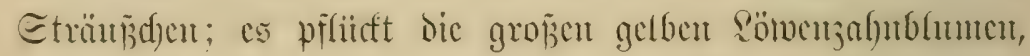

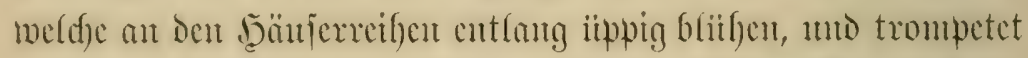
auf Deren hohlen, bitternildfigen Eticlen. Dem Sinto ijt bie Etraje erit red)t licb, ment jic nicht blos obse Etcimpflajter ijt.

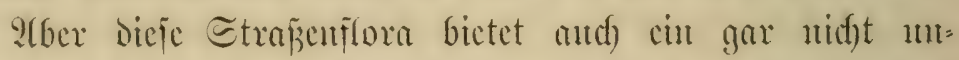

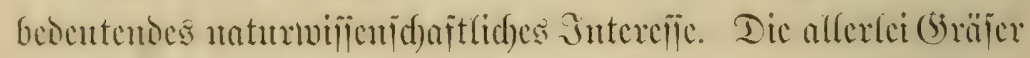

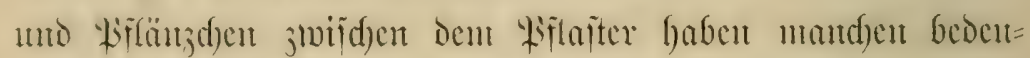

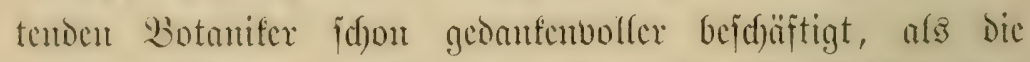

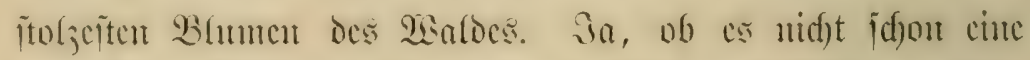

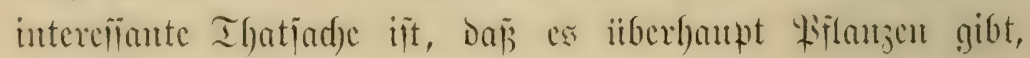

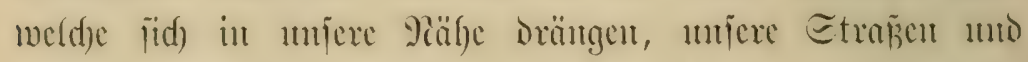

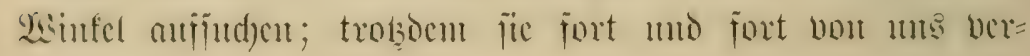




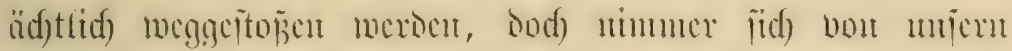

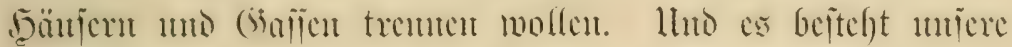

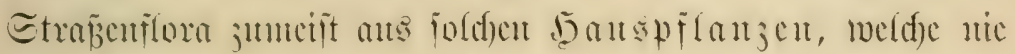

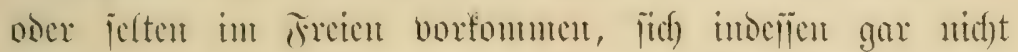

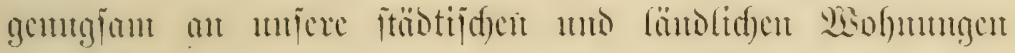

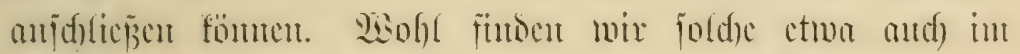

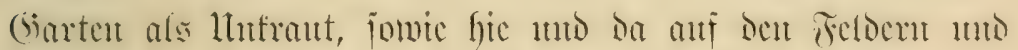

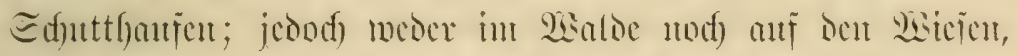

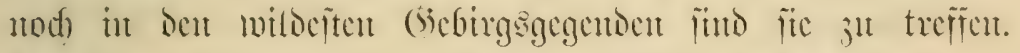

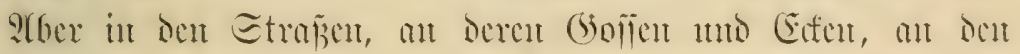

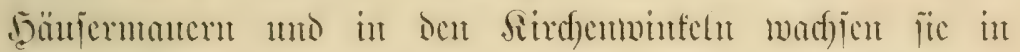

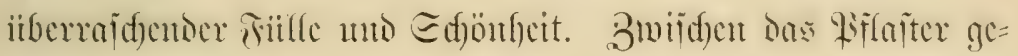

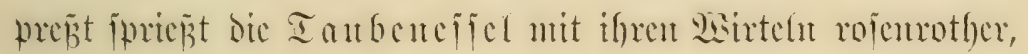

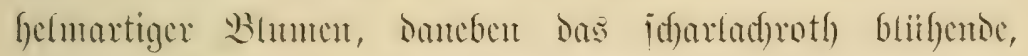

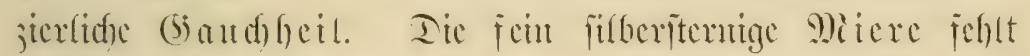

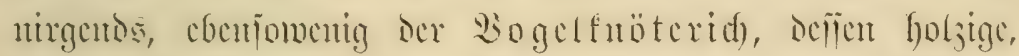

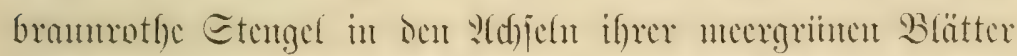

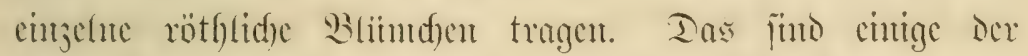
flchblumigen Bierden, melde bem Etrapengrafe ïberall cin=

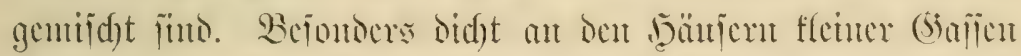

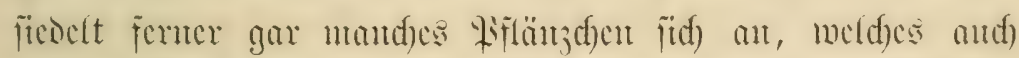

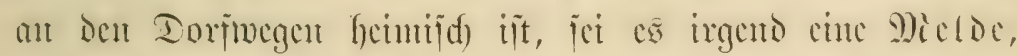

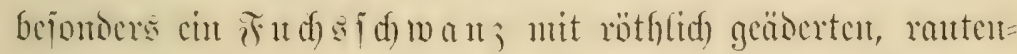
förmigen Blättern, Die b̧äringsmeloe mit bent jo cigentlyim=

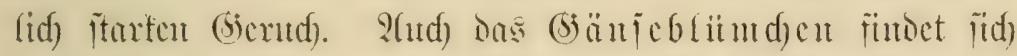

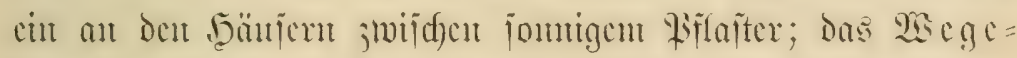
breit mit jemer flad)en grojen Blattrojette ijt mit den

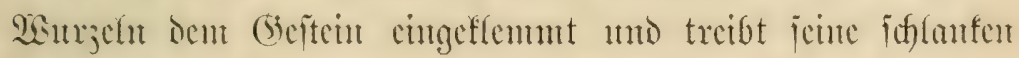
grïnen $2(e$ fren bis itber fingerfod) empor. 


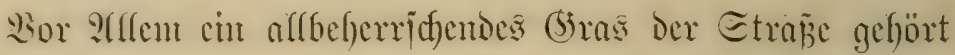

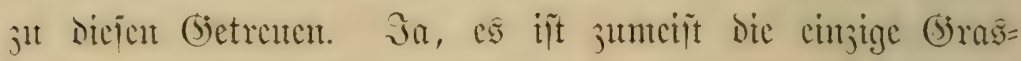

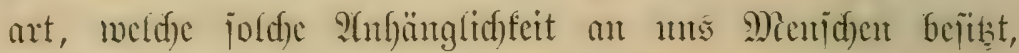

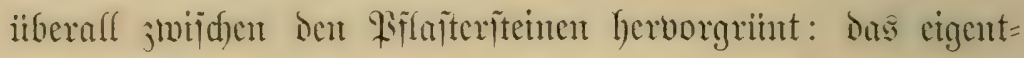

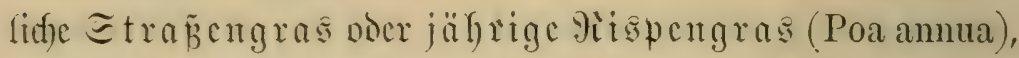

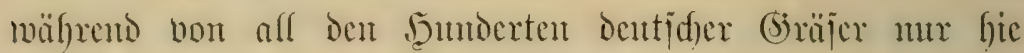

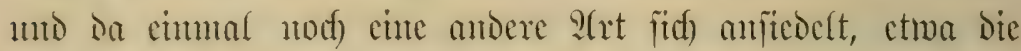
Poa trivialis, doer die Miäljegerite (Hordeum murinum).

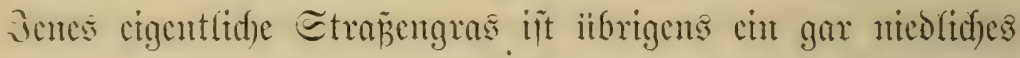

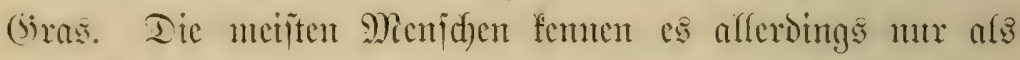
jwij̄den Den Etenten cingeflemmte grašblättrige Bitid)el, aber haben nie anf deren etwa fügerf)ohen, bïmmen, ferjengeraben

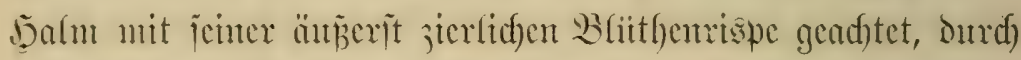

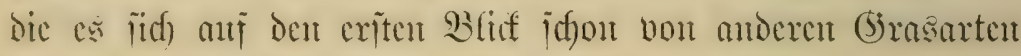

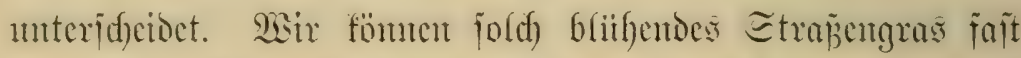

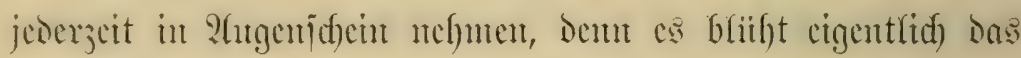
ganje Jabre munterbrod)en, bour exften Trithling an, oft

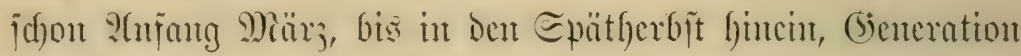

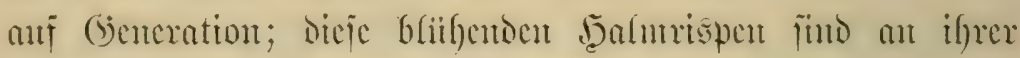

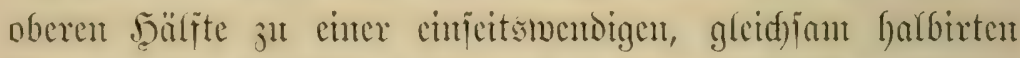

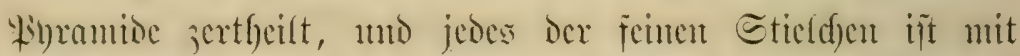

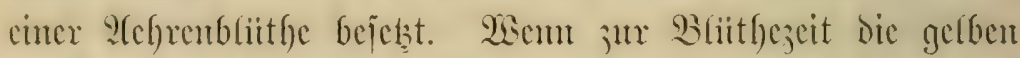
Etanbbentel berantsangen, jo ijt's cin mirtlid) nicolidges Pirlänjd)en.

Wic fonmen aber jente Sirüterdent mo dicjes (biras Daju, mit joldser borlicbe fid) anf unferen gepflafterten Straj̧en

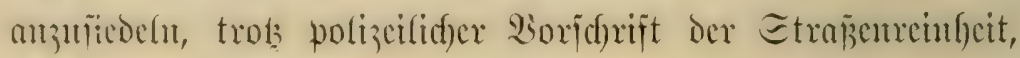

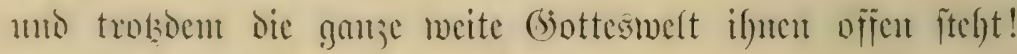

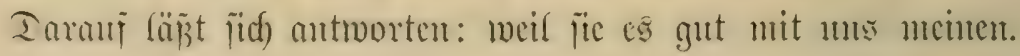




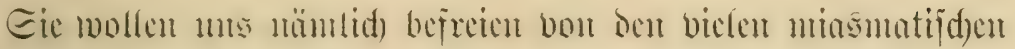

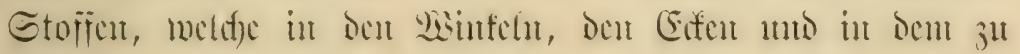

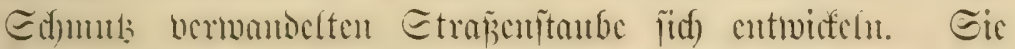

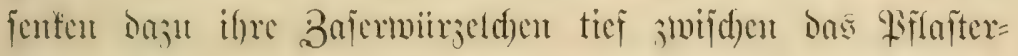

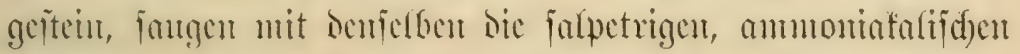

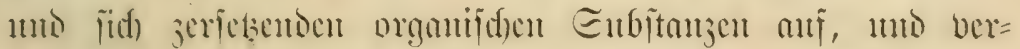

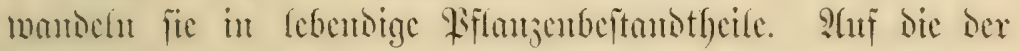
menfflid)en (sejuntheit fentolid)en Stoffe funt fie eben an=

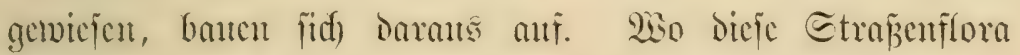
fid) cinfuntet, fömten wir beshalb mit (semijheit and) ant=

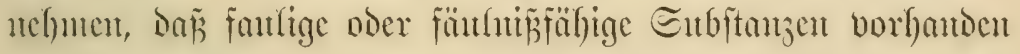

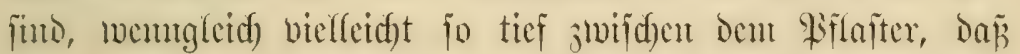
wir mit affer Desinfeftion jic ndd)t wegid)affen fömten; aber

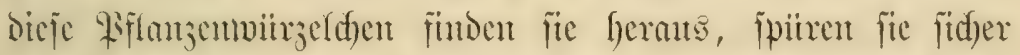
ani mos bejreien uns jegensreidg bon senjelben. Sic fins

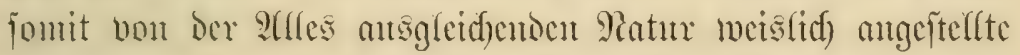

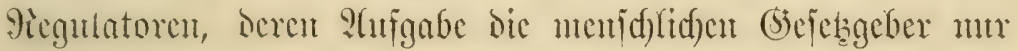

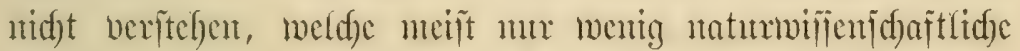

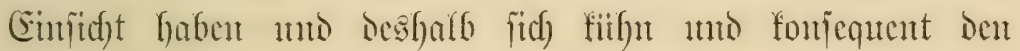

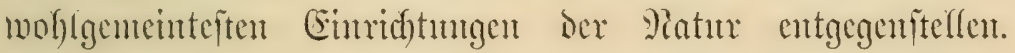

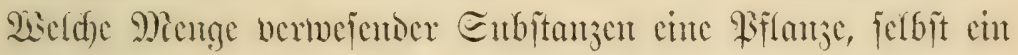

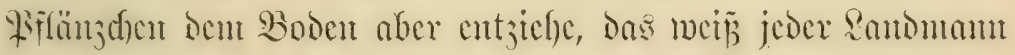
3t fagen, der bald fente Ernte melgr haben witroc, wem er feinen Dintuger anf bie Felder bringen wollte, Den Der Diunger

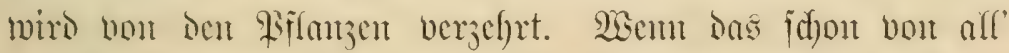

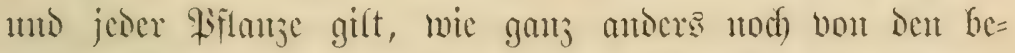

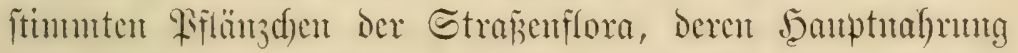

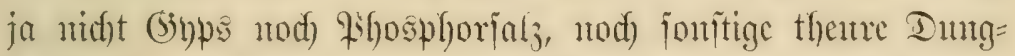
ftoffe f̈ms, fondern cben bor affent falpetrige umo ammto= 


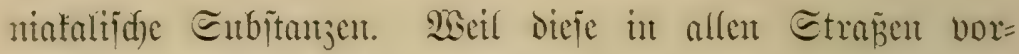
fandenten 巨tofje Durd) jene (Siräjer abjorbirt nuto unjdäblid) gemadft werdon, jo vegetiren bie leksteren ja mud mur an

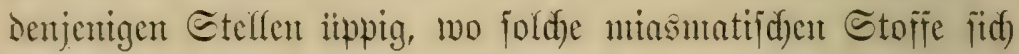

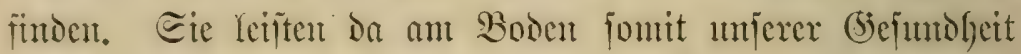
ähnlidge Diente als die Bäume, mit Denen jede Stadtver=

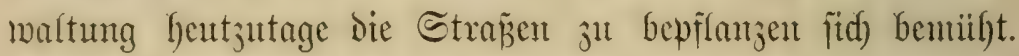
Niag mant in நerbjt, wo die Etragenflora abfitubt und Der

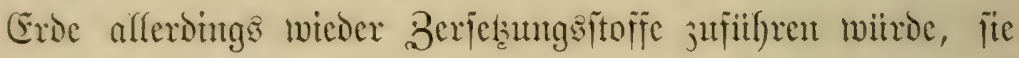
inmerfin Ivegitippen; aber in Der grituen Jafresjeit lafic

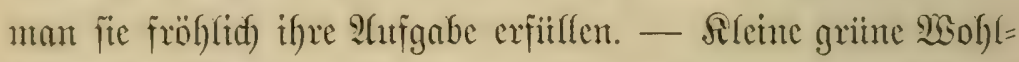
thäter Der Menjdent ifr, wix wollen eud preijent wegent curer

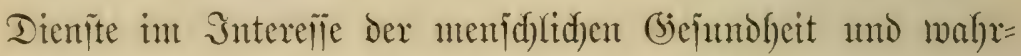

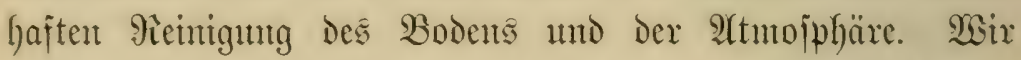

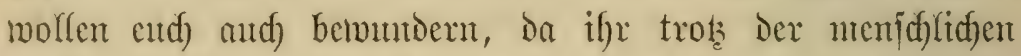
Berfermung in eurer $\mathfrak{A}$ ufgabe end) utdst ine madjen lapit, unermüblich immer wieder bervoriprient, wo dic thöridfte

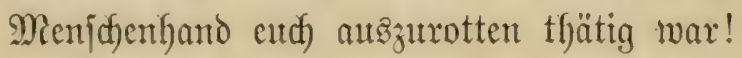

\section{5.}

\section{寻entrdje Gartenblumen.}

\section{gir}

Etanche der in den Dörfern vorfonmenten, jogentanten

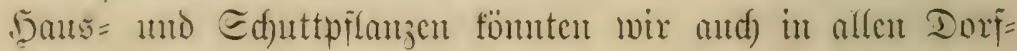
gärten, jelbît in Den (järten der 巨tä̀te finton. "Ilntrant"

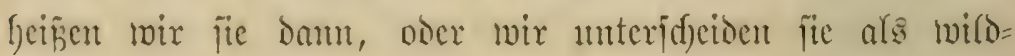




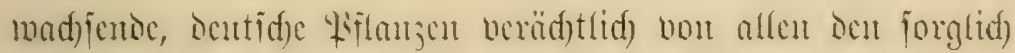

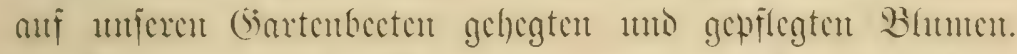
Ireten mir in don (siarten cin, m aber and) énige miperer

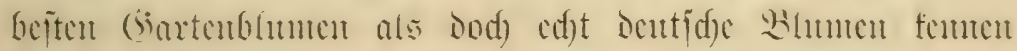
jult lerment

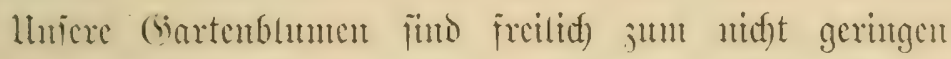

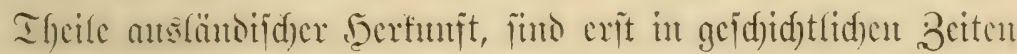

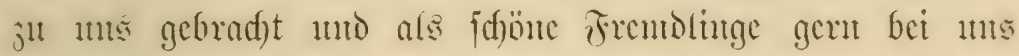

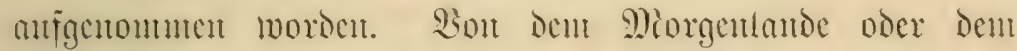

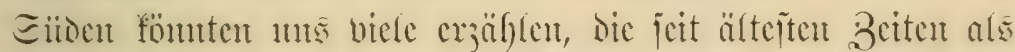
Bierffor bet uns in Egren gehalten wuroen. Woun jenfeit

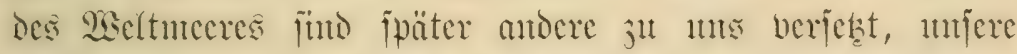
(biärten ju jieren, and and) dicje blitfenden Fremolutge, meld)e wix mit trenex Riffege in unjeren (bärten hegen, füt jum

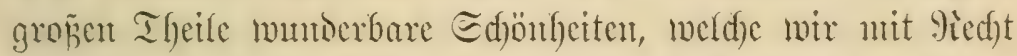

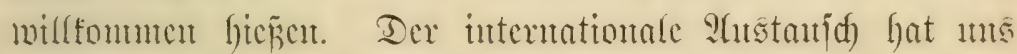

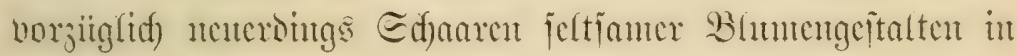
trantcite Pä̈lje gebradet: Die rojige Diftutra, bie Demero=

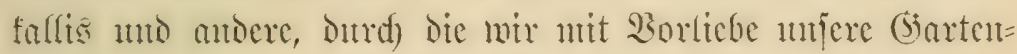
flor bermefren.

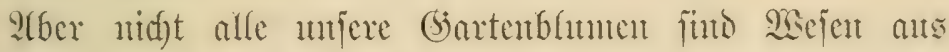

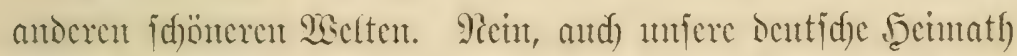

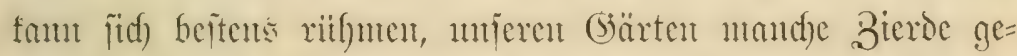

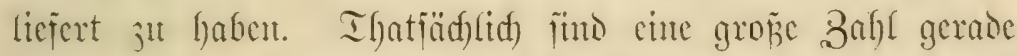

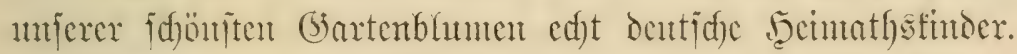
Shre Dentidhe Serfunt ijt Bielon mur unbefannt, weil ntan

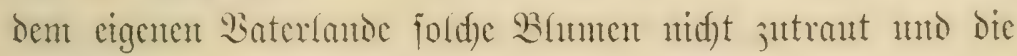

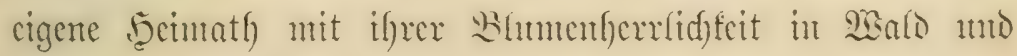
Flur, anf Bergen und in I I älern gar nidgt fent. 


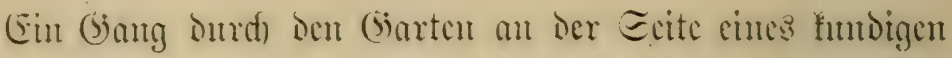

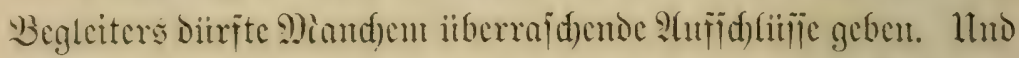

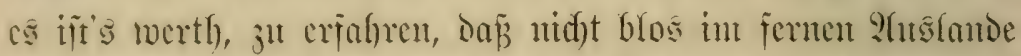

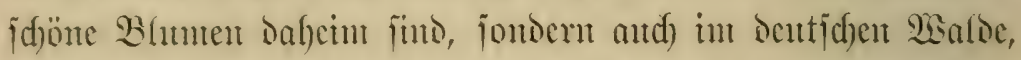

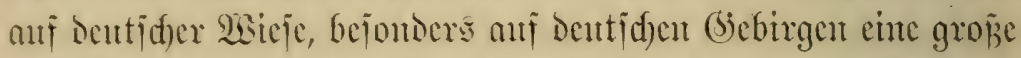

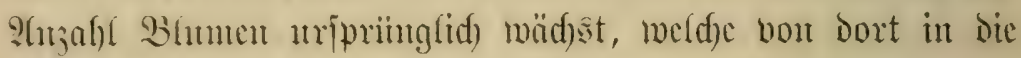

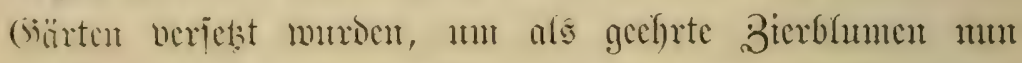

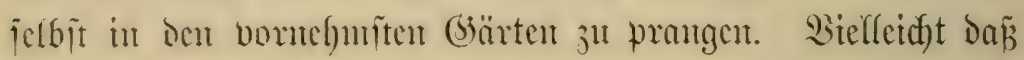

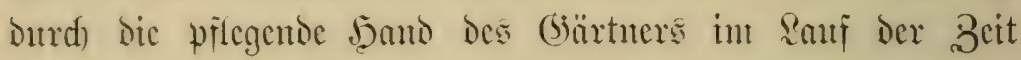
Dieje noer jene ctrwas grö̈er unt volfer getworden ift; aber Die meijten find bis in die feinften Bïge linem nod) diejelben Blumen, als welde fie in freicr Viatur dem 2 sanderer be=

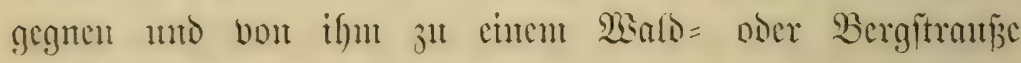
gepfliitit weroent.

Ed)on im Frithling begriipen wix in unferem (siarten edf)t

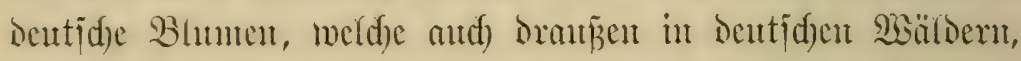

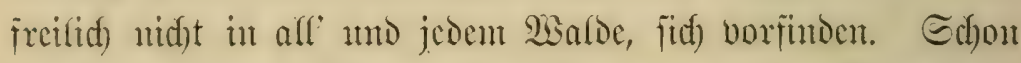
bie erifte blitlentoc Sartenblume, meldac wir and gern in

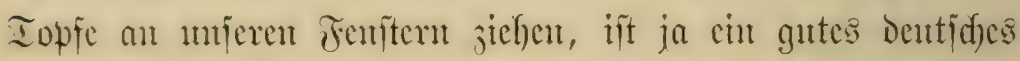

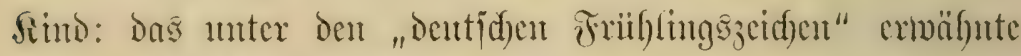

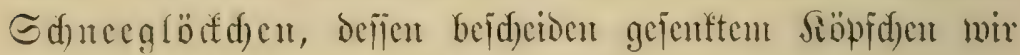

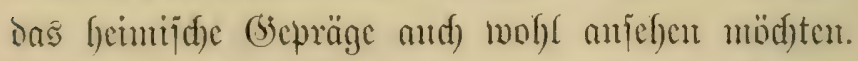

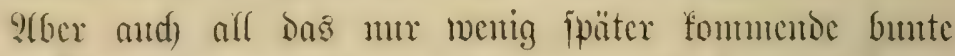

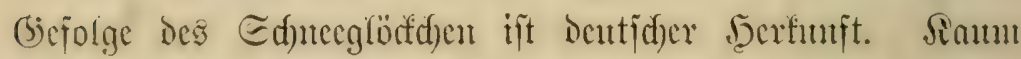

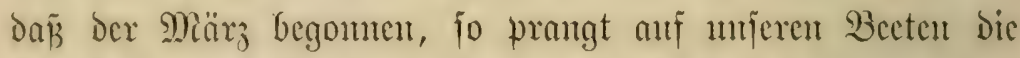
Daplue, and Eetidelbait ober Sellerbals gentant, mit

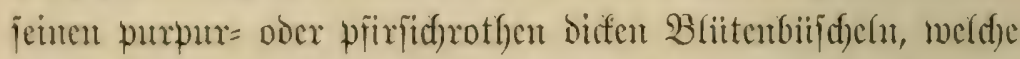

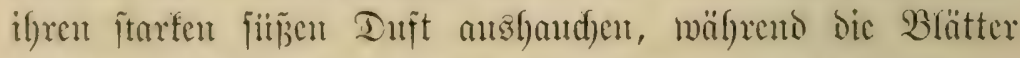

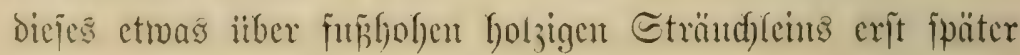




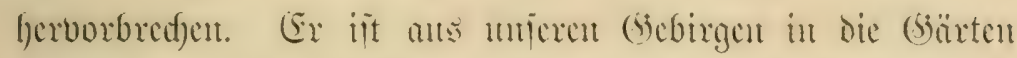

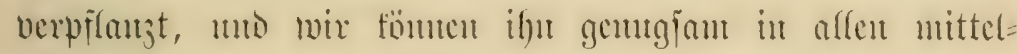
unto fitboentidfen (sobirgen, dajelbjt bejondocs an troctenen

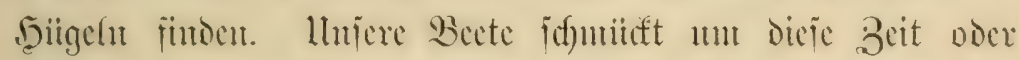

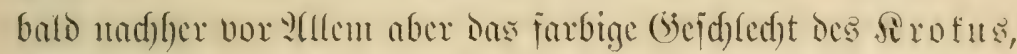

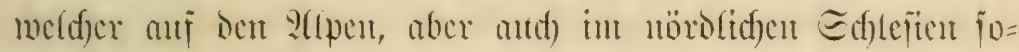

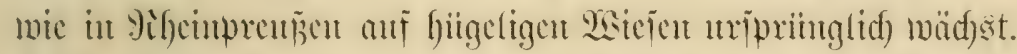

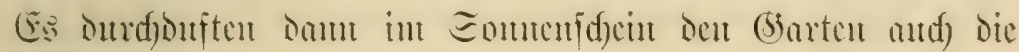

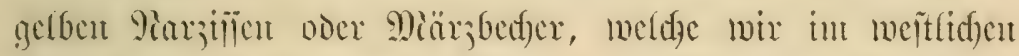

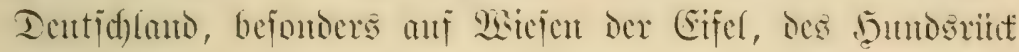

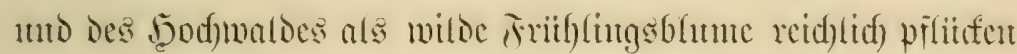

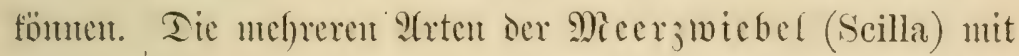

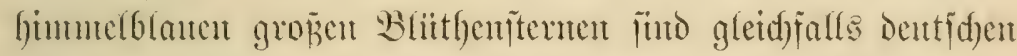

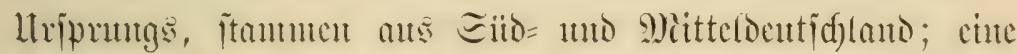

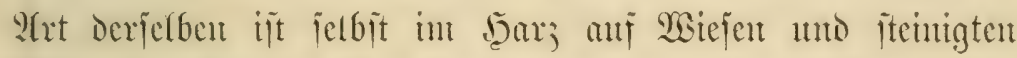

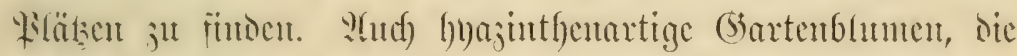

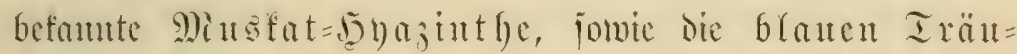
befden fommen in gan; Deutidgland jeritrent auf Wiejen,

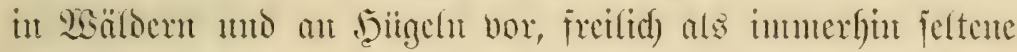

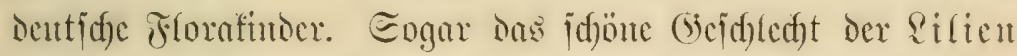

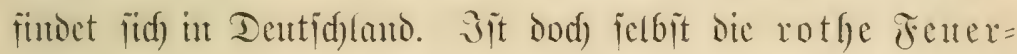

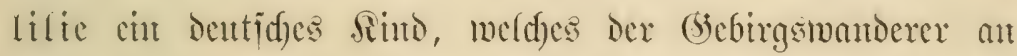

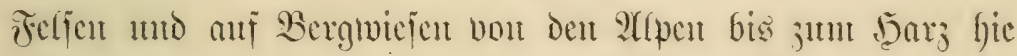

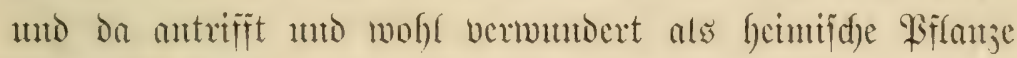

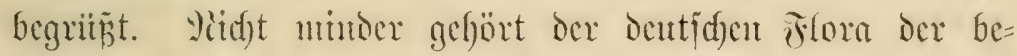
licbte I itrfenfund (Lilium Nartagon) an, unto jwar nidgt blos in (şebirgen an itcintgten, gebitjdigen 2tbfängen

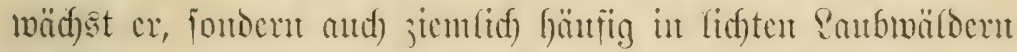

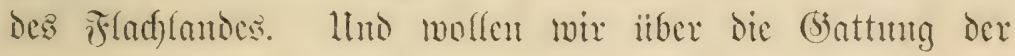




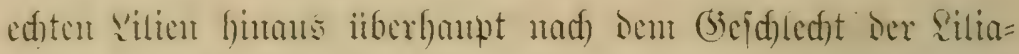
cent fragen, io fömen wir uns nod) gar mand)er colen Blame

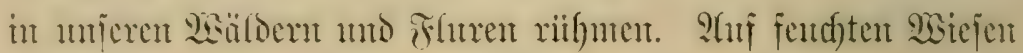

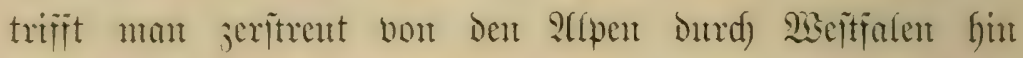

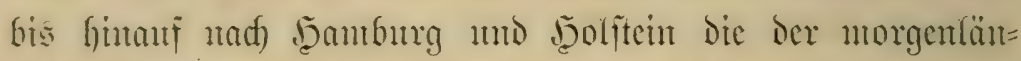

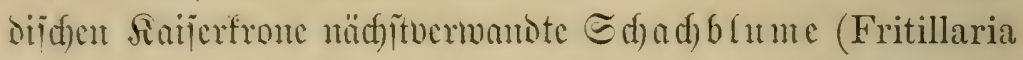

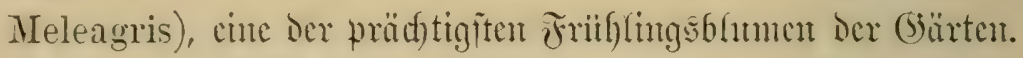

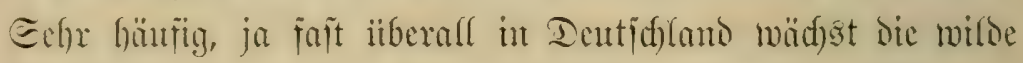
Iulpe mit grojer, lenteftento gelber, jogar suffiger Blument=

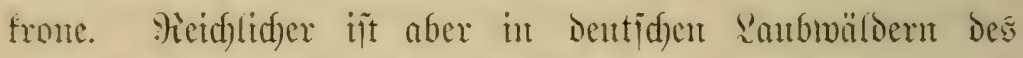

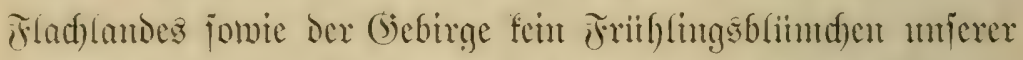
(bärten norbandon, als das blane \&eberblitmdan (Hepatica),

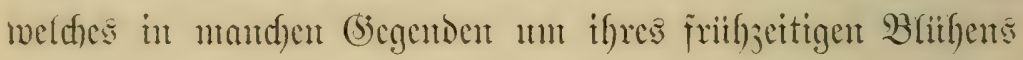

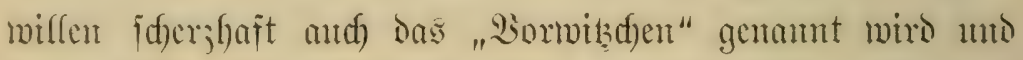

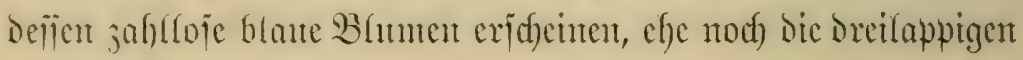
Blätter j̈tdtbar ïno, weld)e exft jum 2(prif hervorfommen, nad)bent die Bummen fajt böllig verbliigt fints. In Waloc

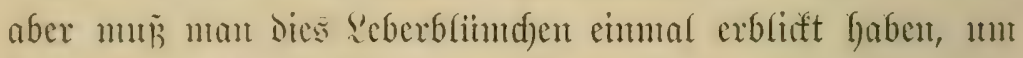

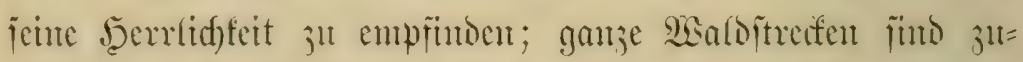

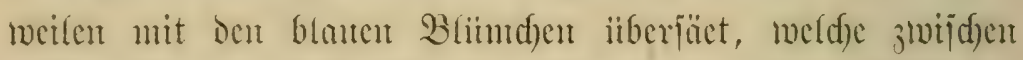

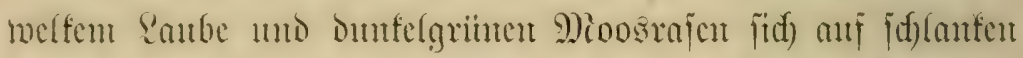
Etieldyen wiegen, während alles Seben umi fie her nod) joläft.

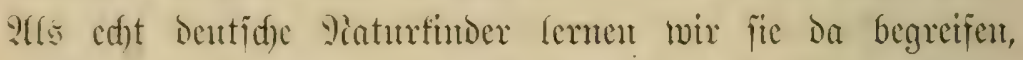

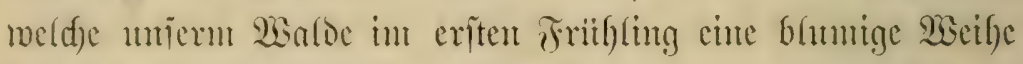

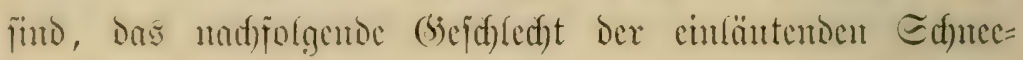
glöctçen.

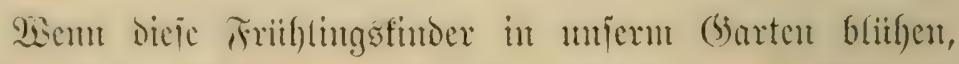

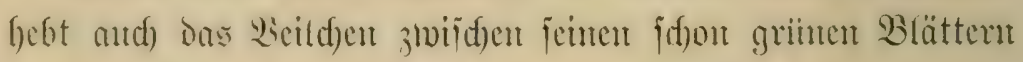

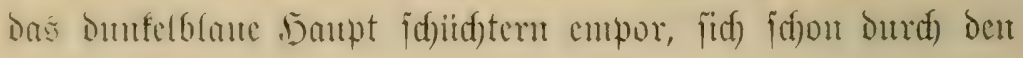




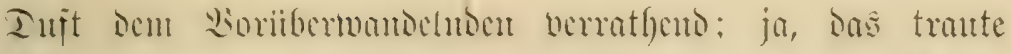
Weilden, meldent wir gern cinte Etätte in Giaten laffent,

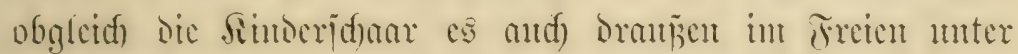

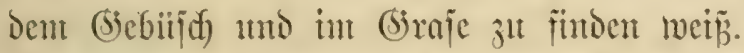

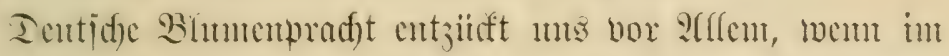

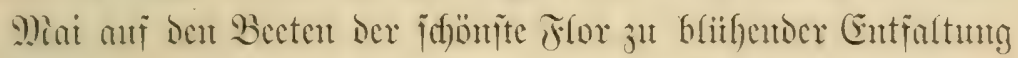

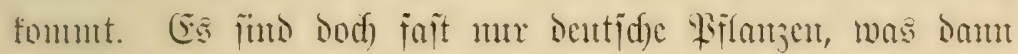
in mieren (jörten prangt umo sujtet. Die Miaiblume,

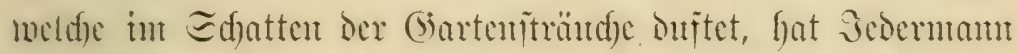

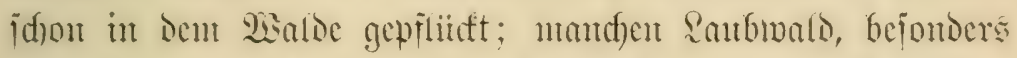

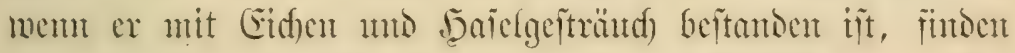

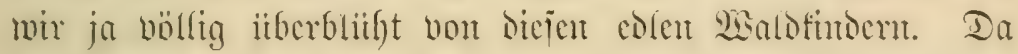

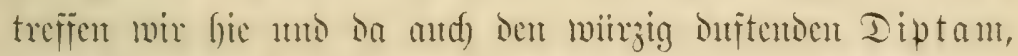
meldyer jwar ju ben ieltencu Fiflanjen unjerex sä̈loer ge=

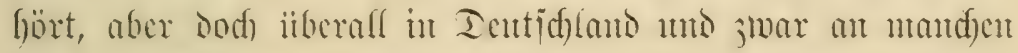

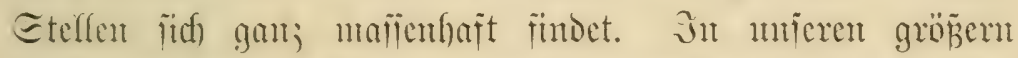

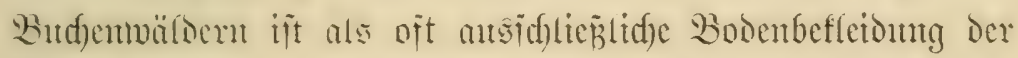
2is alometiter ju trefien, mit dent wir gern die jefattigen

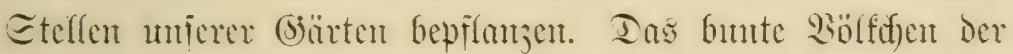

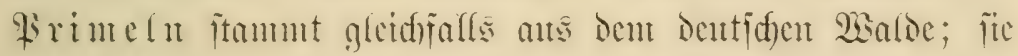

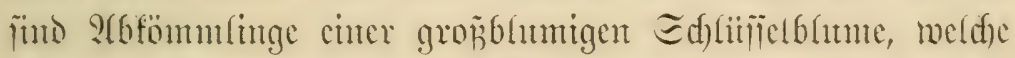

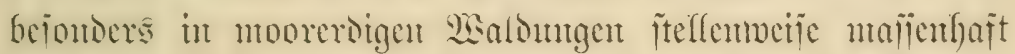

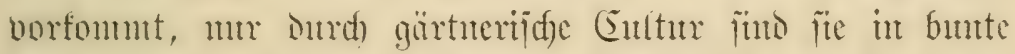

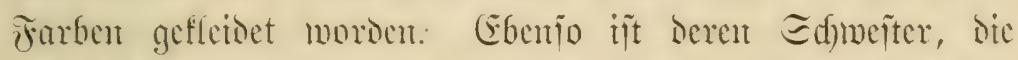

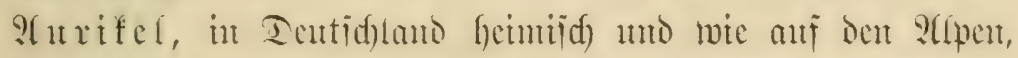

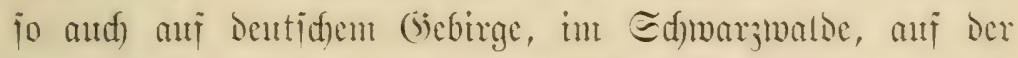

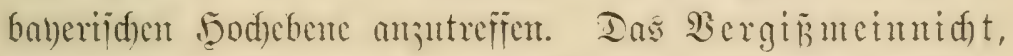
meldes jeocu (siarten verjert, ijt gleidjalls ans beutidden

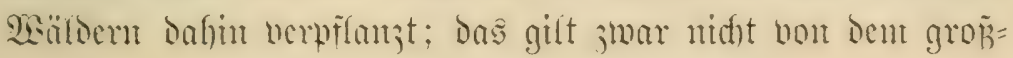




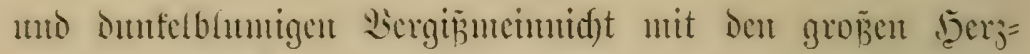
blättem, weldes gax fem cigentlidjes Bergin̄memutdet ijt, jondent bas (5cdenfemeit (Omphalodes verna) ınd nu

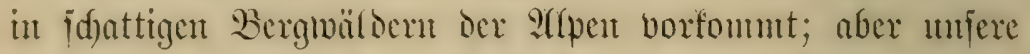
(särten verjiert and ch joldes mit etwas tlemeren uno belleren Bäintdyen, melde den Boden mantd)er Sanbwäliser

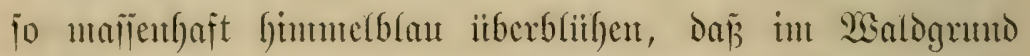

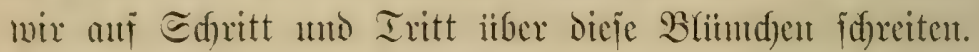

?(ntoere Bicrblumen, weld)e int Mai unfere Beete idnuitifen, felfen allerdings in dem deutfiden; bejonders den nordoentidgen

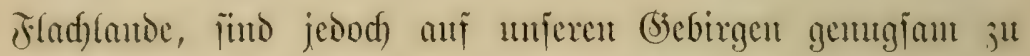
Бanfe. Das gilt etwa von der arfelei oder gefpornten

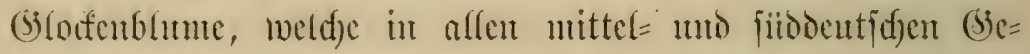

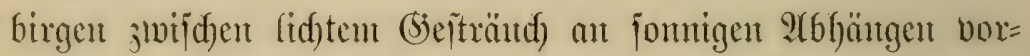
fonmut. Ebenfo treffen wix da das (5hriftophstrant; felbit

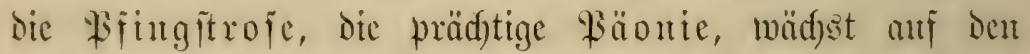
Bergen Eitdontid)(ands, bejonders in Sberbanern, Ivenngleid)

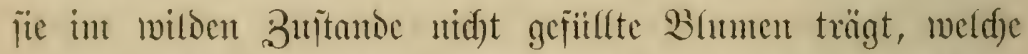
exit die (sultur in uneren (särten if)r gegeben hat. 2fud) dic mantigfadyen 2trten des Eteinbred), Die Horjellan= oder

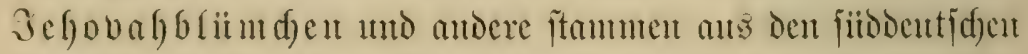
2(rpen, two fie in Den Yitben des Felsgeitems wad)jen. Dex (5oldact, als duftige belbueigerl bout altersher cine werth)=

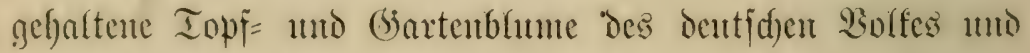
vou unjeren Didfern bejutgeh, wäd)st an Felfen, (Sentäncx

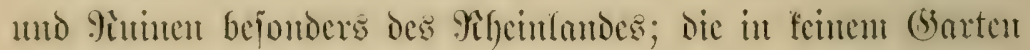
felfende zevfole blitht jwijhen Geröll mo an Felfen des

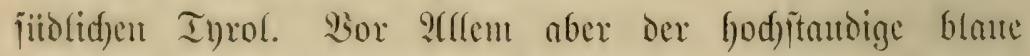

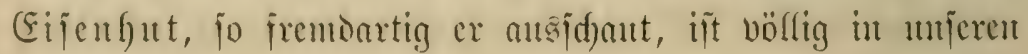


Gebirgen ju Dante; in Den Pl(pen ijt ar befonders in Den

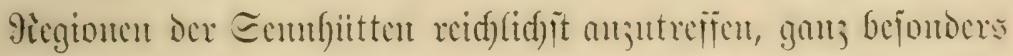

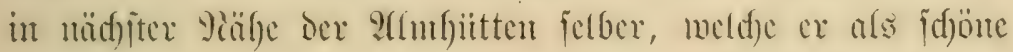

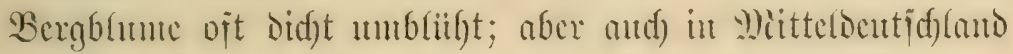

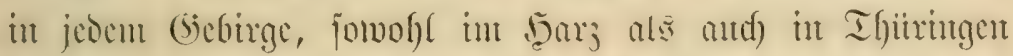
begegnet ex uns fje mo da. Freilife itberall ba ijt $\mathrm{cr}$ cine immerfin jeltene B̧hme, jumal dicjer giftigen, abor als

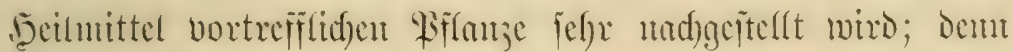

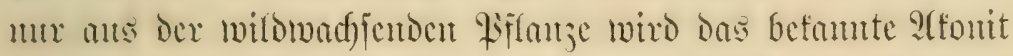
gejogen, da fie anf unjeren Sulturlande ifre ftarfe Siraft

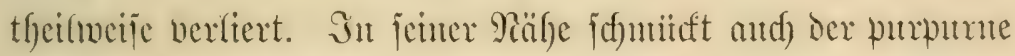

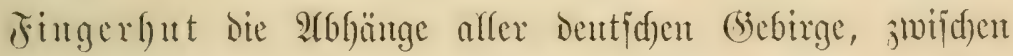

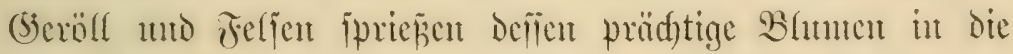

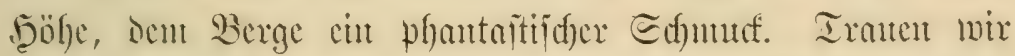

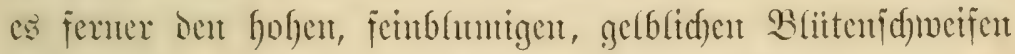

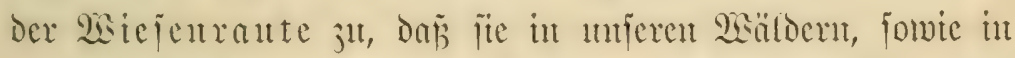

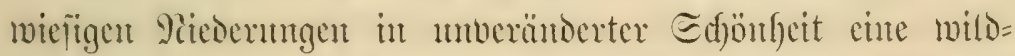

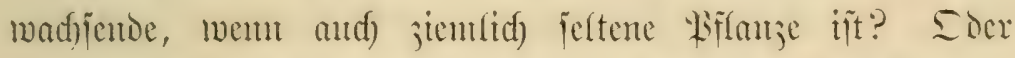
möd)ten wir bon ben nefreren unferex 2 nemonen es glatben,

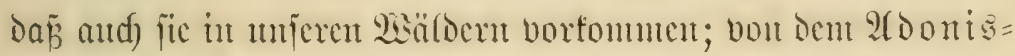

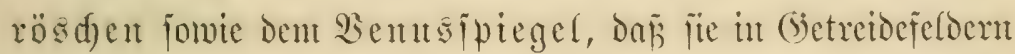

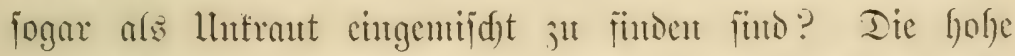
ramunfelägnlidfe, gelbe Iroltblane ferner ift in ganj Ientidf(ant), bejombers in Nordoentiff(and anf najien Wiejen

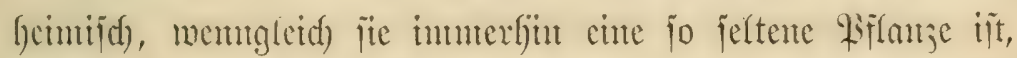
Daj dex Botanifer anjubelt, jo oft ex fic cimmal antrifit.

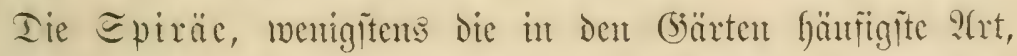

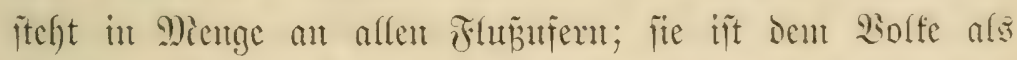

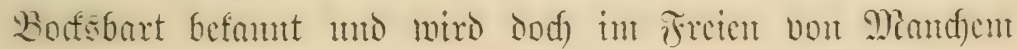


faun angejofjen, Der fie in Fentem (sarten werth) hält, obglecid)

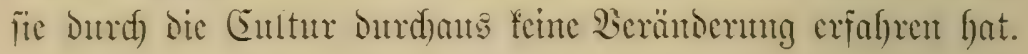
Iic Eiegumr; ober ber antermanusharnifd) (Gladiolus)

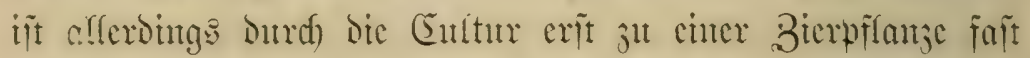
erfen Pantges gemorien, aber f̈ndet fidf mit ben hohen,

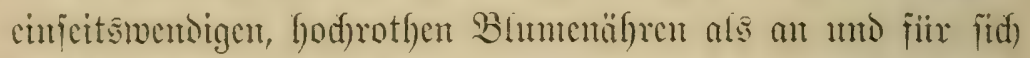

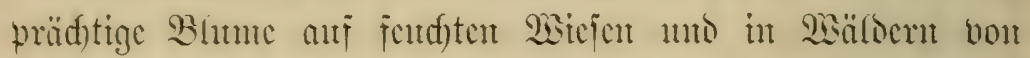

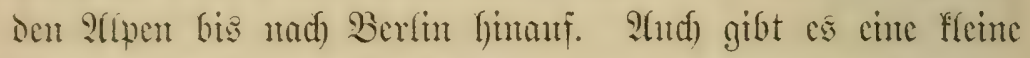

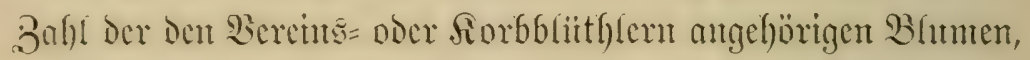
weldye ans ber freten bentidyen Siatur in unfere Särten ber=

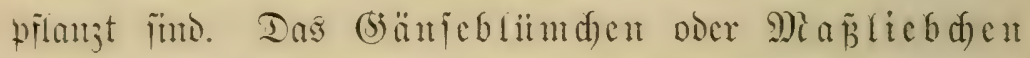

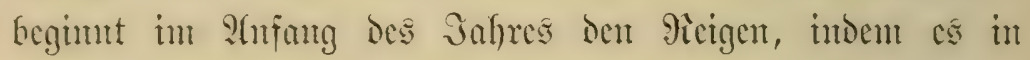

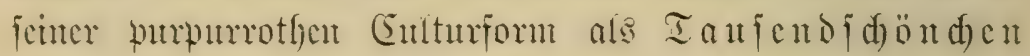

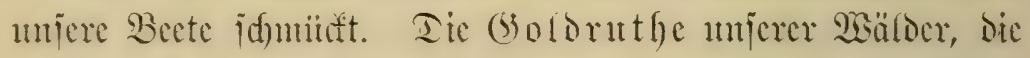

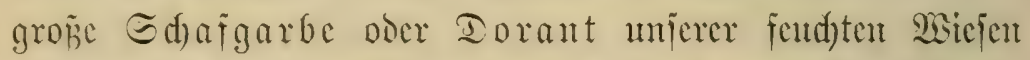

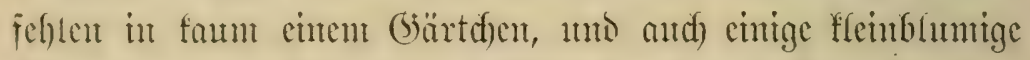

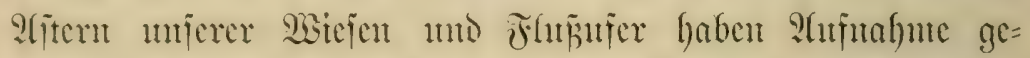

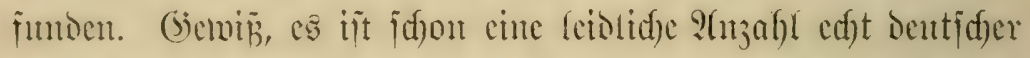

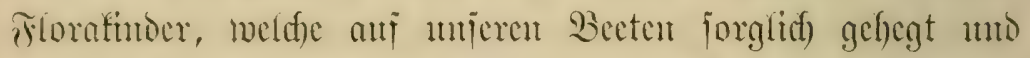
gepflegt werden uno an Eubönfit mo ?tod mis nidft ge=

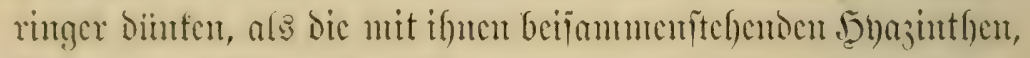

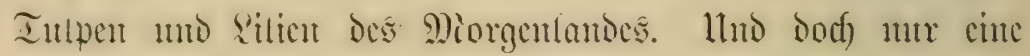

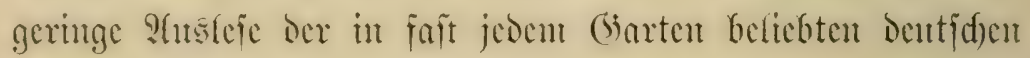
Bierblunten ijt in Dent Šorliegendent gegeben.

Erit woun der Epätionmer beginnt, treten dic dentidfen

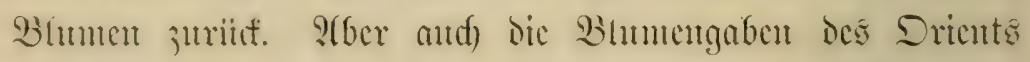
find dan fiir un ju Enoc. Co begint in ben Eommer=

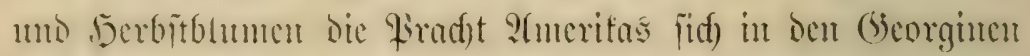

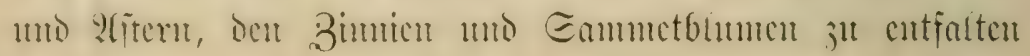




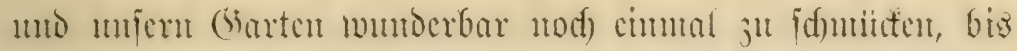

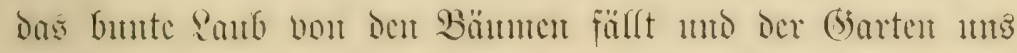

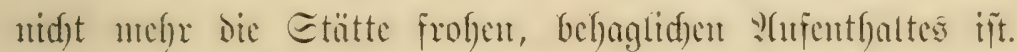
Pfber ber Garten bijut trob all biejer foüten farbegliibenden 3humenprad)t des Epätjonmers uns Dod) fdö̈ner in dent

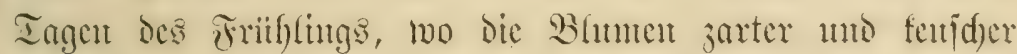

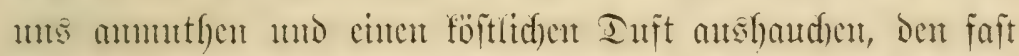
feinc der trantsoceantijdfen Eommerblumen bat. Wisir wollen

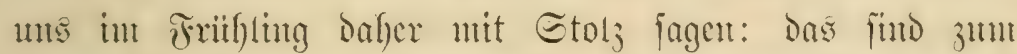

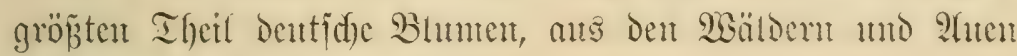
in umiere Särten verickst!

\section{6.

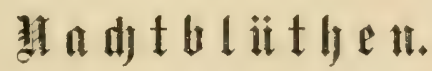

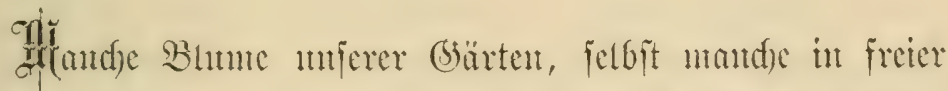

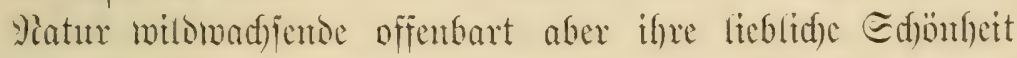
erft, weut ber Iag boritfer ijt uns ber s(bents futt. Frenen

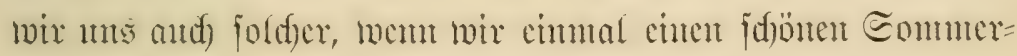
abento unter Den Bhamen in Garten verbringen!

Ermadft Dod) mit Dent ?fbend crît mandes bolfe Seben

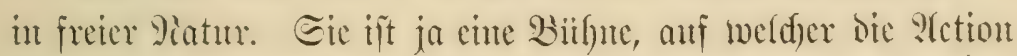

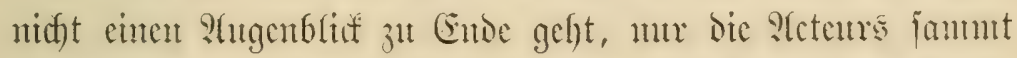

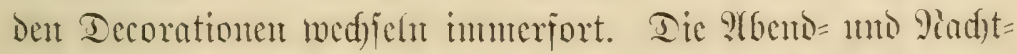
fenen aber fint bie uninterefijanteften nidyt. ?tu bie Etelle

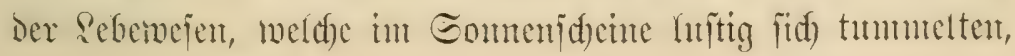


treten mit hereintbredgender Iäntnterung wicocr antore, weldhe mun und jwar jun Theil bis in die Rad)t fintein in gleidfer

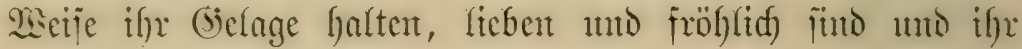

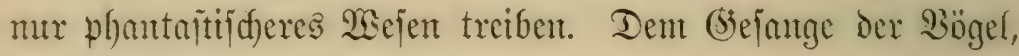
Dem Exumimen, Eummen uno Bijden der Inginjecten folgt

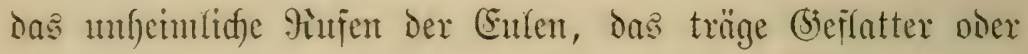
pfeilartige நujden der 2(bento = und ?ad)tfalter, und mit

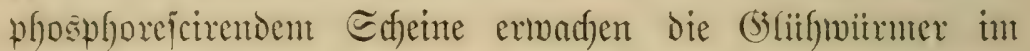
bethauten siraje.

24ud) Das B(ïlen und Duften der Piflanjentwelt ruft

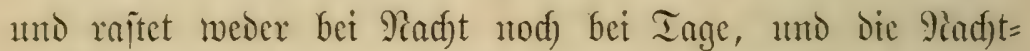
blunten blïfen und Duften nidgt weniger föftidf, ja oft nod).

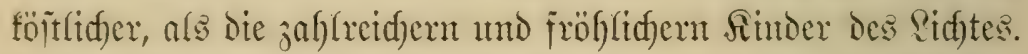
Fait alle einfeimijden deutjdyen B(umen fd) lajen böllig cin,

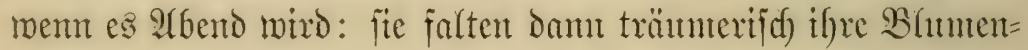

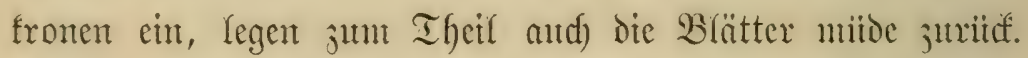
Andere wieder wadjen Iag mo Padjt in munterbrodjenter

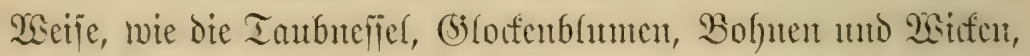

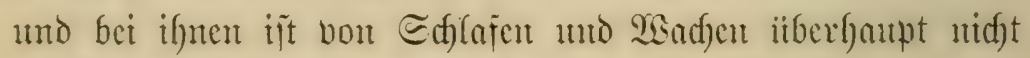
zu reden: fie find Snmertvad)e.

Siejentigen Biffanjen, weld)e wixflid) ant Iage wnfen unto

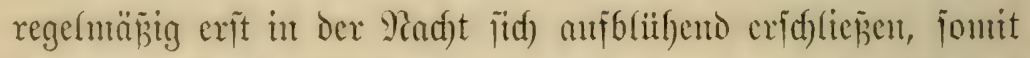

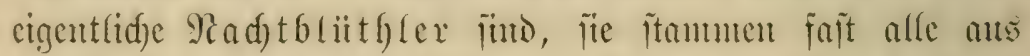
fernen fremben sändern. Bumcijt ans atmerifo find fie jtt

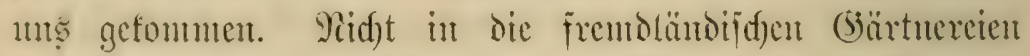

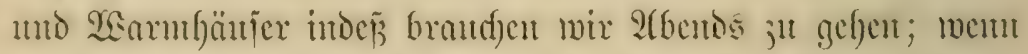
bort and) die idjönte alfer anterifanijd)en Jiad)tblitflerinten, Die hefre Rönigin ber liad)t bor 2 flem ju fudfen ijt. atmerifa bat mit jeiner Hiflanzenfflor befanntlid) nidjt mu 


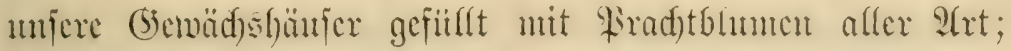
and in mferen (sö̈rten erblitfen mo Dufften mand anceri=

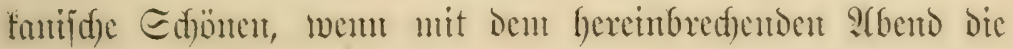

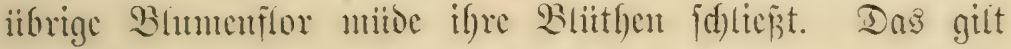

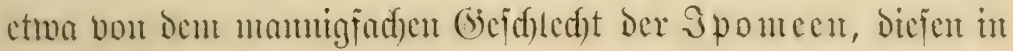

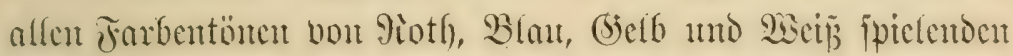

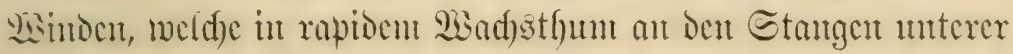
Sauben unt 23erantos entporflettem mo dicje mit grofien

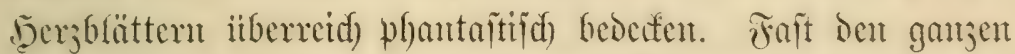
Ing ïber fint fie jedod) bliitgenlos; will eas aber Irbent werden, jo bredfen ibberall janberifd fdjöne, groje Tridferbsithen anf,

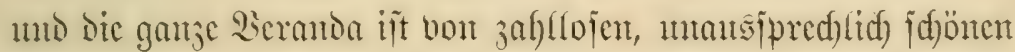
Tarbenblumen wie mit jarten Sampions befängt. Eic blühen bic Padft fintourd) immer job(reidfer, inmex volfer auf, mo went wir Miorgen in Den (Barten fonmen, jo prangen fite

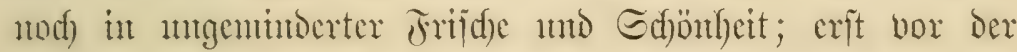
fteigenden Eonte welfent fie einte nad) dex antornt Dahin, bis um Dic Mittagsftumbe affe berblitht, cingejdyumpft uno nabeju veridivitnden fint.

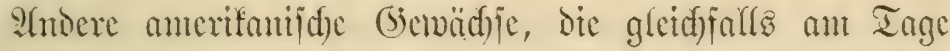

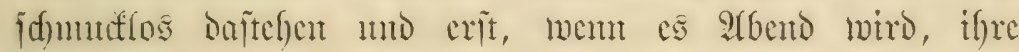

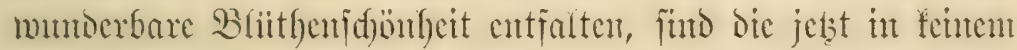
(Sarten felfenden Salappen, beren gelbe, magentarothe oder

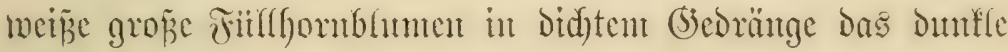

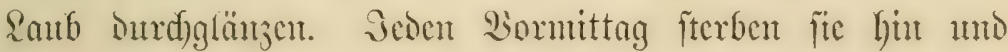
jeden atbento werden fite wod)enlang ourch inmex nete Fillfe erietst. Co aud dic idulifblättrigen Irabescantien aus

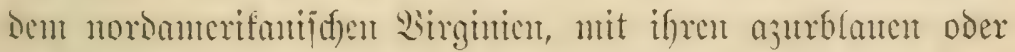
purpurvioletten zarten B(umentiönd)en. 


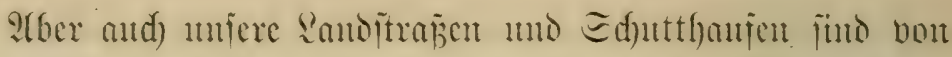
PAncrita ber mit lluträntern mand)erled 2 (rt befäet, bou benen mandfes bei uns fo luytig vegetirt, Daj cs guten Dentid)en

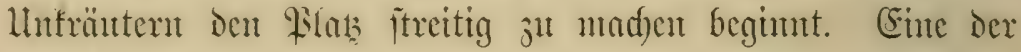
föjtlidjen Siad)tbliithlerinmen prangt hentjutage iiberall in

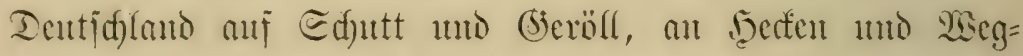

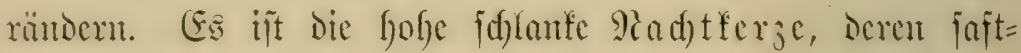

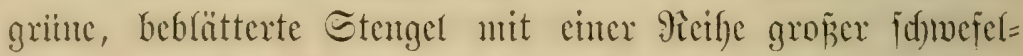
gelber jartonftiger Bumen gefrönt jimb. Wer fic ant Tage fieft, wo nux felten che Bhume Daxan blïht, fdumiäht wohl

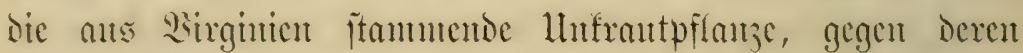

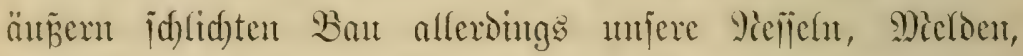
filetten no Dijteln viel anfefnlidere, malerijdere (benëd)je

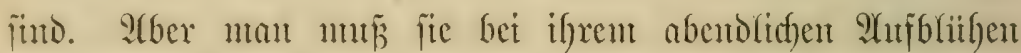
belaujd)en, unt fie jut wïrdigen. Sn Sult umo ?luguft fommt

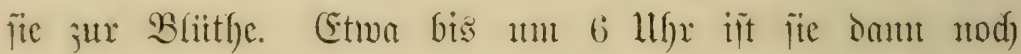

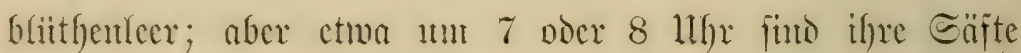

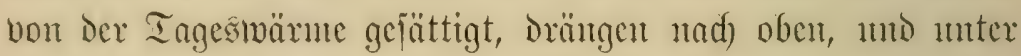

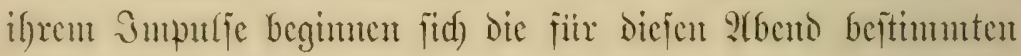

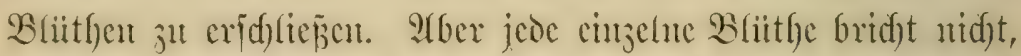

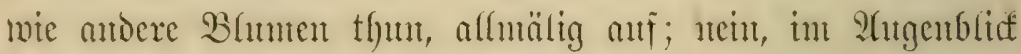
ipringt wic anf 3antberwort ber Seld) anf, who mut bebnt

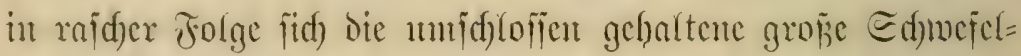
bhume meiter mo weitex, uno uad) nidft cher Minute ift fie unter unjeren 2 atgen rabartig völlig juritufgejd)lagen. In ïberjd)wängliden Drängen biegt jie if)re Bhumenblätter fajt

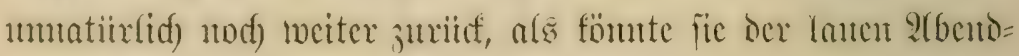
fuît nid)t genutg ifre Suncrites entgegenbreiten. Who cine

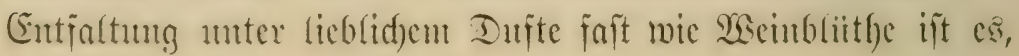




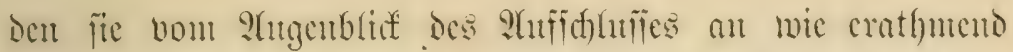

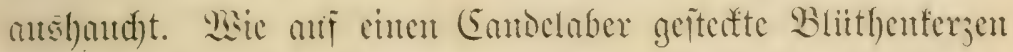

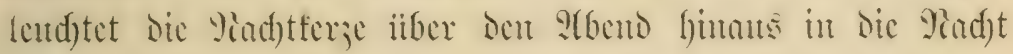
fincin mit ifgen intenju glänjenden (5ielb. So bei ciner Irt (Oenothera macrocarpa) ijt vout Forfudern, dic Hibcr Bas fogentante Seudfent ocr Wiflatjen cingehento Beobad)tungen

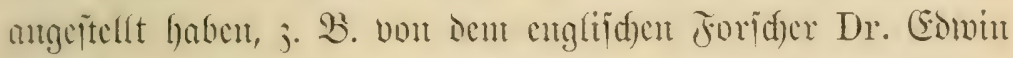
Sanfejter, felbjt ch blibartiges Wettertendften mal)rgentonmen

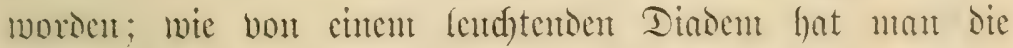

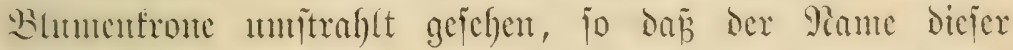

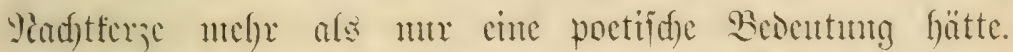

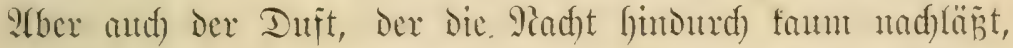

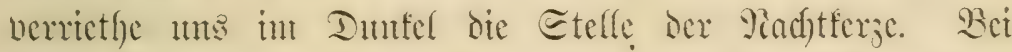
träftigen Excuthlaren fömen wir wodbentang jeden atbent uts

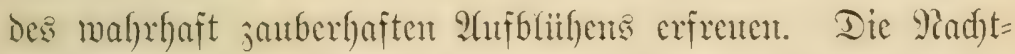

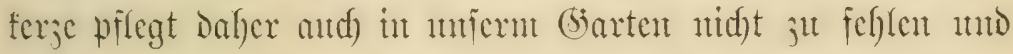
ijt in ser That bon llutrant jur Bierpflanje cxhoben. Mebrere bejondere alrten werden in mieren Bhumengärten jeit lüngerer Beit jobn gepflegt uno prangen felbit in Iöpfen, bor $2(l e m$ die jdöne Oenothera muricata. Ja, Dem poetifden

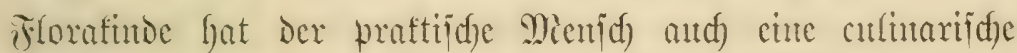

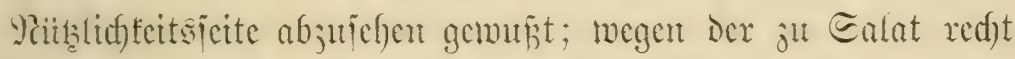
gut verwendbaren diffen Simmel hat ntan Die Tadhtferge näntlif) hie tnto da in (somiifegärten gepflegt und ift fie unter Den Pianten Siapontica in Dicfer Bejiehnng vielfach befant. wer ite in feinem Garten hat, wird aber un ifrer Blithe

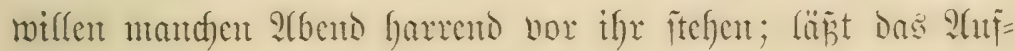
blitfen cimmal jul lange warten, jo fant mant es auth jelbit ber=

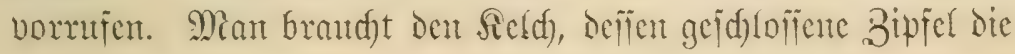


Bhmmentrone an ber Entfaltming nod) bindom, mu an ber Epibe cin wentg ju löjen, atıb alsbalo fah)ren bann anter deu Drängen

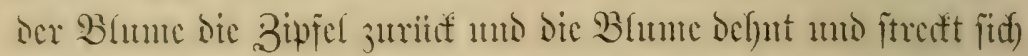

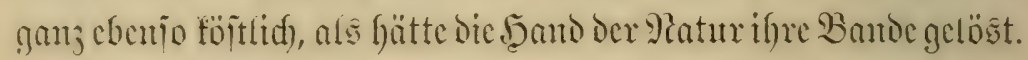

20 möd)te aber in cintem dentichen (barten, und wenn

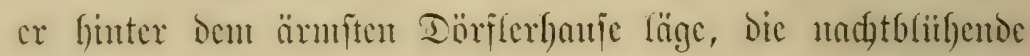
É(f)weiter fellen, seren unjere Boorfabren fdon fid) gefreut

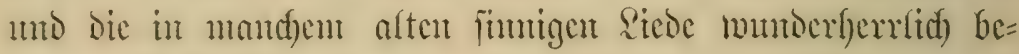

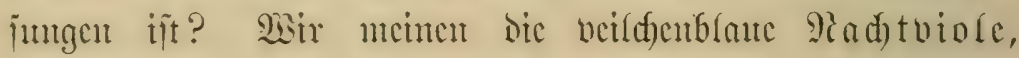

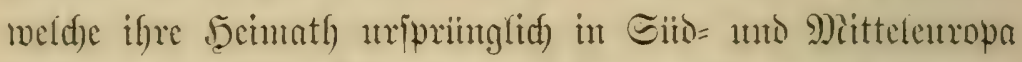

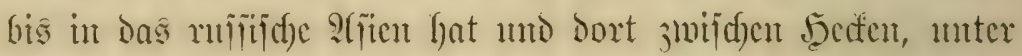

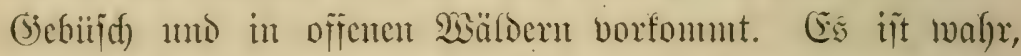
ilgre Edjönfeit wiro iiberboten won mandfer fremblämsifd)en

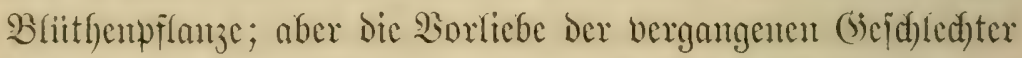

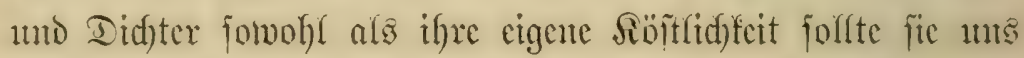
immerfort licben mb hegen faffen. Sic blitht bei Ing anto

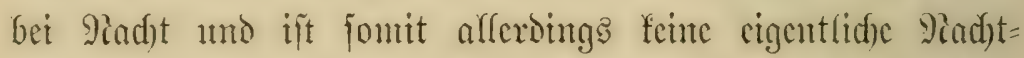
briithlerin; aber mem ser Duft ber Pifflanzen, wie die Dideter

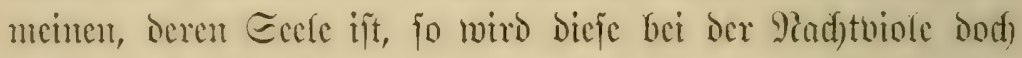

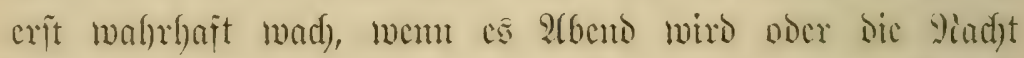
fonment. Iamm fitrönt jic, bic an Iage mur geringen (sicrud) gat, ifren Inff, ifre Eccle in dic lanc Dumfelleit ans,

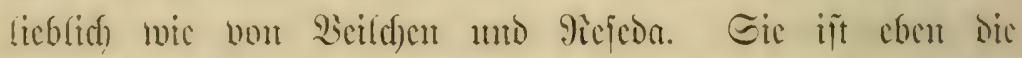

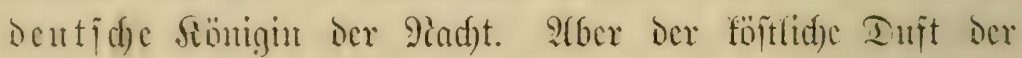

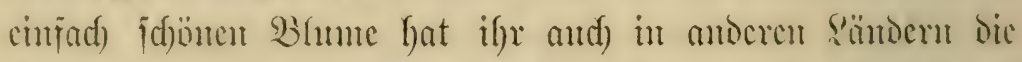

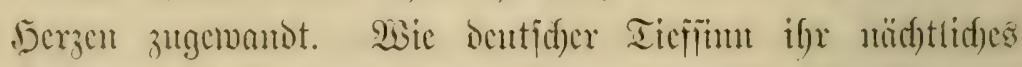

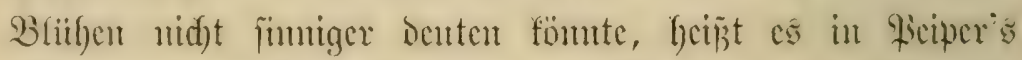
"Etimmen des Drient" unter den "2Buten ans der Eprende

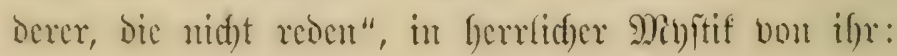


Dả̉ idf am Tag meinen 2(ether verbehle, Ind die गadat jur (Entid)leierung mähle, Das gejuiteht, weil die Madjt es ift, Wo bie Qiebe ifre gebeimen 3ujanmentünjte feiert

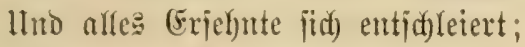
Jern ift Der beobarftende Feind, פgägrent Der Freutu uns ericheint, Ilto twent ex ipridjt: bedarf einer meine Spenden?

Da habe id bic (jemofnheit, meine Sellfzer औts Boten зи ifm з̆ jenden.

Itnd meine Demuth mũ fin bei feimer (Frhabentheit für mid) verwenten.

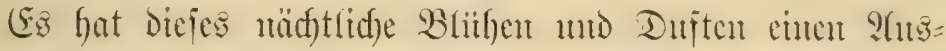

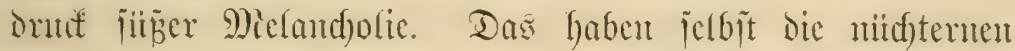
Botanffer empfumben, welche entigen joldfer un ?(bentos duften= Den Bhtnten Den Beinamen tristis (Hesperis tristis, Gladiolus tristis, Pelargonium triste) gegeben unt fie jomit als bie Iranrigen noer Edfwermitthigen bejeidnet haben. Jntefïen

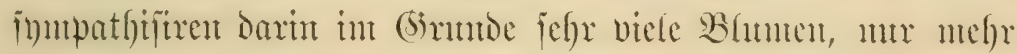

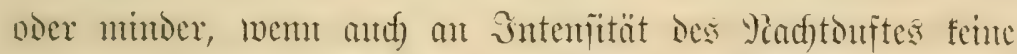

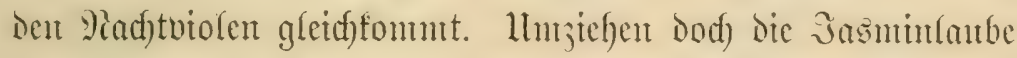

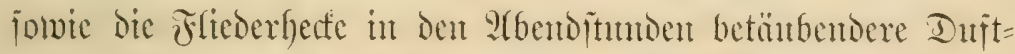

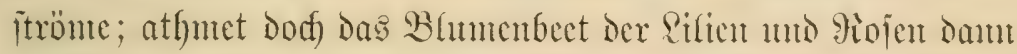

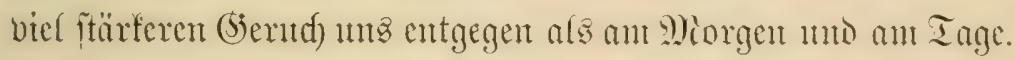
llnt) went in lauter, fpäter Dänmeritnnde bon allen Beeten,

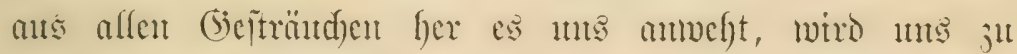

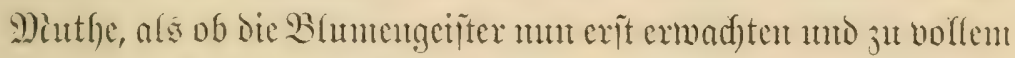
Seben gelangten, die jüb̈nen E(fen, weld)e nad) alter Sage im

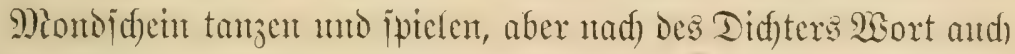

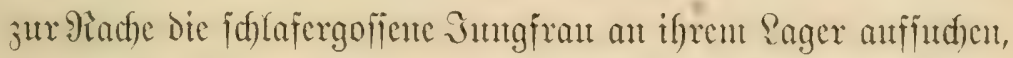

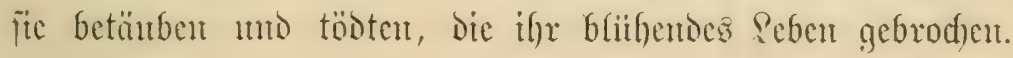




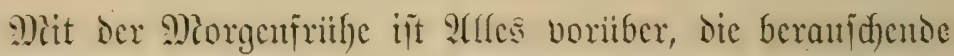
Mirflidfeit unto unfere cigene gehobene poetifde Etmunntg. Die Eome geht jo beritändig anf, fiifle Ihoutropien hangen

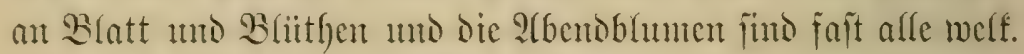
Gs mar eben cin Eommennd)tstranm, Den wir in frijder Morgenluft faum nod) begreifen. 


\section{VII.

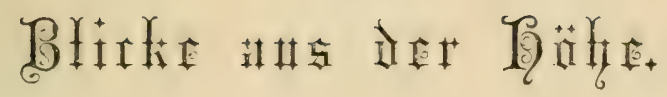

Saft ou ben Blict vont Einzelnen über oas Banze erhobert,

Don oer befक̆ränfenden 2äh' weit in die ferne hinaus:

Siebend fehrit ou juräd und weift nun verftändig зu loben,

Was in ocr Ecimath dir blüht, nahe ocm eigenen Faus!

p. 自. 
$\mathfrak{U}_{\mathrm{i}}$

5 scientia amabilis, die "liebenswürbige $\mathfrak{W}$ iffenfifaft", wird die $\mathfrak{B O}_{0}$

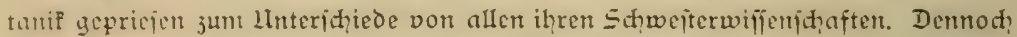
idgeint fie auch einige redht unliebenswüroige 2lufgaben zu haben, infofern ihre Jünger die Blumen anatontif unterjuchen, die Jahl der Staubgefäbe und anderer Blüthentheile pedantifh einregiffriten, unter bent Mifroffop das Jell= gewebe beurtheilen, defien Inhalt demifh prüfen. Sogar die methode der Etatiftif wird anf die holden florenfinder angewendet, und viele oer begeifterteften Botanifer haben feine widhtigere Bemïhung auf ihren wanderungen durch die blühende Zatur, als tabellarifhes Journal über die aufgefundenen Blumen $3^{\text {u }}$

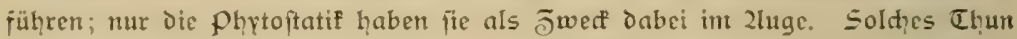
nöhte freilich unliebenswürdig genug crihcinen! Ja, wenn wir es im einzulnen falle beobaḑten; aber all bieje ThätigPeit erit liefert ein immer flareres geiftiges Bild der Blumenwelt und des Blumenlebens, welfhen fhlieglich wohl Ziemand jeine freudige Theilnahnte verfagen wird. - So begreifen wir auh die von einem Bilohauer auf ben Steinblocf geführten Sđläge anfangs niđht, nur er jelbft freut fid dabei in Eeift fhon des werdenden Bebildes; allmälig erit lemen auch wir fein Wirfen verftehen und ehren, wem wir dic Joeen des Meifters ju ahnen beginten, oder gar in Dollendung fein werf vor uns fteht. Die Zatur ift allerdings an fid fhon ein Dollfontmenes, aber es hat die Wiffertidiaft nad?: zumeifen ihre idealen J̈üge und das fhöne Befüge des Banzen. Dic deutidye Blumenwelt eriheint injonderheit durdq die Bentühung der Statiftif erft in ihrem vollen, herrlidyer Werthe.

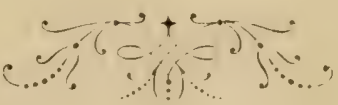




\section{9alumen und Bnjlet.}

as follen Blumen wohl mit Bahlen ju thun haben;

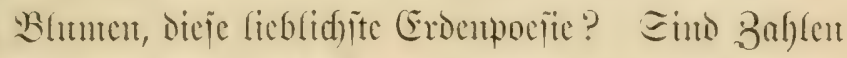

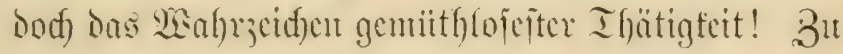

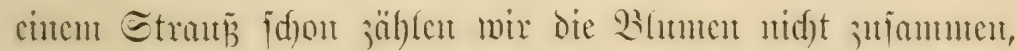

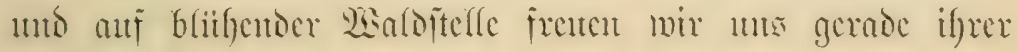
unzählbaren Mienge.

Dian verurthetle bic armen Bahlen aber ntdyt untgefjört!

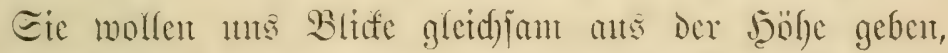

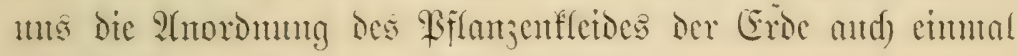
int Bujanmentange iiberjonaten lafien.

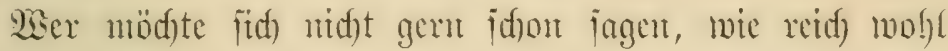

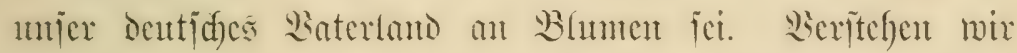

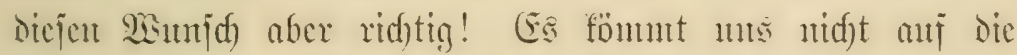

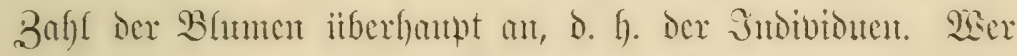

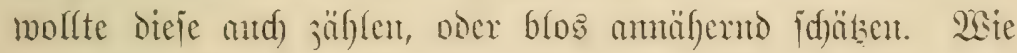

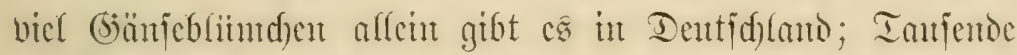

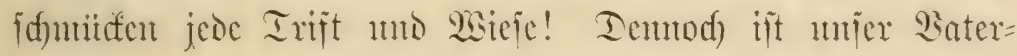
land thatiädlid) jehr arm an (siänjeblimudjen, dem cis cut=

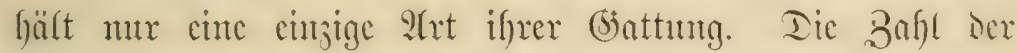

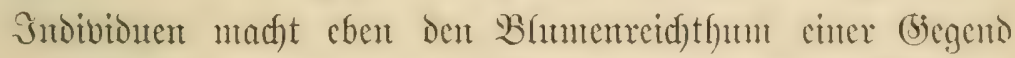

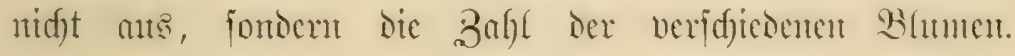




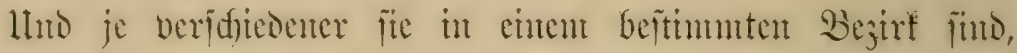

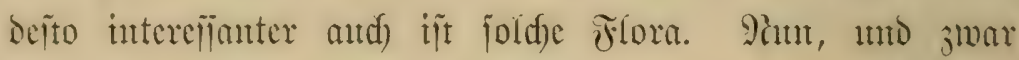

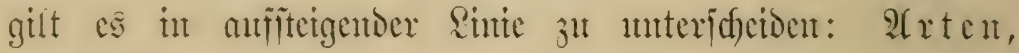
(b)attungen uto Fantlien. - Bead)ten wir cumal bie

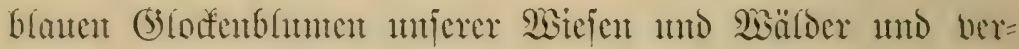

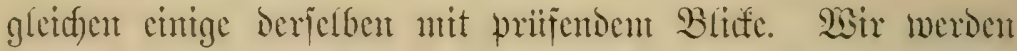

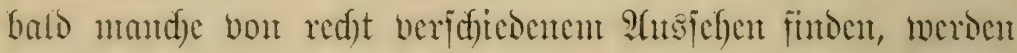

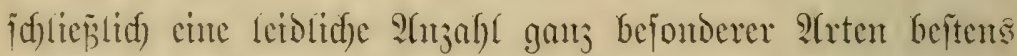

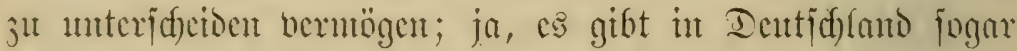

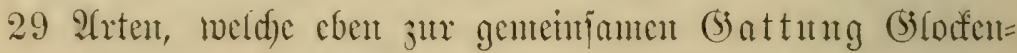
blunte gefören. Wollten wir mu aber in cinter Ffora blos

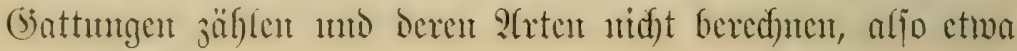

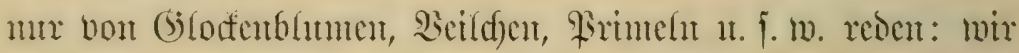

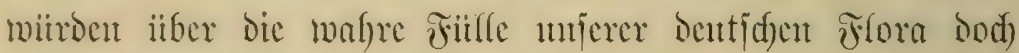
แns fein lletfeil biloen fömen, sem jede der 636 Gattungen

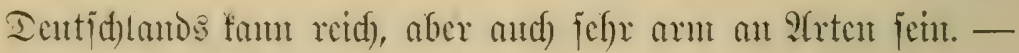

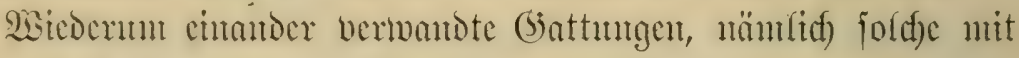

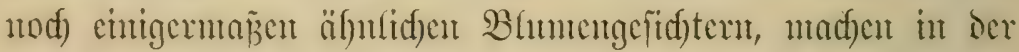

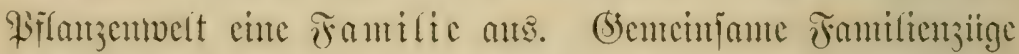

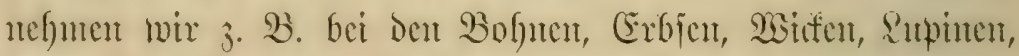

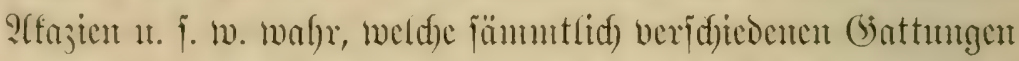

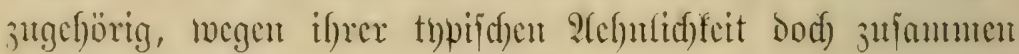

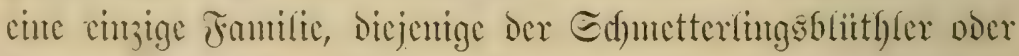

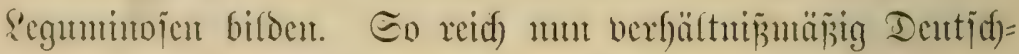

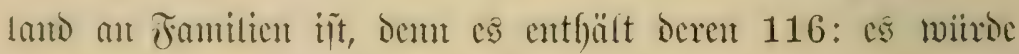

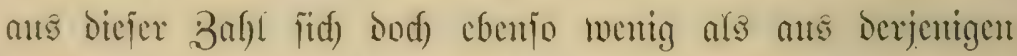

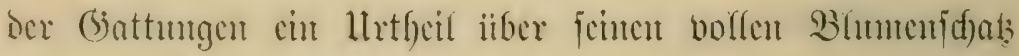
gemimen lafjest. Es gibt ja Fantilien mit mur cincr Gat=

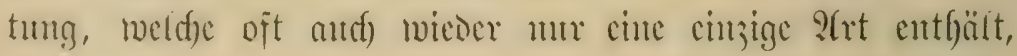


3. 2. Die Fantlie Der Tammenmedel, oder Der Gagel; wicderum baben andore Famtlien jahlretde (5attungen, jede biejer Gat=

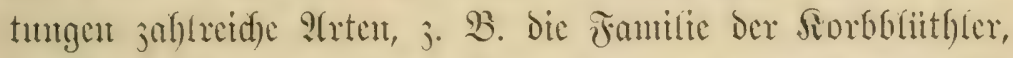

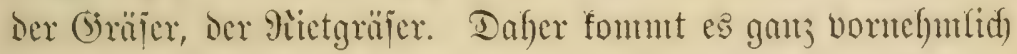
anf bie Bafi ber âten an, went wir nad dem Bejtand ciner Flora fragen.

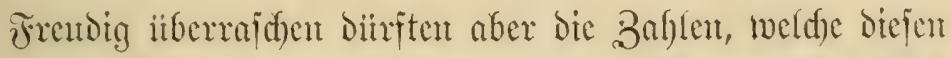

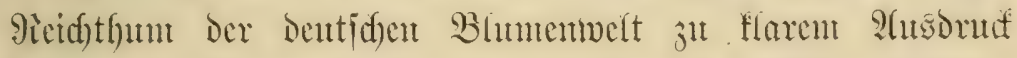

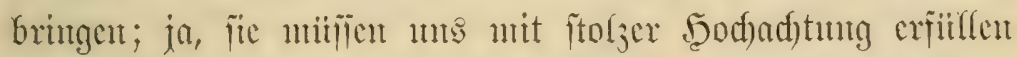

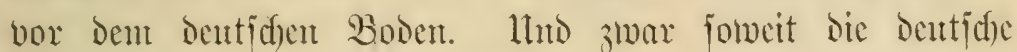

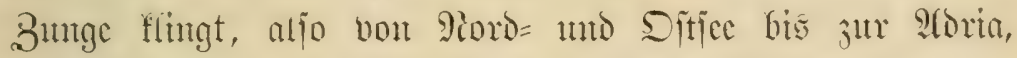

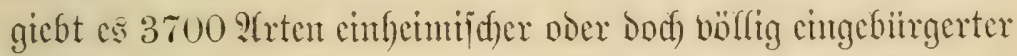

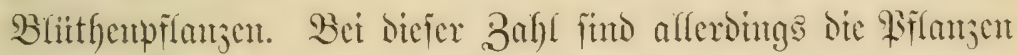

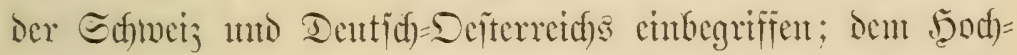

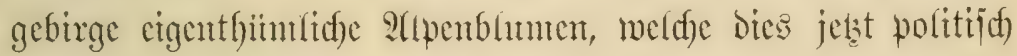

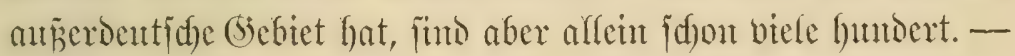

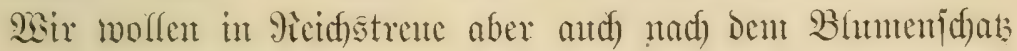

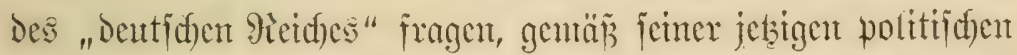

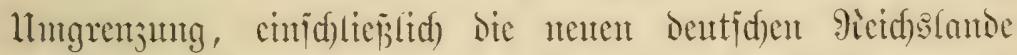

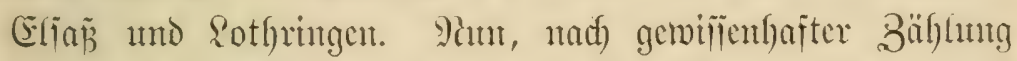

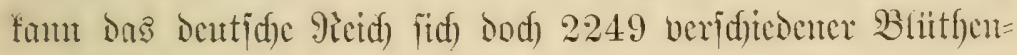
pflangen rithmen. Mise(d) gutes Theil immerfin nod) gegen= ïber den im Sanjen 10,000 Blïtbenpflanjen des gejammten Gitropa!

Bablen reden auperdent Deutlich bon dent Borrang Deutid)= lands vor mandien Padibarländern. Einglanto hat mu 1300,

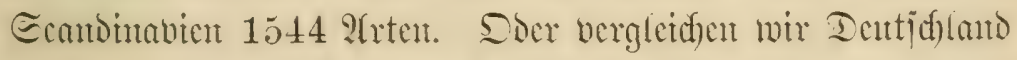
nit viel grö̈eren Pändermafjen. Mian pflegt dic ganje curo= 


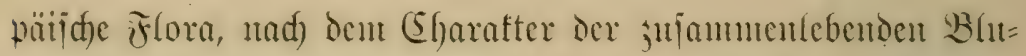

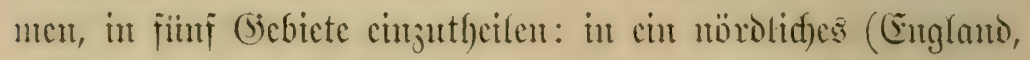

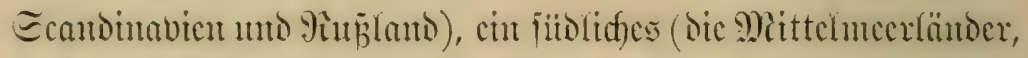

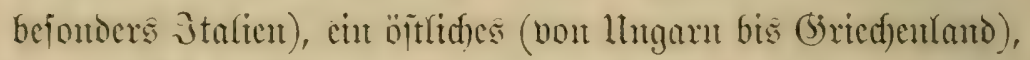
cin mejtidses (Epanien and Frantreid)) und cin central=

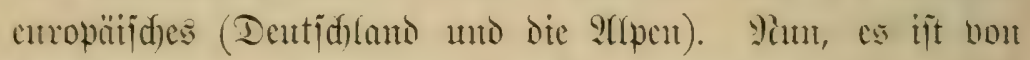

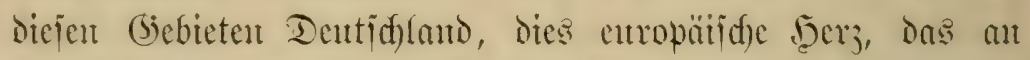

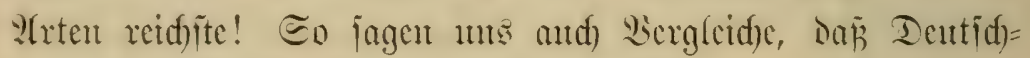
land wirtlid cin blunturcides Sand jet.

Sieje blithenton Sdjäbe finton jidd freilid) in feinex

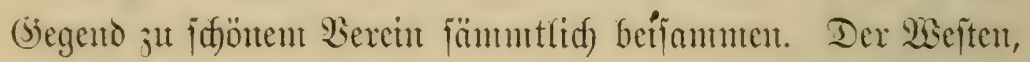
bejonters die gibeinprovinj, hat einten andern floriftifden Charafter als Der Siten; wieder die norobentidye fandige Ticiebene cinten antern, als bie ntittel= mono jitodentiden (Sebirge;

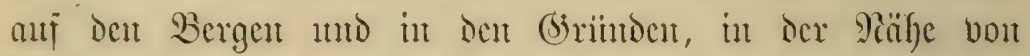

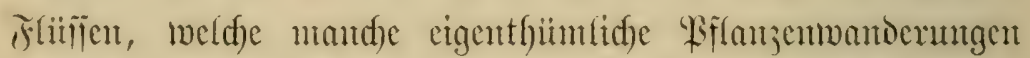
veranlajien, anj Salfgebirgen, auj Emoftcin, auf Ealzboben, - iiberall da treten bercingelt melur oder minder eigenartige

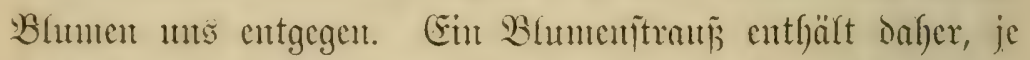
nad) bent dentid)en Eantoc, in weldfem ex gepflitift wurde, chinge bejondere $\mathfrak{B}$ hmen, die man anderwärts gar nicht fent. Jn den nordoentiffen Eanogegenden briifen alferorten, als die gencunten Bhumen der Flur, dic gelbe $\$$ mmortelle (IIclichrysum arenarium), die (5rasuelfe (Almeria vulgaris), dic 260 ffulld) (Euphorbia Cyparissias); in den

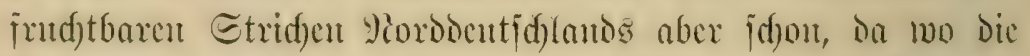

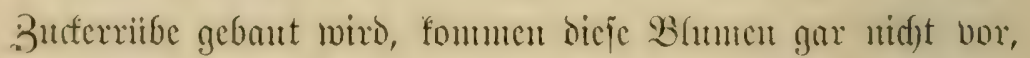

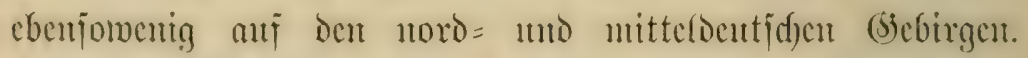
Erit in ben jandigen (Ebenen Eiidocntid)Lants jeigen fie jidd) 


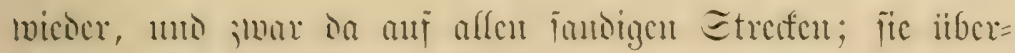

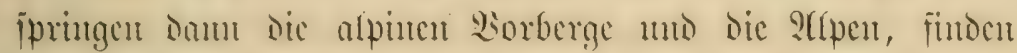

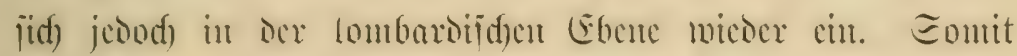

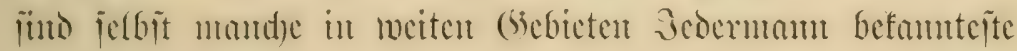

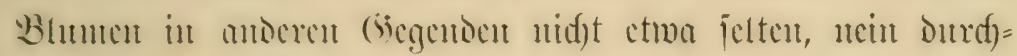

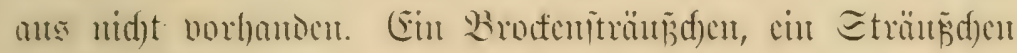

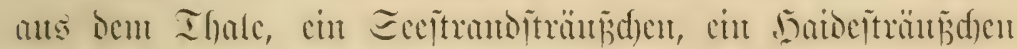

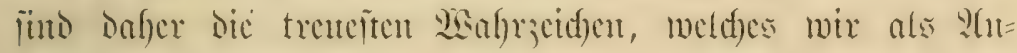
benfen von einer Feife mit in die Deimath nelymen. -

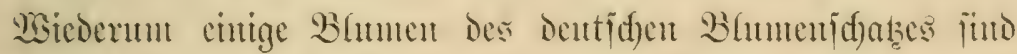

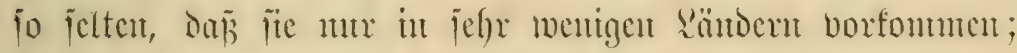
ja, mand)e baben mu cincu cmjigen batiogen Etandort.

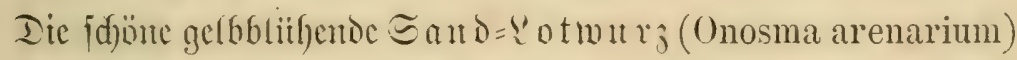

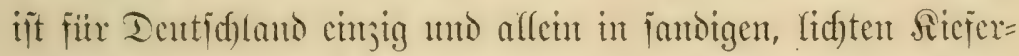

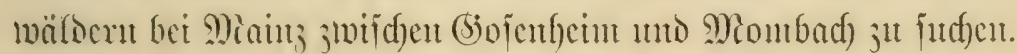

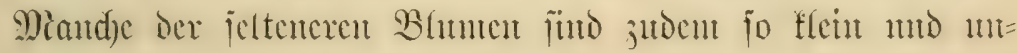

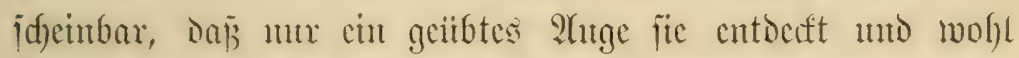

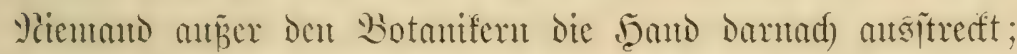

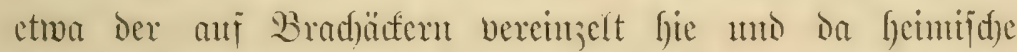
Sileiuliug (C'entunculus minimus) hat foum ïber énen Boll lange, flembeblätterte Etengeldyen, verjiert mit winjigen

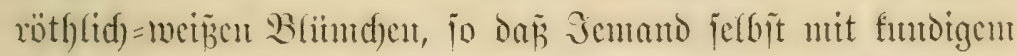

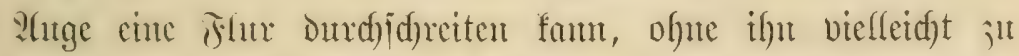

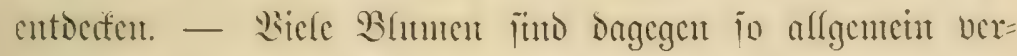

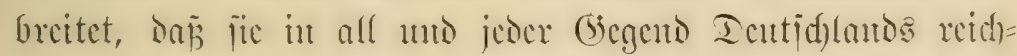

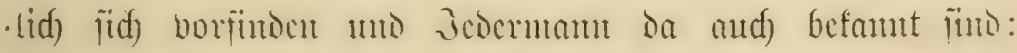

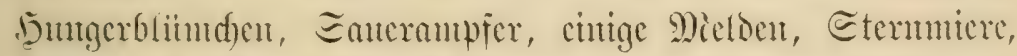
Pugentroit, Wiogelfutöterid), Wegcbreit uno anoere, bejoutors

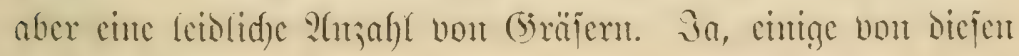


fommen als seltbitrger jogar in alfen Erotheilen in gleider

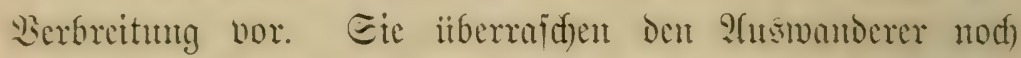
jenjeit des Dcents im ferniten Wejten, in Eolorabo, Brajilten,

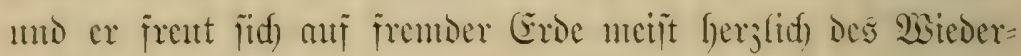
jehens biejer Bhlunen, bie or fdyon in ber finbleit anf ben Geimathlichen Fुturen jah.

In weldem Piaje bie cinjelnen bentid)en Sanditridse an

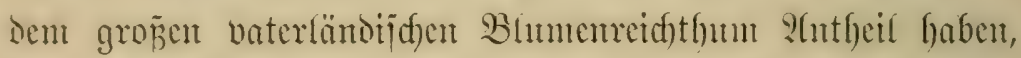

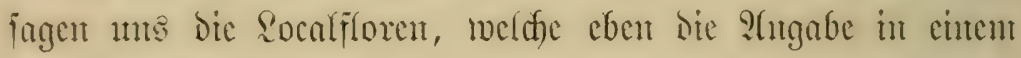

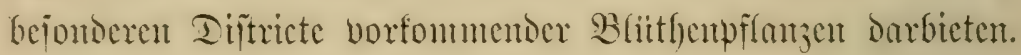
Ier ganje Sar; hat (uad) Dampe) Deren 1275 ?(rten. E5

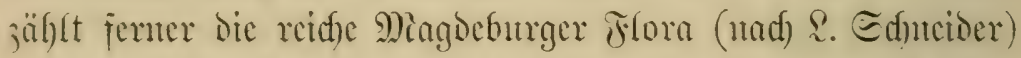
in cinten Bejirf von ctma 100 Dutadratmeilen genta 1235

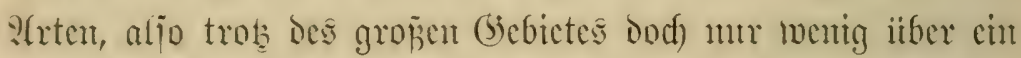

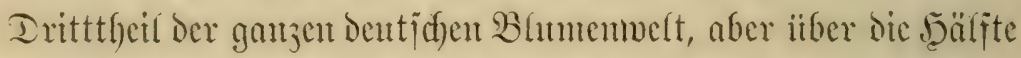
Derjenigen des dentidyen gecides. Die cin cbenjo umpangreidyes

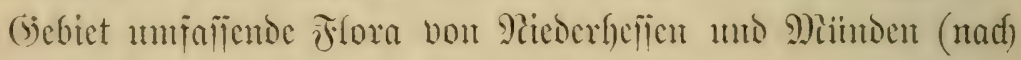

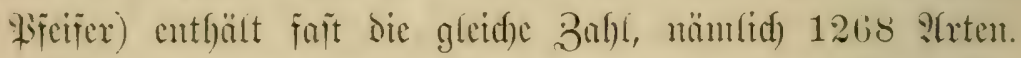

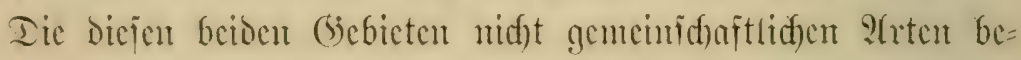

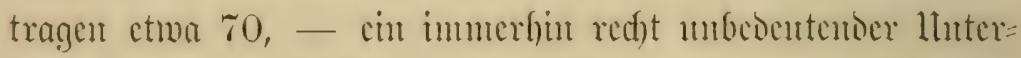

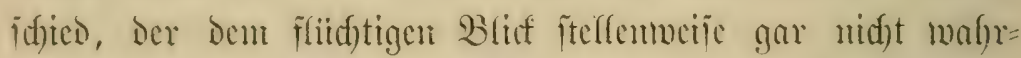
nebmbar wiro. 2(ber bergleidfen wir cimmal juet entfernteft

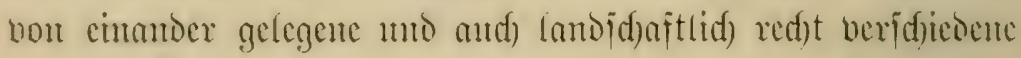

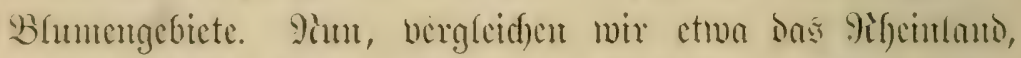

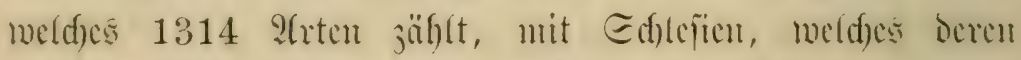
1409 entbält. 23(mmenarten, meld)e beiben (Bebieten genren=

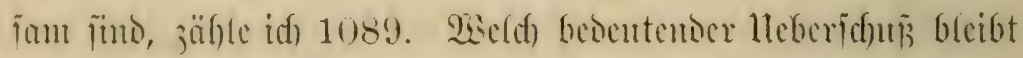
da bou joldsen, melde etwa im sibeintanoe borfommen, ba=

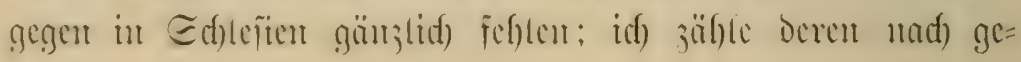




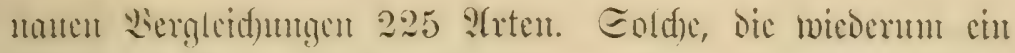

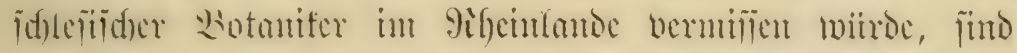

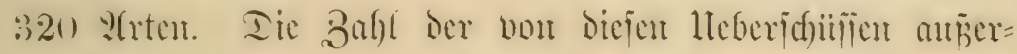

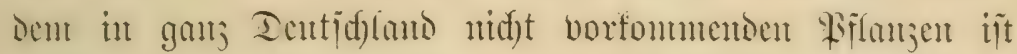

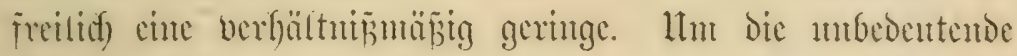

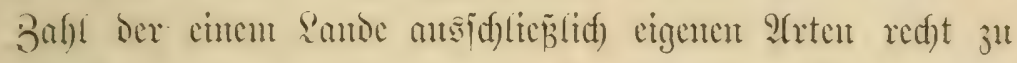

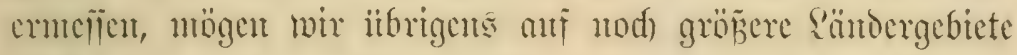

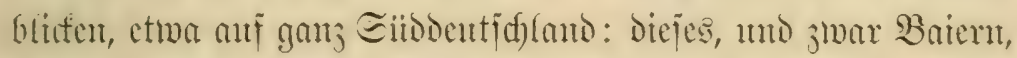

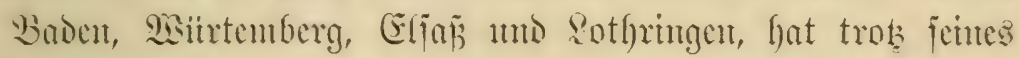
wejentlid) anderen Silima ond) mus 82 S(rten, weldye im

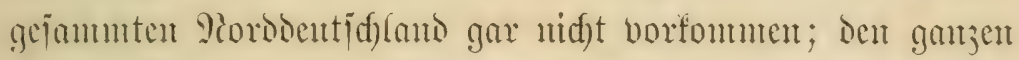

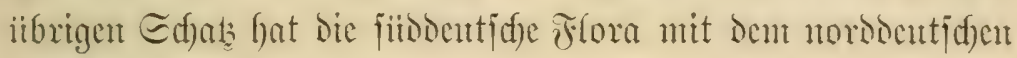
Reidislande gememfant.

Das î̃t der Befumb grof̈er Gebiete!

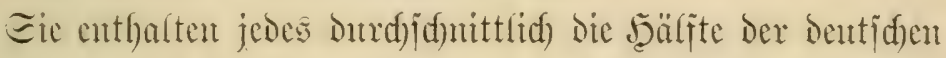

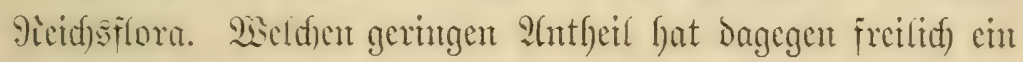

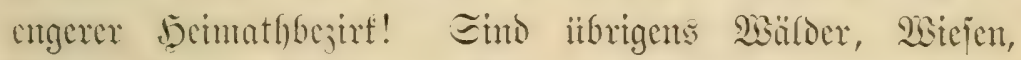

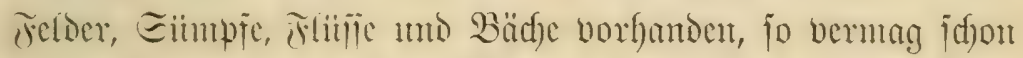

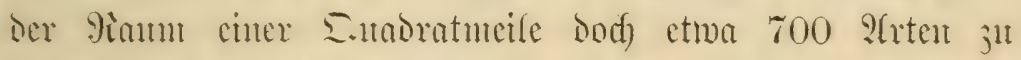
cutbalten. Sirt aber gleidartiges Terrain, etwa unt Felo=

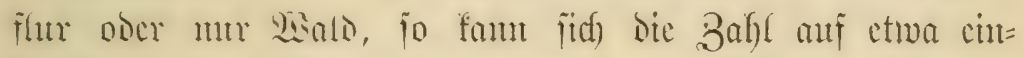

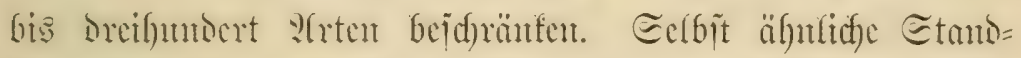

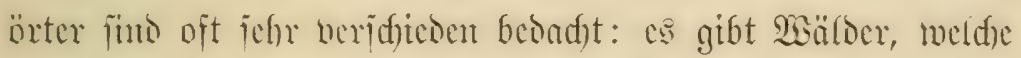
bei nucilengrojem llmpange dod) faum 30 arten jählent,

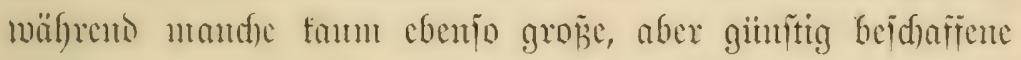

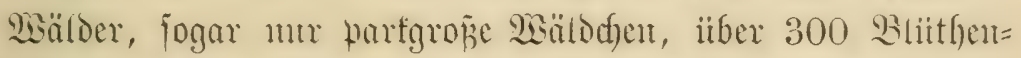

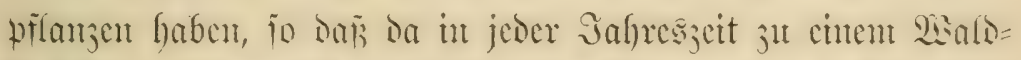

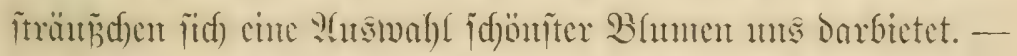

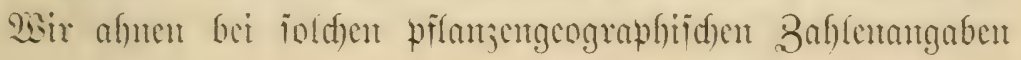




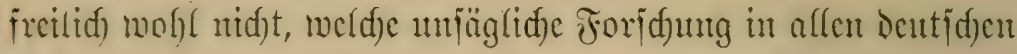
(Gebietent nötfig war, un dicfe lidgten lleberblicfe des Dentid)en

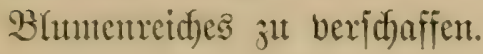

Bablen unt fömten ums ferner jagen, weldfe Blumen

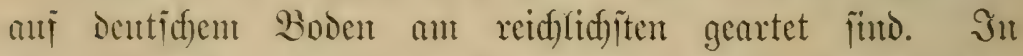

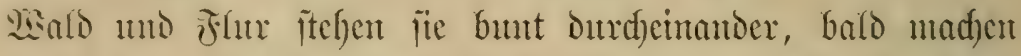

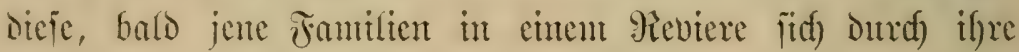

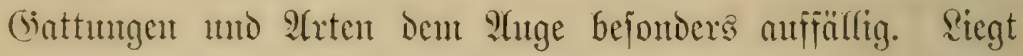

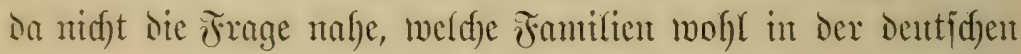
Flora iiberfantpt vortwiegen? Coll es j. B. mel)r Atrten von (5rä̈jert geben, oder bon fiorbblittflem, oder von Doldenblïtflem, oder von Rippentiithlem, oder von Mielfen?

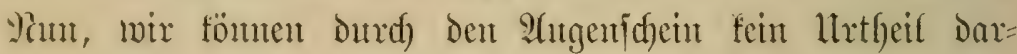

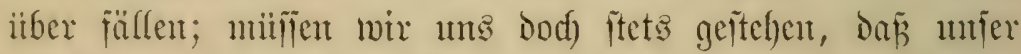
Bliff eint gegen das banje nur ent begrenjtes bebict

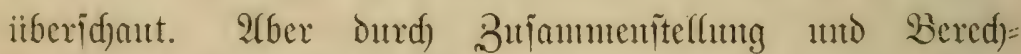

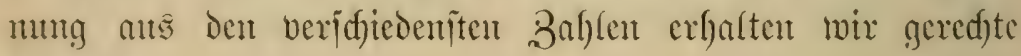
2ntwort.

Sìn, id) jälfe fïr. das gantje, niddt blos das poli=

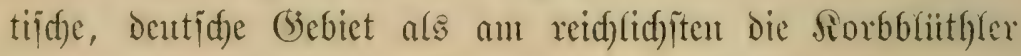
vertreten nit 180, die Sirentjb(iitf)(er nit 117, dic 9itete mit 114, die E(f)metter(ingsb(iit)(er mit 110, die Dolden= bliithler mit 88, die Sippentbeiithler mit 78, die 9ia

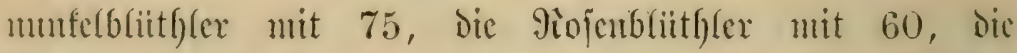
Erdjiocen mit 48 2frten. Betrad)ten wir unt cumeal bas

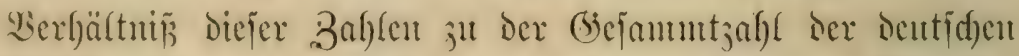

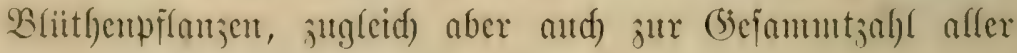
B(iithenpflanjen auf Eronen. 
Es verbalten fid

$$
\begin{aligned}
& \text { zur. Sejauntzabl zur Gejammtzabl } \\
& \text { ber beutiden. alfer Bliutbentplanjen } \\
& \text { Brïtbenffan;en: auf Erben: }
\end{aligned}
$$

Dic Rorbbiuthler

$$
=1: 15,4=1: 8,2
$$

$$
\begin{aligned}
& \text { " Siräjer } \\
& =1: 20,5=1: 12,3 \\
& \text { " Ireuzbliutbler } \\
& =1: 32,5=1: 18,2 \\
& \text { " Rivete } \\
& =1: 32,3=1: 18,6 \\
& \text { "Sdymetterfingabliutblex }=1: 33,5=1: 18,4 \\
& \text { "Doldenbliuthler } \\
& =1: 42,0=1: 22,8 \\
& \text { " Rippenbliutblex } \\
& =-1: 47,4=1: 24,7 \\
& \text { " Riamunfelbliatller } \\
& =1: 49,3 \\
& =1: 30,6 \\
& \text { " Rojenbliutbler } \\
& =1: 61,9=1: 19,0 \\
& \text { " Srditiben } \\
& =1: 77,0 \\
& =1: 47,4 \text {. }
\end{aligned}
$$

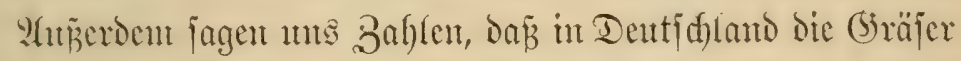
แmo butntgen Siräuter gegen Jolggewädje meit mefre vor= herriffen, als in anderen, bejonters fïblidfen Rändern. (5ianz

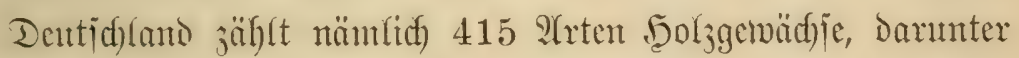

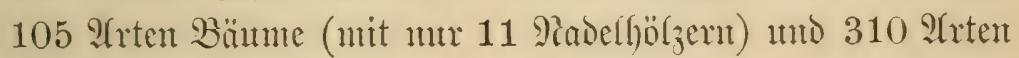

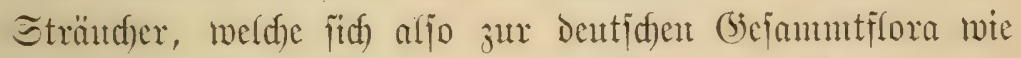

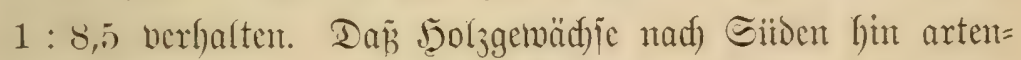
reidfer werden, Deutet iibrigents Deutjoland felbyt an, in defien jitilidyen Theilen etwa 14 bolzarten mebr auftreten, als in Den nörolichen.

Eo geben Bablen gar manche lebenswolle Blite anf

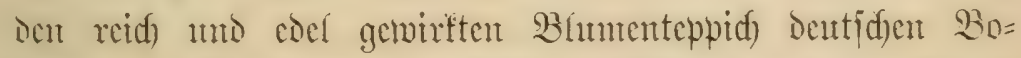

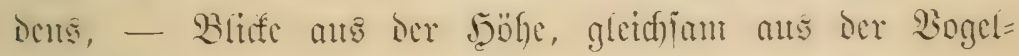
peripective. 
Ans folder böbe verlangt es ats, mitoer herabjutteigen

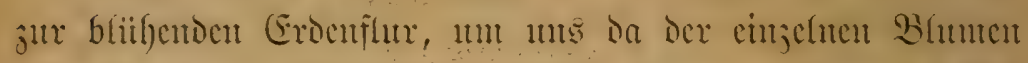

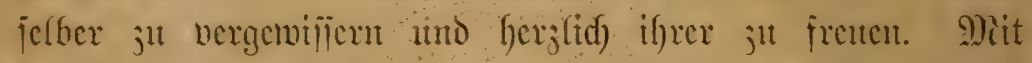

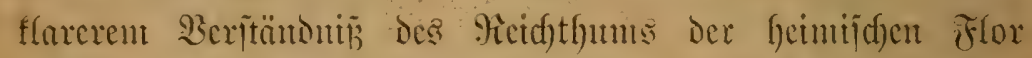

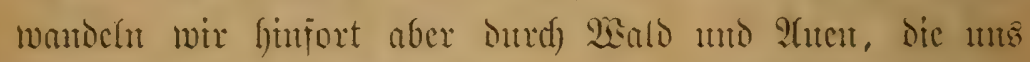
immer bon netent fagen wolfen, - was mir faben an unferer Dentiden Bamenturlt.

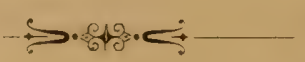




Kummer, Paul/Deutsche Blumenwelt in Char

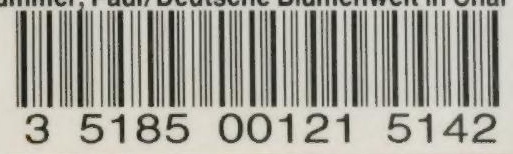




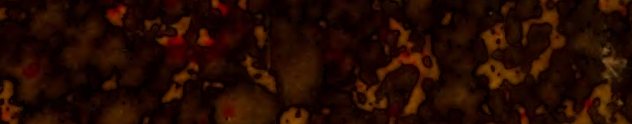

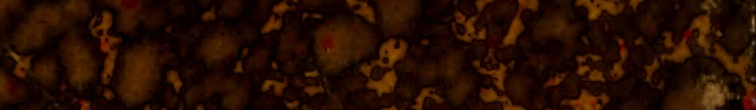

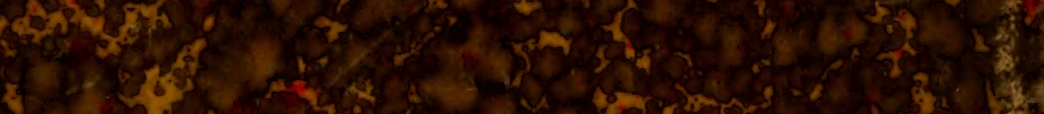

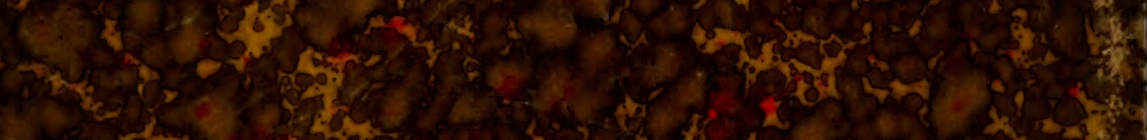

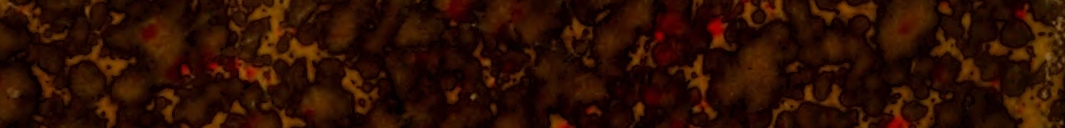

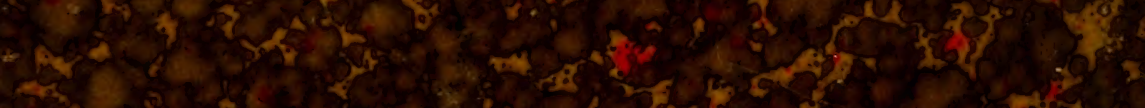
5. 2. 30.73

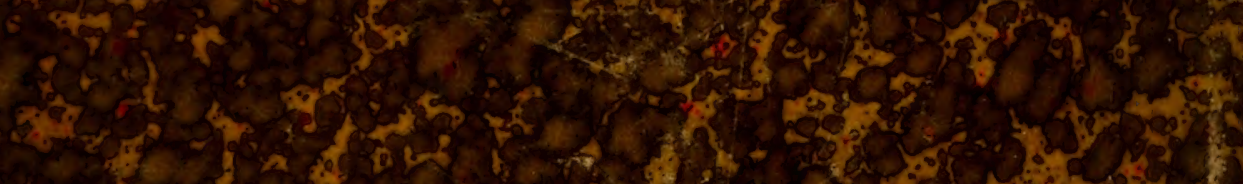

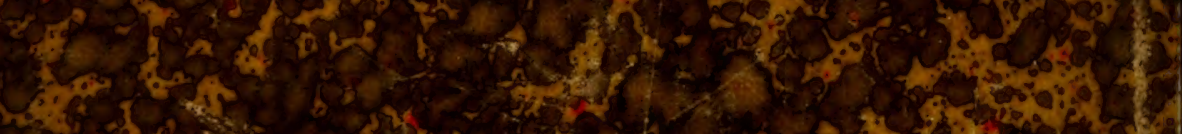

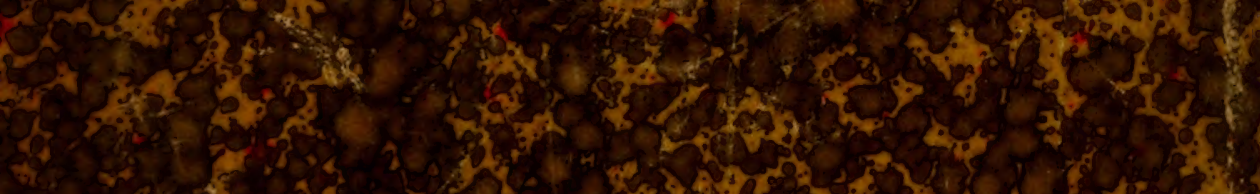

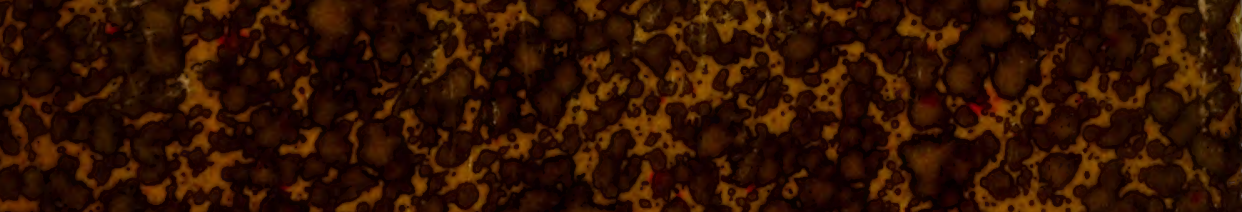

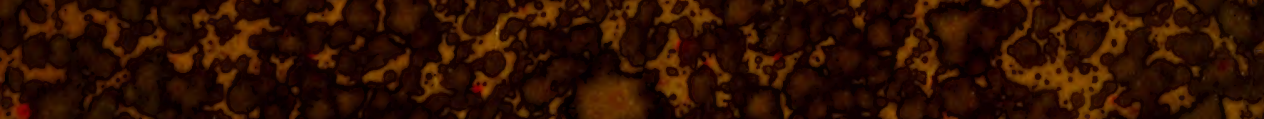
(1) S. 1.0.

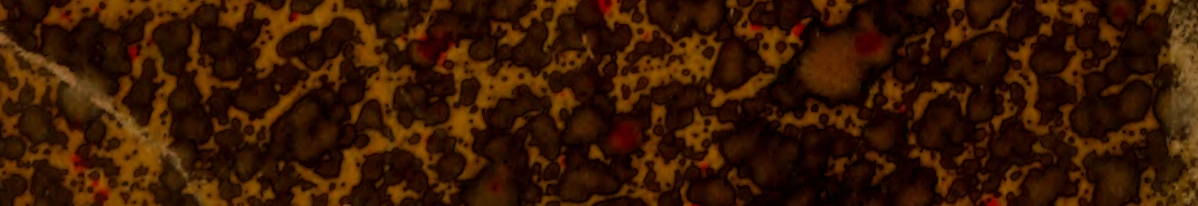

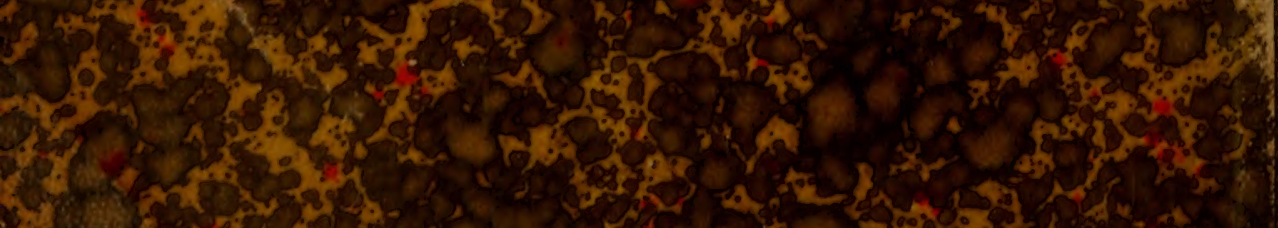

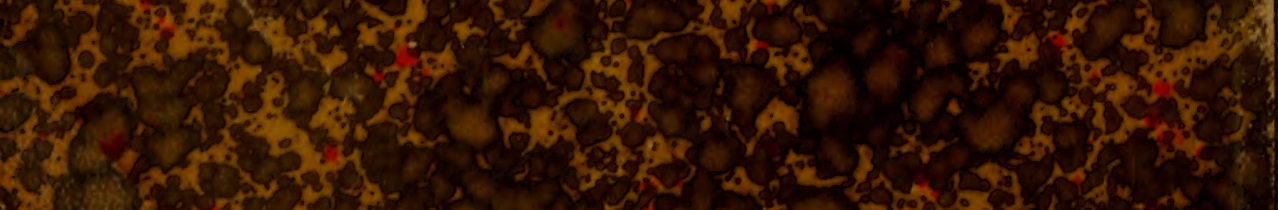

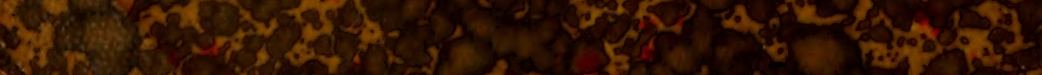

